PNL-7613

UC-903

\title{
Limited Site Investigation of Landfills 1 and 4, Fort Lewis, Washington
}
G. V. Last
K. R. Olsen
P. A. Eddy
J. R. Raymond
S. P. Airhart
D. R. Dahl

August 1990

Prepared for the U.S. Army, I Corps

and Fort Lewis, Washington

under a Related Services Agreement

with the U.S. Department of Energy

Contract DE-AC06-76RLO 1830

Pacific Northwest Laboratory

Operated for the U.S. Department of Energy

by Battelle Memorial Institute

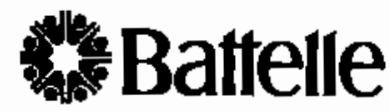




\title{
DISCLAIMER
}

This report was prepared as an account of work sponsored by an agency of the United States Government. Neither the United States Government nor any agency thereof, nor Battelle Memorial Institute, nor any of their employees, makes any warranty, expressed of implied, or assumes any legal liability or responsibility for the accuracy, completeness, or usefulness of any information, apparatus, product, or process disclosed, or represents that its use would not infringe privately owned rights. Relerence herein to any specific commercial product, process, or service by trade name, trademark, manuiacturer, or olherwise does not necessarily constitute or imply its endorsement, recommendation, or favoring by the United States Government or any agency thereof, or Battelle Memorial Institute. The views and opinions of authors expressed herein do not necessarily state or reflect those of the United States Government or any agency thereof.

\author{
PACIFIC NORTHWEST LABORATORY \\ operated by \\ BATTELLE MEMORIAL INSTITUTE \\ for the \\ UNITED STATES DEPARTMENT OF ENERGY \\ under Contract DE-AC06-76RLO 1830
}

\author{
Printed in the United Slates of America \\ Availahte to DOE and DOE contractors from the \\ Office of Scientific and Technical Information, P.O. Box 62, Oak Ridge, TN 37831; \\ prices available from (615) 576-8401. FTS 626-8401.
}

Availabie to the public from the National Technical information Service, U.S. Department of Commerce, 5205 Port Royal Rd., Springfield, VA 22161. 
PNC -7613

UC -903

LIMITED SITE INVESTIGATION OF

LANDFILLS 1 AND 4,

FORT LEWIS, WASHINGTON

G. V. Last

P. A. Eddy

S. P. Airhart

K. R. Olsen

J. R. Raymond

D. R. Dahl

August 1990

Prepared for

the U.S. Army, I Corps and Fort Lewis, Washington under Related Services Agreement with the U.S. Department of Energy

Contract DE-AC06-76RLO 1830

Interagency Agreement 11832B

Pacific Northwest Laboratory

Richland, Washington 99352 



\section{SUMMARY}

Fort Lewis has been in operation since 1917, when the citizens of Pierce County donated 77,320 acres of land to the Federal government for the establishment of a permanent military base. During the period of its operation, the Fort has provided facilities for troop induction, training, embarkation, and debarkation. The normal operation of this facility has included the disposal of generated waste into landfill sites and disposal pits.

The information presented in this report was collected during Timited site investigation activities conducted in the vicinity of Landfills 1 and 4 at Fort Lewis between April and December 1988. The purpose of this work was to provide a means of detecting and evaluating the impacts of these inactive landfills on ground-water quality and adjacent lands. This effort included the design and construction of ground-water monitoring systems for compliance with applicable Federal and State regulations governing Resource Conservation and Recovery Act (RCRA)-type landfi]ls.

Landfill 1 operated as a trench cut-and-fill operation for the disposal of sanitary solid waste unt $j$ at least late 1951. The landfill was then used for surface dumping of construction rubble. Waste materials exposed at the surface include broken concrete, broken asphalt, and various types of wood. Aerial photographs taken in 1951 appear to indicate that additional areas were used for open-pit dumping (waste materials unknown), burning, and storage. Unauthorized surface dumping of trash wood and paper, old asphalt shingles, various automobile parts, and Christmas trees has also occurred.

Landfill 4 also operated as a trench cut-and-fill operation for the disposal of sanitary solid waste until at least 1967. Four separate trench areas were interpreted from aerial photographs of the site. Waste materials exposed at the surface consisted of household waste, automobile parts, construction rubble, tires, and various large metal objects. Surface dumping of tree stumps and some unauthorized dumping have also occurred at this site. This site has also been used for training of heavy equipment and military vehicle operators. 
Three principal activities were conducted during this limited site investigation. The first activity involved 1) field reconnaissance of each landfil1, 2) visual inspection of monitoring wells and collection of water levels from the existing monitoring wells, and 3) personal interviews with Fort Lewis Directorate of Engineering and Housing (DEH) employees concerning the operational history and waste inventory of the landfilis and with the U.S. Army Corps of Engineers concerning aerial photographs and the hydrogeology of the area. The second activity, conducted in July 1988, involved surface geophysical surveys to determine the physical boundaries of Landfill 4 . A ground-penetrating radar unit, ground conductivity surveys, and metal detector surveys were used to delineate the waste boundaries. The third activity, conducted between August and October 1988, involved insta1ling 10 new monitoring wells, remediating 8 existing wells installed in 1981 and 1984, and abandoning 1 existing (1981) well.

Boreholes for the 10 new wells were drilled using either cable-tool or hollow-stem auger methods. Sediment samples were collected and classified in the field throughout the drilling effort. Generally, the sediment from the different boreholes consisted of sandy gravel to muddy sandy gravel with minor layers of sand and clay. On completion of the drilling, eight of the boreholes were logged using a gamma-ray (natural-gamma) geophysical probe. Water-level data from Landfill 1 indicate that ground-water flow is toward the northeast. Water-level data from Landfill 4 suggest that ground-water flow is to the north away from Sequalitchew Lake.

Ground-water samples were collected from both existing (1981 and 1984) wells and the newly installed (1988) wells. Five field sampling events were conducted commencing in late August 1988 and ending in mid-December 1988. The analytical results from the water samples indicate that the ground water in and around Landfill l contains limited contamination. Contaminants may include volatile organic compounds and nitrate. The primary concern in the area around Landfill 1 was the determination that ground water from two wells may contain cis-1,2-dichloroethylene and 1,1,1-trichloroethylene above drinking water standards. Nitrate levels in the downgradient wells were greater than those in upgradient wells and exceeded drinking water standards in some of the less-representative samples. Analyses of ground-water samples from 
wells in and around Landfill 4 indicate several contaminants may be present. These include volatile organic compounds (principally cis-1,2-dichloroethylene and 1,1,1-trichloroethylene), coliform, oil and grease, and perhaps some metals (iron and magnesium). The primary concern in the area around Landfill 4 was the determination that ground water from five wells contained cis-1,2-dich7oroethylene and 1,1,1-trichloroethylene above drinking water standards. The source of contaminants beneath either landfill cannot yet be identified. Insufficient data exist to disprove or confirm either landfill as possible contributors. 


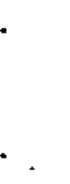




\section{CONTENTS}

SUMMARY ...............................

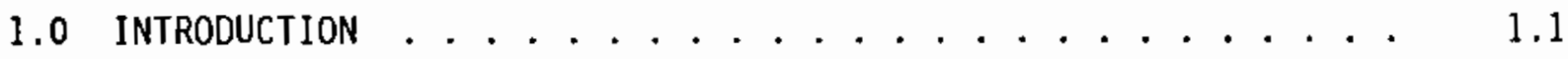

2.0 SITE BACKGROUND . . . . . . . . . . . . . . . 2.1

2.1 SITE LOCATION AND LAYOUT .............. 2.1

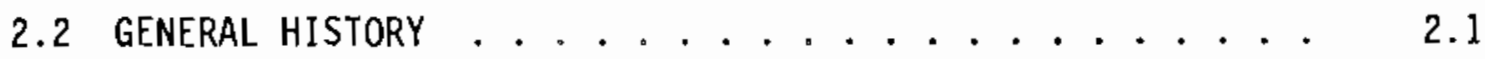

2.3 AERIAL PHOTOGRAPHY AND SITE MAPS ........... 2.3

2.4 HISTORY OF LANDFILLS I AND $4 \ldots \ldots \ldots \ldots$

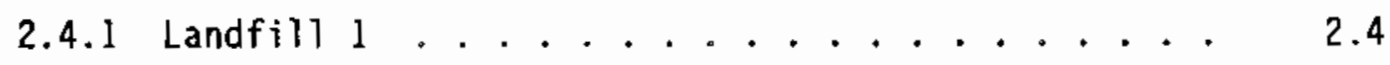

2.4 .2 Landfill 4 . . . . . . . . . . . . 2.4

2.5 PERMIT AND REGULATORY HISTORY . . . . . . . . . . 2.5

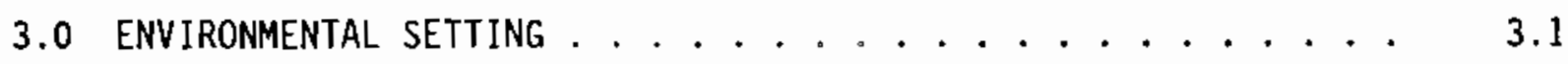

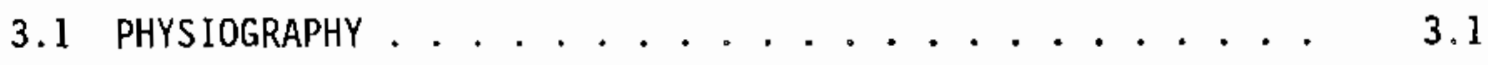

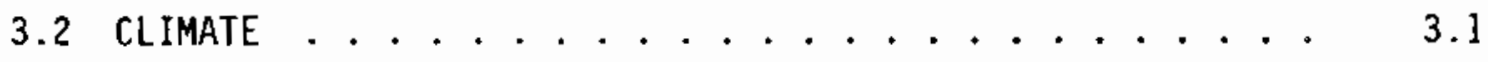

3.3 REGIONAL GEOLOGY . . . . . . . . . . . . . . . . 3.4

3.3.1 Kitsap Formation . . . . . . . . 3.6

3.3 .2 Vashon Drift . . . . . . . . . 3.6

3.4 REGIONAL HYDROLOGY . . . . . . . . . . . . 3.8

3.4 .1 Surface Water . . . . . . . . 3.8

3.4 .2 Ground water ............. 3.9

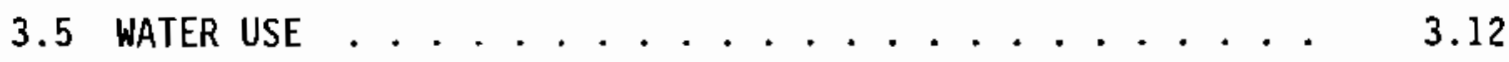

3.6 CRITICAL HABITATS . . . . . . . . . . . . . 3.13

4.0 SITE INVESTIGATION METHODS . . . . . . . . . . . 4.1

4.1 LOCATING LANDFILL BOUNDARIES . . . . . . . . . 4.1

4.1.1 Ground-Penetrating Radar ............ 4. 4.1 
4.1.2 Ground-Conductivity Meter .......... 4.2

4.1 .3 Metal Detector ............. 4.2

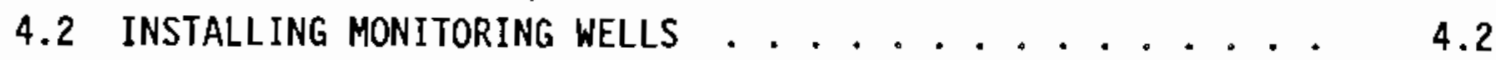

4.2.1 Drilling Methods ............. 4.3

4.2.2 Geologic Sampling and Sediment Characterization Methods ................. 4,3

4.2 .3 Monitoring Well Construction . . . . . . 4.5

4.2.4 Gamma-Ray Borehole Geophysical Logging . . . . . 4.7

4.2.5 Water-Level Measurement . . . . . . . . 4.7

4.2.6 Well Development Methods . . . . . . . 4 4.8

4.2 .7 Surveying . . . . . . . . . . 4.8

4.3 REMEDIATING AND ABANDONING WELLS .......... 4.11

4.4 GROUND-WATER SAMPLING . . . . . . . . . . 4.13

4.4 .1 August Sampling ............. 4.16

4.4 .2 September Sampling ............ . 4.16

4.4 .3 Early October Sampling .......... 4.17

4.4.4 Late October Sampling ........... 4. 4.18

4.4.5 December Sampling ............. . 4.18

4.5 ANALYZING GROUND-WATER SAMPLES . . . . . . . . . 4.19

4.6 quality ASSURANCE .................. 4.20

4.7 QUALITY CONTROL . . . . . . . . . . . 4.22

5.0 EVALUATION OF LANDFILL $1 \ldots \ldots \ldots . \ldots . \ldots . \ldots . \ldots$

5.1 LOCATION OF LANDFILL I BOUNDARIES .......... 5.1

5.2 GEOLOGIC FRAMEWORK . . . . . . . . . . . . . 5.3

5.3 GROUND-WATER FLOW ........................... 5.11

5.4 GROUND-WATER QUALITY . . . . . . . . . . . 5.15 
5.5 EVALUATION OF THE MONITORING NETWORK . . . . . . . 5.21

6.0 EVALUATION OF LANDFILL $4 \ldots \ldots \ldots . \ldots . \ldots . \ldots . \ldots$

6.1 LOCATION OF LANDFILL 4 BOUNDARIES .......... 6.1

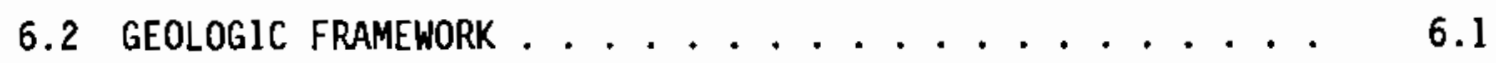

6.3 GROUND-WATER FLOW . . . . . . . . . . . . 6.4

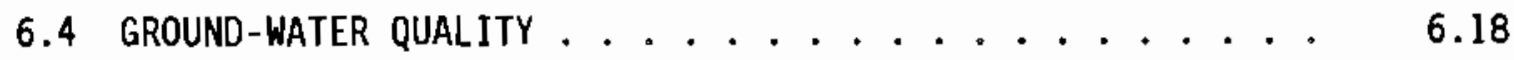

6.5 EVALUATION OF THE MONITORING NETWORK . . . . . . 6.23

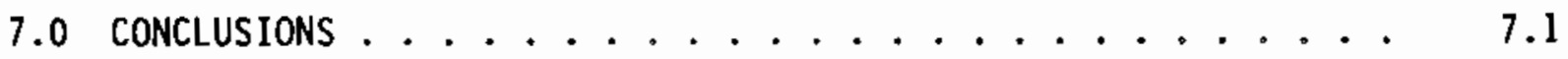

7.1 OVERALL SITE INVESTIGATION .................... 7.1

7.2 STATUS 0 LANDFILL $1 \ldots \ldots \ldots \ldots . \ldots . \ldots . \ldots . \ldots$

7.3 STATUS OF LANDFILL $4 \ldots \ldots \ldots \ldots . \ldots . \ldots . \ldots . \ldots$

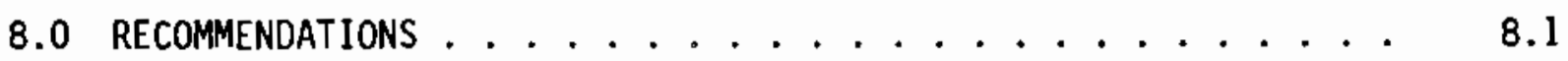

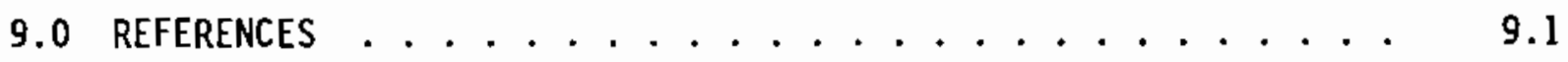

APPENDIX A - RESULTS OF GEOPHYSICAL SURVEYS AT LANDFILL $4 \ldots \ldots$. . . A.l

APPENDIX B - WELL SPECIFICATIONS .............. B.I

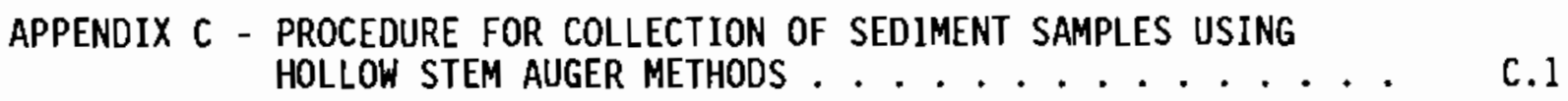

APPENDIX D - GEOLOGIC SAMPLING AND WELL CONSTRUCTION
DOCUMENTATION PROCEDURE . ............ D. . . .

APPENDIX E - WELL COMPLETION REPORT, AS BUILT DIAGRAM, NATURAL-
GAMMA LOGS, AND DRILL LOGS OF THE NEW WELLS ... . E. .

APPENDIX $F$ - MOISTURE CONTENT OF SEDIMENT SAMPLES . . . . . . F.I

APPENDIX G - WATER-LEVEL MEASUREMENT PROCEDURE . . . . . . . G.1

APPENDIX H - WELL LOCATION AND ElEVATION SURVEY DATA . . . . . H.l

APPENDIX I - DRILL LOGS OF EXISTING WELLS AT LANDFILL

APPENDIX $\mathrm{J}$ - GROUND-WATER SAMPLE ANALYSES PROCEDURES . . . . . . J J.l

APPENDIX K - GROUND-WATER SAMPLE ANALYSES RESULTS . . . . . . K. K 


\section{FIGURES}

1.1 Location Map of Fort Lewis . . . . . . . . . . 1.2

2.1 Location of Landfills 1 and 4 at Fort Lewis, Washington . . . 2.2

3.1 Location of the Tacoma Upland . . . . . . . . . 3.2

3.2 Location of Streams, Lakes, and Cultural Features within the Tacoma Upland and Adjacent Areas ............... 3.3

3.3 Upper Stratigraphic Column for the Fort Lewis Area . . . . 3.7

3.4 Regional Potentiometric Surface Map of the Uppermost Unconfined Aquifer Beneath the Tacoma Upland ....... 3.11

4.1 Ternary Diagram for Naming Sediments . . . . . . . . 4.4

4.2 Schematic Design of the Newly Installed Monitoring Wells at Landfills l and 4 ................. 4.6

5.1 Location of Landfill I Boundaries and Historical Configurations ................. 5.2

5.2 Location of Monitoring Wells Around Landfill l . . . . . . 5.4

5.3 Subsurface Correlation Chart of Well LF1-PNL1 . . . . . . 5.6

5.4 Subsurface Correlation Chart of Well LFI-PNL2 . . . . . . . 5.7

5.5 Subsurface Correlation Chart of Well LF1-PNL3 . . . . . . 5.8

5.6 Subsurface Correlation Chart of Well LFI-PNL4 . . . . . . . 5.9

5.7 Generalized Geologic Cross Section Through Landfill 1 . . . 5.10

5.8 Water-Table Surface Beneath Landfill 1 as Interpreted from Water Levels Measured on October 25, 19B8 . . . . . . . . 5.12

5.9 Water-Table Surface Beneath Landfjl1 1 as Interpreted from Water Levels Measured on December 14, 19B8 . . . . . . . 5.13

5.10 Generalized Ground-Water Flow Directions and Location of Upgradient and Downgradient Wells at Landfill l ...... 5.14

6.1 Location of Surface Geophysical Surveys, Showing Location of Suspected Waste Materials . . . . . . . . . . . 6.2

6.2 Boundaries and Configuration of Landfill $4 \ldots \ldots . . . . .6 .3$ 
6.3 Location of Monitoring Wells Around Landfill 4 . . . . . 6.5

6.4 Subsurface Correlation Chart for Well LF4-PNL1 . . . . . . 6.7

6.5 Subsurface Correlation Chart for Well LF4-PNL2 . . . . . . 6.8

6.6 Subsurface Correlation Chart for Well LF4-PNL3 . . . . . 6.9

6.7 Subsurface Correlation Chart for Well LF4-PNL4 . . . . . 6.10

6.8 Subsurface Correlation Chart for Well LF4-PNL5 . . . . . . 6.11

6.9 Subsurface Correlation Chart for Well LF4-PNL6 . . . . . . 6.12

6.10 Generalized Geologic Cross Section Through Landfill $4 \ldots . . .6 .13$

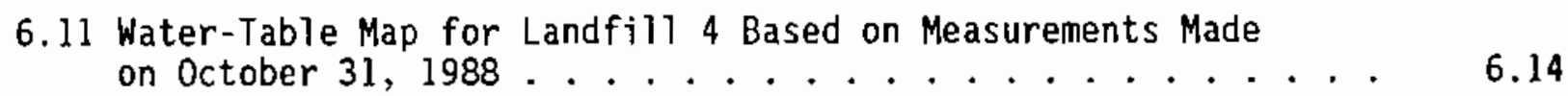

6.12 Water-Table Surface Beneath Landfill 4 as Interpreted from Water Leveis Measured on December 15, 1988 ........ 6.15

6.13 Generalized Ground-Water Flow Directions and Location of Upgradient and Downgradient Wells at Landfill 4 ...... 6.17

6.14 TCE Concentration Ranges Based on Analyses of September Through December Sampling Events .......... 6.24 


\section{TABLES}

2.1 Aerial Photographs Available for Landfills 1 and $4 \ldots 2.3$

3.1 Climatic and Stratigraphic Units in the Tacoma Upland . . . 3.5

4.1 Development Information for Wells at Landfill l . . . . . 4.9

4.2 Development Information for Wells at Landfill $4 \ldots . . . .4 .10$

4.3 Landfill 1 Ground-Water Sampling History . . . . . . . . . 4.14

4.4 Landfill 4 Ground-Water Sampling History . . . . . . . . 4.15

4.5 List of Analytical Methods Used on the Fort Lewis 4.20

4.6 Comparison of U.S. Geological Survey Standard Concentration
with Am Test, Inc., Results.................... 4.23

4.7 Comparison of the U.S. Geological Survey Standard
Concentration With PNL Results . . . . . . . . . 4.24

5.1 Well Completion Data for Landfill 1 . . . . . . . . 5.5

5.2 Water-Level Measurements from Landfill l . . . . . . . 5.16

5.3 Analytical Results of All Ground-Water Samples Collected
Near Landfill . . . . . . . . . . . . . 5.17

5.4 Volatile Organic Compound Concentrations in All Ground-Water Samples from Landfill 1.............. 5.19

6.1 Well Completion Data for Landfill $4 \ldots \ldots .6 . \ldots 6$

6.2 Water-Level Summary for Wells Near Landfill $4 \ldots . . . . .6 .19$

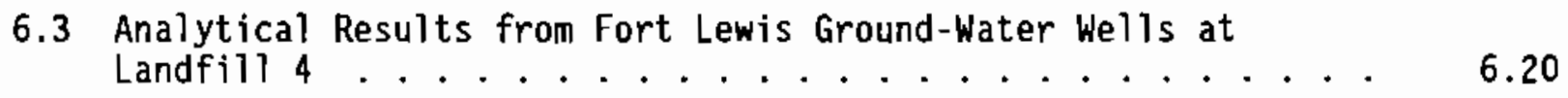

6.4 Analytical Results for Volatile Organic Compounds at
Landfill $4 . . . .6$ 
1. 


\subsection{INTRODUCTION}

The 86,000 -acre Fort Lewis Facility (the Fort) is located approximately 12 miles southwest of Tacoma, Washington, and 18 miles northeast of 0lympia, Washington (Figure 1.1). The Fort has been an army facility since 1917 with onsite industrial operations including maintenance of aircraft and vehicles and repairs and refurbishing of weapons.

During the Fort's more than 70 years of operation, several landfill sites were used for the disposal of large quantities of solid waste material. These waste materials slowly degrade and/or mobilize in the subsurface and can result in the release of contaminants through the vadose zone and into shallow underlying aquifer systems.

Information presented in this report was collected by the Pacific Northwest Laboratory (PNL) (a) at the request of the U.S. Army. Work was performed under a related services agreement with the U.S. Department of Energy (Contract DE-AC06-76RLO 1830 Interagency Agreement 11832B). This information was collected during site investigations conducted at Landfills 1 and 4 located on the Fort. The goal of the site investigations was to provide a means for detecting and evaluating the impacts of inactive Landfills 1 and 4 on the ground water and adjacent lands. This goal was to be achieved through the design and construction of ground-water monitoring systems at the landfills that would comply with applicable federal and state regulations.

Three principal activities were conducted at the landfills. The first activity involved 1) field reconnaissance of both landfills to determine the types of materials present and the areal extent of each landfill, 2) visual inspection of existing monitoring wells to determine their usefulness and for the collection of water-level data, and 3) interviewing Fort Lewis Directorate of Engineering and Housing (DEH) employees concerning the operation, use, and waste inventories of the landfills and the U.S. Army Corps of Engineers personnel concerning available aerial photographs and existing hydrogeologic

(a) The Pacific Northwest Laboratory is operated by Battelle Memorial Institute for the U.S. Department of Energy under Contract DE-AC06-76RLO 1830 . 


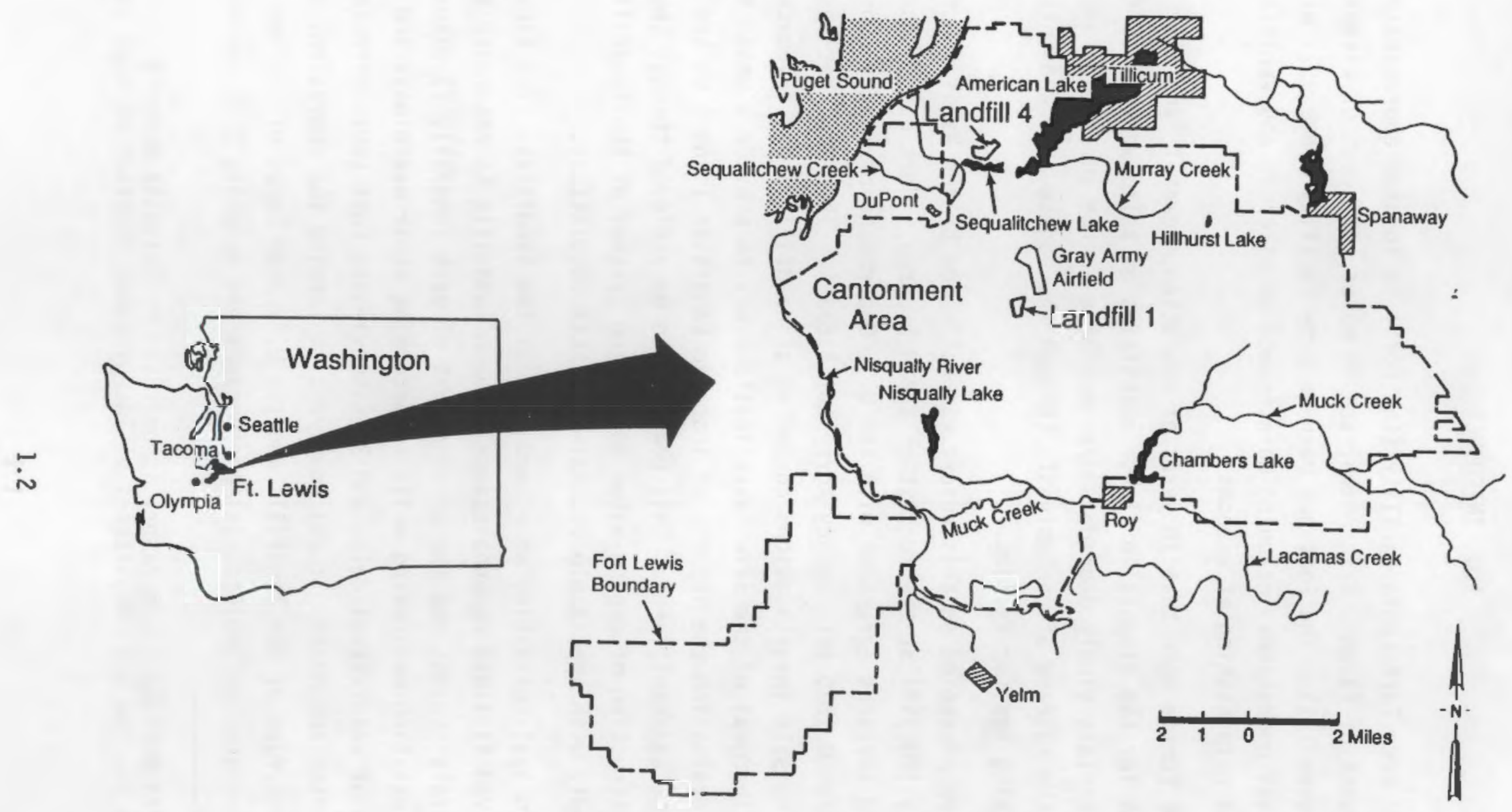

FIGURE 1.1. Location Map of Fort Lewis 
information. The second activity was to perform surface geophysical surveys over Landfill 4 to determine its physical boundaries. Ground-penetrating radar, ground conductivity surveys, and metal detector surveys were conducted in July 1988. The third activity, performed in August through October 1988, consisted of drilling and installing 10 new wells, remediating 8 existing wells installed in 1981 and 1984, and abandoning 1 of the 1981 wells. Sediment samples were collected at 5 - $\mathrm{ft}$ intervals throughout the drilling effort. These sediments were generally classified by using the percentages of gravel, sand, and mud (silt plus clay) found in the sample. Five ground-water sampling events were performed, each involving all or portions of the 10 new (1988) wells and 8 existing (1981 and 1984) wells.

This report contains background information on the Fort and its environmental setting. The report also discusses the investigative methods used in the study, the specific hydrogeologic characteristics of the landfills, and the possible impacts of these landfills on the ground-water quality. 

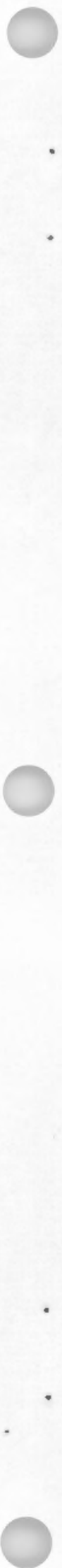


\subsection{SITE BACKGROUND}

This chapter describes the location and physical layout of the Fort and Landfills 1 and 4, the history of the Fort, the photographs and sketches available for these landfills, the operational history of the landfills, and the permit and regulatory history of the landfills.

\subsection{SITE LOCATION AND LAYOUT}

The Fort is located along Interstate 5, approximately 12 miles southwest of Tacoma, Washington, and 18 miles northeast of 01 ympia, Washington (see Figures 1.1 and 2.1). The Fort encompasses approximately 86,000 acres and is configured in a general hourglass shape, with its long axis oriented northeast to southwest. The Nisqually River crosses the fort at its narrowest point, dividing the Fort into northeastern and southwestern sectors. The northeastern sector is the largest and contains the two landfills of interest (Landfills 1 and 4 ). These two landfills are located on opposite sides of the west-southwest to east-northeast-oriented Interstate 5 , which cuts through this portion of the Fort (Figure 2.1). Landfill 1 is located south of Interstate 5, approximately 0.5 mile southwest of Gray Army Airfield. Landfill 4 is located north of Interstate 5 , immediately north of Sequalitchew Lake.

\subsection{GENERAL HISTORY}

The Fort is an active facility that has been owned and operated by the U.S. Army since 1917. McMaster (1982) indicated that this facility serves as the focal point for induction, training, embarkation, and debarkation of troops in the northwest. Activities at this installation include the operational use, handling, storage, and disposal of various hazardous materials. A summary of these activities, taken from McMaster (1982), is provided below.

Industrial operations at the Fort include vehicle and aircraft maintenance, weapons repair and refinishing, and limited furniture refinishing. Vehicle and aircraft maintenance activities currently use 1,1,1trichloroethane (TCA) in degreaser baths and caustic sodium hydroxide for 


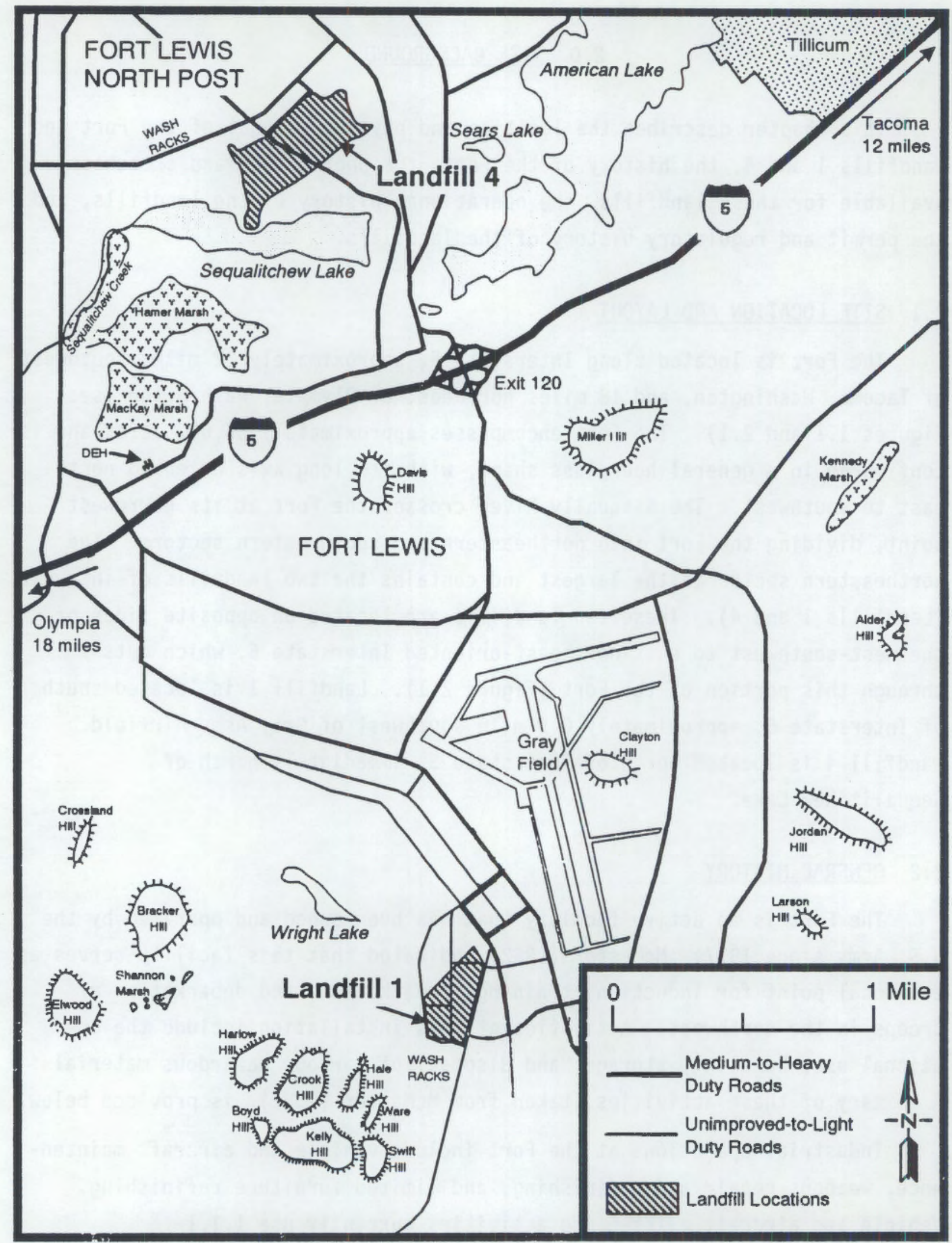

FIGURE 2.1. Location of Landfills 1 and 4 at Fort Lewis, Washington 
for paint stripping. Trichloroethylene (TCE) was used at Fort Lewis until the mid-1970s, when its use was discontinued. Small-armament maintenance activities involve sand blasting, degreasing, acid rinsing, and oil dipping. Furniture refinishing involves the use of commercial paint strippers, paint, and varnish.

Hazardous materials have been and are currently handled and/or stored at numerous locations throughout the Fort. These materials include 1) pesticides (insecticides, herbicides, fungicides, and rodenticides), 2) polychlorinated biphenyls (PCBs), 3) industrial and laboratory chemicals, 4) radiological materials, and 5) petroleum, oils, and lubricants.

Waste disposal and treatment operations have been and are currently being performed at the Fort. These operations involve 1) offsite and onsite industrial waste treatment and disposal, 2) onsite waste water treatment, 3) solid waste landfill disposal, 4) explosive ordnance demolition, and 5) demilitarization activities.

\subsection{AERIAL PHOTOGRAPHY AND SITE MAPS}

Two sets of aerial photographs of Landfill 1 and three sets of Landfill 4 were available for review (Table 2.1). No drawing could be found of these landfills, other than some general site maps. These aerial photographs and site maps can be obtained from the Engineering Division, Survey Branch, Seattle District, U.S. Army Corps of Engineers, Seattle, Washington.

IABLE 2.1. Aerial Photographs Available for Landfills 1 and 4

\begin{tabular}{|c|c|c|c|}
\hline \multirow{2}{*}{$\frac{\text { Landfill }}{1}$} & \multicolumn{2}{|c|}{ Aerial Photograph Set } & \multirow{2}{*}{$\frac{\text { Date }}{7-21-51}$} \\
\hline & 2 & -- 113,114 and 115 & \\
\hline \multirow{4}{*}{4} & $\mathrm{KP}-70$ & $\begin{array}{l}--7-12-13 \text { and }-14 \\
7-13-18\end{array}$ & $5-24-70$ \\
\hline & 1 & -- 172 through 176 & $7-21-51$ \\
\hline & $\mathrm{KP}-70$ & $\begin{array}{l}-7-11-18 \text { and }-19 \\
7-12-19\end{array}$ & $5-24-70$ \\
\hline & $\mathrm{NW}-\mathrm{C}-7$ & $--15 A-24$ and -25 & $7-15-76$ \\
\hline
\end{tabular}




\subsection{HISTORY OF LANDFILLS 1 AND 4}

Landfills 1 and 4 are two of 14 reported solid waste landfills/disposal sites that have been used at the Fort (McMaster 1982). McMaster reported that both these landfills were used for the disposal of "sanitary" wastes. Landfill 1 was reportedly used between 1946 and 1951, and Landfill 4 was reportedly used between 1951 and 1967 (McMaster 1982). No records or firsthand personal accounts could be obtained to estimate the waste constituents or inventories of these landfills. Those staff interviewed from the DEH believed that Landfill 1 was used primarily for surface dumping of construction debris and that Landfill 4 was used primarily for disposal of domestic garbage. Additional interpretations of the disposal history and waste inventory are based on available aerial photographs and visual observations of the landfills.

\subsubsection{Landfill 1}

Aerial photographs taken in July 1951 indicate that a portion of Landfill 1 was still in operation at that time. However, this trench cutand-fill operation may have been discontinued in late 1951, as reported by McMaster (1982). Several isolated disposal pits and/or burn pits near this trench/fill area can also be observed in these photographs. Aerial photographs taken in May 1970 suggest that the landfill may have been extended farther to the southwest, possibly by overbank dumping along the western escarpment created by the later stages of the trenching operation. Some surface storage was also indicated near the southern tip of the landfill.

Field inspections made during May through July 1988 found evidence of more recent (post-1970) surface dumping of (primarily) construction rubble. Waste materials observed at the surface included sand and gravel, broken cement, broken asphalt, old concrete structures, and various types of wood debris. Unauthorized surface dumping of trash wood and paper, old asphalt shingles, various automobile parts, and Christmas trees was also apparent.

\subsubsection{Landfill 4}

Aerial photographs taken in May 1970 show that operations at Landfill 4 had apparently ceased. Other aerial photographs taken in July 1976 show no 
change in the landfill configuration since the 1970 photographs. Thus, it is believed that the landfill was used before 1970 and perhaps closed in 1967 as reported by McMaster (1982). The photographs suggest at least four trench cut-and-fill areas:

Visual inspection and surface geophysical surveys made during May through July 1988 suggest numerous isolated open-pit dumps surrounding these trench areas. Waste materials observed on the landfill surface included domestic garbage, automobile parts, industrial debris (steel cables, reinforced concrete), tires, and asphalt. More recent surface dumping of tree stumps and some unauthorized surface dumping of trash wood and paper, automobile parts, old lumber, trees, barbed wire, etc., were also observed. This site has also been used for heavy equipment and military vehicle operator training.

\subsection{PERMIT AND REGULATORY HISTORY}

McMaster (1982) indicated that the Fort has filed a Notification of Hazardous Waste Activity under the Resource Conservation and Recovery Act (RCRA) (40 CFR 265) and has received U.S. Environmental Protection Agency (EPA) identification number WA 9214053465 for generation, storage, and treatment of hazardous waste. The wastes listed on the notification include solvents, battery acid waste, pesticides, and explosive waste. The Fort has applied for a RCRA permit to cover a hazardous waste storage facility located on the Fort Lewis Logistics Center.

No operating permits are known to be in place for Landfills 1 and 4. As part of the Installation Restoration Program and to monitor past disposal sites, four monitoring wells were installed around Landfill 1 in 1984, and five wells were installed around Landfill 4 in 1981. 

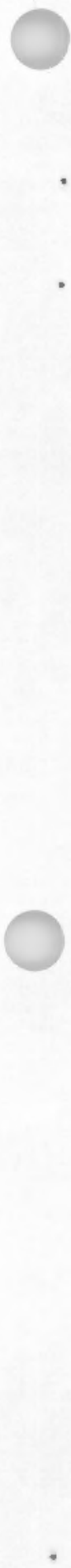


\subsection{ENVIRONMENTAL SETTING}

To thoroughly investigate the landfills, the environmental setting was characterized including physiography, climate, regional geology and hydrology, water use, and critical habitats.

\subsection{PHYSIOGRAPHY}

The two landfills of interest are located within the Tacoma Upland subarea (Figure 3.1) of the central Puget Sound Lowland as defined by the U.S. Geological Survey (USGS 1962). The Puget Sound Lowland is a broad plain that extends from the Cascade Range on the east to the 01ympic Mountains and Willapa Hills on the west, and into the Willamette Valley to the south. The Tacoma Upland is located on a poorly drained drift plain that ranges from sea level to approximately $600 \mathrm{ft}$ above mean sea level (ms1).

The topography of the Tacoma Upland is a product of processes from the most recent (Vashon) Pleistocene glaciation and subsequent Recent glaciofluvial deposition and erosion; it is characterized by rolling hills (moraines and drumlins) and depressions (kettles) that are scattered across the plain. Numerous lakes and swamps exist in the depressions.

Two major rivers edge the Tacoma Upland. The Puyallup River forms the northeastern and eastern boundaries of the Tacoma Upland, and the Nisqually River forms the southwestern boundary. A number of small creeks and streams drain the interior of the Tacoma Upland. Figure 3.2 shows locations of streams, lakes, and cultural features within the Tacoma Upland and adjacent areas. Landfill 1 lies within the Murray Creek subbasin, and Landfill 4 is in the Sequalitchew Creek/Lake subbasin.

\subsection{CLIMATE}

The climate of the study area is moderate/humid, with winter temperatures generally above freezing and summer temperatures generally below $80^{\circ} \mathrm{F}$. Mean annual precipitation in the lowland is approximately $40 \mathrm{in./yr}$, with 

R.1E.
R.2E.
R.3E.
R.4E.
R.5E.

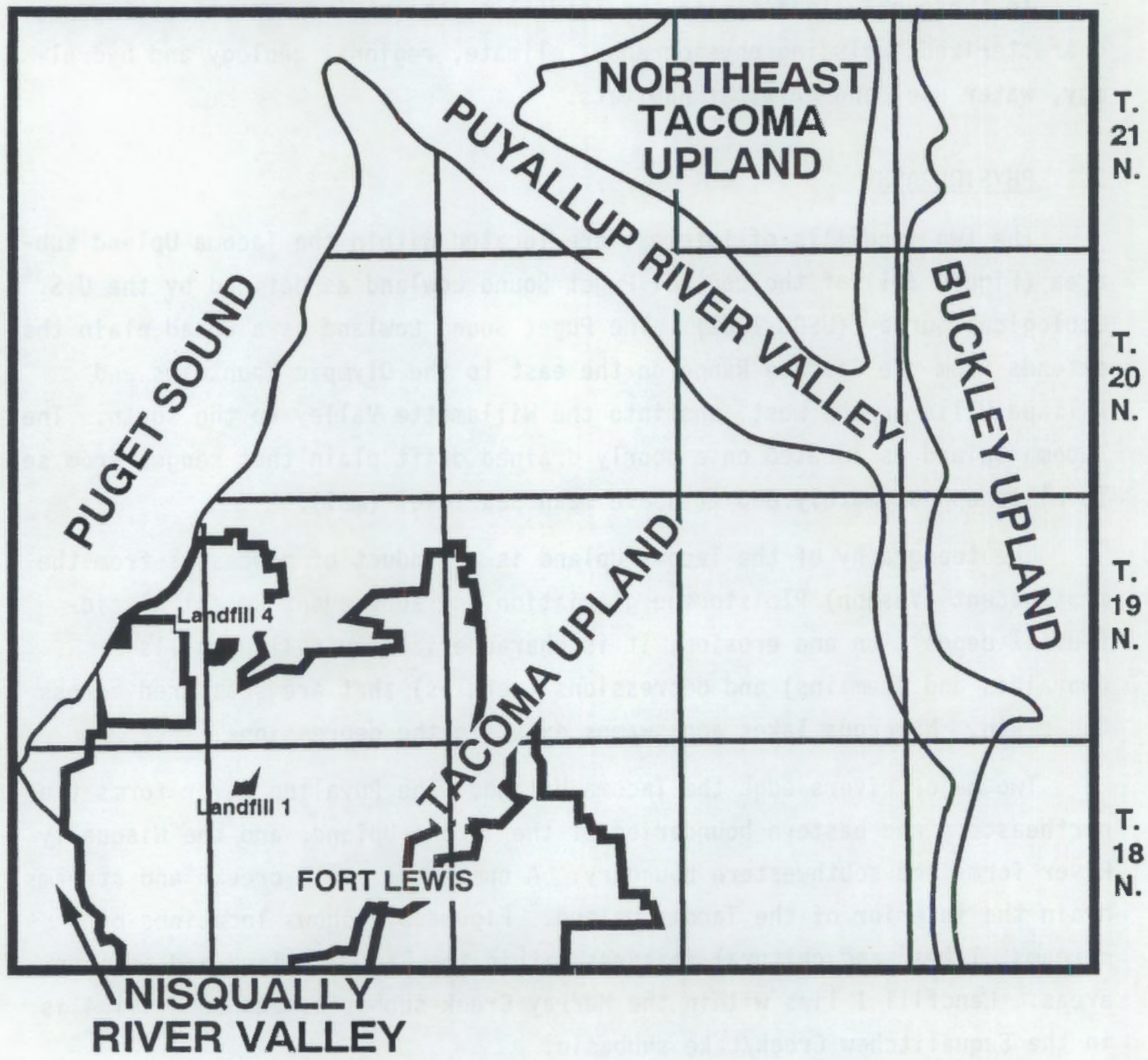

FIGURE 3.1. Location of the Tacoma Upland (from USGS 1962) 


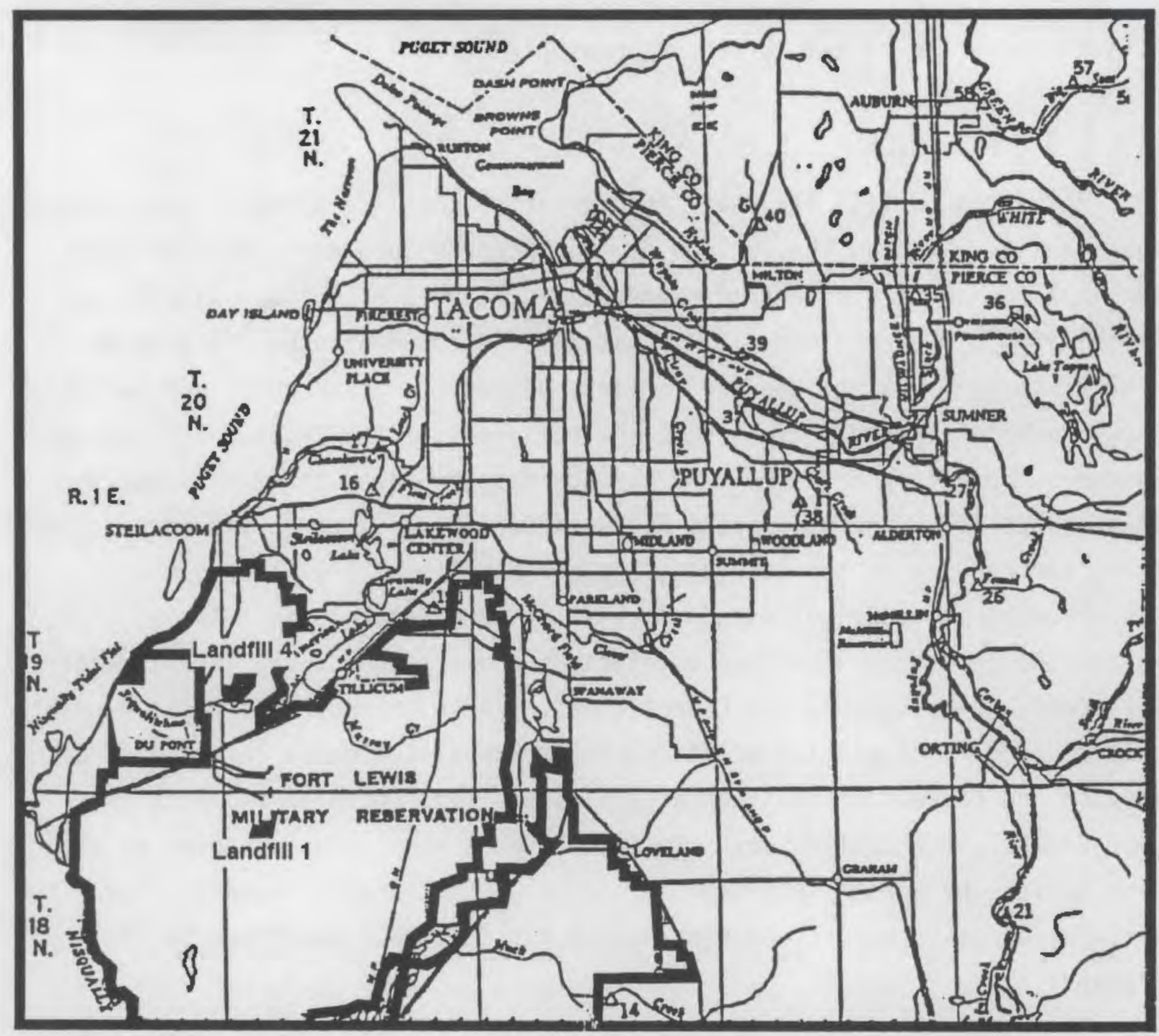

FIGURE 3.2. Location of Streams, Lakes, and Cultural Features Within the Tacoma Upland and Adjacent Areas (from USGS 1962) 
most of the rain falling during the spring and winter months. Puget Sound, the Pacific Ocean, and the mountain ranges to the west provide moderating influences on the climate of the Tacoma Upland.

\subsection{REGIONAL GEOLOGY}

The Tacoma Upland lies near the center of the Tacoma low, a deep structural basin. Data on the geology of the Tacoma Upland were compiled from publications on this area (Brown and Caldwell 1983; USGS 1962; Walters and Kimme 1 1968). Bedrock underlying this area is assumed to be the same or similar to the Tertiary and pre-Tertiary volcanic, metamorphic, and consolidated sedimentary rocks exposed at the periphery of the Puget Sound Lowland. However, these rock units have no surface exposure near the landfills, and they do not occur in the deepest wells (>2000 ft) drilled in the area. Overlying the bedrock in the Tacoma Upland are more than $2000 \mathrm{ft}$ of semiconsolidated and unconsolidated clay, silt, sand, gravel, and glacial till. These sediments were deposited by glaciers and streams during late Tertiary (Pliocene), Pleistocene, and Recent times. The Pliocene/Pleistocene deposits consist largely of glacial drift and interglacial sediments laid down from advance and retreat of continental ice sheets that occupied the area from approximately 2.5 million years to 11,000 years ago. Final shaping of the land surface by stream incision of valleys and subsequent deposition of alluvial deposits occurred during Recent times $(11,000$ years ago to the present).

The Pleistocene glacial and interglacial intervals are of most interest in the present study because the resulting sediments deposited by these events make up the majority of the subsurface geologic materials, and these sediments provide the framework for the ground-water regime in the Tacoma Upland. Four Pleistocene glaciation intervals have been recognized in the Tacoma Upland. These are, from oldest to youngest (Crandall et a1. 1958) 1) Orting, 2) Stuck, 3) Salmon Springs (and upper Salmon Springs), and 4) Vashon [possibly a stade within the broader "Fraser" glaciation described by Armstrong et a1. (1965)]. Interglacial episodes, where the area was free from ice for an extended time, follow each glaciation. Table 3.1 (modified 
IABLE 3.1. Climatic and Stratigraphif Units in the Tacoma Upland (all Pleistocene Epoch)(a)

Climatic Unit

Vashon Glaciation

Olympia Interglaciation

Upper Salmon Springs Glaciation

Unnamed Interglaciation

Salmon Springs Glaciation

Puyallup Interglaciation

Stuck Glaciation

Alderton Interglaciation

Orting Glaciation
Stratigraphic Unit

Vashon Drift

Steilacoom Gravels

Vashon Recessional Outwash

Vashon Till

Vashon Advance Outwash

Colvos (Esperance) Sand

None identified

None identified

Kitsap Formation

Salmon Springs Drift

Puyallup Formation

Stuck Drift

Alderton Formation

Orting Drift

(a) Modified from Brown and Caldwell (1983).

from Brown and Caldwell 1983) shows the relationships and names of the climatic units and resultant geologic (stratigraphic) units in the Tacoma Upland.

The glacial drift units generally consist of three depositional facies: 1) outwash deposited by advancing glaciers, 2) till deposited by stagnant glaciers, and 3) outwash deposited by receding glaciers. The outwash deposits (advance and recessional) typically consist of well-sorted gravel or stratified sand and gravel. The tills generally consist of semiconsolidated, poorly sorted gravel in a silty/clayey matrix.

The interglacial units generally consist of fluvial and lacustrine deposits. Sands, silts, and clays deposited in lakes or over broad alluvial plains are most common, al though gravels occur locally in mainstream channels. 
The upper two units, the Kitsap Formation and the Vashon Drift, are the most significant to this study, because they are believed to control the uppermost aquifer beneath the Fort. Figure 3.3 illustrates a generalized geologic column of these two formations.

\subsubsection{Kitsap Formation}

The Kitsap Formation, overlying the Salmon Springs Drift, is widespread. It is of nonglacial origin (lacustrine and fluvial) and consists of clay, silt, and fine sand. The Kitsap Formation is typically 50 to $150 \mathrm{ft}$ thick in the Tacoma Upland.

\subsubsection{Vashon Drift}

The Vashon Drift is the youngest glacial deposit in the area and is exposed at the surface. Several subunits have been described within the Vashon Drift. These are in ascending order (oldest to youngest) Colvos (Esperance) Sand, Vashon Advance Outwash, Vashon Ti11, Vashon Recessional Outwash, and Steilacoom Gravels.

The Colvos (Esperance) Sand overlying the Kitsap Formation, al though considered an advance outwash deposit, is mapped and defined independently because of its easily identified characteristics of a 10ose, well-sorted sand with occasional gravel occurrences (and a basal clay section in some areas). The Colvos Sand is up to $150 \mathrm{ft}$ thick in the Upland area.

Overlying the Colvos Sand is the Vashon Advance Outwash. The advance outwash is a stratified, well-sorted pebble- to cobble-sized gravel that is present under most of the Tacoma Upland in thicknesses of 25 to $50 \mathrm{ft}$.

The Vashon Till overlies the Vashon Advance Outwash in much of the Tacoma Upland. The till may be present as a semiconsolidated matrix of silt and clay with intercalated sand and gravel, or it may take the form of unconsolidated, unstratified gravel, sand, and silt. Although the till may be as much as $100 \mathrm{ft}$ thick in places, it more typically is from 5 to $30 \mathrm{ft}$ thick. 


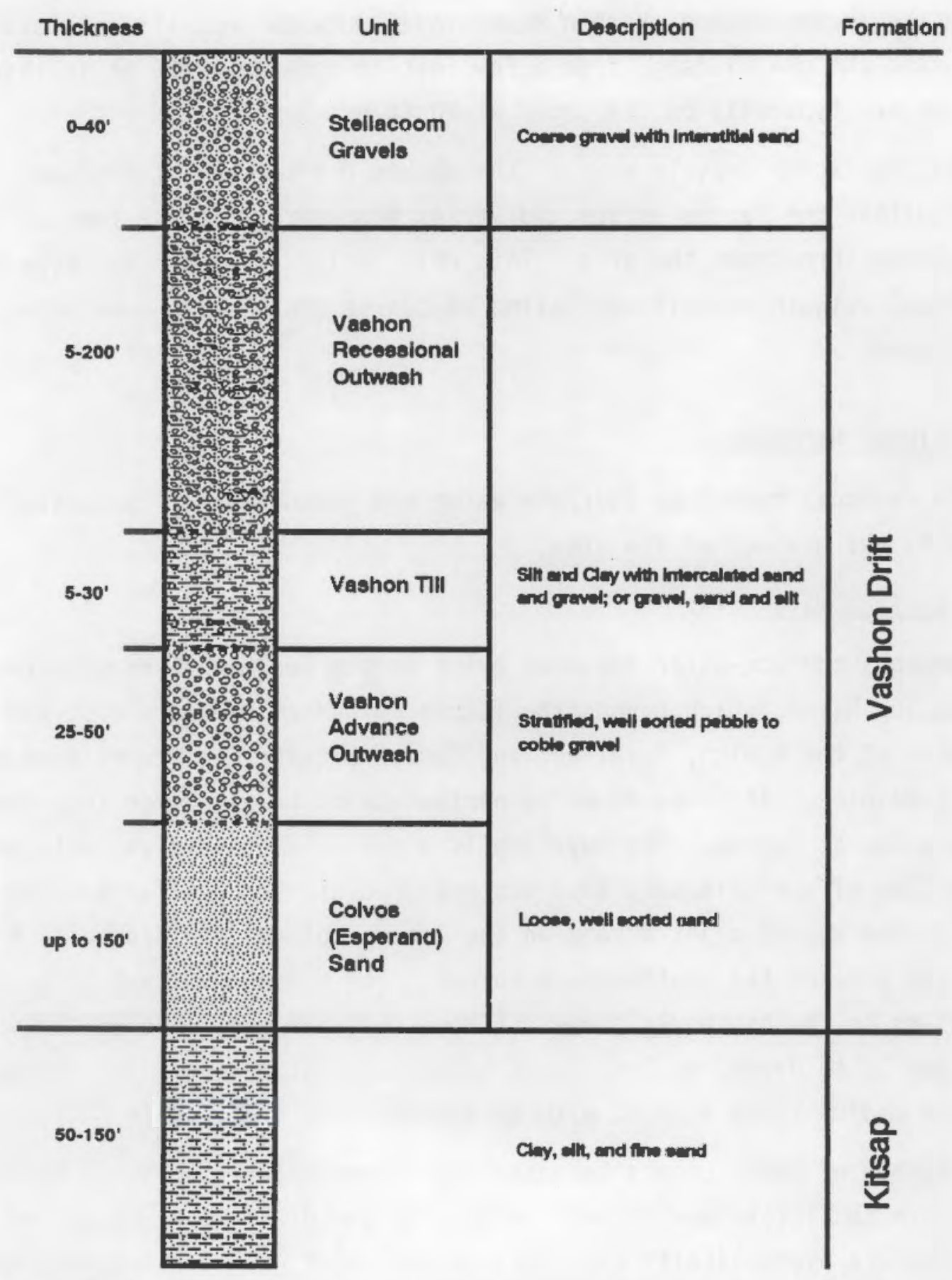

FIGURE 3.3. Upper Stratigraphic Column for the Fort Lewis Area 
In the Tacoma Upland, Vashon Recessional Outwash deposits consisting of coarse sand and gravel range from a few feet to more than $200 \mathrm{ft}$ in thickness, but are typically on the order of $20 \mathrm{ft}$ or so thick.

The Steilacoom Gravels unit of the Vashon Drift covers the ground surface within the Tacoma Upland and varies from zero to a few tens of feet in thickness throughout the area. This unit is locally a special type of recessional outwash deposit consisting of coarse gravel with some interstitial sand.

\subsection{REGIONAL HYDROLOGY}

The regional hydrology (surface water and ground water) is controlled largely by the geology of the area.

\subsubsection{Surface Water}

Numerous surface-water features exist in the Tacoma Upland (Figure 3.2). The Puyallup River, which bounds the Tacoma Upland on the northeast and east, originates at the Mowich, Puyallup, and Tahoma Glaciers on the western slopes of Mount Rainier. It flows 46 miles northwestward to discharge into Commencement Bay at Tacoma. The Puyallup is a medium-sized stream, with an average flow of approximately 3300 cfs measured at the Puyallup gauging station. The second major stream in the Tacoma Upland, the Nisqually River, bounds the area on the southwestern corner. The Nisqually River originates at glaciers on the southerly slopes of Mount Rainier and winds westerly to northwesterly to discharge into Puget Sound south of DuPont. The Nisqually also is a medium-sized stream, with an average flow of $1370 \mathrm{cfs}$.

A number of small creeks traverse the Tacoma Upland. Most of these streams are small (average flows of $<100 \mathrm{cfs}$ ) and drain local subbasins. The creeks are hydraulically connected to the upper ground-water aquifers in the area, in some places gaining water from and in other locations contributing water to the aquifers.

Murray Creek is a small, ungauged creek that originates in Kinsey Marsh located on the Fort east of Madigan General Hospital and the Logistics Center (Mount Rainier Ordnance Depot). Murray Creek runs westerly for approximately 
4 miles and discharges into American Lake. Sequalitchew Creek is a small ungauged stream that serves as the outlet of Sequalitchew Lake and runs westerly for a distance of approximately 2.5 miles to discharge into Puget Sound near the Nisqually tidal flat.

Numerous springs exist throughout the Tacoma Upland. The springs are located along the Puget Sound Bluffs, along the creek and river channels, and along the edges of the deeper kettle depressions. The springs deliver a significant amount of ground water to the surface stream, lake, and swamp environments. Springs in the Tacoma Upland discharge an estimated 100,000 acre-ft/yr (Walters and Kimmel 1968). Many springs discharge at rates greater than $1 \mathrm{cfs}$.

Lakes are the most prominent hydrologic features in the western part of the Tacoma Upland. Major lakes (>100 acres in area) are American, Steilacoom, Spanaway, Gravelly, and Sequalitchew. These lakes are the surface expressions of the shallow ground-water system and occur in the deepest topographic depressions (kettles). The bottoms of the major lakes are in the coarse Steilacoom Gravels, allowing interchange of the surface and ground waters.

\subsubsection{Ground Water}

The thick sequence of unconsolidated glacial, fluvial, and lacustrine sediments underlying the Tacoma Upland provides the framework for groundwater systems with extremely productive aquifers (water-bearing geologic media). Ground-water recharge from precipitation in the Tacoma Upland is estimated at approximately 400,000 acre-ft/yr (USGS 1962). Ground-water discharge occurs through springs and seeps, which eventually discharge into the Puyallup and Nisqually rivers or directly into Puget Sound along the boundaries of the Tacoma Upland.

The coarse sands and gravels of the Vashon Recessional Outwash are the most productive aquifers in the Tacoma Upland. This outwash often lies in the vadose zone (above the zone of saturation and above the water table) and, thus, does not produce ground water. However, where the outwash is below the water table, it is highly productive. The Vashon Recessional Outwash aquifer 
is highly susceptible to contamination from surface sources because of its high permeability and near-surface location.

The Vashon Till, which lies beneath most of the Tacoma Upland, is not a particularly productive aquifer. Where it lies below the zone of saturation and consists of mostly sand and gravel at least $20 \mathrm{ft}$ thick, it will yield sufficient water to wells for single or small group domestic supply of a few to a few tens of gallons per minute. In most places, however, the Vashon Till consists mostly of silt and clay and can be considered an aquiclude (or aquitard) that protects the underlying water-bearing zones from surface or near-surface sources of contamination.

The Vashon Advance Outwash is generally below the water table; thus, it provides a productive and important aquifer in the Tacoma Upland. It exhibits extremely high transmissivities (up to and exceeding $2 \mathrm{million} \mathrm{gpd} / \mathrm{ft}$ ). Wells completed in the Vashon Advance Outwash can deliver water in amounts up to several thousand gallons per minute. The Vashon Advance Outwash aquifer is moderately isolated from surface contamination where it is overlain by low-permeability materials of the Vashon Till.

The contact between the Vashon glacial deposits and the underlying preVashon unconsolidated sediments is unconformable throughout most of the Tacoma Upland; thus, the contact ranges from $700 \mathrm{ft}$ above $\mathrm{ms} 1$ to more than $300 \mathrm{ft}$ below msl. The sand and gravel aquifers of the pre-Vashon unconsolidated deposits (undefined as to formation) have considerably lower transmissivities than the Vashon Advance Outwash aquifers. Transmissivity appears to range from approximately 10,000 to $30,000 \mathrm{gpd} / \mathrm{ft}$ (USGS 1962), and wells constructed in the pre-Vashon deposits can be adequate for moderately large water supplies. Wells yielding up to $1000 \mathrm{gpm}$ have been completed in these deposits, but yields of a few hundred gallons per minute from wells completed in the pre-Vashon deposits are more common.

Figure 3.4 (after USGS 1962) shows the potentiometric surface (contour lines of water-table elevation above $\mathrm{ms} 1$ ) of the upper, unconfined aquifer located primarily in the Vashon Drift of the Tacoma Upland. Also shown on the figure are general directions of ground-water flow near the study areas. 


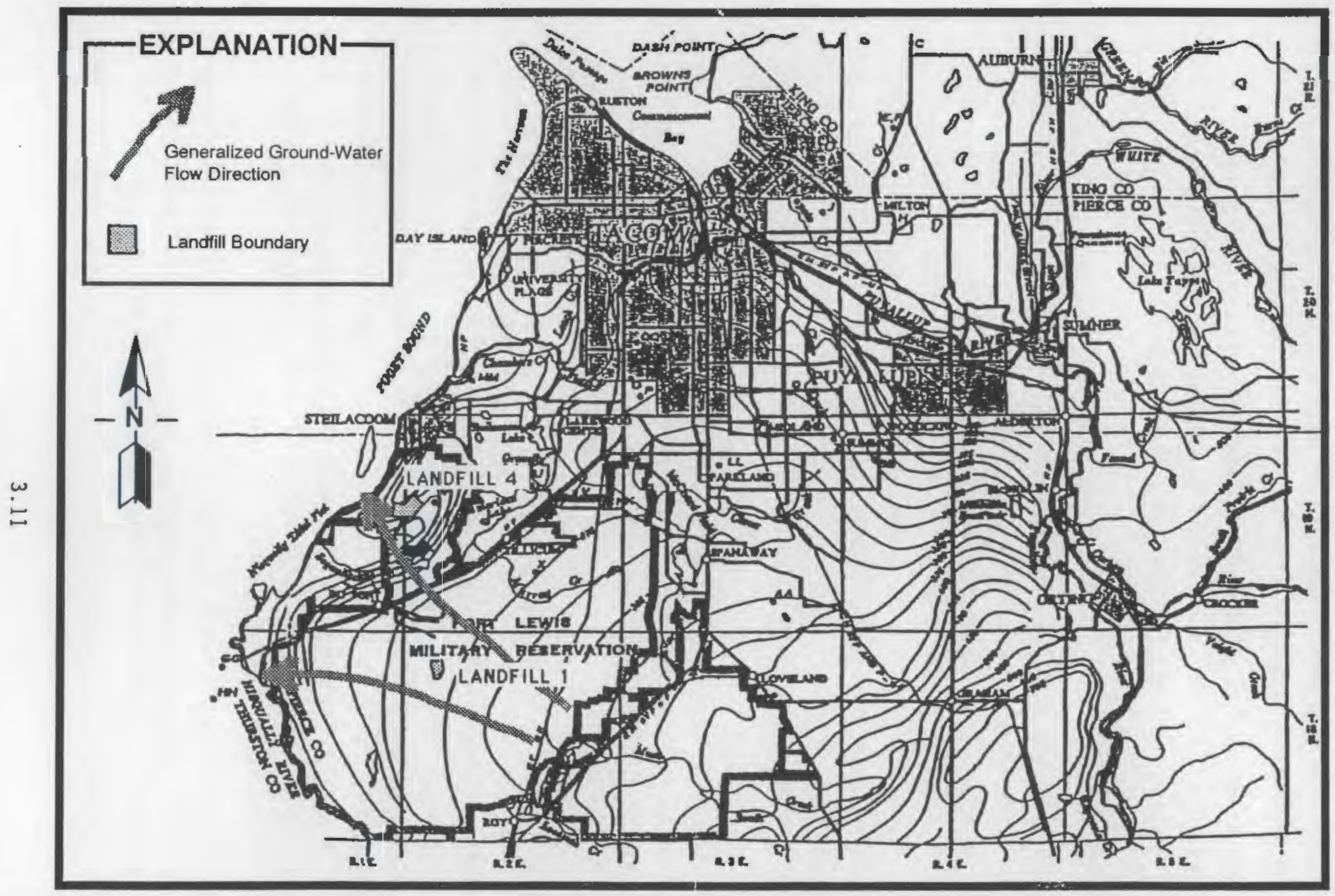

FIGURE 3.4. Regional Potentiometric Surface Map of the Uppermost Unconfined Aquifer Beneath the Tacoma Upland 
Ground-water flow is generally westerly and northwesterly. Ground water in this area ultimately discharges into Puget Sound or the Nisqually River.

The availability of ground water in the pre-Tertiary and Tertiary formations underlying the Tacoma Upland is unknown (but probably smal1), because no wells penetrate to these zones in the area.

\subsection{WATER USE}

Until the early 1900s, ground water was the sole source for most water supplies in the Tacoma Upland. With exception of the City of Tacoma, which started importing large amounts of water from the Green River in 1913, the area still relies on ground water to meet most of the various demands and needs for water. Major users of ground water adjacent to the landfills of interest are the military installations [Camp Murray, Fort Lewis, McChord Air Force Base, Madigan General Hospital, Logistics Center (Mount Rainier Ordnance Depot), etc.], and the communities of DuPont, Tillicum, and Lakewood.

The Lakewood Water District, which supplies municipal and domestic water for much of the American Lake area (including Tillicum), uses an average of approximately 12 million gpd of ground water (Ecology and Environment, Inc. 1986). McChord Air Force Base uses an average of 1.8 million gpd of ground water.

In 1982, six wells and Sequalitchew Spring provided the principal water supply for Fort Lewis. Other smaller wells are used that are not connected to the main supply system. The amount of water used per day for the main supply system varies from 5 to 23 million gpd (an average of approximately 7.8 million gpd) (McMaster 1982). Of this amount, Sequalitchew Spring may supply from 5.5 to 9 million gpd. Although the spring technically may be considered "ground water," the water derived from this source is actually subflow from adjacent American Lake.

Shallow wells completed in the Vashon Drift may be subject to contamination from surface and near-surface sources. Some of the older municipal water-supply wells and most of the wells used for domestic water supply in the Tacoma Upland are relatively shallow and do derive ground water from the 
Vashon Drift aquifers. Wells completed below the Vashon Drift and that produce water from the lower aquifers in the area are generally isolated and protected from sources of surface and near-surface contamination.

Most of the wells adjacent to Landfills 1 and 4 are believed to be completed far below the Vashon Drift, and, thus, should not be affected by past waste disposal activities. Sequalitchew Spring is an exception, as this water source could be affected by surface contamination and also may be affected by changes in adjacent lake-water quality.

\subsection{CRITICAL HABITATS}

No known threatened or endangered species of plants or animals exist within the vicinity of Landfills 1 and 4 . Additions are made to both the Federal and State lists when new species are approved for addition, and these lists should be checked periodically. There are also no wildlife refuges or game preserves within this area. At present, there are no known critical habitats. Two active bald eagle nests are present, although both are outside the landfill areas: one near Camp Murray and one near the Veteran's Hospital. One inactive nest is known, which is also outside the study areas.

Coho salmon and rainbow trout are reared in pens within Sequalitchew Lake under an ongoing cooperative agreement with the State of Washington.

Migratory waterfowl, primarily ducks and geese, are found at American and Sequalitchew lakes during the migration season. A certain number of ducks, geese, and coots winter at these lakes. Wright Lake is also used by waterfowl, but goes dry in some years.

A number of sites of former cultural activity are known, particularly around the lake shores. Any major construction or modification activities would require more detailed reconnaissances, but the scope of activities under this study should not affect any of these sites. 

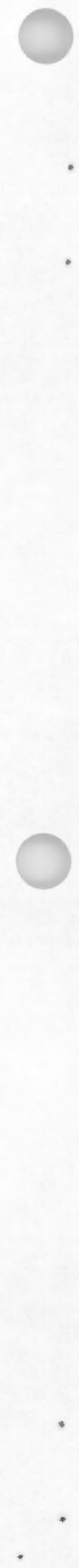


\subsection{SITE INVESTIGATION METHODS}

Investigations at the two landfills included activities aimed at locating the landfill boundaries, installing new ground-water monitoring wells, remediating existing monitoring wells, sampling the ground-water, and analyzing samples. The methods used in these investigations are described below, along with quality assurance and control activities.

\subsection{LOCATING LANDFILL BOUNDARIES}

To locate landfill boundaries, numerous aerial photographs of the two landfills were examined, and the physical features of the landfills were plotted on base maps. Two sets of aerial photographs were available for Landfill 1. These were taken in July 1951 and May 1970. Three sets of aerial photographs were available for Landfill 4. These were taken in July 1951, May 1970, and July 1976 (see Table 2.1).

Because aerial photographs did not provide sufficient information to determine the boundaries of Landfill 4, surface geophysical surveys were conducted on July 18 and 19 and July 27 and 28, 1988. Geophysical data were collected along 30 traverse lines located in one area where the boundary was fairly well known and three areas where the boundaries were poorly known. These lines varied in length from 200 to $1200 \mathrm{ft}$ and were marked with wooden stakes at $100-\mathrm{ft}$ intervals along their lengths. The locations of the stakes were established using a Brunton Compass and pacing. Geophysical-sensing instruments used in this survey were 1) a ground-penetrating radar unit, 2) a ground-conductivity meter, and 3) a metal detector. Each of these instruments is discussed below. An informal report on the results of this survey is presented in Appendix A.

\subsubsection{Ground-Penetrating Radar}

The ground-penetrating radar unit used in this survey was a Geophysical Survey Systems, Inc. (GSSI), Model SIR 7 with a $120-\mathrm{MHz}$ (nominal) antenna (GSSI Mode1 3110) that transmits and receives radar signals in a frequency band of approximately 50 to $250 \mathrm{MHz}$. The antenna, mounted on a fiber-glass sled, was pulled along the survey lines by a small all-terrain vehicle (ATV). 
Radar signals reflected from subsurface objects or interfaces were detected by the antenna and were transmitted by cable to control and recording modules mounted on the ATV. There, the signals were amplified, digitized, and recorded on digital tape cartridges for later playback and processing. This instrument exhibits good spatial resolution and can produce quasi-pictorial images of both metallic and nonmetallic objects or materials in the ground.

\subsubsection{Ground-Conductivity Meter}

The ground-conductivity meter used in the survey was a Geonics EM31 electromagnetic induction sensor. This hand-carried device operates by transmitting an oscillatory magnetic field into the ground, then detecting the secondary magnetic field produced by the electric currents that are induced in the ground. The device exhibits relatively poor spatial resolution, but can respond to both metallic and nonmetallic waste deposits. The data produced by this instrument were digitized and recorded by a small data recorder carried by the operator. The data were subsequently transferred to a computer for scaling and display.

\subsubsection{Metal Detector}

The metal detector used in the survey was a Fisher M-Scope, Model TW- 6 . This instrument was originally marketed as a pipe and cable detector, but has proven to be effective in detecting buried metallic waste materials at depths of a few feet. The metal detector was carried by hand along the survey lines until a response was obtained. The location of the response was then recorded in a field notebook.

\subsection{INSTALLING MONITORING WELLS}

Ten ground-water monitoring wells were installed from August through October 1988 as part of this study ( $\mathrm{six}$ in the vicinity of Landfill 4 and four near Landfill 1). These wells were designated by the corresponding landfill number, the designation "PNL," and the consecutive number at the time they were drilled (e.g., LF1-PNL2 corresponds to the second well drilled during the PNL study at Landfill 1). The new wells were installed by Onwego Drilling Co., Kennewick, Washington. Specifications for these wells are provided in Appendix B and are consistent with the requirements of 
WAC 173-160. This section describes the methods used for drilling, sediment sampling, construction, geophysical logging, development, and surveying of the new wells.

\subsubsection{Drilling Methods}

Boreholes, for installation of the monitoring wells, were drilled with continuous flight, hollow-stem auger and cable-tool methods. The augerdrilled boreholes were drilled using 8-in.-OD, 4-in.-ID (outside and inside diameter) hollow-stem auger flights. The boreholes drilled with a cable-tool rig used either hard-tool or drive-barrel methods. Hard-tool drilling consisted of driving a solid metal bit into the sediments, breaking them up, and mixing them with added water (obtained from a nearby fire hydrant) to form a slurry, which was then bailed out of the borehole. After reaching the desired sampling depth, the borehole was bailed clean so that sediment samples could be collected. When using the drive-barrel method, a drive barrel (a short length of heavy-walled pipe) was driven into the sediments and withdrawn, and the sediments were removed from the drive barrel.

\subsubsection{Geologic Sampling and Sediment Characterization Methods}

Sediment samples were collected at 5-ft intervals or at major 1ithologic changes encountered during drilling. The samples were collected using a 2-in. split-spoon sampler when auger drilling, a 4-in. split-spoon sampler when hard-tool drilling, and directly from the drive barrel when drive-barrel drilling. The procedure used for collecting split-spoon samples is provided in Appendix C. These samples were photographed, and subsamples were collected in glass, pint jars. The samples have been placed in storage for a permanent record of the sediments encountered during drilling.

Once the sample was photographed, it was physically described by a wellsite geologist, and the information was recorded on daily drill logs. The sediments were characterized based on estimated particle-size distribution, sorting, gross mineralogy, clast roundness, general color of the fines, reaction to $10 \%$ hydrochloric acid ( $\mathrm{HCl}$ ), consolidation of the formation, changes from the previous sample, and any unusual findings. These samples were collected and documented in accordance with the procedure provided in Appendix $D$ and the guidance found in Last and Liikala (1987). The as-built diagrams 
found in Appendix E show the geologic sediment classification names given to the sediments at each sample interval at each well. The criteria used by the well-site geologist to classify the sediments are based on the ternary diagram shown in Figure 4.1.

In addition to sampling the sediment for physical characterization, sediment moisture samples were collected for information on the moisture profile of the unsaturated zone. These samples were collected immediately after the sampler was pulled from the borehole. The samples were placed in airtight tin containers, sealed with Teflon tape, labeled, and enclosed in plastic bags to prevent moisture loss. The samples were kept shaded and transferred at the end of the week to a refrigerator at PNL's Richland,

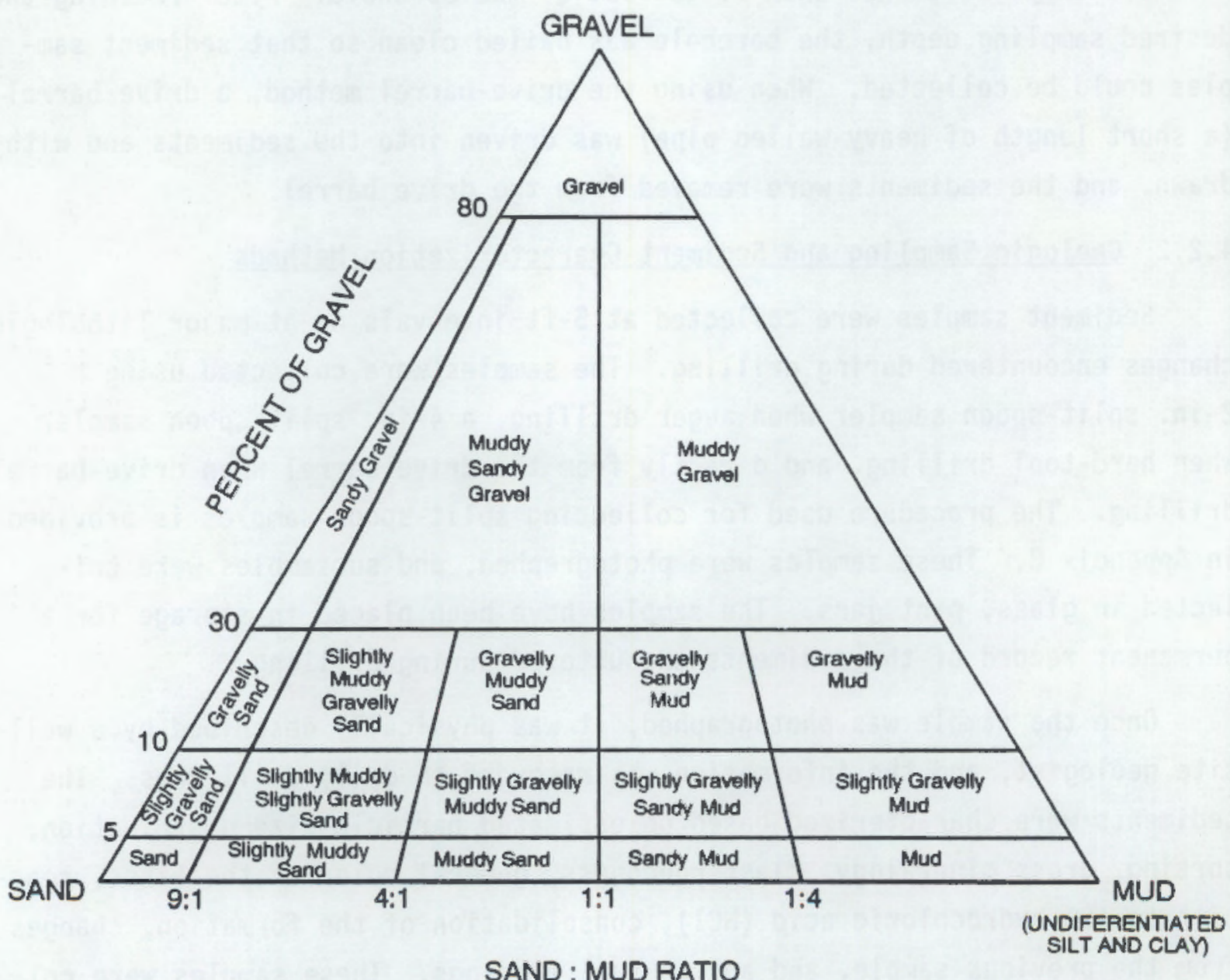

FIGURE 4.1. Ternary Diagram for Naming Sediments (from Folk 1974) 
Washington, location. Within a few days after their arrival, the samples were removed from the plastic bags, weighed, oven-dried at $105^{\circ} \mathrm{C}$ for $24 \mathrm{hr}$, and, finally, reweighed. This routine is in accordance with ASTM procedure D 2216 (ASTM 1986). These data are presented in Appendix F.

Water-level measurements were taken by a well-site geologist using an electrical tape (E-tape) during drilling. These measurements provided information on the static water level that was then used to determine the placement depth of the screened interval. These measurements can be found on the drilling logs in Appendix E.

\subsubsection{Monitoring Well Construction}

The monitoring wells were constructed as shown in Figure 4.2. These wells were constructed with polyvinyl chloride (PVC) casing (schedule 40, ASTM 1785) and $15 \mathrm{ft}$ of slotted PVC screen (20 slot, 0.02-in. opening). The screen was placed with $5 \mathrm{ft}$ extending above and $10 \mathrm{ft}$ below the static water level. This design was selected to compensate for fluctuations in the water leve1. Only $10 \mathrm{ft}$ of screen were installed in well LF4-PNL4 because of a thin saturated zone encountered above a clay/silt layer. In the auger boreholes, wells were constructed with 2-in.-dia PVC casing and screen (because of the small inside diameter of the auger flights), while those constructed in cable-tool boreholes were constructed with 4-in.-dia PVC. After installing the PVC casing and screen, silica sand was installed in the annulus to approximately 3 to $5 \mathrm{ft}$ above the top of the PVC screen. Three to $5 \mathrm{ft}$ of 0.25 -in. bentonite pellets were then placed on top of the sandpack. The remaining annulus, to approximately 1 to $3 \mathrm{ft}$ below land surface, was sealed using medium-sized bentonite chunks. Note that the annular seal materials were added while the auger flights or temporary casing (depending on drilling method) were extracted from the ground. The field geologist and driller carefully monitored the annular seal placement during completion, so that the natural formation would not cave around the PVC and thus jeopardize the integrity of the annular seal. Once the casing or auger flights were completely removed, a 1 - to 3 -ft-deep void around the PVC remained. A 5-ft-long, 6-in.-dia protective steel surface casing was installed around the PVC, and a 4 -ft by $4-\mathrm{ft}$ concrete surface pad and surface seal were poured in 


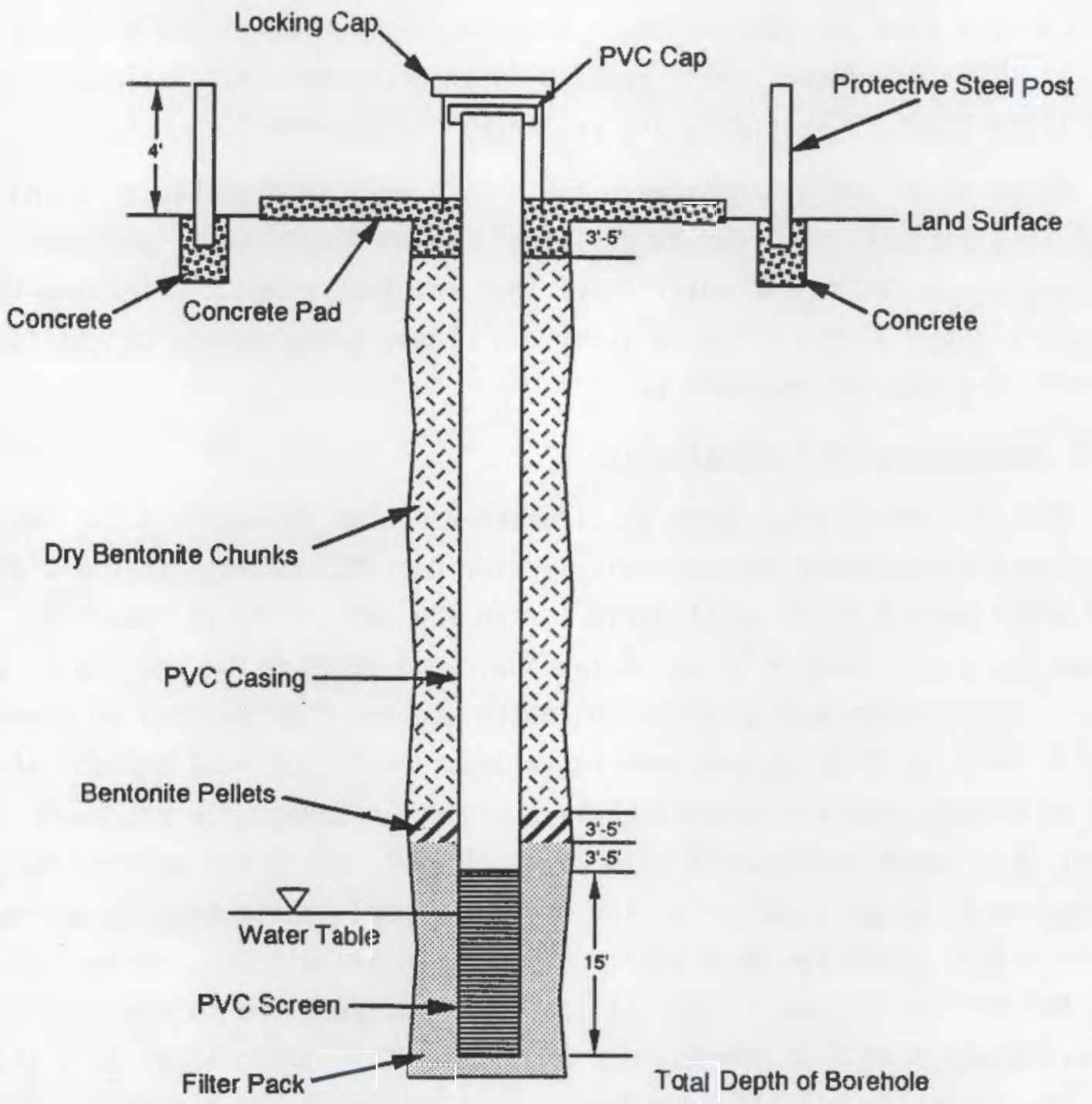

FIGURE 4.2. Schematic Design of the Newly Installed (1988) Monitoring Wells at Landfills 1 and 4

the remaining void. Pre-mix concrete or Portland cement mixed with local sand and gravel was used for the concrete pad and surface seal.

A brass marker provided by the U.S. Army Corps of Engineers was placed in each pad and embossed with the well identification number on completion of the surveying effort (see Subsection 4.2.7). Four protective posts were placed in concrete around each well. Finally, the four protective posts and 
the surface protective casing were painted safety yellow, and an aluminum cap and lock were attached to the top of the surface protective casing.

A field geologist documented the construction of each well on daily drill logs, well completion inspection forms, and as-built diagrams. The procedure used to collect and document drilling data is provided in Appendix D. The we1l completion inspection forms, as-built diagrams, and daily logs for each new (1988) well are presented in Appendix $E$.

\subsubsection{Gamma-Ray Borehole Geophysical Logging}

Gamma-ray borehole geophysical logging was conducted when the borehole reached maximum depth and before the well was completed. There were two exceptions to this: we1l LF4-PNL1 was logged after well completion, and well LF4-PNL3 was not logged.

The boreholes were logged using a gross-gamma probe. This probe is designed to measure the naturally occurring gamma radiation found in the sediments in the borehole. This probe is most useful in identifying clay or clay-rich zones that characteristically contain elevated concentrations of natural gamma-emitting radioactive isotopes, such as potassium-40. Sand and gravel, on the other hand, can be identified by a low natural-gamma response. Results of the natural-gamma logging are useful in confirming the lithologic divisions identified by the well-site geologist and in identifying the location of low-permeability (clay-rich) zones. The results of the naturalgamma logging are presented with the as-built diagrams in Appendix E.

\subsubsection{Water-Level Measurement}

Periodic water-level measurements were taken on all newly completed (1988) and existing (1981 and 1984) wells on the same day. These measurements were made in duplicate using either a steel tape or an electrical tape, according to the PNL procedure (provided in Appendix G). These measurements can be found in the discussions on ground-water flow in the evaluation of each landfill. 


\subsubsection{We11 Development Methods}

The new 2-in.-dia wells were developed using a l-in.-dia dart bailer, air-lifting, and/or a HydroStar positive displacement pump (product of Instrumentation Northwest, Incorporated, Redmond, Washington). The HydroStar pump (discharging at a rate of 3 to $5 \mathrm{gpm}$ ) proved to be the most effective in developing these small-diameter wells.

The new 4-in.-dia wells and existing Landfill 4 (1981) wells were developed using a dart bailer and a 1/3-h.p. submersible pump (discharging at a rate of 10 to $12 \mathrm{gpm}$ ). A HydroStar pump was also used in well LF1-PNL3; however, the low permeability sediments encountered by this well yielded too little water to enable adequate development. The existing wells at Landfill 1 (1984) were not re-developed as part of this study because no subsurface remediation was performed on these wells.

A11 new wells except LF1-PNL3 were developed until the turbidity of the discharge water was visibly low or absent. This was determined by visually examining water discharged into a clean white 5-gal bucket. Tables 4.1 and 4.2 provide a summary of the well development activities associated with each we11. These tables include information on the date the wells were structurally complete, the date well development started, the date it was completed, the total number of saturated zone well volumes extracted during development, and the final water quality description. Further discussion of well development activities is provided in Section 4.4.

\subsubsection{Surveying}

Existing wells near Landfills 1 (1984 wells) and 4 (1981 wells) were initially surveyed in 1988 and 1982, respectively. These surveys were conducted by the Seattle District, U.S. Army Corps of Engineers, using the Washington State coordinate system for horizontal control and the National Geodetic Vertical Datum 1927 (NGVD 1927) for vertical control. Elevations for the 1981 wells were surveyed to the top of the casing. Those for the 1984 wells were surveyed to a 2- by $2-\mathrm{ft}$ wooden hub installed $2 \mathrm{ft}$ from the well. All horizontal locations were surveyed to the center of each casing. 
TABLE 4.1. Development Information for Wells at Landfill 1

\begin{tabular}{|c|c|c|c|c|c|c|c|}
\hline \multirow[b]{2}{*}{ Well Number } & \multirow[b]{2}{*}{$\begin{array}{c}\text { Well } \\
\text { Diameter } \\
\text { (in.) }\end{array}$} & \multirow[b]{2}{*}{$\begin{array}{c}\text { Date } \\
\text { Well } \\
\text { Completed }\end{array}$} & \multicolumn{5}{|c|}{ WELL DEVELOPMENT HISTORY } \\
\hline & & & $\begin{array}{c}\text { Date } \\
\text { Development } \\
\text { Started }\end{array}$ & $\begin{array}{c}\text { Date } \\
\text { Development } \\
\text { Completed }\end{array}$ & $\begin{array}{c}\text { Develpment } \\
\text { Methods } \\
\text { Used }\end{array}$ & $\begin{array}{l}\text { Total Well } \\
\text { Volumes } \\
\text { Removed }\end{array}$ & Final Water Quality \\
\hline 84-CD-LF-1* & 4 & $11-05-84$ & NA & NA & NA & NA & NA \\
\hline 84-CD-LF-2 ${ }^{\star}$ & 4 & $10-31-84$ & NA & NA & NA & NA & NA \\
\hline 84-CD-LF-3* & 4 & $11-09-84$ & NA & NA & NA & NA & NA \\
\hline 84-CD-LF-4* & 4 & $11-15-84$ & NA & NA & NA & NA & NA \\
\hline LF1-PNL1 & 2 & $08-19-88$ & $08-25-88$ & $12-13-80$ & $\begin{array}{c}\text { Bailer } \\
\text { Hydrostar }\end{array}$ & 341 & Slightly Turbid \\
\hline LF1-PNL2 & 2 & $08-19-88$ & $08-17-88$ & $09-08-88$ & $\begin{array}{c}\text { Istr. NW pump } \\
\text { Bailer } \\
\text { Hydrostar }\end{array}$ & 287 & Slightly Turbid \\
\hline LF1-PNL3 & 4 & $08-25-88$ & $08-24-88$ & ND & $\begin{array}{c}\text { Submersible } \\
\text { Bailer } \\
\text { Hydrostar }\end{array}$ & $\begin{array}{c}7 \\
\text { (distilled water } \\
\text { added) }\end{array}$ & Very Turbid \\
\hline LF1-PNL4 & 4 & $09-01-88$ & $08-31-88$ & $09-08-88$ & $\begin{array}{c}\text { Bailer } \\
\text { Submersible }\end{array}$ & 48 & Clean \\
\hline
\end{tabular}

* These wells were not re-developed as part of this study.

NA: Not Applicable.

ND: Not Adequately Developed. 
TABLE 4.2. Development Information for Wells at Landfill 4

\begin{tabular}{|c|c|c|c|c|c|c|c|}
\hline \multirow[b]{2}{*}{ Well Number } & \multirow[b]{2}{*}{$\begin{array}{c}\text { Well } \\
\text { Diameter } \\
\text { (in.) }\end{array}$} & \multirow[b]{2}{*}{$\begin{array}{c}\text { Date } \\
\text { Completed }\end{array}$} & \multicolumn{5}{|c|}{ WELL DEVELOPMENT HISTORY } \\
\hline & & & $\begin{array}{c}\text { Date } \\
\text { Development } \\
\text { Started }\end{array}$ & $\begin{array}{c}\text { Date } \\
\text { Development } \\
\text { Completed }\end{array}$ & $\begin{array}{l}\text { Develpment } \\
\text { Methods } \\
\text { Used }\end{array}$ & $\begin{array}{l}\text { Total Well } \\
\text { Volumes } \\
\text { Removed }\end{array}$ & Final Water Quality Description \\
\hline LF 4-1* & 4 & $04-28-81$ & $10-30-88$ & $10-30-88$ & $\begin{array}{c}\text { Bailer } \\
\text { Submersible }\end{array}$ & 80 & Clear \\
\hline LF4-2* & 4 & $04-29-81$ & $10-30-88$ & $10-30-88$ & $\begin{array}{c}\text { Bailer } \\
\text { Submersible }\end{array}$ & 40 & Clear \\
\hline LF 4-4* & 4 & $05-01-81$ & $10-30-88$ & $10-30-88$ & $\begin{array}{c}\text { Bailer } \\
\text { Submersible }\end{array}$ & 110 & Clear \\
\hline LF4-11* & 4 & $05-12 \cdot 81$ & $10-30-88$ & $10-30-88$ & $\begin{array}{c}\text { Bailer } \\
\text { Submersible }\end{array}$ & 61 & Clear \\
\hline LF4-PNL1 & 4 & $08 \cdot 09-88$ & $08-08-88$ & $09-01-88$ & $\begin{array}{c}\text { Bailer } \\
\text { Submersible }\end{array}$ & 218 & Clear \\
\hline LF4-PNL2 & 2 & $08-09-88$ & $08+23-88$ & $09-07-88$ & $\begin{array}{l}\text { Bailer } \\
\text { Hydrostar }\end{array}$ & 30 & Clear \\
\hline LF4-PNL3 & 2 & $08 \cdot 09-88$ & $08-08-88$ & $12 \cdot 14-88$ & $\begin{array}{l}\text { Air Litt } \\
\text { Bailed } \\
\text { Hydrostar }\end{array}$ & 187 & Clear \\
\hline LF4-PNL4 & 4 & $10-05-88$ & $10-06-88$ & $10-06-88$ & Submersible & 57.5 & Clear \\
\hline LF 4-PNL5 & 4 & $10-07-88$ & $10-07-88$ & $10-14-88$ & $\begin{array}{c}\text { Bailer } \\
\text { Submersib!e }\end{array}$ & 151 & Clear \\
\hline LF4-PNL6 & 4 & $10-12-88$ & $10-12-88$ & $10-14-88$ & $\begin{array}{c}\text { Bailer } \\
\text { Submersible }\end{array}$ & 70 & Clear \\
\hline
\end{tabular}

- These wells were re-developed as part of this studiy. 
A71 new (1988) and existing (1981 and 1984) wells were surveyed in the spring of 1989 by Horton Dennis and Associates, Inc., using the Washington State coordinate system for horizontal control and NGVD 1929 for vertical control. Horizontal and vertical values were established for the center of the brass marker installed in each well pad. Vertical values were also established for the north rim of the PVC well casing located inside each carbon-steel protective casing. An error was observed in the horizontal location of well LF4-PNL5. It was also noted that the identification of welTs LF4-1 and LF4-2 were backwards. These errors were later corrected via personal communication with Ms. Joan Shafer (U.S. Army Corps of Engineers Seattle district). These data are provided in Appendix $H$.

\subsection{REMEDIATING AND ABANDONING WELLS}

In addition to the drilling and installation of the new monitoring wells, remediation was performed on the existing wells near Landfills 1 and 4. The existing wells were geophysically logged (by the methods noted above) before remediation.

The existing wells near Landfill I were drilled in 1984 by Richardson Drilling. The wells were drilled using a cable-tool rig and were completed with 4-in.-dia PVC casing and 20-slot PVC screen. The original drilling logs for the drilling and completion of these wells are found in Appendix I.

These wells (identified as 84-CD-LF-1, -LF-2, $-L F-3$, and $-L F-4$ ) were in good physical condition, except for the surface completion. All of the wells had a carbon steel protective casing and locking well cap; however, none of the wells had a concrete surface pad or protective posts, and wel1 84-CD-LF-1 had a very loose protective surface casing. The remedial work performed at each of these wells consisted of installing l) a 4-ft by 4-ft concrete surface pad, 2) four protective posts around the pad, 3) a brass marker in the concrete pad, and 4) an aluminum cap with lock at the top of the protective surface casing. The protective posts and casing were painted safety yellow. The protective surface casing on well $84-C D-L F-1$ was reset in concrete before placing the surface pad. Drill logs of the remedial work are also presented in Appendix I. 
The existing wells at Landfill 4, identified as Fort Lewis 1, 2, 3, 4, and 11 (referred to in this report as LF4-1, -2, -3, -4 and -11), were drilled in 1981 by Tacoma Pump and Drilling Co., Inc., Graham, Washington. The wells were drilled with a rotary rig and were completed using 4-in.-dia, schedule-40 PVC pipe. The PVC sections were connected by nonthreaded sliptype couplings, and it appears that glue was used in connecting the male/ female ends. The screened intervals were perforated by drilling evenly spaced, 0.25-in. holes in the PVC casing. No plug or end cap was installed in the bottom of the perforated section. More information on the completion of these wells can be found in the original drilling logs in Appendix I. Except for the pipe in well LF4-3, the PVC pipe in these wells appeared to be in good condition.

The overall physical condition of these wells was fair to very poor, and remedial work was necessary both above and below ground. Well LF4-3 had $30 \mathrm{ft}$ of 8-in.-dia carbon steel casing in the ground, and the PVC was broken apart approximately $21 \mathrm{ft}$ below land surface. There were approximately $13 \mathrm{ft}$ of bentonite annular seal extending from the top of the perforated PVC pipe to $29 \mathrm{ft}$ below ground. The water bailed from inside the PVC pipe was very stagnant smelling and contained pieces of wood and decayed animal and insect remains. This well was abandoned in accordance with WAC-173-160-915 as follows: 1) the PVC casing and screen were speared and removed from the hole, 2) medium-sized bentonite chunks were emplaced in the borehole as the 8-in. carbon steel casing was removed, 3 ) a cement grout with $5 \%$ bentonite was set in the upper $6.5 \mathrm{ft}$ of the borehole to provide a rigid surface sea1, and 4) a 2 -ft by 2-ft concrete pad with a 6 -in. surface casing (2.5-ft stickup) was emplaced to permanently mark the abandoned well.

The remaining 1981 wells at Landfill 4 were in fair physical condition. These wells required removal of the 8 -in. carbon steel casing and installation of the bentonite annular seal as the steel casing was removed and the 4-in. PVC casing was left in place. Bentonite slurry was required in two of the wells, because the dry bentonite tended to form a bridge at the bottom of the casing. Finally, a 1 - to 2-ft cement surface seal was poured, and the surface completion work (pad, posts, brass marker, cap, and painting) was performed. 
The only complication encountered during the remediation effort was at well LF4-11. At this well, the PVC casing was pulled back approximately $8 \mathrm{ft}$ in an attempt to remove a bentonite plug that had formed inside the PVC. The benonite was successfully washed out of the PVC casing by jetting with clean drilling water. Pulling back the PVC casing should not pose any problems, however, because the top of the perforated PVC casing is still below the bentonite seal. Drill logs kept during the remediation work are included in Appendix I.

The four existing (1981) wells at Landfill 4 were redeveioped using a dart bailer and 1/3-h.p. submersible pump. None of the existing (1984) wells at Landfill 1 were redeveloped because of time constraints.

\subsection{GROUND-WATER SAMPLING}

Ground-water samples were collected from the new (1988) and existing (1981 and 1984) wells around Landfills 1 and 4. Two sets of samples were collected from each new (1988) well, and one set of samples was collected from the existing (1984) wells around Landfill 1. Three sets of samples were collected from all new (1988) and existing (1984) wells around Landfill 4 except well LF4-PNL6, which was sampled only twice. All samples were collected during five separate sampling events: 1) August, 2) September, 3) early October, 4) late October, and 5) December 1988. The August, September, and early october sampling events were conducted only to indicate what chemical constituents might be present in each well and to assist in identifying the need and location of additional wells. The 1ate October and December sampling events were conducted to evaluate the concentrations and distribution of potential contaminants beneath the landfills. The groundwater sampling procedures followed during each sampling event are presented in Appendix $\mathrm{J}$.

A description of each sampling event is provided below. Tables 4.3 and 4.4 summarize the sampling history for each landfill. These tables provide the sampling dates, indicate if the well had been developed before sampling, the sampling methods used, the number of saturated well volumes purged before sampling, and a qualitative estimate of the sample's representativeness. A 
IABLE 4.3. Landfill l Ground-Water Sampling History

\begin{tabular}{|c|c|c|c|c|c|}
\hline Well Number & $\begin{array}{l}\text { Sampling } \\
\text { Date }\end{array}$ & $\begin{array}{l}\text { Development } \\
\text { Complete? }\end{array}$ & $\begin{array}{l}\text { Sample } \\
\text { Collection } \\
\text { Method }\end{array}$ & $\begin{array}{c}\text { Well } \\
\text { Volumes } \\
\text { Purged }\end{array}$ & $\begin{array}{c}\text { Sample } \\
\text { Represntativeness }\end{array}$ \\
\hline 84-CD-LF-1 & $12-13-88$ & NR & Bailer & 0.4 & Fair \\
\hline 84-CD-LF-2 & $12-14-88$ & NR & Bailer & 0.4 & Fair \\
\hline 84-CD-LF-3 & $12-14-88$ & NR & Bailer & 0.4 & Fair \\
\hline 84-CD-LF-4 & $12-14-88$ & NR & Bailer & 0.4 & Fair \\
\hline LF1-PNL1 & $\begin{array}{l}08-24-88 \\
12-13-88\end{array}$ & $\begin{array}{l}\text { No } \\
\text { Yes }\end{array}$ & $\begin{array}{l}\text { Bailer } \\
\text { Hydrostar }\end{array}$ & $\begin{array}{l}1.7 \\
341\end{array}$ & $\begin{array}{l}\text { Poor } \\
\text { Good (water turbid) }\end{array}$ \\
\hline LF1-PNL2 & $\begin{array}{l}09-08-88 \\
12-14-88\end{array}$ & $\begin{array}{l}\text { Yes } \\
\text { Yes }\end{array}$ & $\begin{array}{l}\text { Hydrostar } \\
\text { Bailer }\end{array}$ & $\begin{array}{l}198 \\
1.7\end{array}$ & $\begin{array}{l}\text { Good (water turbid) } \\
\text { Good }\end{array}$ \\
\hline LF1-PNL3 & $\begin{array}{l}09-09-88 \\
12-14-88\end{array}$ & $\begin{array}{l}\text { No } \\
\text { No }\end{array}$ & $\begin{array}{l}\text { Hydrostar } \\
\text { Bailer }\end{array}$ & $\begin{array}{c}4 \\
0.4\end{array}$ & $\begin{array}{l}\text { Poor } \\
\text { Poor }\end{array}$ \\
\hline L.F1-PNL4 & $\begin{array}{l}09-08-88 \\
12-13-88\end{array}$ & $\begin{array}{l}\text { Yes } \\
\text { Yes }\end{array}$ & $\begin{array}{l}\text { Hydrostar } \\
\text { Bailer }\end{array}$ & $\begin{array}{l}12 \\
0.4\end{array}$ & $\begin{array}{l}\text { Very Good } \\
\text { Good }\end{array}$ \\
\hline
\end{tabular}

NR: No Record. These wells were not developed as part of this study. 
TABLE 4.4. Landfill 4 Ground-Water Sampling History

\begin{tabular}{|c|c|c|c|c|c|}
\hline Well Number & $\begin{array}{l}\text { Sampling } \\
\text { Date }\end{array}$ & $\begin{array}{l}\text { Development } \\
\text { Complete? }\end{array}$ & $\begin{array}{l}\text { Sample } \\
\text { Collection } \\
\text { Method }\end{array}$ & $\begin{array}{c}\text { Well } \\
\text { Volumes } \\
\text { Purged }\end{array}$ & $\begin{array}{c}\text { Sample } \\
\text { Represntativeness }\end{array}$ \\
\hline LF4-1 & $\begin{array}{l}10-06-88 \\
10-30-88 \\
12-15-88\end{array}$ & $\begin{array}{l}\text { No } \\
\text { Yes } \\
\text { Yes }\end{array}$ & $\begin{array}{c}\text { Bailer } \\
\text { Submersible } \\
\text { Bailer }\end{array}$ & $\begin{array}{l}0.4 \\
15 \\
0.4\end{array}$ & $\begin{array}{l}\text { Poor } \\
\text { Very Good } \\
\text { Good }\end{array}$ \\
\hline LF4-2 & $\begin{array}{l}10-06-89 \\
10-30-88 \\
12-14-88\end{array}$ & $\begin{array}{l}\text { No } \\
\text { Yes } \\
\text { Yes }\end{array}$ & $\begin{array}{c}\text { Bailer } \\
\text { Submersible } \\
\text { Bailer }\end{array}$ & $\begin{array}{l}0.4 \\
80 \\
0.4\end{array}$ & $\begin{array}{l}\text { Poor } \\
\text { Very Good } \\
\text { Good }\end{array}$ \\
\hline LF4-4 & $\begin{array}{l}10-06-88 \\
10-30-88 \\
12-15-88\end{array}$ & $\begin{array}{l}\text { No } \\
\text { Yes } \\
\text { Yes }\end{array}$ & $\begin{array}{c}\text { Bailer } \\
\text { Submersible } \\
\text { Bailer }\end{array}$ & $\begin{array}{l}0.4 \\
77 \\
0.4\end{array}$ & $\begin{array}{l}\text { Poor } \\
\text { Very Good } \\
\text { Good }\end{array}$ \\
\hline LF4-11 & $\begin{array}{l}10-06-88 \\
10-30-88 \\
12-15-88\end{array}$ & $\begin{array}{l}\text { No } \\
\text { Yes } \\
\text { Yes }\end{array}$ & $\begin{array}{c}\text { Bailer } \\
\text { Submersible } \\
\text { Bailer }\end{array}$ & $\begin{array}{l}0.4 \\
21 \\
0.4\end{array}$ & $\begin{array}{l}\text { Poor } \\
\text { Very Good } \\
\text { Good }\end{array}$ \\
\hline LF4-PNL1 & $\begin{array}{l}08-24-88 \\
10-06-88 \\
12-15-88\end{array}$ & $\begin{array}{l}\text { No } \\
\text { Yes } \\
\text { Yes }\end{array}$ & $\begin{array}{l}\text { Bailer } \\
\text { Bailer } \\
\text { Bailer }\end{array}$ & $\begin{array}{l}0.4 \\
0.4 \\
0.4\end{array}$ & $\begin{array}{l}\text { Poor } \\
\text { Good } \\
\text { Good }\end{array}$ \\
\hline LF4-PNL2 & $\begin{array}{l}09-08-88 \\
10-06-88 \\
12-15-88\end{array}$ & $\begin{array}{l}\text { Yes } \\
\text { Yes } \\
\text { Yes }\end{array}$ & $\begin{array}{l}\text { Hydrostar } \\
\text { Bailer } \\
\text { Bailer }\end{array}$ & $\begin{array}{l}28 \\
1.7 \\
1.7\end{array}$ & $\begin{array}{l}\text { Very Good } \\
\text { Good } \\
\text { Good }\end{array}$ \\
\hline LF4-PNL3 & $\begin{array}{l}08-24-88 \\
10-06-88 \\
12-14-88\end{array}$ & $\begin{array}{l}\text { No } \\
\text { No } \\
\text { Yes }\end{array}$ & $\begin{array}{l}\text { Bailer } \\
\text { Bailer } \\
\text { Hydrostar }\end{array}$ & $\begin{array}{l}1.7 \\
1.7 \\
184\end{array}$ & $\begin{array}{l}\text { Poor } \\
\text { Poor } \\
\text { Very Good }\end{array}$ \\
\hline LF4-PNL4 & $\begin{array}{l}10-06-88 \\
10-30-88 \\
12-15-88\end{array}$ & $\begin{array}{l}\text { Yes } \\
\text { Yes } \\
\text { Yes }\end{array}$ & $\begin{array}{c}\text { Bailer } \\
\text { Submersible } \\
\text { Bailer }\end{array}$ & $\begin{array}{l}56 \\
15 \\
0.4\end{array}$ & $\begin{array}{l}\text { Very Good } \\
\text { Very Good } \\
\text { Good }\end{array}$ \\
\hline LF4-PNL5 & $\begin{array}{l}10-07-88 \\
10-30-88 \\
12-15-88\end{array}$ & $\begin{array}{l}\text { No } \\
\text { Yes } \\
\text { Yes }\end{array}$ & $\begin{array}{c}\text { Steel Bailer } \\
\text { Submersible } \\
\text { Bailer }\end{array}$ & $\begin{array}{l}21 \\
20 \\
0.4\end{array}$ & $\begin{array}{l}\text { Good (water turbid) } \\
\text { Very Good } \\
\text { Good }\end{array}$ \\
\hline LF4-PNL6 & $\begin{array}{l}10-30-88 \\
12-15-88\end{array}$ & $\begin{array}{l}\text { Yes } \\
\text { Yes }\end{array}$ & $\begin{array}{c}\text { Submersible } \\
\text { Bailer }\end{array}$ & $\begin{array}{l}27 \\
0.4\end{array}$ & $\begin{array}{l}\text { Very Good } \\
\text { Good }\end{array}$ \\
\hline
\end{tabular}

NR: No Record 
sample was considered very good if the well had been developed and at least three well volumes purged before sample collection. A sample was still considered good if the well was developed but had not been purged before sample collection. All other samples are considered poor.

\subsubsection{Auqust Sampling}

The purpose of the August sampling event was simply to obtain an initial indication of the chemical constituents in the ground-water beneath various portions of Landfill 4. This information would then be used in selecting the location of additional wells. The objective was to collect ground-water samples from the first three new (1988) wells installed around Landfill 4. However, one of these wells (LF4-PNL2) produced very turbid water, and a lot of mud was recovered during the sampling attempt. Sampling of this well was delayed. A newly completed well from Landfill l (LFl-PNL1) was sampled just to use the sample bottles and to make the sampling trip more worthwhile.

Ground-water samples were collected from wells LF4-PNL1, LF4-PNL3, and LF1-PNL1 on August 24, 1988, shortly after construction and bai7ing, but before the wells were fully developed. The samples were collected using dedicated Tefion bailers, which were filled with well water and emptied three times before taking samples. This comprised the only purging performed before sample collection; the purging amounted to approximately 1.7 well volumes for the 2-in.-dia wells and 0.4 well volumes for the 4-in.-dia wells. Single temperature and $\mathrm{pH}$ measurements were recorded on the field record sheet for each well sampled. A duplicate sample was collected at we11 LFl-PNL1 for quality assurance purposes.

\subsubsection{September Sampling}

The September sampling event was conducted to obtain an indication of the chemical constituents present beneath various portions of Landfill 1 to assist in identifying the need and location for additional wells. The objective was to collect an initial set of ground-water samples from the first four new (1988) wells installed around Landfill 1. However, one of these new wells (LFI-PNL1) had been sampled in late August, and the one new Landfill 4 well (LF4-PNL2), not sampled in August as originally scheduled, was sampled instead. 
Four we11s (LF1-PNL2, LF1-PNL3, LF1-PNL4, and LF4-PNL2) were sampled September 8 and 9, 1988, using a different HydroStar sampling pump at each well. These newly completed wells were still undergoing development. Well LF1-PNL4 had been fully developed using a 1/3-h.p. submersible pump. Approximately $80 \mathrm{gal}$ of water (12 well volumes) were purged from this well using the HydroStar pump before sampling. Wells LF1-PNL2 and LF4-PNL2 were fully developed using the HydroStar pump immediately before sampling. Approximately 325 gal (198 well volumes) and 46 gal (28 well volumes), respectively, were pumped from these wells. Although several attempts were made to develop well LF1-PNL3 via bailing and the HydroStar pump immediately before sampling, the low yield of the well prevented its full development (refer to Subsection 4.2.6 for further information). This well was nearly completely evacuated and allowed to recover three times over the 2-day period just before sampling. Ground-water samples were collected at the end of the final recovery.

A series of field $\mathrm{pH}$, temperature, and conductivity measurements were recorded during the purging/development of all four wells. A duplicate sample was collected at well LF1-PNL4.

\subsubsection{Early October Sampling}

The early October sampling event was conducted to indicate the areal extent of volatile organic constituents in the ground-water beneath Landfill 4 and to address the need for and location of any additional wells. The objective was to collect a spatially complete set of samples from wells around Landfill 4, using all six new (1988) and four existing (1981) wells.

The early October sampling event was conducted on October 6 and 7, 1988. Eight wel1s (LF4-PNL1, LF4-PNL2, LF4-PNL3, LF4-PNL4, LF4-1, LF4-2, LF4-4, and LF4-11) were sampled using a Teflon bailer, and one well (LF4-PNL5) was sampled using the 2.5-in.-dia carbon steel dart bailer being used for initial well development. Many of these wells still had not been completely developed before initiating this sampling. Three wells (LF4-PNLI, LF4-PNL2, and LF4-PNL4) had been fully developed. Before sampling each well, the bailer was filled and emptied with well water three times, providing a limited purging of the wel1. The degree of this purging is estimated at 
approximately 1.7 we 11 volumes for each 2 -in.-dia well and 0.4 well volumes for each 4-in.-dia well. Two wells, LF4-PNL4 and LF4-PNL5, had been extensively purged by well development activities within $24 \mathrm{hr}$ of sample collection. Following collection of the samples at each well, the used bailer was rinsed three times with distilled water. Field $\mathrm{pH}$, temperature, and specific conductivity measurements were not deemed pertinent to the objectives of this sampling event and were not collected. A field blank sample was collected at we11 LF4-PNL2.

\subsubsection{Late October Sampling}

The late October sampling event was conducted to complete the hydrochemical analyses around Landfill 4 to assist the determination of the concentrations and distribution of selected chemical constituents, primarily anions and cations. The objective was to collect samples from these wells for which these analyses did not already exist.

On October 30, 1988, seven wells from Landfill 4 (LF4-PNL4, LF4-PNL5, LF4-PNL6, LF4-1, LF4-2, LF4-4, and LF4-11). were sampled using a 1/3-h.p. submersible pump. The submersible pump was used to also completely develop and/or purge the wells before sampling. Volumes of water pumped before sampling range from approximately $100 \mathrm{gal}$ (15 well volumes) at wells LF4-1 and LF4-PNL4 to approximately 520 gal (80 well volumes) at well LF4-2. The submersible pump was steam cleaned between wells. Field $\mathrm{pH}$, temperature, and specific conductivity measurements were mistakenly omitted. Duplicate samples were taken at wells LF4-PNL1 and LF4-PNL4.

\subsubsection{December Sampling}

The December sampling event was conducted to provide spatially complete, time-equivalent sets of representative samples from each 7 andfill to compare selected chemical constituents. In addition, chemical data from Sequalitchew Lake were collected to evaluate the potential impact on ground-water quality near Landfill 4.

All 18 existing (1981 and 1984) and new (1988) wells from Landfills I and 4 , as well as Sequalitchew Lake, were sampled December 13 through 15, 1988. The samples were collected using dedicated and/or decontaminated 
Teflon bailers similar to the early October sampling event. Those samples at wells LF1-PNL1 and LF4-PNL3 were collected with HydroStar pumps. Approximately 556 gal ( 341 well volumes) and $300 \mathrm{gal}$ (184 well volumes) of water were pumped from wells LFI-PNLI and LF4-PNL3, respectively, before sampling. A sample was taken from Sequalitchew Lake using a Tefion bailer. The sample was taken from a barge $\mathbf{- 3 0 0}$ yd from the east shore (Spring Station).

Three quality control samples including a duplicate sample at well LFI-PNL1, a field blank sample, and a sample containing standard solutions were taken during this sampling event.

\subsection{ANALYZING GROUND-WATER SAMPLES}

The procedures used to analyze for ground-water quality parameters are listed in Table 4.5 and provided in Appendix J. In addition to the listed procedures, the anaiysis of the volatile organic samples at PNL was conducted according to the method provided in Appendix J. In brief, water samples were analyzed with a Hewlett-Packard Model 5880A gas chromatograph (GC). The GC was equipped with two identical J\&W DB-624 $30-\mathrm{m} \times 0.53-\mathrm{mm}$ fused-silica capillary columns. The DB-624 columns were coated with a cross-1inked and bonded stationary phase comprising cyanopropyl, phenyl, and dimethylsiloxane. The two columns were teed together at the inlet and routed to separate electron capture and flame ionization detectors. Sample introduction was via a Tekmar Mode1 LSC-3 purge and trap unit. The LSC-3 contained a Tenax sorption trap. Samples were thermally desorbed from the Tenax trap and transferred to the columns through a heated transfer 1ine. The Hewlett-Packard Model 5880A $G C$ was equipped with two separate integrators to simultaneously integrate data from both detectors.

Nearly all sample analyses were performed by Am Test, Inc., Redmond, Washington, during the first four fieid sampling events. Am Test results are presented in their entirety in Appendix $K$. Only volatile organic analyses (VOA) on the October 6, 1988, samples from Landfill 4 were performed by PNL. Samples from the December sampling were sent to both Am Test and PNL. Semivolatile organic constituents, coliform, and oil and grease analyses were conducted by Am Test. PNL analyzed for pH, specific conductivity, volatile 
IABLE 4.5. List of Analytical Methods Used on the Fort Lewis Ground-Water Study

\begin{tabular}{|c|c|}
\hline Parameter & Analytical Method Reference (a) \\
\hline Field pH & PNL Procedures \\
\hline Field Conductivity & PNL Procedures \\
\hline Laboratory $\mathrm{pH}$ & EPA Method 150.1 \\
\hline Laboratory Conductivity & EPA Method 120.1 \\
\hline Pesticides, Herbicides & EPA Method 608 \\
\hline PCBs & EPA Method 608 \\
\hline Coliform & Standard Methods 909 \\
\hline $0 i 1$ and Grease & EPA Method 413.2 \\
\hline Total Organic Halogens & EPA Method 450.1 \\
\hline Volatile Organic Analysis & EPA Method 601 \\
\hline Total Dissolved Solids & EPA Method 160.1 \\
\hline Total Organic Carbon & EPA Method 415.2 \\
\hline ICAP Metals & EPA Method 200.7 \\
\hline Cyanide & EPA Method 335.3 \\
\hline Anions $\left(\mathrm{F}^{-}, \mathrm{Cl}^{-}, \mathrm{NO}_{3}{ }^{-}, \mathrm{SO}_{4}{ }^{\circ}\right)$ & EPA Method 300.1 \\
\hline Phenol & EPA Method 420.1 \\
\hline
\end{tabular}

(a) Detection limits reported in results tables and/or actual analytical reports (Appendix $K$ ).

organic constituents, alkalinity, anions, total dissoived solids, and filtered and unfiltered metals analyzed by the inductively coupled argon plasma method (ICAP metals). These results are presented in the text of the report.

\subsection{QUALITY ASSURANCE}

The primary PNL Quatity Assurance (QA) Program is based on ANSI/ASME NQA-1, Quality Assurance Program Requirements for Nuclear Facilities, as it is applicable to the technical services and research and development performed by PNL. Under the PNL QA Program, each project is classified into one of three impact levels (I, II, or 11I); lmpact Level I possesses the 
greatest potential impact in the event of failure, based on such areas as safety, data defensability, programmatic, and regulatory concerns. Written QA plans are developed for projects classified as Impact Level I or II, specifically identifying applicable elements of the PNL QA Program. Projects classified as Impact Level III are performed in accordance with the minimum standards for all work at PNL specified in the PNL Good Practices Standard and do not require a project-specific QA PIan. The work performed in this investigation was classified as Impact Level II.

Because of its relevance in the acquisition of environmental data, a $Q A$ Project Plan was developed for this investigation, in accordance with the criteria in EPA QAMS-005/80, Guidelines and Specifications for Preparing Quality Assurance Project PIans, with elements of the PNL QA Program incorporated to supplement areas not adequately addressed by the EPA program.

Written technical procedures used by PNL's Environmental Monitoring Program (PNL 1989) were employed to collect and characterize drilling data and collect water-samples.

Only qualified, professional geologists were used in the oversight of well-installation activities, and the logging and characterization of soils encountered during well construction as required by the EPA/RCRA Ground-Water Monitoring Technical Enforcement Guidance Document, OSWER-9950.1 (EPA 1986). All PNL staff performing work at the sites received a minimum of 40 -hr prior training in hazardous material handling as required by 29 CFR 1910.

Applicable QA requirements were passed on to subtier organizations. Procurement controls were implemented to verify that suppliers and subcontractors were capable of providing quality services. This control included a pre-award evaluation of the analytical laboratory performing analyses of ground-water samples by the PNL Quality Control Section to verify the laboratory's ability to meet the QA requirements passed on.

The PNL QA Department audited both drilling and sampling to verify conformance with drilling specification and sampling procedure requirements. The data quality and traceability of the results package were also audited. 
Deficiencies encountered were documented, along with an assessment of their root cause, impact on results, and corrective actions designed to preclude recurrence.

\subsection{QUALITY CONTROL}

In contrast to inspecting hardware items to verify they met the dimensional requirements of a specification, data cannot be inspected as they are generated. To inspect data, indirect techniques must be used, such as the preparation and analysis of quality control samples. Analyzing these samples along with the unknown samples gives an indication of the quality of data being generated by a given analytical process in terms of accuracy and precision. Quality control measures implemented included the collection and analysis of duplicate, blank, and standard solution samples.

During the August 24 sampling event, a set of blanks was prepared for all the laboratory parameters analyzed by Am Test, Inc. No contamination was observed in the blank samples. A blind duplicate sample was also prepared from one of the wells (LF1-PNL1) chosen at random and submitted to the analytical laboratory. The results from this analysis can be seen in the data tables contained in Chapter 5.0 of this report. In general, the comparison is acceptable, but could be better. The coliform, total dissolved solids, sulfate, iron, barium, manganese, calcium, and sodium did not compare we 11 . The discrepancies in the iron, barium, manganese, calcium, and sodium results are probably caused by the high solids (mud) content in the water sample. This well had not been adequateiy developed before sampling (refer to Subsection 4.4). If the well were less turbid, the results may have been more comparable. The reason for the discrepancy in the coliform, total dissolved solids, and sulfate results is unknown, but could also be related to the high turbidity of the water samples. A blind USGS ground-water standard was also submitted for analysis. The results of the analys is can be seen in Table 4.6. In general, the Am Test results compare well with the USGS value. However, the Am Test iron and chromium values deviated significantly from the actual value. This discrepancy cannot be explained by the results, except that the concentrations in the sample are approaching the detection limit of the analytical method. 
TABLE 4.6. Comparison of U.S. Geologica] Survey Standard Concentration with Am Test, Inc., Results (ppm)

$\begin{array}{lccc} & \text { AM Test Result } & & \text { USGS Va]ue } \\ \text { Iron } & <0.01 & & 0.042 \pm 0.010 \\ \text { Barium } & 0.044 & & 0.040 \pm 0.002 \\ \text { Cadmium } & <0.002 & & <0.002 \\ \text { Manganese } & 0.009 & 0.008 \pm 0.001 \\ \text { Calcium } & 55 & 54.8 \pm 2.2 \\ \text { Silver } & <0.01 & <0.01 \\ \text { Sodium } & 110 & 107.0 \pm 3.0 \\ \text { Chromium } & 0.022 & 0.008 \pm 0.002 \\ \text { Paliadium } & <0.02 & <0.02 \\ \text { Arsenic } & <0.002 & 0.003 \pm 0.001\end{array}$

During the September 8 and 9 sampling event, a duplicate sample from well LFl-PNL4 was submitted to the laboratory for analysis. The results of this analysis can be seen in the data tables contained in Chapter 5.0 of this report. In general, the results of this analysis were considerably better than the August sample results. Most parameters were in good agreement with each other. Because of an oversight, no blank or standards were submitted with the samples during this sampling episode.

During the October 6 sampling event, only samples for VOA were collected from the wells in the Landfill 4 area. These samples were analyzed at PNL. $A$ blind blank was submitted for analysis with the samples. No contamination was observed in the blank sample.

During the October 30 sampling event, three wells were sampled in the Landfill 4 area. These ground-water samples were analyzed by Am Test. A duplicate sample was collected from well LF4-PNL4. The results of the duplicate analysis are reported in Chapter 6.0 with the analytical results from Landfill 4.

During the December 13 through 15 sampling event, wells at both the landfills were sampled. These samples were analyzed by Am Test, Inc., for semivolatile organic constituents, coliform, and oil and grease. Samples 
were analyzed by PNL for volatile organic compounds, total organic carbon, $\mathrm{pH}$, conductivity, and filtered and unfiltered ICAP metals. A duplicate sample was collected from well LFI-PNLI. A comparison of the results from the duplicate analysis is reported in Chapter 5.0 with the analytical results from Landfill 1. The results from the duplicate analysis agreed very well. A blank sample was generated in the field and sent to each laboratory for the respective analyses. The analytical results from the blank indicate a problem with the oil and grease analysis. Am Test reported $8.3 \mathrm{ppm}$ of $0 i 1$ and grease in the blank sample. The lowest concentration reported for that suite of samples was $8.2 \mathrm{ppm}$. These results indicate either a blank or laboratory contamination problem. No other problems were observed with the analytical results from the blank analysis. A USGS sample was submitted to the PNL laboratory conducting inorganic analysis. Table 4.7 compares the analytical results with the certified USGS value. The results of the analysis compare very well with the USGS reported results.

TABLE 4.7. Comparison of the U.S. Geological Survey Standard Concentration With PNL Results

PNL Results USGS value.

$\mathrm{pH}$

Conductivity

Chloride $\left(\mathrm{Cl}^{-}\right)$

Sulfate $\left(\mathrm{SO}_{4}=\right)$

Alkalinity $\left(\mathrm{CaCO}_{3}\right)$
8.21

$2410 \mu \pi n h / \mathrm{cm}$

$79 \mathrm{ppm}$

$1130 \mathrm{ppm}$

$132.5 \mathrm{ppm}$
$8.2 \pm 0.1$

$2451 \pm 150 \mu \mathrm{mho} / \mathrm{cm}$

$79.6 \pm 3.5 \mathrm{ppm}$

$1170 \pm 33.5 \mathrm{ppm}$

$132.5 \pm 4.0 \mathrm{ppm}$ 


\subsection{EVALUATION OF LANDFILL 1}

Using the information gained from examining aerial photographs, characterizing the environmental setting, and drilling and sampling, the boundaries of Landfill 1 were located and the geologic framework, ground-water flow characteristics, ground-water quality, and monitoring well network were evaluated.

\subsection{LOCATION OF LANDFILL 1 BOUNDARIES}

The boundaries of Landfill 1 were defined by visual interpretation of available aerial photographs and by field reconnaissance. Aerial photographs taken in July 1951 show a portion of Landfill I as still active and also show that the landfill was originally operated as a trench cut-and-fill-type operation. The aerial photographs also show two open-pit dumps, of isolated extent, and one suspected burn area located to the west of the trenching operation. These landfill features were located on a map, based on their correlation to cultural features (i.e., roads and buildings; Figure 5.1). Aerial photographs taken in May 1970 show that Landfill 1 was extended to the southwest, possibly by overbank dumping along the western escarpment created during the trenching operation (see Figure 5.1). An area of surface dumping/storage to the south was also indicated in these photographs. The interpreted boundaries indicate that the trenching area extended under the road (South 22nd Street) and parking lot of the tank repair shop to the north. The area where isolated pit dumping and burning were observed in historical photographs appears to be located under the tank repair shop and other maintenance yards to the west.

Field reconnaissance found areas of subsidence in the northern portion of Landfi11 1 and along South 22nd Street that runs through this portion of the landfill. These areas of subsidence are thought to be associated with the decay and compaction of waste materials in the trenches, thus confirming that the landfill extended under at least a portion of South 22nd Street. Surface dumping of construction rubble was also apparent; this dumping must have occurred after the May 1970 photographs were taken. Also, the topography of the site suggests that overbank dumping may also have occurred after 


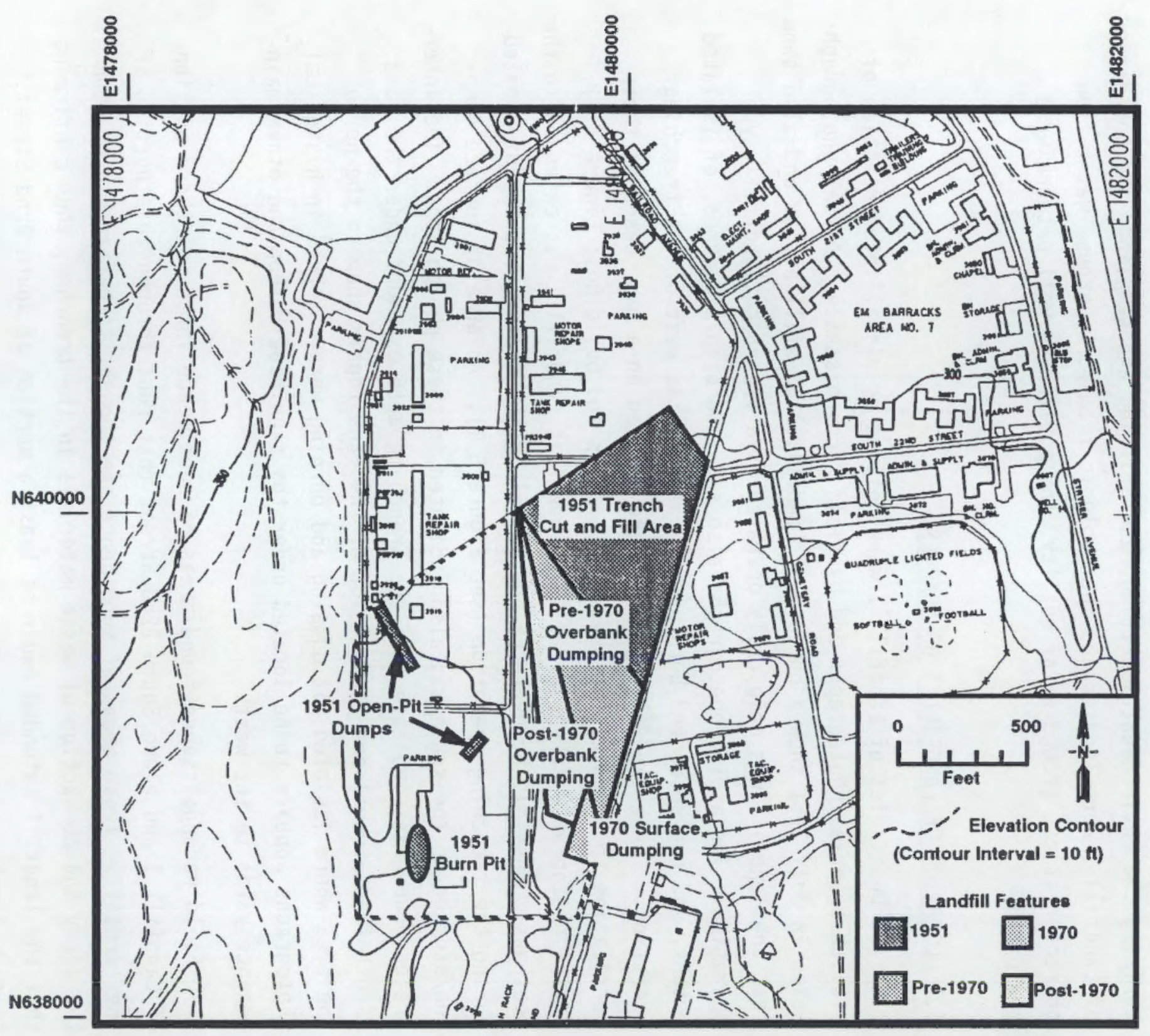

FIGURE 5.1. Location of Landfill 1 Boundaries and Historical Configurations 
May 1970, extending the embankment farther to the west. Figure 5.1 illustrates the current interpretation of Landfill 1 and of the suspected area of isolated pit dumping and burning.

\subsection{GEOLOGIC FRAMEWORK}

The stratigraphy beneath Landfill 1 has been interpreted primarily from field analysis of split-spoon and core-barrel samples collected during the installation of four new (1988) ground-water monitoring wells. These wells ranged in depth from 33 to $48 \mathrm{ft}$ below land surface. The actual field records are presented in Appendix E. Additional geologic information was gathered from drilling logs compiled during earlier (1984) drilling operations (Appendix I). The location of the new and existing wells is shown in Figure 5.2, and the well completion data are summarized in Table 5.1. Subsurface data collected from each new (1988) borehole are summarized in

Figures 5.3 through 5.6. A generalized geologic cross section through the site is provided in Figure 5.7.

The sediments encountered in the new (1988) boreholes generally consist of poorly sorted, muddy sandy gravel with minor layers of gravel and sandy gravel. The general classification name (i.e., muddy sandy gravel) follows the ternary classification system of Folk (1974) (see Figure 4.1) and is based on a field estimate of the grain-size distribution comprising each sediment sample. These sediments are believed to be part of the Vashon Drift Formation of Walters and Kimmel (1968) and equivalent to hydrostratigraphic layer A of Brown and Caldwell (1983). Ground-water lies within this unit at depths between 20 and $40 \mathrm{ft}$ below land surface.

Based on information gathered from an existing production well drilled in this area, it appears that a clay/silt layer, interpreted to be the top of the Kitsap Formation, is located approximately $70 \mathrm{ft}$ below land surface. This is considered to be the contact between hydrostratigraphic layers $A$ and B of Brown and Cadwell (1983). Assuming this layer is continuous, it most likely represents a hydrologic boundary and would, therefore, represent the bottom of the uppermost aquifer. 


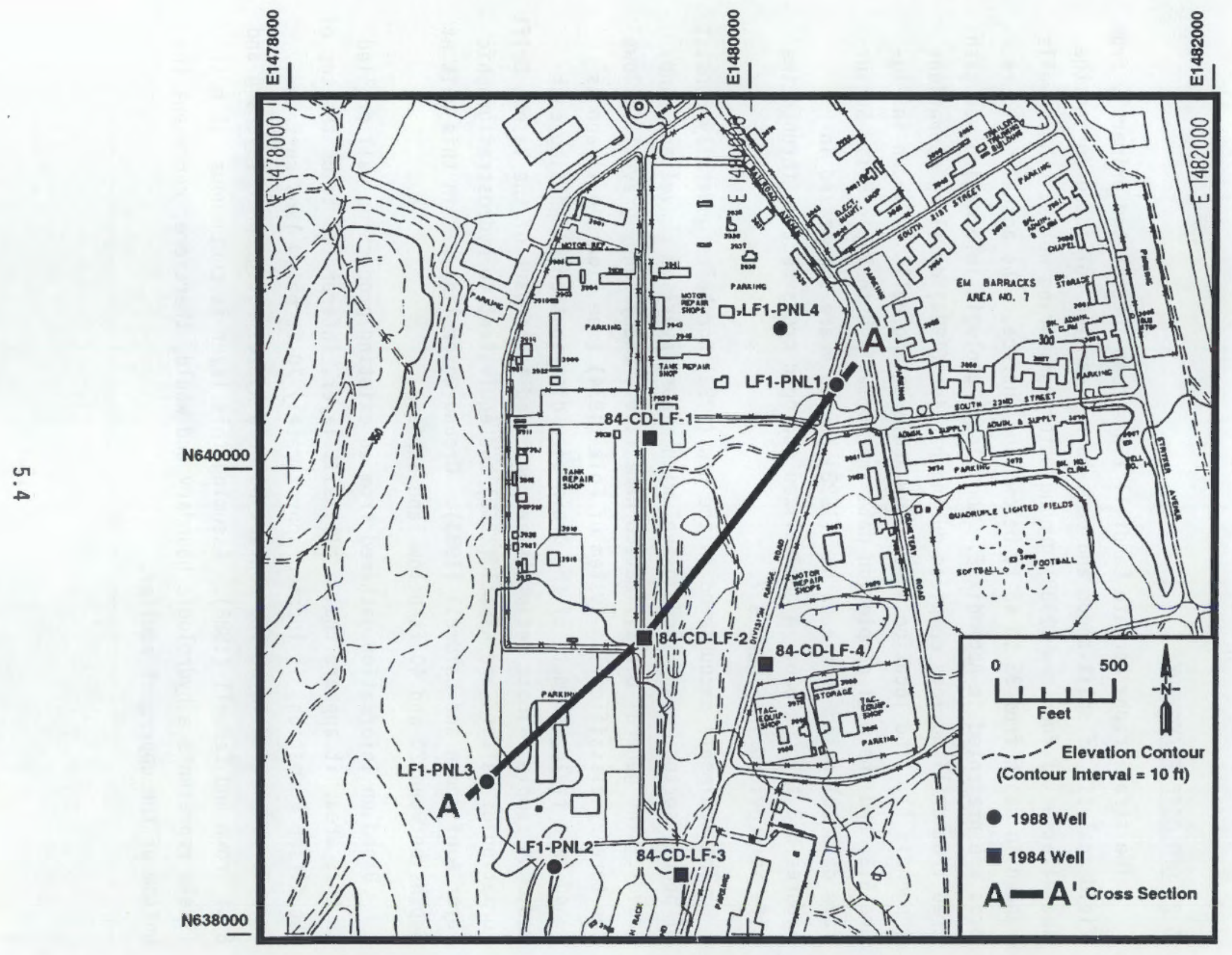

FIGURE 5.2. Location of Monitoring Wells Around Landfill 1 
TABLE 5.1. Well Completion Data for Landfill 1

\begin{tabular}{|c|c|c|c|c|c|c|c|}
\hline Well Number & $\begin{array}{c}\text { Washington State } \\
\text { Coordinates } \\
\text { North,East }\end{array}$ & $\mid \begin{array}{c}\text { PVC Casing } \\
\text { Elevation } \\
\text { (ft-MSL) (a) }\end{array}$ & $\begin{array}{l}\text { Drilled } \\
\text { Depth } \\
\text { (ft below } \\
\text { land)(b) }\end{array}$ & $\begin{array}{l}\text { Depth to } \\
\text { Water } \\
\text { (ft below } \\
\text { land)(b) }\end{array}$ & $\begin{array}{l}\text { Well } \\
\text { Diameter } \\
\text { (in.) }\end{array}$ & $\begin{array}{l}\text { Screened } \\
\text { Interval } \\
\text { (ft below } \\
\text { land)(c) }\end{array}$ & $\begin{array}{c}\text { Date } \\
\text { Completed }\end{array}$ \\
\hline 84-CD-LF-1 & 640126,1479595 & 303.64 & 61.0 & 28 & 4 & $20-60$ & $11-05-84$ \\
\hline 84-CD-LF-2 & 639271,1479573 & 303.48 & 61.0 & 23 & 4 & $20-60$ & $10-31-84$ \\
\hline 84-CD-LF-3 & 638243,1479727 & 297.69 & 61.0 & 21 & 4 & $20 \cdot 60$ & $11-09-84$ \\
\hline 84-CD-LF-4 & 639159,1480091 & 312.59 & 61.0 & 39 & 4 & $20-60$ & $11-15-84$ \\
\hline LF1-PNL1 & 640371,1480403 & 308.66 & 48.0 & 38 & 2 & $33-48$ & $08-19-88$ \\
\hline LF1-PNL2 & 638285,1479185 & 298.37 & 33.0 & 21.5 & 2 & $17-32$ & $08-19-88$ \\
\hline LF1-PNL3 & 638634,1478888 & 307.74 & 40.5 & 28.5 & 4 & $24-39$ & $08-25-88$ \\
\hline LF1-PNL4 & 640612,1480157 & 305.00 & 44.0 & 34.0 & 4 & $29-44$ & $09-01-88$ \\
\hline
\end{tabular}

(a) All elevations are to the north rim of the PVC casing.

(b) Measurements are to the nearest $0.5 \mathrm{ft}$.

(c) Measurements are rounded down to the nearest foot. 


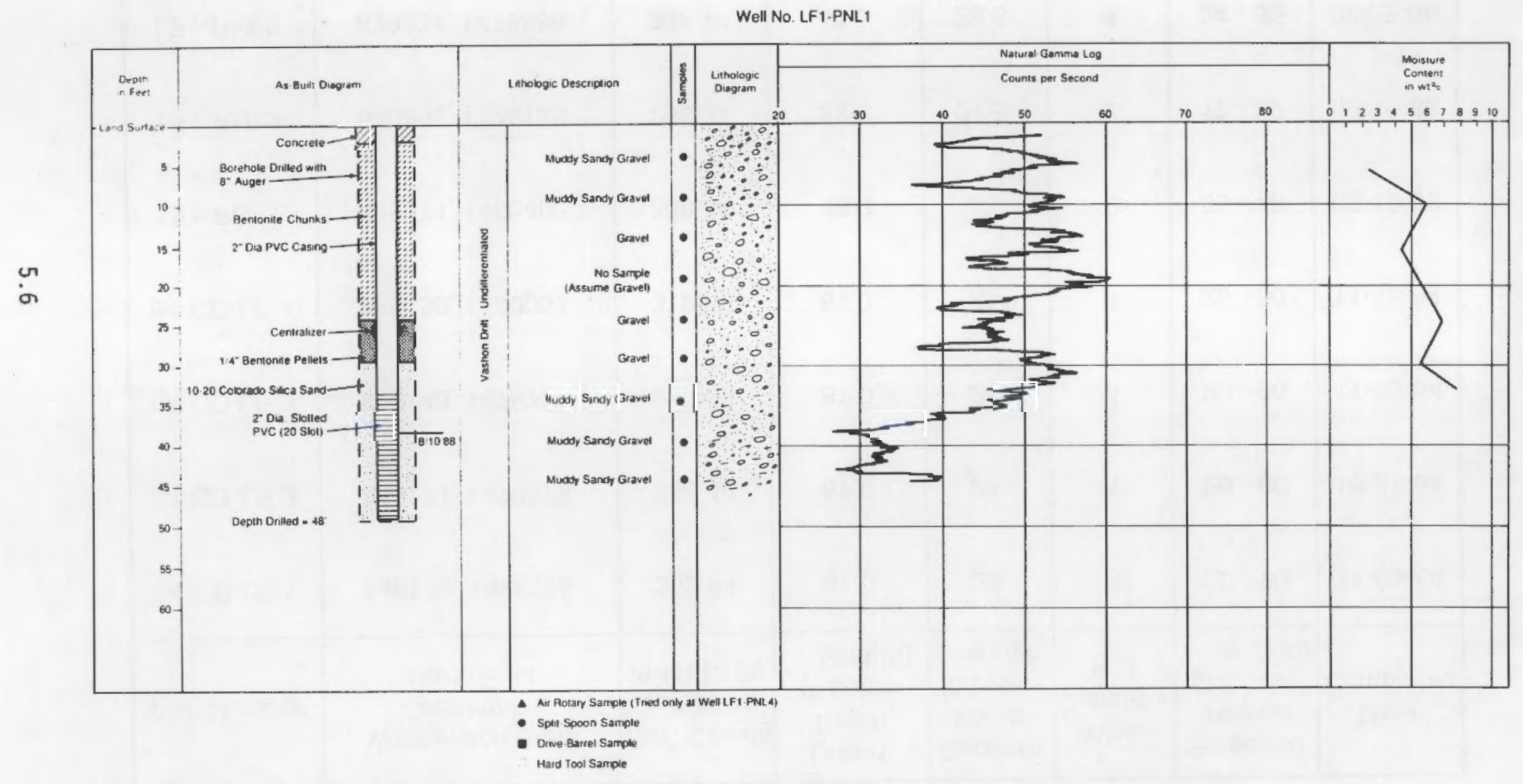

FIGURE 5.3. Subsurface Correlation Chart of Well LF1-PNL1 


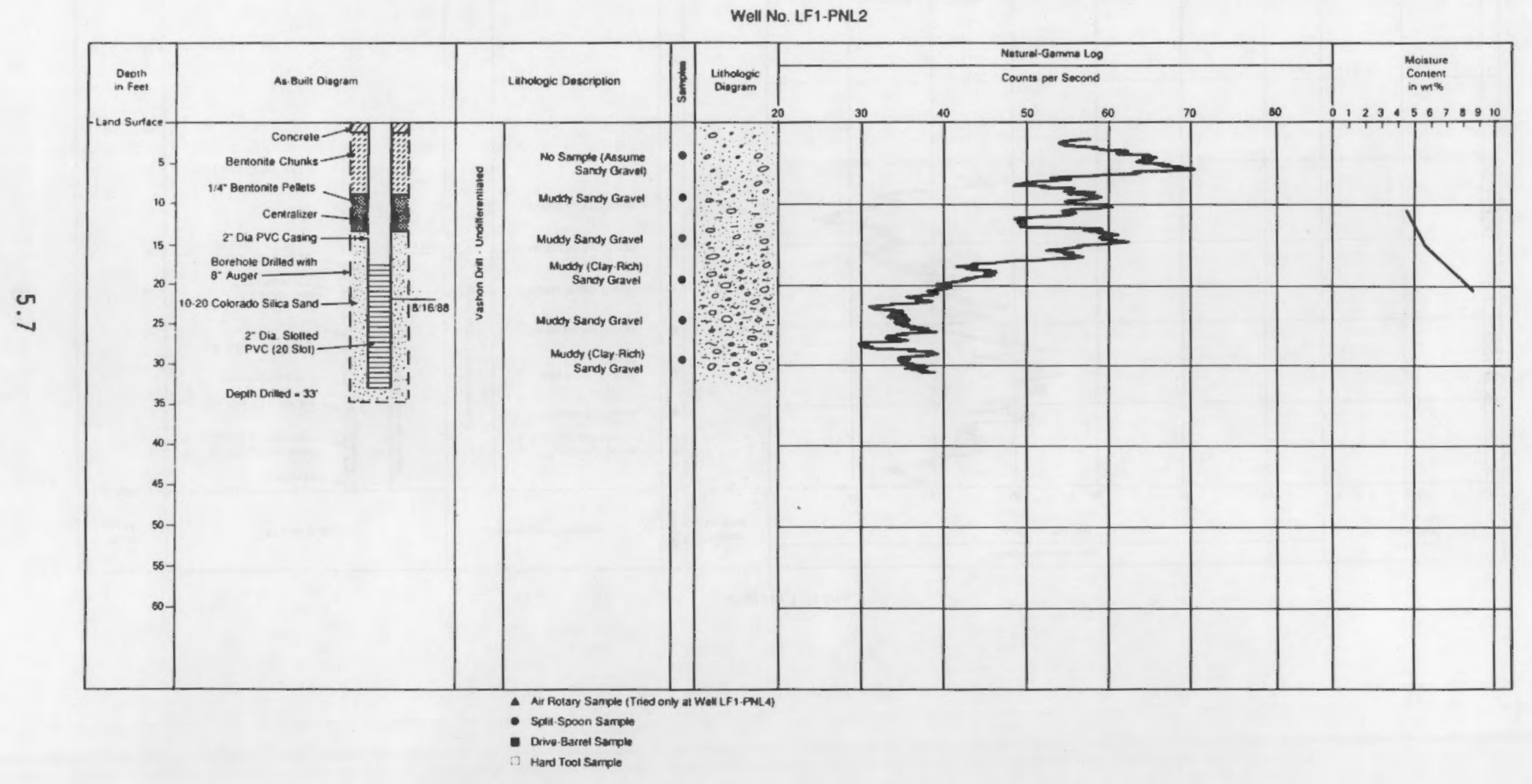

FIGURE 5.4. Subsurface Correlation Chart of Well LF1-PNL2 
Well No. LF1-PNL3

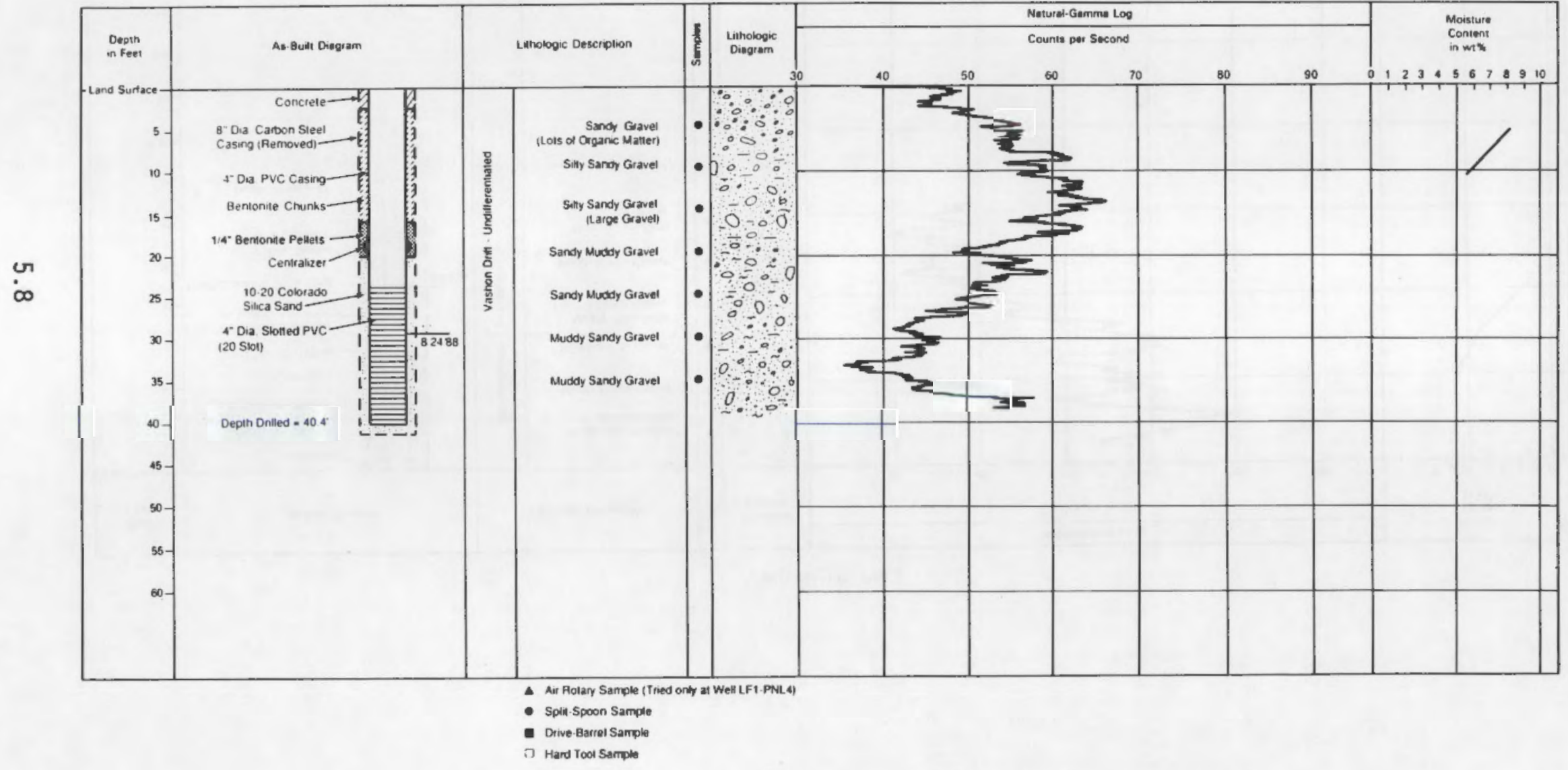

FIGURE 5.5. Subsurface Correlation Chart of Well LF1-PNL3 


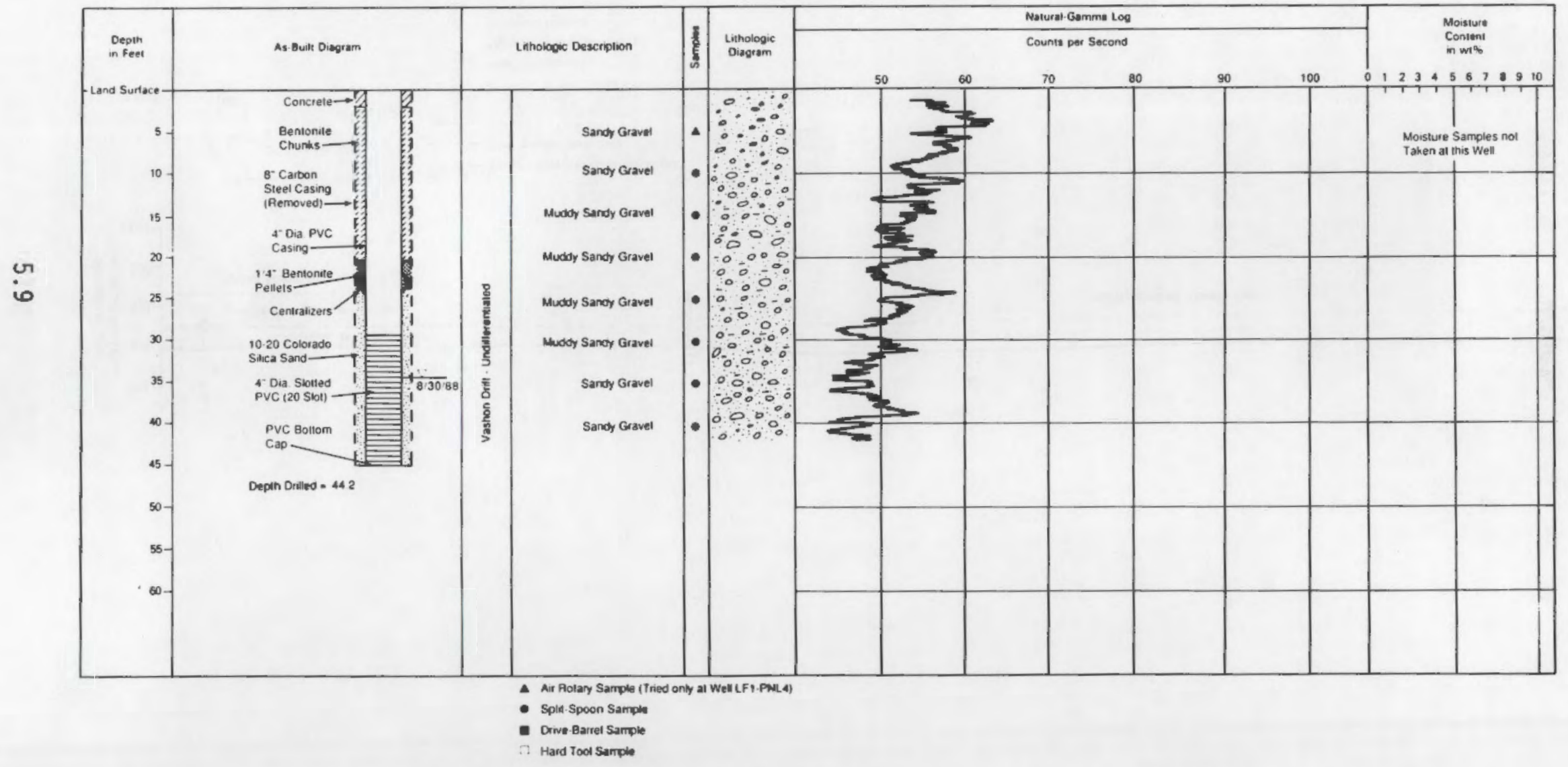

FIGURE 5.6. Subsurface Correlation Chart of Well LF1-PNL4 


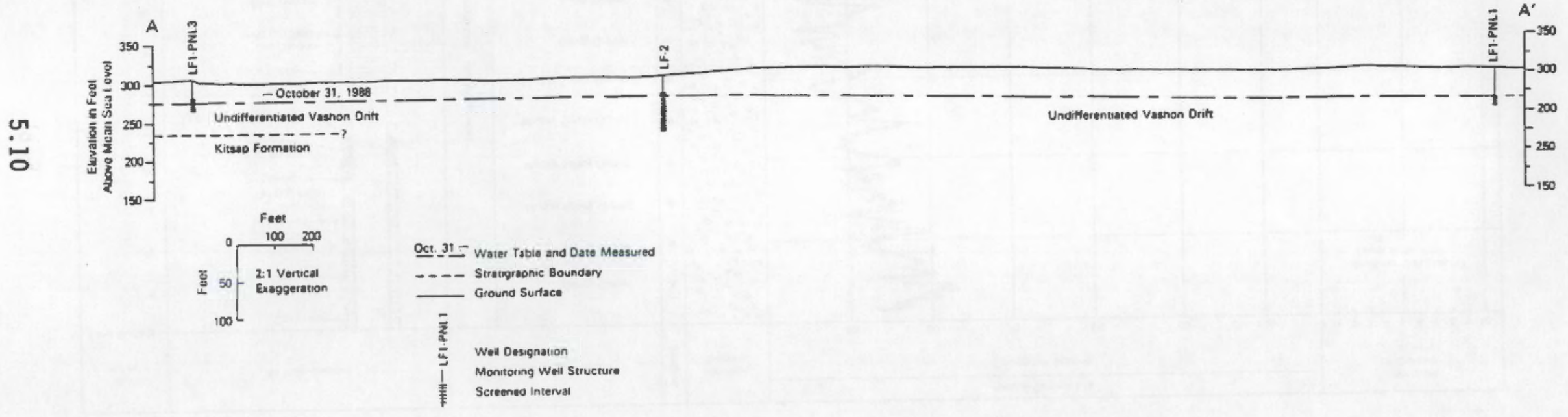

FIGURE 5.7. Generalized Geologic Cross Section Through Landfill 1 


\subsection{GROUND-WATER FLOW}

Ground-water flow in the vicinity of Landfill 1 is dominated by a local ground-water mound superimposed on a general, easterly sloping ground-water surface. Figures 5.8 and 5.9 illustrate the configuration of the water table as interpreted from water-level measurements collected on October 25 and December 14, 1988, respectively. The ground-water gradient across the landfill ranges from approximately 2 to $7 \mathrm{ft} / 500 \mathrm{ft}$ or 0.004 to $0.14 \mathrm{ft} / \mathrm{ft}$.

The localized ground-water mound near the center of the landfill results from artificial recharge from an unlined holding pond located immediately southwest of well 84-CD-LF-2. It is believed that this pond receives precipitation runoff from the parking areas to the north and west. Thus, recharge is expected to be intermittent. This pond may also be a potential source of contaminants upgradient of the Landfill 1 trenching area (see Figures 5.8 and 5.9).

The general easterly ground-water flow direction is opposite from the regional westerly gradient depicted in Figure 3.4. However, this easterly flow is interpreted to relect natural recharge from surface runoff and precipitation along the nearby steep-sided hills (interpreted to be drumlins) just west of the landfill. As ground water moves east away from the hills, it will likely turn to the north and west, eventually aligning with the westerly regional gradient depicted in Figure 3.4 .

Figure 5.10 illustrates the generalized ground-water flow directions and locations of upgradient and downgradient monitoring wells surrounding Landfill 1. Three monitoring we1ls (LF1-PNL2, LF1-PNL3, and 84-CD-LF-1) are interpreted to be hydraulically upgradient of Landfill 1 and the associated open-pit dumping areas. The five remaining wells are interpreted to be either directly or indirectly downgradient of these waste sites. Variations in water levels, particularly those caused by changes in the local groundwater mound, influence the downgradient status of these wells. Wells LF1PNL1 and 84-CD-LF-2 are the most directly downgradient and are the most likely to have been affected by waste-disposal activities in and around Landfill 1. 


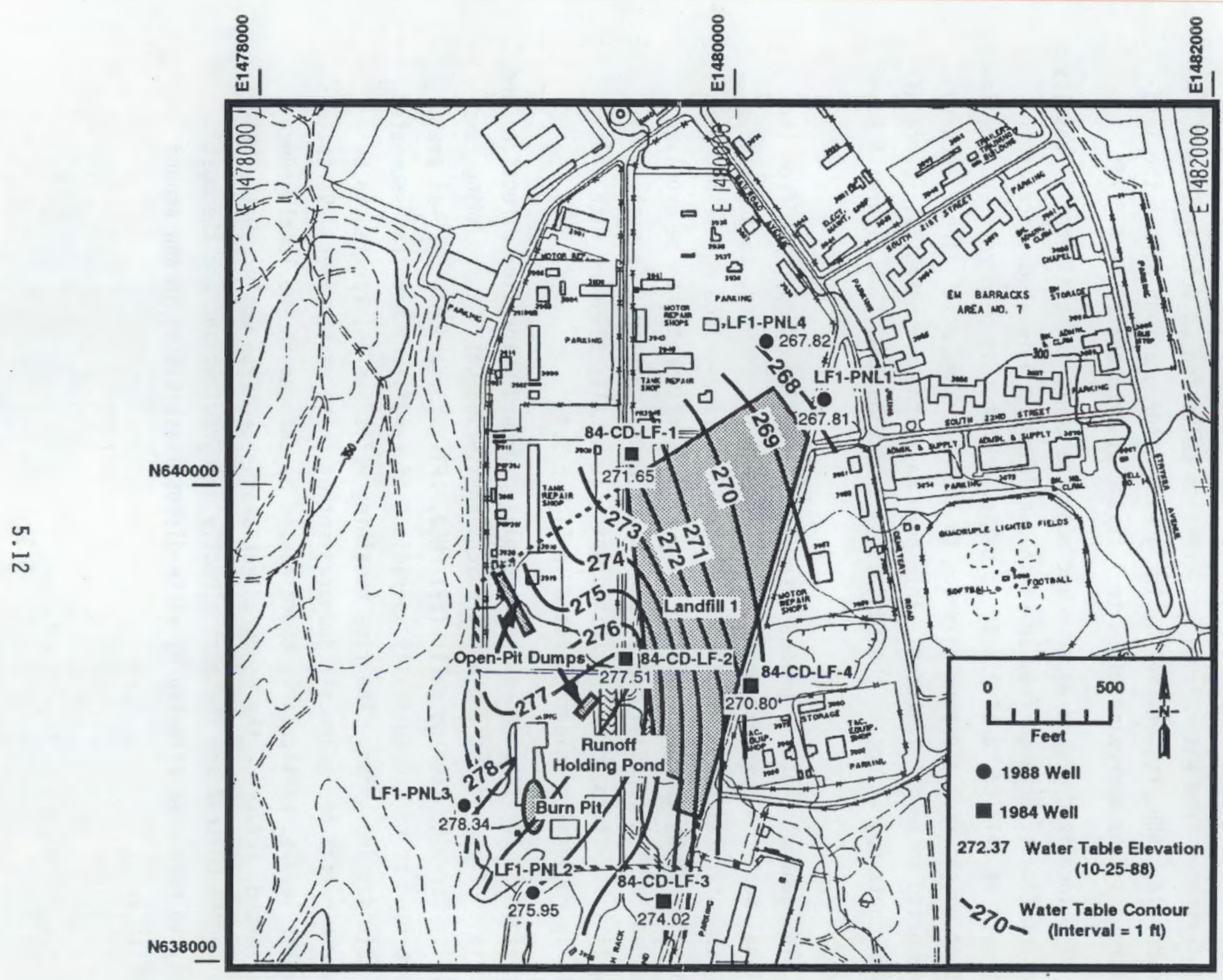

FIGURE 5.8. Water-Table Surface Beneath Landfill 1 as Interpreted from Water Levels Measured on October 25, 1988 


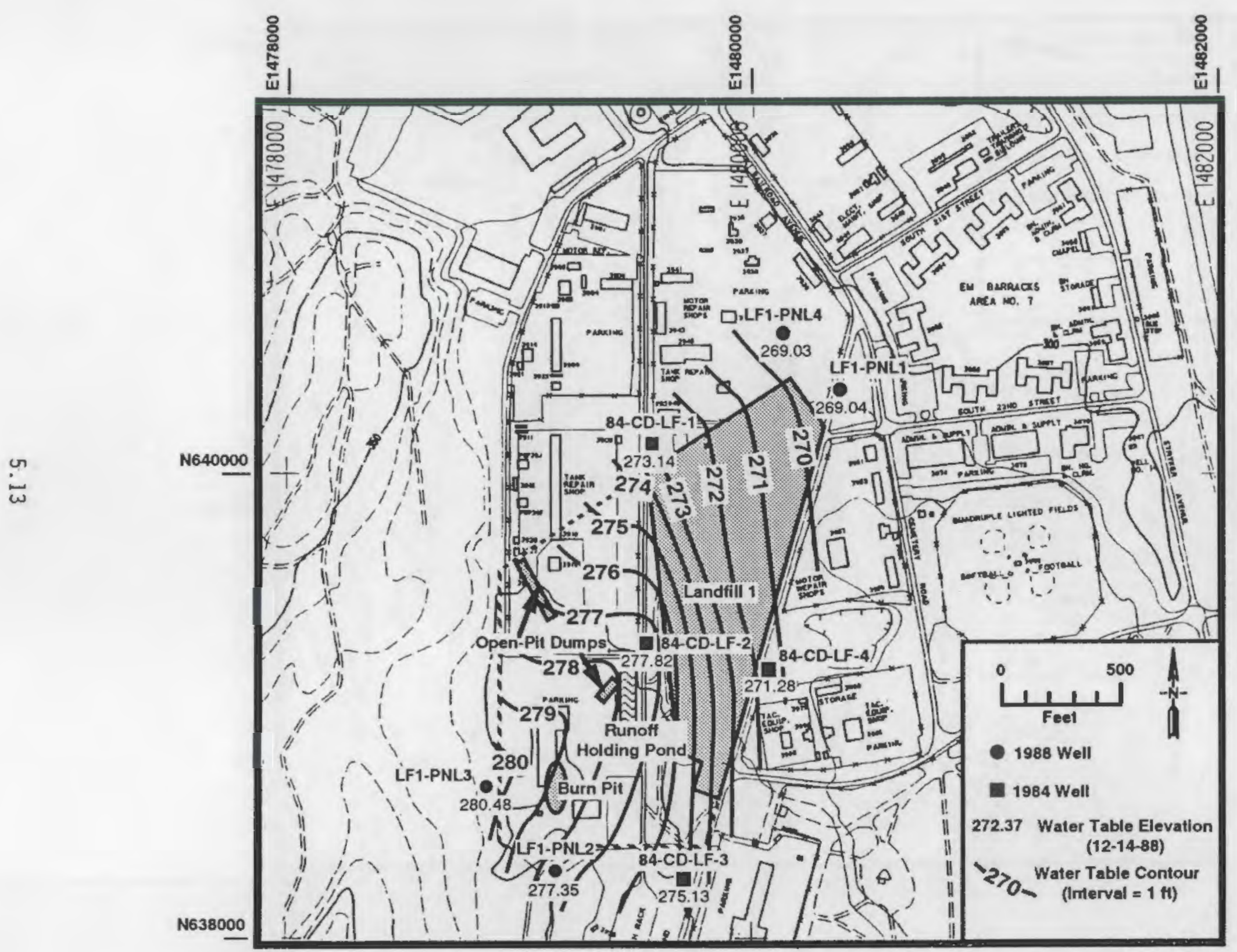

FIGURE 5.9. Water-Table Surface Beneath Landfill l as Interpreted from Water Levels Measured on December 14, 1988 


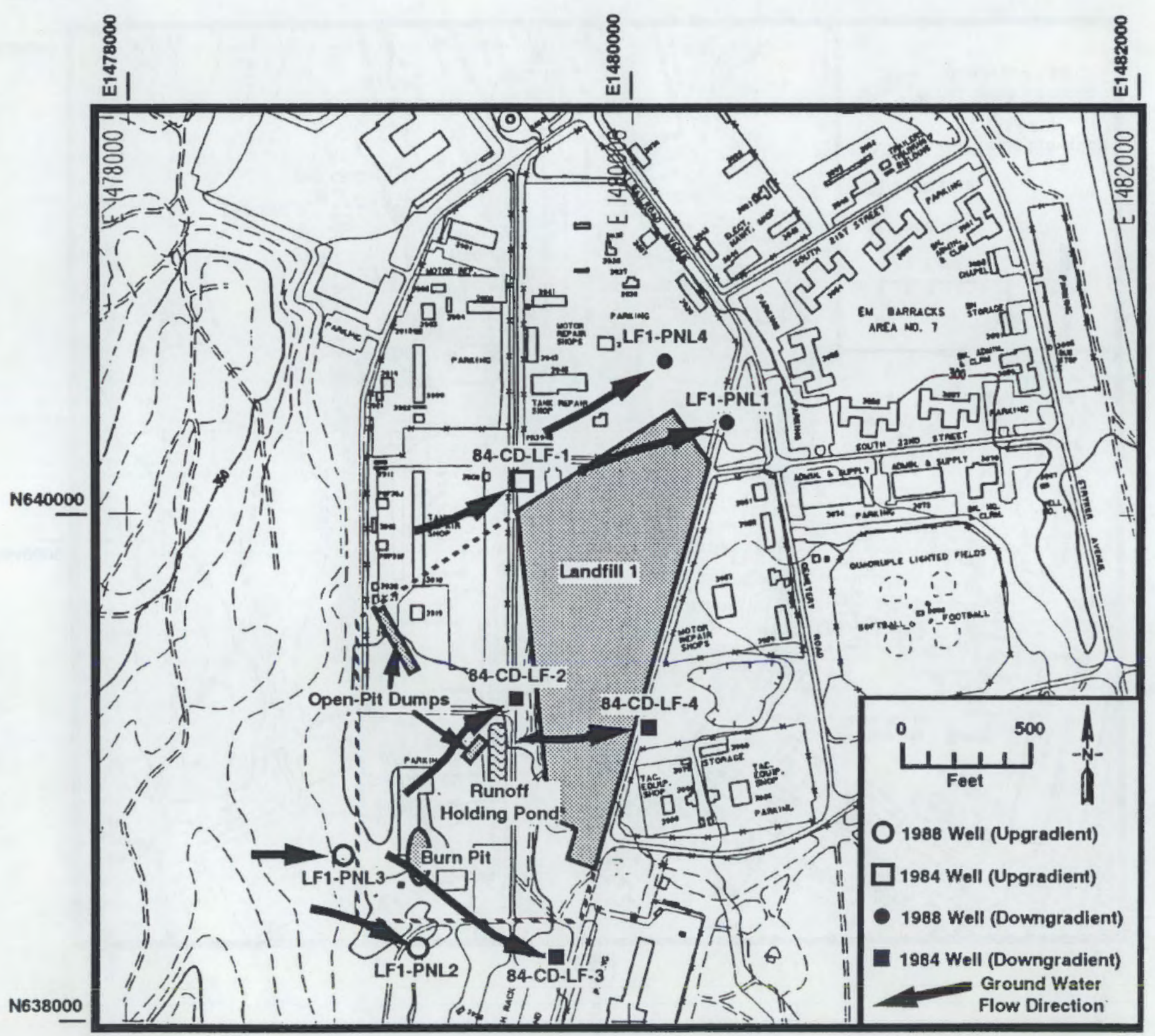

FIGURE 5.10. Generalized Ground-Water Flow Directions and Location of Upgradient and Downgradient Wells at Landfill 1 
Over the 8-month time frame of this study (Spring to Winter of 1988), water levels fluctuated approximately 1.5 to $2.5 \mathrm{ft}$. Table 5.2 provides a summary of the water-level measurements collected between April and December 1988.

\subsection{GROUND-WATER QUALITY}

Ground-water samples were collected from each of the four existing (1981) wells and the four newly installed (1988) wells surrounding Landfill 1. At least one sample was collected from each well. The representativeness of these samples varies according to the well development and purging history of each well before sample collection. Wells LF1-PNL1, LF1PNL2, and LF1-PNL4 produced good to very good samples after development. Wells $84-C D-L F-1,-2,-3$, and -4 were not developed nor extensively purged during this study and produced only fair samples. Well LFl-PNL3 is completed in a low-yielding portion of the aquifer and produced only poor samples. Refer to Subsection 4.2 .6 and Section 4.4 for further details.

Analytical results for the complete set of ground-water samples collected from the vicinity of Landfill 1 are summarized in Tables 5.3 and 5.4. The analytical reports received from AM Test are provided in Appendix K. As stated above, the representativeness of the ground-water samples varies. Some of the analytical results may be an artifact of the drilling, well construction, poor well development, and/or lack of adequate purging before sampling; these results may not accurately represent the ground-water quality. Thus, these results should be considered preliminary. Results from the good and very good ground-water samples (Tables 5.3 and 5.4) are considered the most representative of ground-water quality beneath Landfill 1. These results indicate the presence of several constituents [some of which exceed either primary or secondary drinking water standards (DWS) or maximum contaminant leve]s (MCL)] that may be of some concern. A discussion of these results is presented below.

Two wells (LF1-PNL2 and LF1-PNL4) produced ground-water samples that contained fecal coliform in excess of the DWS. However, both these samples 
TABLE 5.2. Water-Level Measurements from Landfill 1

\begin{tabular}{|c|c|c|c|c|c|}
\hline Well Number & $\begin{array}{l}6 \text {-in. Casing } \\
\text { Elevation } \\
\text { above ms } 1 \text { (a) } \\
\end{array}$ & $\begin{array}{c}\text { Measurement } \\
\text { Date }\end{array}$ & $\begin{array}{c}\text { Depth to } \\
\text { Water, } \\
\mathrm{ft}\end{array}$ & $\begin{array}{c}\text { Type of } \\
\text { Measurement }\end{array}$ & $\begin{array}{c}\text { Head } \\
\text { Elevation, } \\
\text { ft above msl } \\
\end{array}$ \\
\hline $84-C D-L F-1$ & $\begin{array}{l}304.41 \text { (b) } \\
304.41 \text { (b) } \\
305.62 \\
305.62 \\
305.62 \\
305.62\end{array}$ & $\begin{array}{l}04 / 20 / 88 \\
08 / 12 / 88 \\
10 / 25 / 88 \\
10 / 31 / 88 \\
12 / 13 / 88 \\
12 / 14 / 88\end{array}$ & $\begin{array}{l}30.49 \\
32.29 \\
33.97 \\
34.16 \\
32.45 \\
32.48\end{array}$ & $\begin{array}{l}\text { ST (c) } \\
\text { ST } \\
\text { ST } \\
\text { ET(d) } \\
\text { ST } \\
\text { ST }\end{array}$ & $\begin{array}{l}273.92 \\
272.12 \\
271.65 \\
271.46 \\
273.17 \\
273.14\end{array}$ \\
\hline $84-C D-L F-2$ & $\begin{array}{l}304.53 \text { (b) } \\
304.53 \text { (b) } \\
304.47 \\
304.47 \\
304.47\end{array}$ & $\begin{array}{l}04 / 20 / 88 \\
08 / 12 / 88 \\
10 / 25 / 88 \\
10 / 31 / 88 \\
12 / 14 / 88\end{array}$ & $\begin{array}{l}26.29 \\
27.26 \\
26.96 \\
27.10 \\
26.65\end{array}$ & $\begin{array}{l}\text { ST } \\
\text { ST } \\
\text { ST } \\
\text { ET } \\
\text { ST }\end{array}$ & $\begin{array}{l}278.24 \\
277.27 \\
277.51 \\
277.37 \\
277.82\end{array}$ \\
\hline $84-C D-L F-3$ & $\begin{array}{l}299.17 \text { (b) } \\
299.17 \text { (b) } \\
299.13 \\
299.13 \\
299.13\end{array}$ & $\begin{array}{l}04 / 20 / 88 \\
08 / 12 / 88 \\
10 / 25 / 8 \mathrm{~B} \\
10 / 31 / 88 \\
12 / 14 / 88\end{array}$ & $\begin{array}{l}24.13 \\
25.62 \\
25.11 \\
25.36 \\
24.00\end{array}$ & $\begin{array}{l}\text { ST } \\
\text { ST } \\
\text { ST } \\
\text { ET } \\
\text { ST }\end{array}$ & $\begin{array}{l}275.04 \\
273.55 \\
274.02 \\
273.77 \\
275.13\end{array}$ \\
\hline $84-C D-L F-4$ & $\begin{array}{l}313.66^{(b)} \\
313.66^{(b)} \\
313.67 \\
313.67 \\
313.67\end{array}$ & $\begin{array}{l}04 / 20 / 88 \\
08 / 12 / 88 \\
10 / 25 / 88 \\
10 / 31 / 88 \\
12 / 14 / 88\end{array}$ & $\begin{array}{l}41.29 \\
42.40 \\
42.87 \\
42.95 \\
42.39\end{array}$ & $\begin{array}{l}\text { ST } \\
\text { ST } \\
\text { ST } \\
\text { ET } \\
\text { ST }\end{array}$ & $\begin{array}{l}272.37 \\
271.26 \\
270.8 \\
270.72 \\
271.28\end{array}$ \\
\hline LF1-PNL1 & $\begin{array}{l}309.36 \\
309.36 \\
309.36 \\
309.36\end{array}$ & $\begin{array}{l}10 / 25 / 88 \\
10 / 31 / 88 \\
12 / 13 / 88 \\
12 / 14 / 88\end{array}$ & $\begin{array}{l}41.55 \\
41.69 \\
40.30 \\
40.32\end{array}$ & $\begin{array}{l}\text { ST } \\
\text { ET } \\
\text { ST } \\
\text { ST }\end{array}$ & $\begin{array}{l}267.81 \\
267.67 \\
269.06 \\
269.04\end{array}$ \\
\hline LF1-PNL2 & $\begin{array}{l}299.22 \\
299.22 \\
299.22\end{array}$ & $\begin{array}{l}10 / 25 / 88 \\
10 / 31 / 88 \\
12 / 14 / 88\end{array}$ & $\begin{array}{l}23.27 \\
23.46 \\
21.87\end{array}$ & $\begin{array}{l}\text { ST } \\
\text { ET } \\
\text { ST }\end{array}$ & $\begin{array}{l}275.95 \\
275.76 \\
277.35\end{array}$ \\
\hline LF1-PNL3 & $\begin{array}{l}308.48 \\
308.48 \\
308.48\end{array}$ & $\begin{array}{l}10 / 25 / 88 \\
10 / 31 / 88 \\
12 / 14 / 88\end{array}$ & $\begin{array}{l}30.14 \\
30.43 \\
28.00\end{array}$ & $\begin{array}{l}\text { ST } \\
\text { ET } \\
\text { ST }\end{array}$ & $\begin{array}{l}278.34 \\
278.05 \\
280.48\end{array}$ \\
\hline LF1-PNL4 & $\begin{array}{l}305.72 \\
305.72 \\
305.72 \\
305.72\end{array}$ & $\begin{array}{l}10 / 25 / 88 \\
10 / 31 / 88 \\
12 / 13 / 88 \\
12 / 14 / 88\end{array}$ & $\begin{array}{l}37.90 \\
38.05 \\
36.67 \\
36.69\end{array}$ & $\begin{array}{l}\text { ST } \\
\text { ET } \\
\text { ST } \\
\text { ST }\end{array}$ & $\begin{array}{l}267.82 \\
267.67 \\
269.05 \\
269.03\end{array}$ \\
\hline
\end{tabular}

(a) Calculated from the 1989 survey of the brass cap installed in each well pad and the measured casing stickup. Variations in the casing elevation can be attributed to remedial actions performed on the 1984 wells as well as variations in the surveying and/or measurement of the casing stickup.

(b) Calculated from the 1988 survey of a 2- by $2-\mathrm{ft}$ wooden survey plug installed $2 \mathrm{ft}$ from each well and the measured casing stickup.

(c) ST = Steel tape.

(d) $E T=$ Electric tape. 
TABLE 5.3. Analytical Results of All Ground-Water Samples Collected Near Landfill 1

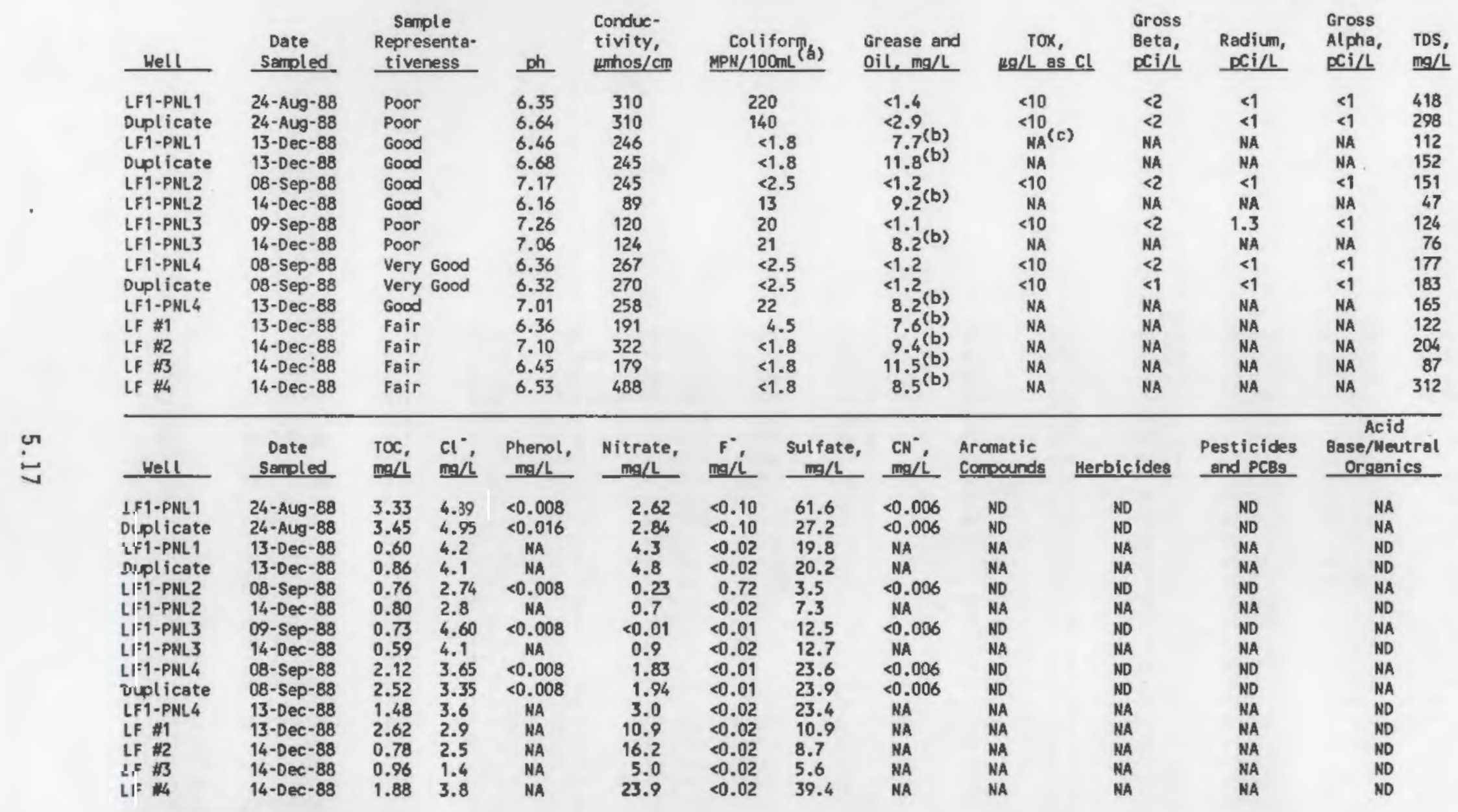

(a) MPN = most probeble number.

(b) Result is suspect, because the sample blank contained $8.2 \mathrm{mg} / \mathrm{L}$ oil and grease.

(c) NA = not analyzed. 
TABLE 5.3. (contd)

Date

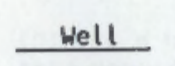

\section{LF1-PNL1} Duplicate LF1-PNL1

Filtered

Duplicate

Filtered

LF1-PNL2

Filtered

filtered

LF1-PNL3

LF1-PNL3

Filtered

LF1-PNL4

Dupl icate

LF1-PNL4

Filtered

$\begin{array}{ll}G & \text { LF \#1 } \\ & \text { Filtered }\end{array}$

泟 $\quad$ Liltered

Filtered

LF $\# 3$

Filtered

LF \#4

Filtered

\begin{tabular}{|c|c|c|c|c|c|c|}
\hline Sampled & Fe, ppm & Ba, ppm & Se, ppm & Cd, Ppm & Mn, ppm & Ca, ppm \\
\hline 24-Aug-88 & 600.0 & 4.00 & $<0.002$ & 0.004 & 20.000 & 210.0 \\
\hline 26 -Aug- 88 & 480.0 & 2.25 & $<0.002$ & 0.005 & 12.800 & 114.0 \\
\hline \multirow[t]{2}{*}{ 13-Dec-88 } & 0.7 & $<0.01$ & $<0.012$ & $<0.0006$ & 0.71 & 21.9 \\
\hline & 0.2 & $<0.01$ & NA & $<0.0005$ & 0.93 & 28.4 \\
\hline \multirow[t]{2}{*}{ 13-Dec-88 } & 0.5 & 0.01 & $<0.012$ & $<0.0006$ & 0.77 & 21.6 \\
\hline & 0.2 & $<0.01$ & NA & $<0.0005$ & 0.89 & 29.6 \\
\hline \multirow[t]{2}{*}{ 08-Sep-88 } & 1.5 & 0.012 & $<0.002$ & $<0.002$ & 0.080 & 23.0 \\
\hline & $<0.1$ & $<0.01$ & NA & $<0.01$ & $<0.01$ & 24.5 \\
\hline \multirow[t]{2}{*}{$14-$ Dec- 88} & 66.5 & 0.34 & $<0.012$ & $<0.0006$ & 1.4 & 19.9 \\
\hline & $<0.2$ & $<0.01$ & NA & $<0.0005$ & $<0.01$ & 10.5 \\
\hline $09-$ sep-88 & 11.0 & 0.078 & $<0.002$ & $<0.002$ & 0.368 & 13.0 \\
\hline \multirow[t]{2}{*}{14 -Dec-88 } & 1.4 & 0.02 & $<0.012$ & $<0.0006$ & 0.25 & 9.0 \\
\hline & $<0.1$ & $<0.01$ & NA & $<0.0005$ & 0.33 & 13.3 \\
\hline \multirow{3}{*}{$\begin{array}{l}\text { 08-Sep- } 88 \\
08-\text { Sep- } 88\end{array}$} & 2.7 & 0.026 & $<0.002$ & $<0.002$ & 1.070 & 30.0 \\
\hline & 3.4 & 0.033 & $<0.002$ & $<0.002$ & 1.000 & 29.0 \\
\hline & 0.6 & $<0.01$ & NA & $<0.01$ & $<0.01$ & 32.7 \\
\hline \multirow{2}{*}{ 13-Dec-88 } & 2.3 & 0.02 & $<0.012$ & $<0.0006$ & $<0.01$ & 23.6 \\
\hline & 1.0 & 0.02 & NA & $<0.0005$ & 1.41 & 33.9 \\
\hline \multirow[t]{2}{*}{ 13-Dec-88 } & 17.9 & 0.10 & $<0.012$ & $<0.0006$ & 0.20 & 19.9 \\
\hline & $<0.1$ & $<0.01$ & NA & $<0.0005$ & 0.03 & 20.4 \\
\hline \multirow[t]{2}{*}{ 14-Dec-88 } & 4.1 & 0.04 & $<0.012$ & 0.0007 & 0.60 & 29.2 \\
\hline & $<0.1$ & $<0.01$ & NA & $<0.0005$ & $<0.01$ & 37.4 \\
\hline \multirow[t]{2}{*}{ 14-Dec-88 } & 8.1 & 0.05 & $<0.012$ & $<0.0006$ & 0.50 & 16.3 \\
\hline & $<0.1$ & $<0.01$ & NA & $<0.0005$ & 0.02 & 19.4 \\
\hline \multirow{2}{*}{$14-$ Dec-88 } & 2.6 & 0.03 & $<0.012$ & 0.0011 & 0.70 & 45.6 \\
\hline & & & NA & $<0.0005$ & 0.63 & 60.2 \\
\hline
\end{tabular}

$\begin{array}{cc}\text { Ag. ppm } & \text { Na, ppm } \\ 0.029 & 24.0 \\ 0.024 & 16.5 \\ \text { NA } & 9.9 \\ \text { NA } & 7.8 \\ \text { NA } & 6.5 \\ \text { NA } & 8.0 \\ <0.010 & 5.3 \\ <0.01 & 8.7 \\ \text { NA } & 10.2 \\ \text { NA } & 12.8 \\ <0.010 & 3.3 \\ \text { NA } & 8.8 \\ \text { HA } & 19.0 \\ <0.010 & 5.3 \\ <0.010 & 4.6 \\ <0.01 & 7.7 \\ \text { NA } & 7.9 \\ \text { NA } & 8.8 \\ \text { NA } & 7.3 \\ \text { NA } & 6.0 \\ \text { NA } & 8.2 \\ \text { NA } & 9.8 \\ \text { NA } & 6.4 \\ \text { NA } & 6.8 \\ \text { NA } & 24.5 \\ \text { NA } & 13.5 \\ & \end{array}$

Cr, ppm
0.680
0.510
$<0.01$
$<0.01$
$<0.01$
$<0.01$
0.073
$<0.01$
0.08
$<0.01$
0.100
$<0.01$
$<0.01$
0.110
0.100
$<0.01$
$<0.01$
$<0.01$
$<0.01$
$<0.01$
$<0.01$
$<0.01$
$<0.01$
$<0.01$
$<0.01$
$<0.01$

Pb, ppm

As, PPm

Hg. Ppm

$<0.02$

$<0.002$

0.0028 $<0.006$

$<0.012<0.0008$

$<0.005$

$<0.006$

$<0.010$

$<0.012$

NA

$<0.02$

$<0.010$

$<0.0008$

$<0.0002$

0.02

NA

$<0.000$

$<0.005$

NA

0.004

NA NA

$\begin{array}{llll}0.100 & <0.02 & 0.004 & <0.0002\end{array}$

$<0.01<0.006$

$<0.005$

MA

$<0.0008$

$<.02$

$<0.002$

NA

$0.01<0.1$

$<0.002<0.0002$

$<0.006$

NA

NA

$<0.005$

$<0.010$

$<0.0008$

NA

$<0.005$

$<0.010$

0.014

$<0.005<0.010$

$<0.010$
$<0.012$

$<0.010$

$<0.010$
$<0.012$

NA
$<0.0008$

NA

$<0.000$

NA
$<0.0008$

NA 
TABLE 5.4. Volatile Organic Compound Concentrations in All Ground-Water Samples from Landfill 1

\begin{tabular}{|c|c|c|c|c|c|c|c|c|}
\hline Well & $\begin{array}{c}\text { Semple } \\
\text { Represent- } \\
\text { ativeness }\end{array}$ & $\begin{array}{c}\text { Date } \\
\text { Sampled } \\
\end{array}$ & Laboratory & $\begin{array}{l}\text { Trans }-1,2 \\
\text { DCE, ppb }\end{array}$ & $\begin{array}{l}\text { Cis }-1,2 \\
\text { DCE,_pob }\end{array}$ & $\begin{array}{l}\text { TCE, } \\
\text { ppob }\end{array}$ & $\begin{array}{c}\text { Benzene, } \\
\text { pob }\end{array}$ & $\begin{array}{l}\text { Carbon Tetra- } \\
\text { chloride, ppb }\end{array}$ \\
\hline LF1-PNL1 & Poor & 24 -Aug- 88 & AM Test & $<0.6$ & - ${ }_{N A}(a)$ & $<0.6$ & NA & $<0.6$ \\
\hline Duplicate & Poor & $24-$ Aug- 88 & AM Test & $<0.6$ & NA & $<0.6$ & NA & $<0.6$ \\
\hline LF1-PNL1 & Good & $13-$-DeC- 88 & PNL & $<0.5$ & $<0.2$ & 0.06 & $<0.5$ & $<0.01$ \\
\hline Duplicate & Good & 13-Dec-88 & PNL & $<0.5$ & $<0.2$ & 0.07 & $<0.5$ & $<0.01$ \\
\hline LF1-PNL2 & Good & $08-5 e p-88$ & AM Test & $<2$ & NA & $<2$ & MA & $<0.6$ \\
\hline LF1-PNL2 & Good & $14-\mathrm{Dec}-88$ & PNL & $<0.5$ & $<0.2$ & 0.16 & $<0.5$ & 0.01 \\
\hline LF1-PNL3 & Poor & $09-$ Sep-88 & AM Test & $<2$ & $\mathrm{NA}$ & $<2$ & NA & $<0.6$ \\
\hline LF1-PNL3 & Poor & 14-Dec-88 & PNL & $<0.5$ & $<0.2$ & $<0.03$ & $<0.5$ & $<0.01$ \\
\hline LF1-PNL4 & Very Good & 08-Sep-88 & AM Test & $<2$ & NA & 2 & NA & $<0.6$ \\
\hline Duplicate & Very Good & $08-$ Sep-88 & AM Test & $<2$ & NA & $<2$ & NA & $<0.6$ \\
\hline LF1-PNL4 & Good & 13-Dec-88 & PNL & $<0.5$ & $<0.2$ & 0.74 & $<0.5$ & $<0.01$ \\
\hline LF \#1 & Fair $r$ & 13 -Dec- 88 & PNL & $<0.5$ & $<0.2$ & $<0.03$ & $<0.5$ & $<0.01$ \\
\hline LF \#2 & Fair & 14 -Dec- 88 & PNL & $<0.5$ & $<0.2$ & 0.25 & $<0.5$ & $<0.01$ \\
\hline LF \#3 & Fair & 14 -Dec-88 & PNL & $<0.5$ & 12.0 & 16.0 & $<0.5$ & 0.05 \\
\hline LF \#4 & Fair & 14-Dec-08 & PNL & $<0.5$ & 17.0 & 7.1 & $<0.5$ & 0.02 \\
\hline
\end{tabular}

(a) NA = not analyzed.

were collected using a Teflon bailer after only a limited amount of purging. Previous samples collected from these wells soon after well development, or extensive purging, yielded no measurable coliform. Although lessrepresentative samples from other wells (Table 5.3) also indicate the presence of coliform, it is not believed that coliform is a constituent of concern.

Chromium exceeded the DWS (0.05 ppm) in unfiltered samples from wells LF1-PNL2 and LF1-PNL4. However, filtered samples from the same wells contained no detectable levels of chromium. This difference suggests that the chromium is associated with particulates suspended in the water samples. Slight turbidity was noted during the collection of samples from well LF1PNL2. Turbidity was also evidenced by high iron content in the December 1988 sample. Thus, the unfiltered samples from this well are not considered representative. The unfiltered chromium values in the December 13,1988, samples from well L.F1-PNL4 fell below the 0.01 detection 1 imit. Although chromium results from other less-representative samples have also indicated the presence of chromium in unfiltered samples, chromium has not been observed in any filtered sample. Thus, chromium is not believed to be a constituent of concern. 
Mercury exceeded the DWS (0.002 ppm) in the December 13, 1988, unfiltered sample from well LF1-PNL2; however, the unfiltered samples from this well are not considered representative as stated above. Although mercury was also observed in the initial (poorly representative) unfiltered sample from wel1 LF1-PNL1, it has not been observed in any other unfiltered samples and is not believed to be a constituent of concern.

The December 1988 samples indicated the presence of oil and grease ranging in concentrations from 7.6 to $11.8 \mathrm{ppm}$ (Table 5.3). However, these values are suspect because the blank sample submitted to the laboratory at this same time was reported to contain $8.2 \mathrm{ppm}$. Furthermore, oil and grease were not detected in any other sample. Thus, oil and grease are not considered to be constituents of concern.

The presence of TCE was observed in several wells. However, concentrations exceed the MCL only in two less-representative samples from wells 84-CD-LF-3 and -LF-4 (Table 5.4). Other volatile organic compounds [cis-1,2dichloroethylene (DCE) and tetrachloromethane (carbon tetrachloride)] were also observed in these less-representative samples. Although these results must be considered tentative, it does appear that volatile organic constituents (principally TCE and DCE) may be present at least at very low concentrations within the aquifer, and thus TCE and DCE may be constituents of concern. Further investigation is needed to verify these results.

No phenols, cyanide, aromatic compounds, herbicides, pesticides, or PCBs have been detected.

Examination of the inorganic constituent results from the upgradient samples (principally from well LF1-PNL2) versus those from the downgradient wells (principally from LF1-PNL1 and -PNL4) indicates slight differences in ground-water chemistry. Specifically, downgradient samples have slightly lower $\mathrm{pH}$ and higher total organic carbon (TOC), sul.fate, and nitrate than the upgradient samples. This contrast suggests that Landfill 1 may have some influence on the ground-water chemistry.

Nitrate concentrations exceeded the DWS (10 ppm) only in lessrepresentative ground-water samples from wells $84-C D-L F-1,-L F-2$, and -LF-4 (Table 5.3); thus, these results should only be considered tentative. 
However, comparison of the nitrate concentrations in the more-representative samples from wells LF1-PNL1, -PNL2, and -PNL4 indicates a higher concentration of nitrate downgradient of the landfill than upgradient. This suggests that nitrate may be originating from the landfill and is therefore a constituent of concern.

\subsection{EVALUATION OF THE MONITORING NETWORK}

Eight monitoring wells (four 1984 wells and four 1988 wells) are located in the vicinity of Landfill 1. Water-level data suggest that three of these wells (LF1-PNL2, LF1-PNL3, and 84-CD-LF-1) are located hydraulically upgradient of the landfill and associated open-pit dumping areas. The five remaining wells are interpreted to be either directly or indirectly downgradient of these waste sites. Fluctuations in the local ground-water mound near well 84-CD-LF-2 can influence the downgradient status of these wells. Wells LFiPNL1 and 84-CD-LF-4 appear to be directly downgradient of the landfill and therefore would be most likely to indicate any ground-water quality impacts from Landfill 1.

Ground-water samples collected from each well varied in representativeness according to the well development and purging history of the well before sample collection. Wells LF1-PNL1, LF1-PNL2, and LF1-PNL4 were extensively developed and produced good to very good samples. Wells $84-C D-L F-1,-2,-3$, and -4 were not developed or extensively purged during this study. Samples from these wells were considered only fair. However, these wells should produce very good samples after an extensive purging is performed at each we11. Well LF1-PNL3 is completed in a low-yielding portion of the aquifer, which prevented adequate development. This well produced only poor (unrepresentative) samples and should be used only for water-level measurements.

The limited analytical results from these ground-water monitoring wells suggest that some volatile organic compounds (principally TCE and DCE) and nitrate are the two chemical constituents of primary concern. In addition, a slight inorganic chemistry difference apparently exists in the upgradient and downgradient wells, suggesting that the landfill (or some other nearby source such as the runoff holding pond) may have some influence on the 
ground-water chemistry. Further investigation is needed to determine the impacts (if any) that Landfill 1 has had on the local ground-water system. 


\subsection{EVALUATION OF LANDFILL 4}

Using the information gained from examining aerial photographs, characterizing the environmental setting, and drilling and sampling, the boundaries of Landfil1 4 were located and the geologic framework, ground-water flow characteristics, ground-water quality, and monitoring well network were evaluated.

\subsection{LOCATION OF LANDFILL 4 BOUNOARIES}

The boundaries of Landfill 4 were determined based on 1) aerial photograph interpretation, 2) field reconnaissance, and 3) surface geophysical surveys. Aerial photographs taken in May 1970 and July 1976 show four distinct areas of trench-type landfill operations. Field reconnaissance allowed mapping of portions of the landfill boundaries based on visual inspection of surface debris and by the topographic expressions of the 1 andfill. Evidence was also found to suggest several areas of localized open-pit dumping and some surface dumping of tree stumps and trash. These field observations were recorded on a 7.5-min quadrangle map of the area. Surface geophysical surveys were conducted over four areas where the landfill boundaries were poorly located. These surveys included the use of 1) a ground-penetrating radar unit, 2) a ground-conductivity meter, and 3) a metal detector. The results of the surveys were summarized on a map of the area (Figure 6.1). The actual data are reported in Appendix A. All aerial photograph, field reconnaissance, and surface geophysical observations were transferred to a common base map to illustrate the current interpretation of Landfill 4 boundaries (Figure 6.2).

\subsection{GEOLOGIC FRAMEWORK}

The stratigraphy beneath Landfill 4 has been interpreted primarily from field analysis of split-spoon and core-barrel samples collected during the installation of six (1988) ground-water monitoring wells. These wells ranged in depth from 32 to $46.5 \mathrm{ft}$ below land surface. The actual field records are provided in Appendix E. Additional geologic information was gathered from 


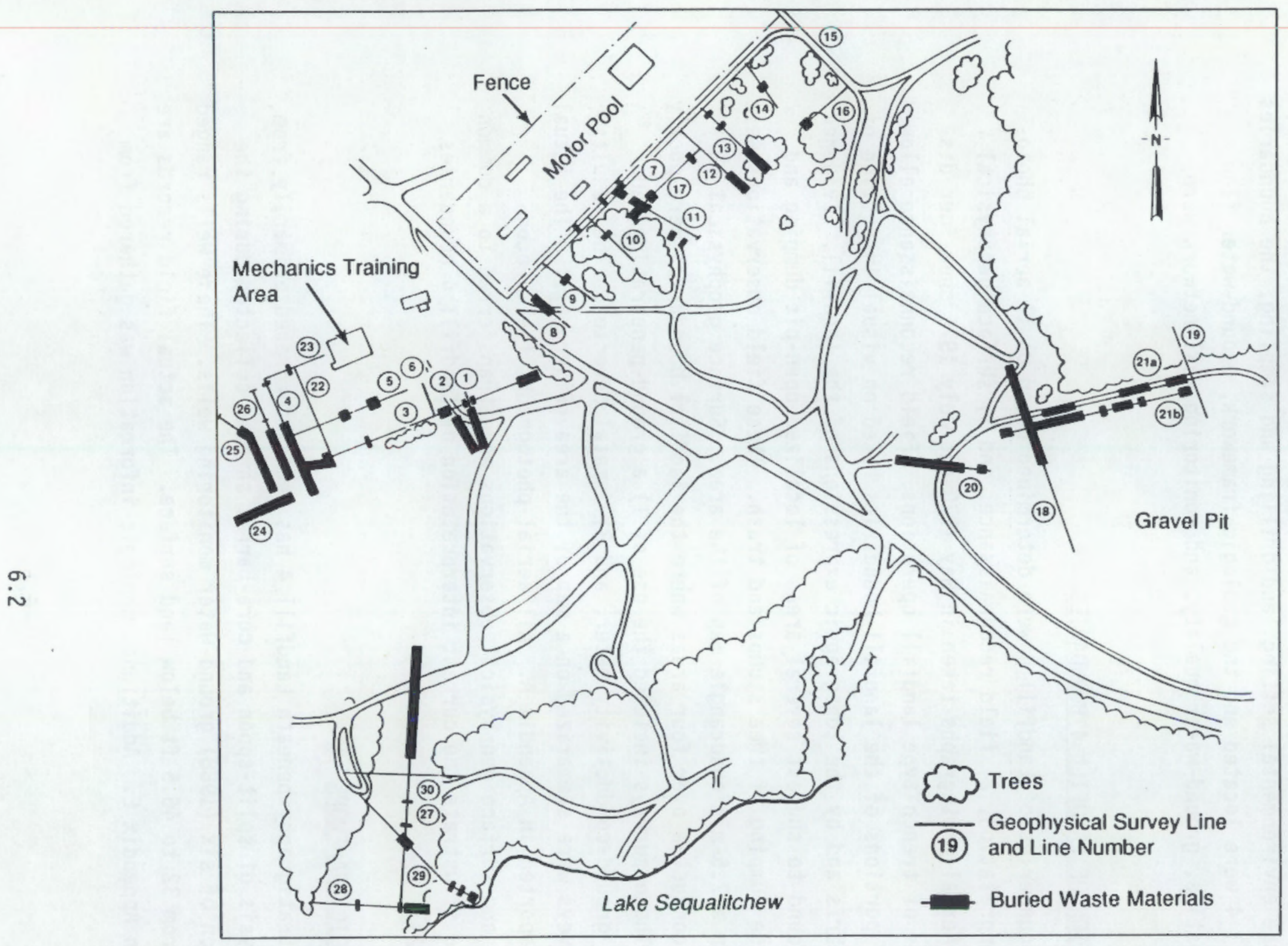

FIGURE 6.1. Location of Surface Geophysical Surveys, Showing Location of Suspected Waste Materials 


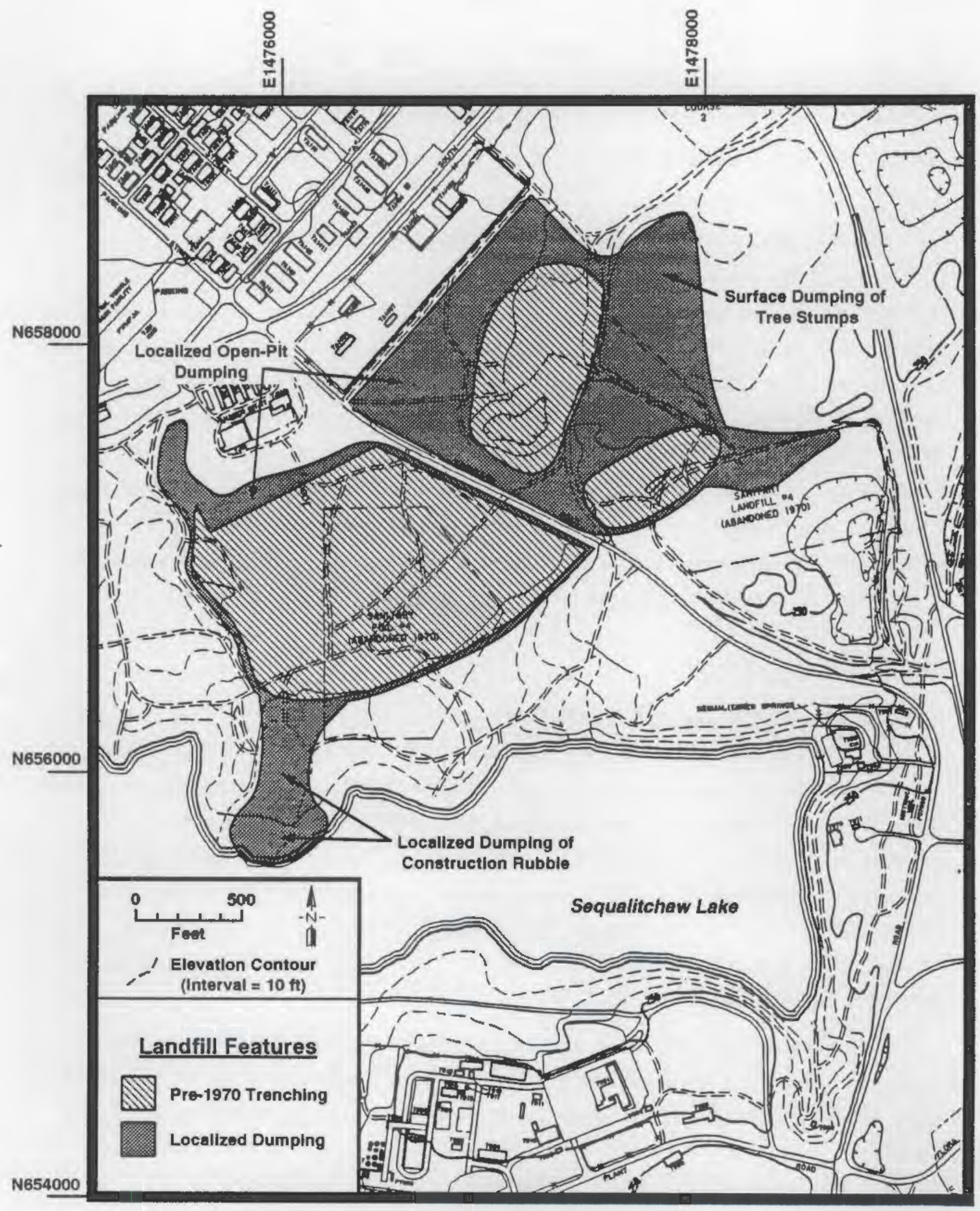

FIGURE 6.2. Boundaries and Configuration of Landfill 4 
drilling logs compiled during earlier (1981) drilling operations (Appendix I). The location of the new (1988) and existing (1981) wells is shown in Figure 6.3, and the well completion data are summarized in Table 6.1. The subsurface data generated from drilling each new borehole are summarized in Figures 6.4 through 6.9.

The sediments encountered in the vicinity of Landfill 4 consist primarily of muddy sandy gravel with minor lenses of sand and gravel. These sediments are considered part of the Vashon Drift Formation. A 3- to 4-ftthick layer of clay/silt was encountered at approximately $31 \mathrm{ft}$ below land surface at well LF4-PNL4. This unit was not encountered in any of the other new wells; however, it is evident in the drill logs for existing (1981) wells LF4-3 and -4. This layer appears to represent the top of the Kitsap Formation and is, therefore, the boundary between hydrostratigraphic units $A$ and $B$ of Brown and Caldwell (1983). The clay layer at well LF4-PNL4 overlies a water-bearing dark-gray to black-colored, fine-grained, silty/sand sequence. A generalized geologic cross section is illustrated in Figure 6.10.

\subsection{GROUND-WATER FLOW}

Ground-water flow in the uppermost aquifer beneath Landfill 4 is generally to the north-northwest. This general north-northwesterly ground-water flow direction is consistent with the regional ground-water gradient depicted by others (see Figure 3.4) and supports the concept that the uppermost ground-water system discharges to Puget Sound. Figures 6.11 and 6.12 illustrate the configuration of the water table as interpreted from water-level measurements recorded on October 31 and December 15, 1988, respectively. The ground-water gradient across the 1 andfill site ranges from very small to nearly $3 \mathrm{ft} / 1000 \mathrm{ft}$ or $0.003 \mathrm{ft} / \mathrm{ft}$.

Two ground-water features appear to have the greatest effect on local flow directions: a gentle ground-water divide located along the far eastern side of the landfill and a ground-water depression located just north of the landfill. The ground-water divide, centered around well LF4-4, cannot be readily explained at this time. However, as with other major lakes in the area, Sequalitchew Lake and nearby American Lake are believed to be 


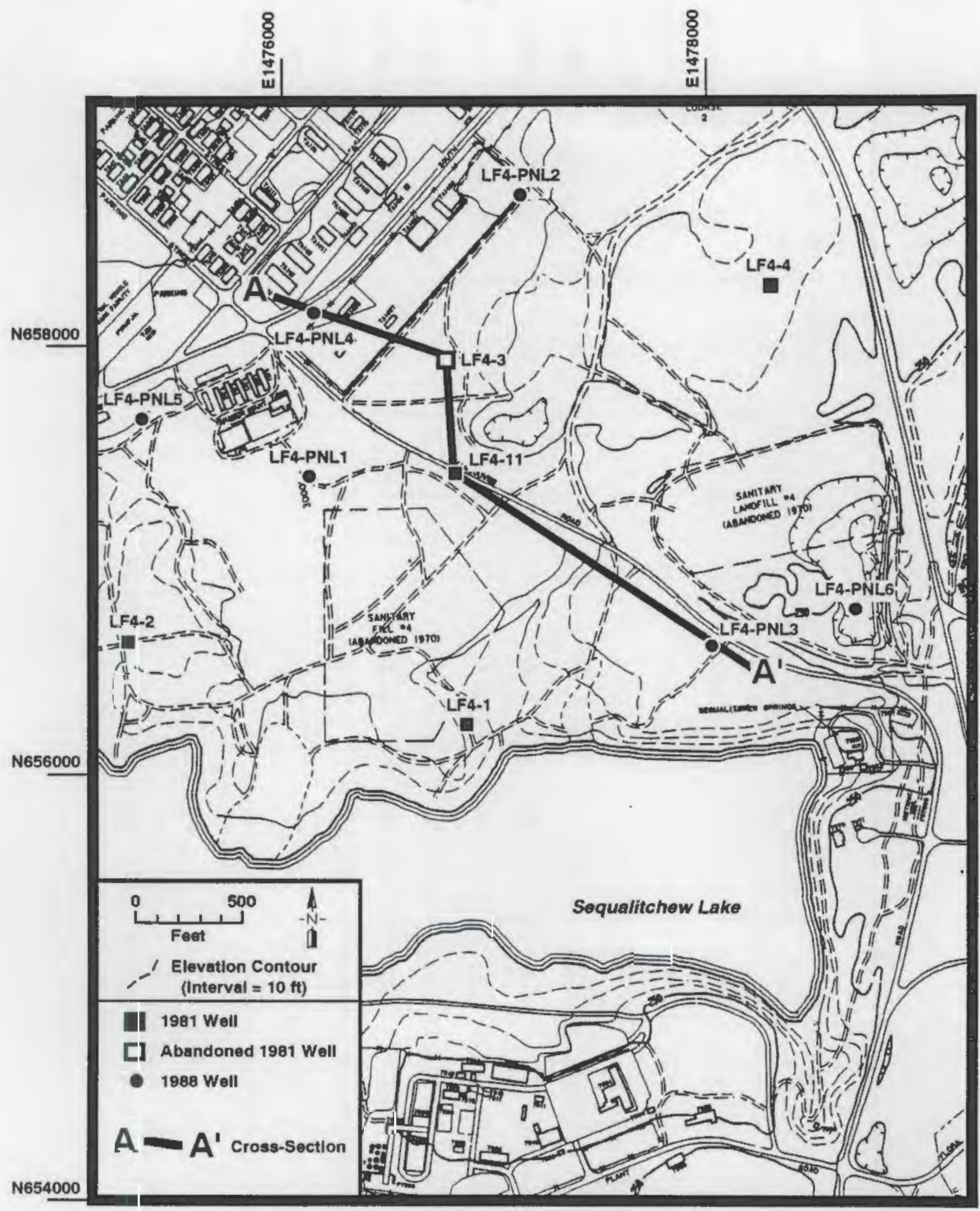

FIGURE 6.3. Location of Monitoring Wells Around Landfill 4 
IABLE 6.1. Well Completion Data for Landfill 4

\begin{tabular}{|l|l|l|l|l|c|c|c|}
\hline Well & \multicolumn{1}{|c|}{$\begin{array}{c}\text { Coordinates } \\
\text { North, East } \\
\text { (State Plane) }\end{array}$} & $\begin{array}{c}\text { Casing } \\
\text { Elevation, } \mathrm{ft} \\
\text { above MSL(a) }\end{array}$ & $\begin{array}{c}\text { Drilled } \\
\text { Depth, } \mathrm{ft} \\
\text { below land(b) }\end{array}$ & $\begin{array}{c}\text { Depth to } \\
\text { Water, } \mathrm{ft}(\mathrm{b}) \\
\text { below land }\end{array}$ & $\begin{array}{c}\text { Well } \\
\text { Diameter, } \\
\text { in. }\end{array}$ & $\begin{array}{c}\text { Screened } \\
\text { Interval, } \mathrm{ft}(\mathrm{c}) \\
\text { below land }\end{array}$ & $\begin{array}{c}\text { Date } \\
\text { Completed }\end{array}$ \\
\hline LF4-1 & 656276,1476795 & 225.37 & 38.0 & 10 & 4 & $22-38$ & $04 / 28 / 81$ \\
LF4-2 & 656697,1475243 & 218.27 & 36.0 & 5 & 4 & $20-36$ & $04 / 29 / 81$ \\
LF4-3(d) & 657932,1476792 & 238.79 & 60.0 & 26 & 4 & $42-58$ & $04 / 30 / 81$ \\
LF4-4 & 658202,1478294 & 235.41 & 98.0 & 27 & 4 & $39-55$ & $05 / 01 / 81$ \\
LF4-11 & 657397,1476814 & 234.05 & 44.0 & 19 & 4 & $30-46$ & $05 / 12 / 81$ \\
LF4-PNL1 & 657439,1476136 & 237.82 & 41.0 & 26.5 & 4 & $22-37$ & $08 / 09 / 88$ \\
LF4-PNL2 & 658639,1477139 & 240.48 & 40.0 & 29 & 2 & $23-38$ & $08 / 09 / 88$ \\
LF4-PNL3 & 656576,1477955 & 246.59 & 46.5 & 34.5 & 2 & $28-43$ & $08 / 09 / 88$ \\
LF4-PNL4 & 658162,1476174 & 235.72 & 39.0 & 26.5 & 4 & $23.5-33.5$ & $10 / 05 / 88$ \\
LF4-PNL5 & 657661,1475355 & 237.46 & 36.0 & 26.5 & 4 & $20.5-35.5$ & $10 / 07 / 88$ \\
LF4-PNL6 & 656714,1478619 & 232.19 & 30.0 & 20 & 4 & $15-30$ & $10 / 12 / 88$ \\
\hline
\end{tabular}
(a) All elevations on the PVC casing are to the north rim.
(b) Measurements are to the nearest $0.5 \mathrm{ft}$.
(c) Measurements are rounded down to the nearest foot or 0.5 foot.
(d) This well is abandoned. This information pertains to the well prior to abandonment. 


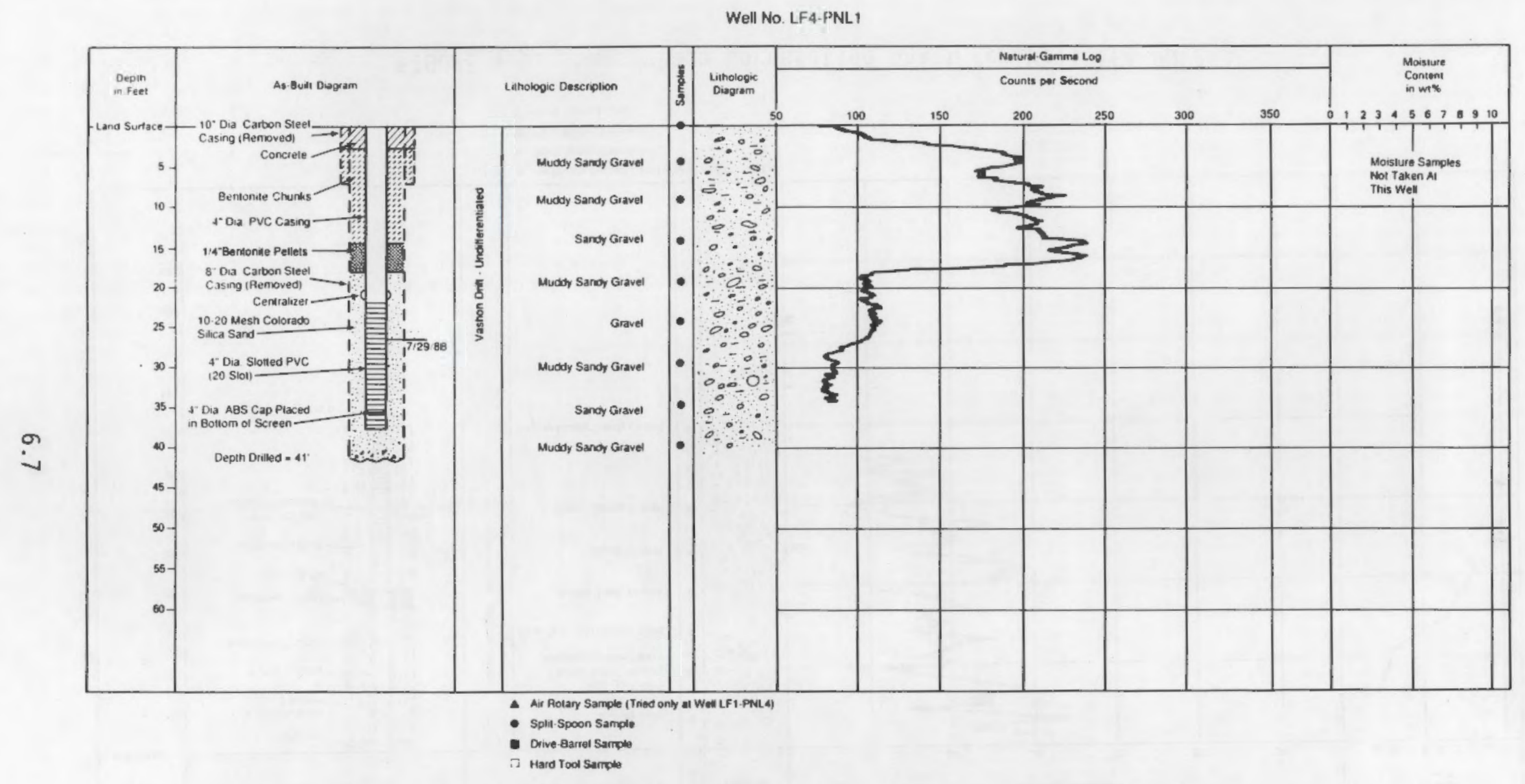

FIGURE 6.4. Subsurface Correlation Chart for Well LF4-PNL1 


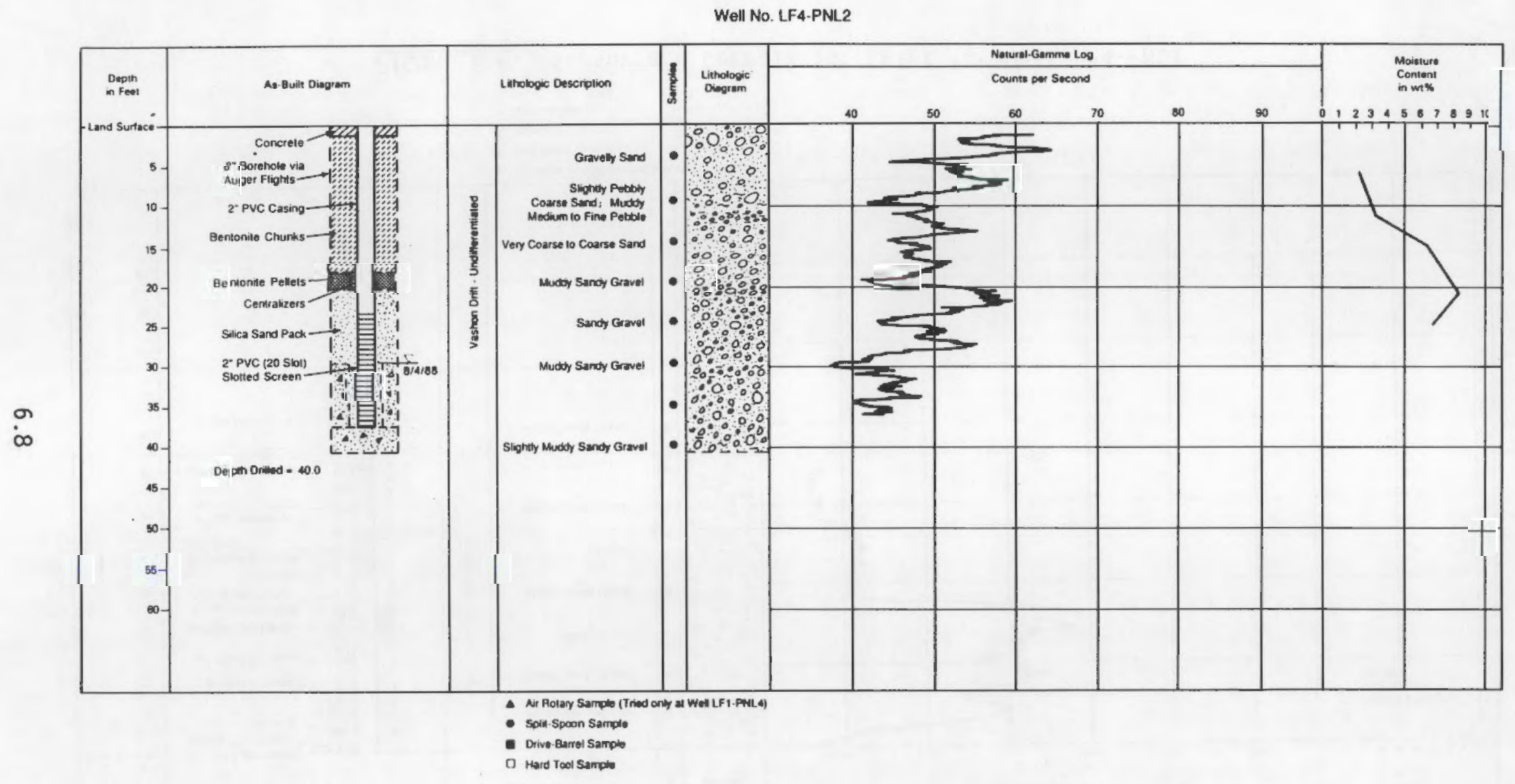

FIGURE 6.5. Subsurface Correlation Chart for Well LF4-PNL2 


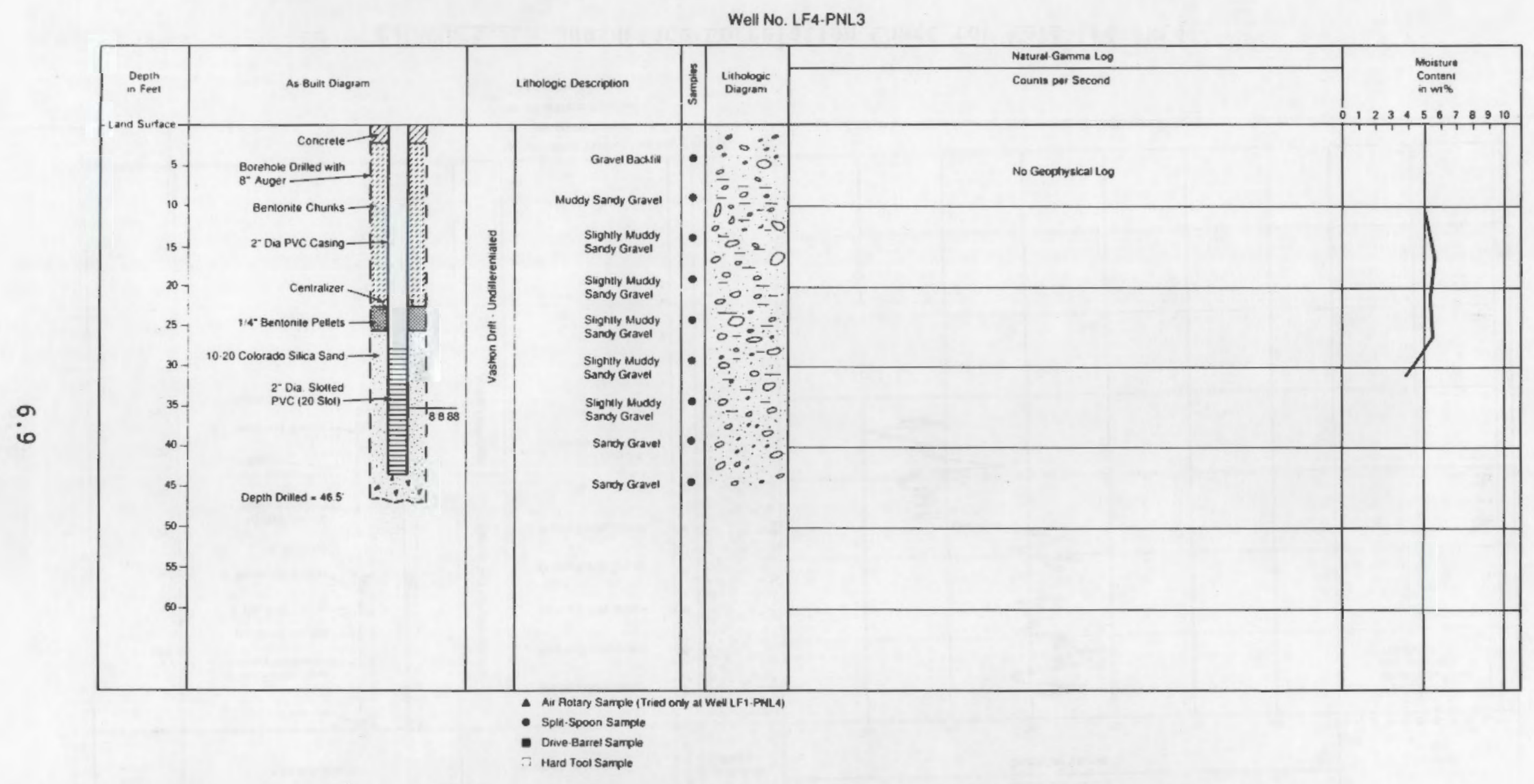

FIGURE 6.6. Subsurface Correlation Chart for Well LF4-PNL3 


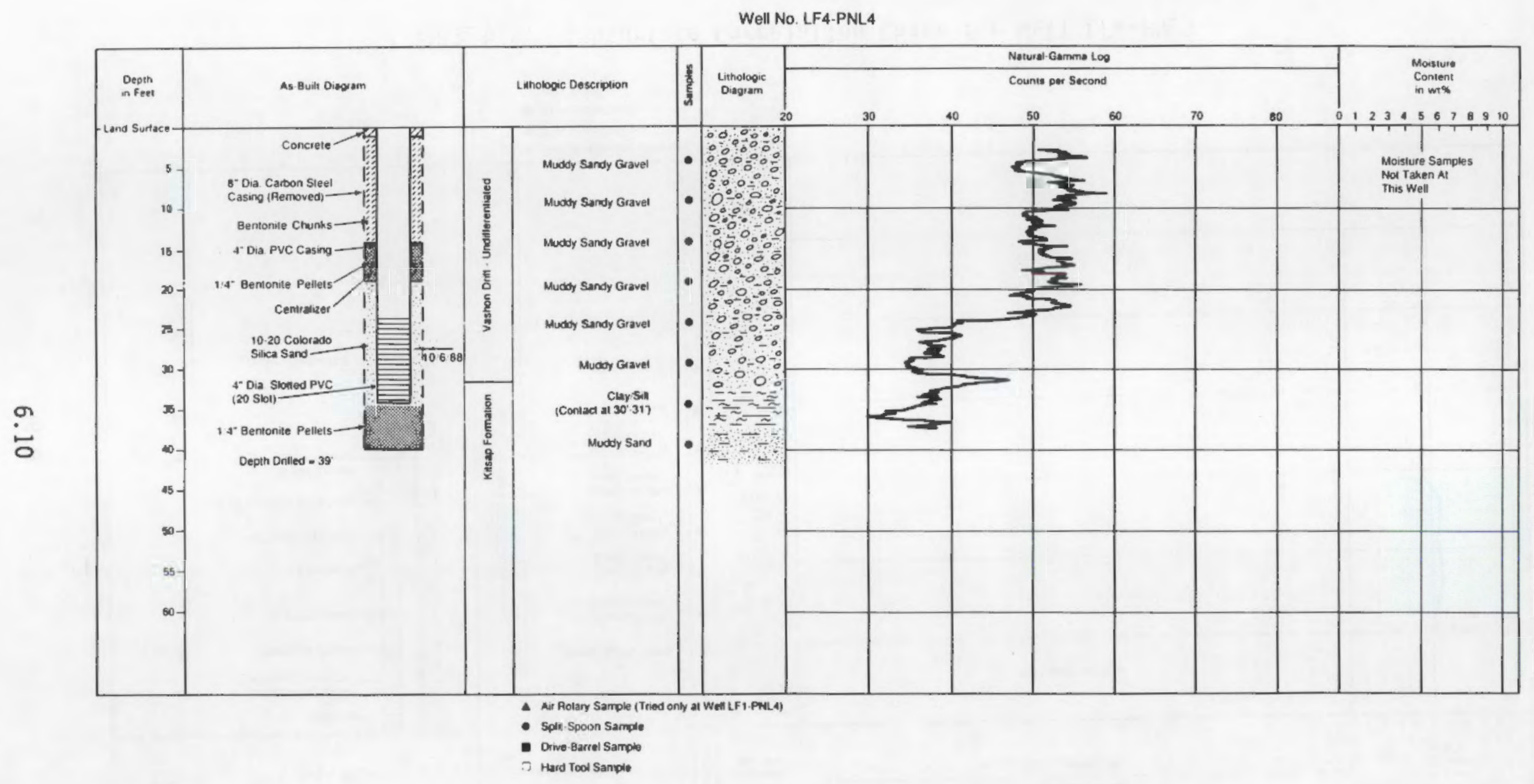

FIGURE 6.7. Subsurface Correlation Chart for Well LF4-PNL4 
Well No. LF4.PNL5

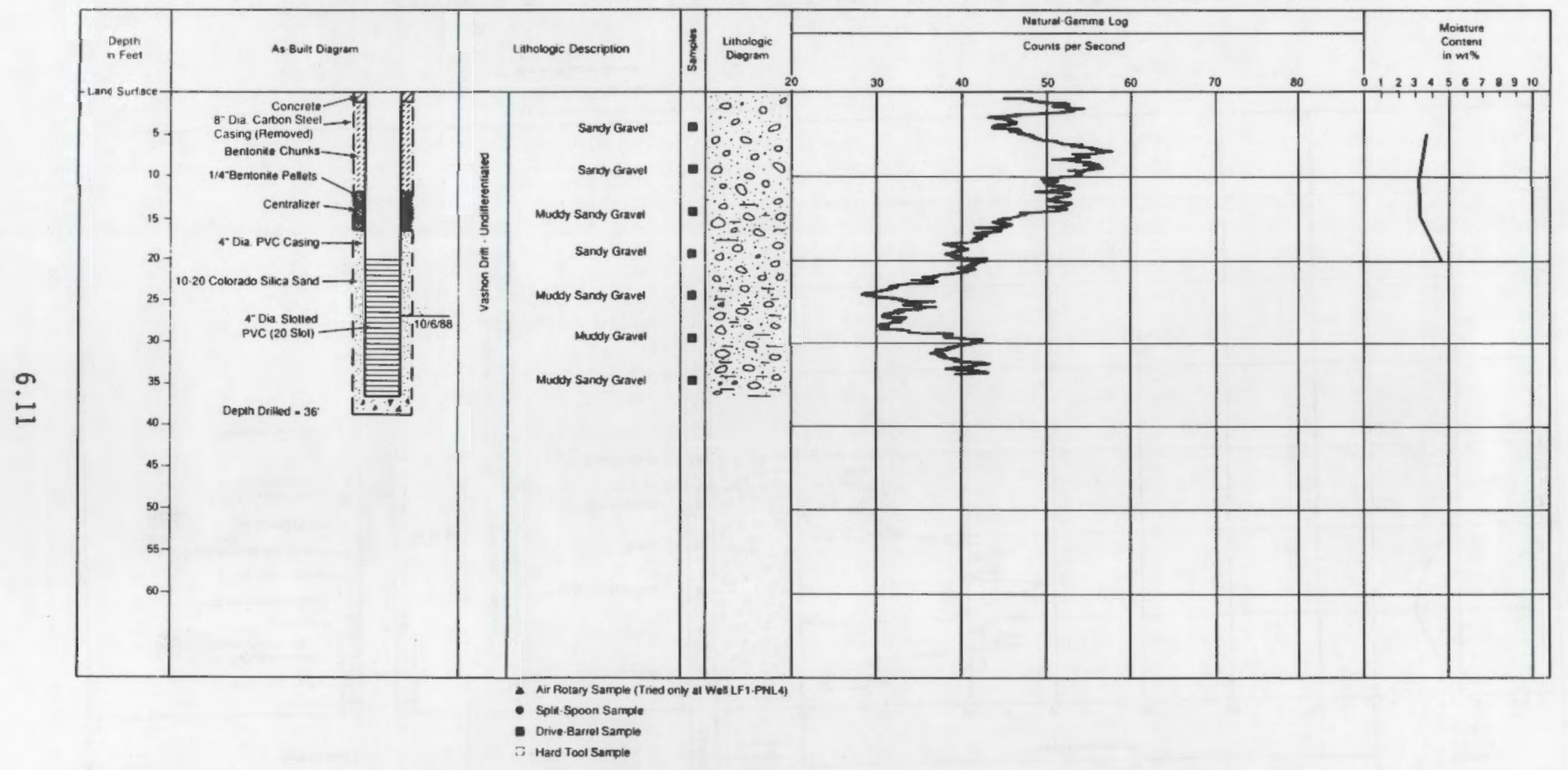

FIGURE 6.8. Subsurface Correlation Chart for Well LF4-PNL5 
Well No. LF4-PNL6

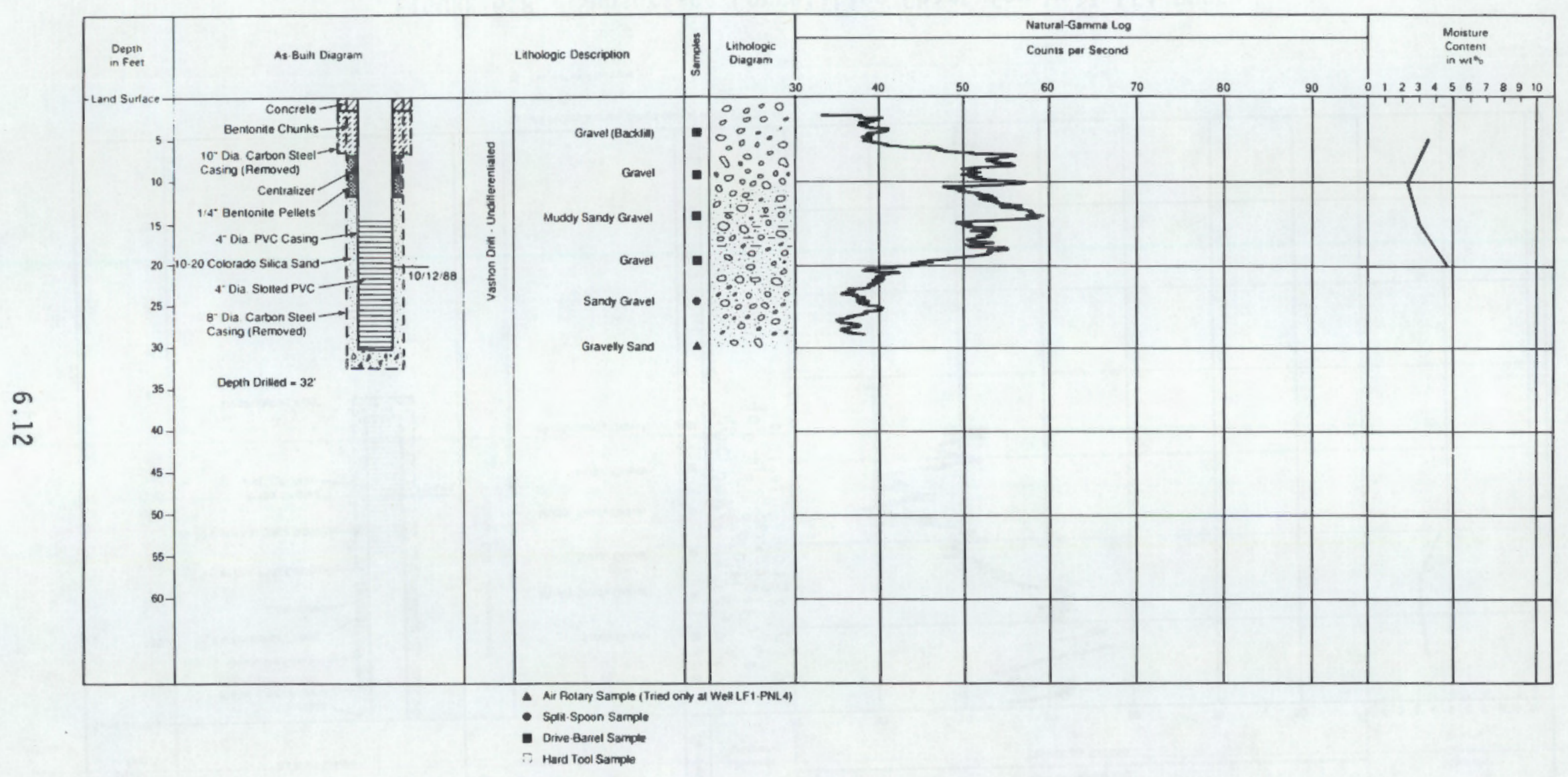

FIGURE 6.9. Subsurface Correlation Chart for Well LF4-PNL6 


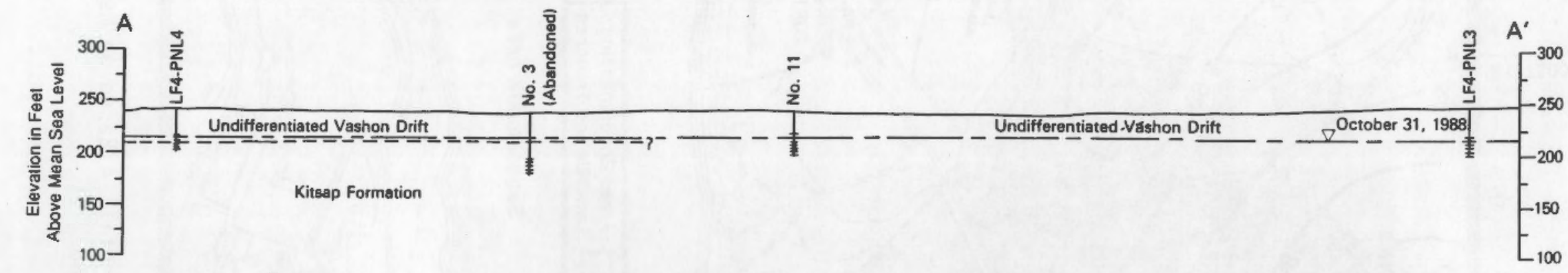

$\stackrel{\sigma}{\omega}$

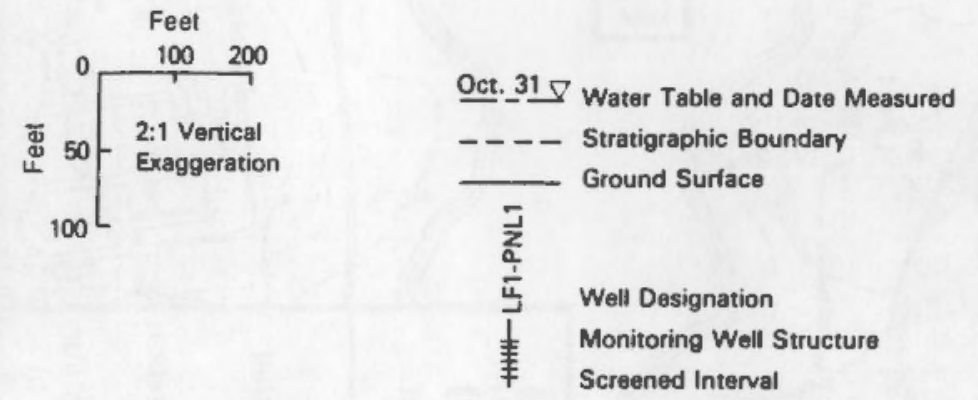

FIGURE 6.10. Generalized Geologic Cross Section Through Landfill 4 


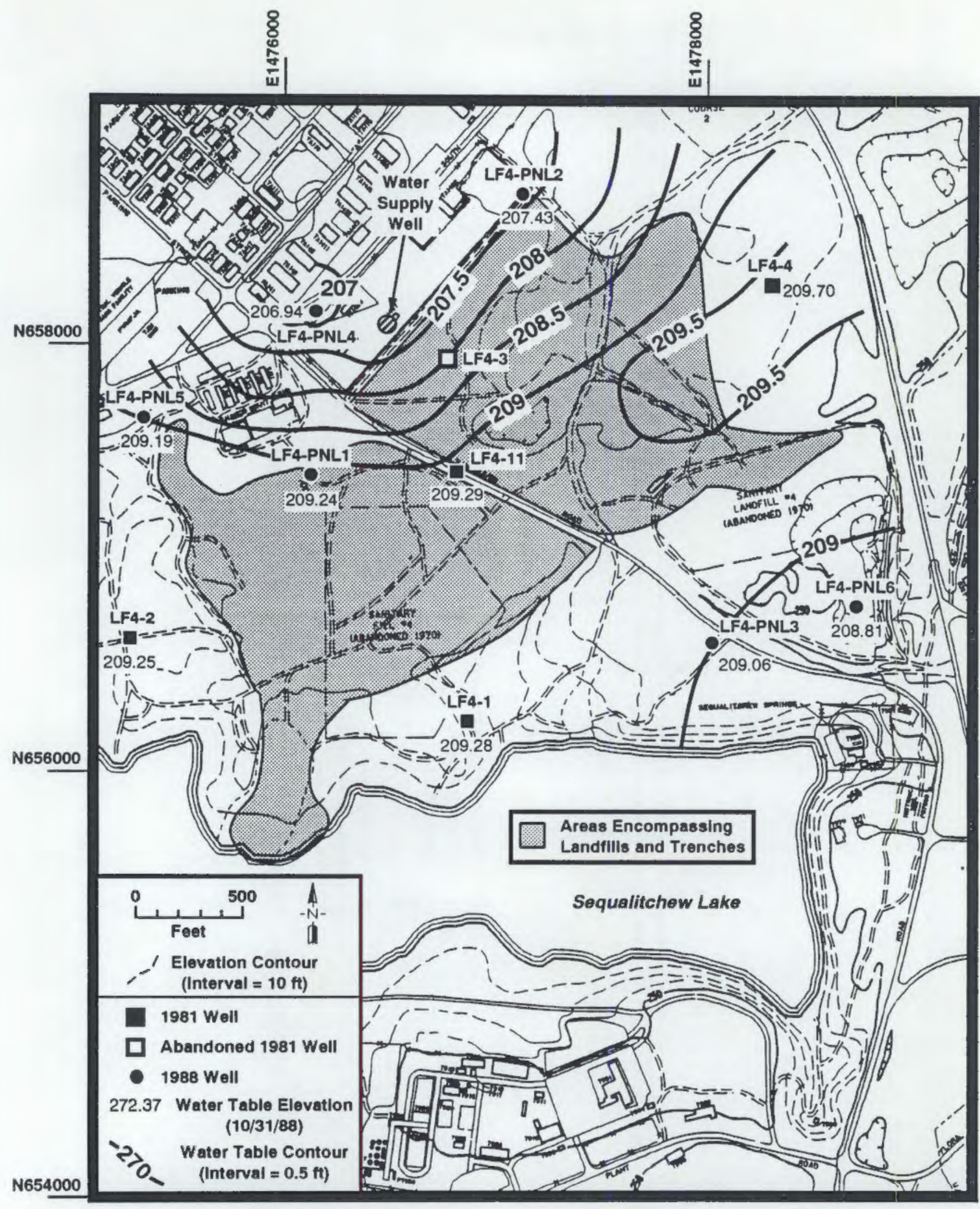

FIGURE 6.11. Water-Table Map for Landfil1 4 Based on Measurements Made on October 31, 1988 


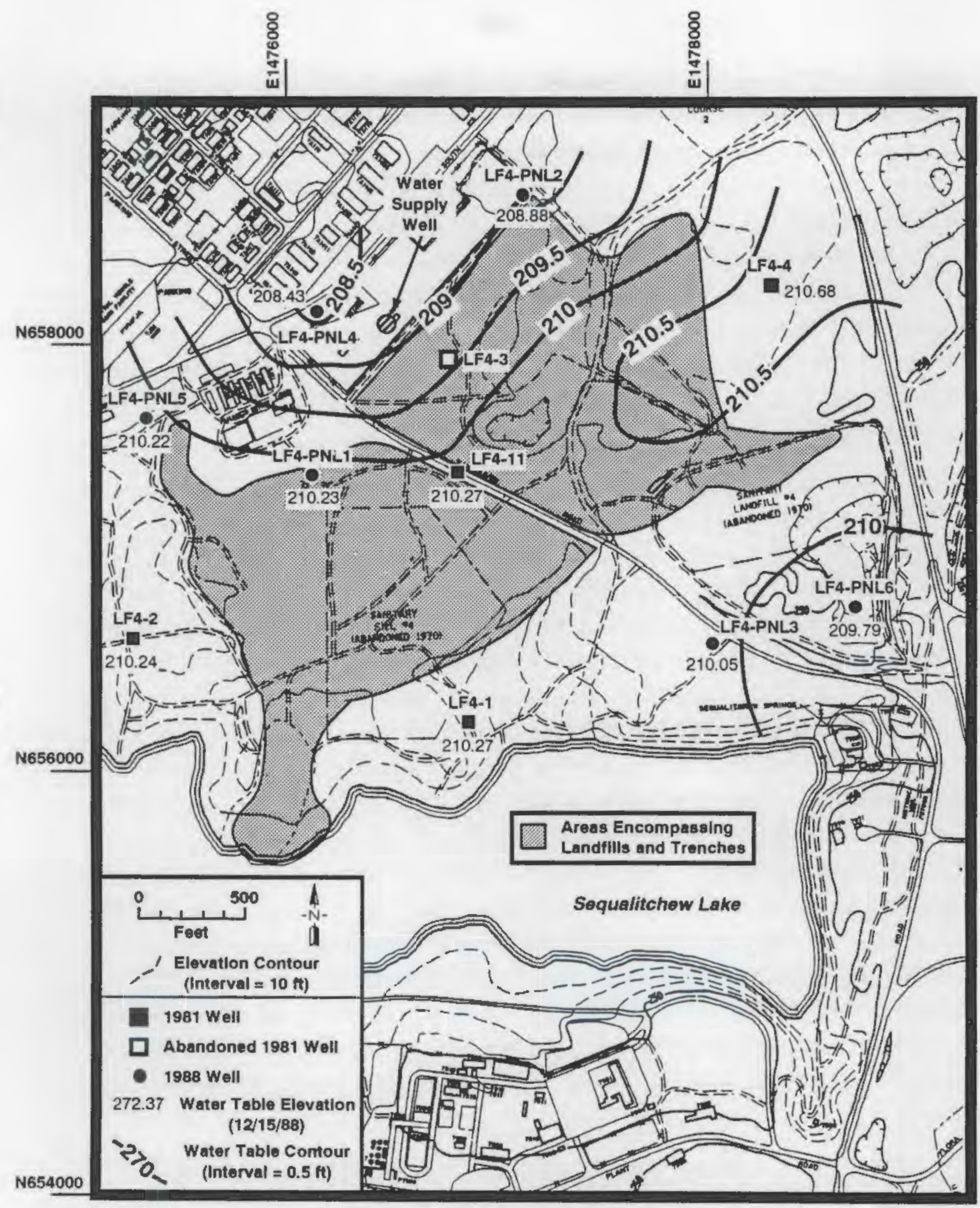

FIGURE 6.12. Water-Table Surface Beneath Landfill 4 as Interpreted from Water Levels Measured on December 15, 1988 
hydraulically connected with the shallow ground-water system beneath Landfill 4, allowing the interchange of surface and ground-water. At times the lake may recharge the local ground-water system, while at other times it may act as a discharge area for local ground water, reversing the local groundwater gradient and creating a temporary ground-water divide, perhaps like that observed in the eastern portion of the landfill area (Figures 6.11 and 6.12). The lower ground-water level at well LF4-PNL4 may not only reflect the natural northerly dipping ground-water surface, but may also reflect the operation of a water supply well located approximately $400 \mathrm{ft}$ to the east (Figures 6.11 and 6.12). The operational history of this water supply well during the time frame of this study has not been investigated.

Figure 6.13 illustrates the generalized ground-water flow directions and location of upgradient and downgradient wells, based on the October 31 and December 15, 1988, water-level measurements. Only one monitoring well, LF4-4, is interpreted to be hydraulically upgradient of Landfill 4 . Wells LF4-1 and LF4-2 (located adjacent to Sequalitchew Lake) may be either upgradient or downgradient of the landfill depending on the relationship between the lake, the shallow aquifer, and the water-table configuration at the time. However, these two wells are expected to reflect the hydrochemistry of the lake more so than that of the aquifer directly underlying the landfill. The same generalizations also apply to wells LF4-PNL3 and LF4-PNL6, which are located over $600 \mathrm{ft}$ southeast (just opposite of the general northwestern ground-water gradient) of the landfill boundary. Although these wells are shown to be downgradient of the landfill at the time of this study, it is expected that their hydrochemistry may be more indicative of the regional aquifer than of the ground water immediately beneath the landfil1. The remaining monitoring wells (LF4-PNL1, LF4-PNL2, LF4-PNL4, LF4-PNL5, and LF4-11) are considered to be truly downgradient of the landfill and are expected to most accurately reflect the ground-water quality beneath Landfill 4.

Variations in ground-water levels, particularly those caused by surface water/ground-water interactions or by the operation of nearby water supply wells, could greatly affect the upgradient/downgradient status of monitoring 


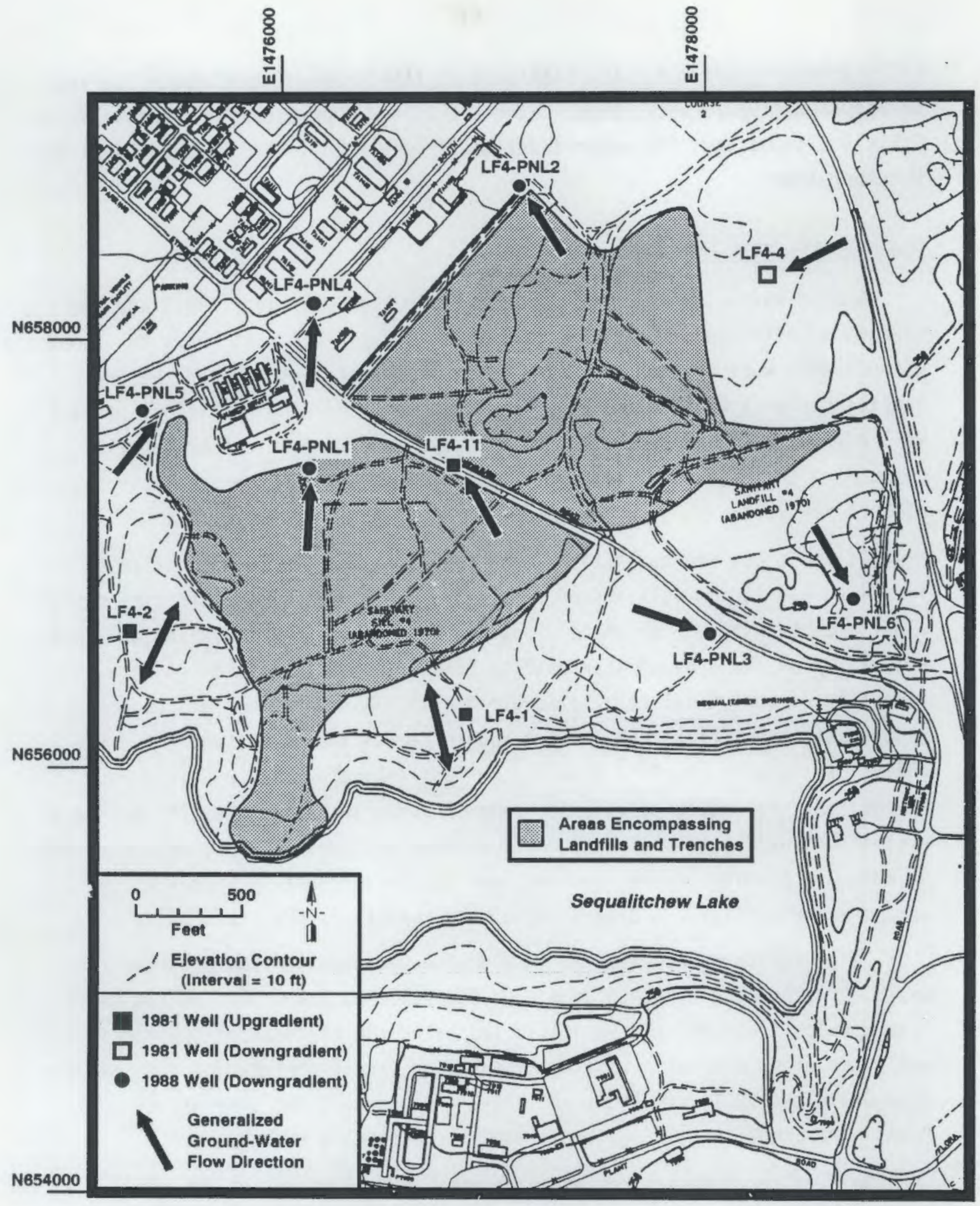

FIGURE 6.13. Generalized Ground-Water Flow Directions and Location of Upgradient and Downgradient Wells at Landfill 4 
wells around Landfill 4. Over the 8-month time frame of this study (Spring to Winter of 1988), water levels have fluctuated over $2.5 \mathrm{ft}$ in some wells. Table 6.2 summarizes the water-level measurements collected between April and December 1988.

\subsection{GROUND-WATER QUALITY}

Ground-water samples were collected from four existing (1981) and the six newly installed (1988) wells surrounding Landfill 4. At least two sampling events were conducted at each well. The representativeness of these samples varies according to the well development and purging history of each well before sample collection. Refer to Section 4.4 for further details concerning the objectives of each sampling event and the representativeness of each sample.

At least one representative (good to very good) sample was collected from each monitoring well around Landfill 4. However, a complete time equivalent set of analyses for these ground-water samples is not available. Thus, evaluation of the ground-water quality beneath Landfill 4 is based on a composite of analytical results acquired primarily in October and December 1988. These analytical results are summarized in Tables 6.3 and 6.4. The analytical reports received from the AM Test laboratory are provided in Appendix $K$. As indicated earlier, some of these analytical results may be an artifact of drilling, well construction, poor well development, and/or a lack of adequate purging before sampling, and may not accurately represent the ground-water quality. A discussion of the results is presented below.

Fecal coliform was detected in ground-water samples from at least two wel1s, LF4-PNL5 and LF4-PNL6, and from Sequalitchew Lake. Dne of the duplicate samples from well LF4-PNL4 also indicated the presence of coliform, while the other did not. Less-representative (and therefore suspect) samples from wells LF4-PNL1, LF4-PNL3, and LF4-2 also suggest the presence of coliform. Thus, coliform is believed to be a constituent of concern.

An unfiltered sample contained chromium in excess of the DWS (0.05 ppm) only in well LF4-PNL2 on one occasion. However, the filtered sample from this same well contained no detectable levels of chromium. This sample had 


\section{IABLE 6.2. Water-Level Summary for Wells Near Landfill 4}

\begin{tabular}{|c|c|c|c|c|c|}
\hline Well & $\begin{array}{c}\text { 6-in. Casing } \\
\text { Elevation, } \\
\text { ft above ms (a) }\end{array}$ & $\begin{array}{c}\text { Measurement } \\
\text { Date }\end{array}$ & $\begin{array}{c}\text { Depth to } \\
\text { Water, } \\
\mathrm{ft} \\
\end{array}$ & $\begin{array}{l}\text { Type of } \\
\text { Measure }\end{array}$ & $\begin{array}{c}\text { Head } \\
\text { Elevation, } \\
\text { ft above mst }\end{array}$ \\
\hline Fort Levis \#1 & $\begin{array}{l}224.69^{(b)} \\
224.69^{(b)} \\
226.70 \\
226.70\end{array}$ & $\begin{array}{l}04 / 20 / 88 \\
08 / 12 / 88 \\
10 / 31 / 88 \\
12 / 15 / 88\end{array}$ & $\begin{array}{l}12.95 \\
15.50 \\
17.42 \\
16.43\end{array}$ & $\begin{array}{l}\mathrm{ST}^{(c)} \\
\mathrm{ST} \\
\mathrm{ET}(\mathrm{d}) \\
\mathrm{ST}\end{array}$ & $\begin{array}{l}211.74 \\
209.19 \\
209.28 \\
210.27\end{array}$ \\
\hline Fort Lewis \#2 & $\begin{array}{l}219.19(b) \\
219.19^{(b)} \\
219.63 \\
219.63\end{array}$ & $\begin{array}{l}04 / 20 / 88 \\
08 / 12 / 88 \\
10 / 31 / 88 \\
12 / 15 / 88\end{array}$ & $\begin{array}{r}7.57 \\
9.97 \\
10.38 \\
9.39\end{array}$ & $\begin{array}{l}\text { ST } \\
\text { ST } \\
\text { ET } \\
\text { ST }\end{array}$ & $\begin{array}{l}211.62 \\
209.22 \\
209.25 \\
210.24\end{array}$ \\
\hline Fort Lewis $\# 3$ & $\begin{array}{l}238.7^{(b)} \\
238.79^{(b)}\end{array}$ & $\begin{array}{l}04 / 20 / 88 \\
08 / 12 / 88\end{array}$ & $\begin{array}{l}27.78 \\
30.29\end{array}$ & $\begin{array}{l}\text { ST } \\
\text { ST }\end{array}$ & $\begin{array}{l}211.01 \\
208.50\end{array}$ \\
\hline Fort Lewis \#4 & $\begin{array}{l}235.93^{(b)} \\
235.93^{(b)} \\
236.78 \\
236.78 \\
236.78\end{array}$ & $\begin{array}{l}04 / 20 / 88 \\
08 / 12 / 88 \\
10 / 31 / 88 \\
11 / 23 / 88 \\
12 / 15 / 88\end{array}$ & $\begin{array}{l}23.12 \\
25.71 \\
27.08 \\
26.50 \\
26.10\end{array}$ & $\begin{array}{l}\text { ST } \\
\text { ST } \\
\text { ET } \\
\text { ST } \\
\text { ST }\end{array}$ & $\begin{array}{l}212.81 \\
210.22 \\
209.70 \\
210.28 \\
210.68\end{array}$ \\
\hline Fort Lewis \#11 & $\begin{array}{l}234.79(b) \\
234.79(b) \\
235.21 \\
235.21\end{array}$ & $\begin{array}{l}04 / 20 / 88 \\
08 / 12 / 88 \\
10 / 31 / 88 \\
12 / 15 / 88\end{array}$ & $\begin{array}{l}23.08 \\
25.58 \\
25.92 \\
24.94\end{array}$ & $\begin{array}{l}\text { ST } \\
\text { ST } \\
\text { ET } \\
\text { ST }\end{array}$ & $\begin{array}{l}211.71 \\
209.21 \\
209.29 \\
210.27\end{array}$ \\
\hline LF4-PNL1 & $\begin{array}{l}238.72 \\
238.72 \\
238.72\end{array}$ & $\begin{array}{l}08 / 12 / 88 \\
10 / 31 / 88 \\
12 / 15 / 88\end{array}$ & $\begin{array}{l}29.45 \\
29.48 \\
28.49\end{array}$ & $\begin{array}{l}\text { ST } \\
\text { ET } \\
\text { ST }\end{array}$ & $\begin{array}{l}209.27 \\
209.24 \\
210.23\end{array}$ \\
\hline LF4-PNL2 & $\begin{array}{l}240.85 \\
240.85 \\
240.85\end{array}$ & $\begin{array}{l}08 / 12 / 88 \\
10 / 31 / 88 \\
12 / 15 / 88\end{array}$ & $\begin{array}{l}32.08 \\
33.42 \\
31.97\end{array}$ & $\begin{array}{l}\text { ST } \\
\text { ET } \\
\text { ST }\end{array}$ & $\begin{array}{l}208.77 \\
207.43 \\
208.88\end{array}$ \\
\hline LF4-PNL3 & $\begin{array}{l}247.18 \\
247.18 \\
247.18 \\
247.18\end{array}$ & $\begin{array}{l}08 / 12 / 88 \\
10 / 31 / 88 \\
12 / 14 / 88 \\
12 / 15 / 88\end{array}$ & $\begin{array}{l}38.24 \\
38.12 \\
37.12 \\
37.13\end{array}$ & $\begin{array}{l}\text { ST } \\
\text { ET } \\
\text { ST } \\
\text { ST }\end{array}$ & $\begin{array}{l}208.94 \\
209.06 \\
210.06 \\
210.05\end{array}$ \\
\hline LF4-PNL4 & $\begin{array}{l}236.68 \\
236.68\end{array}$ & $\begin{array}{l}10 / 31 / 88 \\
12 / 15 / 88\end{array}$ & $\begin{array}{l}29.74 \\
28.25\end{array}$ & $\begin{array}{l}\text { ET } \\
\text { ST }\end{array}$ & $\begin{array}{l}206.94 \\
208.43\end{array}$ \\
\hline LF4-PNL5 & $\begin{array}{l}238.30 \\
238.30\end{array}$ & $\begin{array}{l}10 / 31 / 88 \\
12 / 15 / 88\end{array}$ & $\begin{array}{l}29.11 \\
28.08\end{array}$ & $\begin{array}{l}\text { ET } \\
\text { ST }\end{array}$ & $\begin{array}{l}209.19 \\
210.22\end{array}$ \\
\hline LF4-PNL6 & $\begin{array}{l}232.56 \\
232.56 \\
232.56\end{array}$ & $\begin{array}{l}10 / 31 / 88 \\
11 / 23 / 88 \\
12 / 15 / 88\end{array}$ & $\begin{array}{l}23.75 \\
23.07 \\
22.77\end{array}$ & $\begin{array}{l}\text { ET } \\
\text { ST } \\
\text { ST }\end{array}$ & $\begin{array}{l}208.81 \\
209.49 \\
209.79\end{array}$ \\
\hline
\end{tabular}

(a) Calculated from the 1989 survey of the bress cap installed in each well pad and the measured casing stickup. Variations in the casing elevation can be attributed to remedial actions performed on the 1981 wells as well as variations in the surveying/measurement of casing stickup.

(b) From the 1982 survey.

(c) ST = steel tape.

(d) $\mathrm{ET}=$ electrical tape. 
TABLE 6.3. Analytical Results from Fort Lewis Ground-Water Wells at Landfill 4

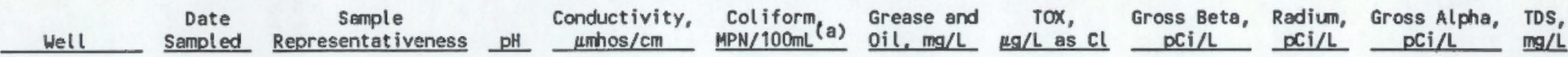

\begin{tabular}{|c|c|c|c|c|c|c|c|c|c|c|c|}
\hline LF4-PNL1 & $08 / 24 / 88$ & Poor & 6.25 & 390 & 1600 & $<1.0$ & 18 & $<2$ & $<1$ & $<1$ & 295 \\
\hline LF4-PNL2 & $09 / 08 / 88$ & Very good & 6.58 & 440 & $<2.5$ & $<1.2$ & $<10$ & $<1$ & $<1$ & $<$ & 296 \\
\hline LF4-PNL3 & $08 / 24 / 88$ & Poor & 6.20 & 138 & 17 & 8.4 & 44. & $<2$ & $<1$ & $<1$ & 524 \\
\hline LF4-PNL3 & $12 / 14 / 88$ & Very good & 6.60 & 131 & $<1.8$ & $8.6^{(b)}$ & $\mathrm{NA}(\mathrm{c})$ & NA & NA & NA & 53 \\
\hline LF4-PNL 4 & $10 / 30 / 88$ & Very good & 6.29 & 260 & $<1.8$ & $<1$ & 94 & $<2$ & $<2$ & $<1$ & 239 \\
\hline Dupl icate & $10 / 30 / 88$ & Very good & 6.35 & 270 & 4.5 & 5.4 & $<10$ & $<2$ & $<2$ & $<1$ & 288 \\
\hline LF4-PNL5 & $10 / 30 / 88$ & Very good & 6.67 & 92 & 23 & 3.2 & 102 & $<2$ & $<2$ & $<1$ & 150 \\
\hline LF4-PNL6 & $10 / 07 / 88$ & Very good & 6.88 & 124 & 49 & 4.2 & 202 & $<2$ & $<2$ & $<1$ & 275 \\
\hline LF4-1 & $10 / 30 / 88$ & Very good & 6.53 & NA & NA & NA & NA & NA & NA & NA & 86 \\
\hline Dupl icate & $10 / 30 / 88$ & Very good & 6.35 & NA & NA & NA & NA & NA & NA & NA & 67 \\
\hline LF4-2 & $10 / 30 / 88$ & Very good & 6.59 & NA & NA & NA & NA & NA & NA & NA & 181 \\
\hline LF $4-2$ & $12 / 14 / 88$ & Good & 6.89 & 186 & 13 & $21.6^{(b)}$ & NA & NA & NA & NA & 135 \\
\hline LF $4-4$ & $10 / 30 / 88$ & Very good & 6.74 & NA & NA & NA & NA & NA & NA & NA & 62 \\
\hline LF4-11 & $10 / 30 / 88$ & Very good & 6.21 & NA & NA & NA & NA & NA & NA & NA & 101 \\
\hline Sequal itchew & $12 / 15 / 88$ & NA & 7.30 & 122 & 17 & $11.8^{(b)}$ & NA & NA & NA & NA & 53 \\
\hline
\end{tabular}

or

$12 / 15 / 88$

NA

Date TOC, $\mathrm{Cl}^{-}$, Phenol, $\mathrm{NO}_{3}^{-}, \mathrm{F}^{*}, \mathrm{SO}_{4}{ }^{=}, \mathrm{CN}^{-}$, Aromatic

Pesticides

Acid-Base-

\begin{tabular}{|c|c|c|c|c|c|c|c|c|}
\hline Well & Samoled & $\mathrm{mg} / \mathrm{L}$ & $\mathrm{mg} / \mathrm{i}$ & mg/L & $\mathrm{mg} \mathrm{m}^{\prime} \mathrm{i}$ & $\mathrm{mg} / \dot{\mathrm{L}}$ & $\mathrm{mg} / \mathrm{L}$ & $\mathrm{mg} / \mathrm{L}$ \\
\hline LF4-PNL1 & $08 / 24 / 88$ & 3.01 & 15.60 & $<0.008$ & $<0.10$ & $<0.10$ & 11.2 & $<0.006$ \\
\hline LF4-PNL2 & $09 / 08 / 88$ & 2.28 & 4.16 & $<0.008$ & $<0.01$ & $<0.01$ & 26.4 & $<0.006$ \\
\hline LF4-PNL3 & $08 / 24 / 88$ & 1.14 & 4.10 & $<0.016$ & 0.76 & $<0.10$ & 10.9 & $<0.006$ \\
\hline LF4-PNL3 & $12 / 14 / 88$ & 0.44 & 3.50 & NA & 3.7 & $<0.20$ & 10.6 & NA \\
\hline LF4-PNL4 & $10 / 30 / 88$ & 1.23 & 3.59 & $<0.008$ & 1.34 & $<0.01$ & 20.5 & $<0.006$ \\
\hline Dupl icate & $10 / 30 / 88$ & 1.26 & 2.32 & $<0.008$ & 1.38 & $<0.01$ & 20.9 & $<0.006$ \\
\hline LF4-PWL5 & $10 / 30 / 88$ & 0.72 & 1.41 & $<0.008$ & 0.416 & $<0.01$ & 7.2 & $<0.006$ \\
\hline LF4-PNL6 & $10 / 07 / 88$ & 0.69 & 2.92 & $<0.008$ & 0.377 & $<0.01$ & 6.9 & $<0.006$ \\
\hline LF 4-1 & $10 / 30 / 88$ & NA & 3.8 & MA & 0.2 & $<0.02$ & 9.3 & NA \\
\hline Dupl icate & $10 / 30 / 88$ & NA & 3.8 & NA & 0.1 & $<0.02$ & 9.8 & NA \\
\hline LF 4-2 & $10 / 30 / 88$ & NA & 4.8 & NA & $<0.1$ & $<0.02$ & 16.4 & NA \\
\hline$L F 4-2$ & $12 / 14 / 88$ & 1.28 & 4.4 & NA & 2.0 & $<0.02$ & 14.4 & NA \\
\hline LF4-4 & $10 / 30 / 88$ & NA & 3.3 & NA & 1.6 & $<0.02$ & 8.7 & NA \\
\hline LF $4=11$ & $10 / 30 / 88$ & NA & 3. & HA & $<0.1$ & $<0.02$ & 19.8 & NA \\
\hline Sequal itch & $12 / 15 / 88$ & 4.07 & & NA & 1.7 & $<0.02$ & 6.7 & NA \\
\hline
\end{tabular}

No (d)
ND
NO
NA
NA
NA
NA
NA
NA
NA
NA
NA
NA
NA
NA

ND
ND
ND
NA
ND
ND
ND
ND
NA
NA
NA
NA
NA
NA
NA

and PCBs

eutral/Organics

Sequalitchew Lake 12/15/88 $4.07 \quad 4.3$

ND
ND
ND
NA
ND
ND
ND
ND
NA
NA
NA
NA
NA
NA
NA

NA
NA
NA
ND
NA
NA
NA
NA
NA
NA
NA
ND
NA
NA
ND


IABLE 6.3. (contd)

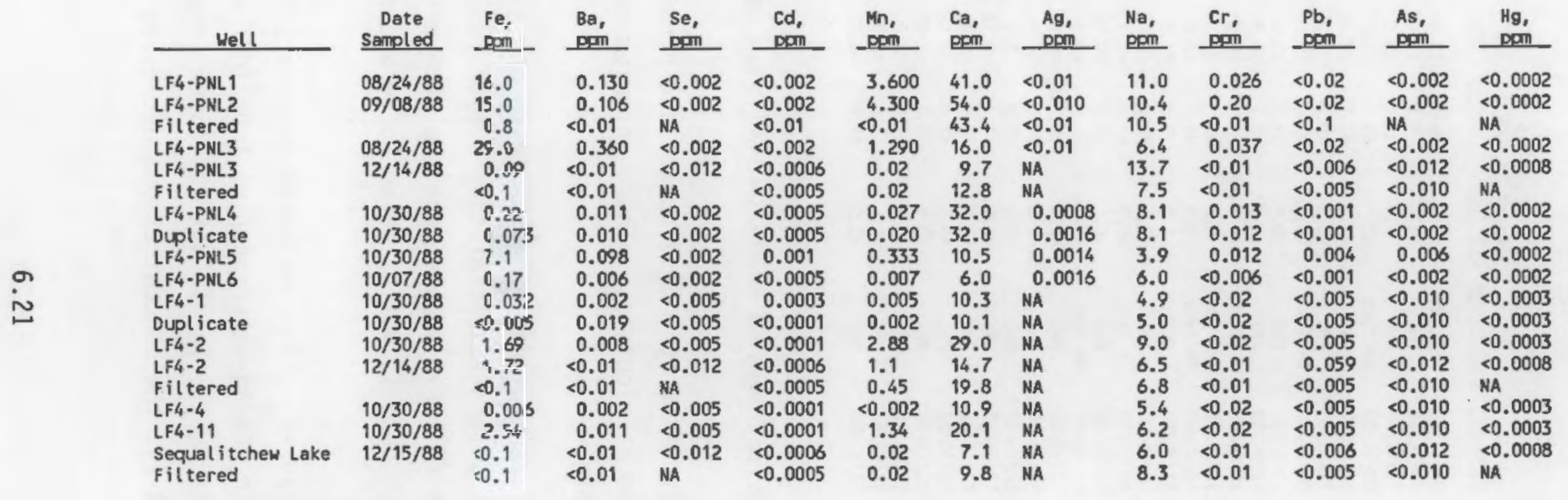

(a) $M P N=$ most probable number.

(b) Analytical result is suspect. Blank sample contained $8.2 \mathrm{mg} / \mathrm{L}$ oil and grease.

(c) $N A=$ not analyzed.

(d) ND $=$ not detected. 
TABLE 6.4. Analytical Results for Volatile Organic Compounds at Landfill 4

\begin{tabular}{|c|c|c|c|c|c|c|c|c|}
\hline Hell & $\begin{array}{c}\text { Sample } \\
\text { Representat iveness }\end{array}$ & $\begin{array}{c}\text { Date } \\
\text { Sampled } \\
\end{array}$ & Laboratory & $\begin{array}{l}\text { Trans-1,2 } \\
\text { DCE,_ppb } \\
\end{array}$ & $\begin{array}{c}\text { Cis }-1,2 \\
\text { DCE, } \\
\text { gpb } \\
\end{array}$ & $\begin{array}{l}\text { TCE, } \\
\text { ppob }\end{array}$ & $\begin{array}{l}\text { Benzene, } \\
\text { ppb }\end{array}$ & $\begin{array}{c}\text { Carbon } \\
\text { Tetrachloride, } \\
\text { pob } \\
\end{array}$ \\
\hline LF4-PNL1 & poor & $08 / 24 / 88$ & AM Test & 0.9 & $\mathrm{MA}^{(a)}$ & 13.9 & $<1.0$ & $<0.6$ \\
\hline LF4-PNL1 & good & $10 / 06 / 88$ & PNL & 1.0 & 9.0 & 17.00 & 1.0 & 0.31 \\
\hline LF4-PNL1 & good & $12 / 12 / 88$ & PNL & 2.4 & 11.0 & 20.0 & 0.9 & $<0.01$ \\
\hline LF4-PNL2 & very good & $09 / 08 / 88$ & AM Test & $<2$ & MA & 5.7 & $<1.0$ & $<0.6$ \\
\hline LF4-PNL2 & good & $10 / 06 / 88$ & PNL & $<0.5$ & $<1.0$ & 0.02 & $<0.5$ & $<0.01$ \\
\hline LF4-PNL2 & good & $12 / 15 / 88$ & PNL & $<0.5$ & $<0.2$ & 0.25 & $<0.9$ & $<0.01$ \\
\hline LF4-PNL3 & poor & $08 / 24 / 88$ & AM Test & $<0.6$ & NA & 13.0 & $<1.0$ & $<0.6$ \\
\hline LF4-PNL3 & poor & $10 / 06 / 88$ & PNL & $<0.5$ & $<1.0$ & 22.0 & $<0.5$ & $<0.01$ \\
\hline LF4-PNL3 & very good & $12 / 14 / 88$ & PNL & $<0.5$ & 2.7 & 32.0 & $<0.5$ & $<0.01$ \\
\hline LF4-PNL4 & very good & $10 / 06 / 88$ & PNL & $<0.5$ & $<1.0$ & 19.0 & $<0.5$ & $<0.01$ \\
\hline $\mathrm{LF}_{4}-\mathrm{PNL}_{4}$ (b) & very good & $10 / 30 / 88$ & AM Test & 0.8 & NA & 22.0 & NA & $<.7$ \\
\hline Dupl icate & very good & $10 / 30 / 88$ & AM Test & 0.8 & NA & 19.0 & NA & $<0.7$ \\
\hline LF4-PNL4 & good & $12 / 15 / 88$ & PNL & 1.5 & 5.3 & 21.0 & $<0.5$ & 0.04 \\
\hline LF4-PNL5 & good & $10 / 06 / 88$ & PNL & $<0.5$ & $<1.0$ & 0.02 & $<0.5$ & $<0.01$ \\
\hline LF4-PNL5 & very good & $10 / 30 / 88$ & AM Test & $<0.7$ & MA & $<0.7$ & NA & $<0.7$ \\
\hline LF4-PNL5 & good & $12 / 15 / 88$ & PNL & $<0.5$ & $<0.2$ & $<0.03$ & $<0.5$ & 0.41 \\
\hline LF4-PNL6 & very good & $10 / 30 / 88$ & AM Test & $<0.7$ & NA & $<0.7$ & NA & $<0.7$ \\
\hline LF4-PNL6 & good & $12 / 15 / 88$ & PNL & $<0.5$ & $<0.2$ & $<0.03$ & $<0.5$ & $<0.01$ \\
\hline LF4-1 & poor & $10 / 06 / 88$ & PNL & $<0.5$ & $<1.0$ & 1.8 & $<0.5$ & $<0.01$ \\
\hline LF4-1 & good & $12 / 15 / 88$ & PNL & $<0.5$ & $<0.2$ & 2.4 & $<0.5$ & $<0.01$ \\
\hline LF4-2 & poor & $10 / 06 / 88$ & PNL & $<0.5$ & $<1.0$ & 0.56 & $<0.5$ & $<0.01$ \\
\hline LF $4-2$ & good & $12 / 14 / 88$ & PNL & $<0.5$ & 0.9 & 0.32 & $<0.5$ & $<0.01$ \\
\hline LF4-4 & poor & $10 / 06 / 88$ & PNL & $<0.5$ & $<1.0$ & $<0.02$ & $<0.5$ & $<0.01$ \\
\hline $\mathrm{LF} 4-4$ & good & $12 / 15 / 88$ & PNL & $<0.5$ & $<0.2$ & $<0.03$ & $<0.5$ & $<0.01$ \\
\hline LF $4-11$ & poor & $10 / 06 / 88$ & PNL & $<0.5$ & $<1.0$ & 5.0 & $<0.5$ & $<0.01$ \\
\hline LF4-11 & good & $12 / 15 / 88$ & PNL & 1.4 & 6.8 & 19.0 & 0.1 & 0.03 \\
\hline $\begin{array}{l}\text { Sequal itchew } \\
\text { Lake }\end{array}$ & NA & $12 / 15 / 88$ & PNL & $<0.5$ & $<1.0$ & $<0.03$ & $<0.5$ & $<0.01$ \\
\hline
\end{tabular}

(a) NA $=$ not analyzed.

(b) This sample also contained $26 \mathrm{ppb}$ of methylene chloride (believed to be a laboratory contaminant).

been collected immediately after well development and probably contained a slight amount of suspended particulates. Thus, chromium is not believed to be a constituent of concern.

Lead [in concentrations exceeding the DWS $(0.05 \mathrm{ppm})$ ] was detected in the unfiltered portion of a less-representative sample (unpurged) from well LF4-2. However, the filtered sample from the same well contained no detectable level of lead. Lead is not believed to be a constituent of concern.

Iron and manganese concentrations exceeded secondary DWS 0.3 and $0.05 \mathrm{ppm}$, respectively) in ground-water samples from four wells (LF4-2, LF4-11, LF4-PNL2, and LF4-PNL5). In all but two cases (iron in well LF4-PNL2 and manganese in well LF4-2), the concentrations decreased below the 
secondary DWS when the samples were filtered. Because of the lack of data to the contrary, iron and manganese are assumed to be constituents of concern at Landfill 4. However, iron and manganese are secondary drinking water parameters.

$0 i 1$ and grease were detected in samples from at least two wells (LF4-PNL5 and LF4-PNL6). One of the duplicate samples from well LF4-PNL4 also indicated the presence of $0 i 1$ and grease, while the other did not. other samples have also indicated the presence of $0 i 1$ and grease; however, the values from the December sampling event are suspect because all samples submitted to the laboratory were found to contain oil and grease, including the blank sample submitted to the laboratory at the same time. These results indicate that $0 i 1$ and grease may be present in the aquifer beneath Landfill 4 and thus are constituents of concern.

Cis-1,2-dichloroethylene and/or TCE were identified in concentrations above MCLs (5 ppb) in ground-water samples from wells LF4-PNL1, -PNL2, -PNL3, -PNL4, and LF4-11 (Table 6.4). The results from wells LF4-PNL1 and LF4-11 are considered less representative because the wells were not purged before sampling. Other less-representative samples from wells LF4-1 and LF4-2 also suggest the presence of these two constituents. Figure 6.14 illustrates the distribution of TCE concentration ranges for the good to very good groundwater samples collected between September and December 1988. Analytical results from wells LF4-PNLI, LF4-PNL4, and LF4-11 also indicate the presence of trans-1,1-dichloroethylene, carbon tetrachloride, and benzene (except well LF4-PNL4). Volatile organic compounds are considered the principal constituents of concern.

No phenols, cyanide, aromatic compounds, herbicides, pesticides, or PCBs were detected.

\subsection{EVALUATION OF THE MONITORING NETWORK}

Ten ground-water monitoring wells (four 1981 wells and six 1988 wells) are located in the vicinity of Landfill 4 . Water-level data from these wells suggest that at least one of these wells (LF4-4) is located hydraulically upgradient of the landfil1. Five wells (LF4-11, LF4-PNL1, LF4-PNL2, 


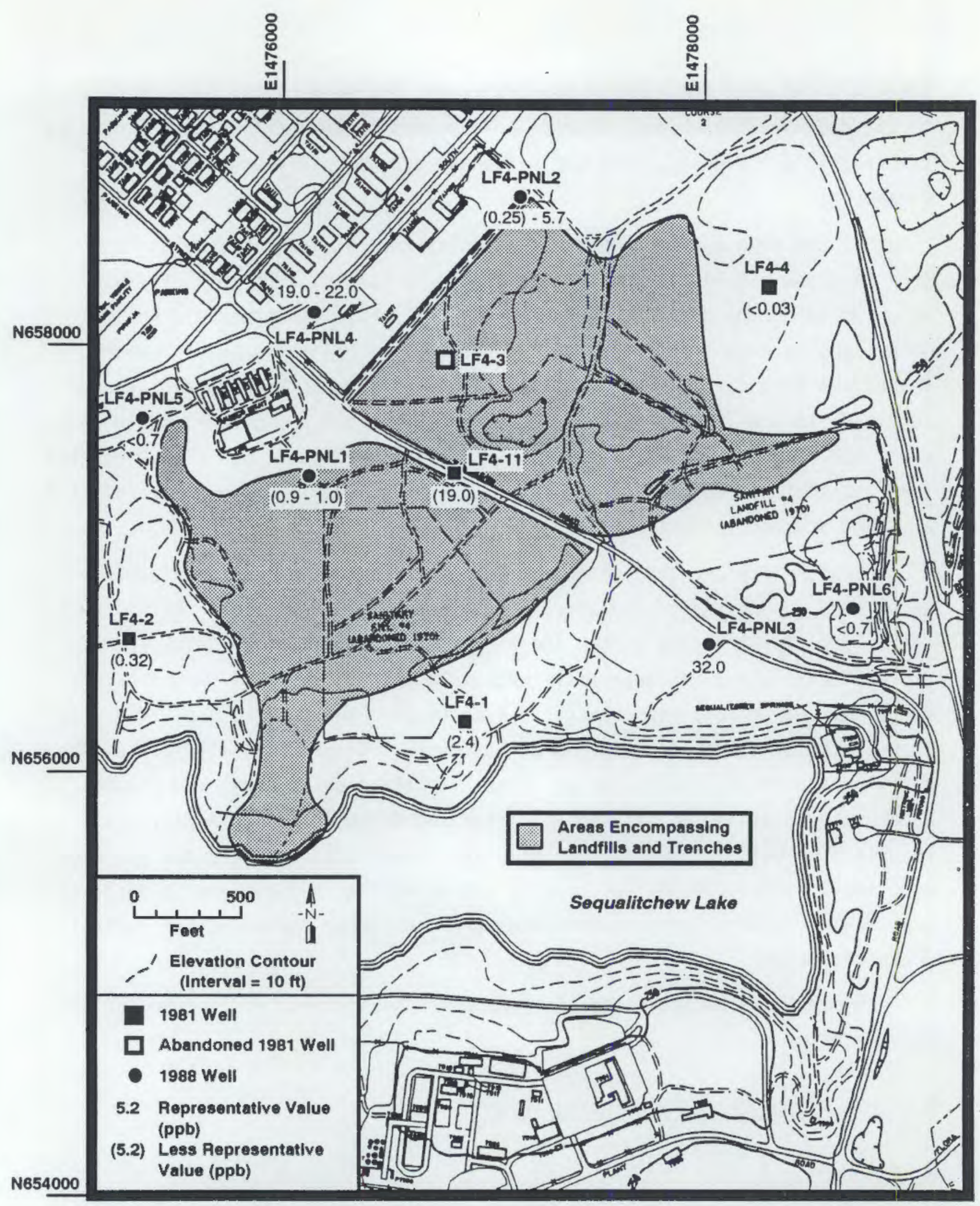

FIGURE 6.14. TCE Concentration Ranges Based on Analyses of September Through December Sampling Events 
LF4-PNL4, and LF4-PNL5) are interpreted to be either directly or indirectly downgradient of the landfill. The four remaining wells (LF4-1, LF4-2, LF4-PNL3, and LF4-PNL6) are considered to be located in areas that at times could be either upgradient or downgradient of the landfill, depending on the hydraulic relationship between Sequalitchew Lake, the shallow aquifer, and the water-table configuration. Variations in the ground-water levels, particularly those influenced by surface water/ground-water interactions and the operation of water supply wells, could greatly affect the upgradient/ downgradient status of these monitoring wells.

Ground-water samples collected from each of these wells varied in representativeness according to the well development and purging history of each well before sample collection. Although it appears that the existing 1981 wells were constructed of glued PVC sections, no analytical results have suggested the presence of organic compounds (Tetrahydrofuran, Methyl Ethy1 Ketone, and (yclohexanone) typically used in this type of glue. All 10 monitoring wells are now considered fully developed and capable of producing representative samples.

The limited analytical results collected to date suggest that coliform, oil and grease, volatile organic compounds (principally TCE and cis-1,2-DCE), and perhaps some metals (iron and manganese) are the chemical constituents of primary interest. A comparison of the upgradient and downgradient wells suggests that the landfill (or perhaps some other nearby source) may have affected the hydrochemistry of the uppermost aquifer. Further investigation is needed to determine the sources and extent of the impact that Landfill 4 may have had on the local ground-water system. 

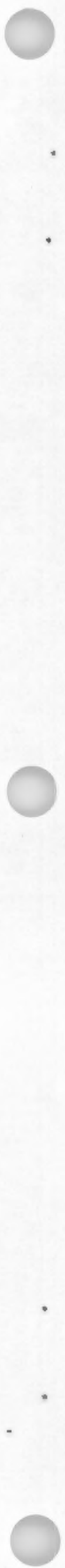


\subsection{CONCLUSIONS}

Severat conclusions were drawn from the results of this study, and each of these is discussed below.

\subsection{OVERALL SITE INVESTIGATION}

- No records are available on the landfill histories and waste inventories, and no employees in the DEH at the Fort had any first-hand knowledge nor could they identify anyone with first-hand knowledge of the landfill operations.

- Aerial photograph interpretation and field reconnaissance proved useful in locating the general boundaries of the landfills.

- Surface-based geophysical-sensing methods of the type used in this. investigation proved useful in locating the boundaries of the 1 andfills where aerial photograph interpretation and field reconnaissance results were uncertain. These methods were successful in locating both large trench-type landfill operations and some localized open-pit dumps.

- The drive-barrel method of cable-tool drilling proved to be more efficient than either hollow-stem auger drilling or the hard-tool method of cable-tool drilling.

- The 4-in. PVC-cased wells proved to be superior to the 2-in. wells, primarily because of their ease of development.

- The ground-water monitoring systems installed at each landfill meet the requirements of 40 CFR 265, subpart F; WAC 173-303; and WAC 173-160. Both of these monitoring systems are considered acceptable for detecting and evaluating the impacts of the inactive landfilis on the ground water and adjacent lands. Thus, the goal of this investigation was achieved. 


\subsection{STATUS OF LANDFILL 1}

- The landfill operation included trench disposal of unknown waste (possibly domestic garbage) and surface dumping of construction rubble (sand and gravel, broken concrete, broken asphalt, etc.).

- Isolated open-pit dumps, burn pits, and surface dumping have also occurred at or near this landfill.

- The boundaries of the 1 andfill extend farther to the north than previously recorded, and an area of isolated open-pit dumping and burning extends to the west of the landfill proper.

- The uppermost aquifer beneath this landfill occurs in the Vashon Drift Formation at a depth of approximately 20 to $40 \mathrm{ft}$. The bottom of the aquifer is considered to be the top of a clay/silt layer at a depth of approximately $70 \mathrm{ft}$. Using the limited waterlevel data available for this site, ground-water flow appears to be to the east and northeast.

- Four new (1988) monitoring wells and four existing (1984) monitoring wells are located around the site. Three monitoring wells are located upgradient of both the open-pit dumping/burning areas and the landfill. The remaining five wells are located either directly or indirectly downgradient of these waste sites. Variations in the water levels, particularly those influenced by the ground-water mound near we11 84-CD-LF-2, can alter the downgradient status of these monitoring wells.

- The representativeness of ground-water samples collected during this study varied according to the development and purging history of the well before sample collection. Thus, much of the analytical data must be considered preliminary and used only for indication purposes.

- Examination of the inorganic results from the upgradient wells versus the downgradient wells suggests that the landfill may have influenced the ground-water chemistry downgradient of the site. 
- Nitrate and volatile organic compounds (principally TCE and DCE) appear to be the two chemical constituents of primary interest. Nitrate exceeded the DWS in wells $89-$ CD-LF-1, -2 , and -4 , while TCE exceeded the MCLs in wells $84-C D-L F-3$ and $-L F-4$.

- Although coliform, chromium, mercury, and oil and grease were detected above DWS in some of these ground-water wells, these samples were not considered to accurately represent the ground-water quality. Thus, none of these constituents is believed to be of concern.

- Wells LF1-PNL1, -PNL2, and -PNL4 were extensively developed and produced representative samples. Well 84-CD-LF-1, -LF-2, -LF-3, and $-\mathrm{LF}-4$ were not deveioped as part of this study but should produce representative samples after a good, extensive purging is performed on each well. Hell LF1-PNL3 produces only poor (unrepresentative) samples and should be used only for water-level measurements.

\subsection{STATUS OF LANDFILL 4}

- Landfill operations included at least four areas of trench disposal and several areas of surface dumping. Waste materials disposed to the trenches are believed to be domestic garbage (glass, tin cans, rubber goods, paper and wood products, automobile parts, etc.). Surface dumping consisted of asphalt and concrete blocks, construction rubble, tree stumps, at least one truck body, household appliances, etc.

- Isolated open-pit dumps and surface dumping have also occurred in areas surrounding the landfill. Some of these dumps may have included petroleum products, as well as trash, automobile parts, and yard debris.

- The boundaries of the landfill area extend farther to the north, east, and west than previously recorded, and perhaps not as far south as previously reported. 
- The uppermost aquifer beneath this Tandfill occurs in the Vashon Drift Formation at a depth of approximateiy 10 to $40 \mathrm{ft}$. The bottom of the aquifer is considered to be the top of the clay/silt of the Kitsap Formation, which lies at a depth between $26 \mathrm{ft}$ to over $45 \mathrm{ft}$. Ground-water flow is primarily to the northnorthwest, under the influence of a fairly flat gradient.

- One monitoring well (LF4-4) is interpreted to be upgradient of the landfi1l, and five wells (LF4-11, LF4-PNL1, LF4-PNL2, LF4-PNL4 and LF4-PNL5) are interpreted to be either directly or indirectly downgradient of the landfill. Four other wells are located in areas that at times may be upgradient while at other times downgradient, depending on the hydraulic relationship between Sequalitchew Lake, the aquifer, and the water-table configuration.

- The representativeness of ground-water samples collected during this study varied according to the development and purging history of the well before sample collection. Therefore, the analytical results from some samples must be considered tentative and used for indication purposes only.

- The limited analytical results collected to date suggest that coliform, oil and grease, volatile organic compounds (principaliy TCE and cis-1,2-DCE) and perhaps some metals (iron and manganese) are the principal chemical constituents of concern at this site.

- Fecal coliform exceeded the DWS in well LF4-PNL2.

- Iron and manganese exceeded the secondary OWS in we11s LF4-2, LF4-11, LF4-PNL2, and LF4-PNL5.

- $0 i 1$ and grease were detected in we1ls LF4-PNL5 and LF4-PNL6, and perhaps LF4-PNL4.

- Cis-1,2-DCE and TCE exceeded the MCLs in wells LF4-PNL1, -PNL2, -PNL3, -PNL4, and LF4-11, and are considered the principal constituents of concern. 
- A comparison of the hydrochemistry found in the downgradient wells versus that found in the upgradient well suggests that the landfill may have affected the hydrochemistry of the uppermost aquifer.

- All 10 of the monitoring wells around Landfill 4 can produce representative samples of the uppermost aquifer and are suitably located to provide adequate monitoring of the landfill. However, additional investigations and sampling will be necessary to determine the sources and extent of impacts attributed to Landfill 4. 


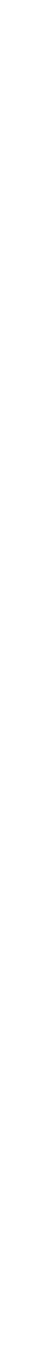




\subsection{RECOMMENDATIONS}

The following recommendations are made concerning future work at these landfills. These are listed in order of highest priority.

1. Collect and analyze ground-water samples from all wells at both landfills on a quarterly basis for a period of 1 year, and perform statistical analyses in accordance with 40 CFR 265, Subpart F. Examine trends in the various contaminants observed at each site. Determine which contaminants remain above DWS or MCLs and re-evaluate the impacts of the landfills on the ground water.

2. Collect periodic and several continuous water-level measurements in wells at both landfills and lakes near Landfill 4 for a period of 1 year to assess the effects of water-level fluctuations and lake/ aquifer interactions on the ground-water flow directions. Determine lake-level elevations by surveying. Evaluate and interpret these data to determine ground-water flow directions with more certainty.

3. Test the ability for soil-gas sampling to locate and track the TCE plume at Landfill 4, and, if successful, locate the source(s) of the TCE.

4. Accurately locate (using aerial photographs, field mapping, and surface geophysics), stake, and survey the locations of the landfill boundaries. Fence off and/or install signs on the landfills to prevent unauthorized dumping. 


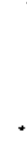




\subsection{REFERENCES}

29 CFR 1910. Occupational Safety and Health Administration, "Hazardous Waste Operations and Emergency Response." U.S. Code of Federal Regulations.

40 CFR 265. U.S. Environmental Protection Agency, "Interim Status Standards for Owners and Operators of Hazardous Waste Treatment, Storage, and Disposal Facilities." U.S. Code of Federal Regulations.

ANSI/ASME (American National Standards Institute/American Society of Mechanical Engineers). 1986. Quality Assurance Program Requirements for Nuclear Facilities. ANSI/ASME NQA-1-1986, American National Standards Institute, New York.

Armstrong, J. E., D. R. Crandall, D. J. Easterbrook, and J. B. NobTe. 1965. "Late Pleistocene Stratigraphy and Chronology in Southwestern British Columbia and Northwestern Washington." Bullet in of the Geological Society of America, $76(3)$.

ASTM (American Society for Testing and Materials). 1986. "Standard Method for Penetration Test and Split-Barrel Sampling of Soils." In 1986 Annual Book of ASTM Standards, American Society for Testing and Materials, Philadelphia, Pennsylvania.

Brown and Caldwe11. 1983. Clover/Chambers Creek Geohydrologic Study. Draft Report Prepared for the Tacoma-Pierce County Health Department, Tacoma, Washington.

Cranda11, D. R., D. R. Mull ineaux, and H. H. Waldron. 1958. "Pleistocene Sequence in Southeastern Part of the Puget Sound Lowland, Washington." American Journal of Science 256.

Ecology and Environment, Inc. 1986. Iillicum Municipal Well Investigation, Iillicum, Washington. TDD R10-8601-02, Prepared for the U.S. Environmental Protection Agency, Region $x$, Seattle, Washington.

EPA (U.S. Environmental Protection Agency). 1986. Resource Conservation and Recovery Act (RCRA) Ground-Water Monitoring Technical Enforcement Guidance Document. OSWER-9950.1, U.S. Environmental Protection Agency, Washington, D.C.

Folk, R. L. 1974. Petrology of Sedimentary Rocks. Hemphill, Austin, Texas. Last, G. V. and T. L. Liikala. 1987. A Field Guide for Well Site Geologists: Cable Tool Drilling. PNL-6392, Pacific Northwest Laboratory, Richland, Washington. 
McMaster, B. N. 1982. Installation Assessment of the Headquarters, I Corps and Fort Lewis, Washington and the Subinstallations Yakima Firing Center, Camp Bonneville, and Vancouver Barrack, Washington, Report No. 325. DRXTHAS-1A-82325, U.S. Army Toxic and Hazardous Materials Agency, Aberdeen Proving Grounds, Maryland.

PNL (Pacific Northwest Laboratory). 1989. Procedures for Ground-Water Investigations. PNL-6894, Pacific Northwest Laboratory, Richland, Washington.

Stanley, T. W., and S. S. Verner. 1983. Interim Guidelines and Specifications for Preparing Quality Assurance Project Plans. QAMS-005/80, EPA-600/4-83-004, U.S. Environmental Protection Agency, Washington, D.C.

USGS (U.S. Geological Survey). 1962. Water Resources of the Tacoma Area, Washington. Water Supply Paper 1499-B, U.S. Geological Survey, Tacoma, Washington.

USGS (U.S. Geological Survey). 1971. Water Resources Data for Washington. Water-Data Report WA-71-1, U.S. Geological Survey, Tacoma, Washington.

WAC 173-160. Washington State Department of Ecology, "Minimum Standards for Construction and Maintenance of Wells." Washington Administrative Code.

WAC 173-303. Washington State Department of Ecology, "Dangerous Waste Regulations." Washington Administrative Code.

Walters, K. L., and G. E. Kimmel. 1968. Ground-Water Occurrence and Stratigraphy of Unconsolidated Deposits, Central Pierce County, Washington. Water Supply Bulletin No. 22, State of Washington Department of Water Resources, 0lympia, Washington. 
APPENDIX A

RESULTS OF GEOPHYSICAL SURVEYS AT LANDFILL 4 


\section{APPENDIX A}

\section{RESULTS DF GEOPHYSICAL SURVEYS AT LANDFILL. 4}

This appendix contains an interna] Pacific Northwest Laboratory memo discussing the results of geophysical surveys conducted at Landfill 4 . Figures cited in the memo are also provided. 


\author{
Date November 7, 1988 \\ To George Last \\ From \\ Subject

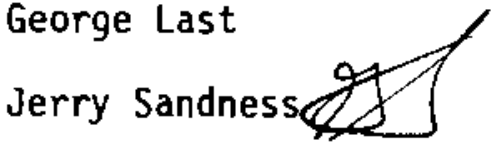 \\ Geophysical Reconnaissance Surveys at Fort Lewis
}

This memo describes the objectives, procedures, and results of a set of geophysical surveys that Kevin Schwartz, Paul Porath, and I performed with your assistance and direction at Fort Lewis, Washington. The work was performed during the periods July 18-19 and July 27-28, 1988.

\title{
$\underline{\text { Objective }}$
}

The objective of our geophysical surveys was to determine the boundaries of waste deposits associated with Landfill 4. This landfill, located just to the north of Lake Sequalitchew, covers an area of at least 60 acres. It is roughly bounded by relatively undisturbed forest on its south and west sides. It is further bounded by a mechanics training area and a motor pool along its northwest edge and by a gravel pit at its east end. The boundary of the waste deposit along the south and west sides of the landfill is easy to locate because the landfill is distinctly elevated relative to the surrounding ground in that area. Elsewhere, however, the topography or appearance of the ground surface does not provide an unambiguous indication as to the precise location of the waste boundary. Near-surface geophysical exploration methods were employed to locate buried waste materials in those areas.

\section{Procedure}

The approach taken in this study was to collect geophysical data along 30 traverse lines. These lines varied in length from a minimum of $200 \mathrm{ft}$ to a maximum of $1200 \mathrm{ft}$ and were marked by wooden stakes at intervals of $100 \mathrm{ft}$. They were concentrated in four areas where the waste boundaries were most obscure and where it seemed important to more accurately define them. These four areas can be identified by reference to the site map of Figure 1 . This map shows the locations of the survey lines, some of the major surface features of the site (e.g., the lake, trees, roads, and buildings), and the results of the geophysical surveys.

The first area (1ines $1-6$ and $22-26$ ) is adjacent to the mechanics training facility near the west end of the landfill. A section of flat ground and a shallow ditch appear to separate that facility from the landfill although some shallow pits have obviously been used for the disposal of small amounts of waste (probably oil, grease, rags, etc.) in the area between the ditch and the buildings of the training facility. The landfill between the ditch and the woods to the south is characterized by many long shallow depressions that seem to be caused by the settling of waste materials in trenches. 
George Last

November 7, 1988

Page 2

The second survey area (1ines 7-17) is adjacent to the motor pool fence at the north side of the landfill. The general appearance of the ground and the presence of two shallow, partially open trenches shows that some waste burial activity has taken place there, apparently in a rather unplanned or random fashion.

The third area is located in the northwest portion of a cleared area that includes an active gravel pit at its east end. No waste materials are evident in the gravel pit, but we could not determine by visual inspection whether waste materials extend into the west end of the cleared area.

The fourth survey area is a strip of bare ground (fili) between the north shore of Lake Sequalitchew and the apparent southwest corner of the landfill. The question is whether waste materials were included in the fill material that was deposited at this site.

Three geophysical sensing instruments were used in this study: 1) a groundpenetrating radar unit, 2) a ground conductivity meter, and 3) a metal detector. These are briefly described in the following paragraphs.

The radar system was a Geophysical Survey Systems, Inc. (GSSI), Model SIR 7 with a 120-MHz (nominal) antenna (GSSI Model 3110) which transmits and receives radar signals in a frequency band of approximately $50-250 \mathrm{MHz}$. The antenna, mounted on a fiberglass sled, was pulled along the survey lines by a small a]l-terrain vehicle (ATV). Radar signals reflected from subsurface objects or interfaces were detected by the antenna and were transmitted by cable to control and recording modules mounted on the ATV. There the signals were amplified, digitized, and recorded on digital tape cartridges for later playback and processing in our laboratory. This instrument exhibits good spatial resolution and can produce quasi-pictorial images of both metallic and nonmetallic objects or materials in the ground.

The ground conductivity meter was a Geonics EMB1 electromagnetic induction (EMI) sensor. This hand-carried device operates by transmitting an oscillatory magnetic field into the ground, then detecting the secondary magnetic field produced by the electric currents that are induced in the ground. It exhibits relatively poor spatial resolution, but can respond to both metallic and nonmetallic waste deposits. The data produced by this instrument were digitized and recorded by a small data recorder carried by the operator. The data were subsequently transferred to a computer for scaling and display.

The metal detector was a Fisher M-Scope, Model TW-6. This instrument was originally marketed as a pipe and cable detector, but has proven to be effective in detecting buried metallic waste materials at depths of a few feet. The metal detector was carried by hand along the survey lines until a response was obtained. The location of the response was then recorded in a field notebook. 
George Last

November 7, 1988

Page 3

Results

Figures 2-6 show the radar data collected in this survey. Each of the small images in these photographs is called a radar profile and represents one survey line. The horizontal dimension in a given profile corresponds to the distance along the survey line. The vertical dimension corresponds to signal travel time or depth. Thus, a radar profile can be regarded as a vertical crosssectional view of the ground under the survey line. The maximum penetration depth shown in these profiles is approximately $16 \mathrm{ft}$. The depth calibration is based on an estimate of the dielectric constant of the ground at this location, so may be in error by 10-15\%. Excavation of an object detected by the radar and measurement of its depth would provide the ground truth needed to improve the accuracy of the depth scale.

Most of the survey lines were traversed twice with the GPR system, once in each direction. Thus, in Figures 2-6, two radar profiles are shown for each of these lines. A pair of profiles for a given line may not be identical because the two traverses were typically made with a lateral offset of a few feet to expand the area covered by the survey.

Our analysis of the radar data was based on a visual interpretation of the profiles. A small, isolated, reflective object in the ground can be identified by a characteristic hyperbolic reflection pattern like the one pointed out in Figure 2. A large deposit of waste material produces a complex reflection pattern consisting of many overlapping hyperbolas. A good example of this is shown in Figure 6, Line 27, where the south edge of the landfill is overlapped by relatively clean fill material. Another type of feature that is displayed in many of the profiles is a sedimentary interface. Good examples are shown in Figure 3 where strongly reflecting subsurface layers are present over most of the 1200-ft length of Line 7. (Note that Line 7 is displayed in two overlapping $1000-\mathrm{ft}$ segments because its total length is too long to be displayed in a single image.)

Figure 4 contains two radar profiles that do not correspond to numbered survey lines. The first of these, labeled "Woods", corresponds to a sinuous path through the trees between the north ends of Lines 18 and 19 (see Figure 1). Scattered debris is present at several points along this line, and a dense landfill-type deposit is present at its west end, from the edge of the trees to the north end of Line 18. The profile labeled "Landfill" corresponds to a short northward traverse from the road spur near the end of Line 20 . Waste materials, including blocks of concrete, are present along most of this line.

The three geophysical survey methods were applied along Line 4 on each of our two visits to the site. On the first visit, we surveyed a $200-\mathrm{ft}$ line (the north half of the $400-\mathrm{ft}$ line shown in Figure 1). The resulting radar and EMI profiles are labeled as Line 4 in Figures 2 and 7 , respectively. We subsequently decided that the line should be extended to the north by an additional $200 \mathrm{ft}$. The resulting full-length profiles are labeled as Line $4+4 X$ in Figures 5 and 13. 
George Last

November 7, 1988

Page 4

The EMI data are plotted in profile form in Figures 7-14. The scale of the vertical axes in these plots is such that the values 2047 and -2048 correspond to positive and negative full-scale responses of the EMBI sensor at an instrument range setting of $10 \mathrm{mmhos} / \mathrm{m}$. However, we did not convert the plotted values to conductivity units because the presence of large amounts of metal in the waste deposits makes that conversion inaccurate, and our purpose was simply to detect the waste materials. For the same reason, we chose to truncate the high-amplitude response peaks in most of the profiles.

The EMI profiles give strong indications of trenches, pits, or waste boundaries on most of the survey lines. Lines 5,6, 19, 23, and 30 are the only lines that do not seem to show compelling evidence of buried waste deposits. (The large peak at the east end of line 23 was produced by a small steel building.) The EMI profile along Line 7 is different from the others in that it exhibits a broad peak that extends over the entire length of the line. The most likely explanation for this result is that it reflects the distribution of metal boats, trailers, and other machinery inside the fenced motor pool area located only $15 \mathrm{ft}$ to the northwest of the survey line. It might also be partly caused by an overhead power line that crosses the survey line at approximately the point where the profile's amplitude is a maximum. A less likely possibility is that the apparent conductivity high is produced by contamination of the groundwater which is probably present at a depth of approximately $25 \mathrm{ft}$ in this area. This explanation is supported somewhat by the fact that the base levels of the profiles for Lines 11 and 12 are slightly elevated relative to the base levels of the profiles for other nearby lines.

The results of the metal detector, EMI, and ground-penetrating radar surveys are summarized on the site map of Figure 1. The broad, dark lines superimposed on the survey lines show the locations of buried waste materials as determined from all three sets of geophysical data. The metal detector and EMI responses were generally consistent with the indications of buried materials or ground disturbance provided by the radar system. However, the radar detected some buried waste materials that did not produce interpretable responses in the other instruments. An example of this is on Line 27 where the radar showed that the waste materials (1andfill deposit) extend approximately $100 \mathrm{ft}$ closer to the lake than was indicated by the metal detector and the EMI sensor.

A visual inspection of the ground surface at several locations at this site provided some insight into the nature of some of the waste materials. For example, the materials found at the ground surface along Line 4 and Lines 2426 are concrete and asphalt blocks containing steel reinforcing rods. We also found this type of material near the lake on Lines 28 and 29 and, as mentioned above, to the north of Line 20 and to the east of Line 18. Domestic garbage is exposed at the edge of the cleared area at the north end of Line 18, and some industrial debris (e.g., steel cable and other pieces of metal) is present along and to the south of Line $21 \mathrm{a}$. 
George Last

November 7, 1988

Page 5

\section{Final Comments}

Surface-based geophysical sensing methods of the types used in this reconnaissance are not normally able to identify the specific materials contained in a buried waste deposit or to determine their toxicity or threat to the environment. These types of information must be determined by sampling or excavation. In this case, our goal was to help in defining locations where groundwater sampling wells could be drilled close to the waste deposits but with a minimum risk of directly encountering buried hazardous waste materiais. This goal was probably achieved even though some areas remain where the locations of the waste boundaries remain uncertain. The areas of greatest uncertainty appear to be: 1) the northeast edge of the landfill between Lines 16 and $21 ; 2)$ the cleared area to the west of the active gravel pit and to the south of Line 21 ; and 3 ) a small triangular area bounded by the mechanics training area, Line 3 , and the highway.

GAS: $k\rceil k$

At tachments 


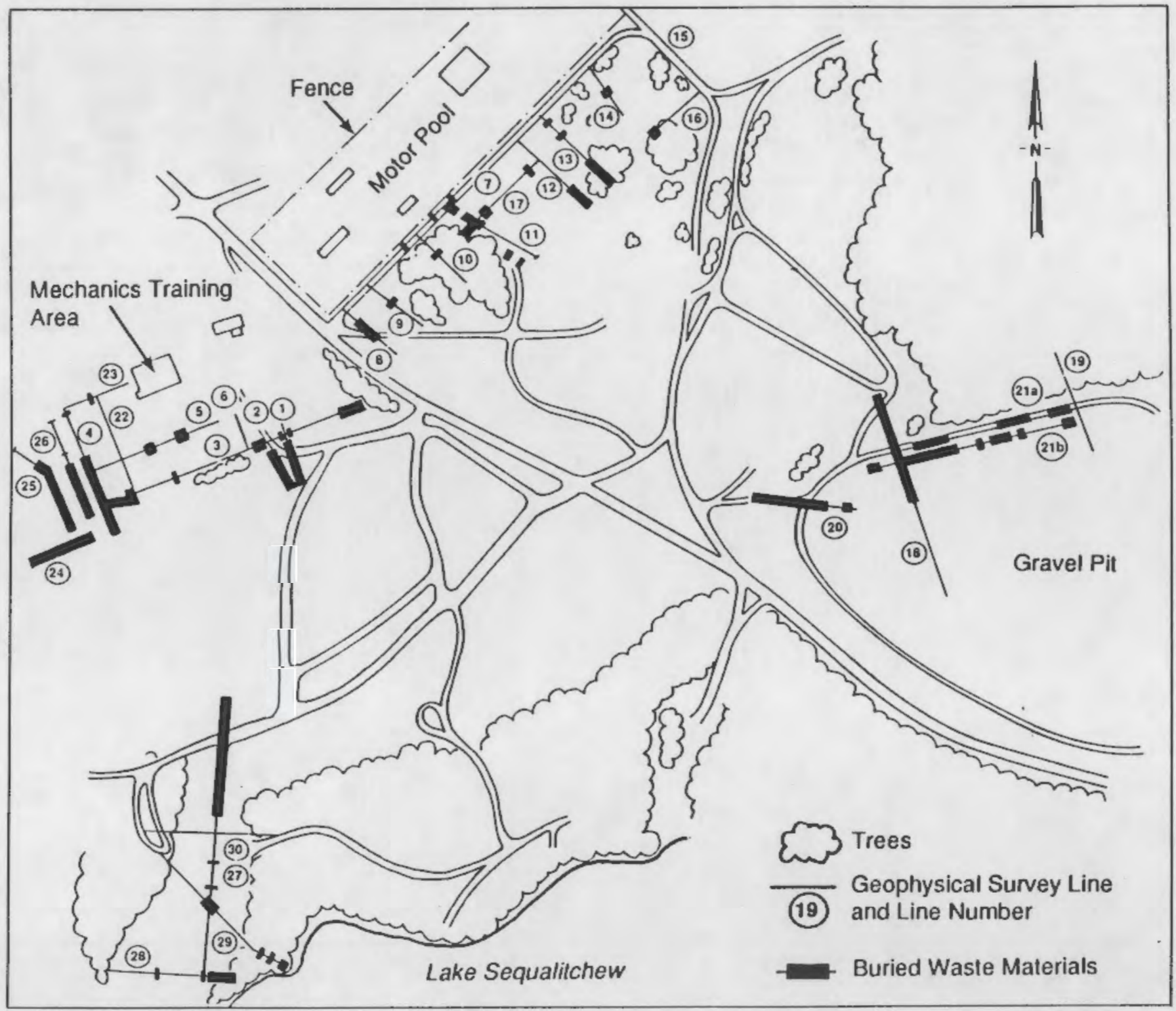

FIGURE 1 

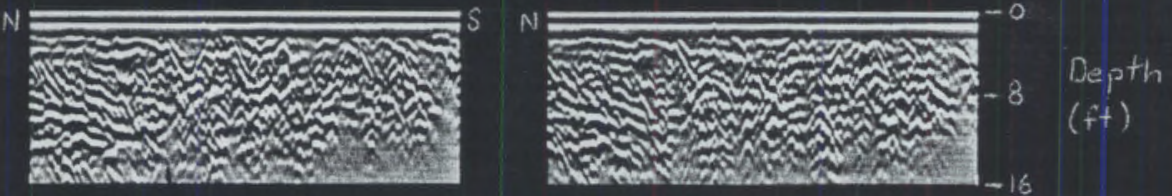

Depth

Hyperbolic

Reflection Pattern

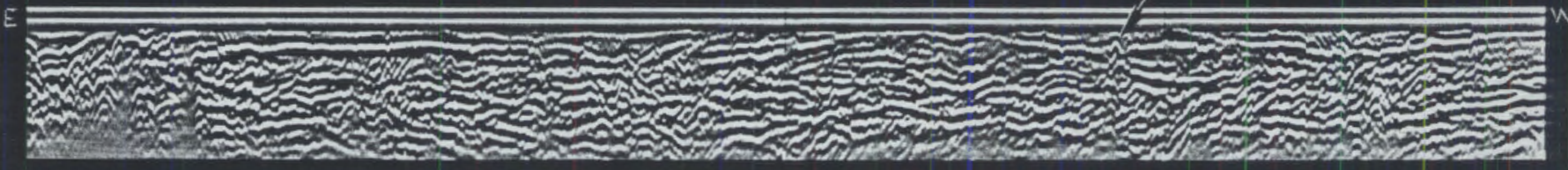

$E$ =

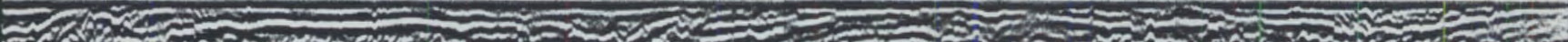

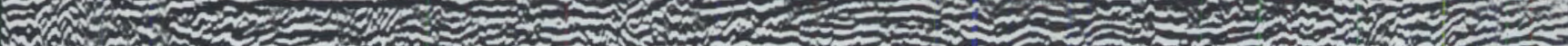

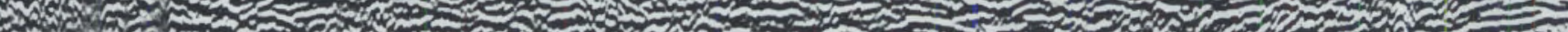

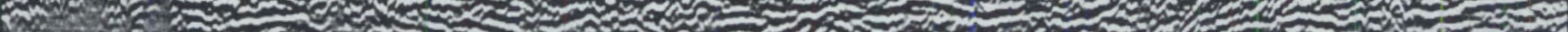

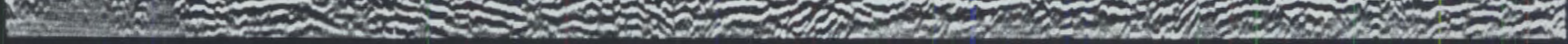
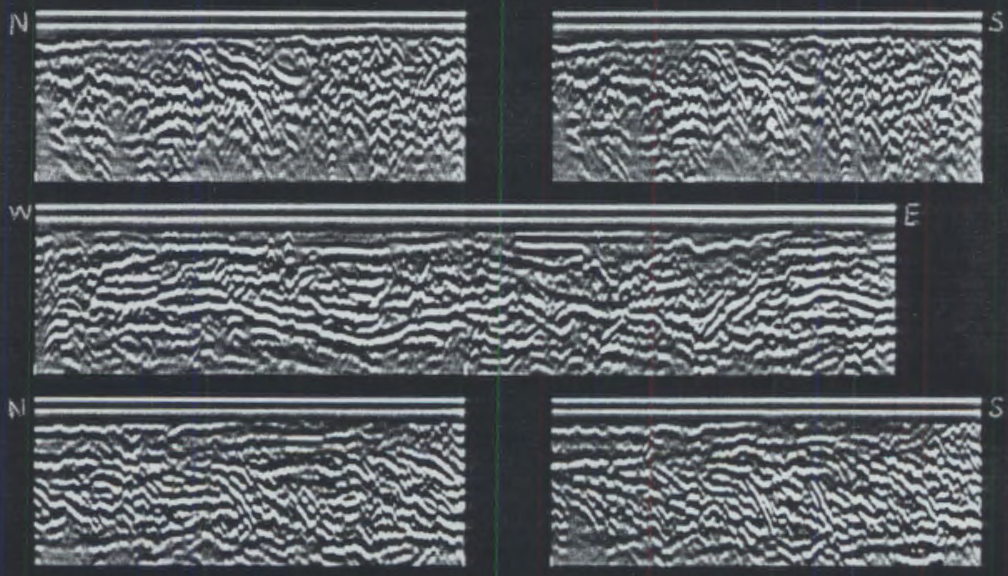


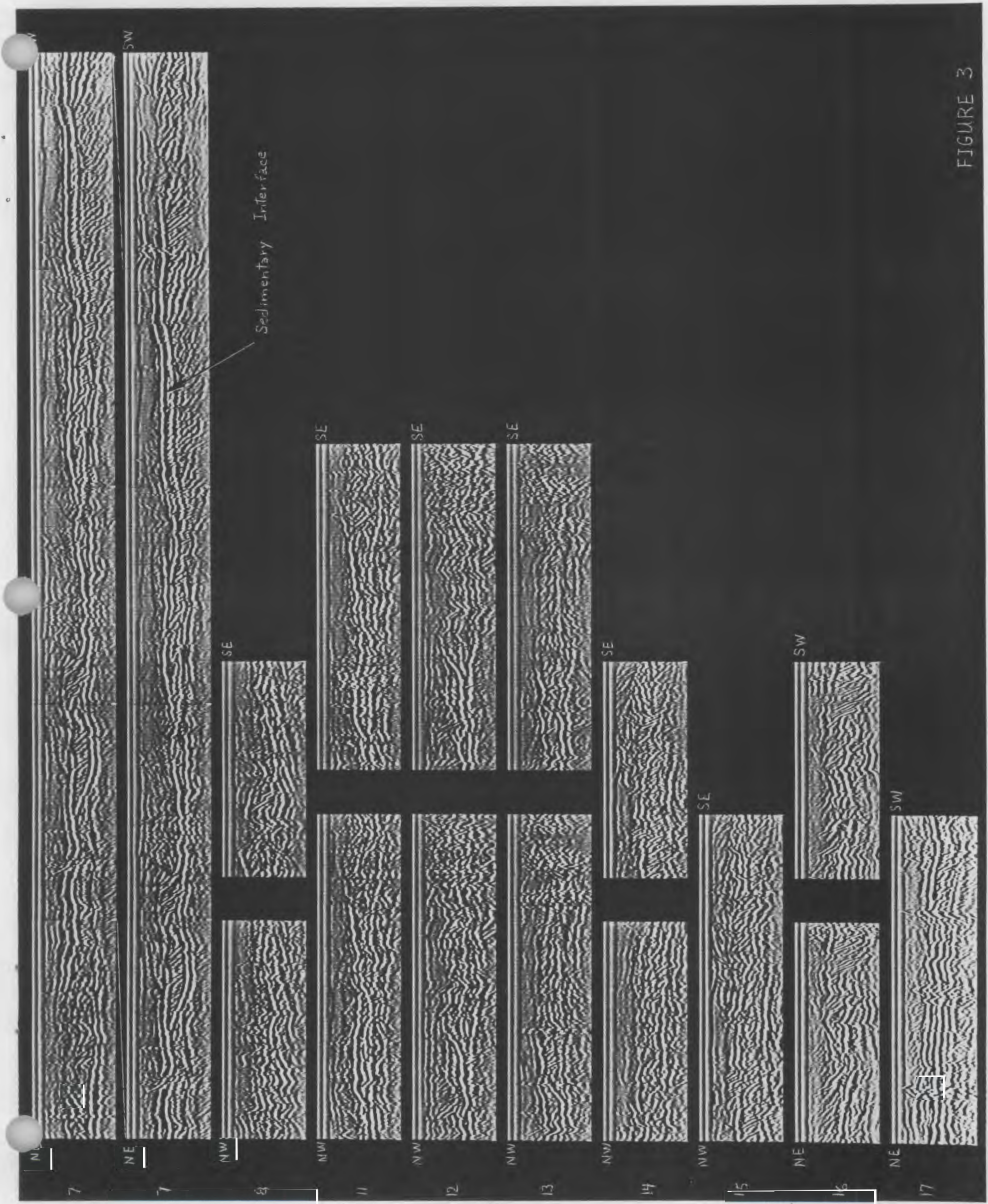


Line

No.

18

NW

S2)

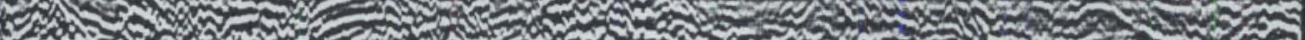

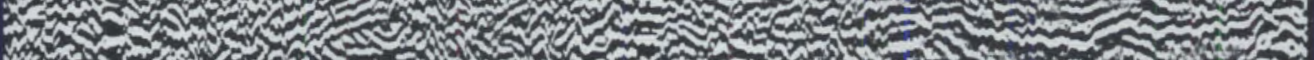
4.

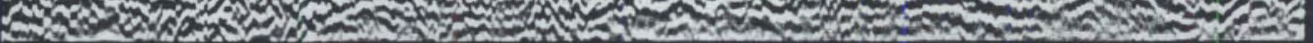

SE

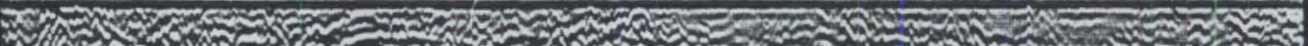

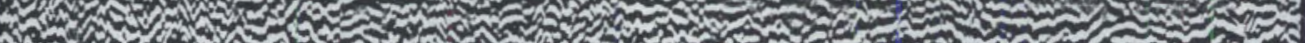

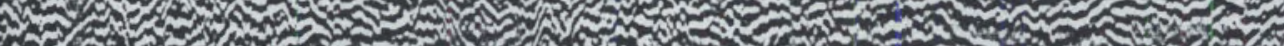

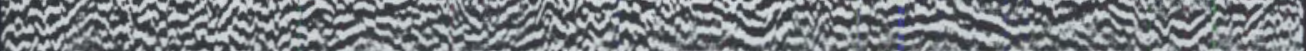

SE

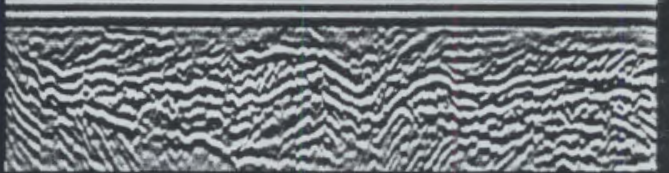

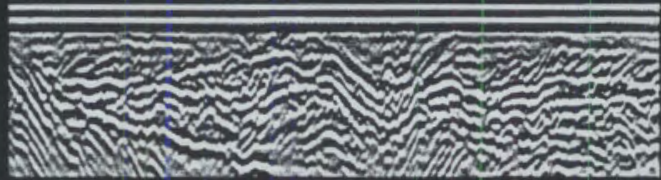

M.

Woods
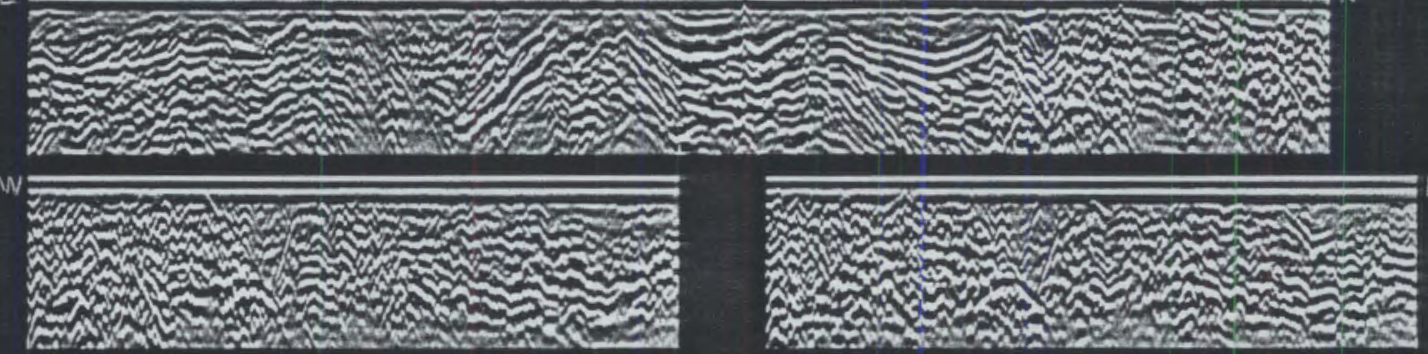

Landfill

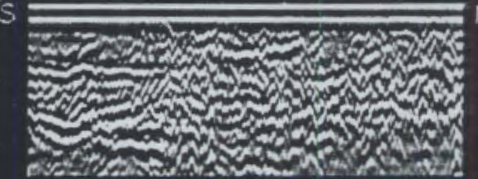

w

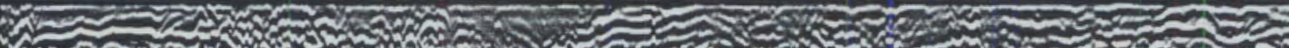

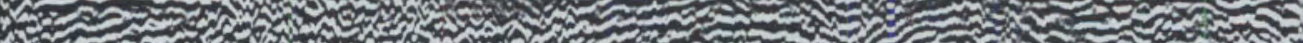

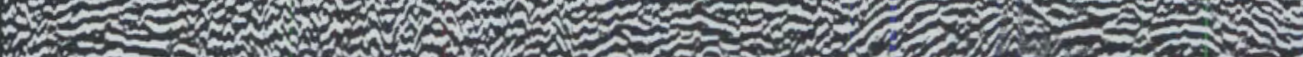
(2) - S

$21 \mathrm{a}$

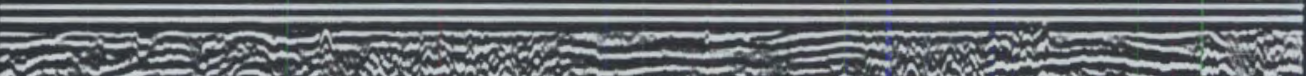

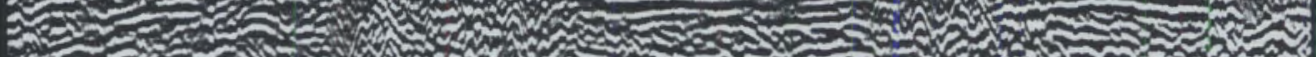

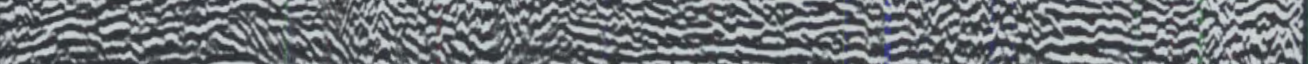

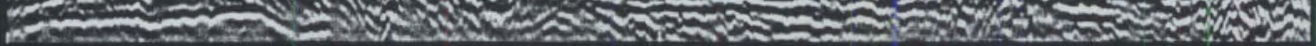



Waste

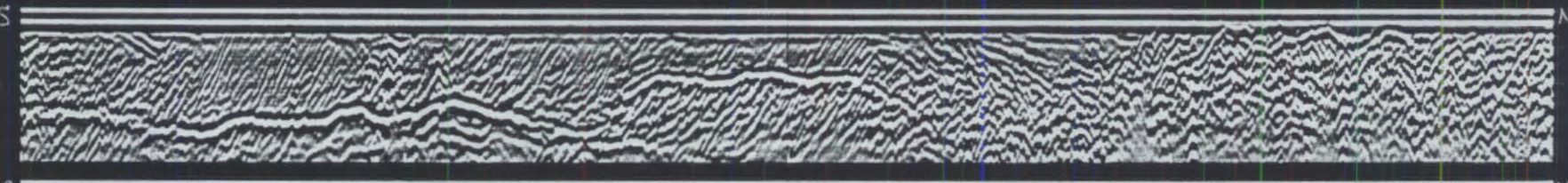
2017)
E

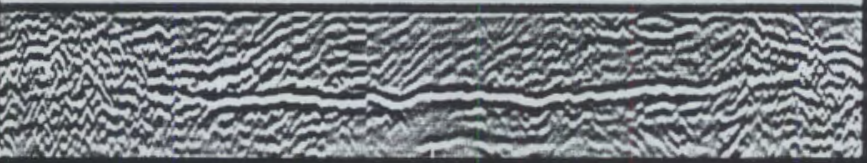

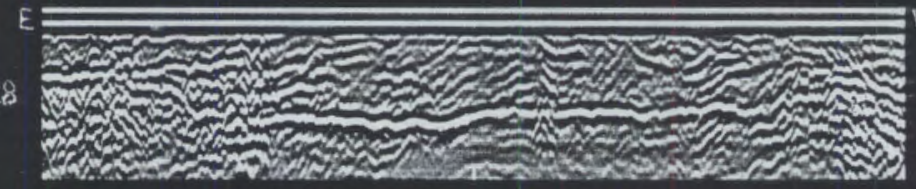

SE

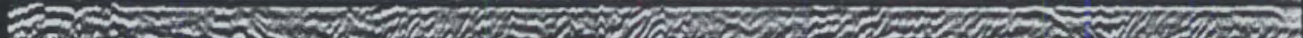

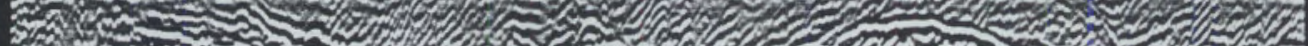

4

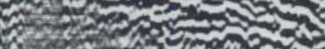

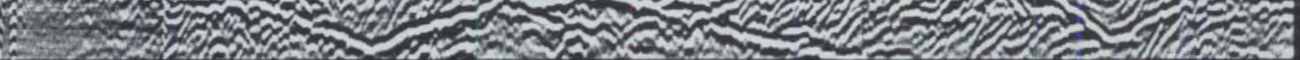
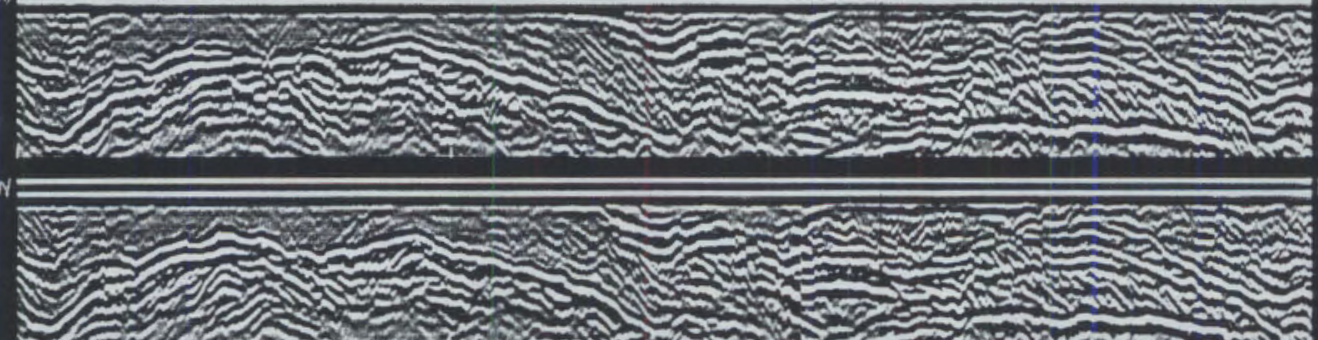
EM31 RESPONSE, LINES 1-6

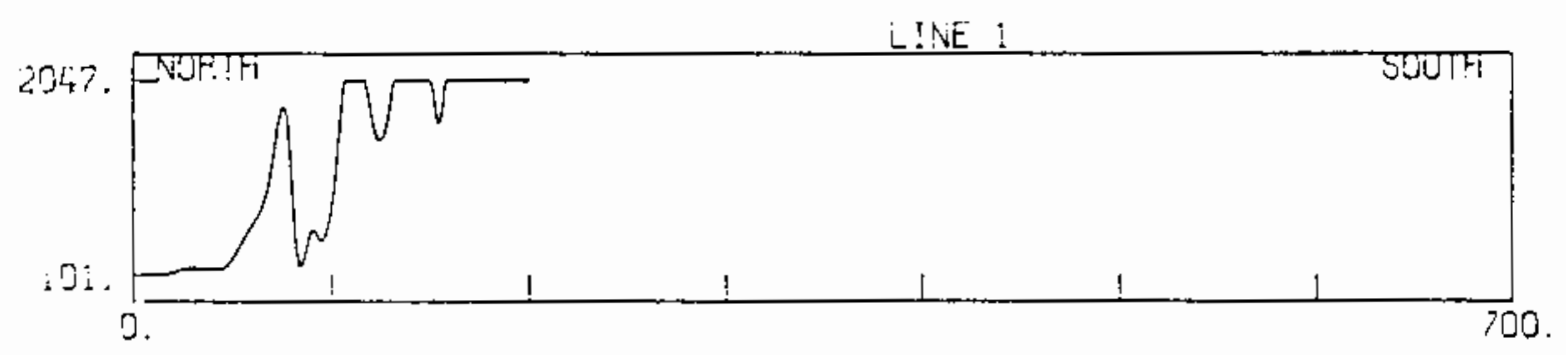

LINE 2

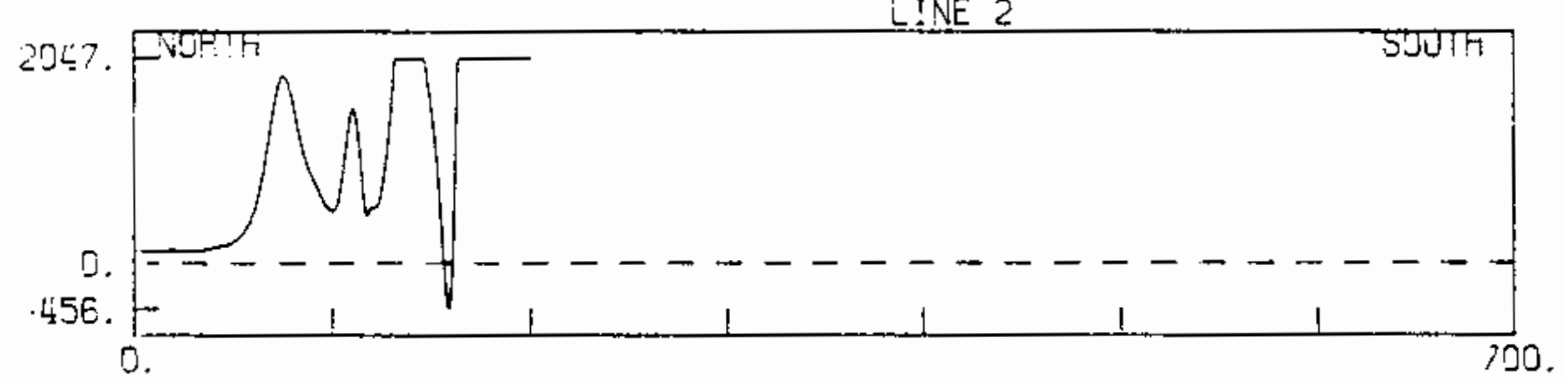

IINE 3
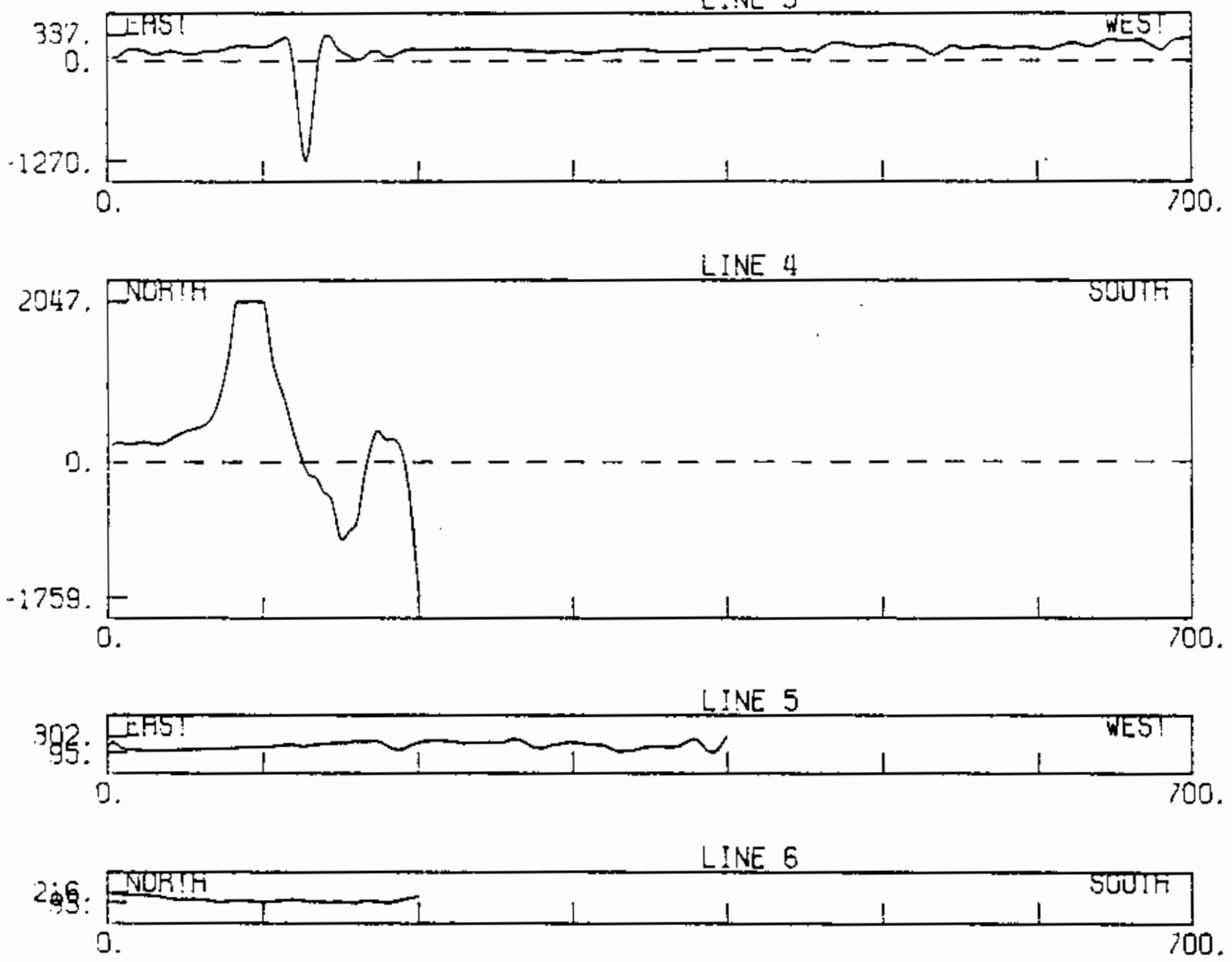


\section{EM3i RESPONSE, LINES $1,2, \& 4$}

L:NE

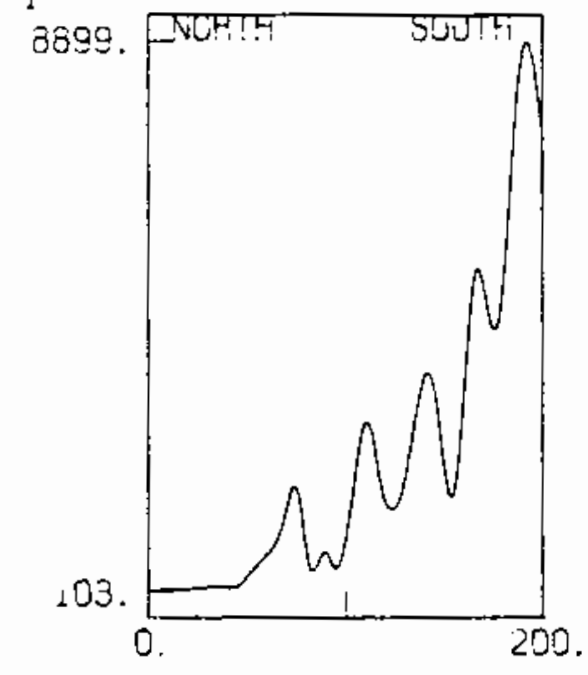

LINE 2

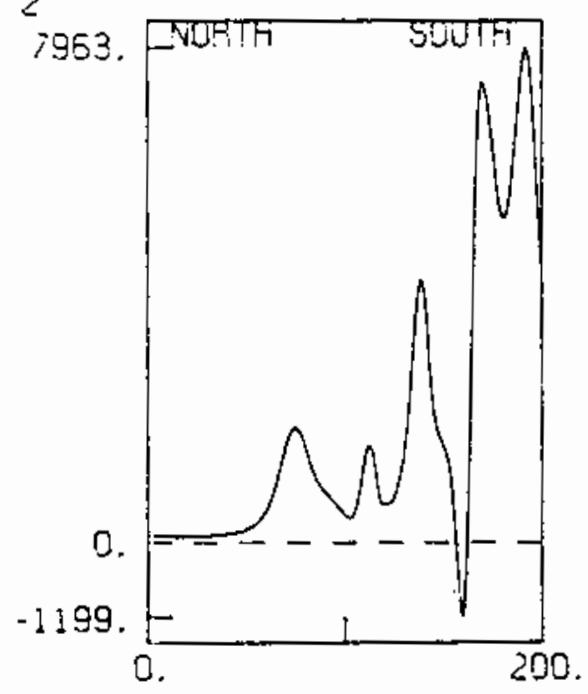

LINE 4

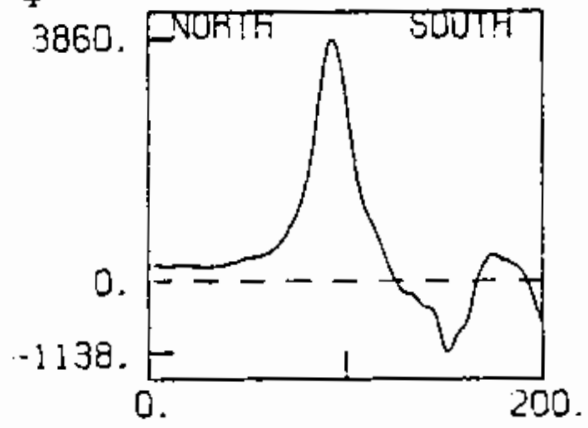

A. 14

FIGURE 8 
EM31 P.ESPONSE, LINES 8-II
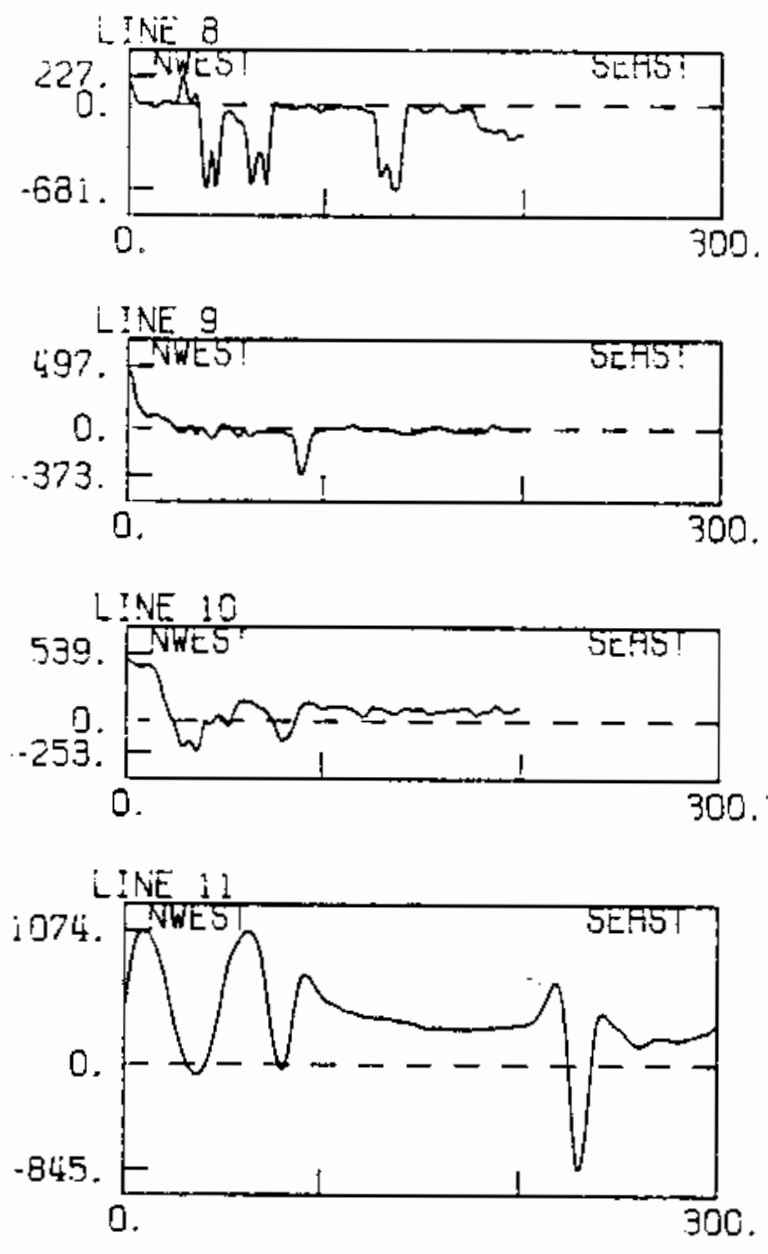
EM3i RESPONSE, LINES 12-I:
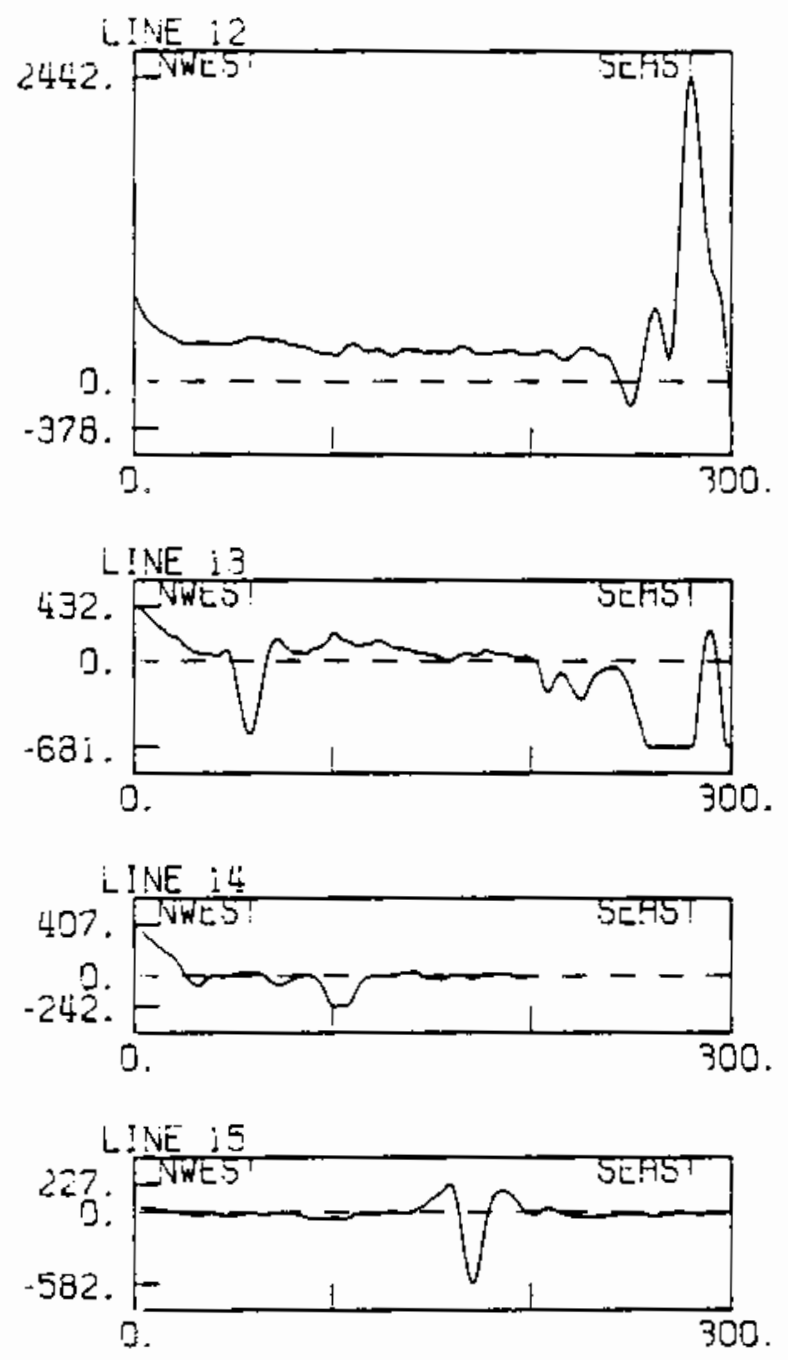

FIGURE 10 
EM31 RESPONSE, LINES 7, 16, \& 17

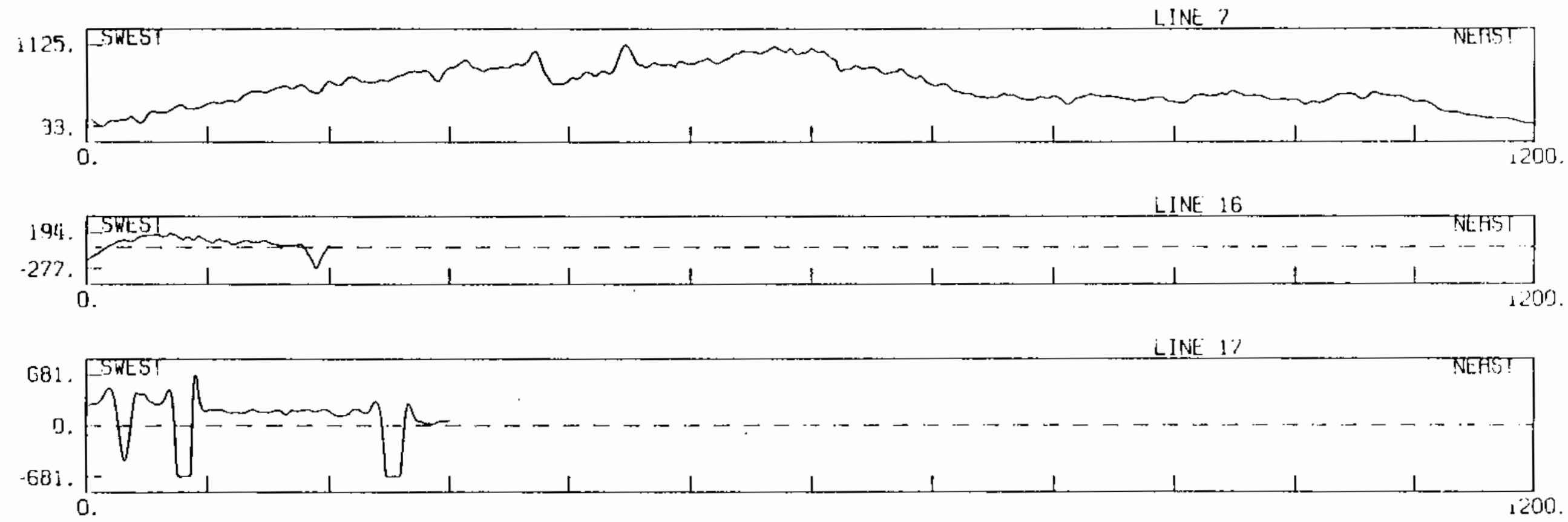


EM31 RiESPONSE, LINES 18-21
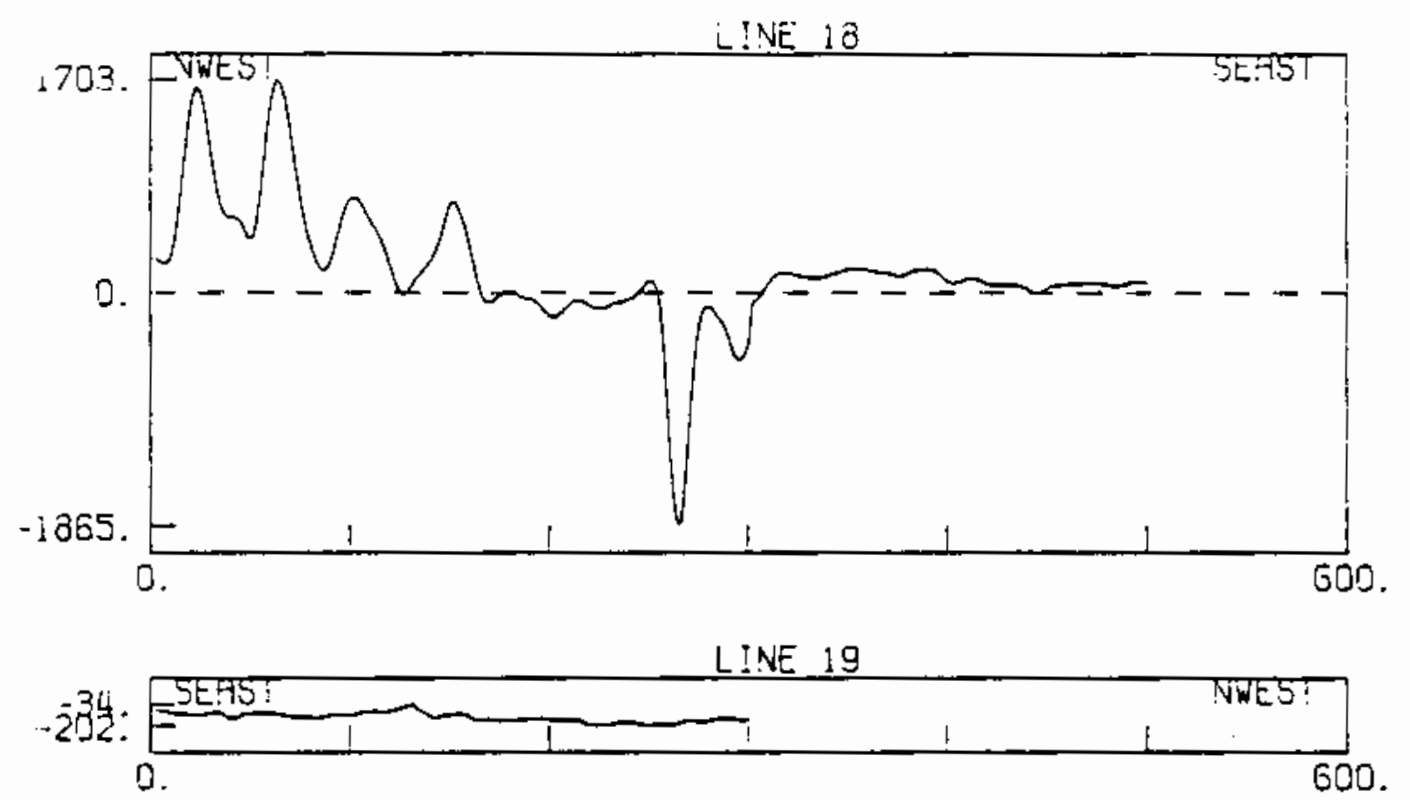

LINE 20

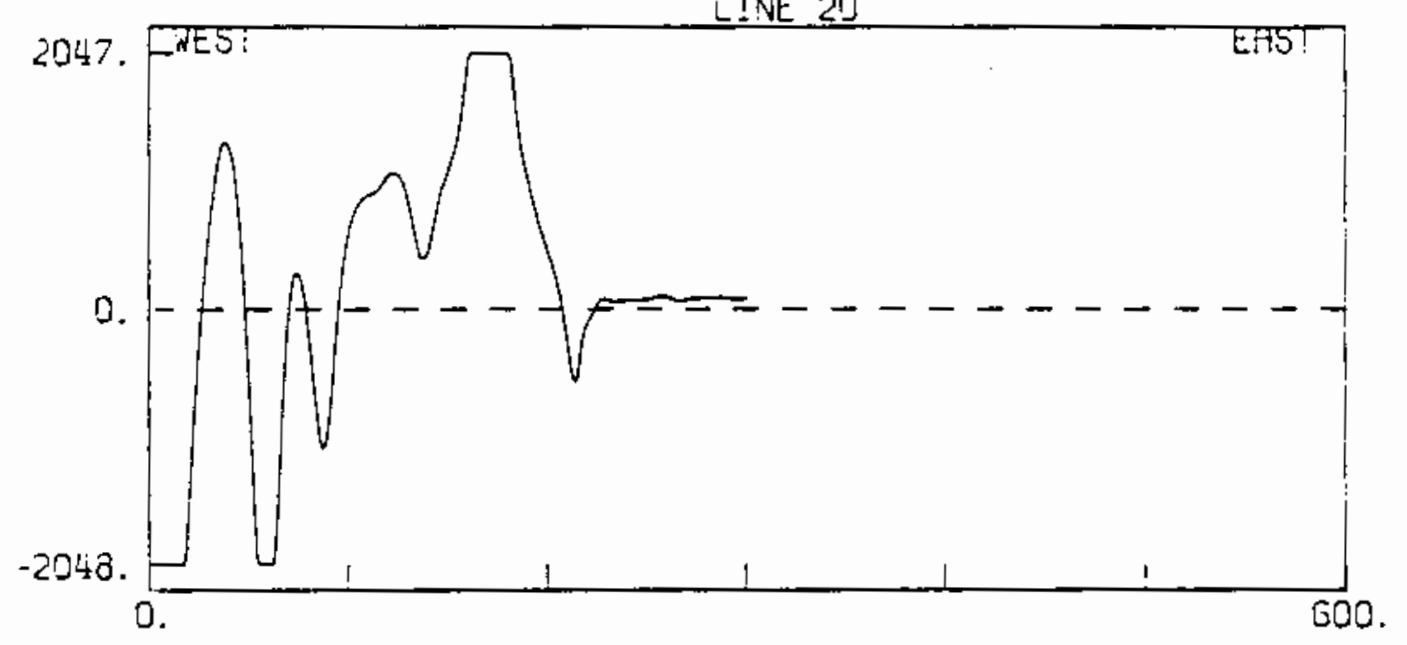

LINE 21

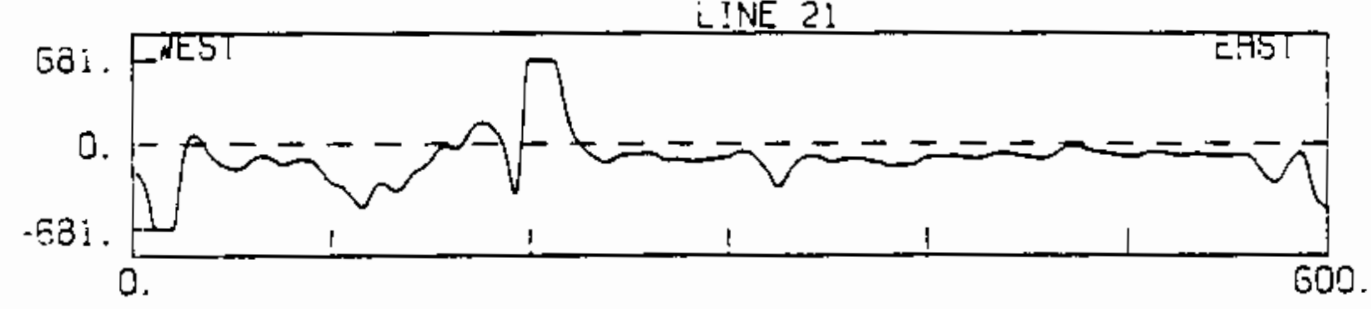

FIGURE 12 
EM31 RESPONSE, LINES 4-4X, 22-25

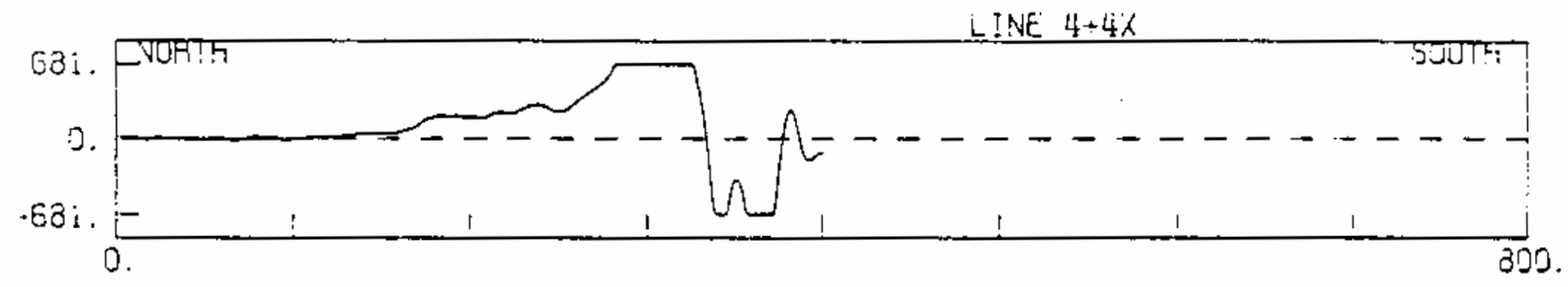

L!NE 22

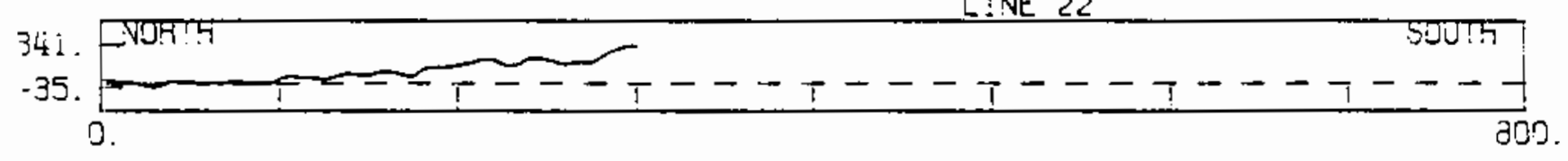

LINE 23
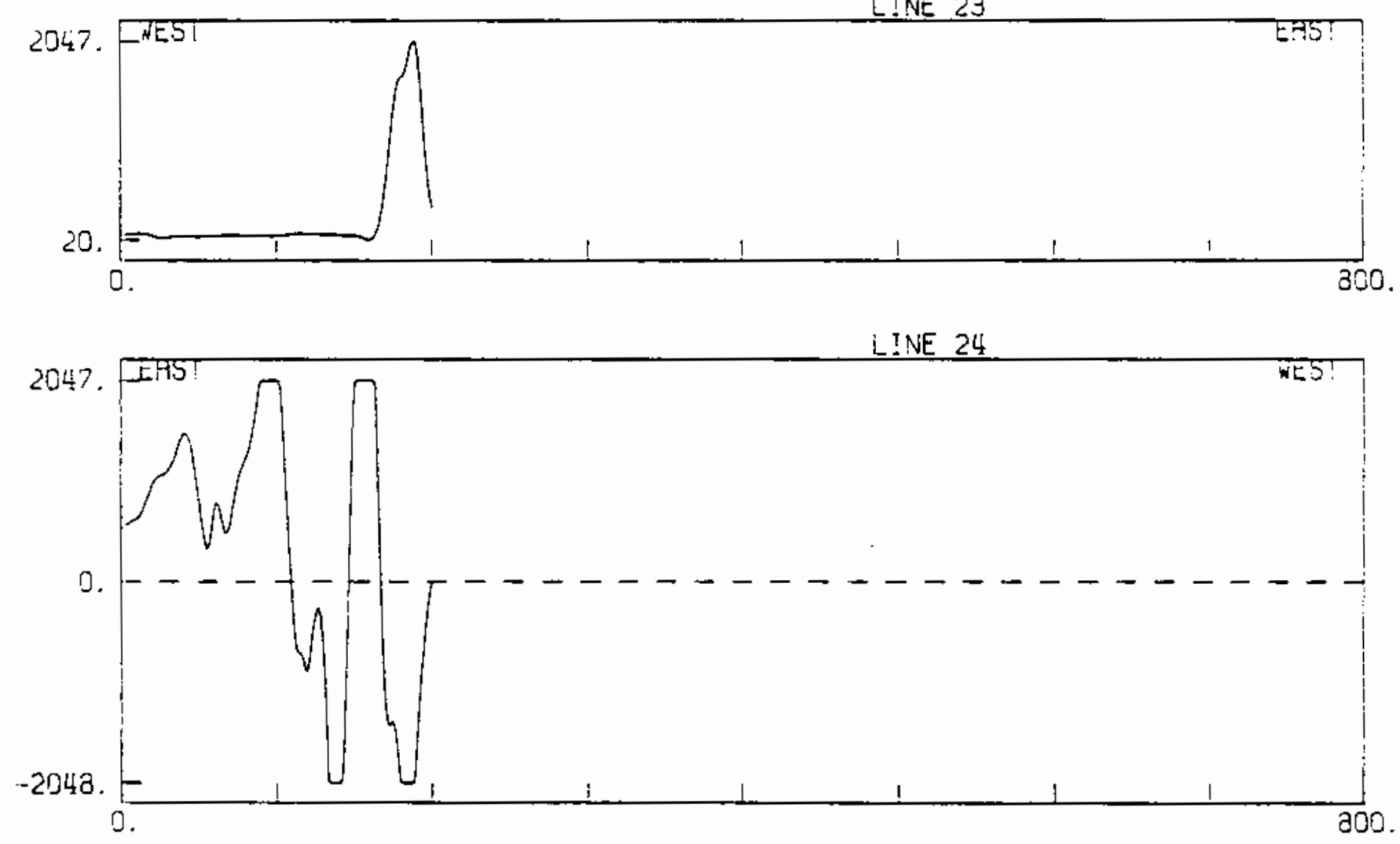

LINE 25

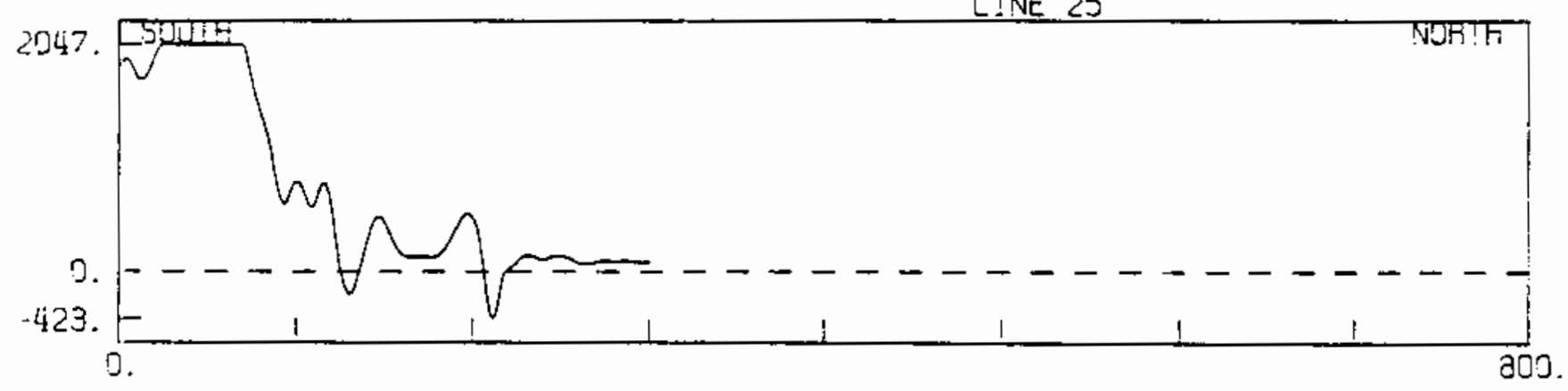


EM31 RESPONSE, LINES 26-30
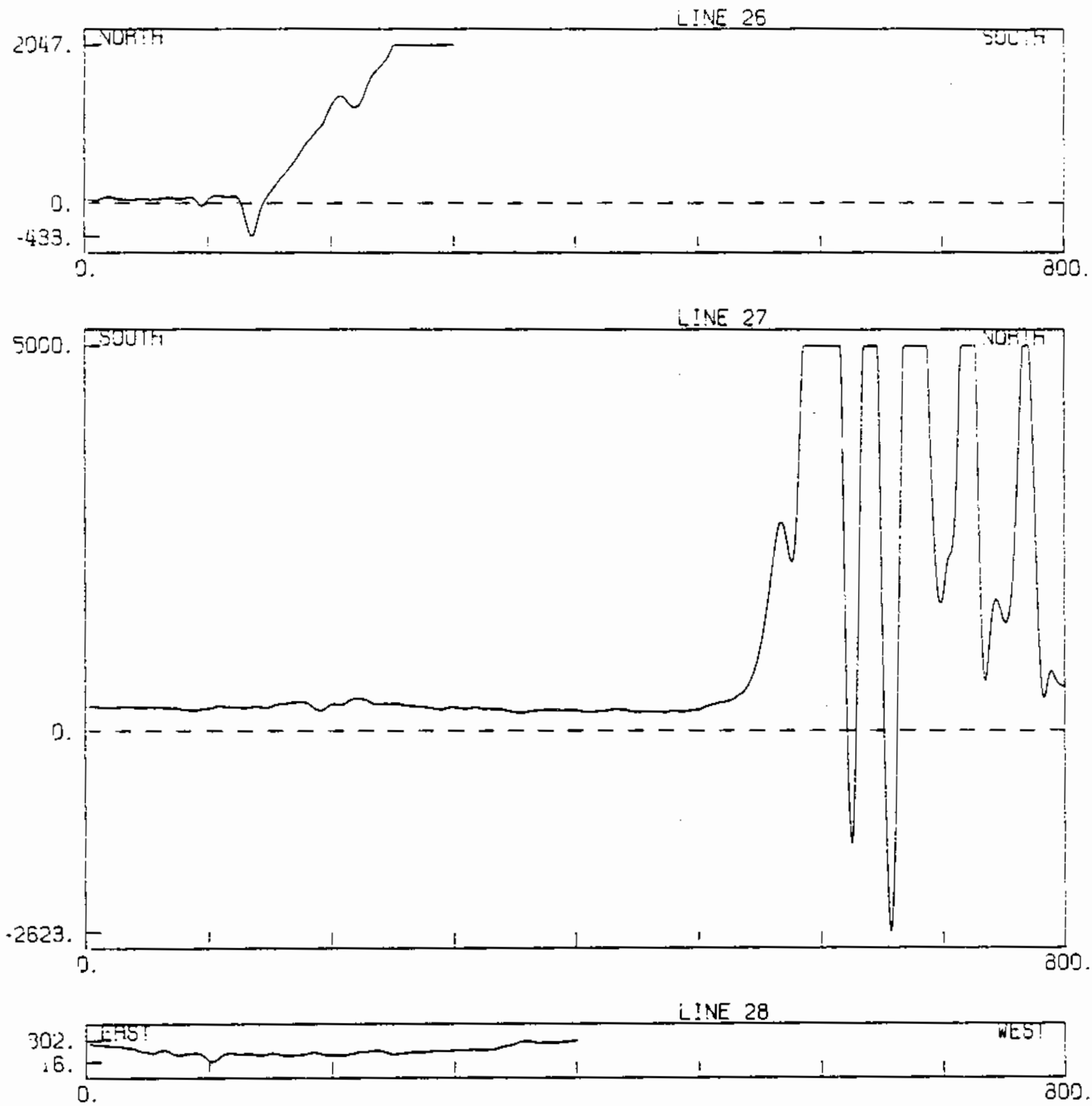

LINE 29

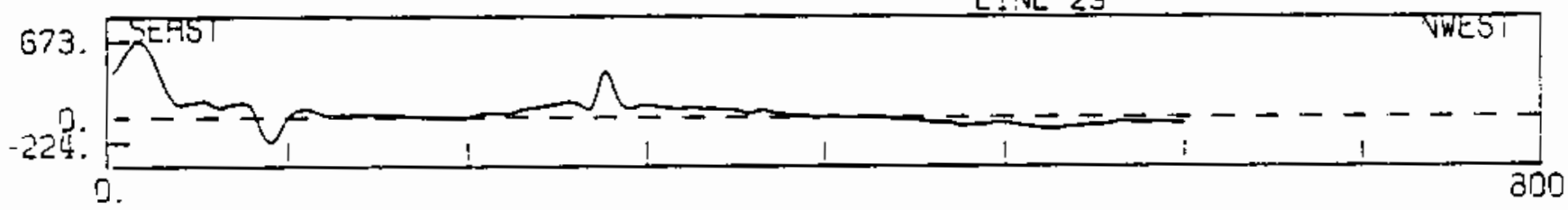

LINE 39

.

D.

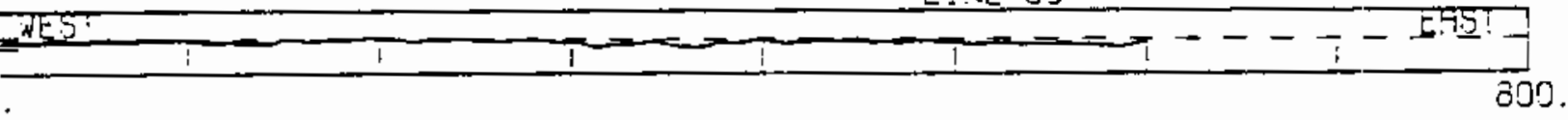


APPENDIX B

WELL SPECIFICATIONS 


\section{APPENDIX B}

\section{WELL SPECIFICATIONS}

These specifications were excerpted from the drilling subcontract. References to other appendixes do not refer to the appendixes of this report. 


\section{DRILLING SPECIFICATION}

\subsection{General}

1.01 Descriotion:

A. Work included:

The effort and materials required for this work include, but are not necessarily Timited to:

1. Drilling, sampling, and installing and developing approximately 23 temporary test holes (TTH), 4 holes during Phase I, and up to 19 holes during Phase II.

2. Furnishing and placing grout, bentonite crumbles, bentonite pellets, and sand pack;

3. Furnishing and installing flush-threaded, Schedule 4D PVC casing, caps, protective steel cover with lockable cap and slotted PVC weil screen; and

4. All other related items indicated in the figures, specified herein, or needed for a complete and proper installation in accordance with the intent of the specifications.

B. Work Location:

All the driling sites are located at fort Lewis, near.Tacoma, WA. The location map is shown in figure 1.

c. Site Access and Security:

The Contractor is responsible for familiarity with access routes for his equipment. Orill rigs may be left on the site overnight, but security and safety of rigs and equipment rests with the contractor.

Access to individual drilling sites is varied, ranging from open fields and parking lots to wooded areas.

D. Working Hours:

Drilling will be carried out during daylight hours, nomally extending from 8:00 a.m. to approximately 5:00 p.m. Pacific Dayight time, a though extended hours may be necessary to complete critical stages of work that are not planned. All work will be completed during the standard five day work week. 
A. Qualifications of Horkmen:

Use sufficient journeyman drillers and a competent supervisor in the execution of this work to ensure proper and adequate installation of items throughout. Provide a complete list of workmen involved and enter those present in the daily log.

B. Compliance with Specifications:

1. Whenever required during progress of the work and after compietion of construction, the contractor shall immediately furnish proof acceptable to Battelle that all items of the TTH's installed equal or exceed all requirements specified for this work.

2. In the event such proof is not available or is not acceptable to Battelle, the contractor may be required to remove the item or $i$ tems and replace 'with material meeting the specified requirements and to repair all damage caused in the removal and replacement, all to be accomplished at no additional cost to Battelle.

3. Ail TTH's shall be constructed and completed in accordance with the intent of Chapter 173-160 WAC, "Minimum Standards for Construction and Maintenance of Hater Hells", and the specifications contained herein. In the event of a conflict, the technical specifications contained herein shall govern.

\subsection{Drawinas:}

A. Intent of Figures:

The Contract figures, (Figures 2 and 3) do not attempt to show exact details of ail piping and items to be installed. Diagrams are schematic and do not attempt to show all fittings, obstructions, flush couplings, or spacing required. Hork measurements shall be verified from field measurements.

B. Shop Drawings:

No shop drawings are required.

C. As-Buitt Drawings:

During progress of the work, the Contractor will maintain an accurate record of all changes made, if any, in the specified installation layout and materials specified herein such as PVC casing, protective steel casing, welds, etc. The contractor will provide as-built drawings upon completion of the contract or provide as-built data to the Battelle Technical Administrator. 


\subsection{Material Handing:}

A. Protection:

The contractor shall use all means necessary to protect well construction materials before, during, and after installation. All materials to be installed will be kept off the ground on stands, racks, or sawhorses to keep them clean. During vehicle transport all materials will be enclosed in the containers in which they were shipped to keep them clean and free from damage.

B. Damage and Repairs:

In the event of damage, the Contractor shall inmediately make all repairs and replacements necessary to the approval of Battelle at no additional cost.

C. Cutting and Water Disposal:

Drill cuttings from the saturated (water bearing) and unsaturated zones shall be contained pending determination of their waste status. Containment shall consist of $7 \mathrm{mil}$ plastic sheeting under and above the cuttings. Cuttings will be retained pending analytical results. It is not necessary to contain water for analysis.

D. Decontamination:

It is the contractor's responsibility to set up and use a decontamination station for cleaning the drill rig(s) and potentially contaminated equipment. All equipment in contact with earthen materiais or borehole fiuids shall be steam-cleaned between each borehole. The contractor shail supply the equipment necessary for this decontamination.

\subsection{Site Conditions:}

A. Site Geology:

The Contractor should anticipate drilling in unconsolidated materials, consisting of clay, silt, sand, gravel, cobbles, and boulders. These materials were deposited in former strean channels and glacial outwash. The distribution of these materials is highly variable.

\section{$\underline{2.00 \text { Materials }}$}

A. General:

Equipment and materials necessary to complete the TTH's in all cases shall include, but are not necessarily limited to:

1. Oriling and completion equipment;

2. Protective steel covers, arc welding equipment, as needed and accessories; 
3. PVC casing, well screen, top caps and bottom plugs;

4. Sand pack;

5. Steam cleaning equipment;

6. Cement and bentonite grouts;

7. Bentonite pellets or crumbles (TTH seal);

8. Approved $A: B: C$ fire extinguisher capable of containing any fire excursion caused by drilling operations;

9. Concrete for TTH pad and steei protective posts; and

10. A1l other materials and supplies for completion of the work.

B. Compliance with Specifications:

When a separate item, which includes the furnishigg of any material, is provided in the proposal schedule, the cost of furnishing, hauling, storing, and handling shall be inciuded in the unit price for that item. When a separate item is not provided in the scheduie for furnishing any material required to be furnished by the Contractor, the cost of furnishing, hauling storing, and handling shall be included in the unit price for the work for which the material is required. Materials furnished by the Contractor shall be of the type and quality described in these specifications. The Contractor shall make a dijigent effort to procure the specified materials from any and all sources, but where materials required by the specifications become unavailable, substitute materiais may be used, provided that no substitute materials shall be used without prior written approval of the Batteile Technical Administrator. Battelle's determination as to whether substitution shall be permitted, and as to what substitute materials may be used, shall be final and conclusive.

\subsection{Drilling and Support Equipment}

The use of one rig is necessary, such as a hollow-stem auger rig capable of drilling to the estimated depth of 40 to 60 feet, but not to exceed 100 feet. The hollow-stem auger type rig, if used, shail be capable of drilling through earthen materials (including clay, silt, sand and gravel) at the site to the estimated depths. The drilling machine for the TTH's must be capable of drilling to the required depth and allow for the collection of samples during dritling. The use of temporary casing will be allowed during the drilling. If methods other than hollow-stem auger are used, then the use of temporary casing during driling is encouraged. The rig must be capable of removing any temporary casing while the permanent PVC casing is in the bore. 5and packing the well screen and sealing and grouting the permanent casing shall be accomplished while temporary casing is being removed. No additives may be used to facjiltate lubrication. The contractor shall soecify the size and capability of the eouioment proposed for use. 
Wherever possible, boreholes shall be drilled using hollow-stem auger flights having a minimum 3.75-in. I.D. and an 8-in. 0.D. for installing nominal 2 in. diameter PVC. If 4 in. diameter PVC is used a minimum 6.D-in. I.D. and 10.5in. 0.D. flights will be required. The rigs shall be capable of collecting continuous core and split-spoon samples. If a split-spoon is used it shall be 2 or $2.5 \mathrm{in}$. diameter and samples should be taken every 5 feet or suspected change in lithology (sand to clay etc.). The lead flight of the augers will be equipped with an appropriate cutting bit to allow penetration into the type of material occurring at the site. Temporary steel casing set in the borehole shall have a minimum inside diameter of 8.0 inches.

\subsection{PVC casing}

Casing for the TTH's shall be new, white, 2-inch or 4-inch diameter, Schedule 40 PVC pipe having no painted surfaces. The 2-inch pipe will be used when drilling is done by auger and 4 -inch will be used for other methods of drilling. Written documentation of the confomance to PS 2170. ASTM 1785 must be provided in the shipping manifest. The minimum pipe section length shail be 2 feet with Viton or Tefion " 0 " rings used at each flush-threaded connection. Dimension of the 2-inch PVC pipe shall be: outside diameter 2.375 inches, inside diameter 2.047 inches, and a wall thickness 0.164 inch. Dimensions of the 4-inch PVC pipe shall be: outside diameter 4.500 inches, inside diameter 4.026 inches, a wall thickness of 0.247 inch. The threading shall conform to ASTM F 480 requirements for flush-threaded, themoplastic well casing and pipe. All casing shall be cleaned and decontaminated after cutting and threading at the factory, using a mild detergent, then isopropyl alcohol or an approved equivalent cleaning procedure; then air dried and finally, hemetically sealed in plastic to minimize potential for contamination prior to installation in the borehole. Chlorinated solvents shall not be used for cleaning pipe or screen. The well casing shall be manufactured by UOP Johnson, St. Paul MN; Timco Manufacturing, Inc., Prairie du Sac, HI; Brainard-Kilman, Tucker, GA; Aardvark Corporation, Puyallup, HA; or an equal approved in advance by the Battelle Technical Administrator.

\subsection{Screen, Casing Connection, End Caps and Pluas}

A. Screen shall be provided in 5 and 10-ft lengths only and shall be 2-inch or 4-inch nominal inside diameter, Scheduie 40 PVC with flush interior to permit passage of a 1.75 inch or 3.76 inch outside diameter bailer. The screen shall be of the continuous wrap or high flow slotted type in order to provide maximum inlet area consistent with strength requirements. At a minimum, the screen shall consist of 4 row, 100 slots per row, factory slotted PVC pipe. The screen shall be manufactured by UOP Johnson, St. Paul MN; Timco Manufacturing, Inc., Prairie du Sac, WI; Brainaro-Kilman, Tucker, GA; Aarovark Corporation, Puyallup, HA; or an equal approved in advance by the Battelle Technical Administrator.

B. PVC casing and screen shall have internal, flush-threaded ends requiring no glue or solvents and providing a smooth; uniform, outside diameter. 
C. The overall screen length and slot size shall be selected so that the average velocity of water entering the screen is $0.1 \mathrm{ft} / \mathrm{second}$. The screen shall provide a minimum of $10 \%$ open area with No. 0.020 siot openings.

0. All screens and casings shall be free of foreign matter (e.g. adhesive tape, labels, soil, grease, etc.) and cleaned and seaied in the same manner as the PVC casing. Screen and casing shall be kept in boxes or on racks until immediately prior to insertion in the TTH.

$\varepsilon$. The bottom of the screen should be placed no more than 3 feet above the bottom of the TTH.

F. Schedule 40 PVC fiush-threaded end caps or plugs to fit the bottom end of the screen and removable Schedule 40 vented, flush-threaded caps for the top of the casing shall be provided. All 10-foot screen bottoms shall be securely fitted at the factory with a flush-threaded bottom cap of the same composition as the screen. Five foot screen sections shall be of flush threaded couplings except for the 6 screens that will be placed in the 60 ft. TTH's. These 5-foot screens will have flush-threaded bottom caps similar to the 10-foot screens. The end cap shall be within 0.5 feet of the open portion in the bottom section of the screen.

G. Silt traps shall not be used.

H. Joints within and between the casing and screen shall be flush-threaded couplings. Heat-weided joints and/or gaskets shall not be used.

I. The tops of all PVC casings shall be installed with maie threads upward so that slip caps placed on top can be easily removed by hand.

2.04 Centering Guides

Centrajizers are required and shall be placed 2.0 feet above the sand pack. 2.05 Filter (Sand) Pack:

The Contractor shall provide and install filter pack below and around the screens. The filter pack around the screen shall consist of a size sufficient to be retained outside of the 0.020 slot screen while passing no more than $1.0 \%$ through the screen. Filter pack material shall consist of kiln dried, uniform, rounded and spherical grains of sand composed of at least $95 \%$ quartz. The filter pack sand shall have a uniformity coefficient (the quotient of the $60 \%$ passing, $D_{60}$ size, divided by the $10 \%$ passing, $D_{10}$ size) between 1.0 and 2.0 . At least $90 \%$ of the particles shall have a Powers roundness of 4 to 6 (i.e., subrounded to well rounded). The filter pack material shall have a U.S. sieve size of 10 to 20 mesh (effective particle size of 1.0 to $1.2 \mathrm{~mm}$ or 0.039 to 0.047 in.). The sand materials shall be packaged in such a way as to prevent contamination and water damage. Each sack must be clearly labeled as to the mesh size of the sand contained. Approved supply sources are Colorado silica Sand, Inc., Colorado Springs, Colorado and the Fountain Sand and Gravel Company, Pueblo, Colorado. 
The use of any brand or variety of filter (sand) pack (other than the two approved sources noted above) must be approved by the Battelle Technical Administrator prior to the arrival on site of the drilling machinery. The following data shall be submitted in writing to the Battelle Technical Administrator as a part of the approval request:

1. Brand name(s)

2. Manufacturer(s)

3. Manufacturer(s) address(es) and telephone number(s)

4. Product description(s) from label(s) or brochure(s)

Allow six (6) working days from the time of receipt and information by Battelle for request evaluation and recomendations.

\subsection{Bentonite Seal}

A. Bentonite is the only sealant allowed. No organic additives shall be used. The use of any brand or variety of bentonite must be approved by the Battelle Technical Administrator prior to the arrival on-site of the drilling machinery. This includes bentonite powders, crumbles, pellets, etc., intended for grout, seais or hole abandonment. The following data shall be submitted in writing to the Battelle Technical Administrator as a part of the approval request:

1. Brand name (s).

2. Manufacturer(s).

3. Manufacturer(s) address (es) and telephone number(s).

4. Product description(s) from label(s) or brochure(s).

Allow six working days from the time of receipt of information by Battelle for request evaluation and recomendation.

B. Bentonite for seais and boring abandonment shail be composed of conmercially available peliets that are $1 / 4$ to $3 / 8$ inch in diameter or chunks, crumbles, or granules. Pellet seals shall be a minimum of $5-\mathrm{ft}$ thick as measured immediately after placement, without allowance for swelling.

C. Bentonite grout, when used in TTH construction shall be a bentonite s)urry grout or its equivajent. Such bentonite grout shall be mixed to approximately 100 pounds per 100 gallons of water, or in accordance with the manufacturers recommendations. Neither additives nor borehole cuttings shall be mixed with the grout. 


\subsection{Pratective Stee 1 Cover}

A. The protective steel cover shall be, black plain-end welded steel pipe conforming to ASTM-53 or A-120 or an approved equal. The pipe section length shall be 5 feet with a locking cap. Size and wall thickness of base shail be 6 -inch minimum inside diameter with a nominal $1 / 4$ inch wall thickness. Steel caps shall have at least a $1 / 8$ inch wall thickness. All welding must be performed in accordance with applicable portions of AHS D1.1. A minimum $1 / 4$ inch to a maximum $1 / 2$ inch diameter hole located approximately 2.5 feet below the top of the steel cap shall serve as a drainage port.

B. Only the outside of the protective casing and covers/caps shall be painted safety yellow with a paint brush (not aerosol can) after installation. The yellow color for finish coats shall be as defined in ANSI 253.1. Safety Color Code for Marking Physical hazards. Primer and paint materials shall conform to the following Federai Specifications:
- Primer for metal parts
$T T-P-645$
- Finish ename]
TT-E-489F, Class A

c. The painting of the TTH designation on the outside of the protective casing shail be performed using black paint and brush. This identification shall be done after the casing is painted as described above. Painting is required to be completed and dry prior to TTH development.

\subsection{Surface TTH Protection}

The final TTH shall be protected from damage by the following:

o A 4-foot by 4-foot by 4-inch thick concrete pad will be installed around the TTH's.

- Four metal posts shall be installed at the corners of the concrete pad. These posts shall be 4 -inch in diameter schedule 40 carbon steel and shall be at least 6 -feet in length of which 3.5 -feet shall be above the pad. The post shall be painted safety yellow in the same manner as the TTH.

- A brass survey marker shail be placed in the surface of the pad. The surface of the survey marker shall be at least 3 in. in diameter to be of sufficient size to be stamped with the TTH number. The approved part number and material supply source is part number 01602 from Allen Precision Equipment, Atlanta, Georgia. Other brass markers may be used, if approved by the Battelie Technical Administrator.

\subsection{Other Materiais}

All other materials, not specifically described but required for a complete and proper installation, shall be new and of first quality of their respective kinds, and subject to approval by the Battelle Technical Administrator. 


\subsection{Health and Safety Equipment}

A. All individual protective clothing including coveralls, gloves, hard hats, steel-toed and shank boots, and related safety equipment shall be provided by the Contractor.

B. Monitoring of drill cuttings shall be carried out by Battelle. This monitoring will be done using an HNU organic vapor analyzer, an LEL O2 combustible gas indicator or similar devices. In the event that significant vapors are detected, work shall be suspended until the nature of the vapors is determined. It is estimated that most delays will be less than an hour, and any delays directed by Battelle will be considered standby time as documented by an approved Standby Order, Appendix C. Recommencement of work may require the use of half-face masks with organic vapor cartridges. All personnel must have had appropriate training in the use of such equipment prior to arrival on site.

\subsection{Execution}

\subsection{Scope:}

This section covers the complete construction and completion of TTH's.

A. Inspection:

The Battelle Technical Administrator shall verify that the TTH's have been constructed in strict accordance with all specifications presented herein.

B. Soil Description:

The Contractor should anticipate drilling in fine-to-coarse-grained material consisting of clay, silt, sand, gravels and cobbles to approximately 40 to 60 feet.

C. Warranty:

The information indicated in the technical section is to used by the contractor to assist him in properly evaluating the amount and character of the work that might be required. Such information is given, however, as being the best factual information available, without the assumption of responsibility as to its accuracy or for any conclusions that the Contractor might draw therefrom.

D. Notice:

The Contractor shall promptly, and before such conditions are disturbed, notify Battelle in writing of: 1) subsurface or latent physical conditions at the site differing materially from those indicated in this contract; or 2) unknowm physical conditions at the site of an unusual nature, differing from those ordinarily encountered and from those generaliy recognized as inherent in the work of the character provided for in the contract. The 
Battelle Technical Administrator shall promptly investigate the conditions and if he finds that such conditions do so materially differ and cause an increase or decrease in the cost of materials or the time required for the perfomance of this contract, he may recommend to the Battelie contract Representative that an equitable adjustment be made and the contract modified accordingly.

E. Site of Work:

The Contractor is cautioned to visit the work site and by his own investigation satisfy himself as to the existing conditions affecting the work to be done under these specifications. If the Contractor chooses not to visit the site, he will never-the-less be charged with knowledge of conditions which a reasonable inspection would have disclosed.

\subsection{Workmanship}

Equipment used for drilling must be in good condition, of adequate size, with competent operators to insure satisfactory well construction within the time limits specified.

Drilling of the TTH's may be done by the hollow-stem auger or percussion tool method. TTH's shall be straight and a minimum of 8 inches in diameter to accomodate the installation of the permanent PVC casing specified, and allow the placement of grout by the tremie method.

\subsection{Construction Record:}

Written daily logs of construction progress shall be kept by the contractor, indicating size and length of casing instalied; character, depth, and thickness of all changes in formation penetrated; water levels during the course of drilling; and any reasons for delays. Daily logs shall be made available to the Battelle Technical Administrator. All log entries shall be traceable to the date, time and workman making the entry. The Drilling Supervisor shall review daily logs and approve them by signature and date at the end of each working day.

\subsection{Casing, Screen and Accessories}

The PVC casing diameter, wall thickness, and other requirements are specified in Sections 2.02 and 2.03. Screen sections are to be joined to casing and each other by flush-threaded joints. Casing and screen shall be set round, plumb and true to line. Casings shall extend at least 24 inches above the concrete pad.

The screens will be ordered in writing by the Contractor in lengths specified in section 2.03. For each of the seventeen forty (40) foot deep TTH's the screen length shall be a total of 15 feet. The 6, 60 foot deep TTH's around landfill $\frac{\|}{\pi l}$ will have a 5-foot screen instalied at the bottom of each TTH. Screens shall be installed by setting them at the depth designated by the Battelie Technical Administrator. Use of other methods will not be permitted without specific approval, in writing, of the Battelle Technical Administrator. 


\subsection{Sand Pack:}

After setting the screen in place, the sand pack, which meets the requirements of Section 2.05, shall be placed without a tremie to surround and extend 5 feet above the top of the screen. Where necessary, a loose fitting surge block or bailer will be used for 10 minutes to settle the sand pack; then additional sand may be added to satisfy the requirements illustrated in figure 3 . Temporary well casing or auger flights shall be withdrawn as the sand pack is being placed.

\subsection{Bentonite Seal:}

Approximateiy 100 ibs. of bentonite pellets shall be placed without using a tremie, inmediately above the sand pack. Pellets are to be added slowly to prevent bridging in the annulus. Bentonite crumbles, chunks, granules, or grout slurry placed above the pellets may be installed immediately after pellets have settied to the bottom of the annulus. If grout is used it shall be placed by pumping through a tremie pipe. The tremie pipe shall extend to the bottom of the annular space. The tremie pipe is to remain submerged during the placement of all grout.

The Contractor shall place the bentonite seal around the TTH's as follows:

A. During removal of any drill casing, the annulus between the borehole and PVC casing shall be filled with bentonite to a point that is always at least two (2) feet above the bottom of the temporary casing, or auger flights.

B. Bentonite grout shall be placed through a tremie pipe (or hose) located just above the top of the seal.

C. After 24 hours the contractor shall check the site for grout settiement and that day pour the concrete well pad.

D. The protective steel casing and posts will then be instailed while the concrete pad is being poured.

\subsection{Protective Steel Cover}

The Contractor shall protect the TTH at all times to prevent tampering or the entrance of foreign matter. After the concrete well pad has been placed and is reasonably firm, the specified protective steel cover (Section 2.07 ) shall be installed.

\subsection{Hell Develooment}

Development of TTH's by pumping, bailing, and surging will be performed until the well water is clear, as determined by the Battelle Technical Administrator. 


\subsection{Cleaning and Decontamination of Equipment and Materiais}

Steam cleaning and rinsing of all drilling equipment including: rigs, water tanks (inside and out), augers, drill casings, rods, samplers, tools, recirculation tanks, etc., shall be done by the Contractor prior to arrival at the site. All equipment used in the borehole shall be cleaned between well sites. Prior to use on site, all casings, augers, and water tanks, etc., shail be devoid both inside and out of any asphaltic, bituminous, or other encrusting or coating materials, grease, grout, soil, etc. Paint, applied by the equipment manufacturer, need not be removed from the drilling equipment. All equipment or portions of equipment that come in contact with water or soil from the borehole must be cleaned between boreholes by brushing and steam cleaning. The residue from steam cleaning does not need to be contained for analysis. A separate steam cleaning site can be established, if appropriate, for each of the two drilling sites.

\subsection{Abandonment of TTH's:}

If, in the judgement of the Battelle Technical Administrator, a TTH should be abandoned for whatever reason, the Contractor will be ordered, in writing on a TTH Abandonment Form, see Appendix $E$, to abandon and fill the hole. If the TTH is to be abandoned, as a result of Contractor error or fault, the Contractor shall immediately make all repairs and work necessary for replacing the abandoned structure to the approval of the Battelle Technical Administrator at no additional cost. If the TTH is abandoned for reasons beyond the control of the Contractor, payment will be made for the actual depth drilied at the rate stated in the Payment Schedule, Appendix G.

The abandonment of any TTH's must be approved by the on-site Battelle Technical Administrator prior to any casing removal, sealing, or backfilling. Abandonment requests must be submitted in writing, see Appendix $E$, and include the following data:
A. Designation of TTH in question.
B. Current status (depth, contents of hole, stratigraphy, water ievel, etc.).
C. Reason for abandonment.
D. Recormendation.

Decisions on abandonment will be made within 4 hours of the request.

Once approved, the TTH to be abandoned shall be sealed by placing bentonite pellets and/or slurry from the bottom of the TTH to the surface. Grout shall be placed via the tremie method until undiluted grout flows from the TTH at the surface. 
After 24 hrs., the Contractor shall check the abandoned site for grout settlement. That day, any settlement depression shall be filled with grout and rechecked 24 hrs later. This process shall be repeated until firm grout remains at the ground surface.

Nomally an abandoned TTH shall be grouted with the well screen and casing in place. However, a lack of data concerning TTH construction or other factors may dictate the removal of the TTH materials and a partial or total hole redrilling prior to the sealing of the TTH site.

\subsection{Site Cleanup and Restoration:}

All structures, utilities and natural vegetation will be protected by the Contractor during the progress of the work. All debris, equipment and unused material will be removed from Fort Lewis by the Contractor. The property will be restored as near as possible to its original condition by the contractor.

Disposal of any contaminated materiais shall be the responsibility of the U.S. Anny.

\subsection{Standby (Delav) Time:}

If it is necessary for the contractor to stop drilling due to waiting for the Battelle Technical Administrator to complete other work, the Contractor will be placed on standby time, in writing, and removed from standby in writing, using the Standby Order form, Appendix C.

\subsection{Special Work}

Conditions at the fort Lewis site may require special work. This work may include special perforating, setting special tools, special testing, minor exploratory drilling, unscheduled rehabilitation, abandonment of pre-existing Weils, or some unforeseen situation. Battelle may order special work using the Special Work Task Order (SWTO) form, Appendix D. 


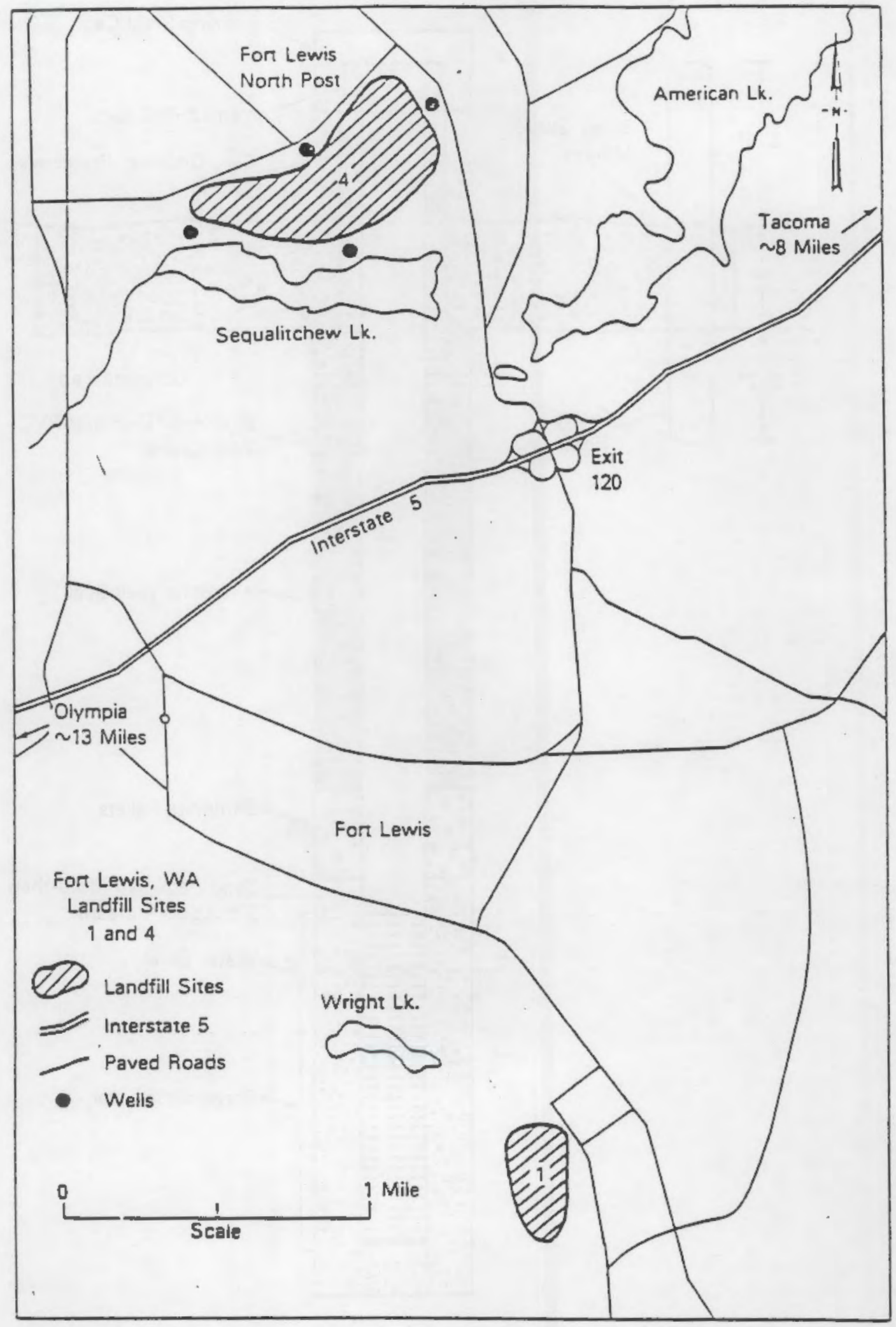

FIGURE 1. Fort Lewis, WA Landfill sites 


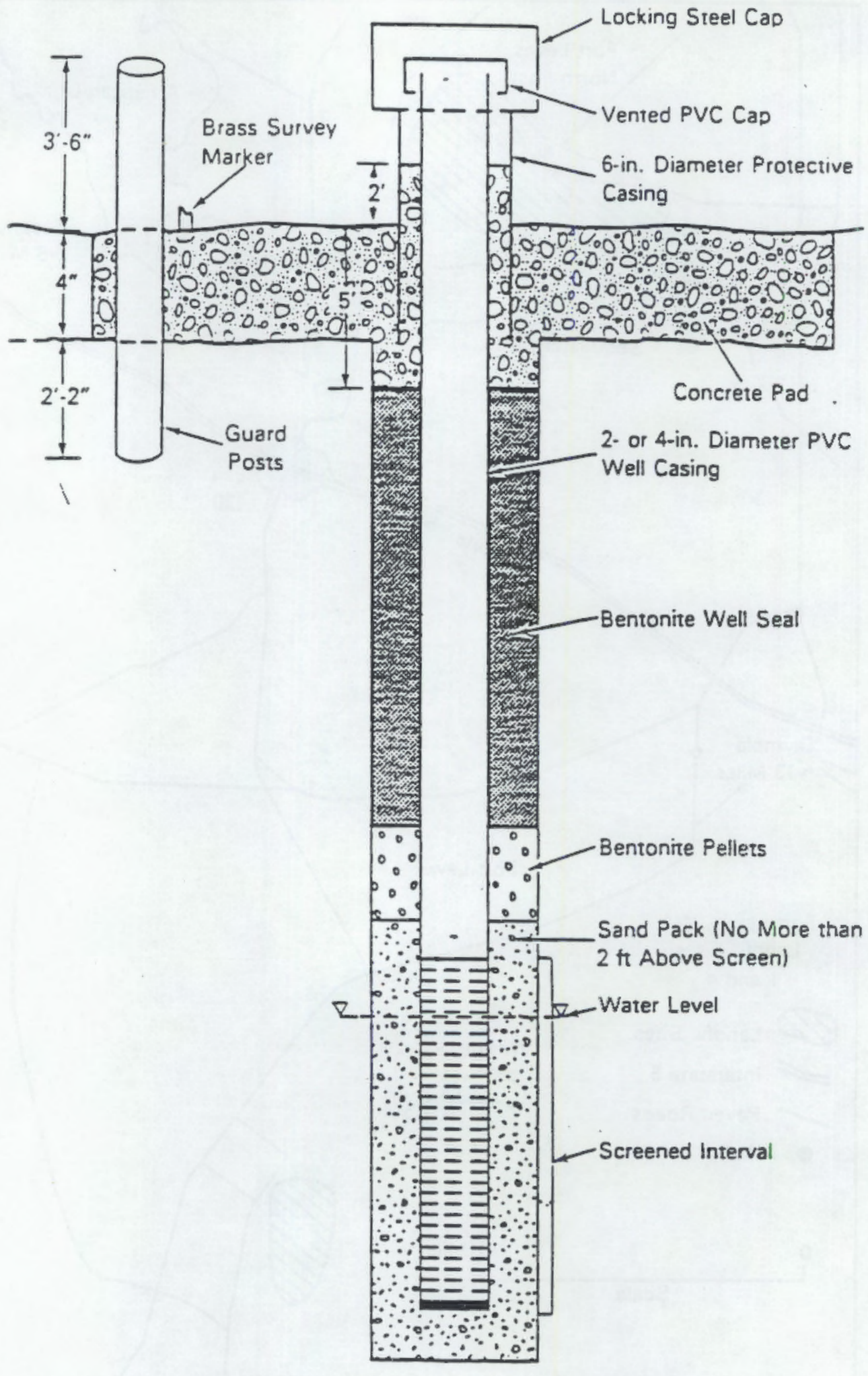

FIGURE 2. As-Built Hell Diagram 


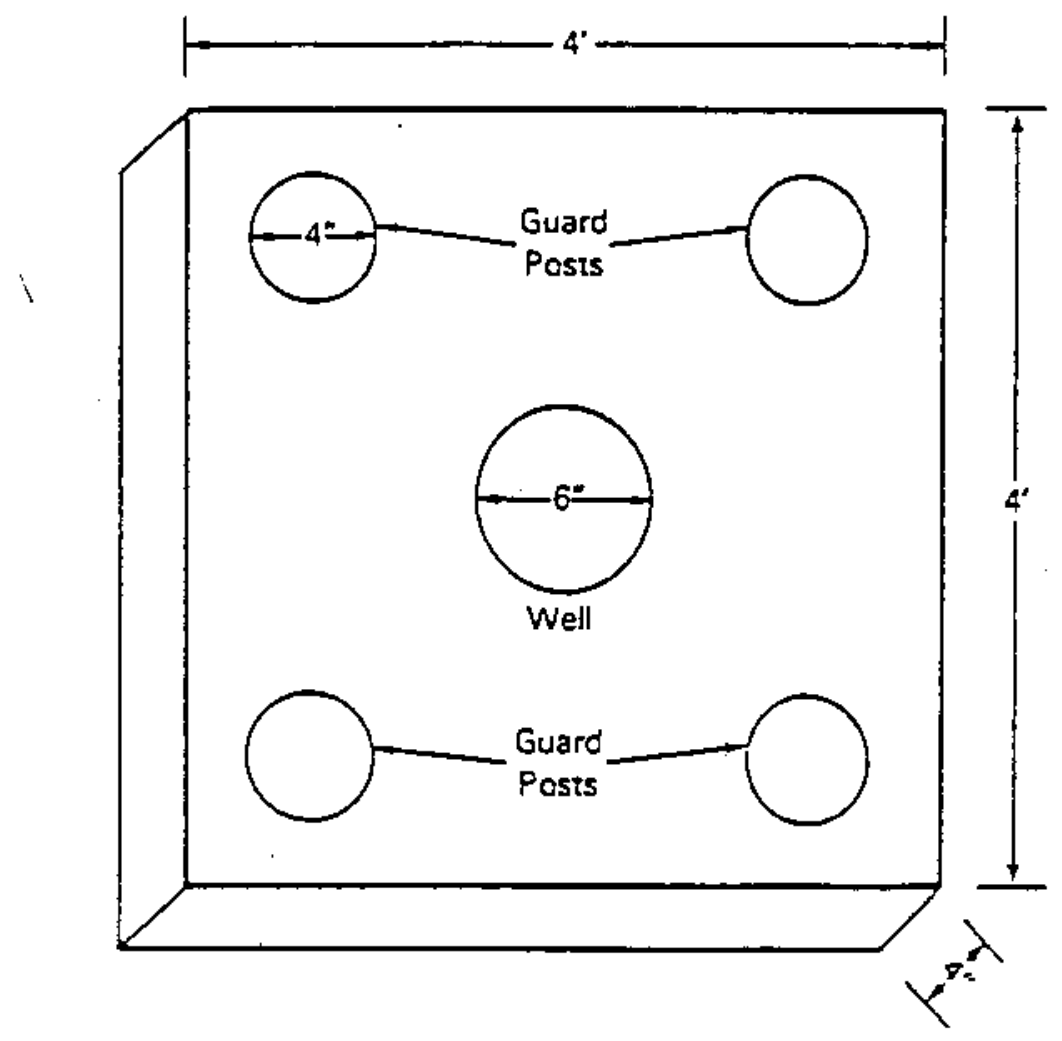

FIGURE 3. Surface Completion 


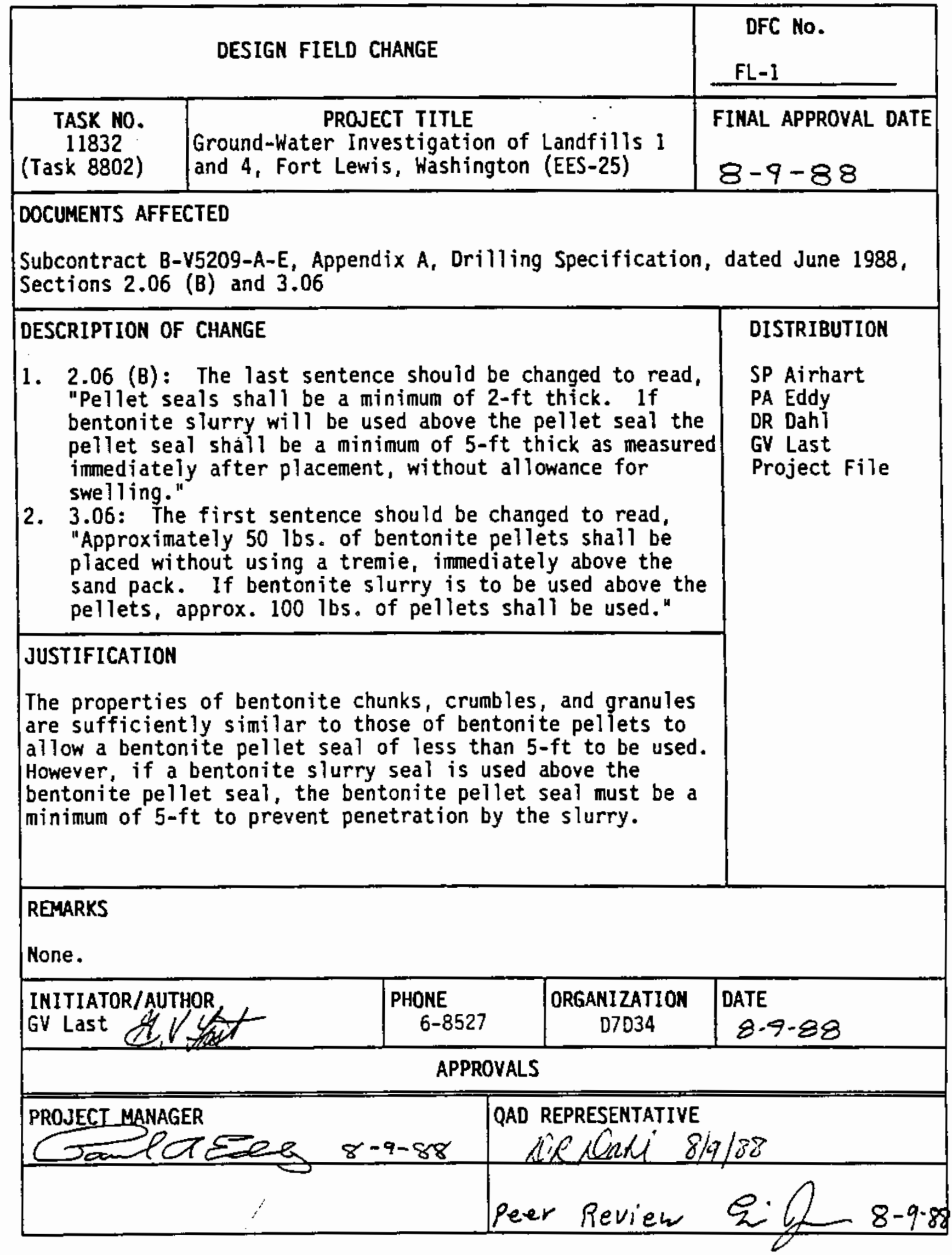




\section{APPENDIX C}

PROCEDURE FOR COLLECTION OF SEDIMENT SAMPLES

USING HOLLOW STEM AUGER METHODS 


\section{TITLE: TP-8, COLLECTION AND DOCUMENTATION OF SEDIMENT SAMPLES RECOVERED} USING HOLLOW STEM AUGER METHODS.

\subsection{APPLICABILITY}

This procedure applies to work perfomed by well site geologists during collection and documentation of sediment samples recovered from boreholes drilled by hollow stem auger methods. This includes both the periodic and continuous sampling methods.

\subsection{DEFINITIONS}

Does not apply.

\subsection{RESPONSIBLE STAFF}

3.1 Project Manager

3.2 QAD Representative (Cognizant QE)

3.3 Principal Investigator and Cognizant Staff

\subsection{Well Site Geologist}

\subsection{PROCEDURE}

4.1 The driller shall advance the hollow stem auger fiights to just above the required sample interval (every $5 \mathrm{ft}$ and/or lithologic changes). If the continuous sampling method is being used, skip to step 4.4 .

4.2 If a pilot bit is not being used while advancing the auger flights, then the driller shall remove any soil or residue that entered the flights during their advancement. The driller shall then lower a clean split-spoon sampler (also referred to as a split-barrel or split-tube sampler) through the hollow stem auger flights to the bottom of the clean borehole using solid drill rods.

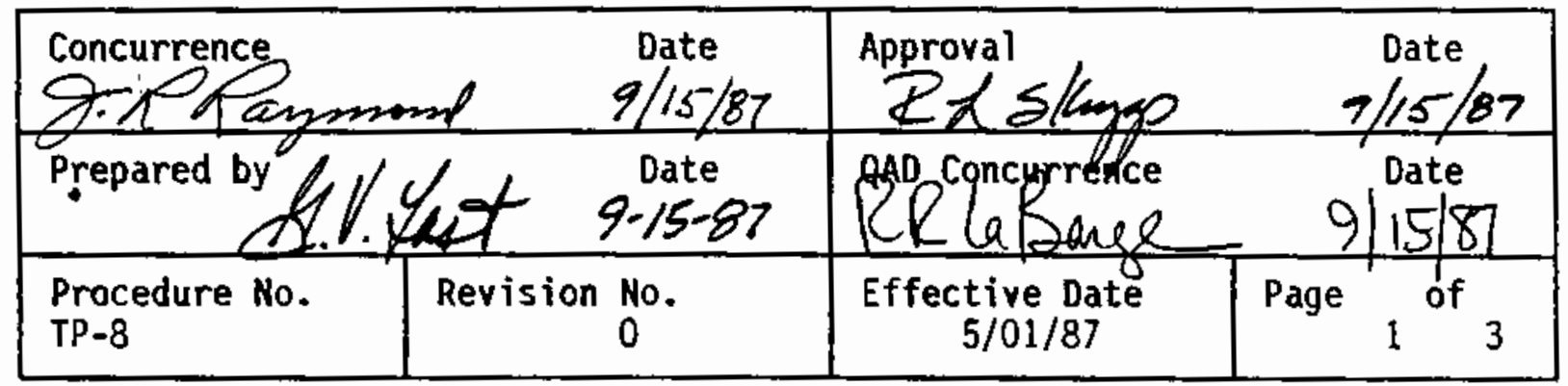

C. 1 


\section{TECHNICAL PROCEDURE}

4.3 The driller shall then use an appropriate drive weight to hammer the sampler into the undisturbed materials (soil) below the borehole. The driller shall not drive the sampler a distance greater than the length of the sampler. The number of hammer blows should be counted and recorded by the well site geologist.

4.4 The driller shall remove the sampler from the auger flights, being careful not to knock the sample out of the sampler. The sampler shall then be carefully removed from the drill rod.

4.5 Carefully remove the drive shoe and the end cap from the sampler. Lay it flat and remove one side of the split-tube sampler.

4.6 With a knife or other suitable object, carefully scrape any smeared material (top $1 / 8-3 / 8$ in.) off the top half of the sample. If this cannot be done without disturbing the sample, and/or if the sample can easily be described without exposing a fresher surface, then disregard this step.

4.7 Examine, physically describe, and photograph (optional) the sample. The physical description shall be recorded in the geologist's log or field notebook and shall include the borehole number, depth of the sampled interval, drill method, wet/dry sample, lithologic description, date and time the sample was collected, and any suitable comments regarding the representativeness of the sample. These descriptions shall be thorough, and all entries will be recorded chronologically. The geologist's $\log$ (or field notebook) shall be signed and dated at the end of each day.

4.8 Carefully remove selected subsamples from the sampler and place them in suitable containers (moisture can, canning jars, etc.). If more than one layer of material is present, then the subsamples shall be collected by partitioning the sample into lithologically similar subsamples. Care should be taken to exclude the top portion of the sample thought to be slough or not representative of the sampled interval. The sample containers shall be labeled with the borehole identification number, the depth interval of the sample or subsample, the date, and the geologist's initials.

4.9 The sampler shall then be scraped clean of all foreign matter and washed with water or a pre-approved detergent solution. The sampler shall then be rinsed clean, and reassembled.

\begin{tabular}{|l|c|c|cc|}
\hline $\begin{array}{l}\text { Procedure No. } \\
\text { TP-8 }\end{array}$ & $\begin{array}{c}\text { Revision No. } \\
0\end{array}$ & $\begin{array}{c}\text { Effective Date } \\
5 / 01 / 87\end{array}$ & Page ${ }^{\text {of }}$ \\
\hline
\end{tabular}

C. 2 


\section{TECHNICAL PROCEDURE}

4.10 Samples shall be labeled "Suspect" and/or discarded if the location and depth of the sample is lost or unknown, or if the representativeness of the sample is in question.

4.11 If physical analyses are to be performed by PNL's Soils Laboratory in the Sigma $V$ building, the samples shall be hand delivered to the Soils Laboratory Coordinator or his representative. When the samples are received, the Soils Lab. Coordinator or his representative will log the samples into a controlled notebook, along with the date and time they were received. The samples will be logged in and always identified by the borehole number and depth as clearly labeled on the sample.

4.12 If chemical analyses are to be performed, then the appropriate sample handling and chain-of-custody procedures shall be followed as 1isted in PNL-MA-580, Environmental Monitoring Procedures.

\begin{tabular}{|l|c|c|c|}
\hline $\begin{array}{l}\text { Procedure No. } \\
\text { TP-8 }\end{array}$ & $\begin{array}{c}\text { Revision No. } \\
0\end{array}$ & $\begin{array}{c}\text { Effective Date } \\
5 / 01 / 87\end{array}$ & Page $3^{\text {of }} 3$ \\
\hline
\end{tabular}


. 
APPENDIX D

GEOLOGIC SAMPLING AND WELL CONSTRUCTION DOCUMENTATION PROCEDURE 


\section{APPENDIX D}

\section{GEOLOGIC SAMPLING AND WELL CONSTRUCTION DOCUMENTATION PROCEDURE}

This appendix contains the procedure for collecting and documenting drilling data that was in place during well drilling at the landfills. 
Procedure for Coilection and Cochmentation of Orfling Data, -

P-6 for RCRA Compliance/Hazartous Matarials Monitoring

\subsection{ORJECTIVE}

Tits procsiure provides standardized methods for the collection and documentation of sediment sampies and well eanstrsction data, for we!ls drilled by the cable tooi method.

\subsection{APPLICABILITI}

This procedure applies to work perioraed by well site geologists during well consțruction.

\subsection{RESPONSIBILITIES}

\subsection{Weil site Gegiodist}

- Collect and dociment drilling data.

\subsection{Sentor Technical Reviewer}

- Reviey and sign/date Well Completion Report/Title IjI Inspection List

- Review and sign/date As-euilt ofagram

- Review and sign/date Orill Legs

\subsection{PROCEDURE}

\subsection{Coilection of Sediment Samoles}

Sediment samples shall be collected at 5-foot interals and changes in formation by the well stte geologist. All drill cuttings to be sanpliei shail be collested from the driller in a 5 galion bueket.

Two pint fars shall then be filled from the bucket. One jar siall be submitted for laboratory analysis and the other ratained for archiving.

A label indicating well number, depth, data, drililing method and initiais of the geologist collecting the sample shali be placad cr eaci sampie far. Well number, depeh and date siall also be written on the lid of each far.

\begin{tabular}{|c|c|c|c|}
\hline $\begin{array}{l}\text { Afprovals: } \\
\text { Project Manag }\end{array}$ & & Ga Rep. AP & $4 f \quad 3 / 16$ \\
\hline$\underset{\mathrm{P}-\sigma}{\text { Frocedura } \mathrm{Na}}$ & $\mid \begin{array}{c}\text { Revision } \\
0\end{array}$ & $\begin{array}{l}\text { loate issued: } \\
\qquad-16-87\end{array}$ & Page 1 o: 3 \\
\hline
\end{tabular}

D.2 
Procadure ior Collection and Documentation of DHll1ng Data, P-6 (continued)

If the well is being drliled with a drive barrel above the watar tabla, a wisture sample shall also be collected from the bucket. Each noistris: sample shall be sealed with white identiflcation tape. The well numicer, depth, date and inftials of the geologist ollecting the sample shall be recorded on the identification tape with indeltbie ink. Each motsturs sample shall then be doubly wrapped in a glastie bag, and taped shut.

The remainder of the samls in the hucket shall be used for sedirent satie description.

\subsection{Documentation of Sefiment Samoles and dell Construction Data}

Sedinent 'sample descriptfons and weil construction data for each well sinall be recoried on a Hell Completion Reportititle III Inspection List (Atiactment 1), As-8uilt Oiagram (Attzchment 2) and Orfll Log (Attachnent j). These data shall be recorded daily by the well site geologist. Monecpilicabie iters shall be designated N/A.

The Hell Completion Report/TItle III Inspertion List provides a conipleta sunmary of weli construction and eompletton data. Data meordar on the Well Completton Report/Title III Inspection List shall inelude: generai profect and well information, drllling method, completion data, casing data, perforations, screen, annular seal, geophystcal logging, aqulier testing and other applicable ttens. Casing data, periorattons, sersen, annuiar seal, geophysical locging, aquifer testing and other apolicable items shall he approved by the well site gegiogist. After ccingletion of the well, an overall review of the bell completion Report/Title III Inspection list shall be periomed by the Sentar Tesintel Reviewer:

The As-auit Diagram is a graphical representation of the well constriction, geologic and hydrologie data. Data resorded on the As-autl of agran shall include: weil number, geologist, page number, constrsction data, depth in feet, gealogic and hydrologte data. After coluletion of the well, an overall review of the As-autlt Diagran siall be perionad by the Senior Teshnical Reviever.

The orfll Log ecntains detalled desctiptfons of the sediment samples and well censtruetion data. Data recorded on the DrflT Log sinall incluce: geologist name, date, rig, well numier, dept at start, depth at fintst, computer numier, projest numer, subegntract numer, total casing, dept.'. drifl method, wet/ary sample, lithologie desceiption iricluding moisturs sample data, tiate, drilling coments and remarks. A new Orill Log sinali be used each day. Aftar ccmpletion of the day's activities, the well sita geologist shall sign and date the Orfll Log. After cempletion of the weil, an overalt revien of the Orill Logs shall be ferionimed by the Senior Technical Reviewer.

\begin{tabular}{|c|c|c|c|}
\hline $\begin{array}{c}\text { Procadure No. } \\
\text { P-o }\end{array}$ & $\begin{array}{c}\text { Reytsion No. } \\
0\end{array}$ & $\begin{array}{c}\text { Issued: } \\
\text { I-16-57 }\end{array}$ & Page 2 of 3 \\
\hline
\end{tabular}


Precedure for Collection and Documentation of Drflling Data, P-6 (contfnued)

4.3 Oescriotion of Sediment Samoles and Well Construction'Data

Detailed descriptions of the sediment samples and well censtruetion data shall be recorded on the Orill Log under "Lithologie Description" by the well site geologist.

Sedinent sample descriptions shall include the following information as a minimus: lithologic name, texture, soriing, gross mineralogy of the framework and matrix, roundness of the franemork and matrix, wet/dry celor, reaction in hydrechloric acld (HCl), consolidation and changes in Tithology.

Well construction data shall include the foliowing inioration as applicable: drill method, drill depth, coupletion depth, drill rate, casing (type, size, depth and lengths), perforations (eype, depth and schedule), screen (type, length, slat size and depth), annular seal (type, interval and volume), packer (type, size and deptb), well development and depth to water.

\subsection{Oata Manaqement}

Aiter completion of the project, the original Weil Completion Report/Titie III Inseection List, As-Built Of agram and Orlli Logs for each well siall be retained by $V$. L. MeGhan of the PNL Geasetences Departuent. A copy of each completed form siall be retained by the PNL Resards Retantion Center.

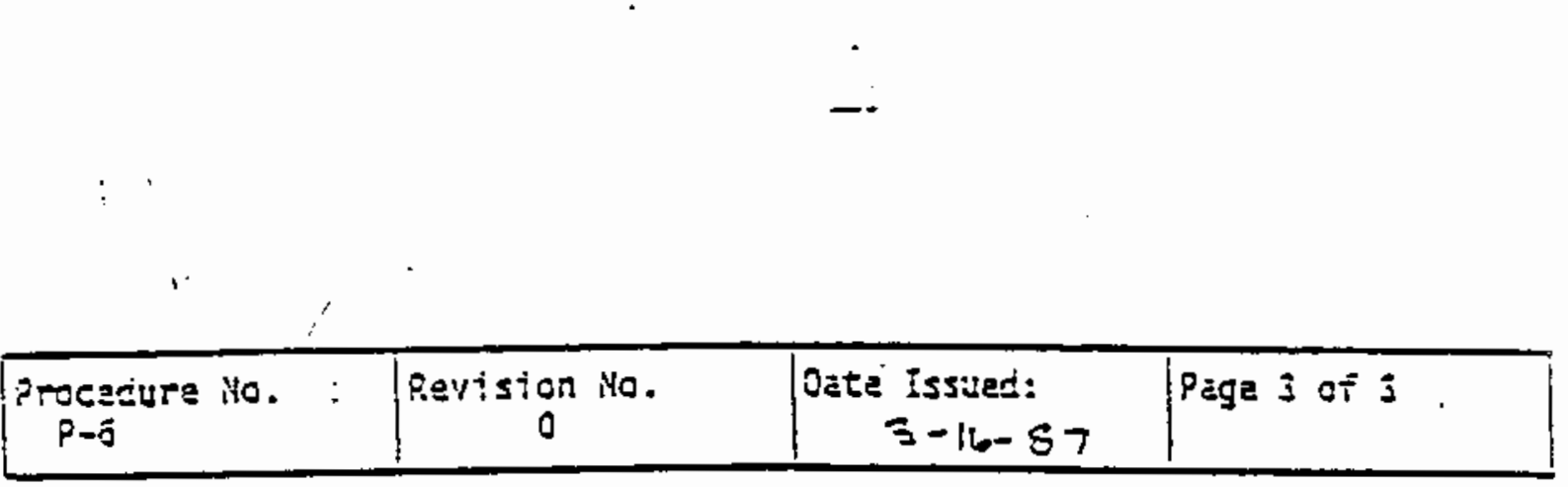


Procedure Mo. 8-5, Pey. No. 0, Attuctinent 1

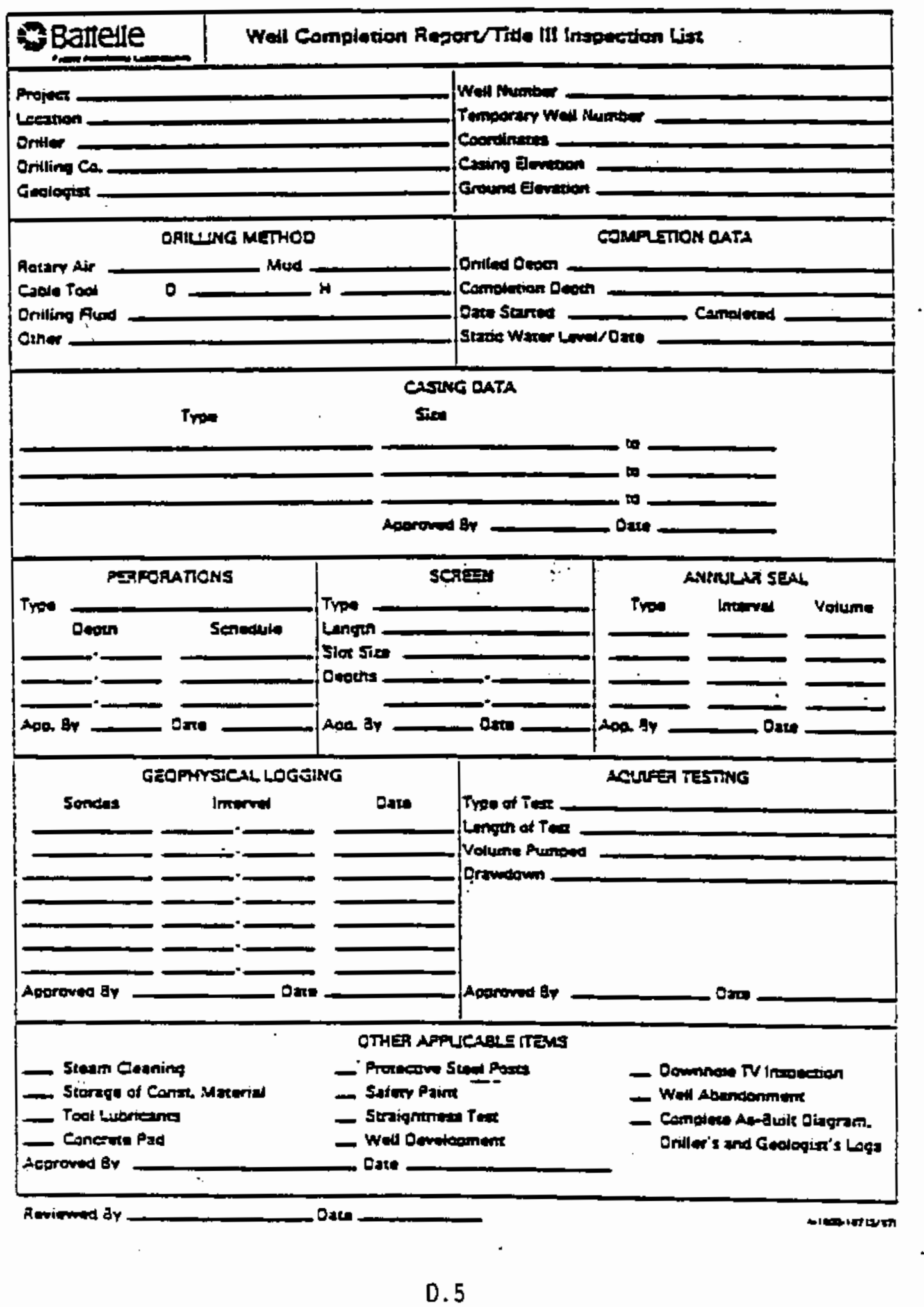


Procedure Ho, P-6, Rey. Mo. 0, Attachment 2

Page 1 of 1

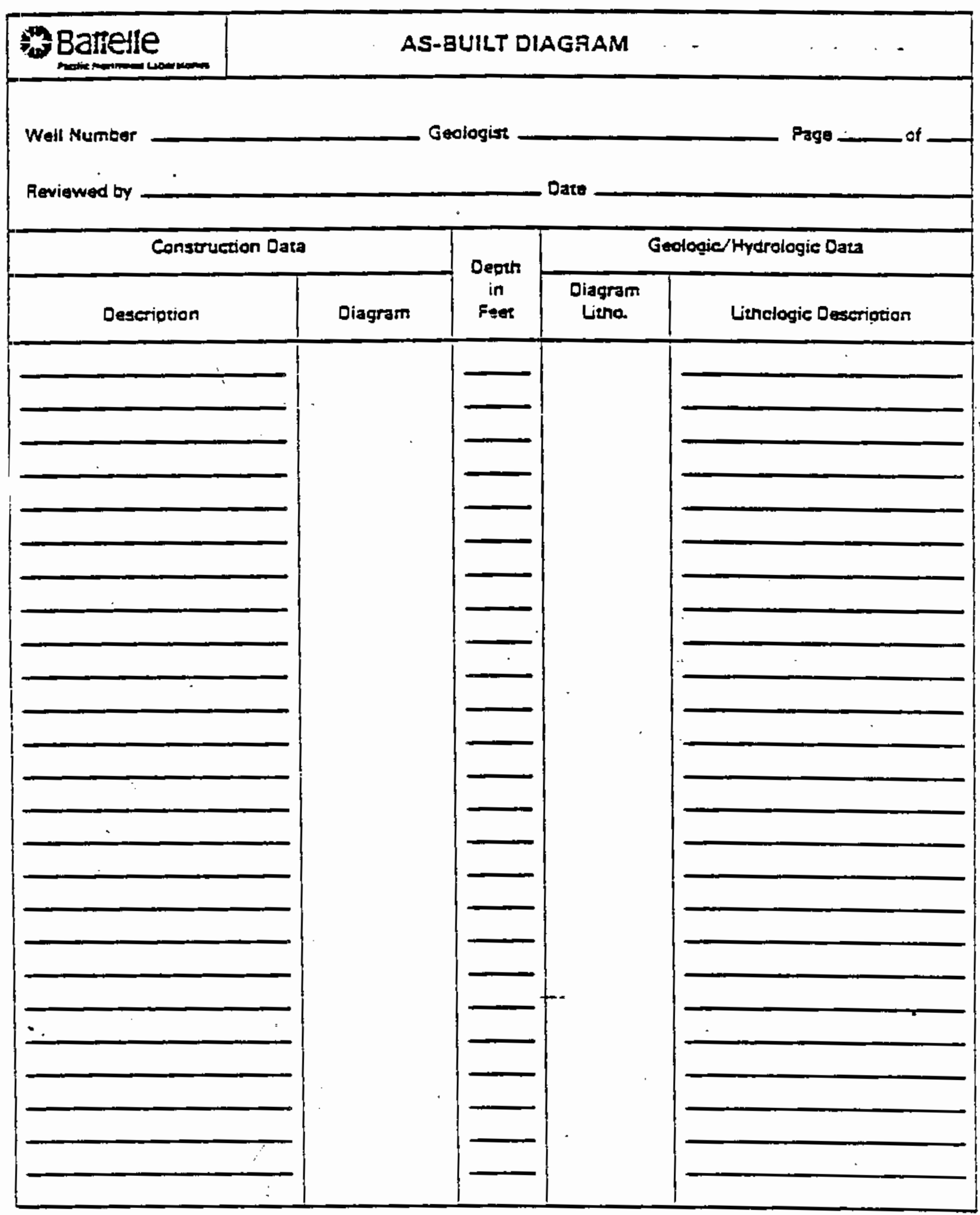




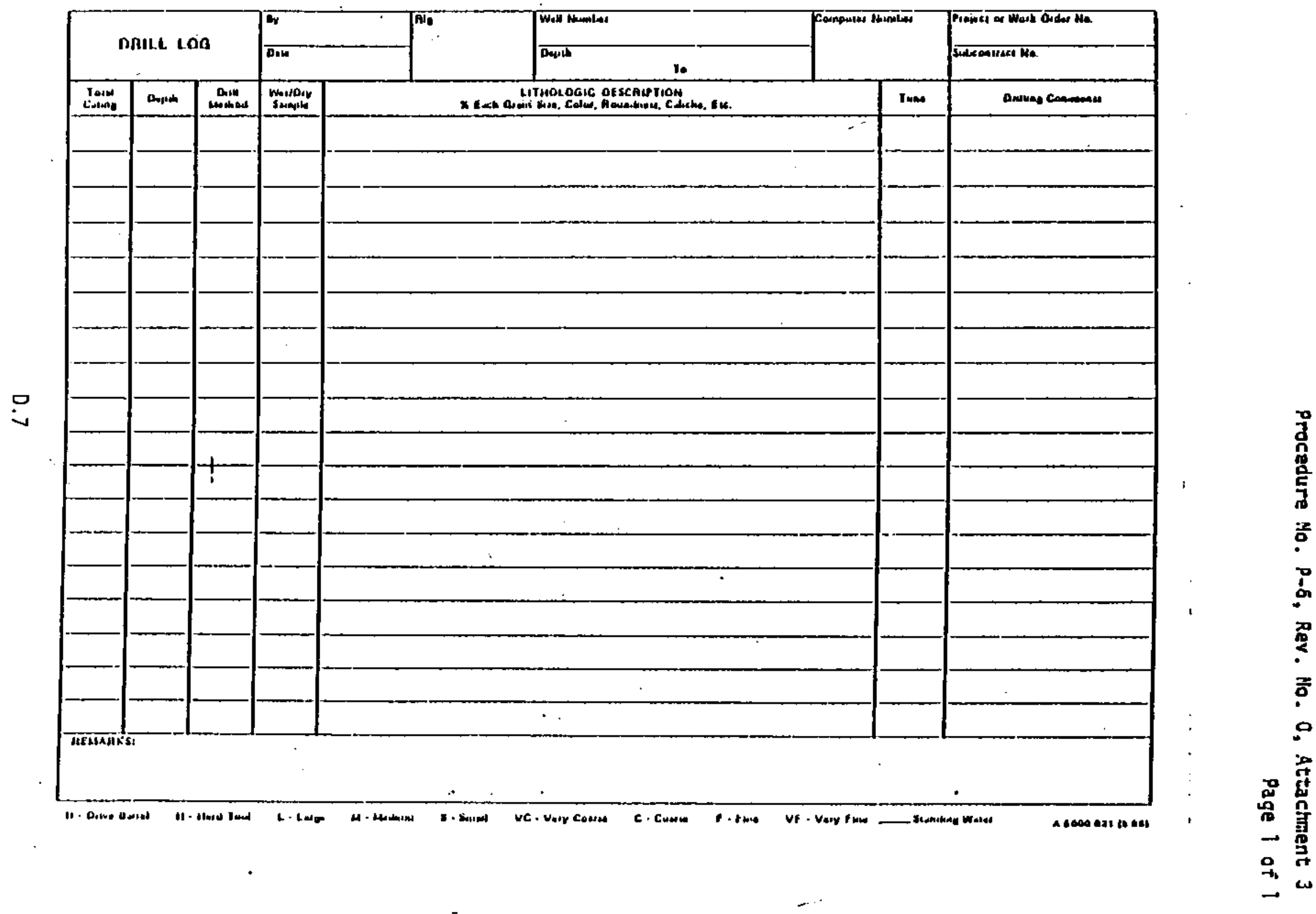



APPENDIX E

WELL COMPLETION REPORT, AS BUILT DIAGRAM, NATURALGAMMA LOGS, AND DRILL LOGS OF THE NEW WELLS 


\section{APPENDIX E}

\section{WELL COMPLETION REPORT, AS BUILT DIAGRAM, NATURAL- GAMMA LOGS, AND DRILL LOGS OF THE NEW WELLS}

This appendix contains field documentation compiled while drilling wells near Landfills 1 and 4 . The wells described include

- for Landfili 1

- LFI-PNL1

- LFI-PNL2

- LF1-PNL3

- LFL-PNL4

- for Landfil1 4

- LF4-PNL1

- LF4-PNL2

- LF4-PNL3

- LF4-PNL4

- LF4-PNL5

- LF4-PNL6

Note: no natural-gamma $\log$ is available for well LF4-PNL3 because no geophysical survey was conducted at that we11. 

WELL LF4-PNL1 



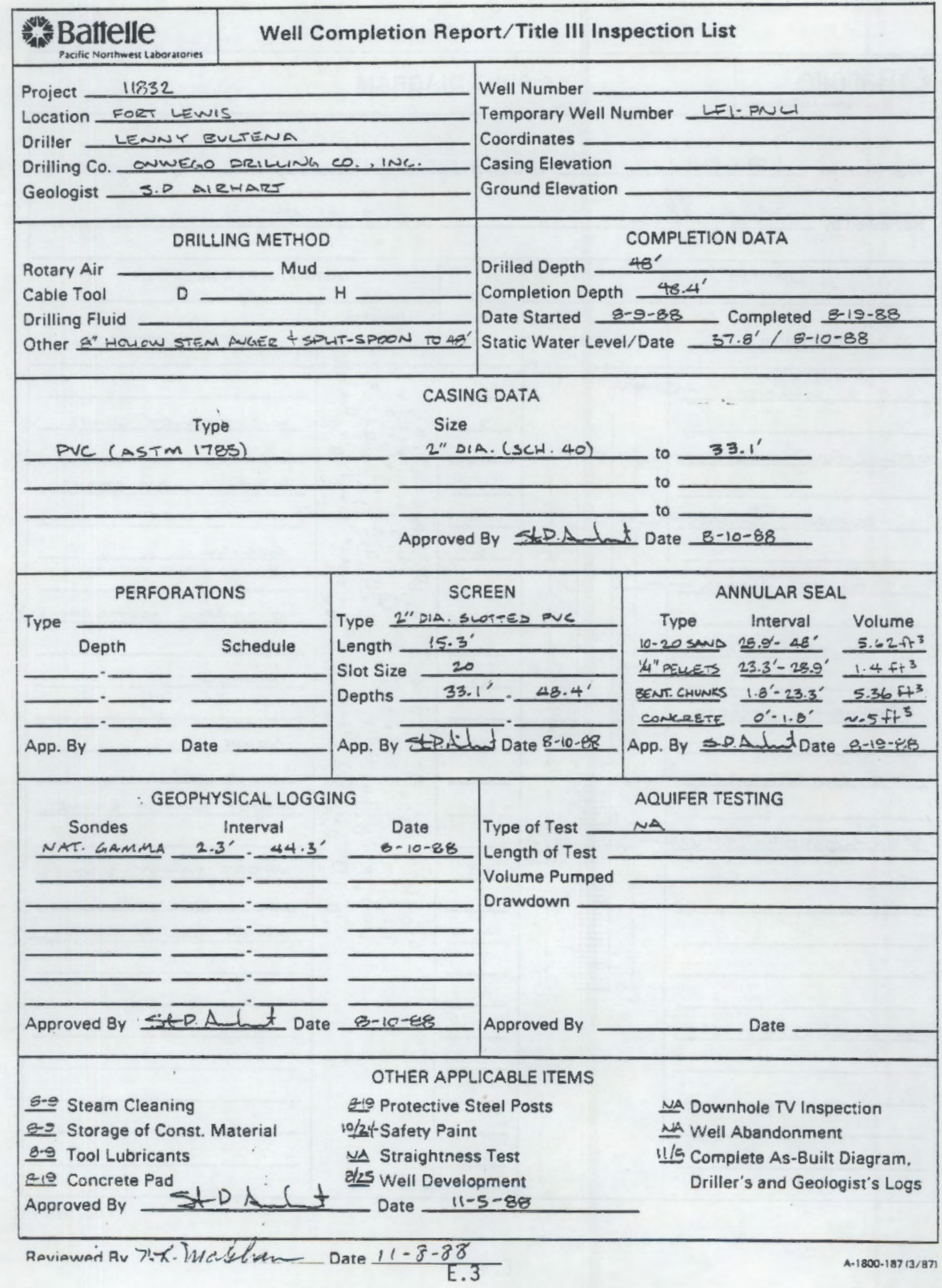




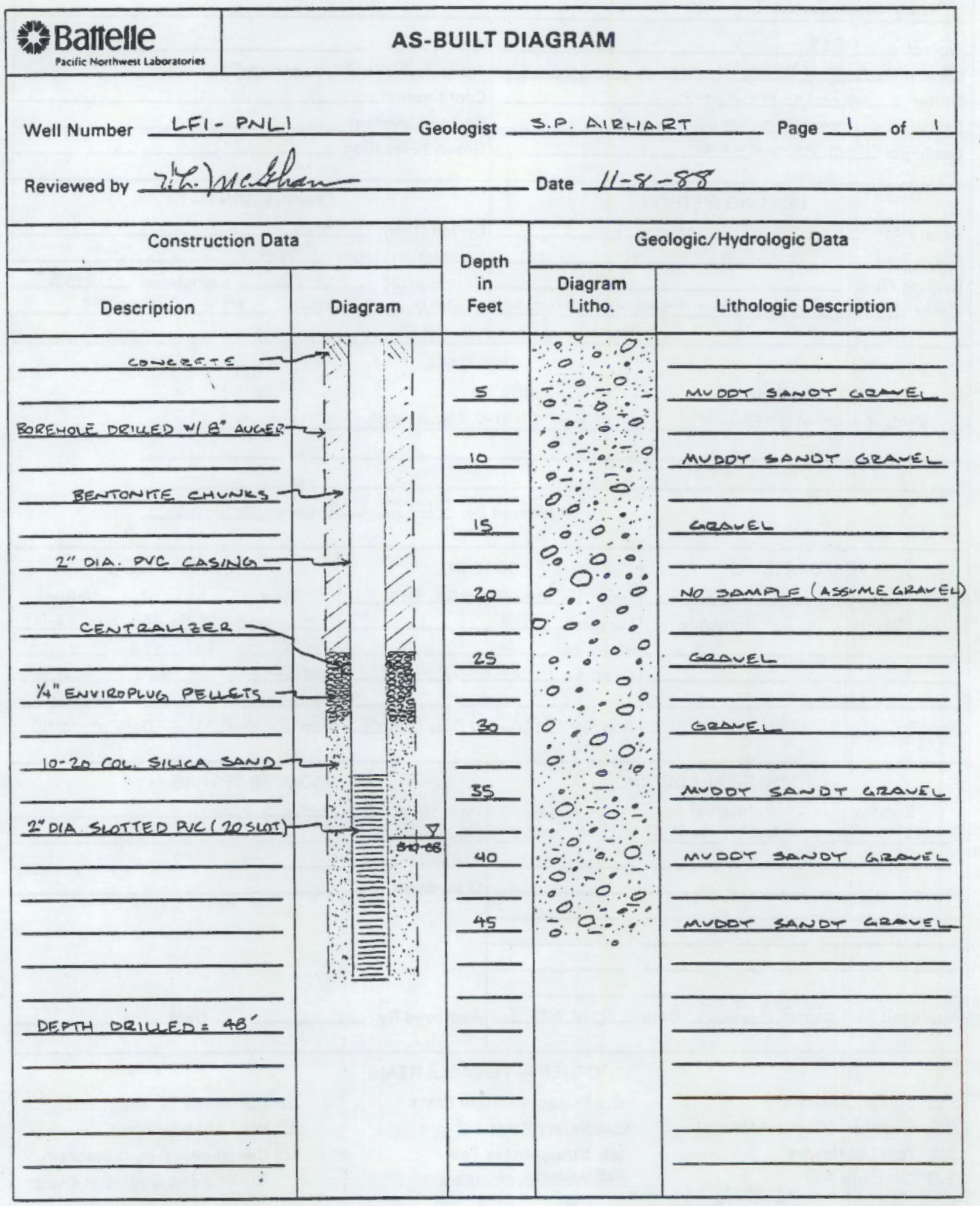

A-1800.186 (3/87)

E. 4 


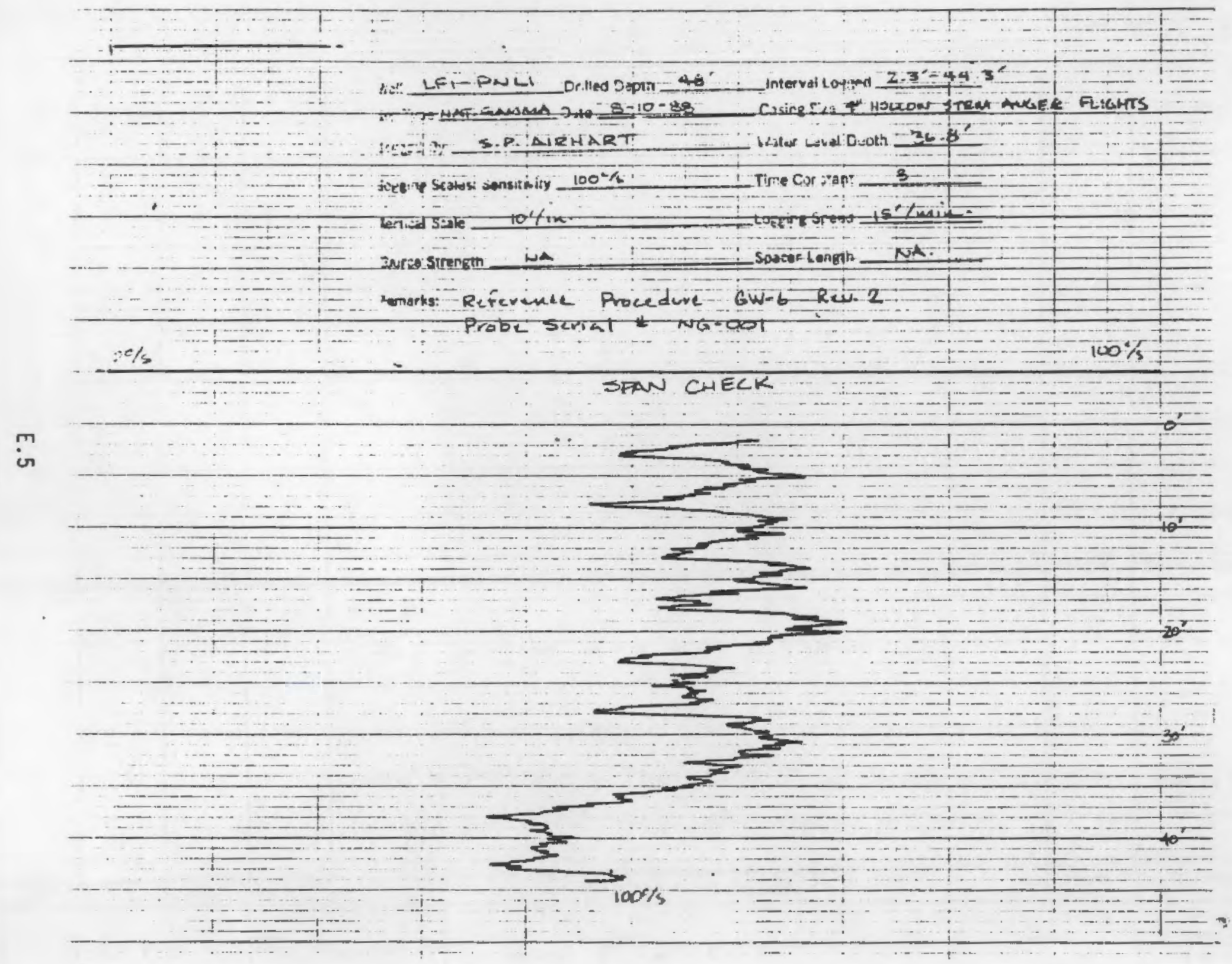


page 1 of 1

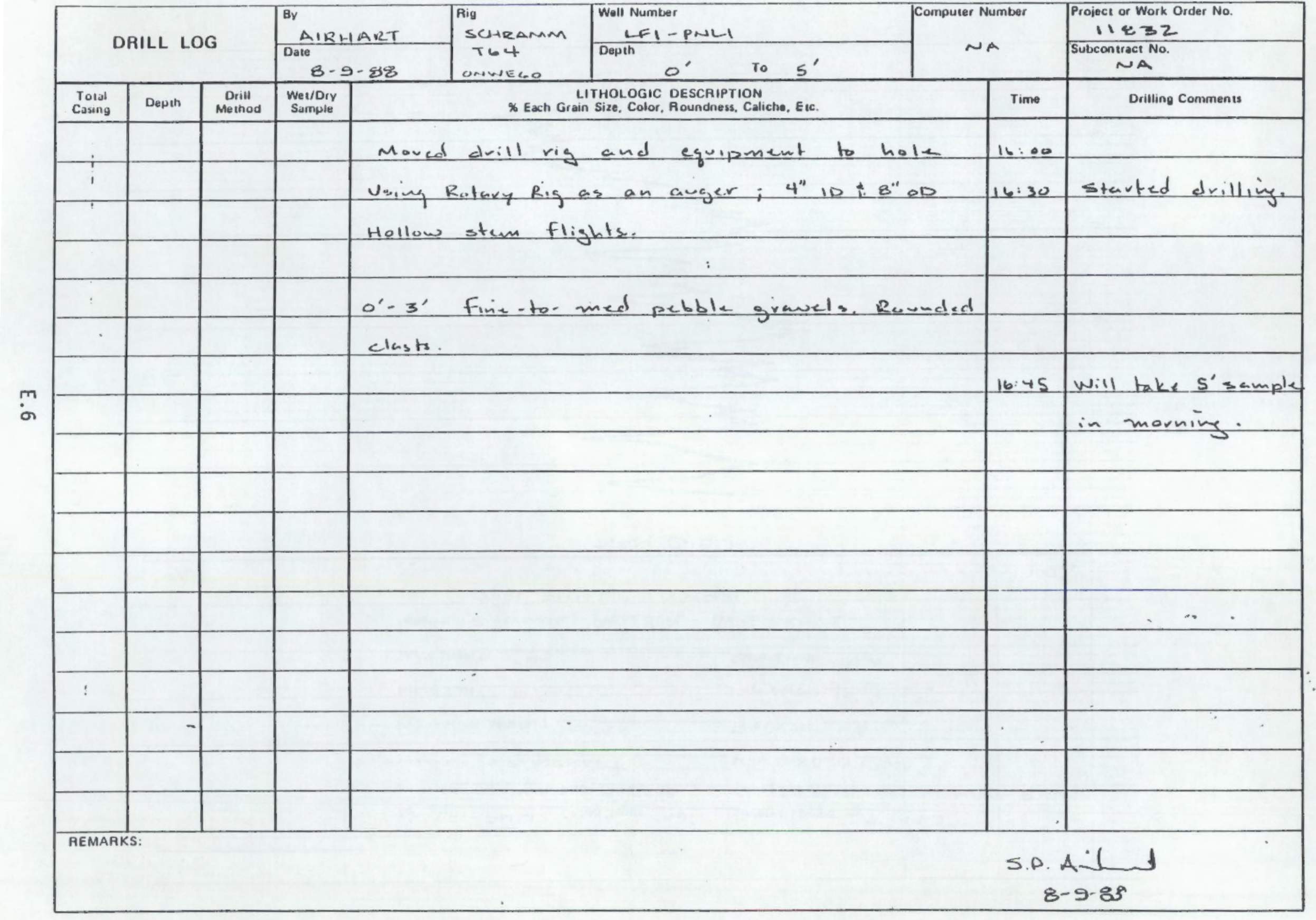

D. Drive Barret 
r......

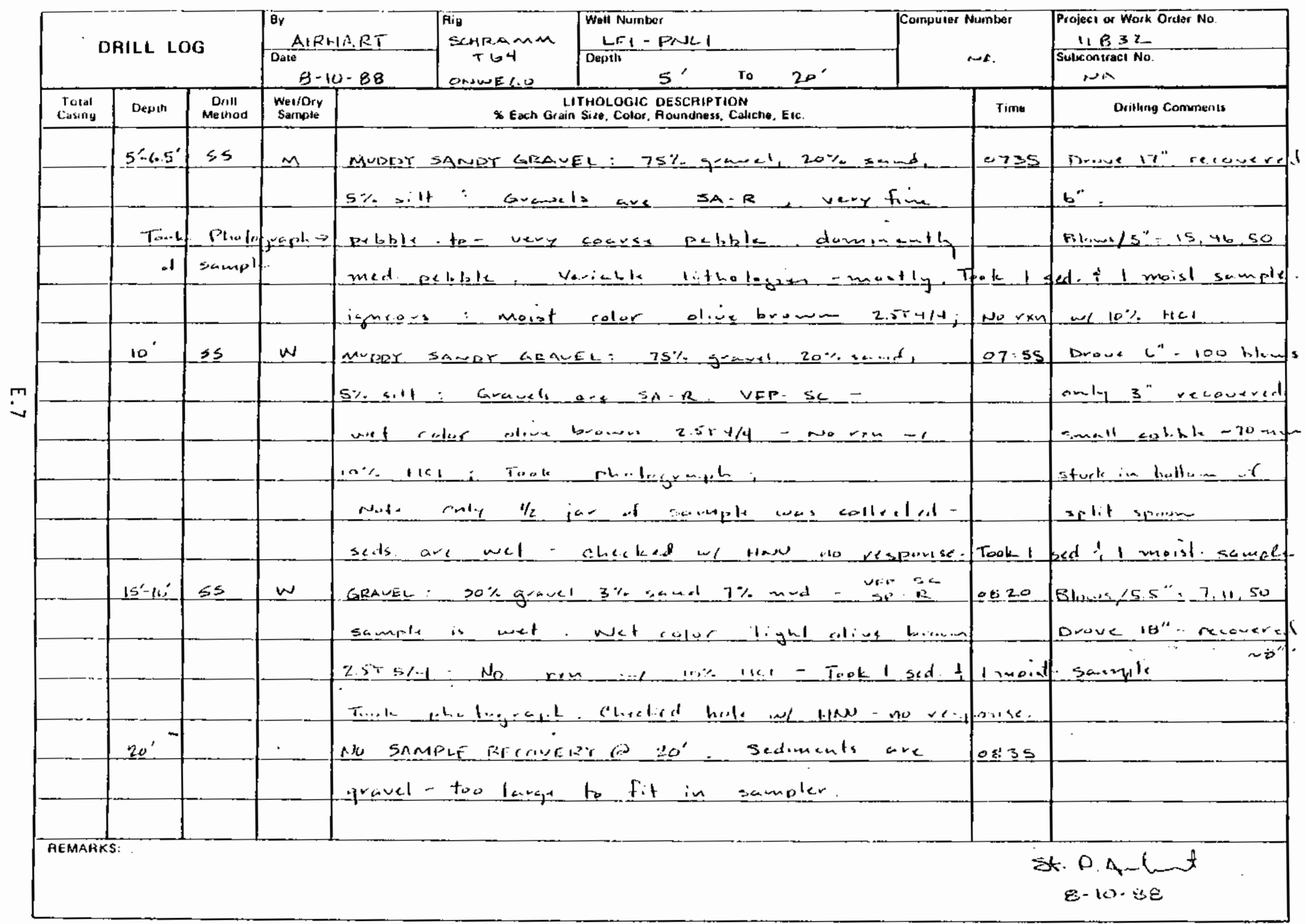

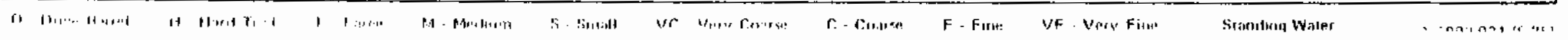




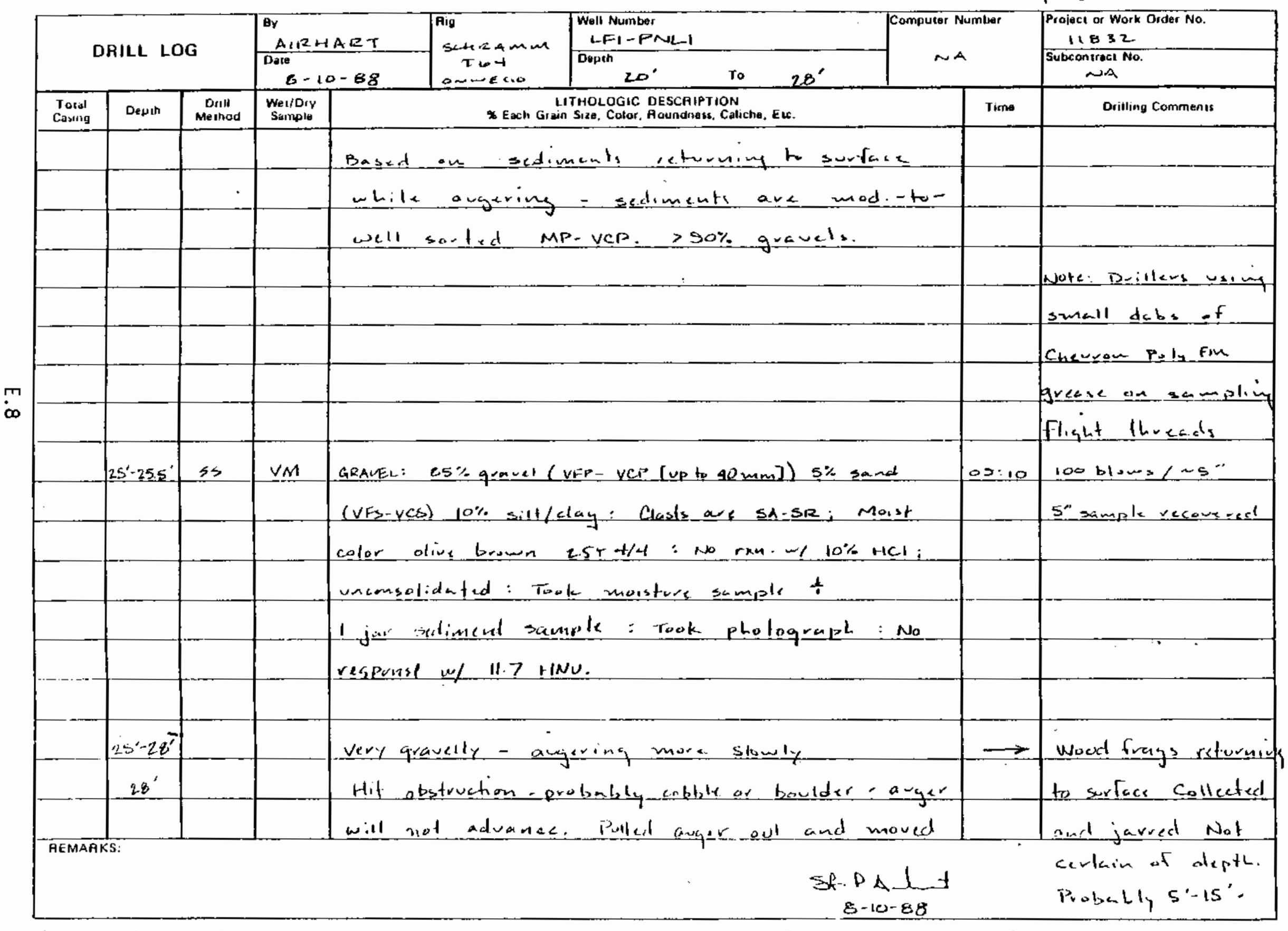


pagt 3 .f 5

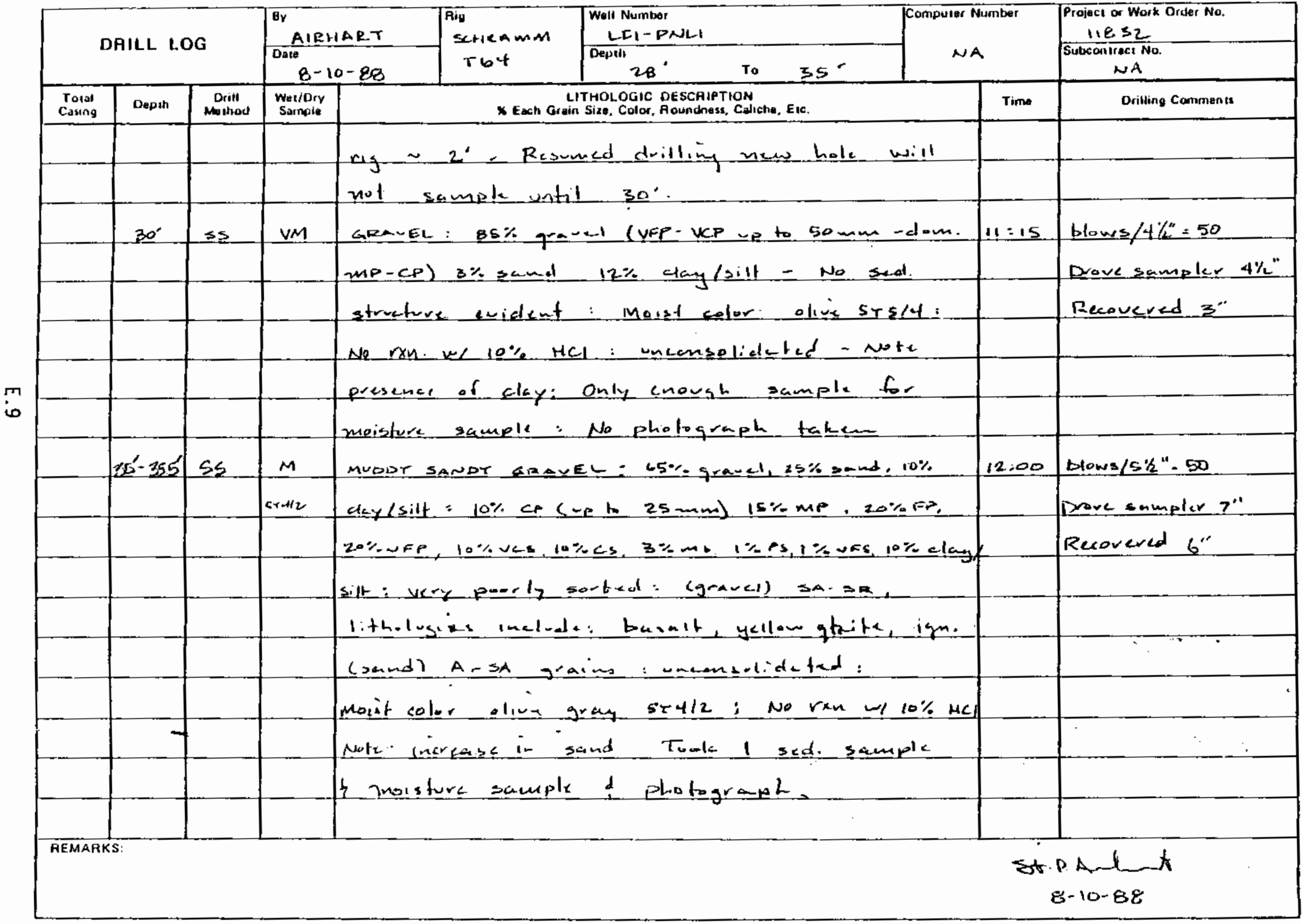

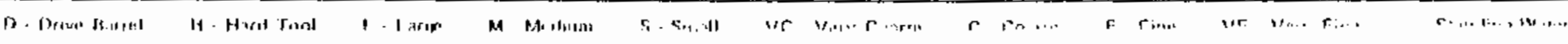


pag 4 of 5

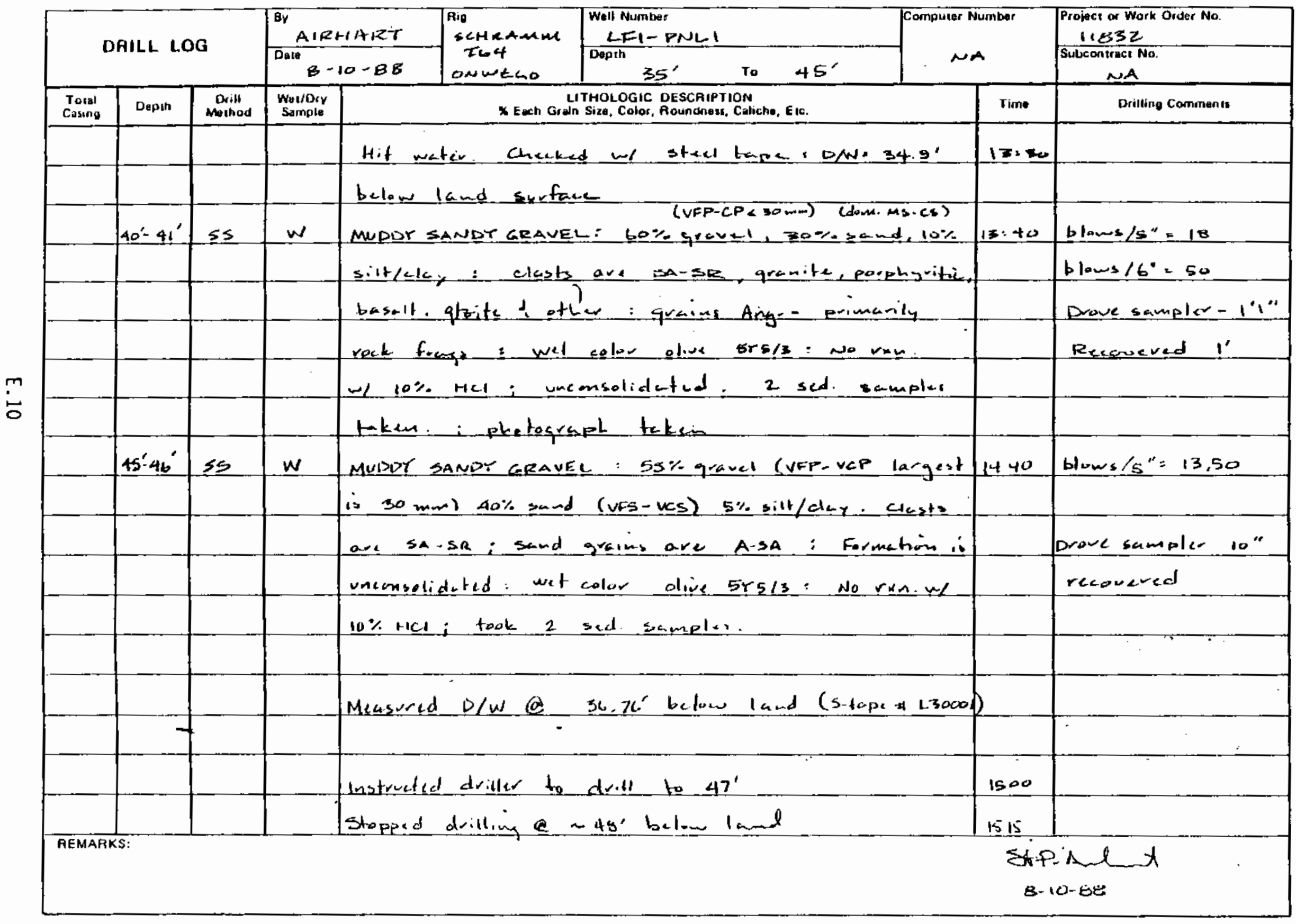

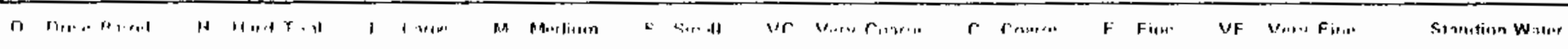




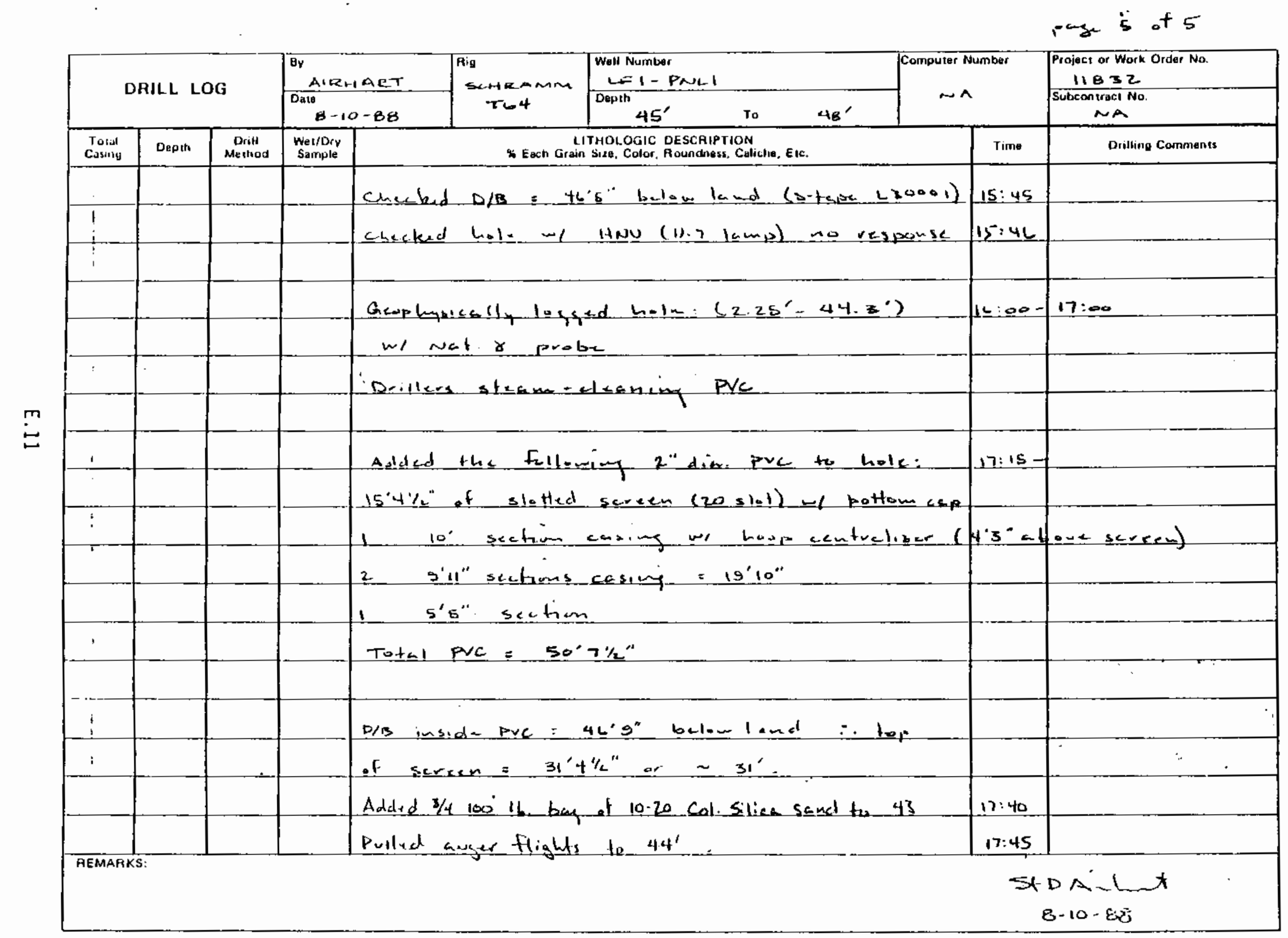

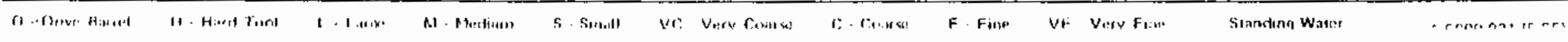


pung 1 of 1

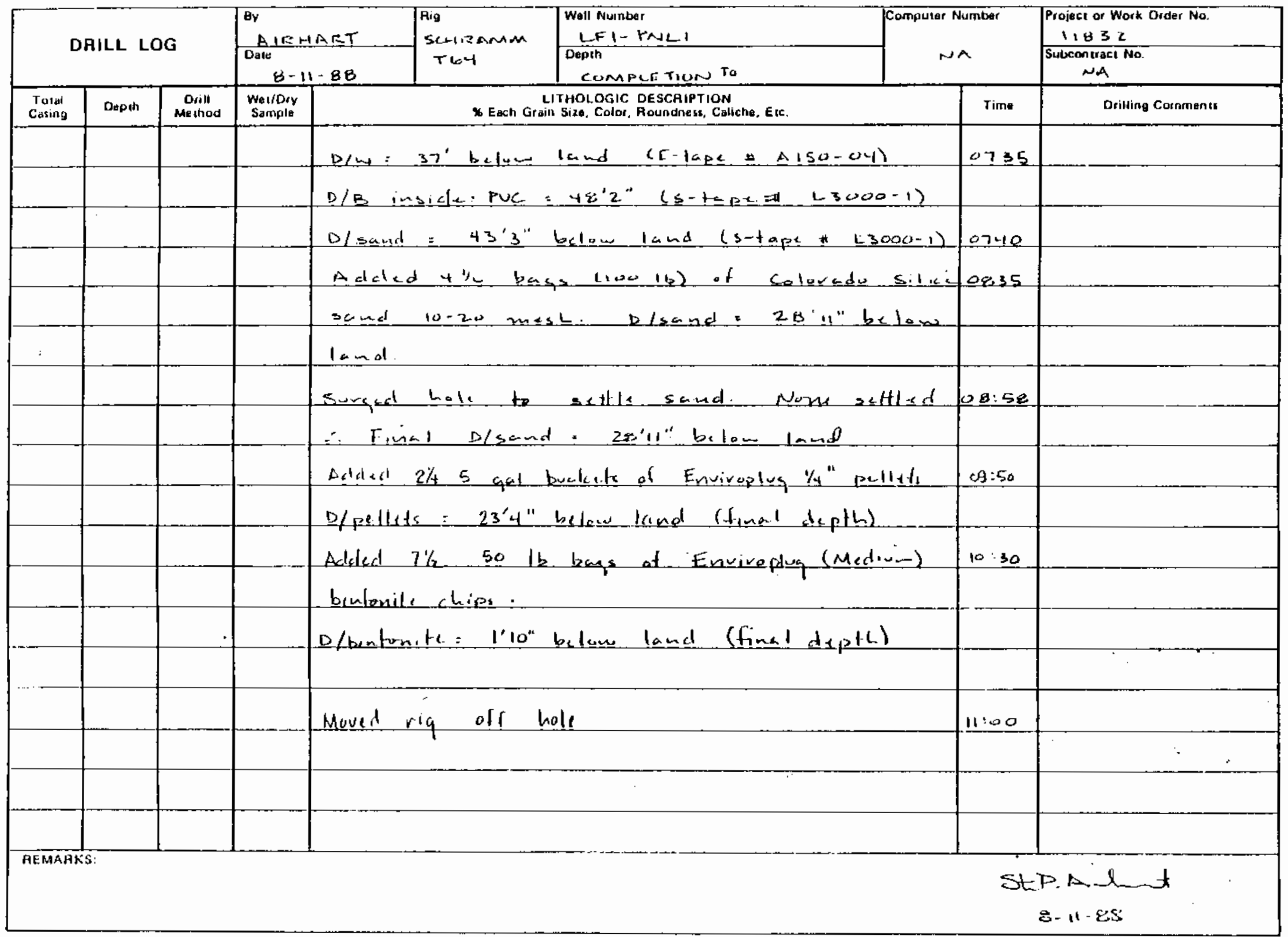




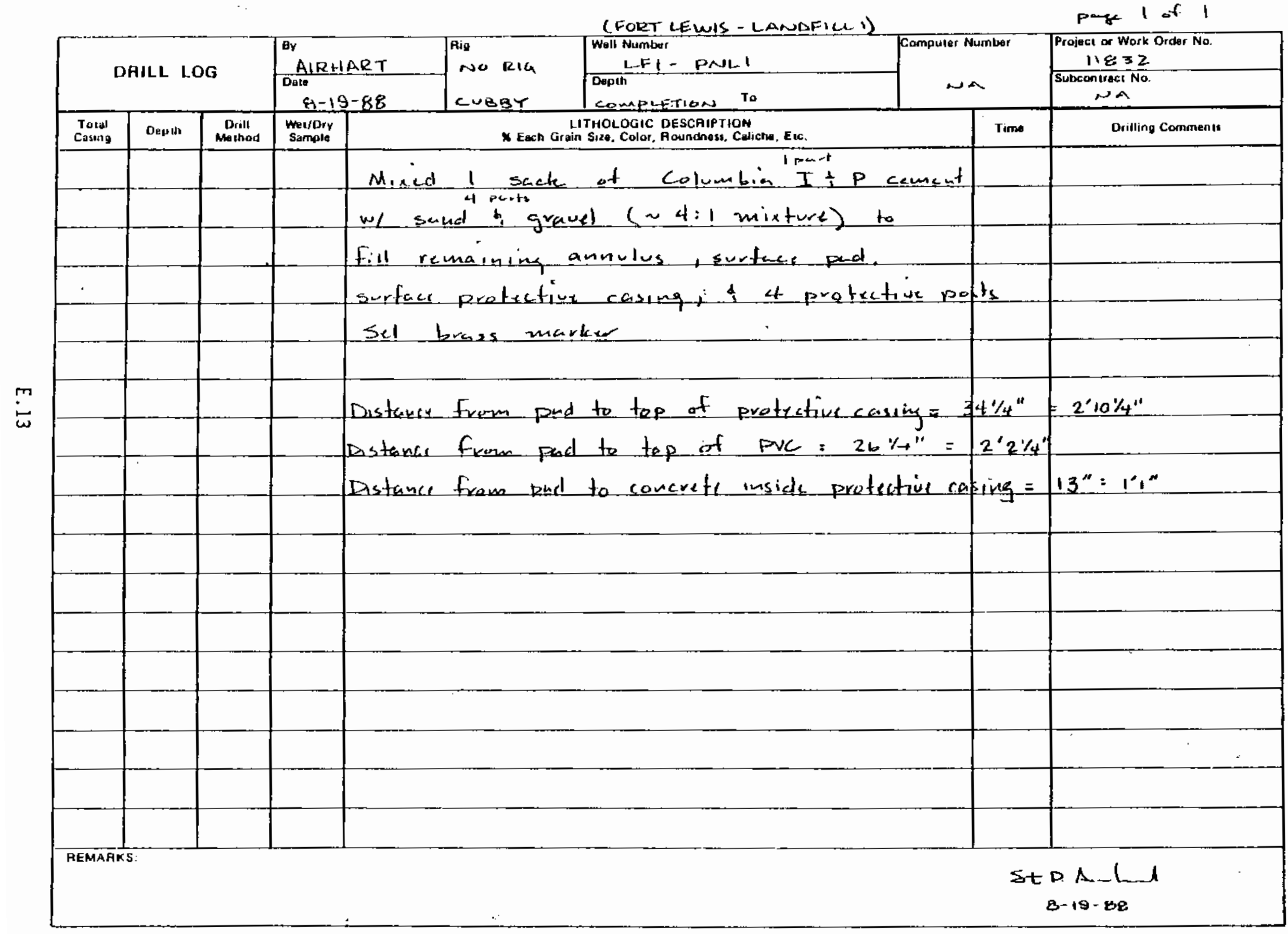




\begin{tabular}{|c|c|c|c|c|c|c|}
\hline \multicolumn{3}{|c|}{ DAILL LOG } & $\begin{array}{l}\text { AIIZHART } \\
\text { Date } \\
9-1-88\end{array}$ & \multirow{2}{*}{ 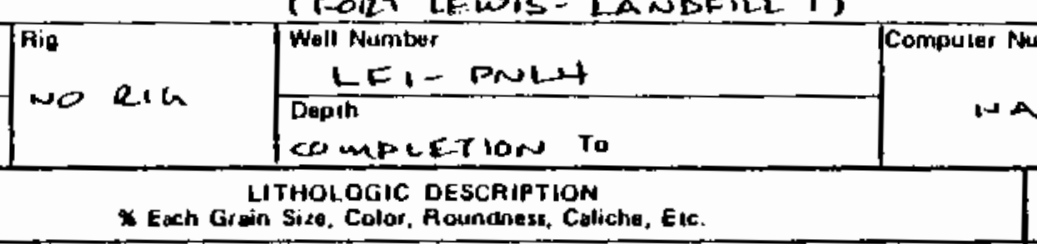 } & & 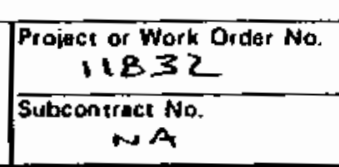 \\
\hline \multirow[t]{2}{*}{$\begin{array}{c}\text { Total } \\
\text { Casiong } \\
\end{array}$} & \multirow[t]{2}{*}{$\overline{\text { Deputh }}$} & $\begin{array}{c}\text { Drill } \\
\text { Metrod } \\
\end{array}$ & \begin{tabular}{|l} 
Wetid ory \\
somplat \\
\end{tabular} & & Time & Drilling Cormmenis \\
\hline & & & & Mixing coment - pourimg eurfacs pad & 0900. & +10.45 \\
\hline & & & & 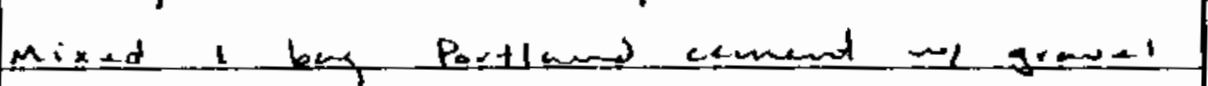 & & \\
\hline & & & & t sand and poxvel surface pud and & & \\
\hline & & & & brise averous protective prests. & & \\
\hline & & & & & & \\
\hline & & & & set brass cap & $10: 45$ & \\
\hline & & & & & & sis \\
\hline & & & & Top of ped to tzop of $b^{\circ}$ protective casing $=249$ & & $\begin{array}{rr}11 . & 2 \\
2 & 40 \\
\end{array}$ \\
\hline & & & & Top of pad to inside protectivi casing $=0.4 c^{\prime}$ & belou & ped 182 \\
\hline & & & & Top of pad to top of Puc $=1.82^{\circ}$ & & \\
\hline & & & & & & \\
\hline & & & & & & \\
\hline & & & & & & \\
\hline & & & & & & \\
\hline & & & & & & \\
\hline & & & & & & \\
\hline & & & & & & \\
\hline & & & & & & \\
\hline AE $\overline{M A A}$ & & & & & $\begin{array}{c}S 6 p \\
9-1\end{array}$ & Ald \\
\hline
\end{tabular}




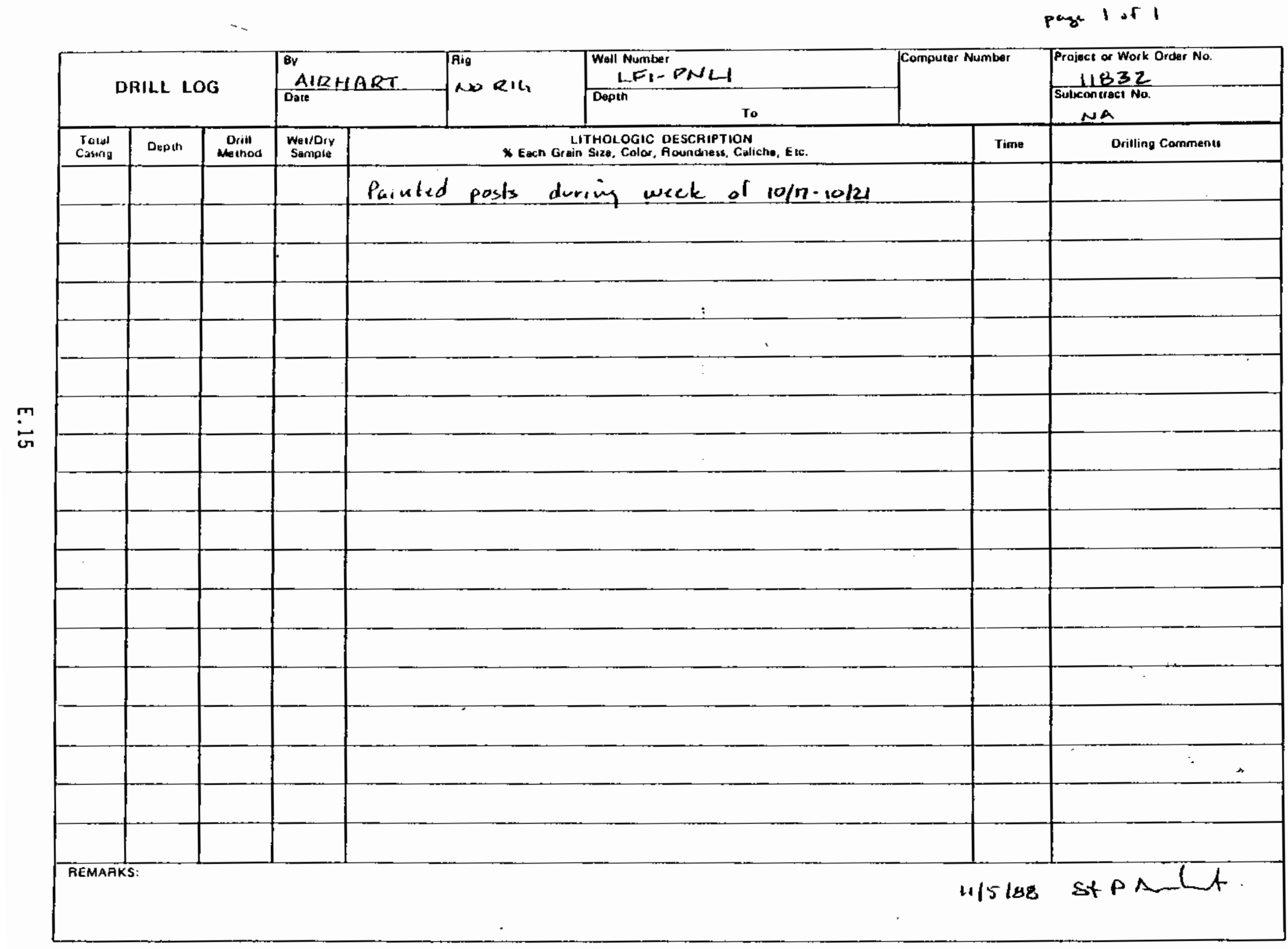



WELL LF4-PNL2 


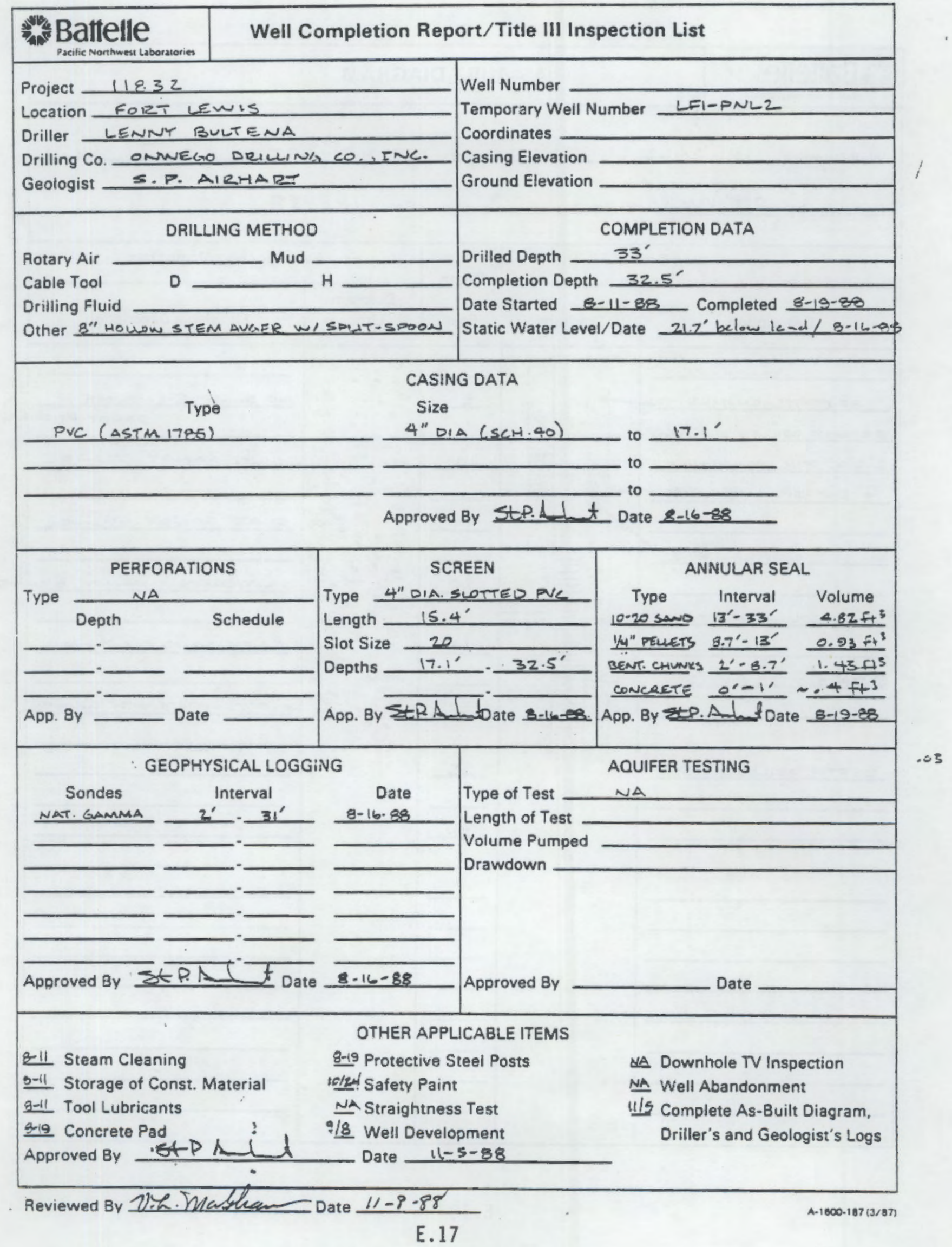




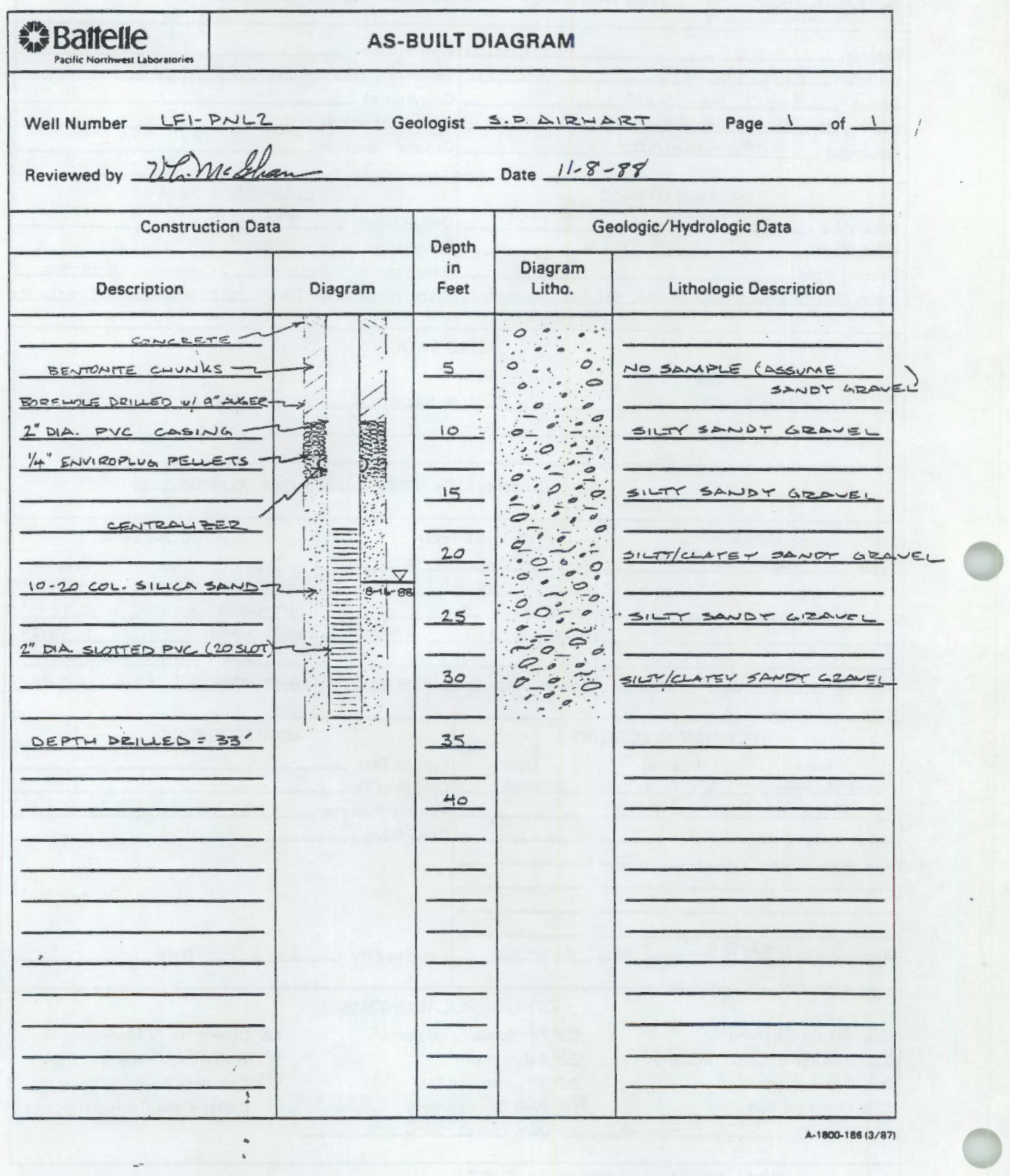




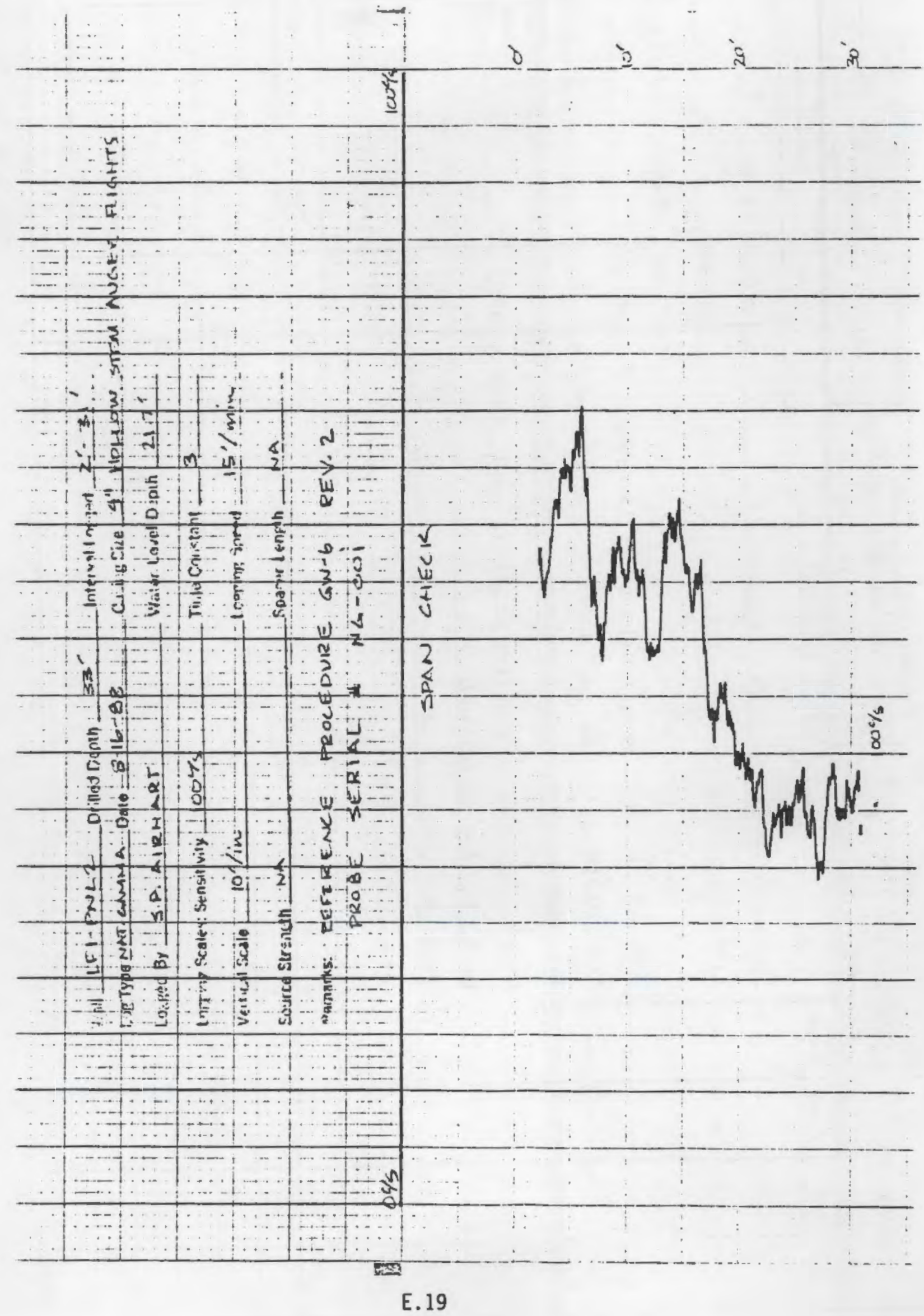


pare 1 of 1

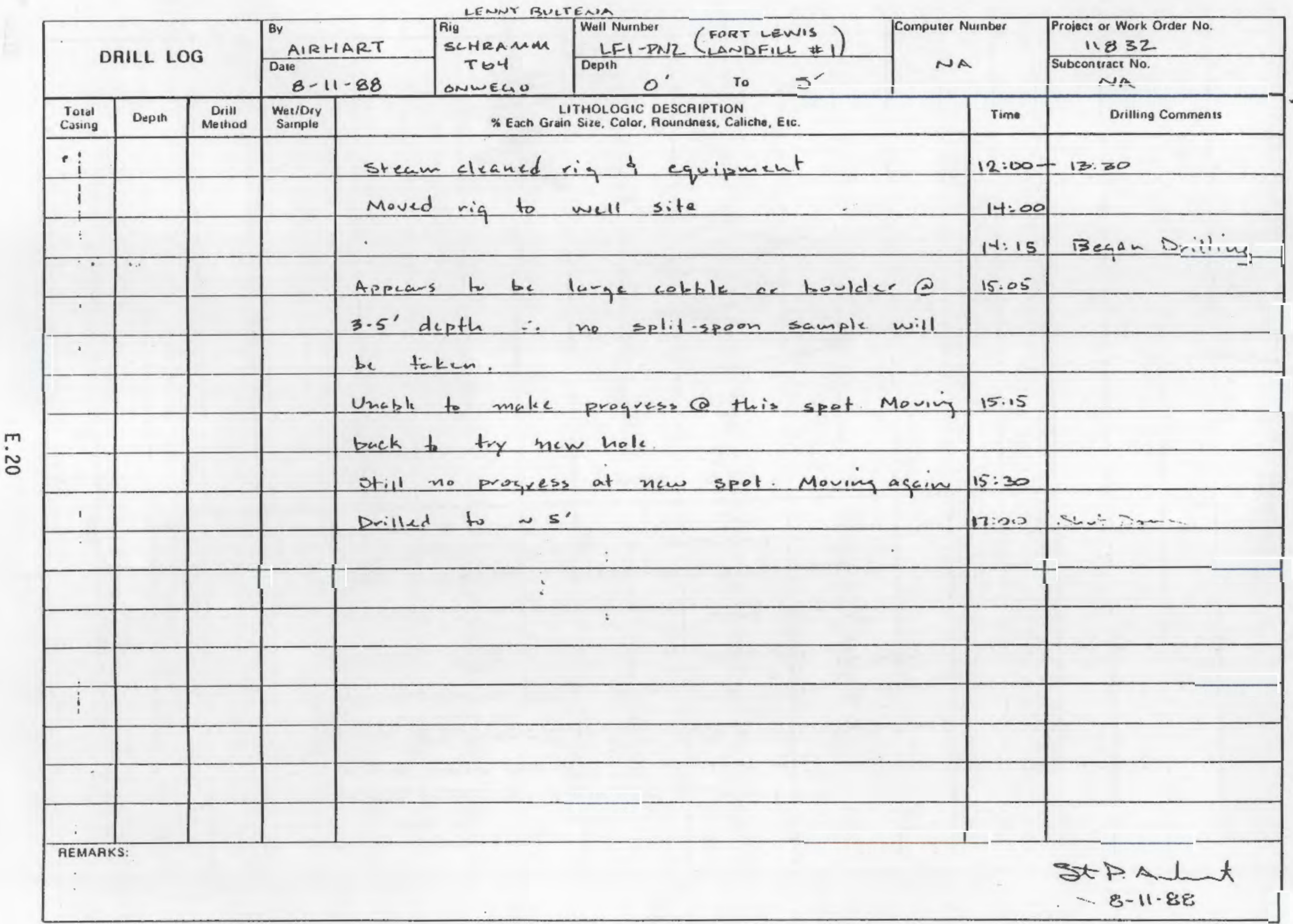
D. Drive Barrel
H. Hard Tool
L. Large
M - Mectium
s - Small
Ve. Voru rasrom 


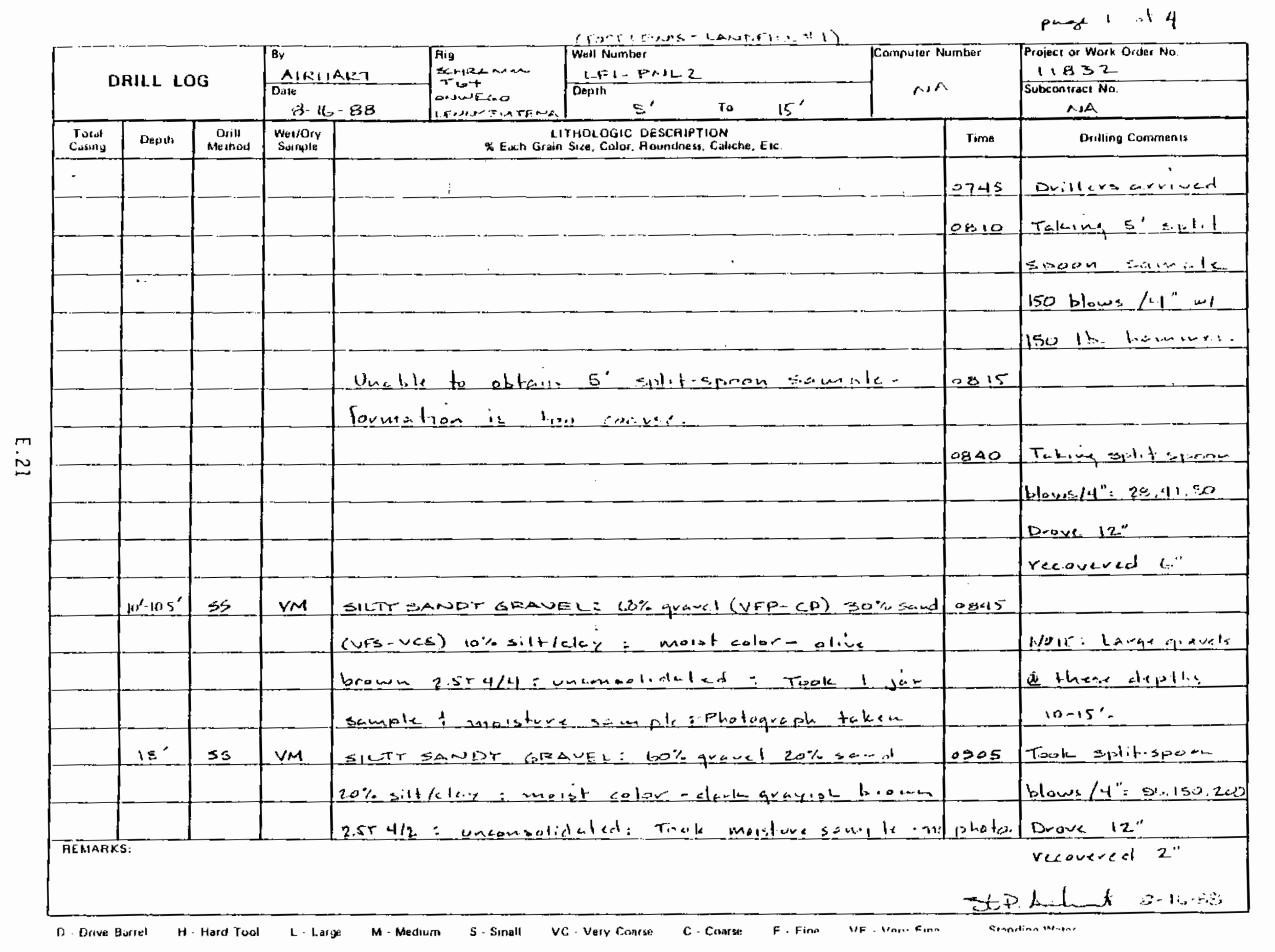




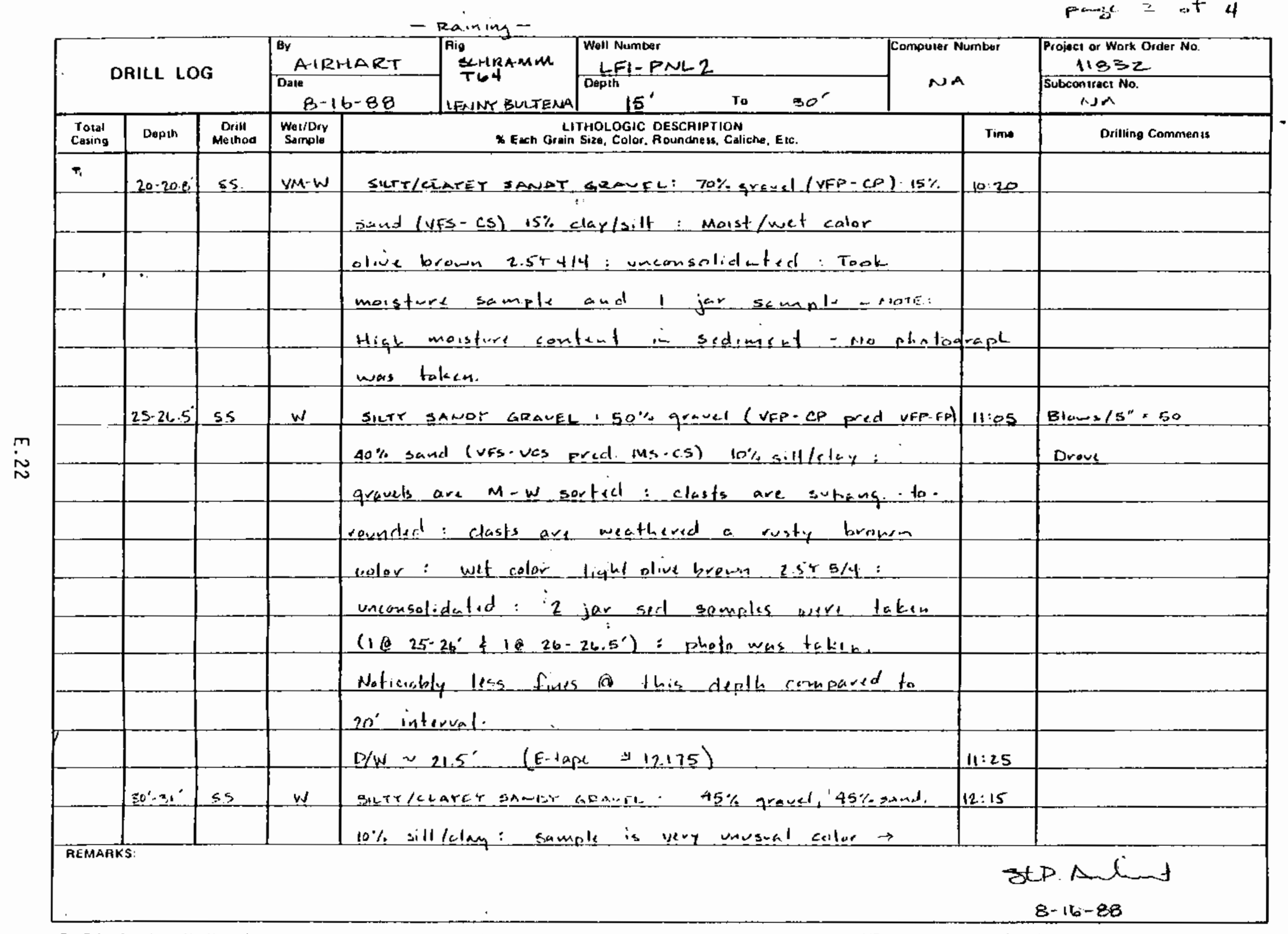

D - Drive Burfel

H. Hóral Tool

L. Lardoe M. Medium

vc very Comse

c. Coarse

we nem rim 
page $\equiv$ - 4

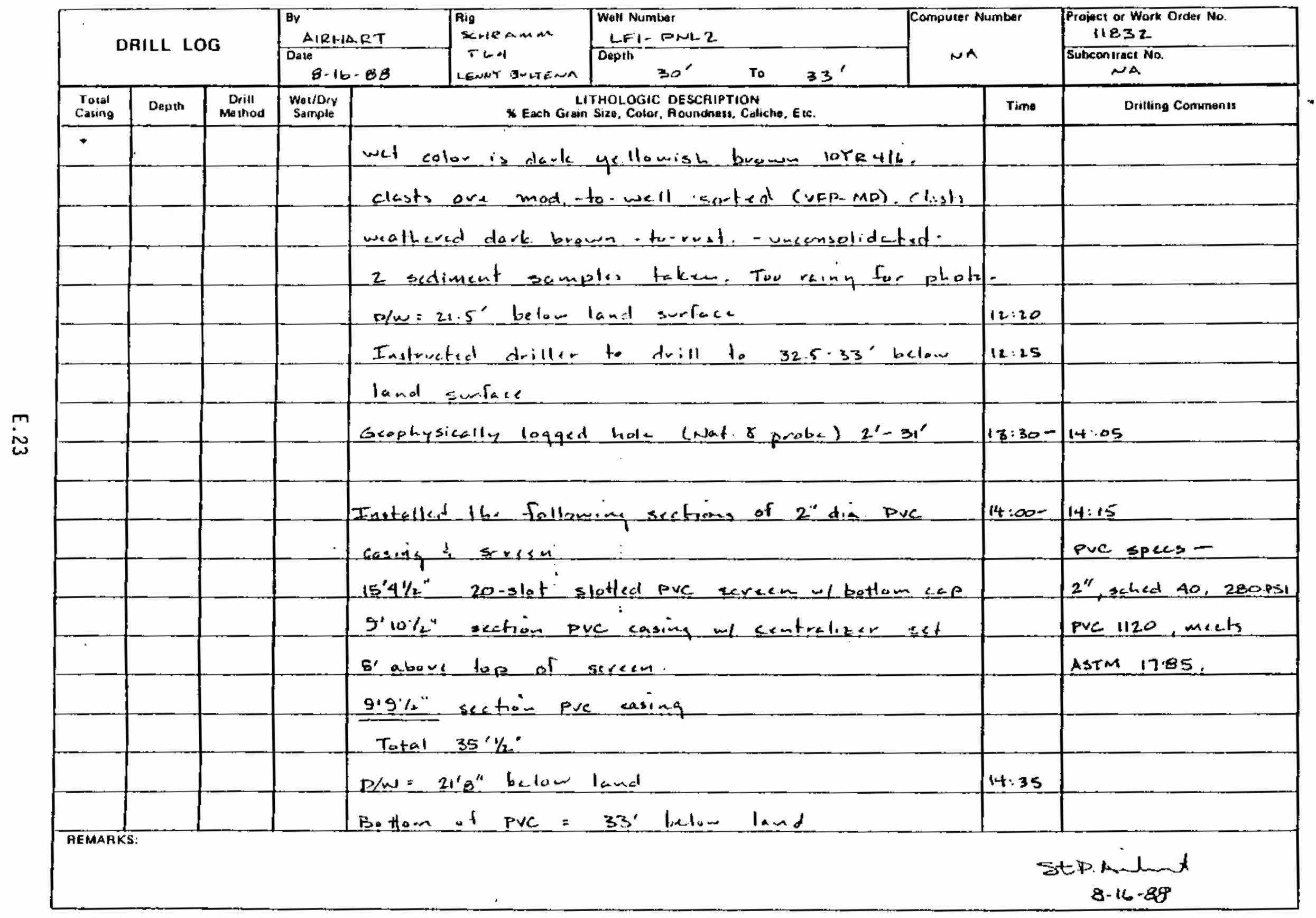

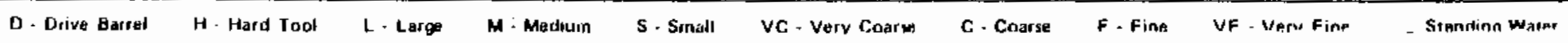


rangt 4 of th

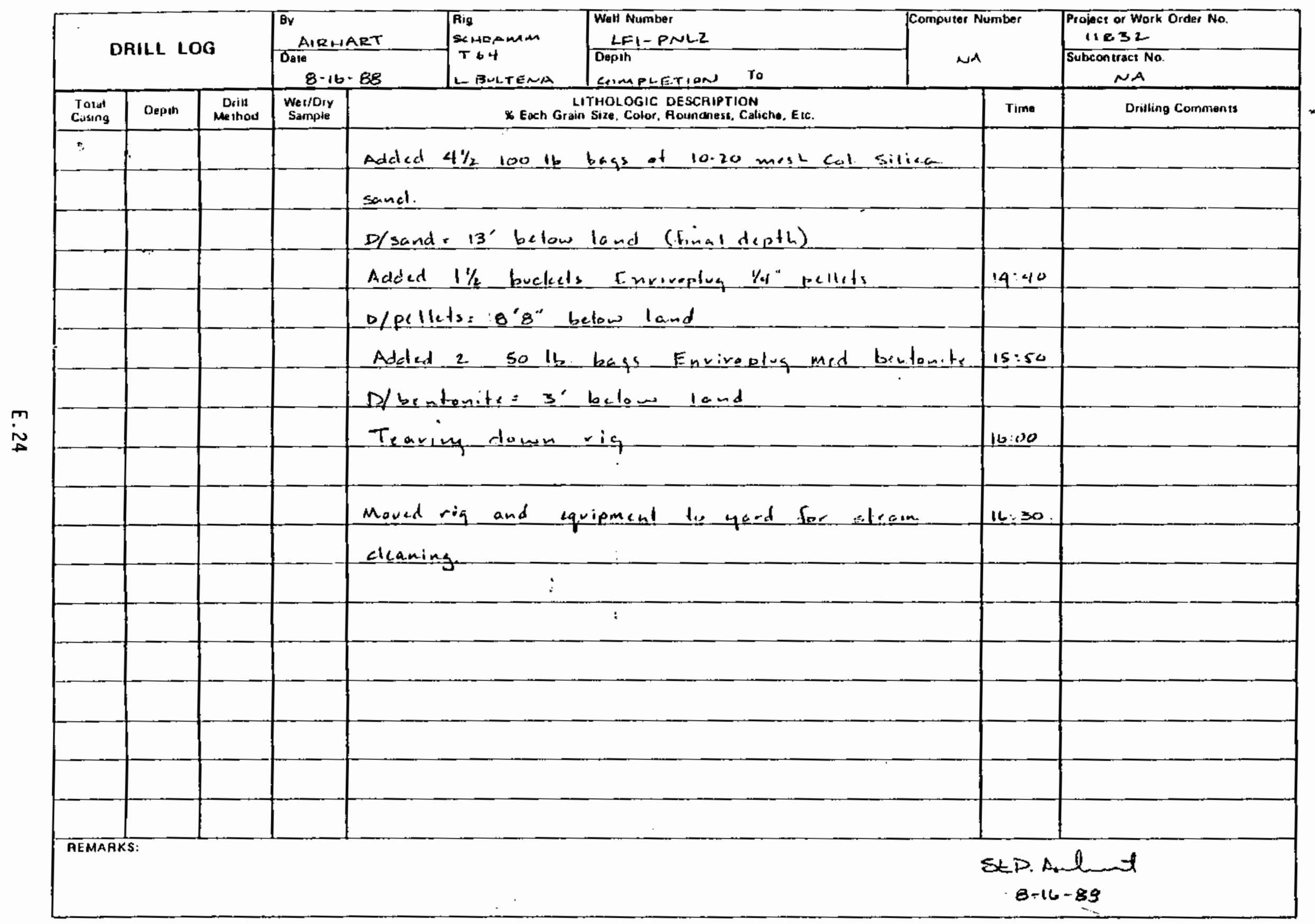

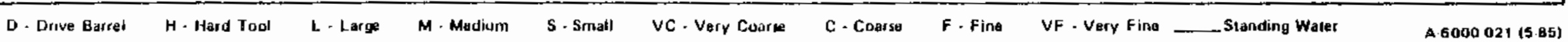




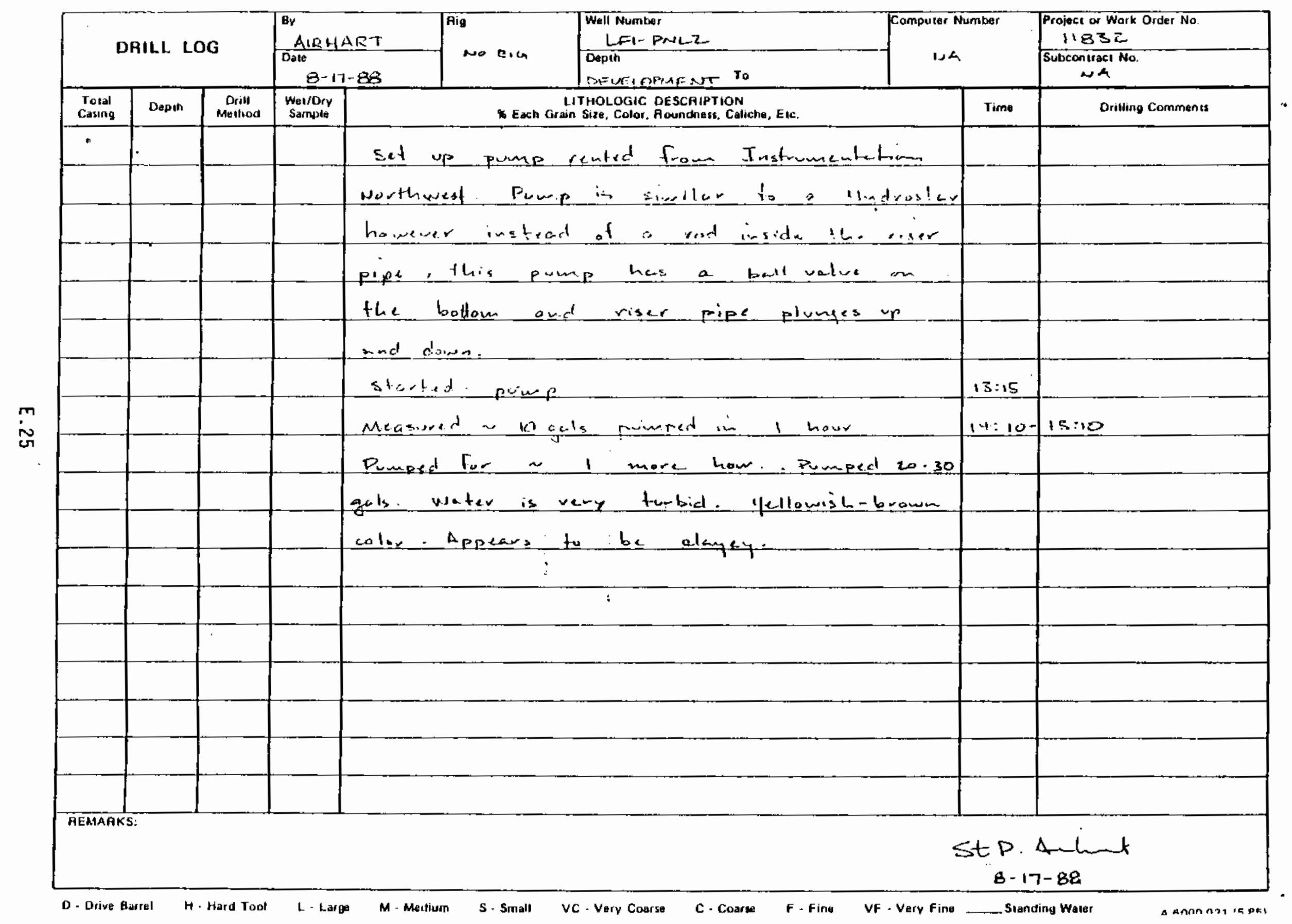


paye 1 of 1

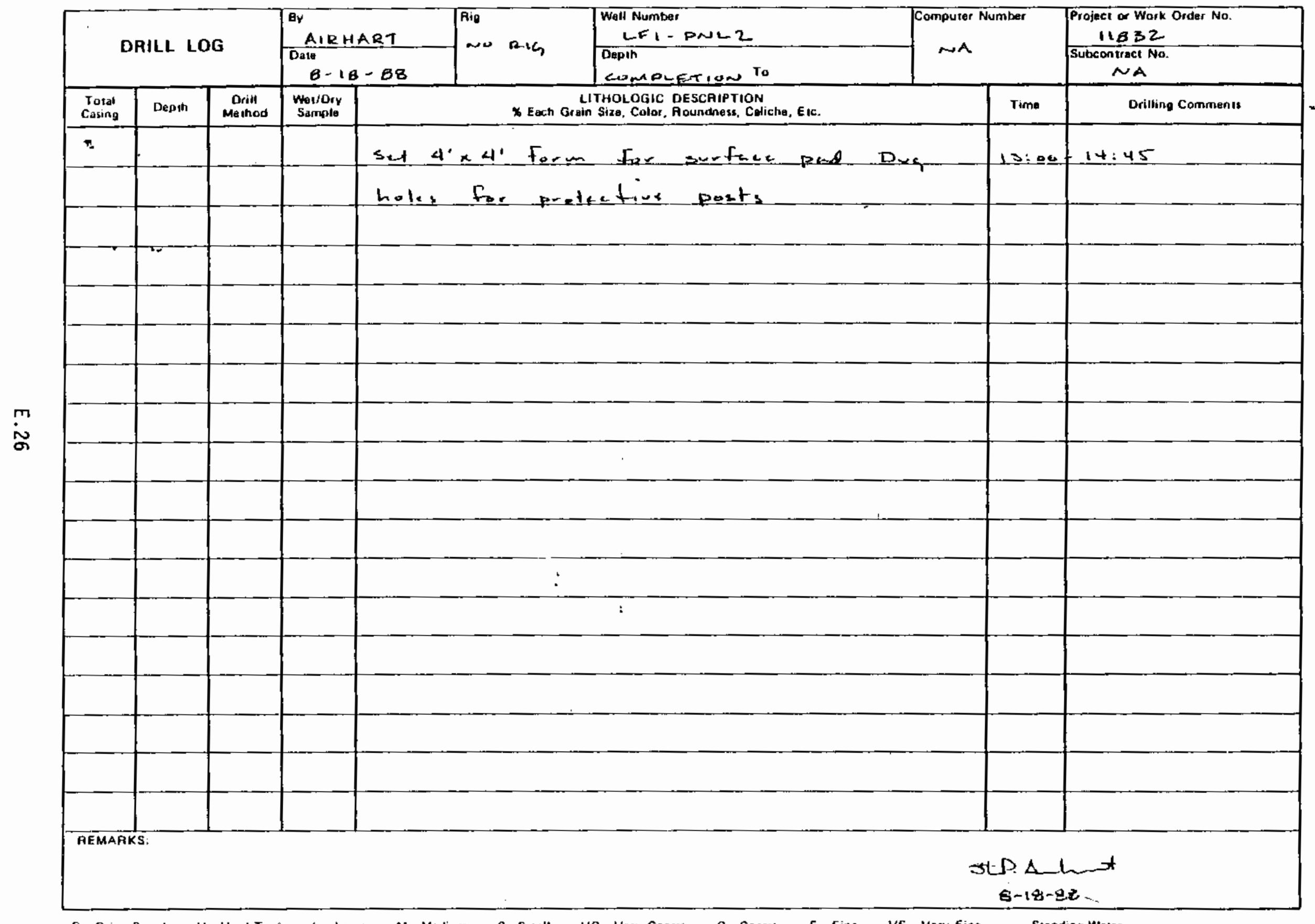
D. Deive Aasrel
H. Hard Tnnt
M. Mestirtm
S - Smalt
Ur, - Uare. renaren
c. rnarse
F. Fims
uf Vone. fins
Stanctinos tatarer 


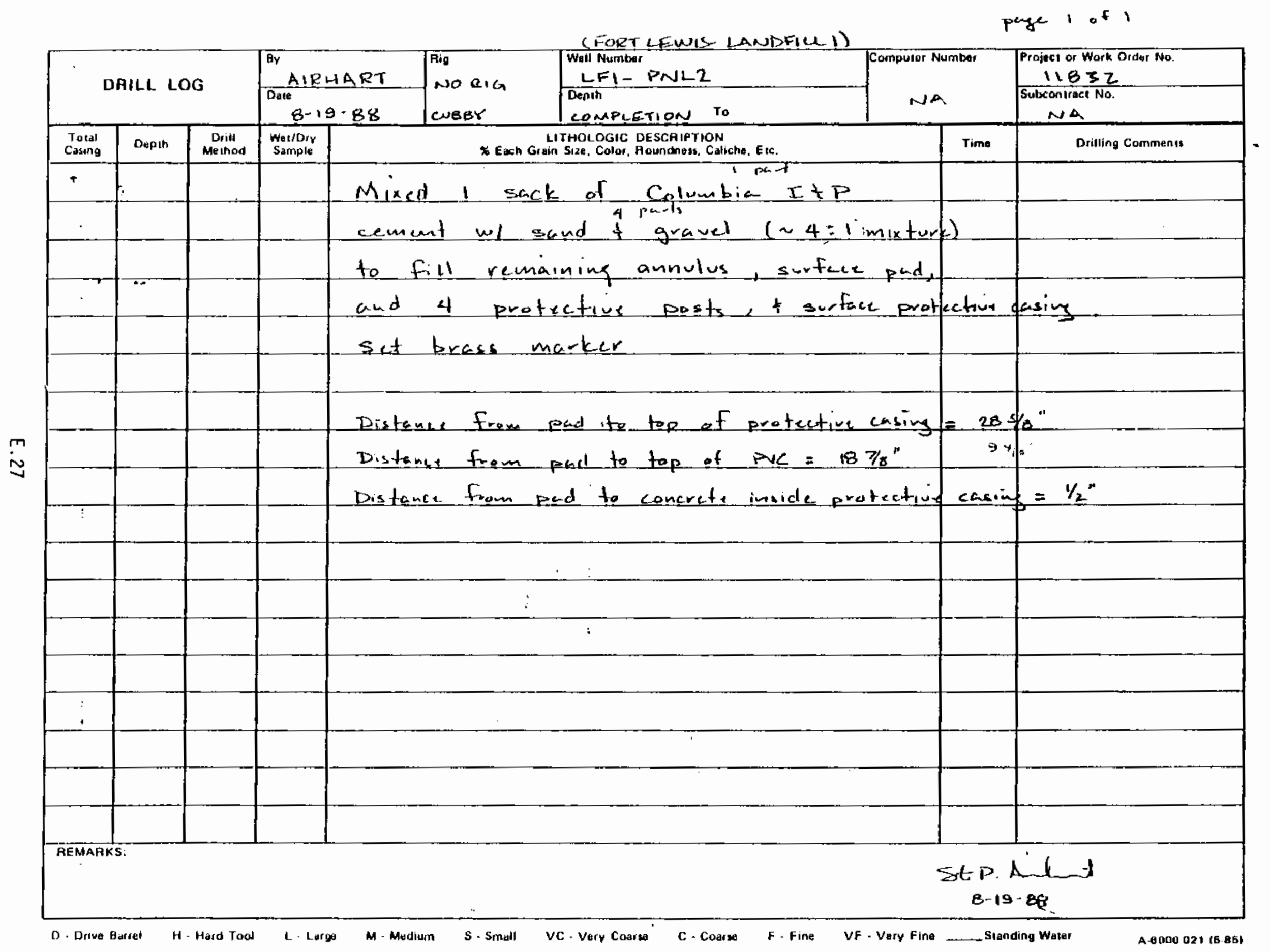


pange : or 1

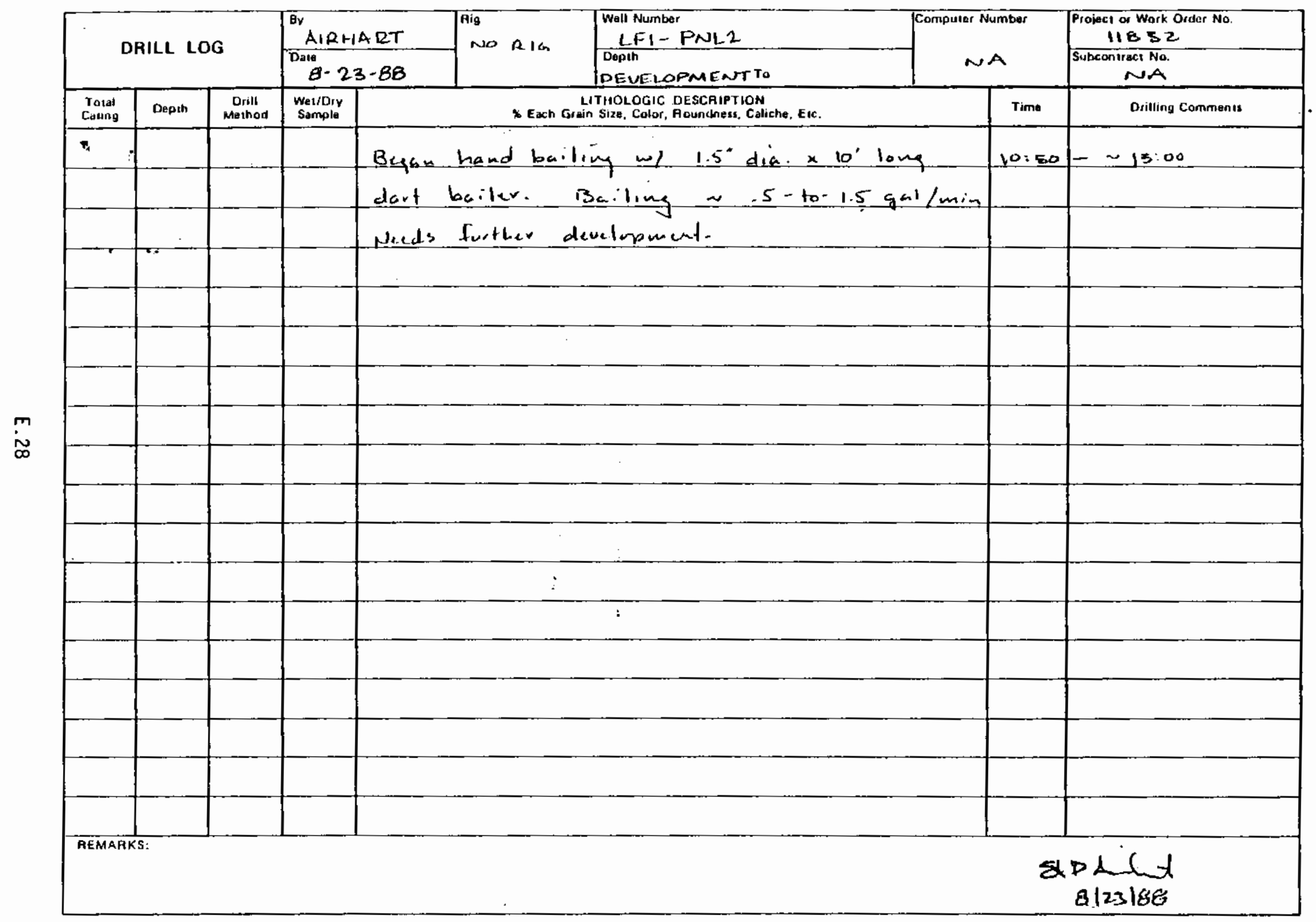

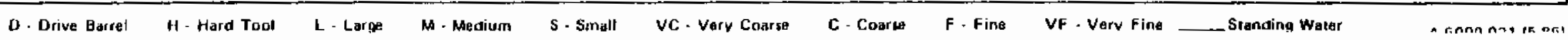


pare 1 of 1

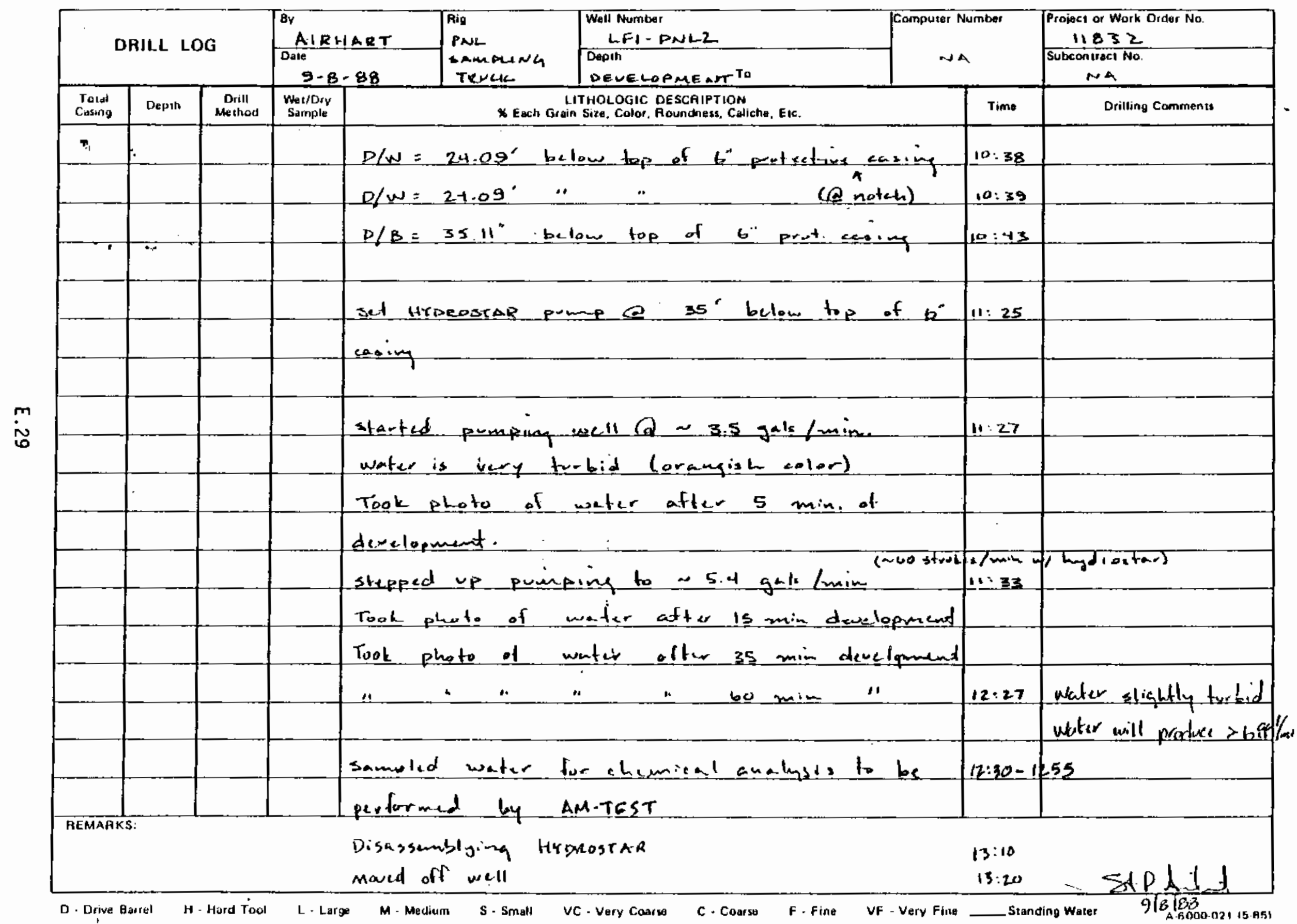


1 of 1

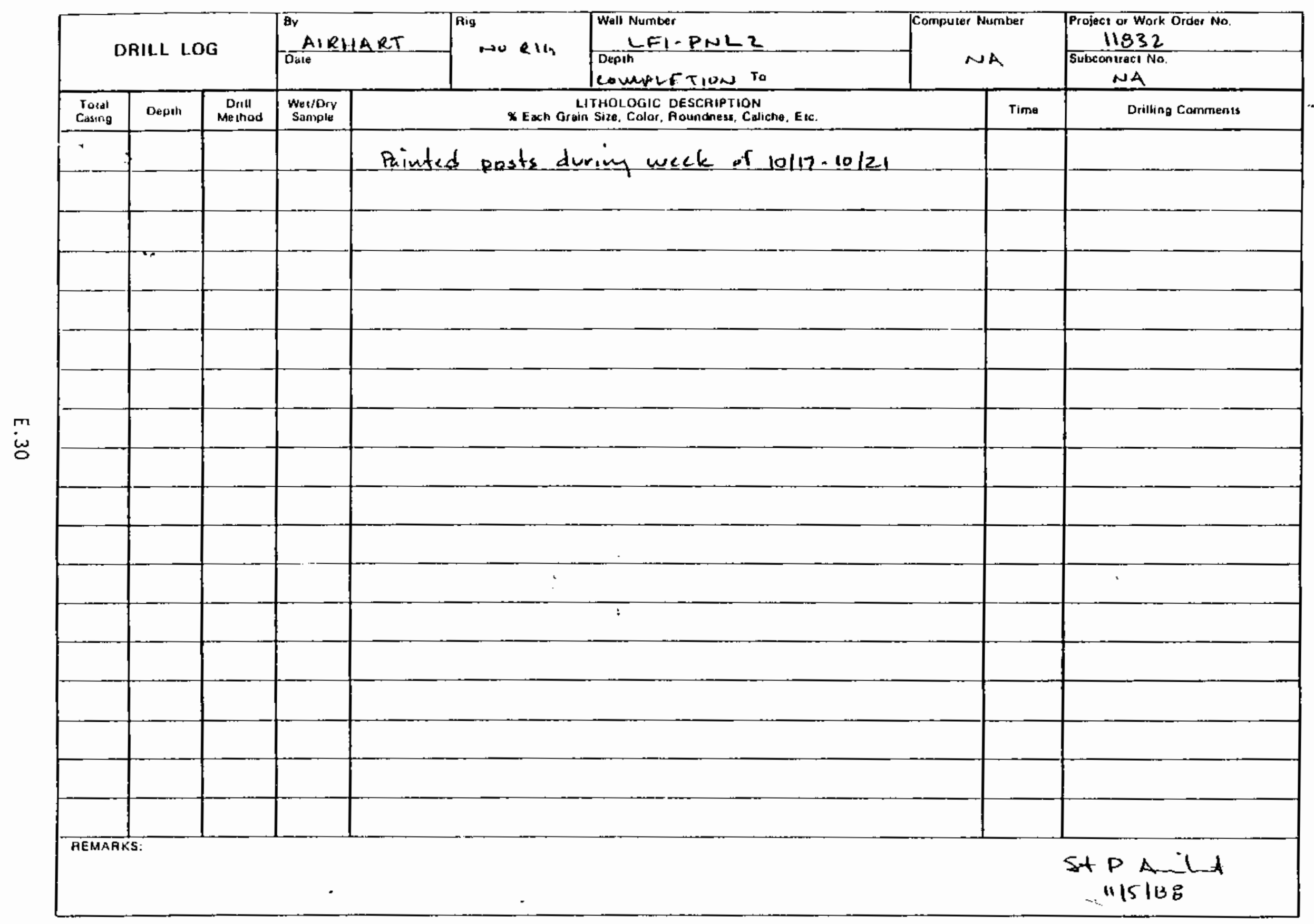
D - Dirve Barrel
H. Hard Toot
L. Lary:
M- Medium
s. Small
VC. Verv Coarse
C. Conrsa
F. Fine VF- V/pen Fine
Sianding ixtatne 
WELL LF1 - PNL3 


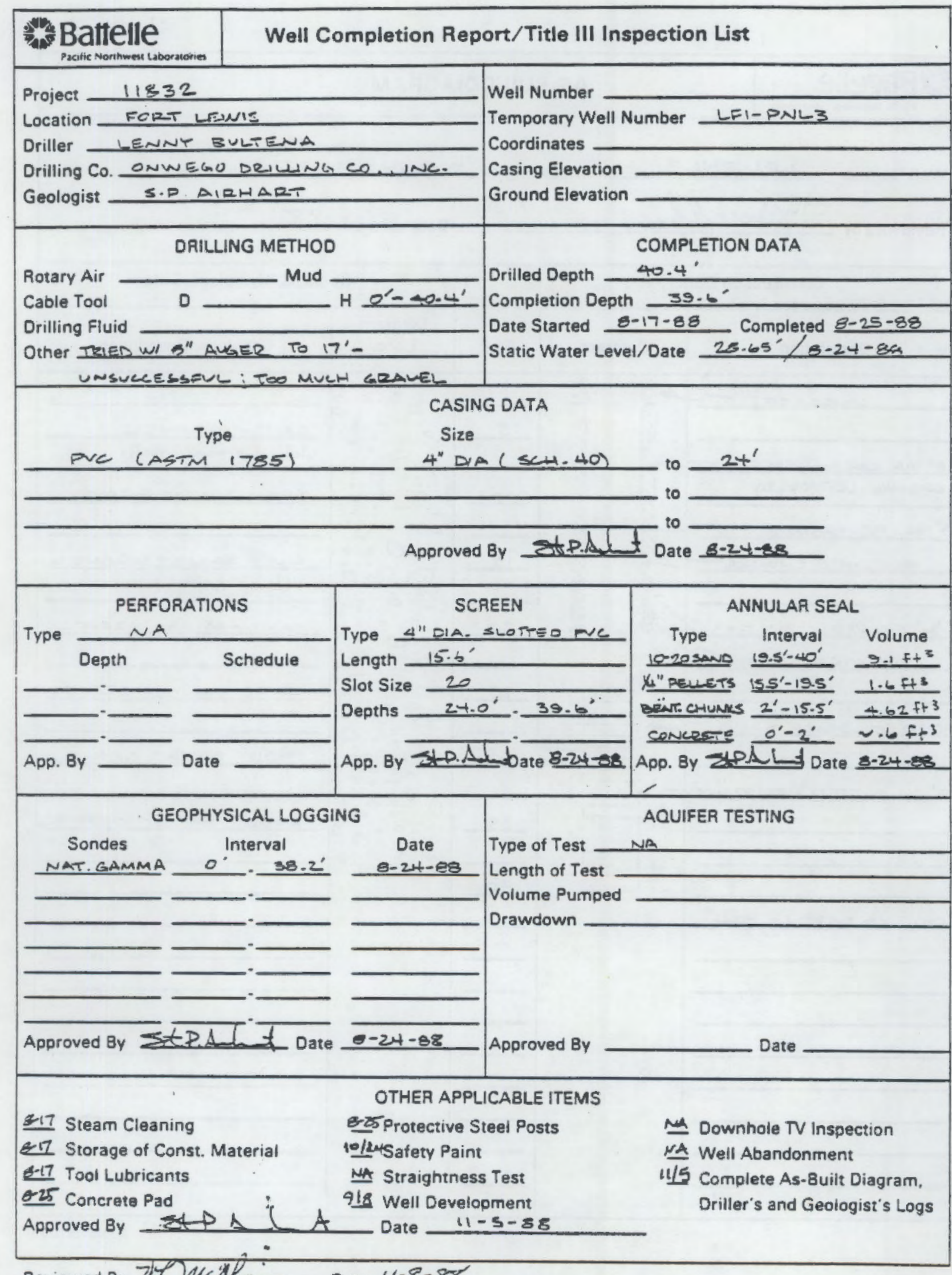

Reviewed By Jih. Whatheic Date $11-8-88$ 


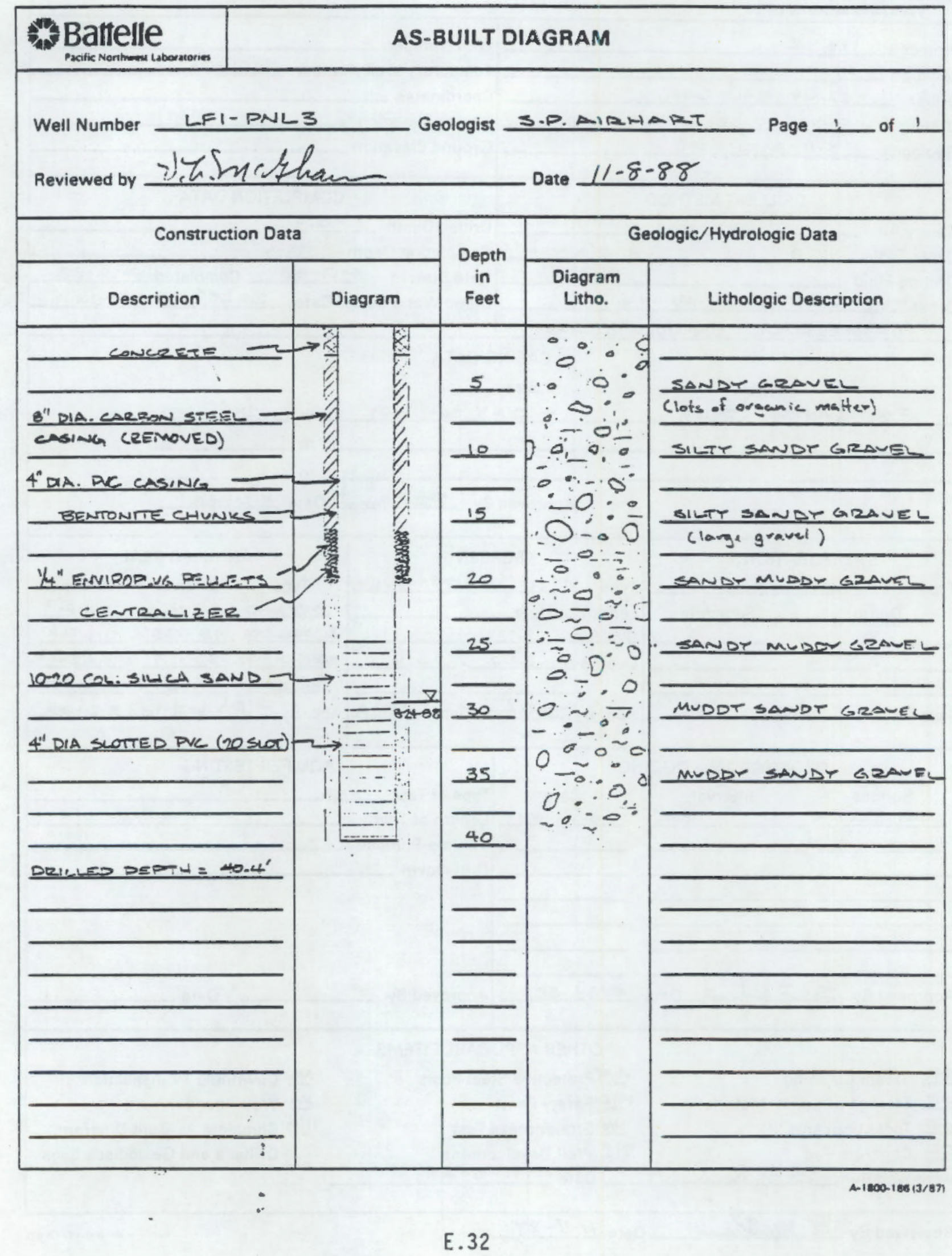




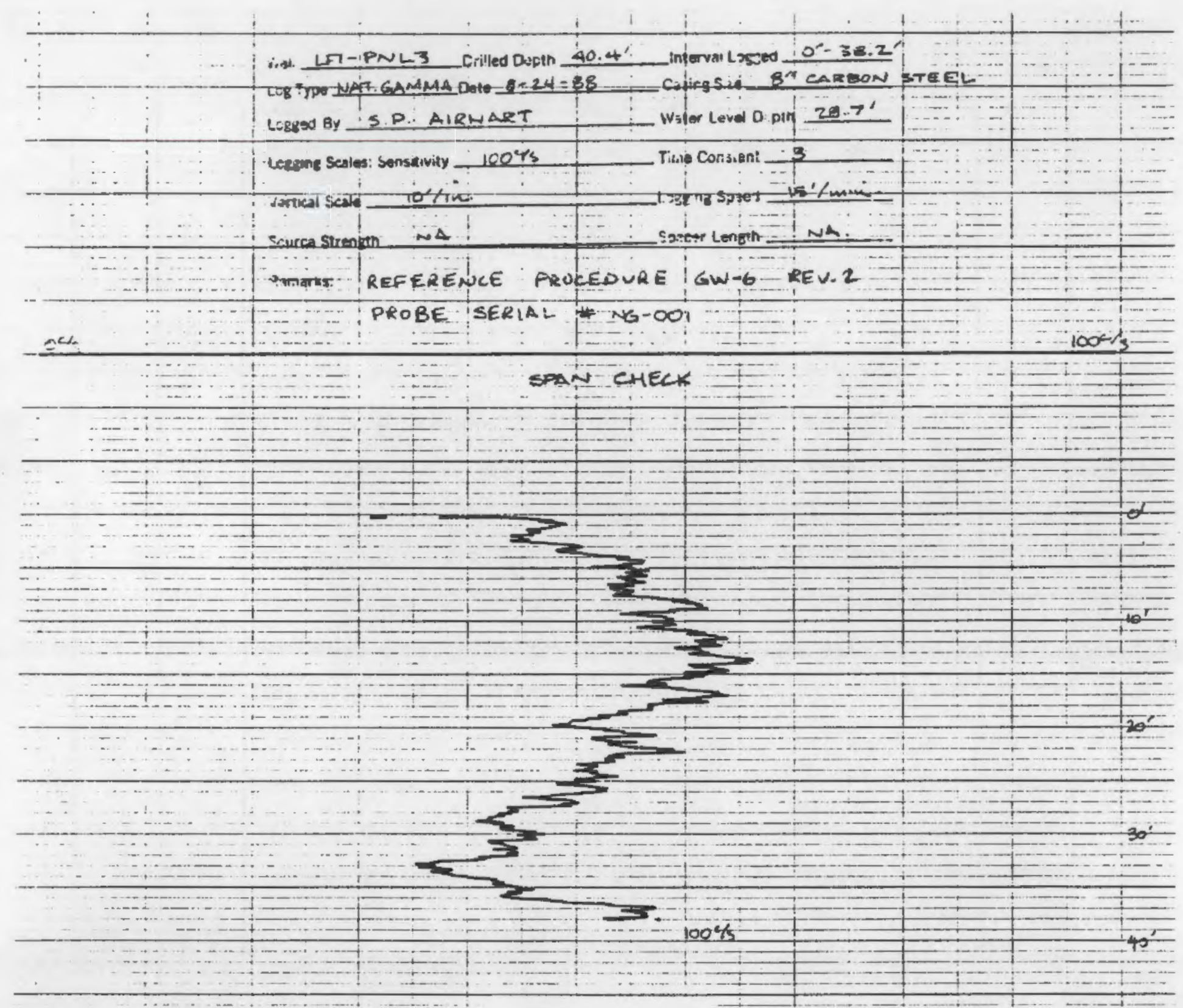




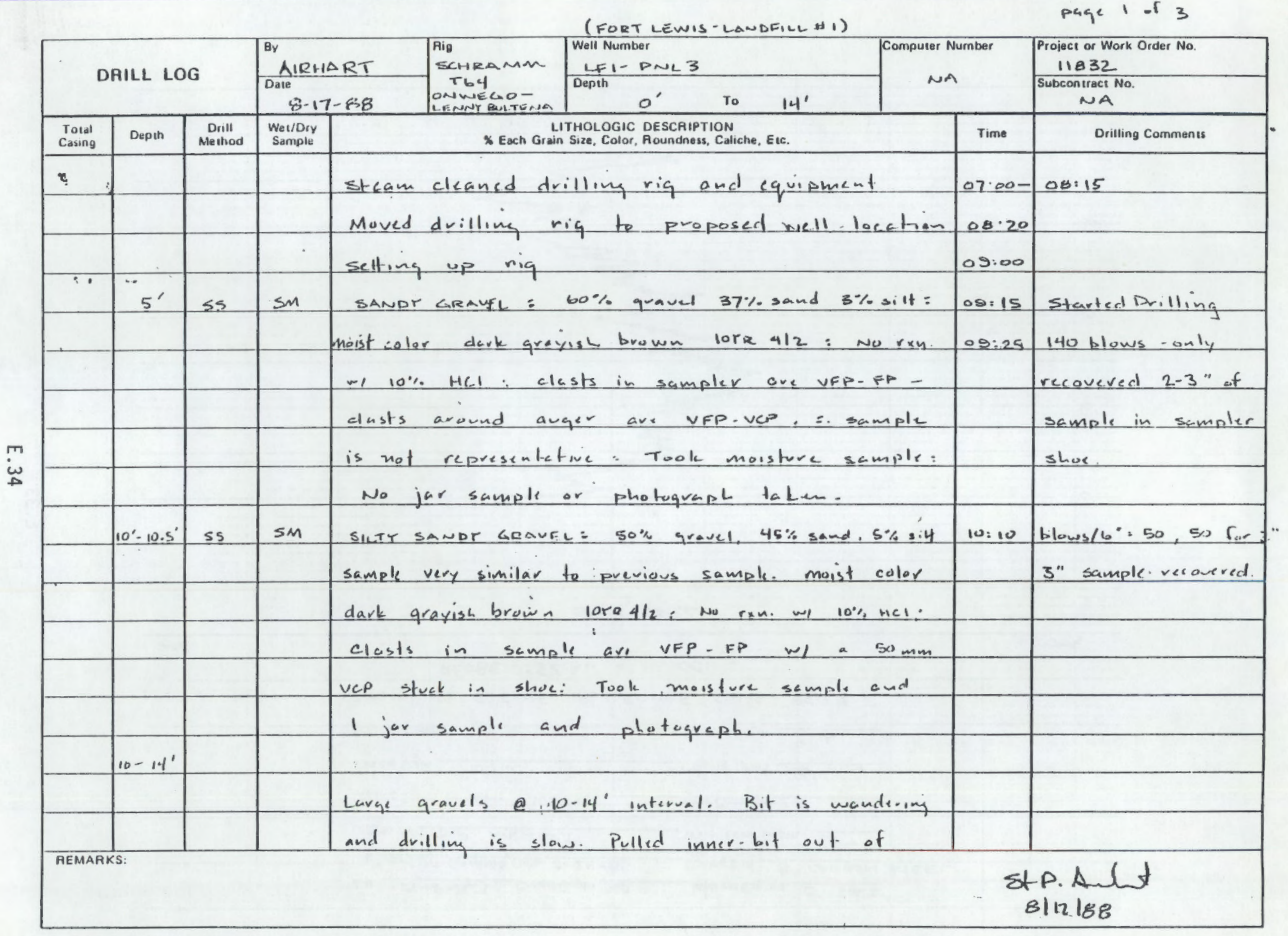

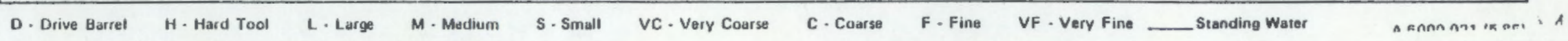


$p+g$ 2.f 3

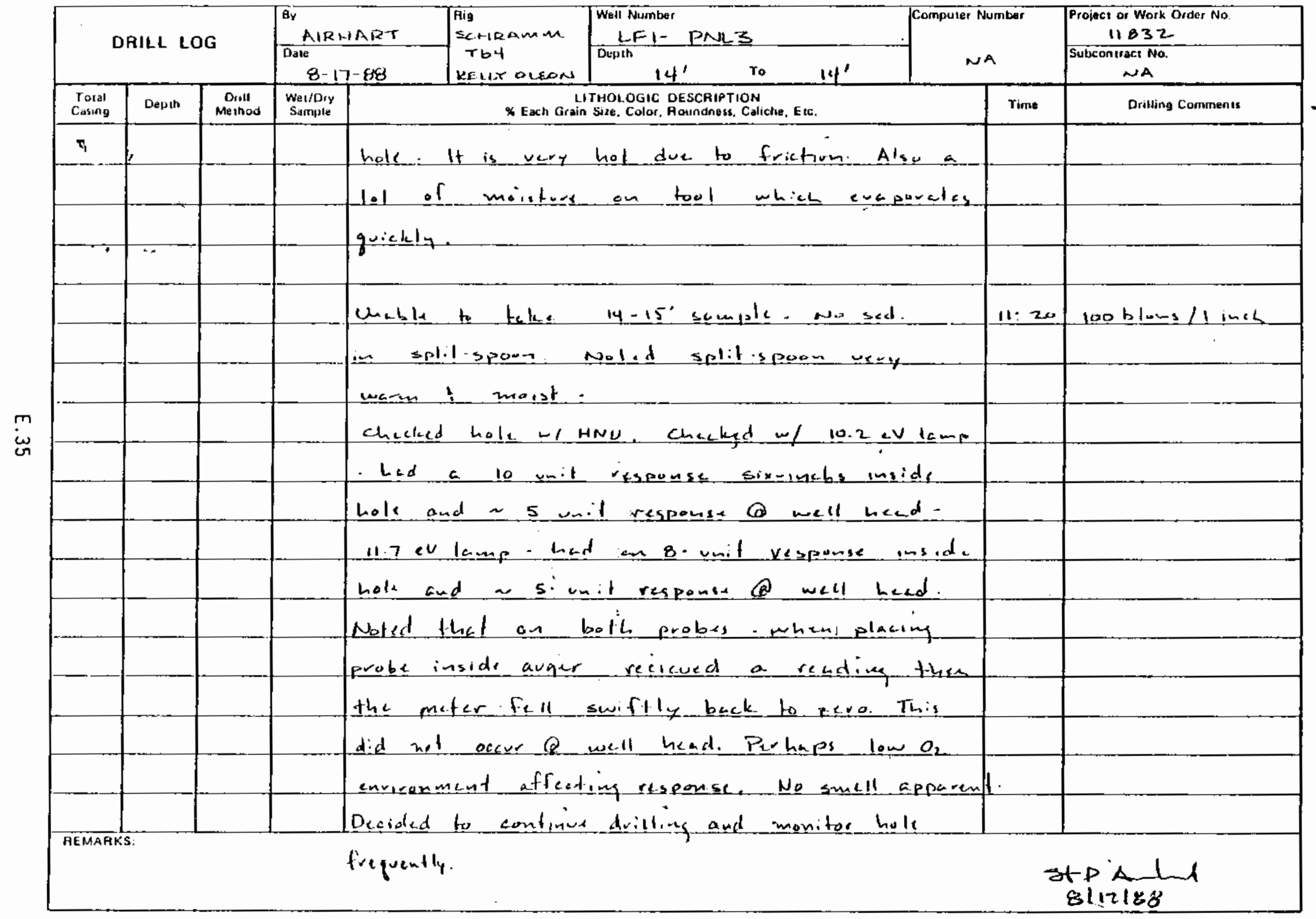

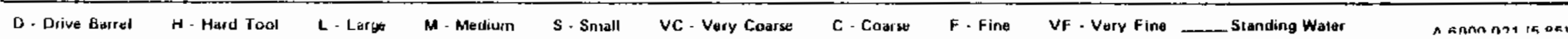


$p+y+3$

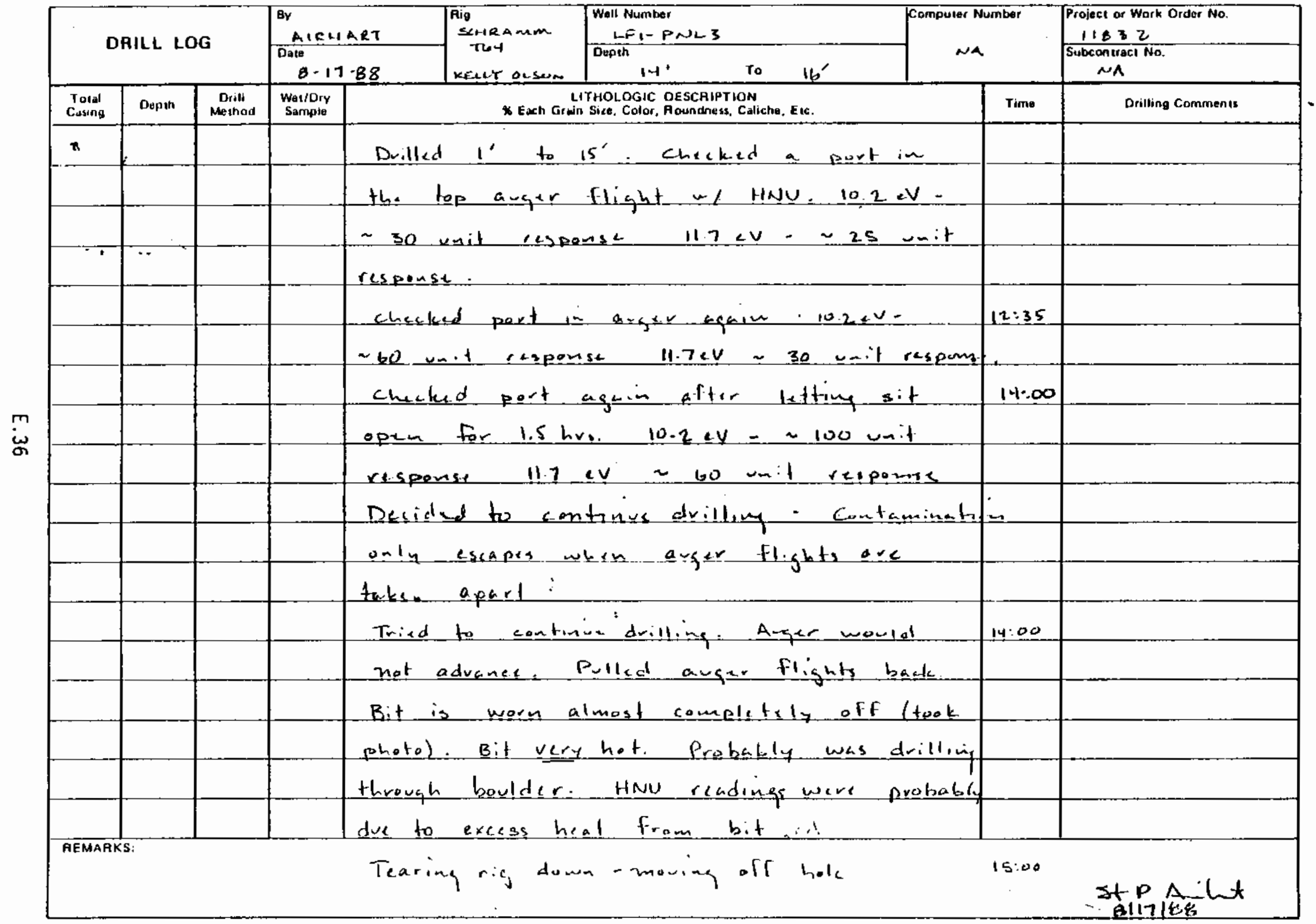

D. Dive Barrel H. Hlard tool L. Large M. Madium S. Sinall VC. Very Coarse C. Coarse F. Fine VF. Very Fine LCStanding Water 


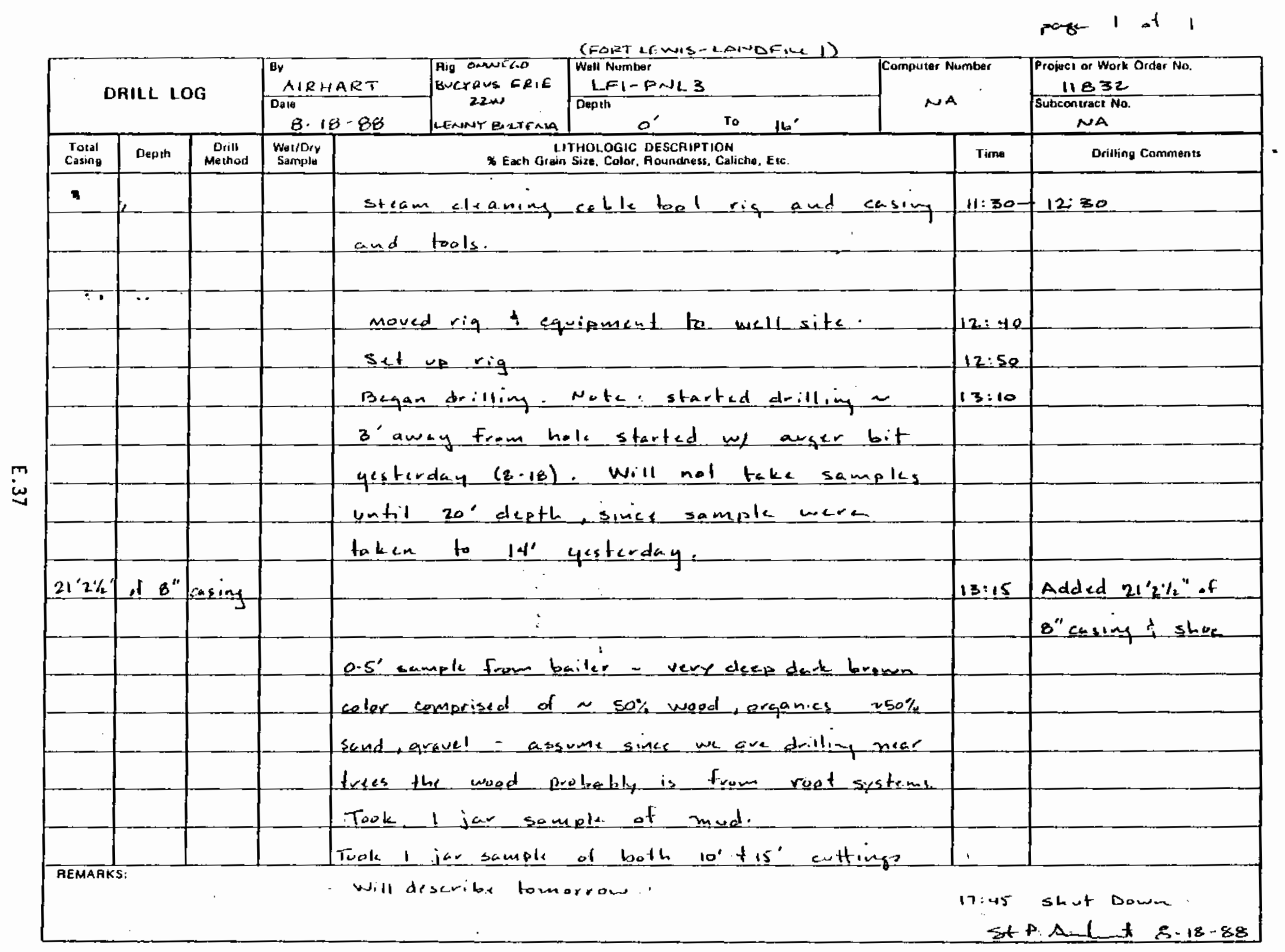

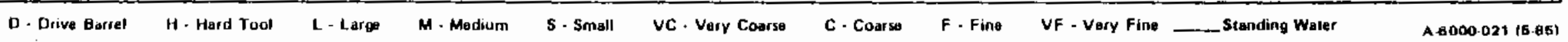


page 1 if 4

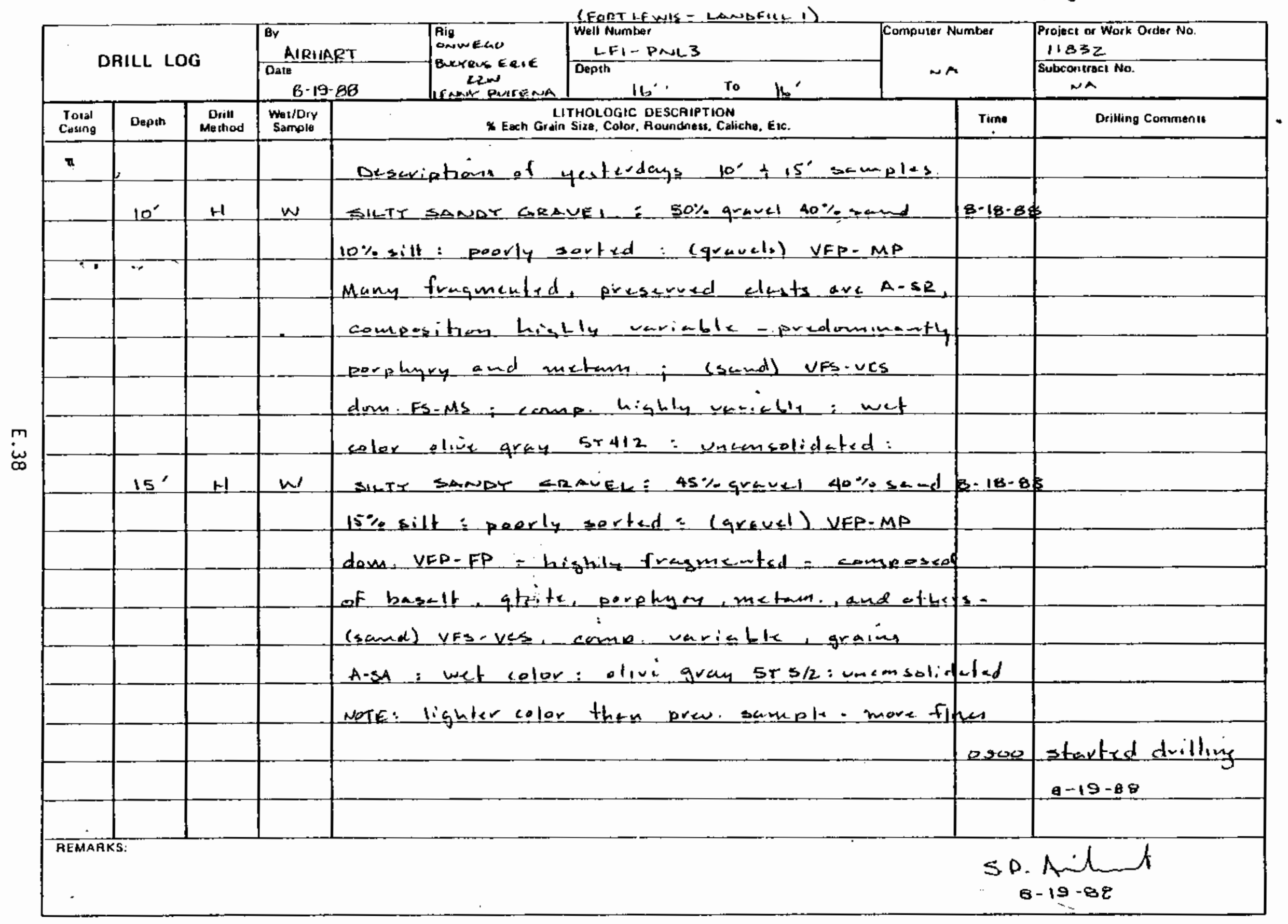

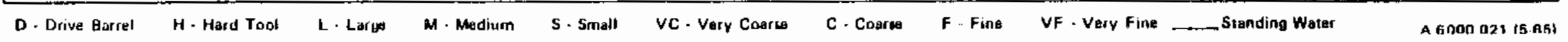




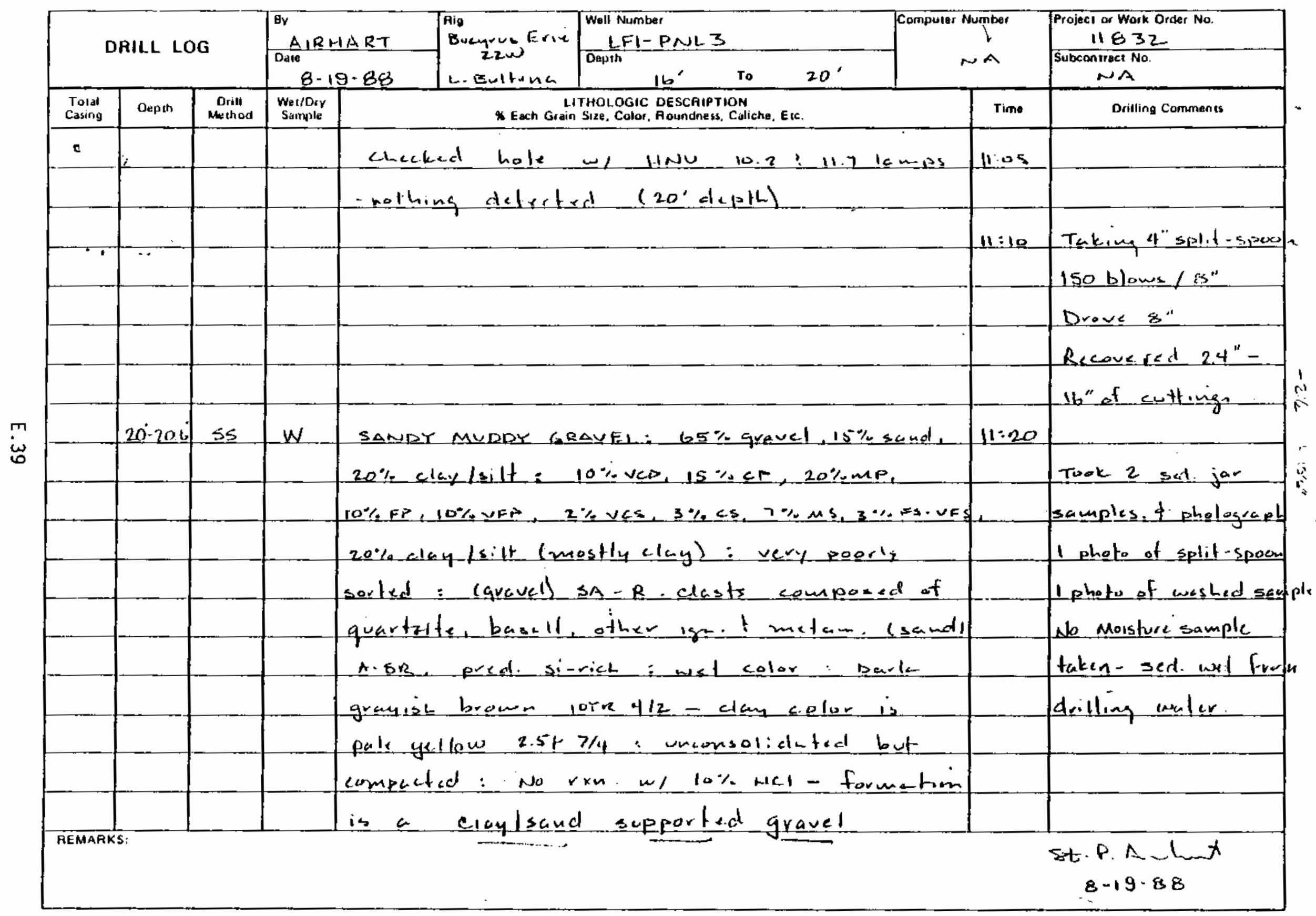

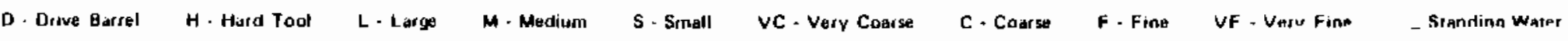


poge 3 it 4

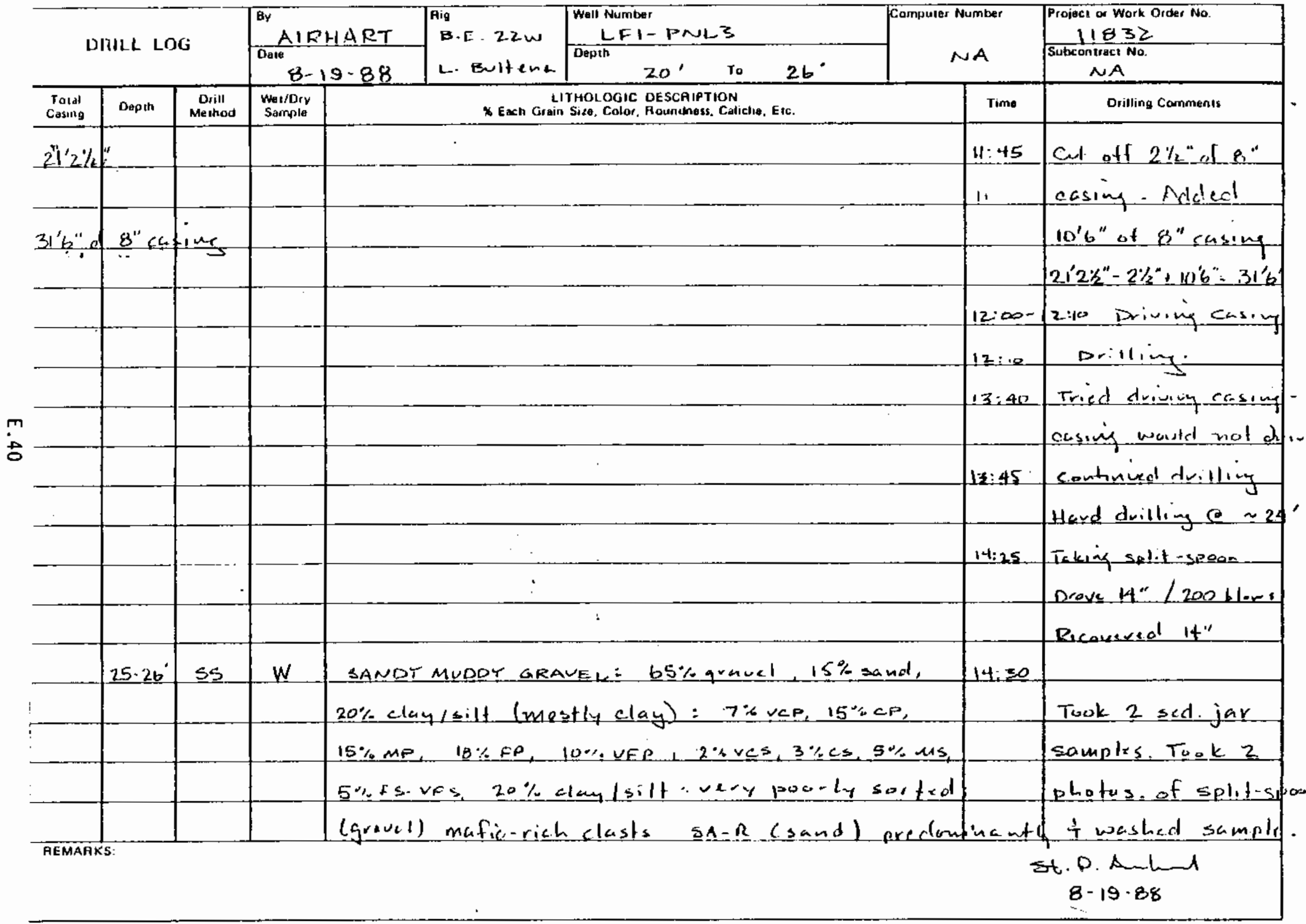

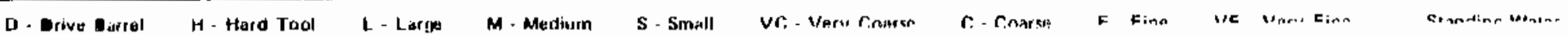


puge 4 of 4

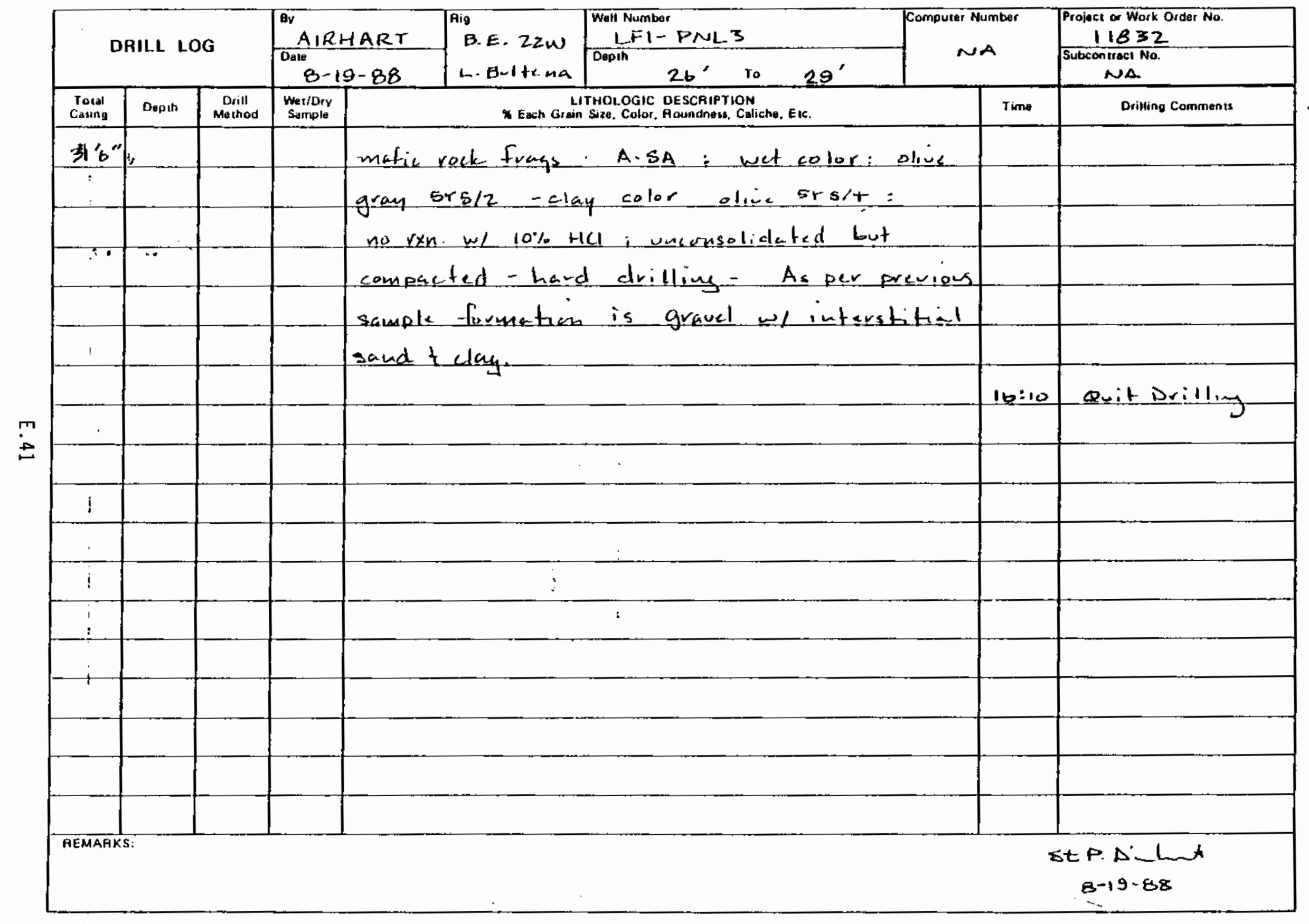

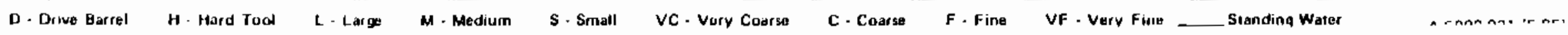




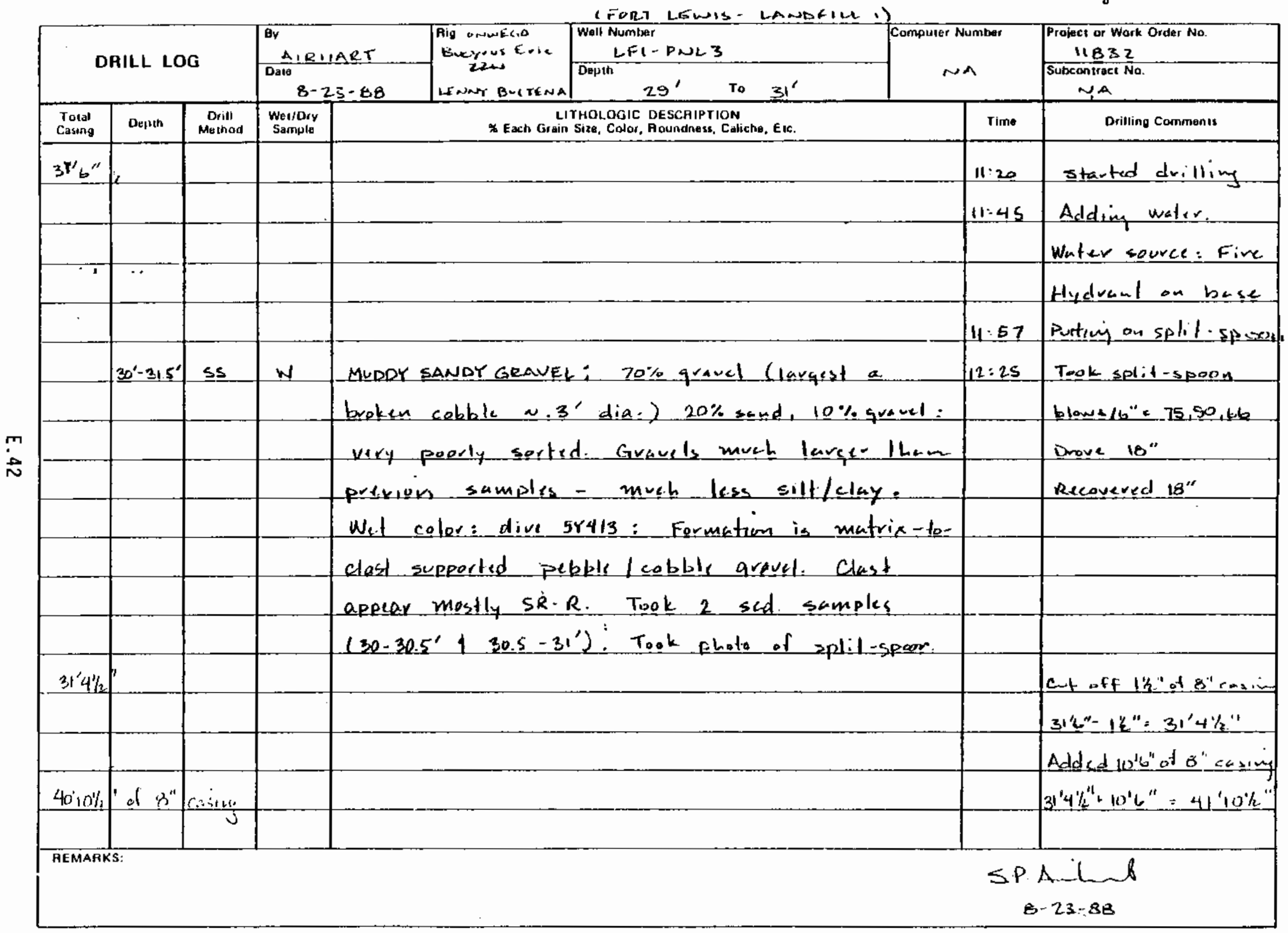

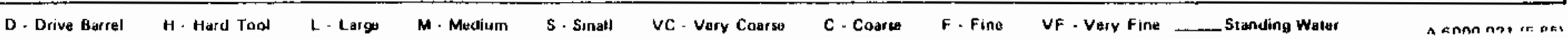


page 2 of 2

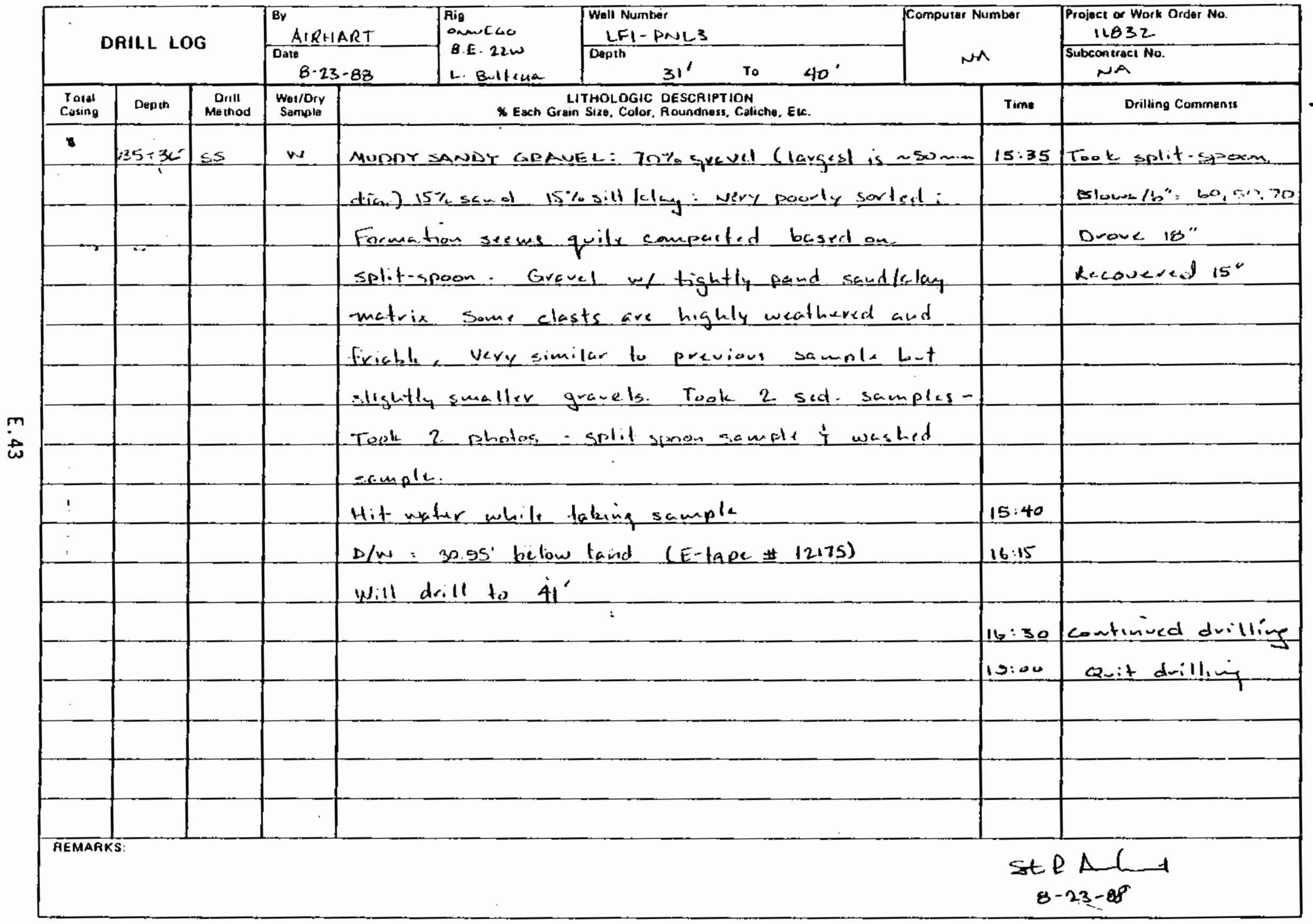
D. Nrive Harral
H. Herd Tans
M4. Anation 


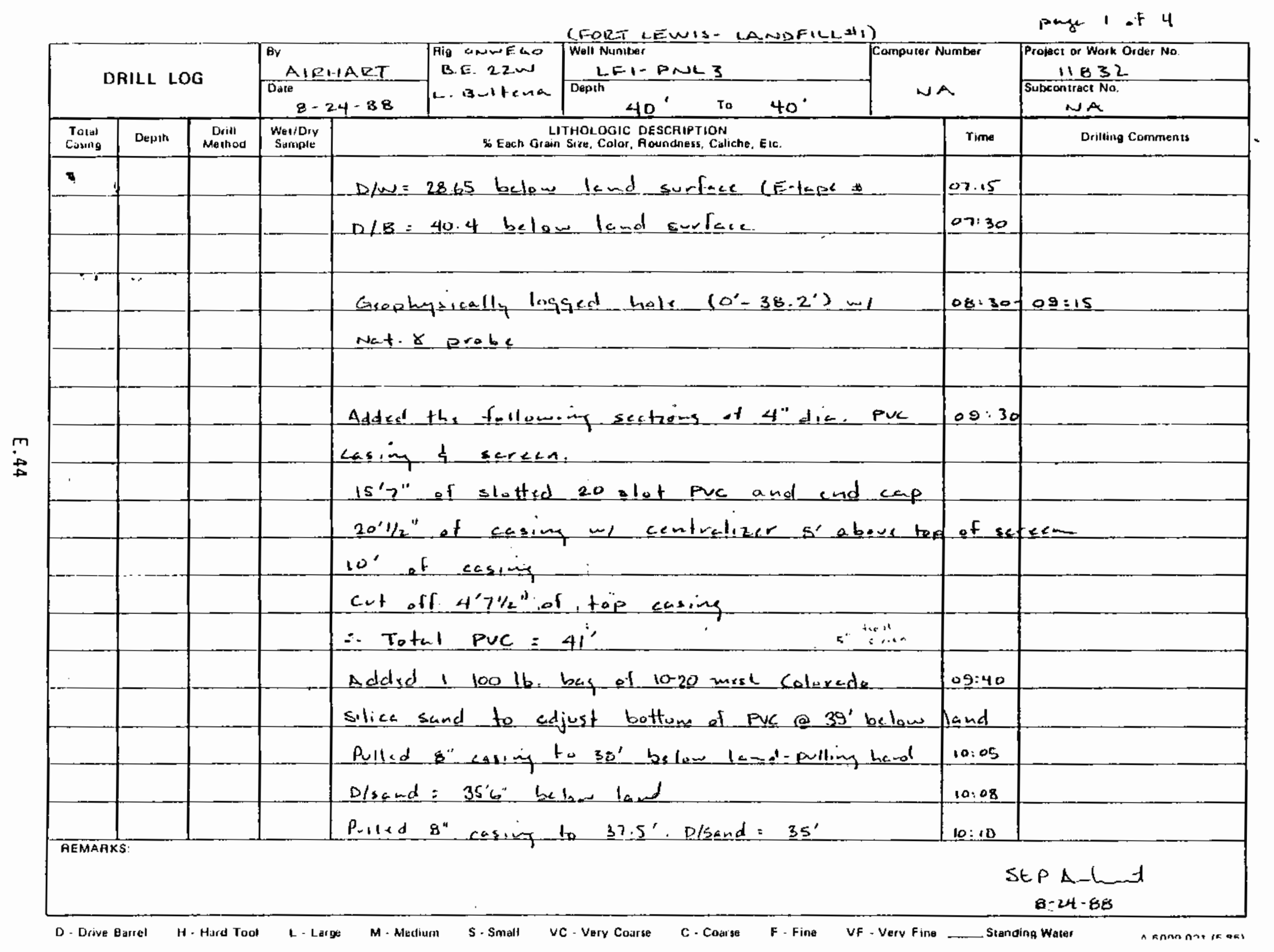


$19+160, d$

prge 2 al 4

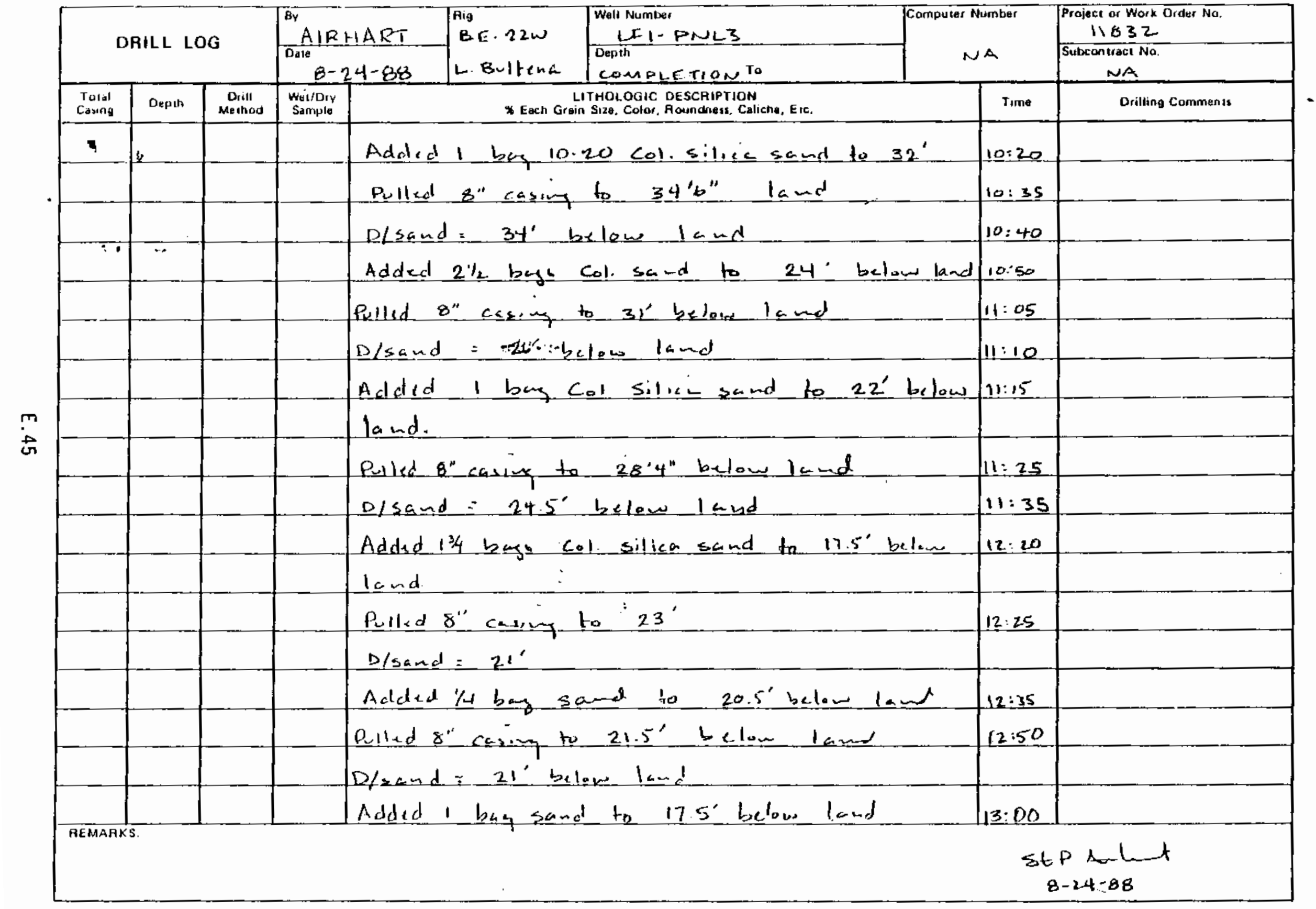

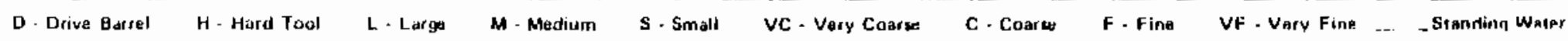


prge 3 - 3

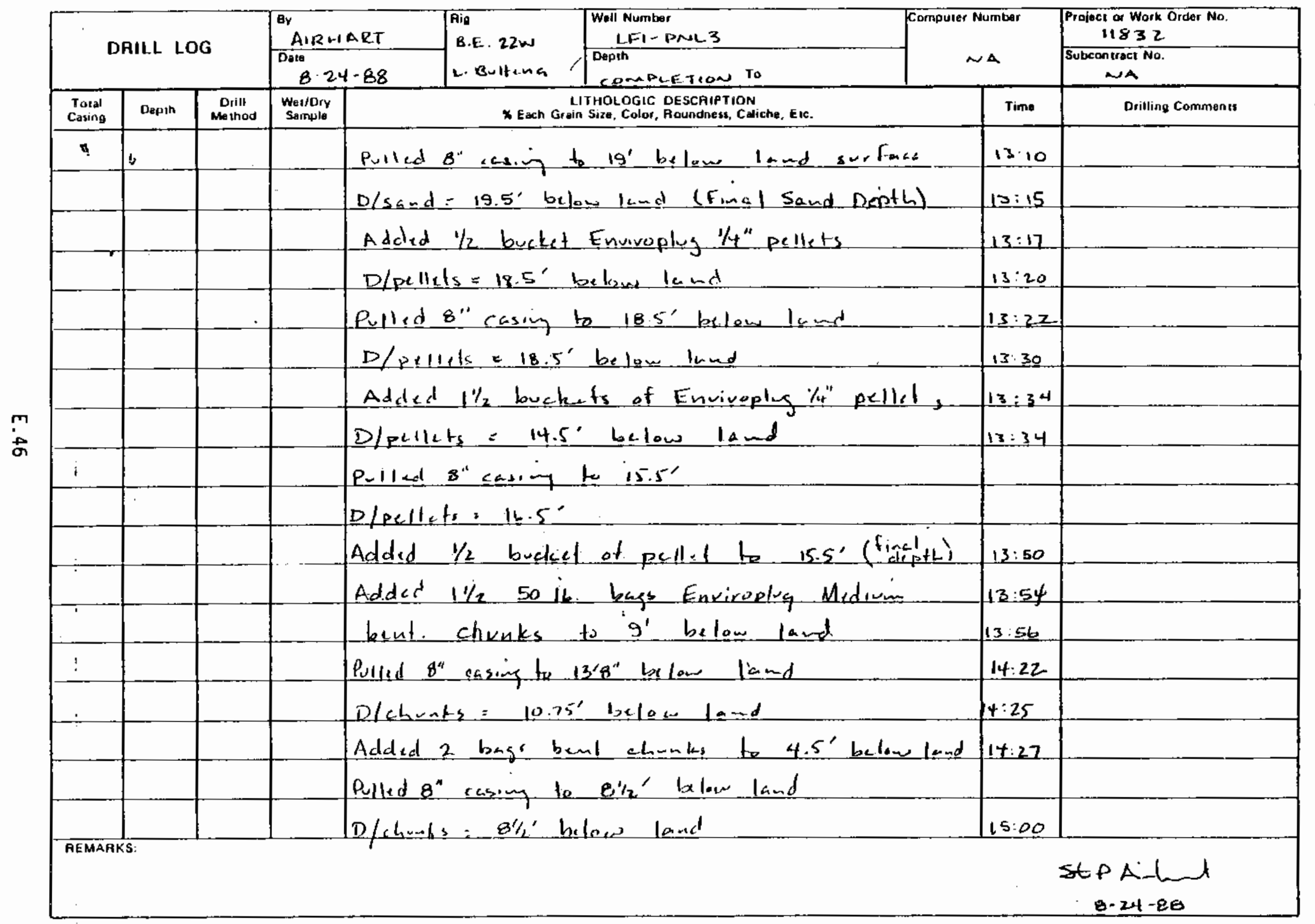


paze 4 of 4

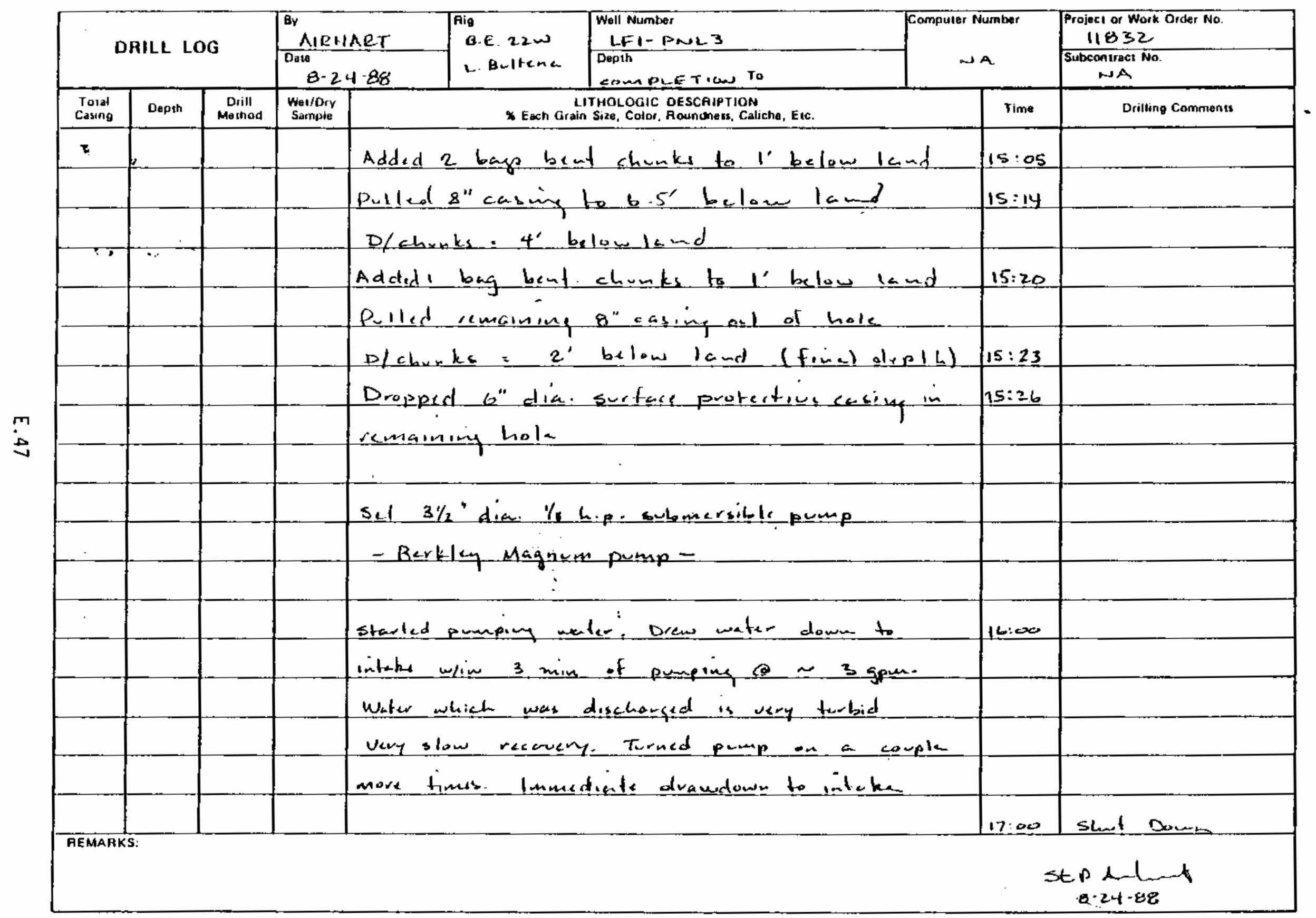

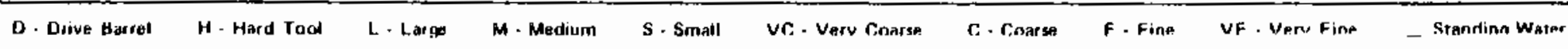




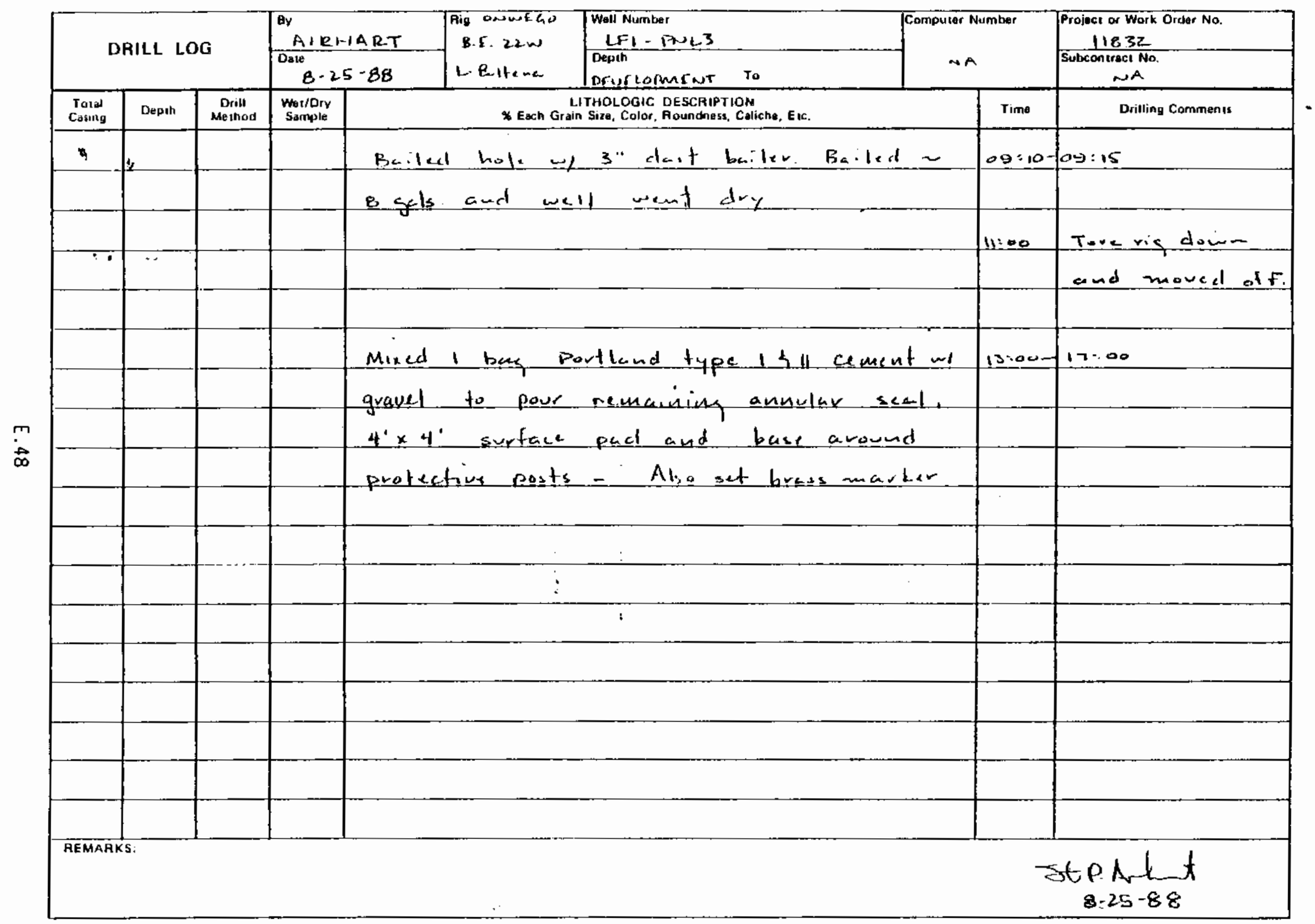
D - Drive Barret
H. Mard Tool
L. Large
M - Medium
S - Small
VC - Verv Coarse
C - Coasse
F. Fina
VF - Very Fine
gramana dere 


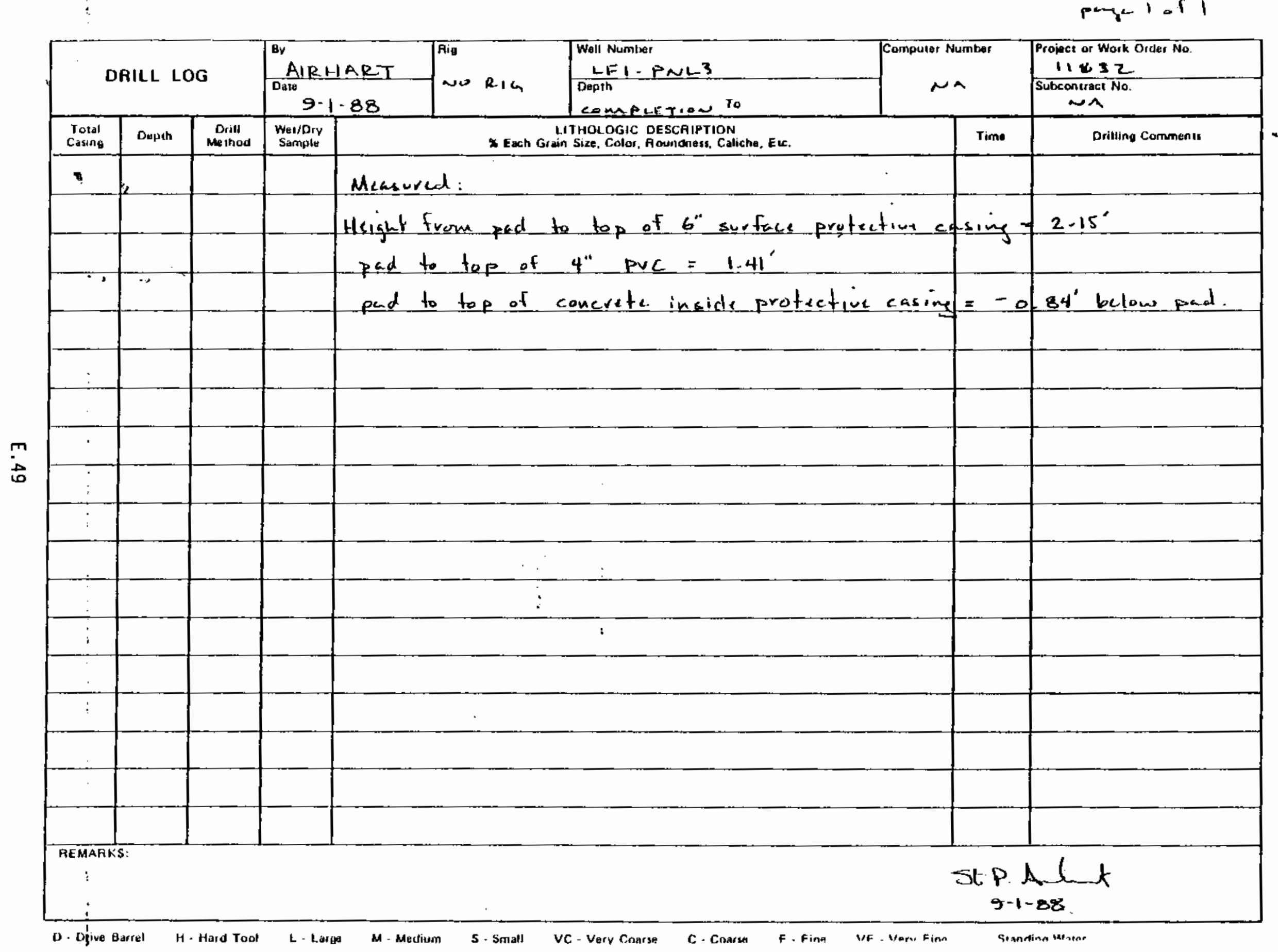


$p-z^{2}+$ if

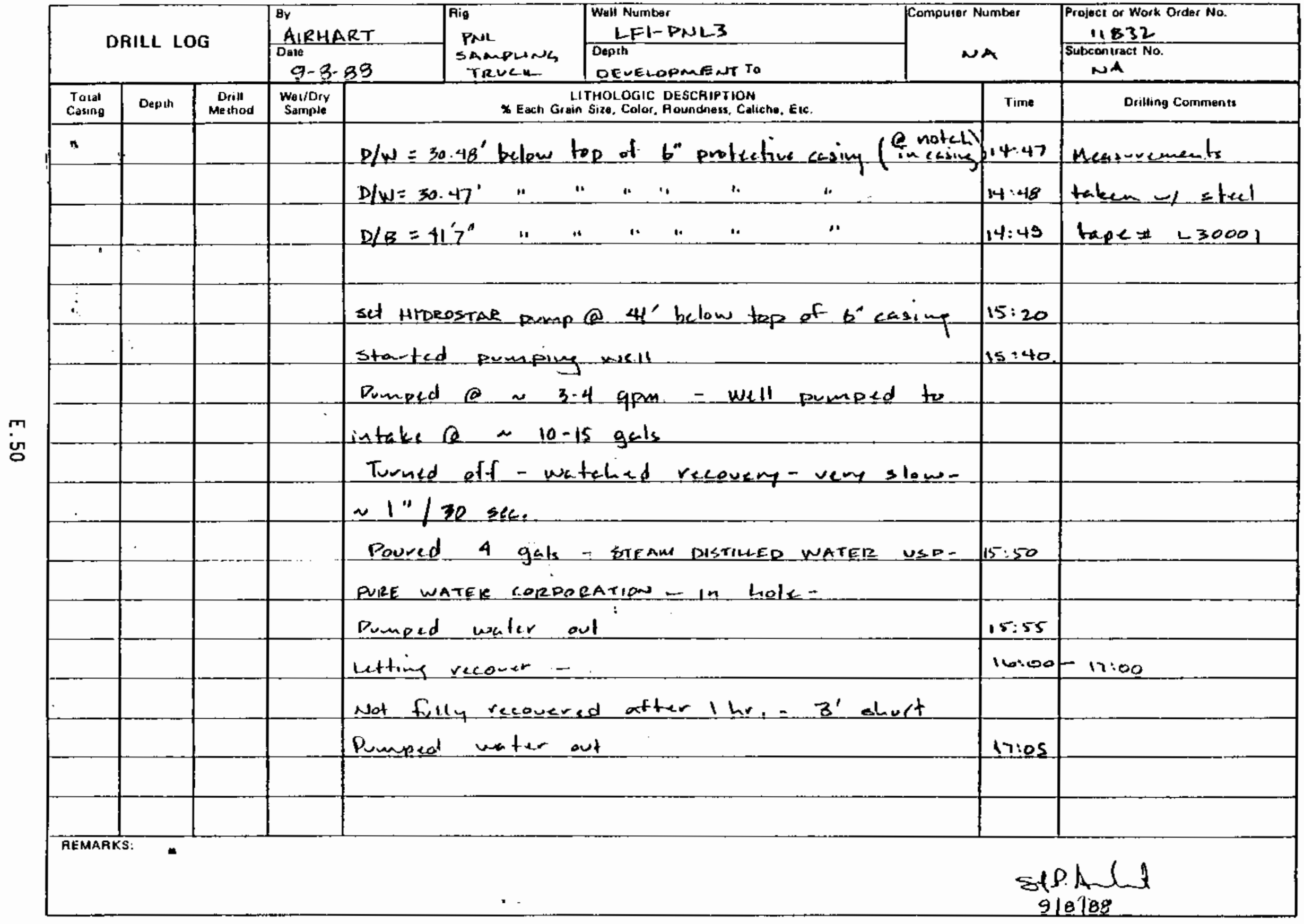

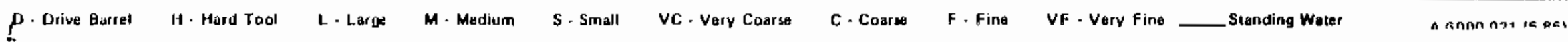


pine 1 -1 1

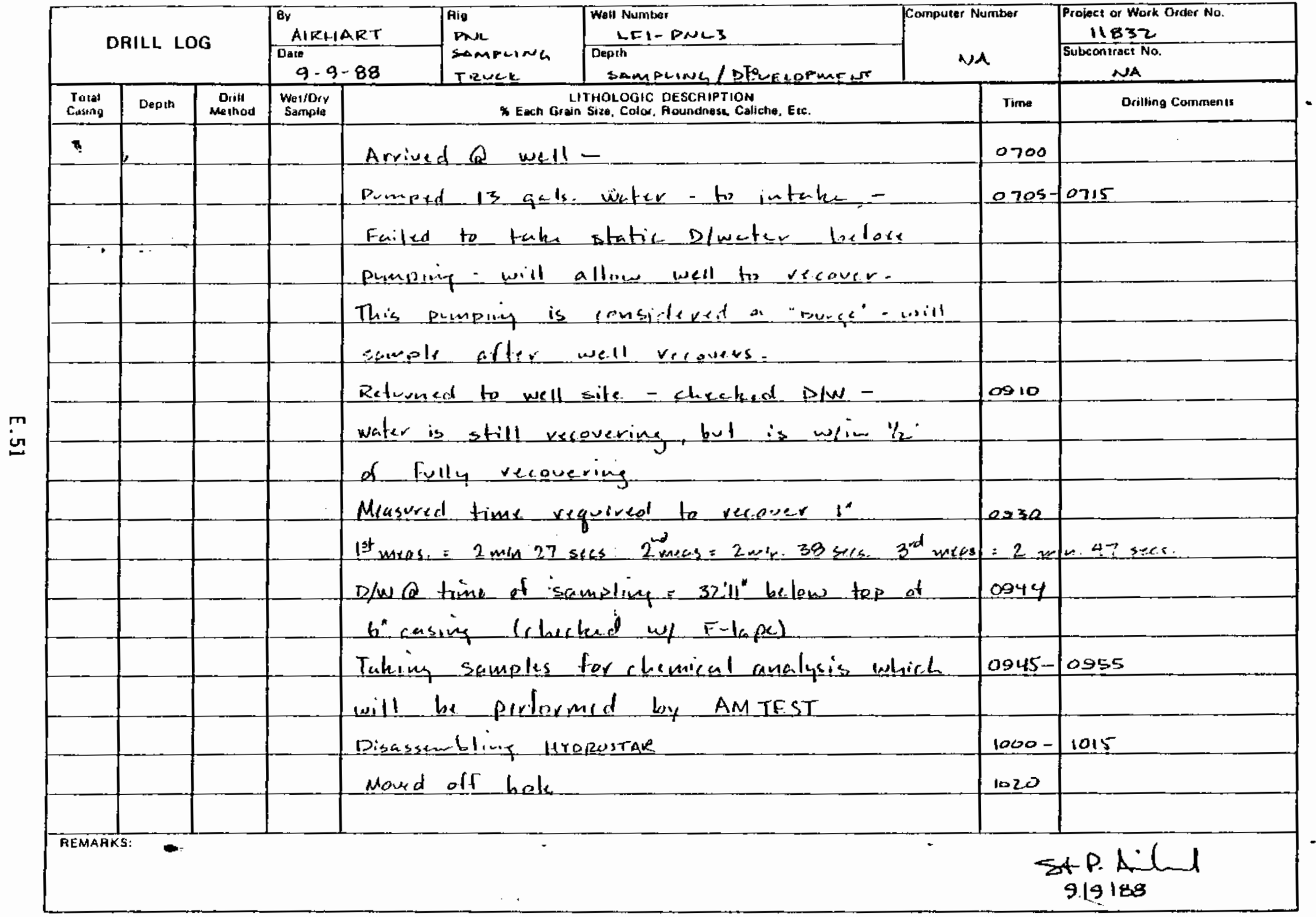
D. Difve Barrei
H. Hasd Tool 
rage 1 of 1

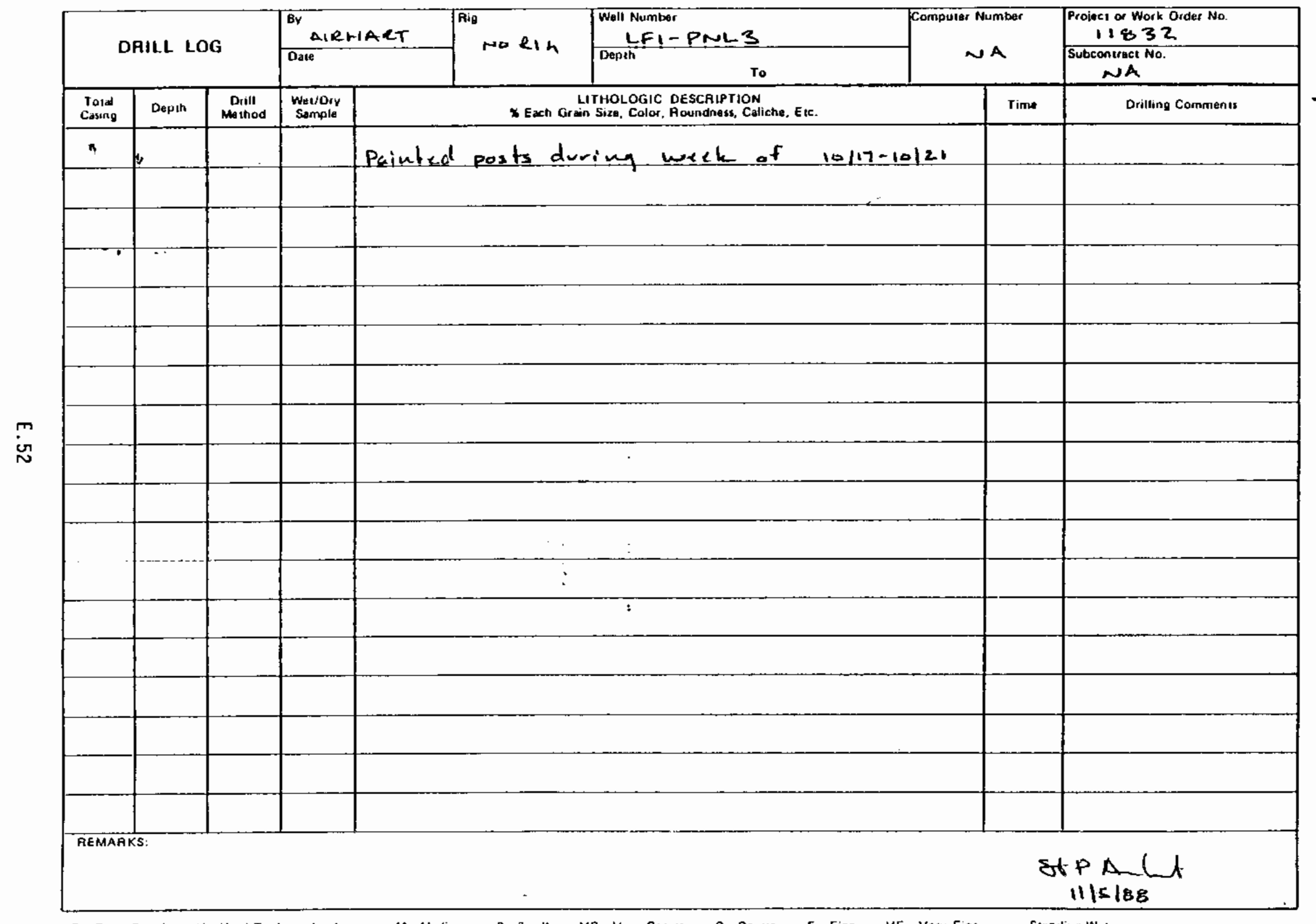
D - Dive Burrel
H - Hard Tood 
WELL LF4-PNL4 


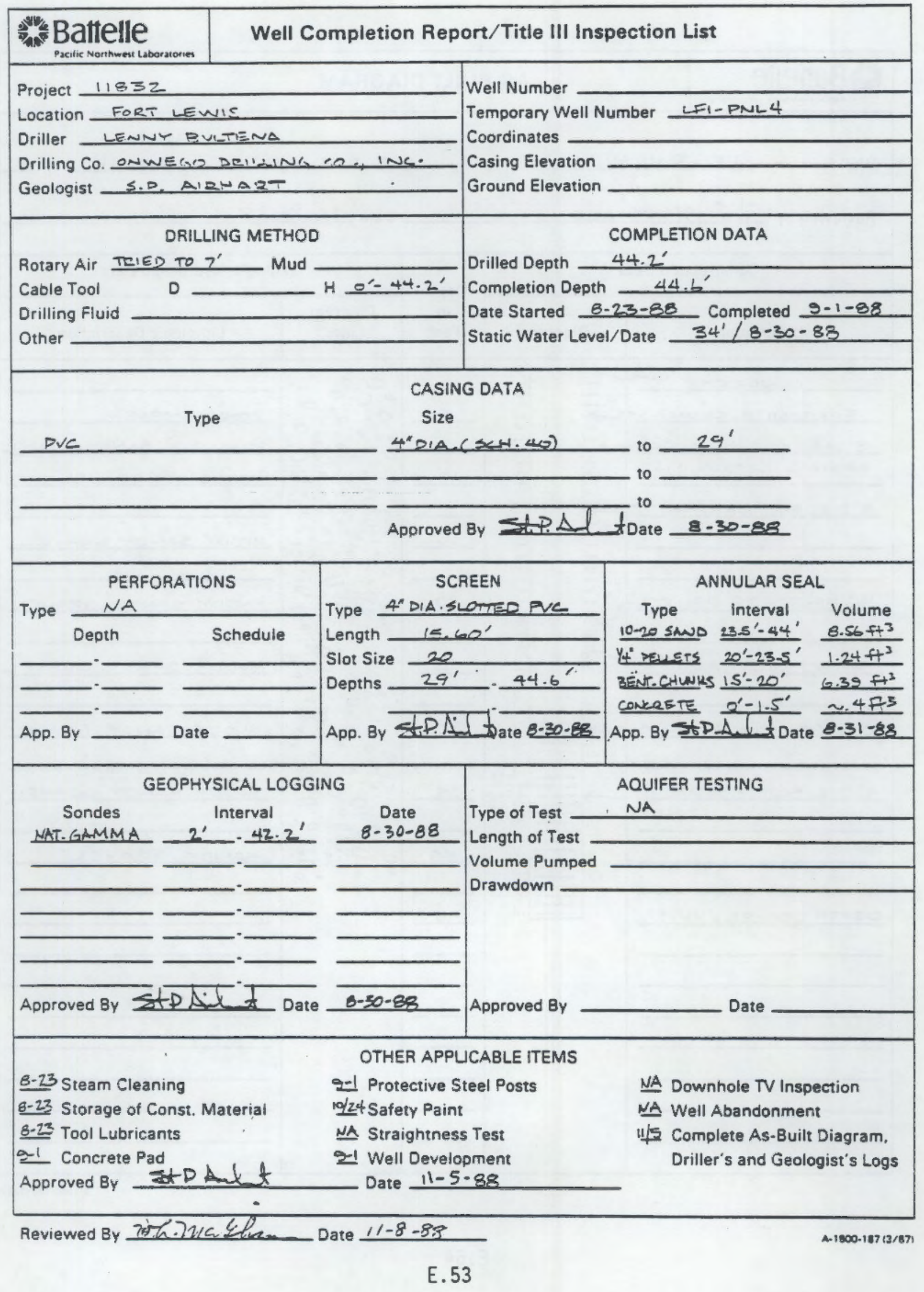




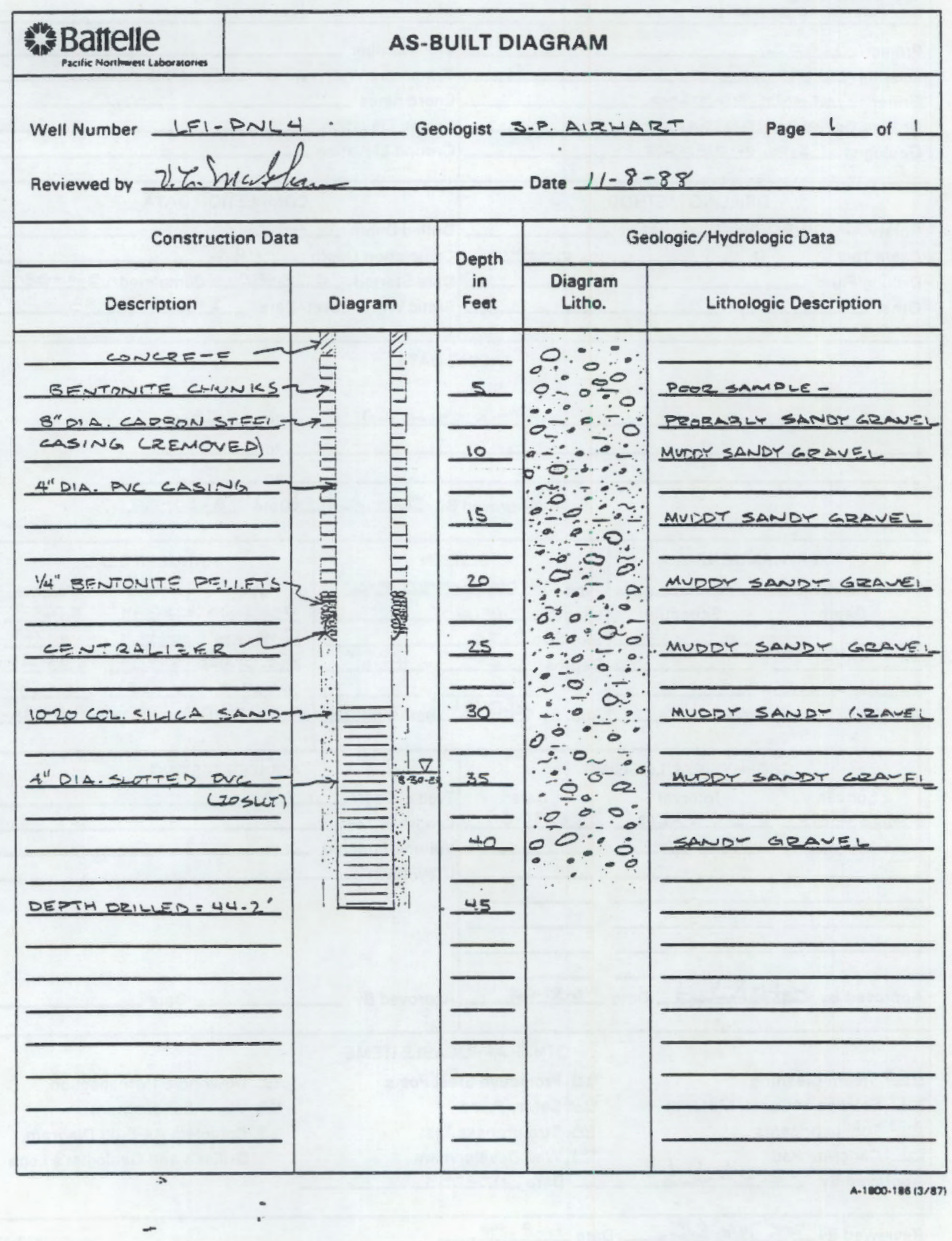




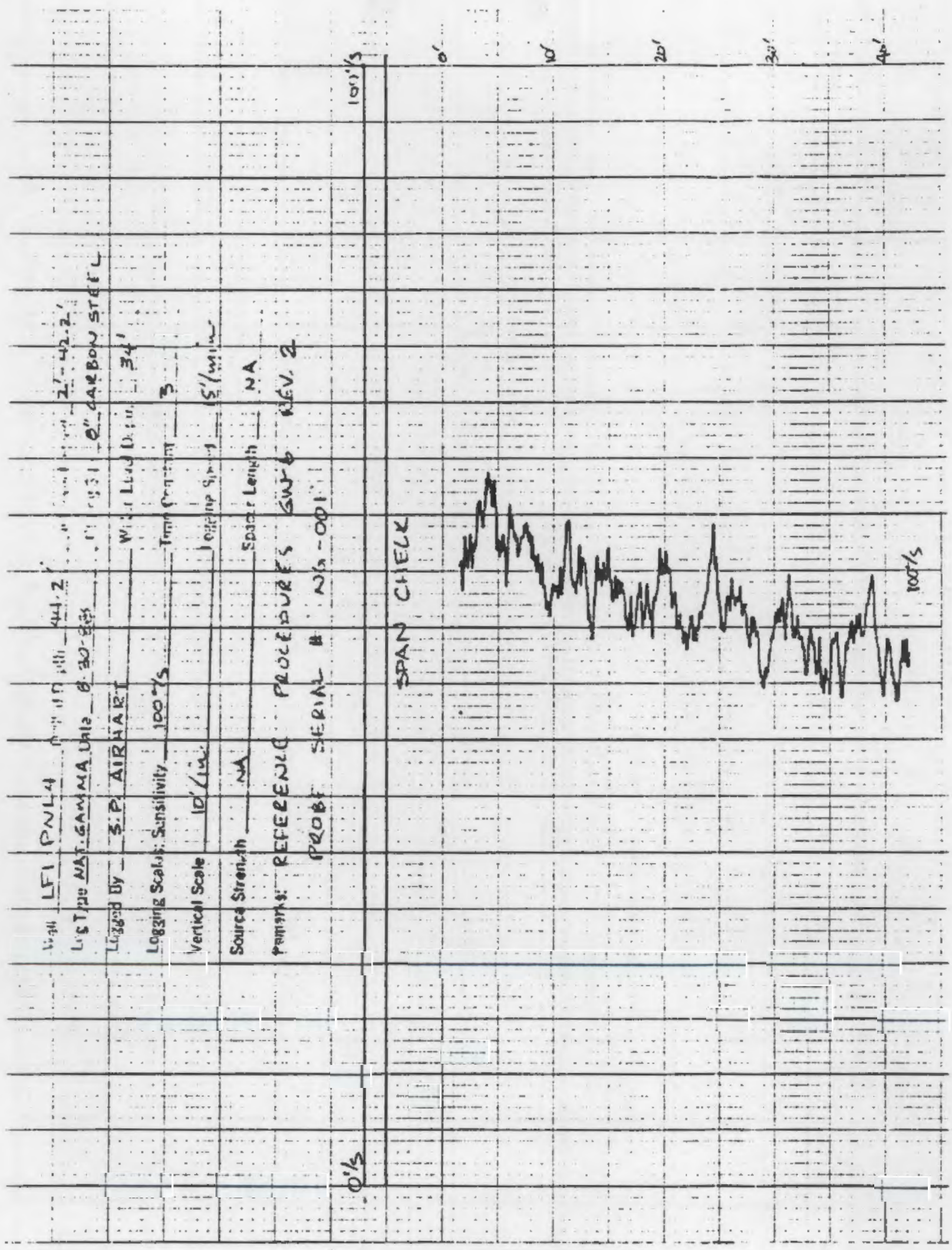




\begin{tabular}{|c|c|c|c|c|c|c|}
\hline \multicolumn{3}{|c|}{ DRILL LOG } & $\begin{array}{l}\text { BV } \\
\text { AIRHAIRT } \\
\text { Date } \\
8-23-88 \\
\end{array}$ & 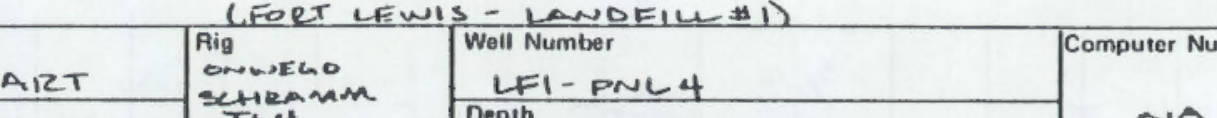 & Computer Number & \multirow{2}{*}{\begin{tabular}{|} 
Project or Work Order No. \\
11832 \\
Subcontract No. \\
$\sim$
\end{tabular}} \\
\hline & & & & \multirow{2}{*}{$\begin{array}{l}\text { LITHOLOGIC DESCAIPTION } \\
\times \text { Each Grain Size, Color, foundoess, Caliche, Etc. } \\
\end{array}$} & & \\
\hline $\begin{array}{l}\text { Tasial } \\
\text { Casing } \\
\end{array}$ & Depth & $\begin{array}{c}\text { Drill } \\
\text { Method } \\
\end{array}$ & $\begin{array}{c}\text { Weit/Ory } \\
\text { Sample }\end{array}$ & & Time & Drilling Comments \\
\hline 1 & & & & steam deaning rig \& equip. - & 0700 & $=0800$ \\
\hline & & & & Note: Using a pressure wash chemical & & \\
\hline . & & & & while steam deaning to aid in degreasing: & & \\
\hline . & & & & Spees en chemical: & & \\
\hline & & & & Manufacturer: Dayton Electric MFg. Co. & & \\
\hline & & & & Chicago Illinois b0648 & & \\
\hline & & & & Product Name: Pressure Washer Chemical No. snf & 132 & \\
\hline & & & & Description : A Non-flammable, biodegraduble and & & \\
\hline & & & & U.S.D.A. approved detergent for cleaning heavy & & \\
\hline & & & & accomulations of grease and oil. Accepted for & & \\
\hline & & & & use in kitchens, feed procrssing equipment ets & & \\
\hline & & & & Contents: Complex Sodium Phosphates, Alkaline & & \\
\hline & & & & Builders and surfactanits. & & \\
\hline & & & & - It was o.k'd to use this product as long & & \\
\hline & & & & as eqipment was rinsed w/ non-detergent & & \\
\hline & & & & woter after cleaning. & & \\
\hline & & & & Moved riq to hole. & $11: \infty, 0$ & \\
\hline AEMAF & & & & Setting up rig (irhramm Tu, Air Rola.., of Tricane (...1) & $11.00-$ & $12: 30$ \\
\hline & & & & & & StP. A thet \\
\hline
\end{tabular}

D. Drive Barrel H. Hard Tool L. Large M-Medium S-Small VC-Verv Conerse C-Coarse F. Finn VF-Verv Fine - Standinn Water 
pant: 2 . 2

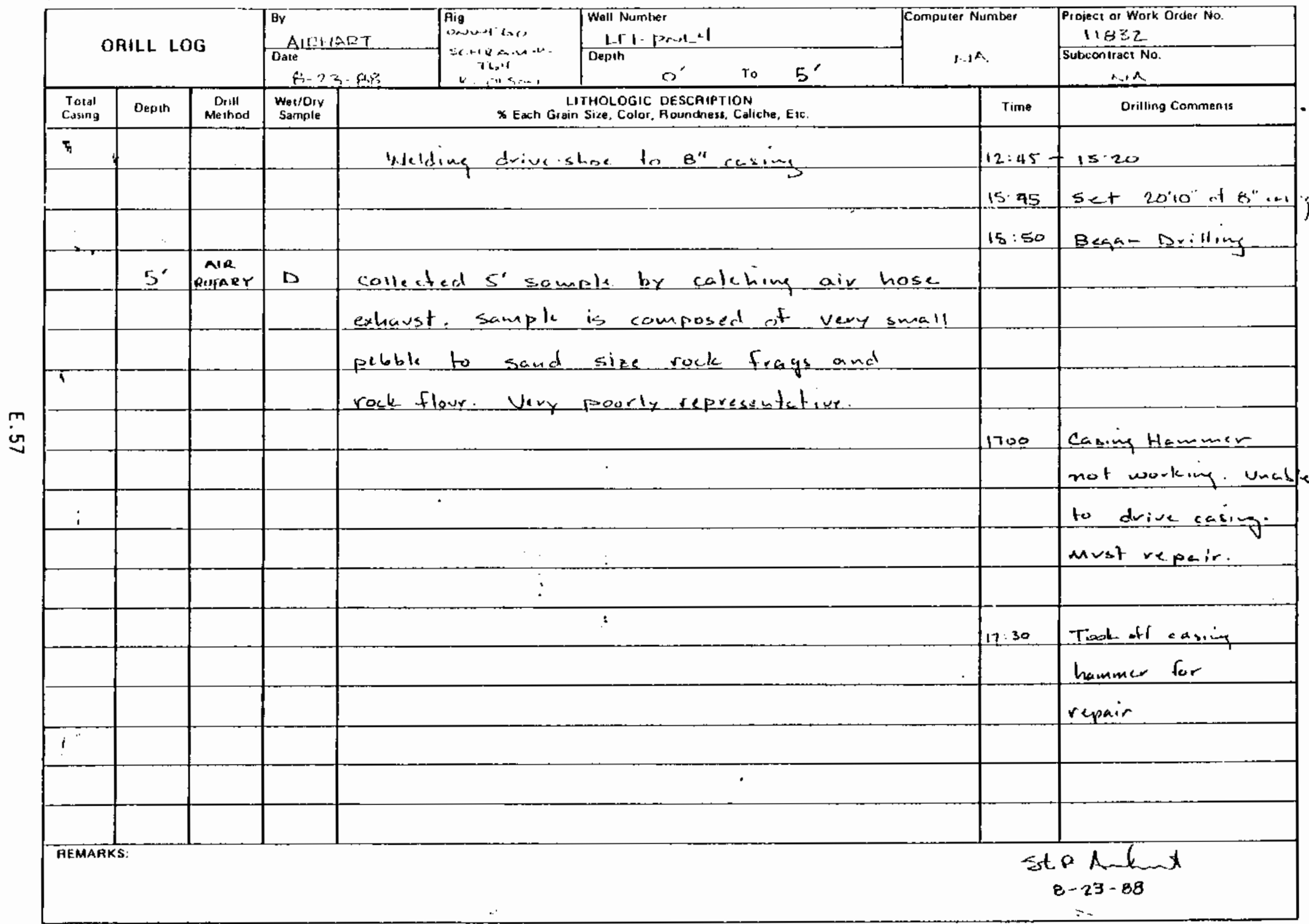

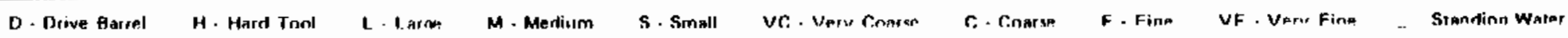


Parz 1 ot 2

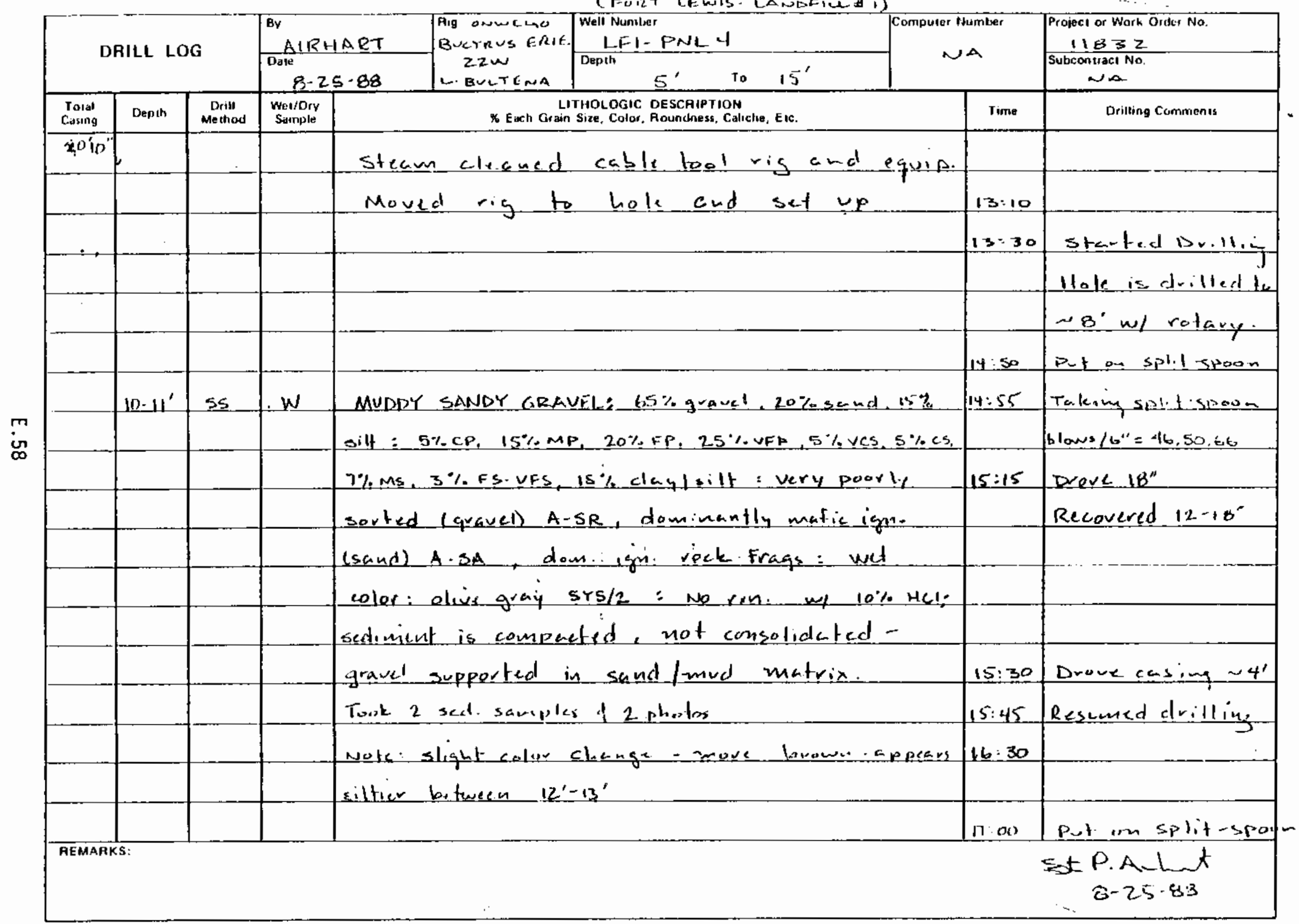

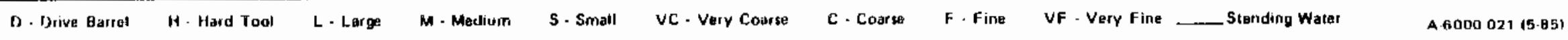


pury 2 if 2

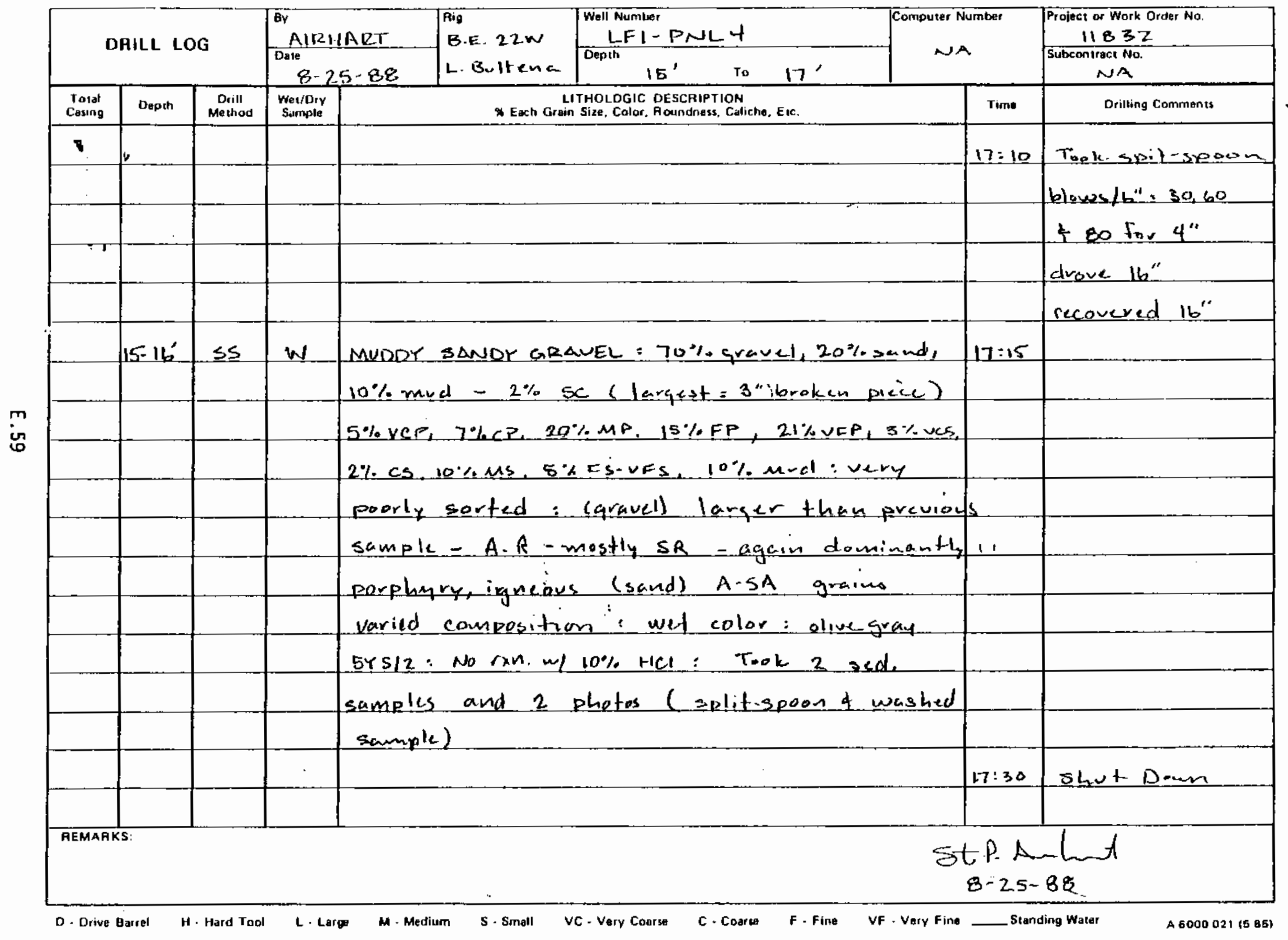




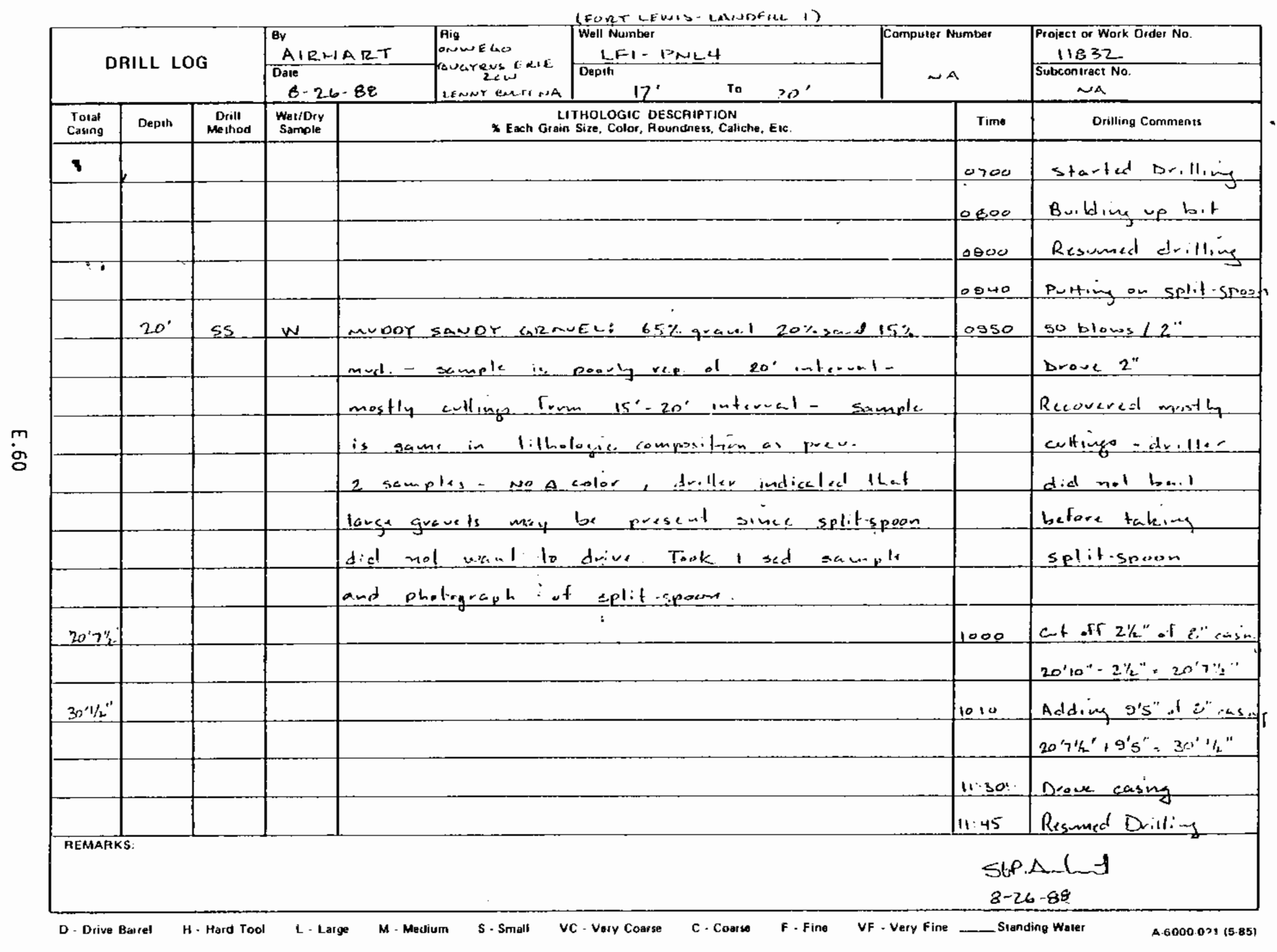


pege $2+14$

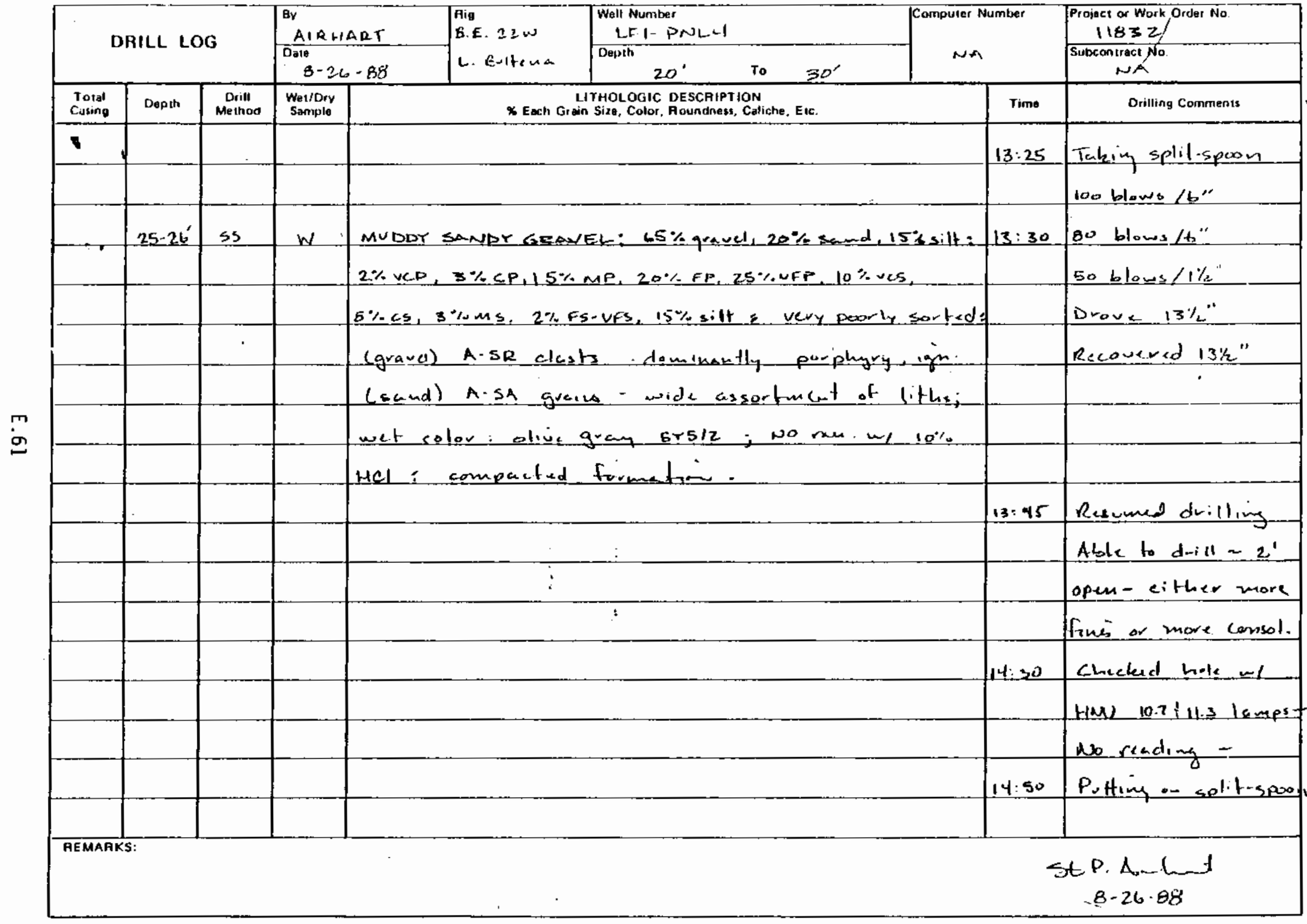
D - Drive Barrel
H. Hard Tood
M. Medium
S. Small
Vr, Vere Gnarse
C. - Conars
F. Fimn
If Y Y w F Finn
4 tantimo Min mor 


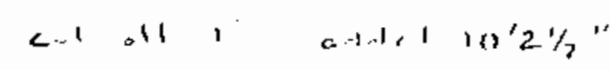

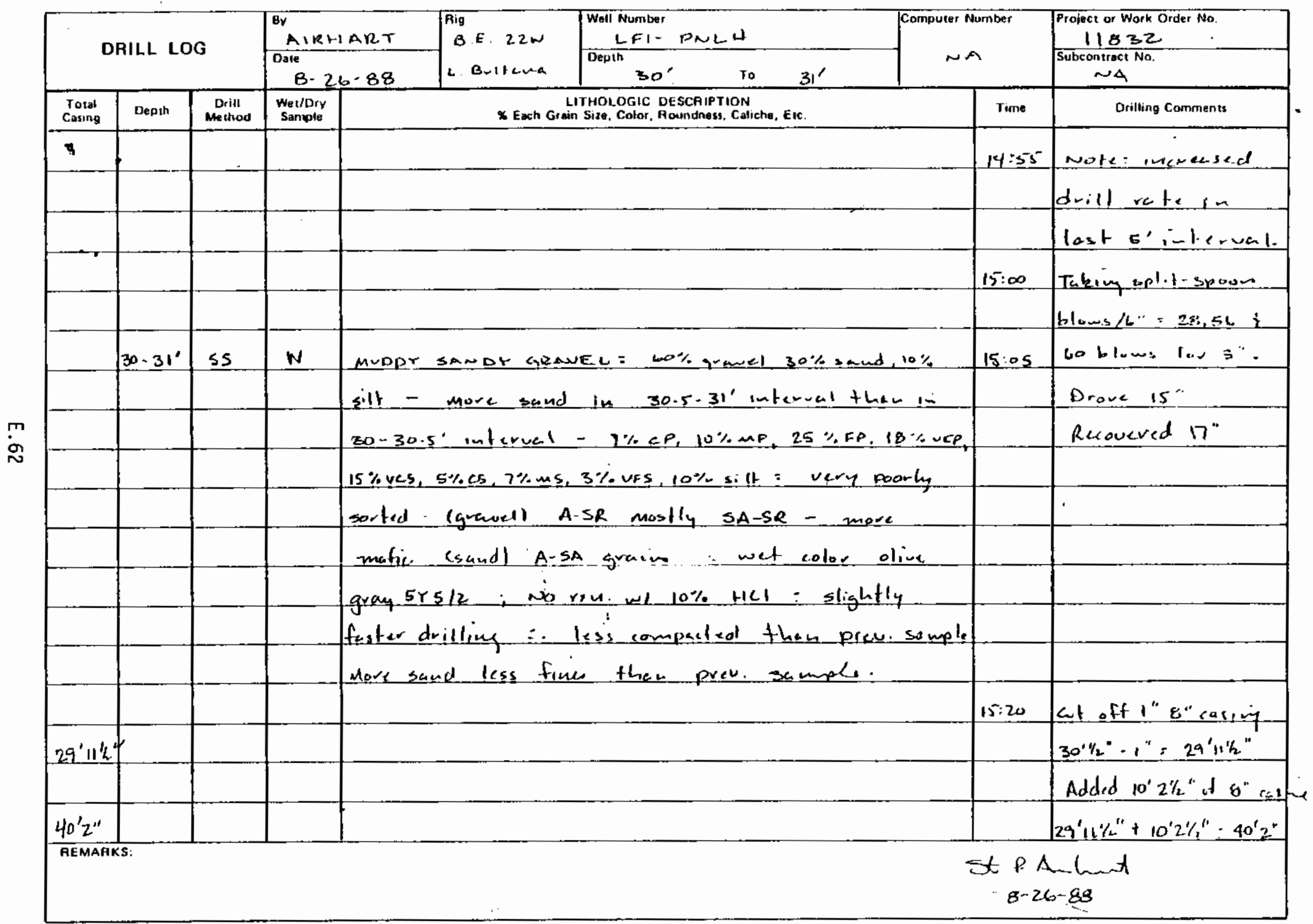

D. Orive Garret

H - Hard Ton

L. L-arne

M-Mertium

Ve, - Whry Corarsp

C. . Cinarse F. Fine

VF . Varu Fino

pare 3 -f 4 
page 4 of 4

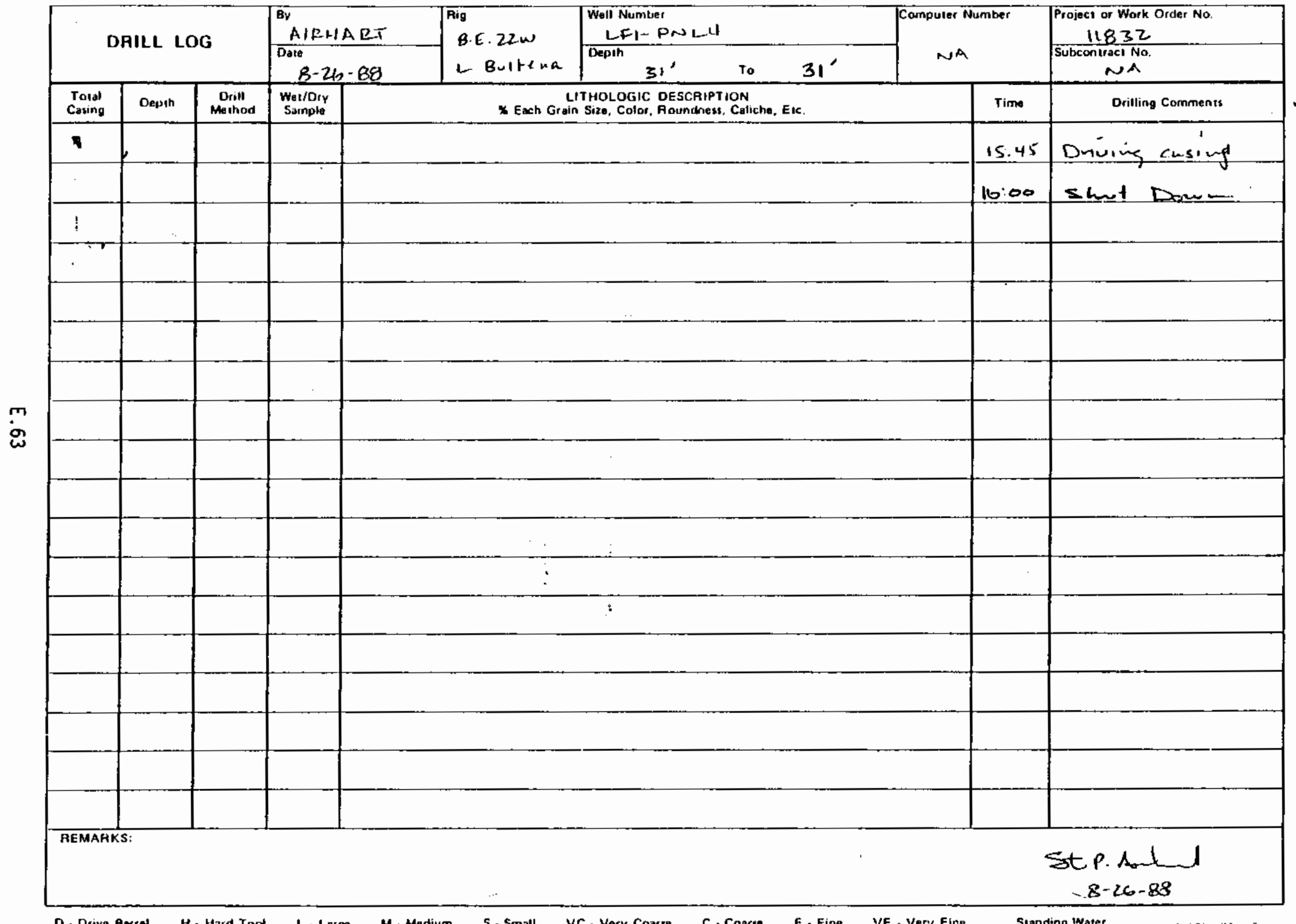

D - Drive Burret

H - Hard Tool

M. Medium

- Small

C. Verv roarsp

c. - Conarse

F - Ver. Finp

Stanninn Watar 


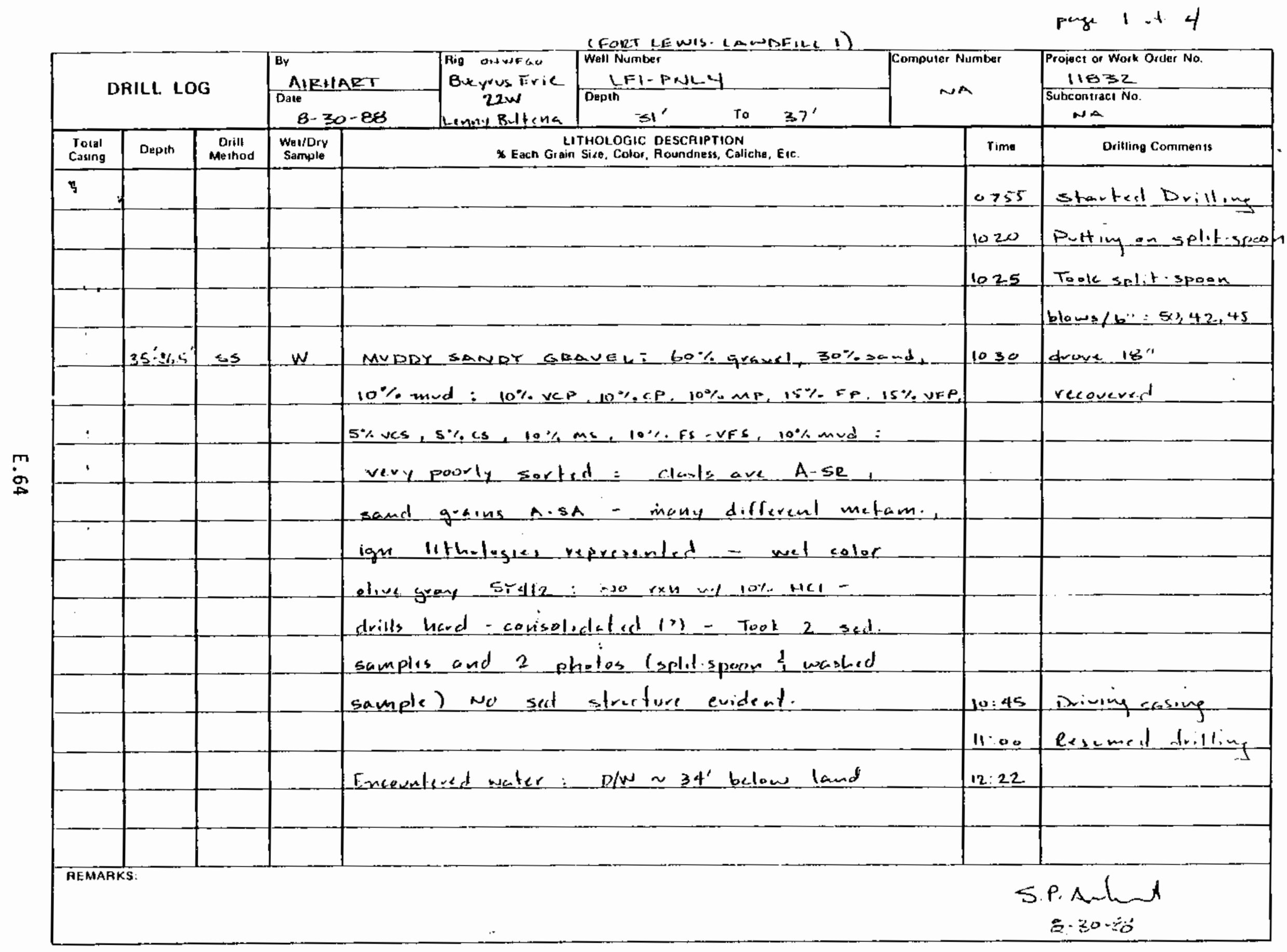




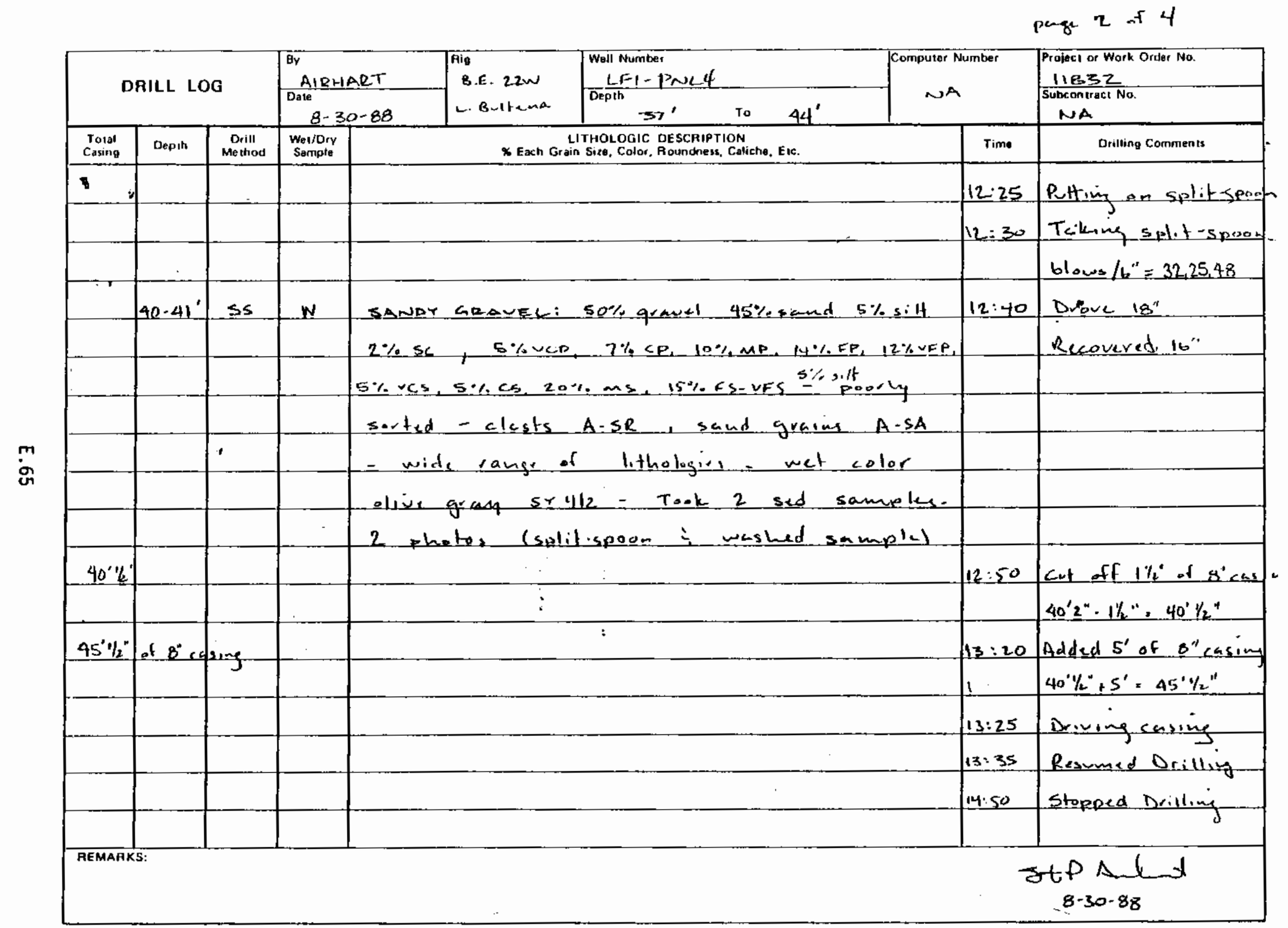

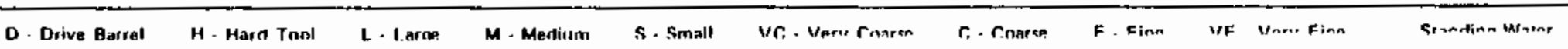




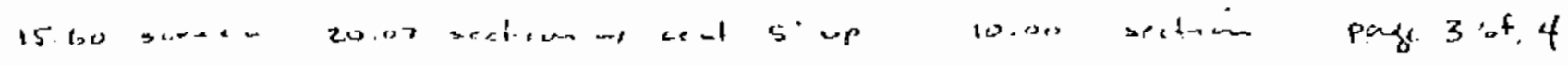

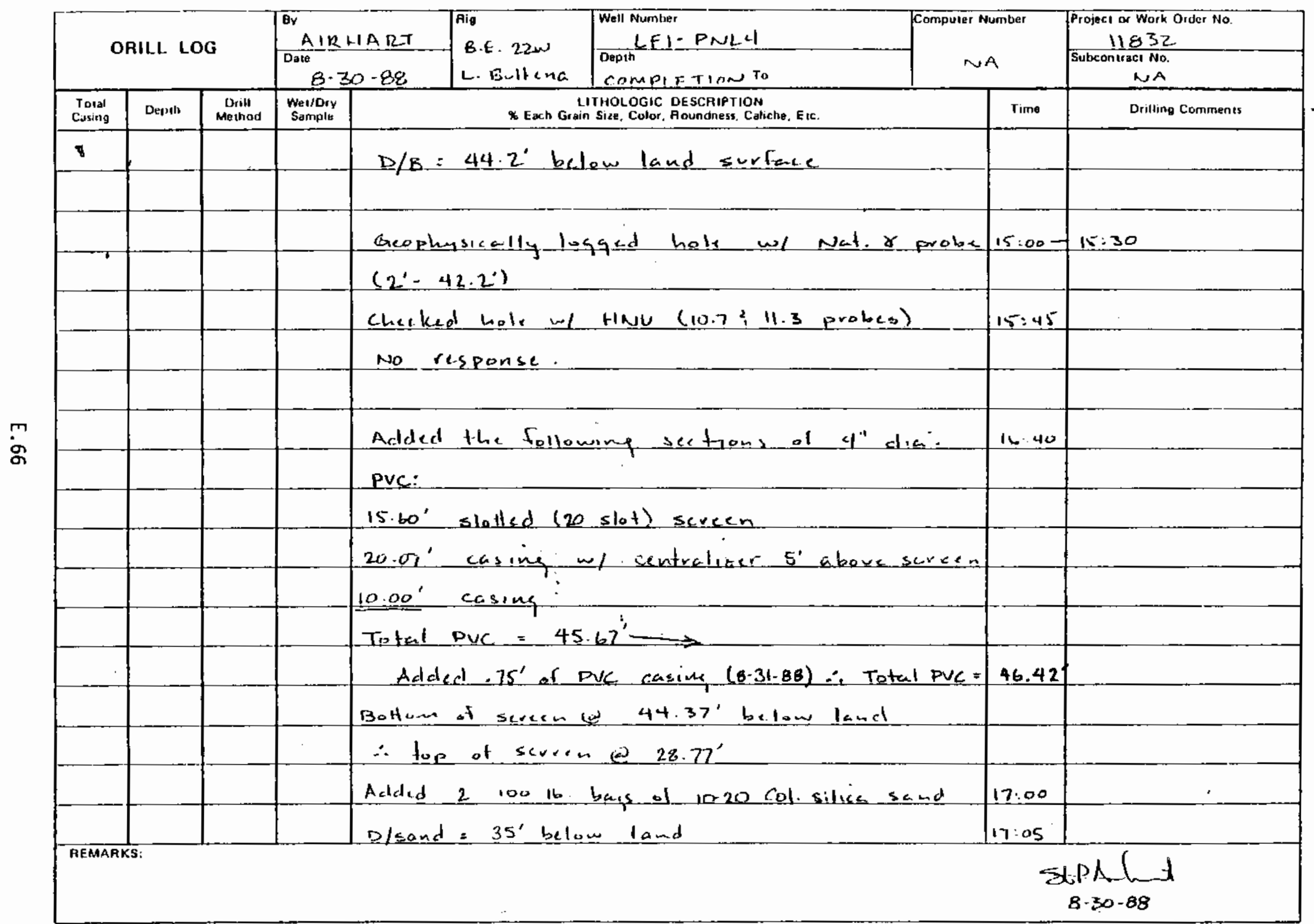

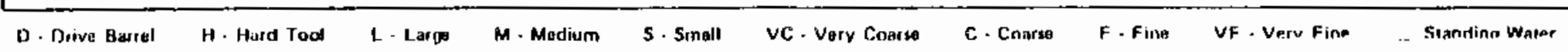


page 4 -f 4

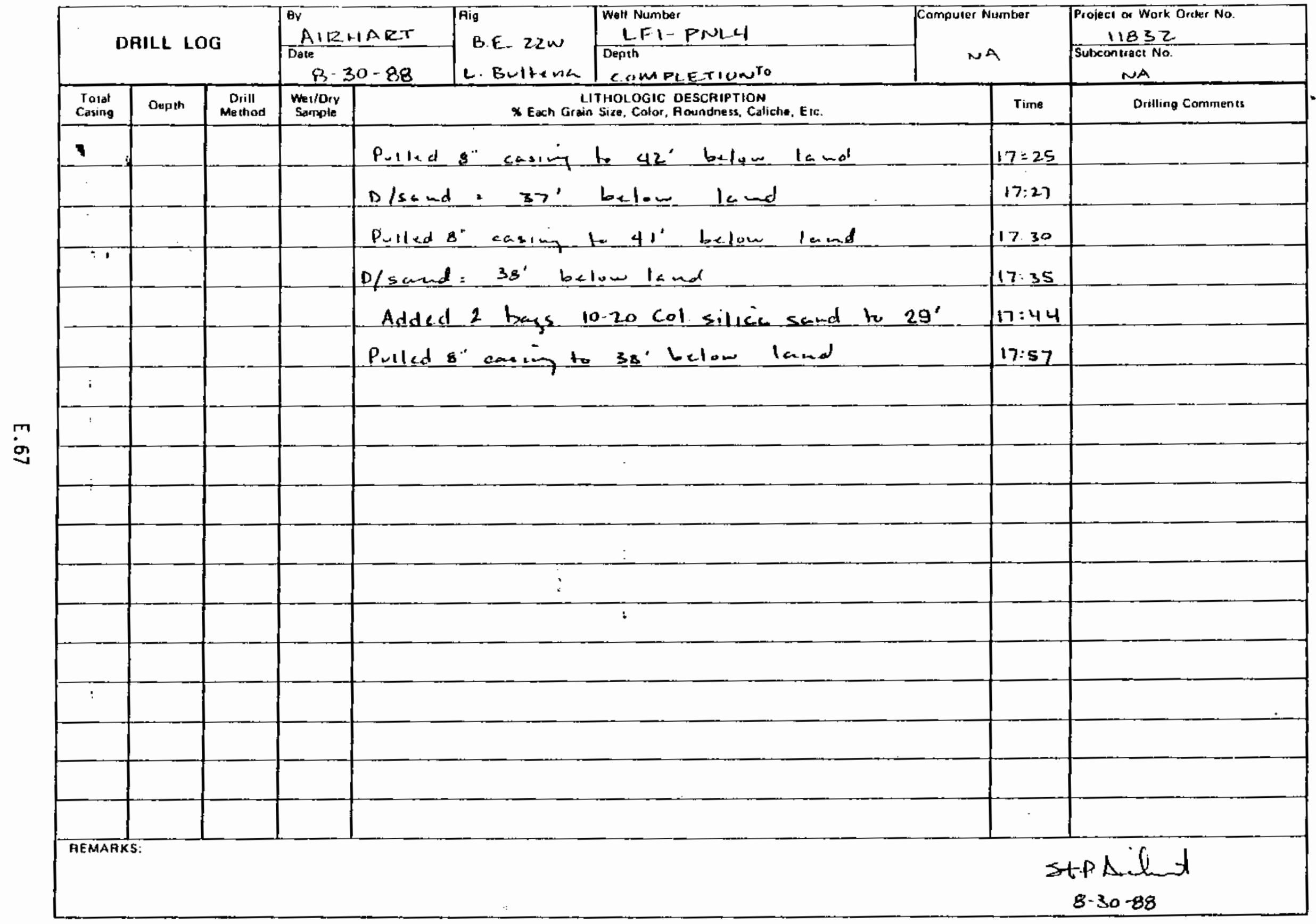

D. Drive Barrel

H - Hard Tool

L. Large

M - Medium

S. Smalt

C. Vatu rearse

r. - Comarse

F. Finn

"re "uncin 
reot 1 .t 2

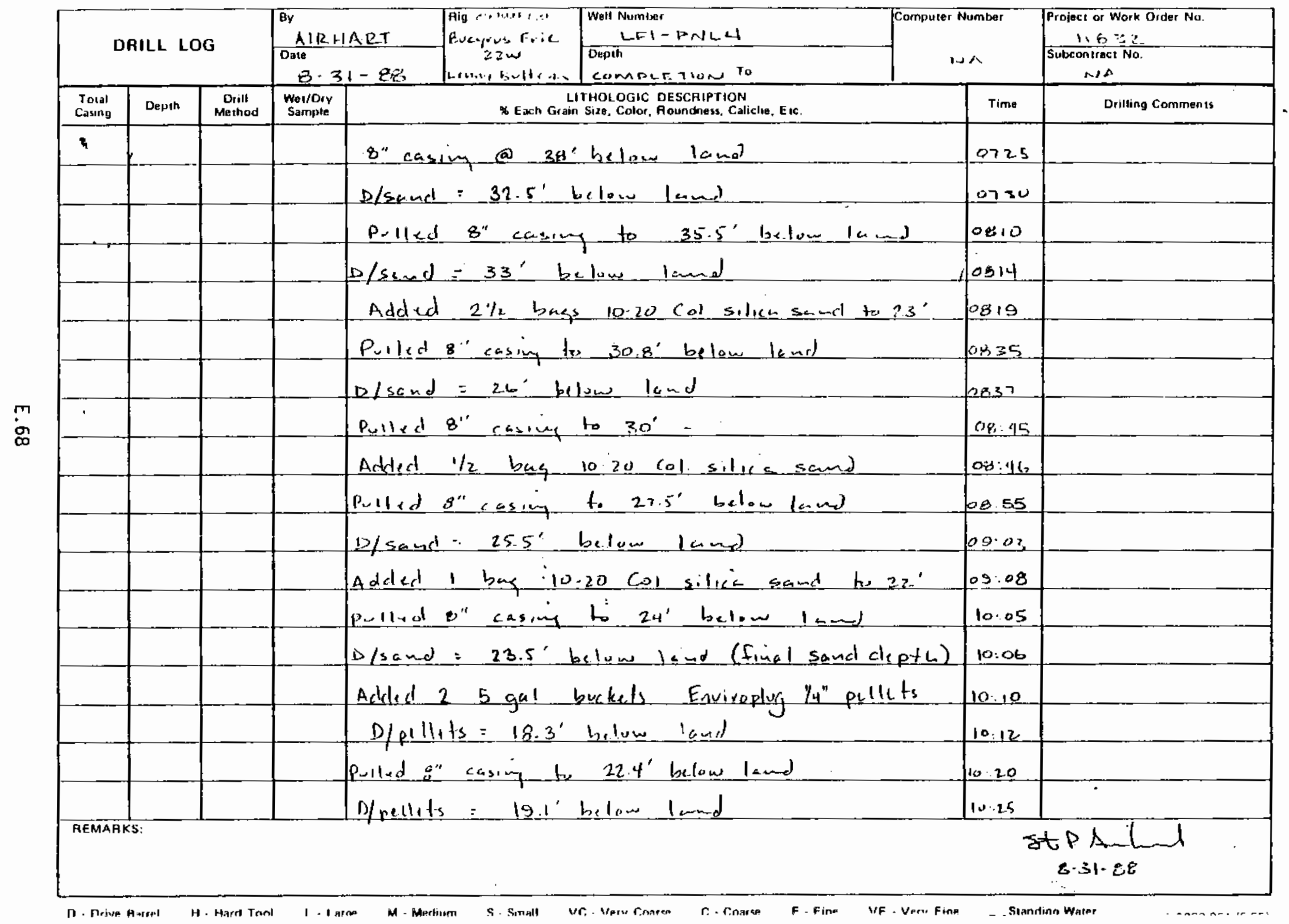


page 2 of 3

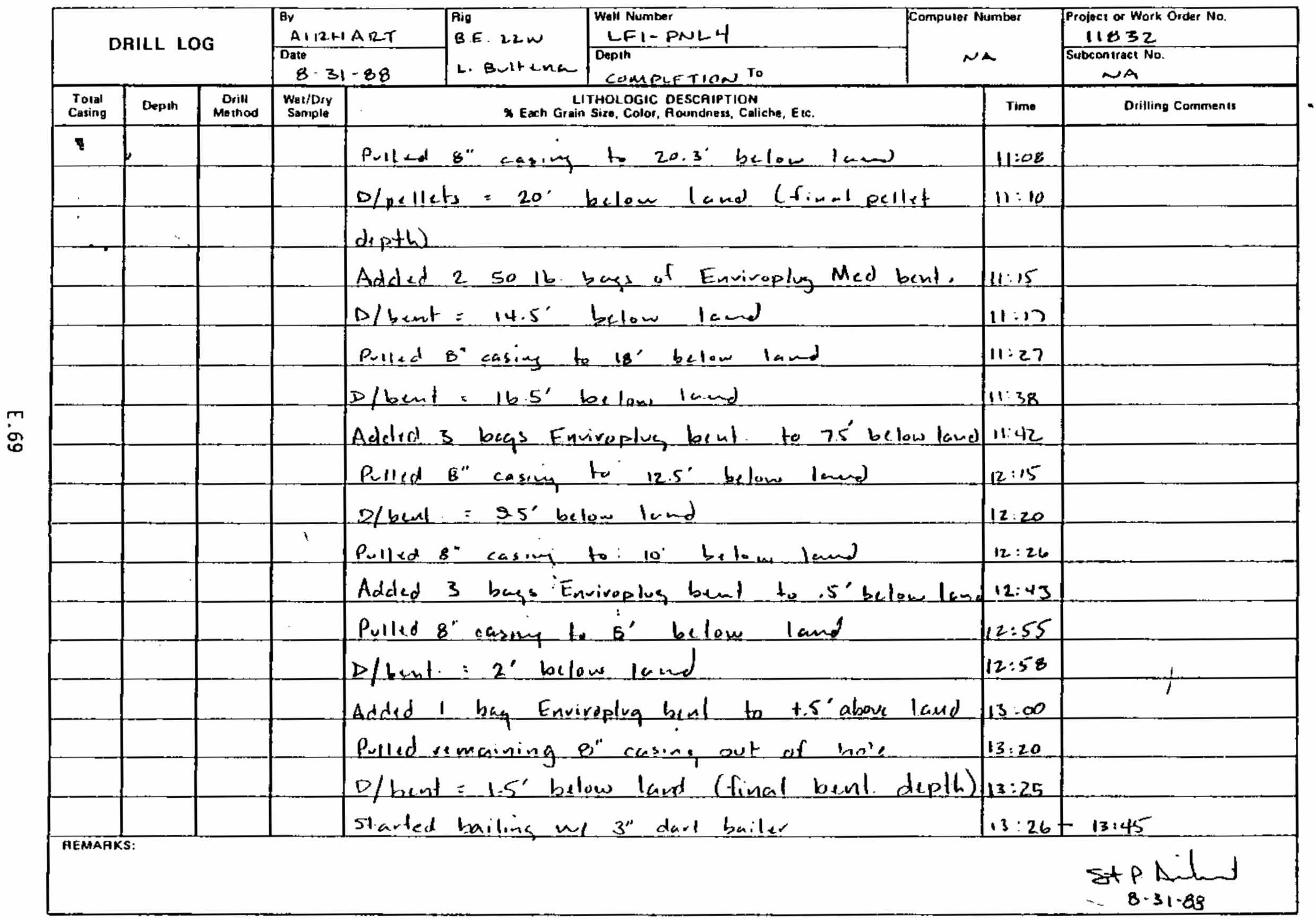




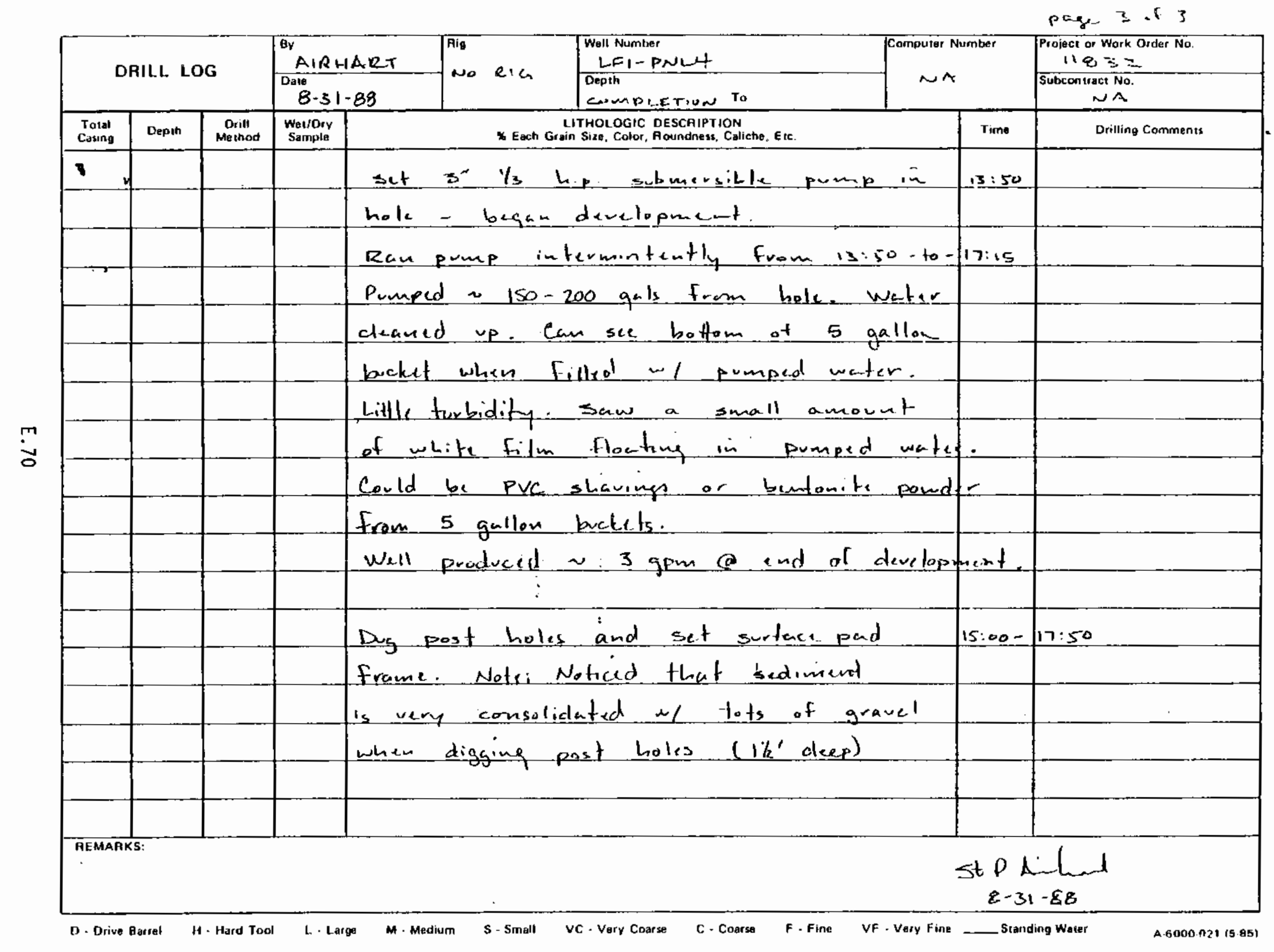


page 1 . I 1

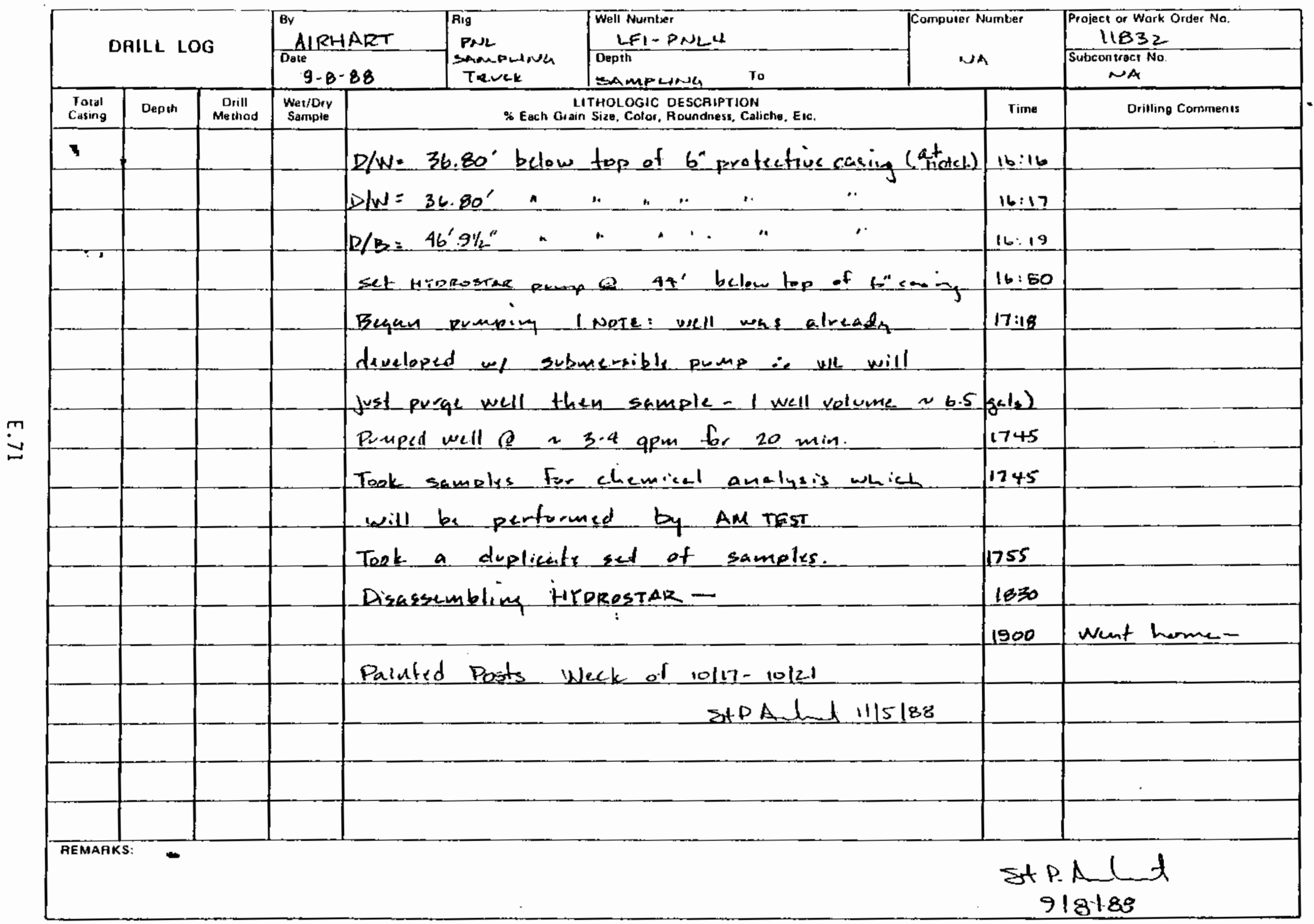

D- Drive Barrel H. Hard Tood L. Large M. Medium S. Small VC-Very Coarte C. Coarse F. Fine VF - Very Fine _C Standing Water 

WELL LF4-PNL1 


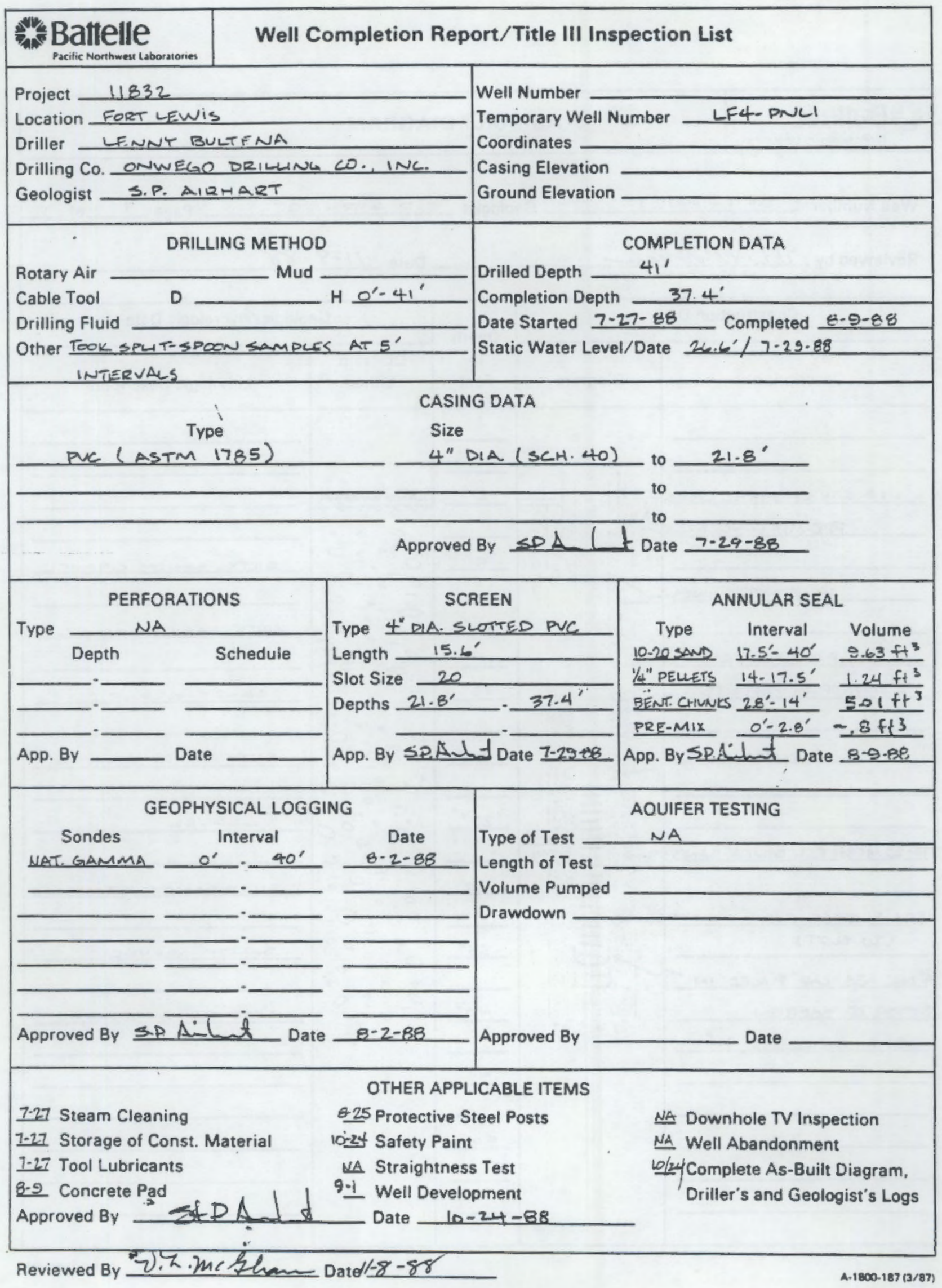




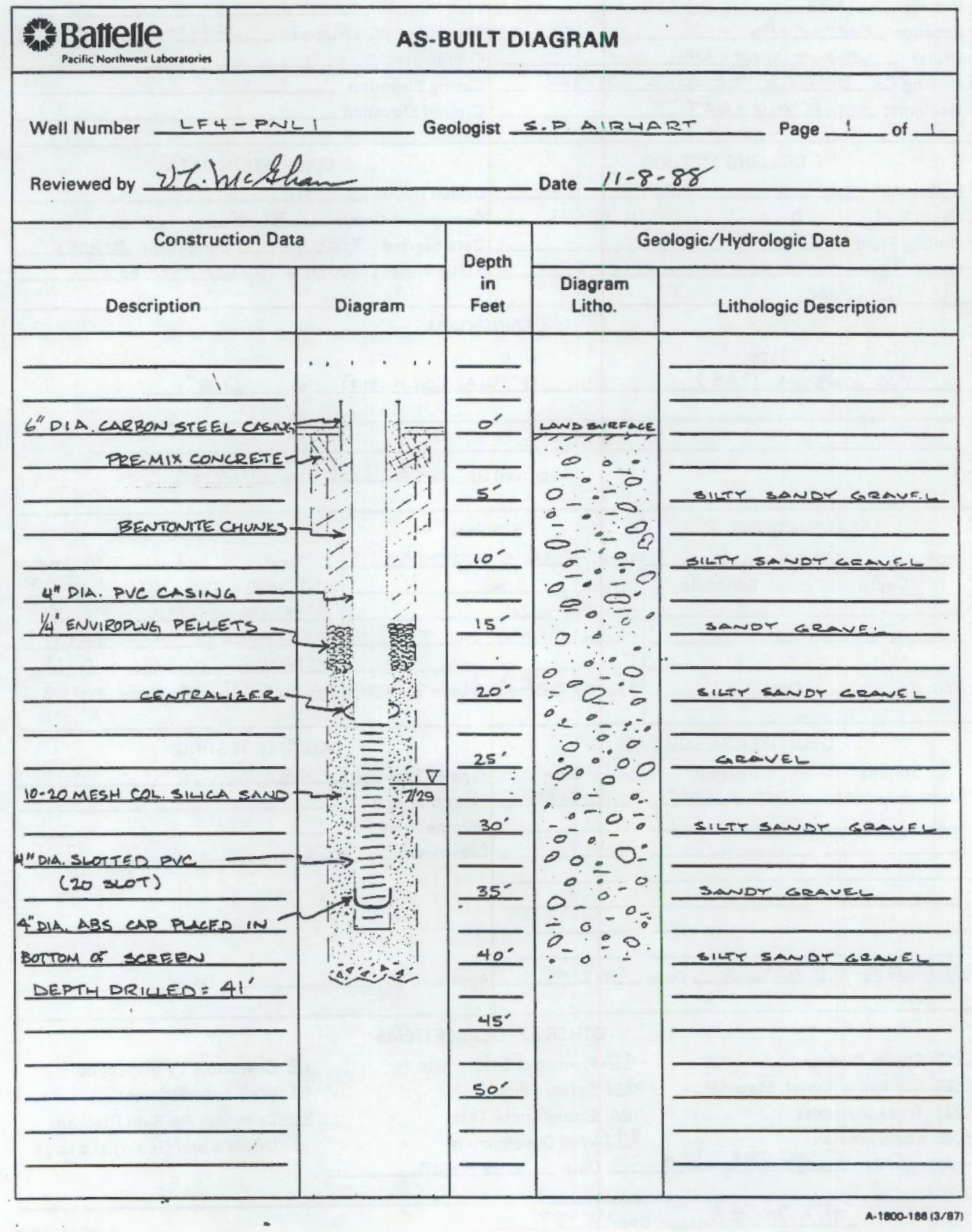




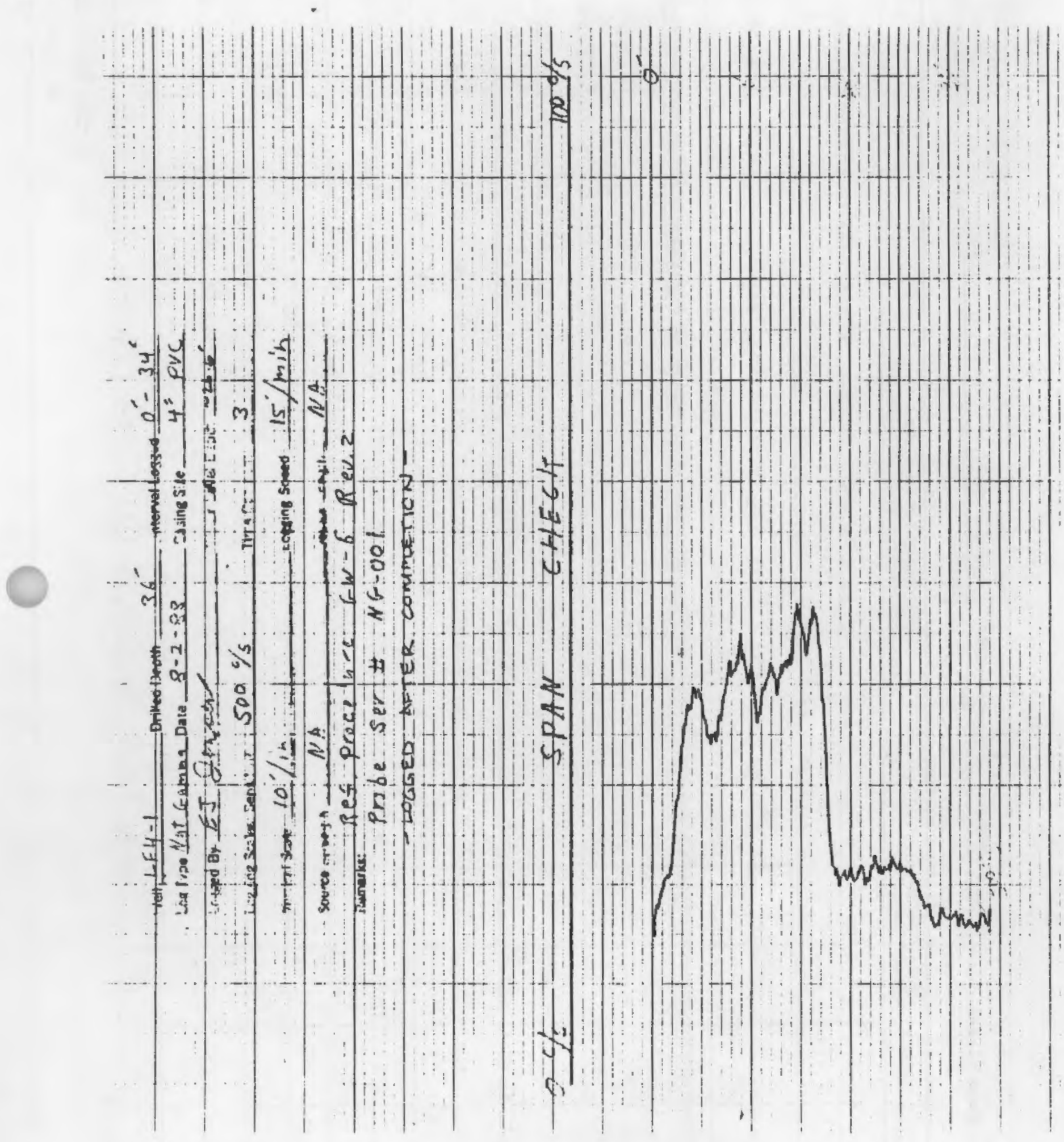


rage 1 of 2

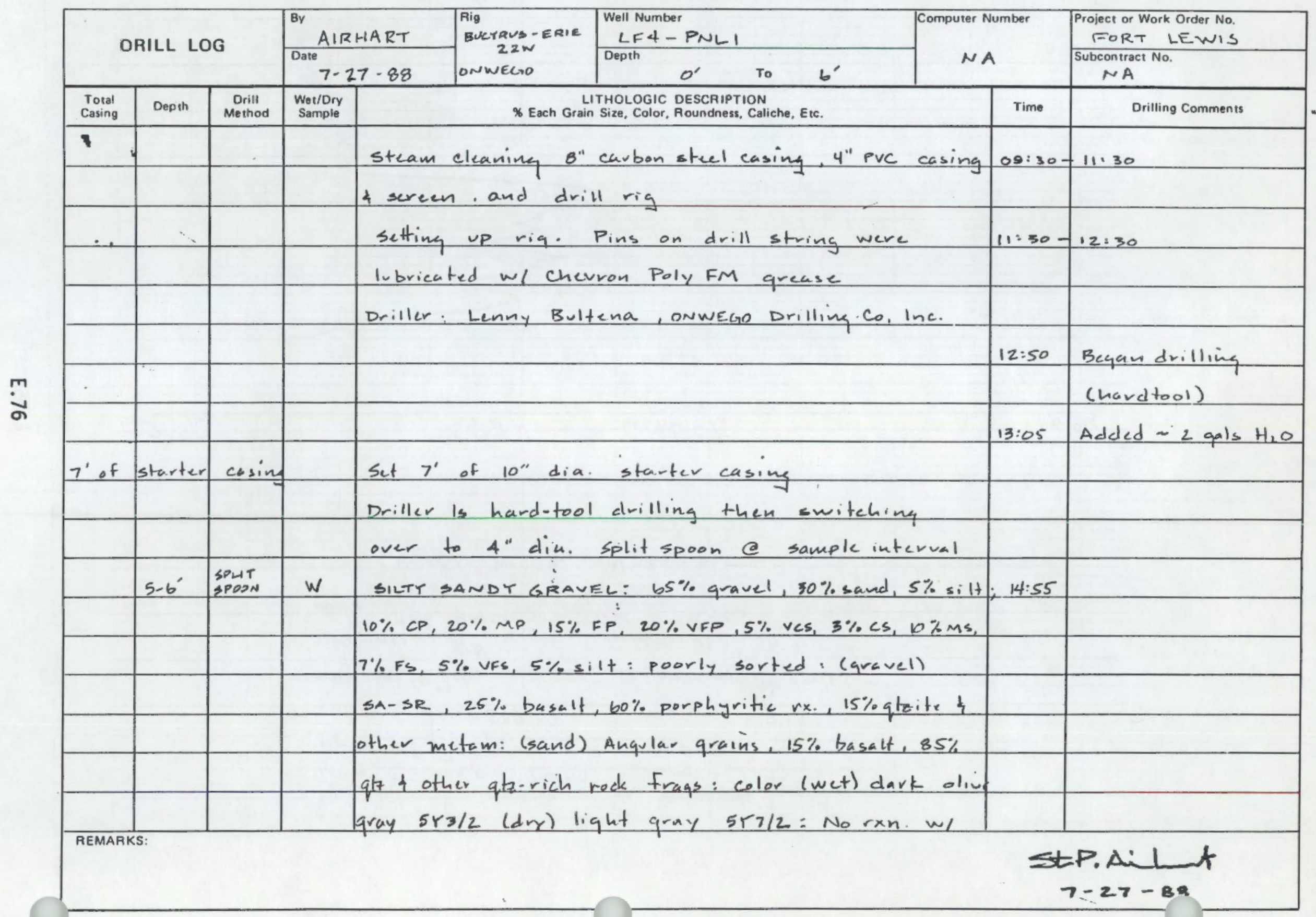


page $z-f 2$

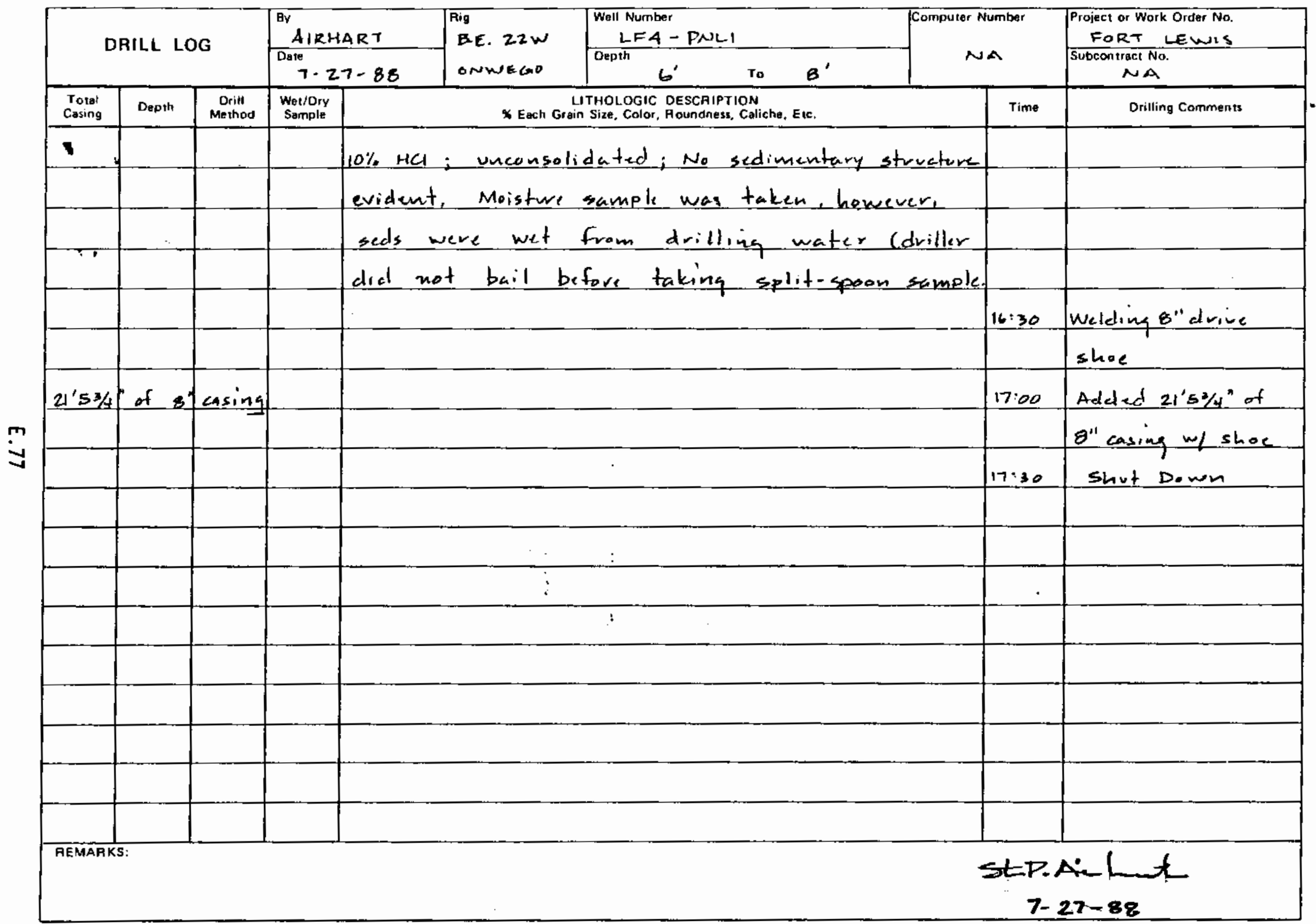

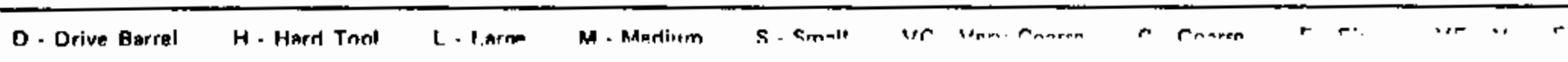


paye ! of 5

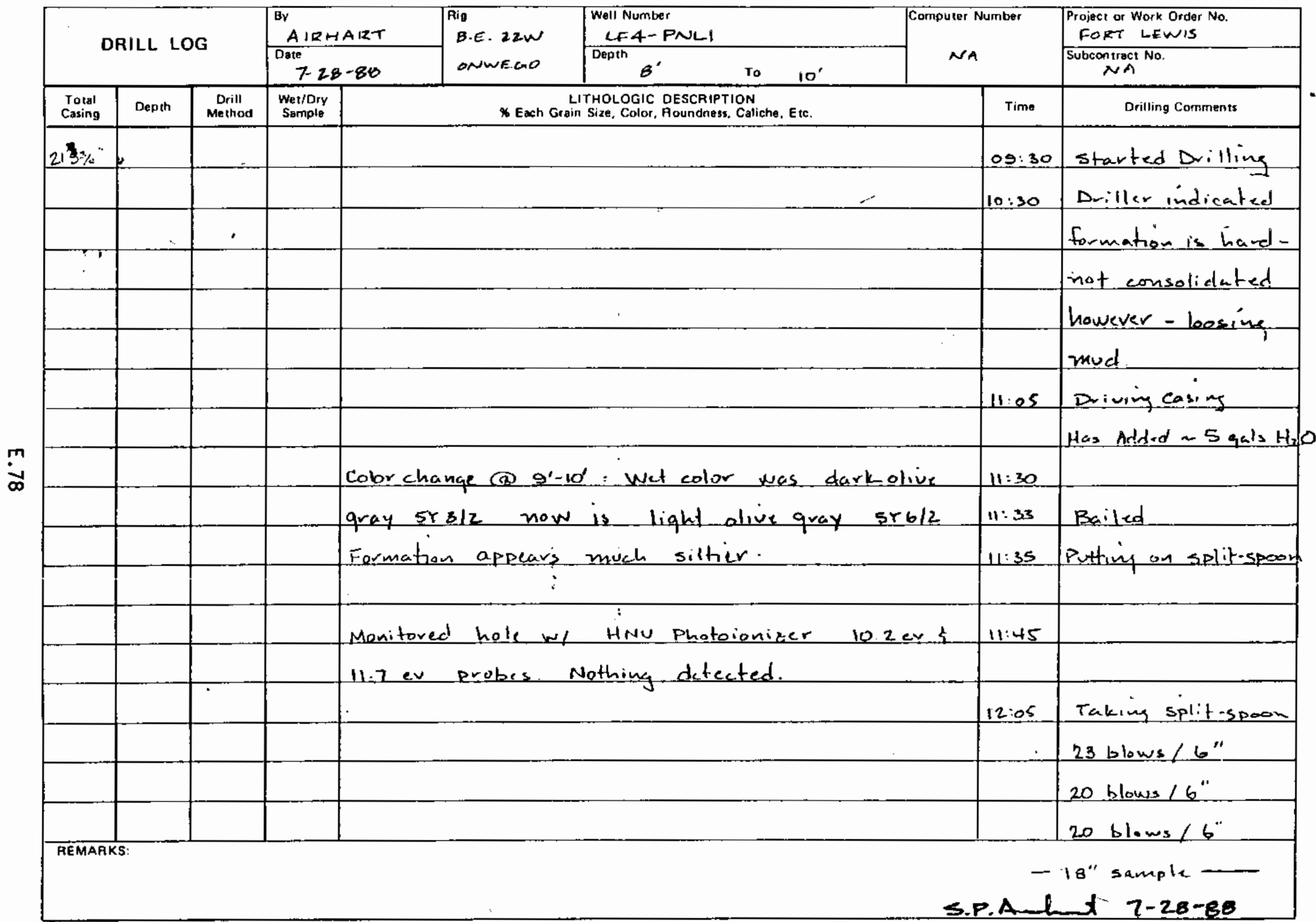




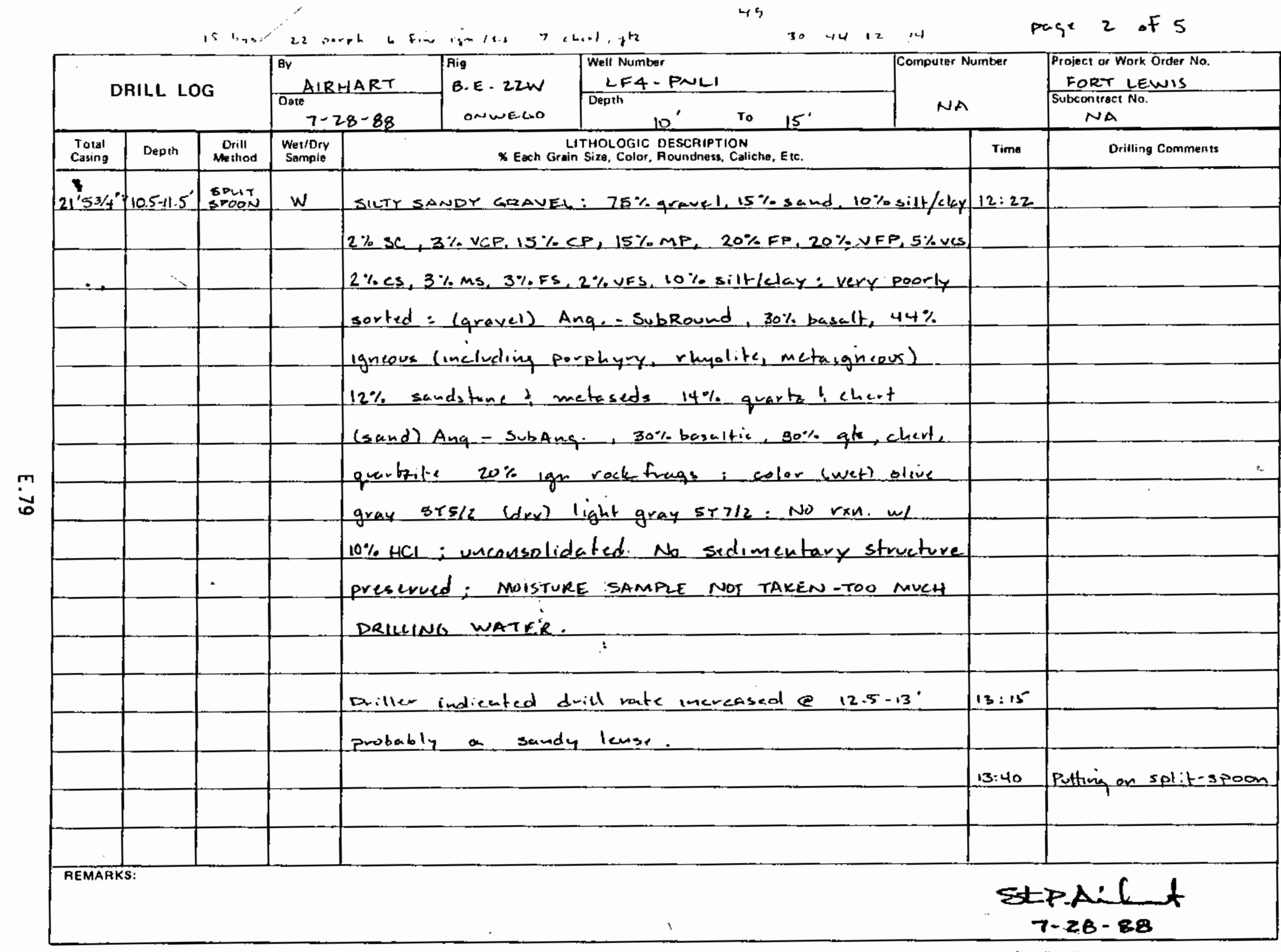




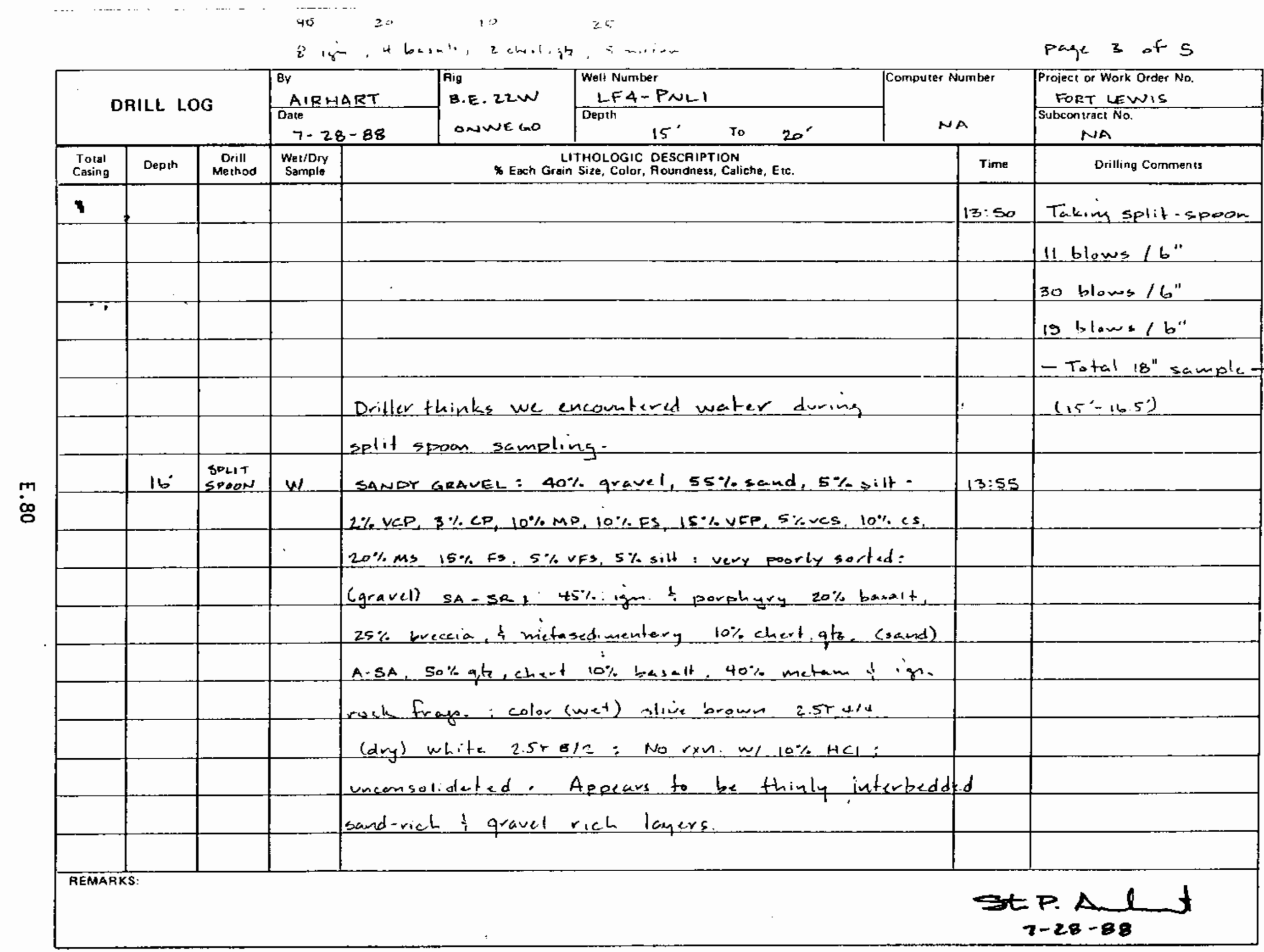

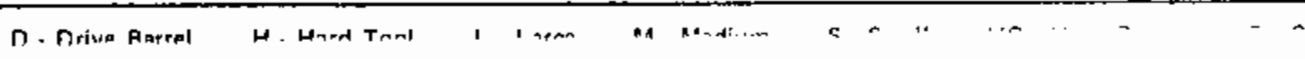




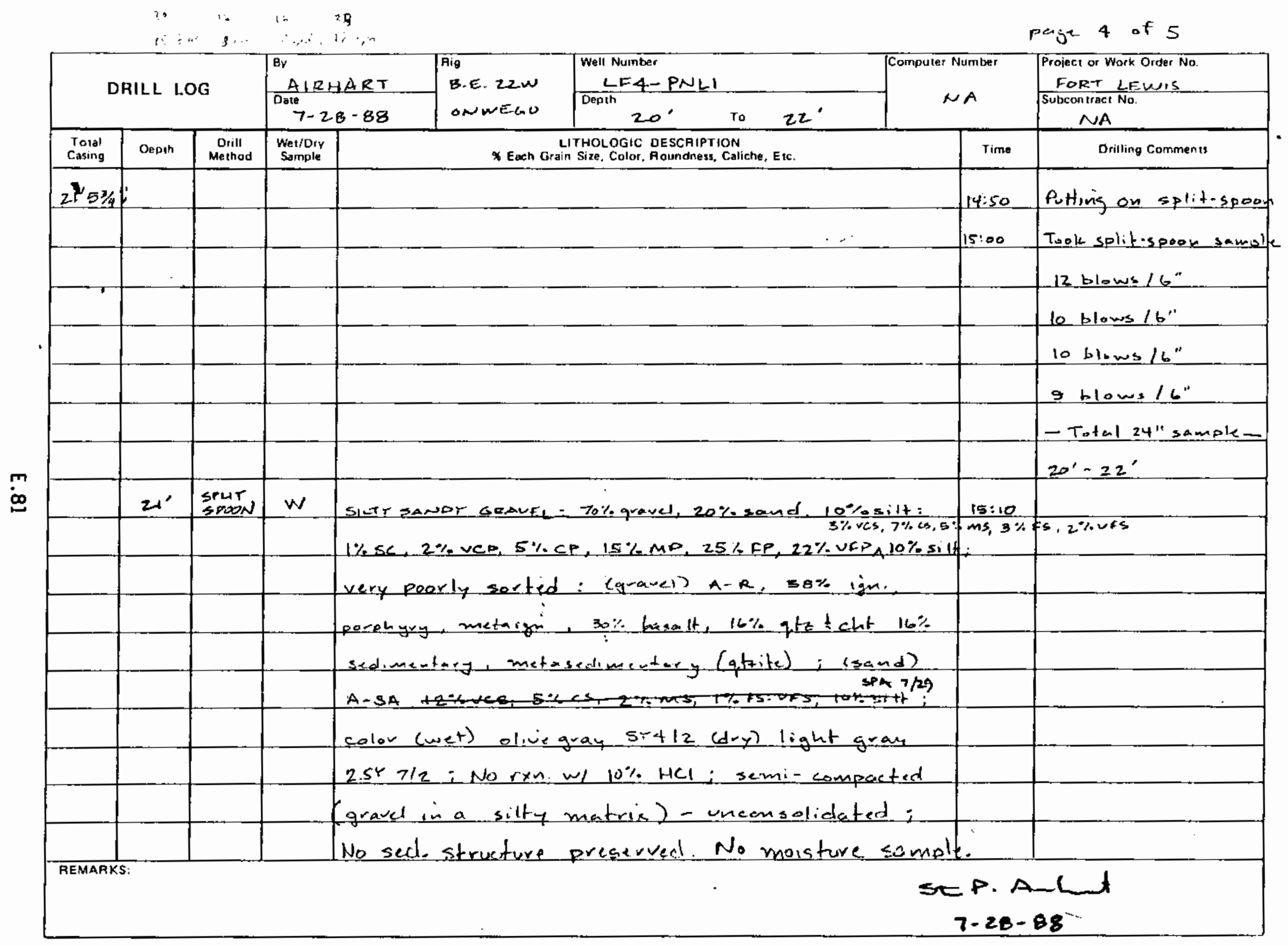

n. Mriwe Aarmi 
Finge $5=55$

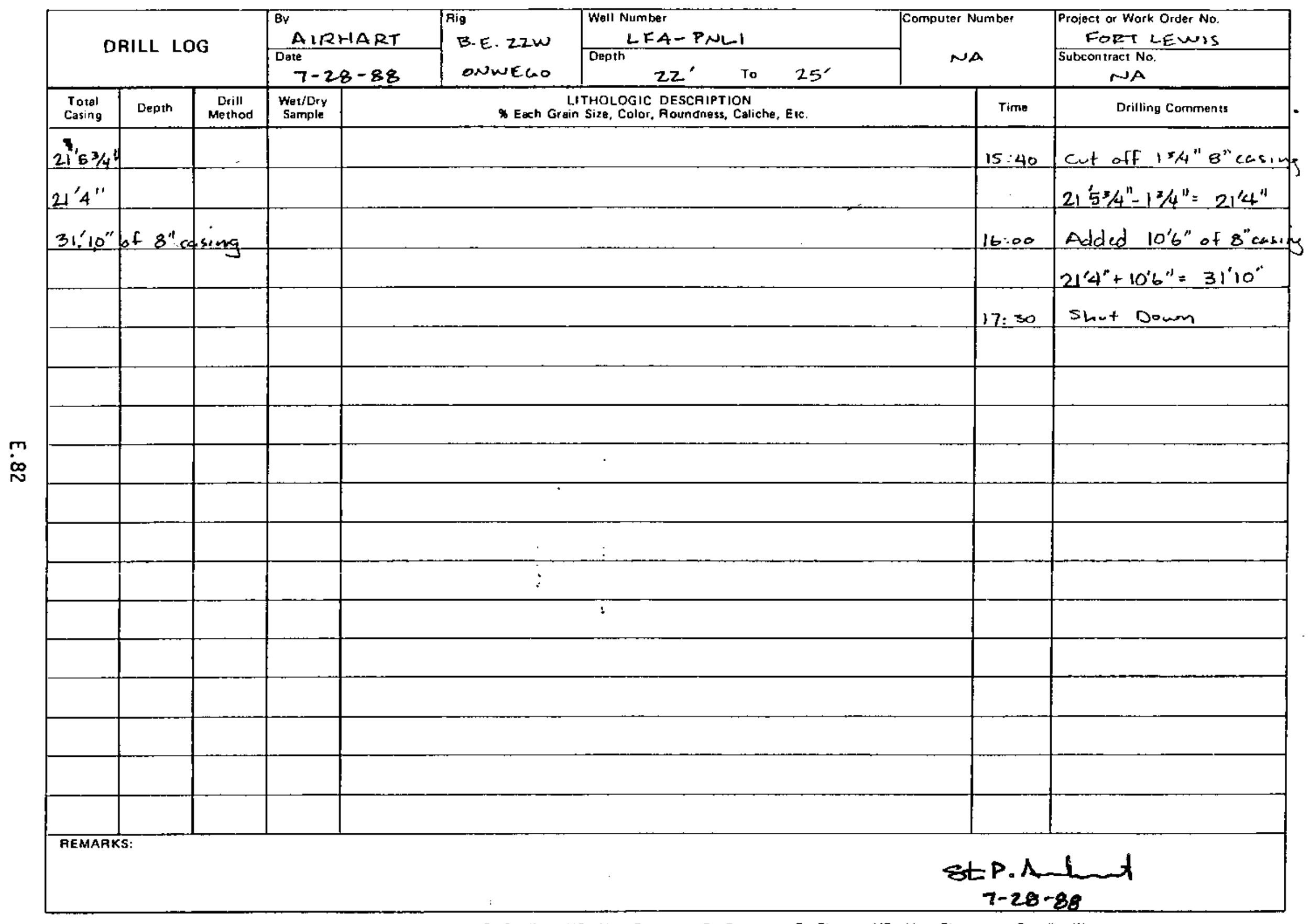
n. Mrive A qural
L. Ware Tanl
M. AAnrti, m
C Small
r. rongren 
poy 1 ot 8

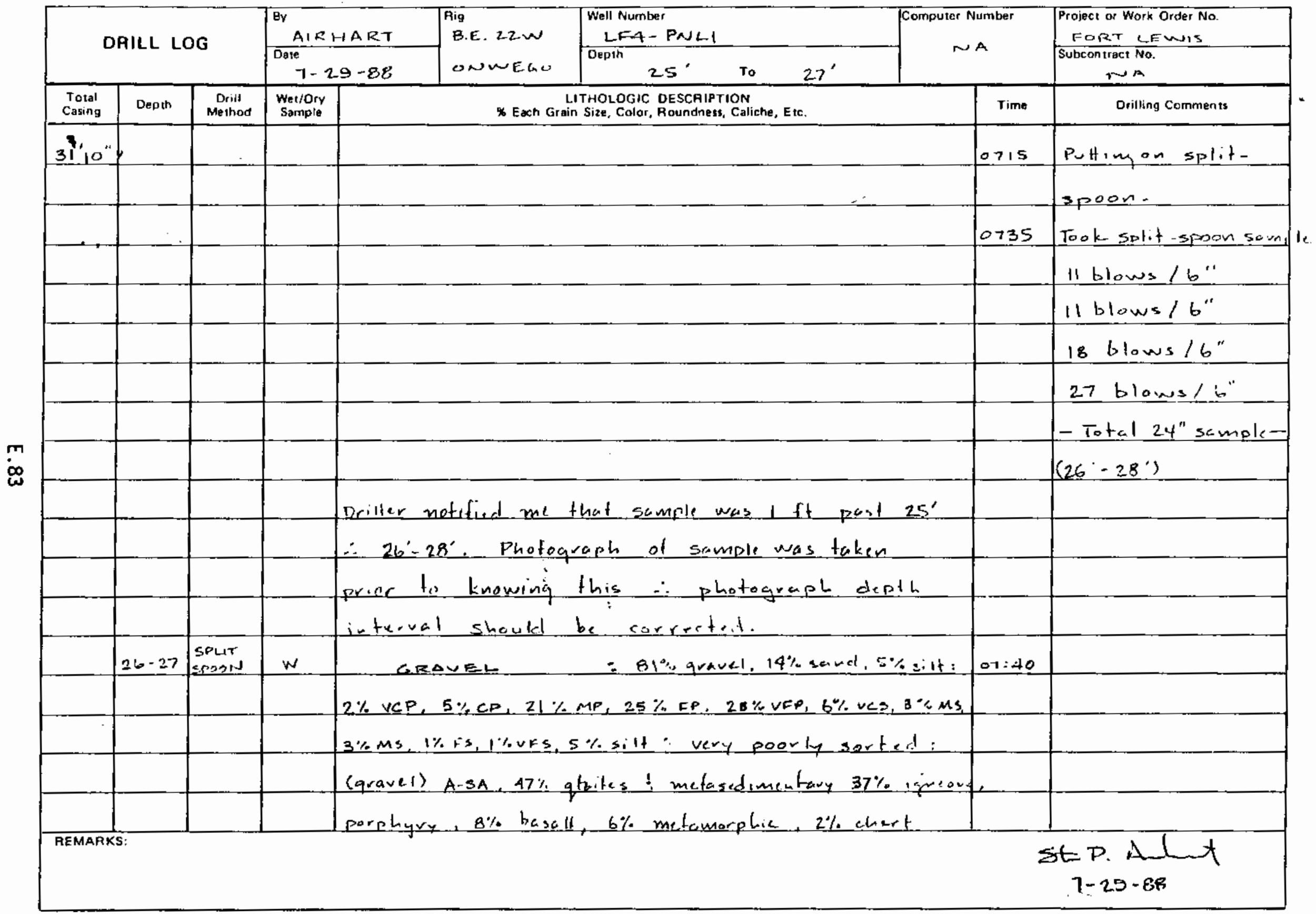

n. nriven Derent 


\begin{tabular}{|c|c|c|c|c|c|c|}
\hline \multirow{2}{*}{\multicolumn{3}{|c|}{ DRILL LOG }} & \multirow{2}{*}{\begin{tabular}{|c|} 
BY \\
AIRHAART \\
Date \\
$7-29-88$ \\
\end{tabular}} & \multirow{2}{*}{\multicolumn{2}{|c|}{ 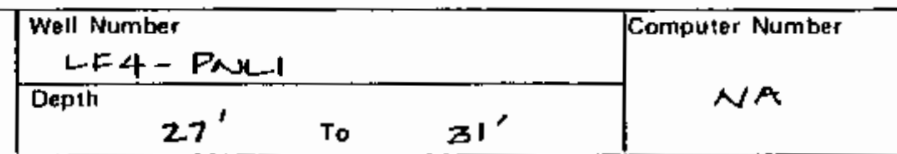 }} & \multirow{3}{*}{ 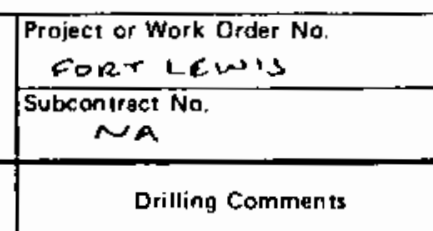 } \\
\hline & & & & & & \\
\hline $\begin{array}{c}\text { Total } \\
\text { Casing }\end{array}$ & Oepin & $\begin{array}{c}\text { Drill } \\
\text { Method }\end{array}$ & $\begin{array}{c}\text { Weatidry } \\
\text { Sampite }\end{array}$ & * Eech Grain SizoL.OGIC OEGSCRIPTION & Time & \\
\hline $31^{\prime \prime 1} 0^{\prime \prime}$ & & & & (sand) A-SA, $70 \%$ glt 1 q q trite, $10 \%$ basclt, $20 \%$ & & \\
\hline & & & & Igne I metan rock frays : coler (wet) olive & & \\
\hline & & & & $5 r 513$ (dru) light gray $5 Y 7 / 2$; No ran w/ $10 \%$ HCl; & & \\
\hline & & & & uncongolidated: No sed structure preserved. No & & \\
\hline & & & & moisture sample taken. Nore: Coarsening & & \\
\hline & & & & evident a end of sample interval (28). & $O B: 30$ & Putting on split-spoon \\
\hline & & & & & le:40 40 & Fook spilt spoon stmpl. \\
\hline & & & & & & 7 bions 16. \\
\hline & & & & . & & 8 blows / 6 " \\
\hline & & & & & & I blowe / 60 \\
\hline & & & &. & & $0+1.02=16^{\circ}$ \\
\hline & & & & $\therefore$ & & - 24" sumple - \\
\hline & & & & 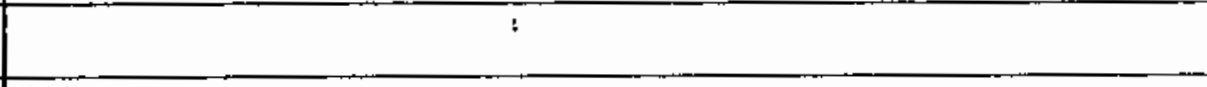 & & $30^{\prime}-32^{\prime}$ \\
\hline & $31^{\prime}$ & $\begin{array}{l}\text { Sput } \\
\text { Spoon } \\
\end{array}$ & $w$ & Sintr SANDT GRAVEL: 70\% quavel, $25 \%$ sand, $5 \%$ silt: & $00: 50$ & \\
\hline & & & & $1 \% \operatorname{VCr}(50 \% \mathrm{~m}), 3 \% \leq P, 5 \%, \mathrm{mr}, 26 \% F P, 35 \%$ VFP, $10 \% \mathrm{kcs}$ & & \\
\hline & & & & $7 \%$ cs $5 \%$ Ms $2 \%$ es $1 \%$ VEs $5 \%$ silt : very poorly & & \\
\hline & & & & sortied : (grave1) $A-S R, 40 \%$ qfaite, $40 \%$ iquerus meta & . & \\
\hline & & & & porphyvy $10 \%$ ahe cht $10 \%$ baselt (sand) A-SA & & \\
\hline AEMAA & & & & & Anc & 21 \\
\hline
\end{tabular}


pay $3 .+B$

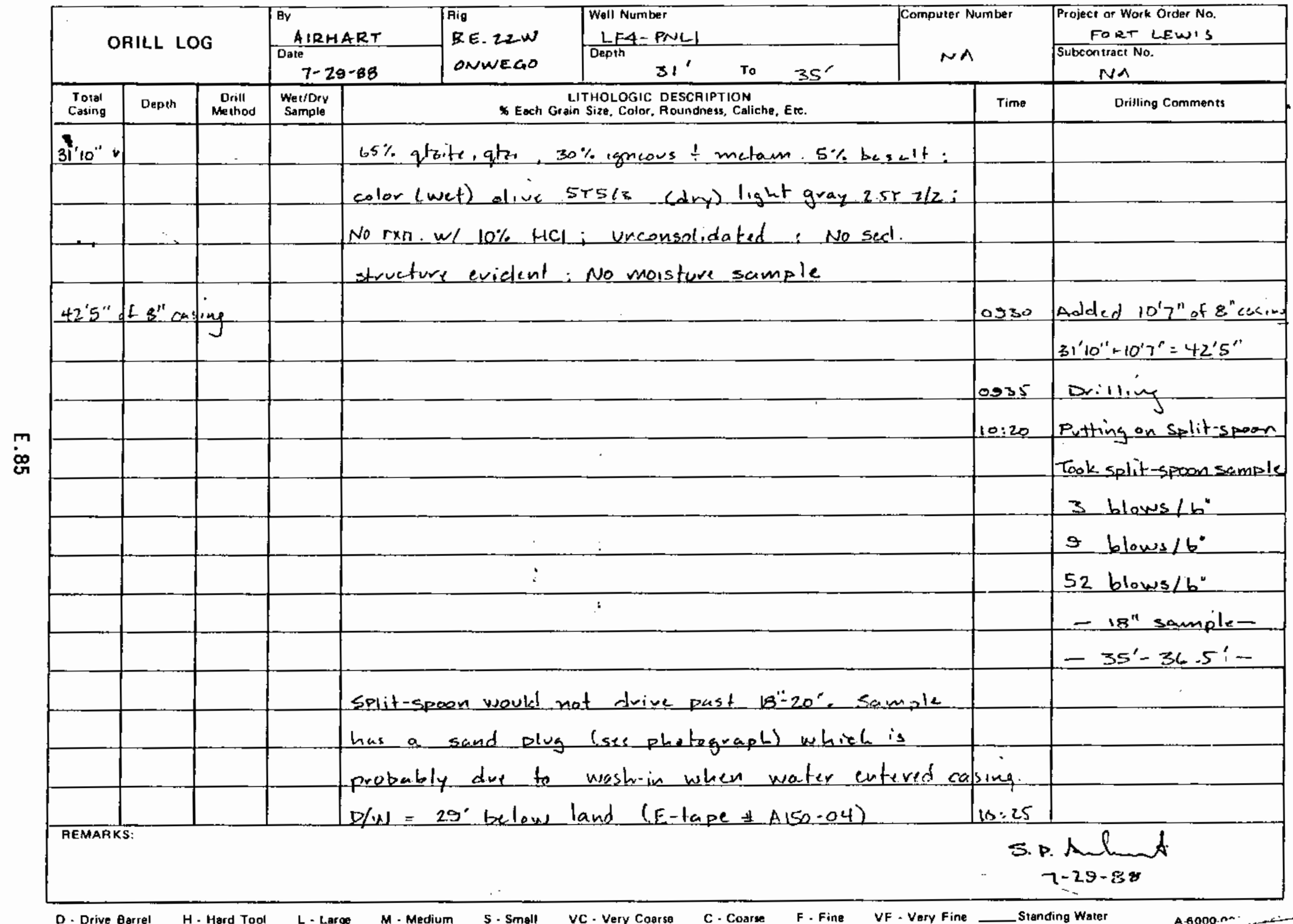


pose 4 of 8

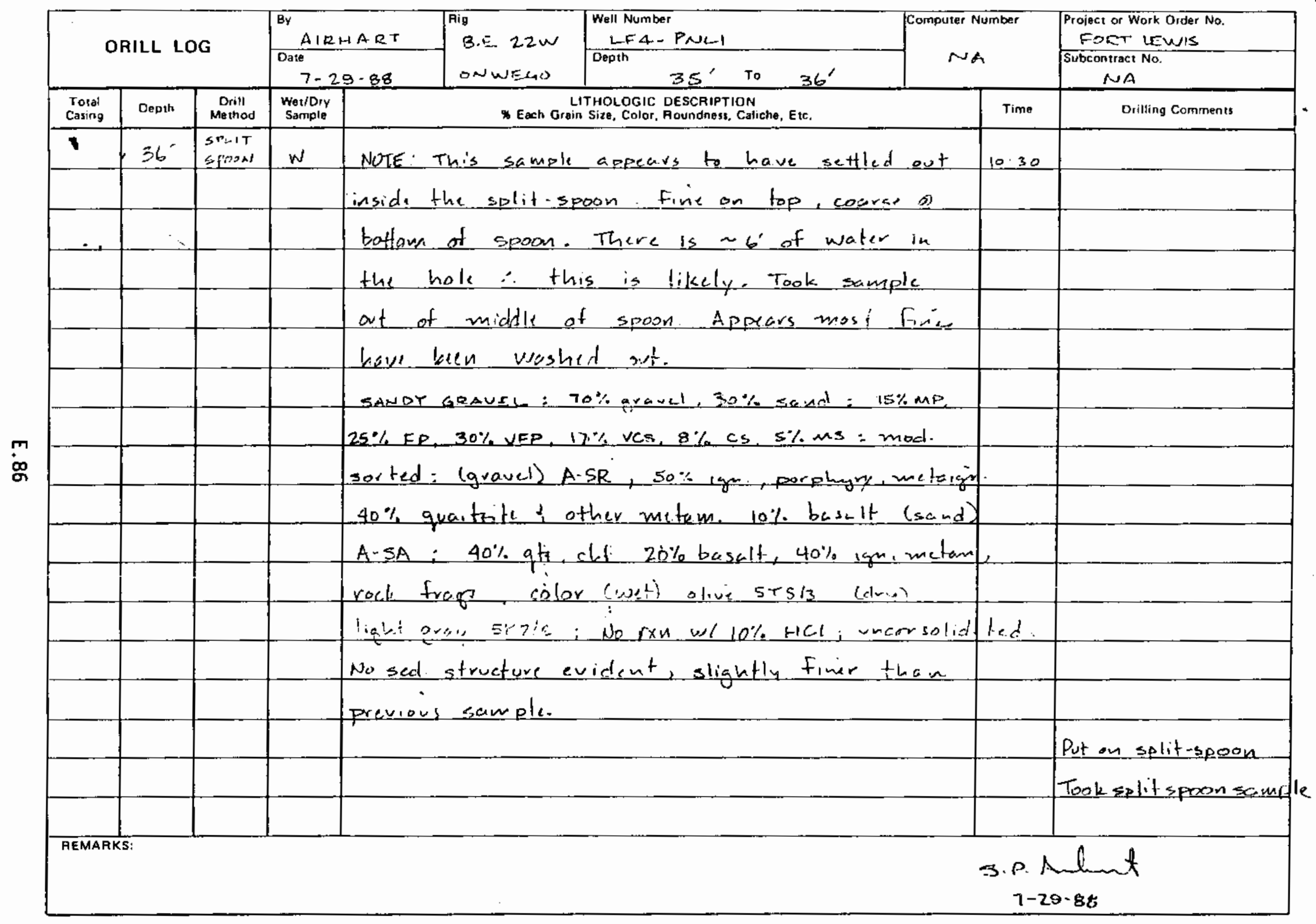




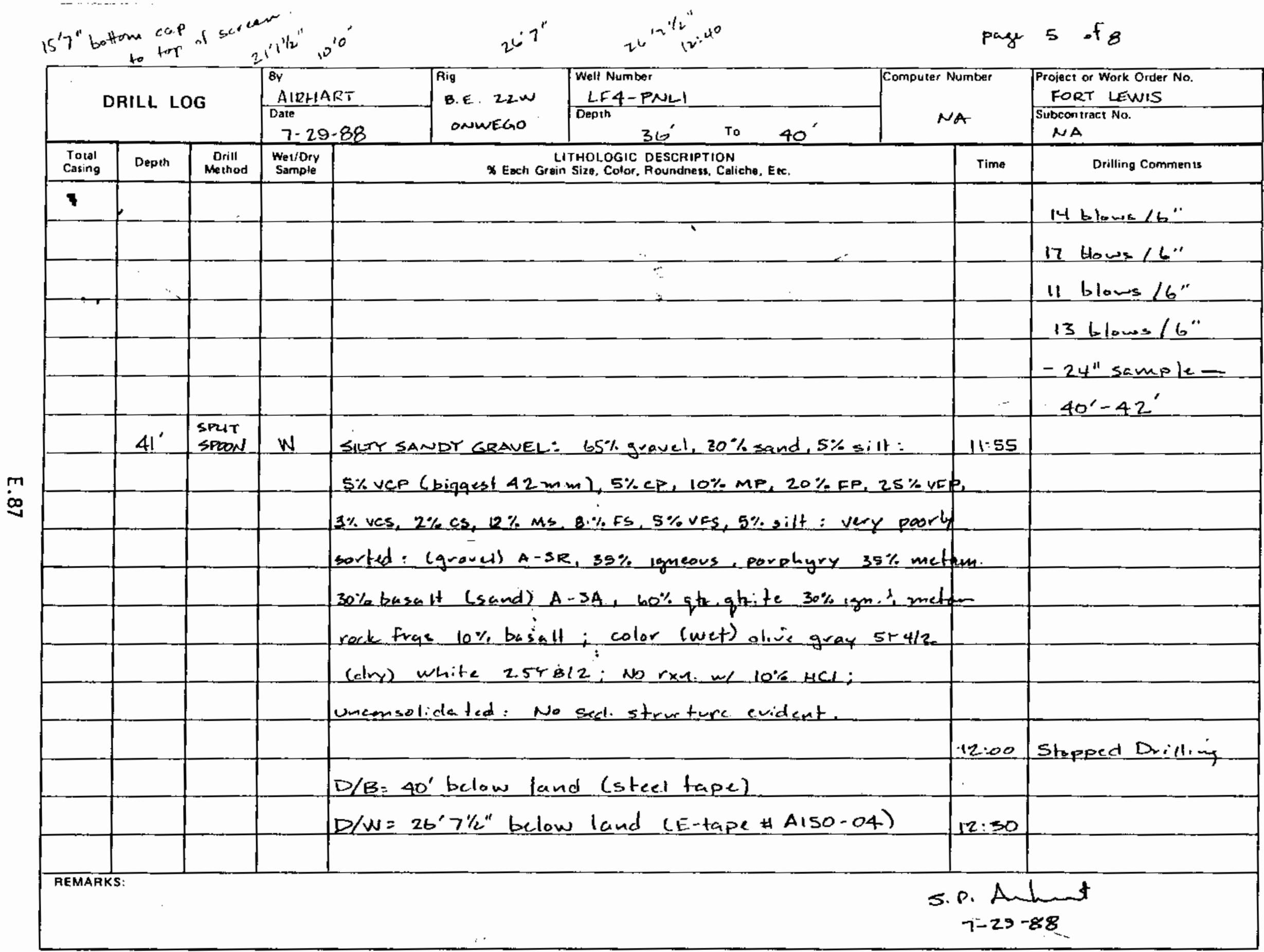

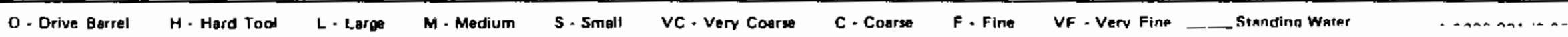




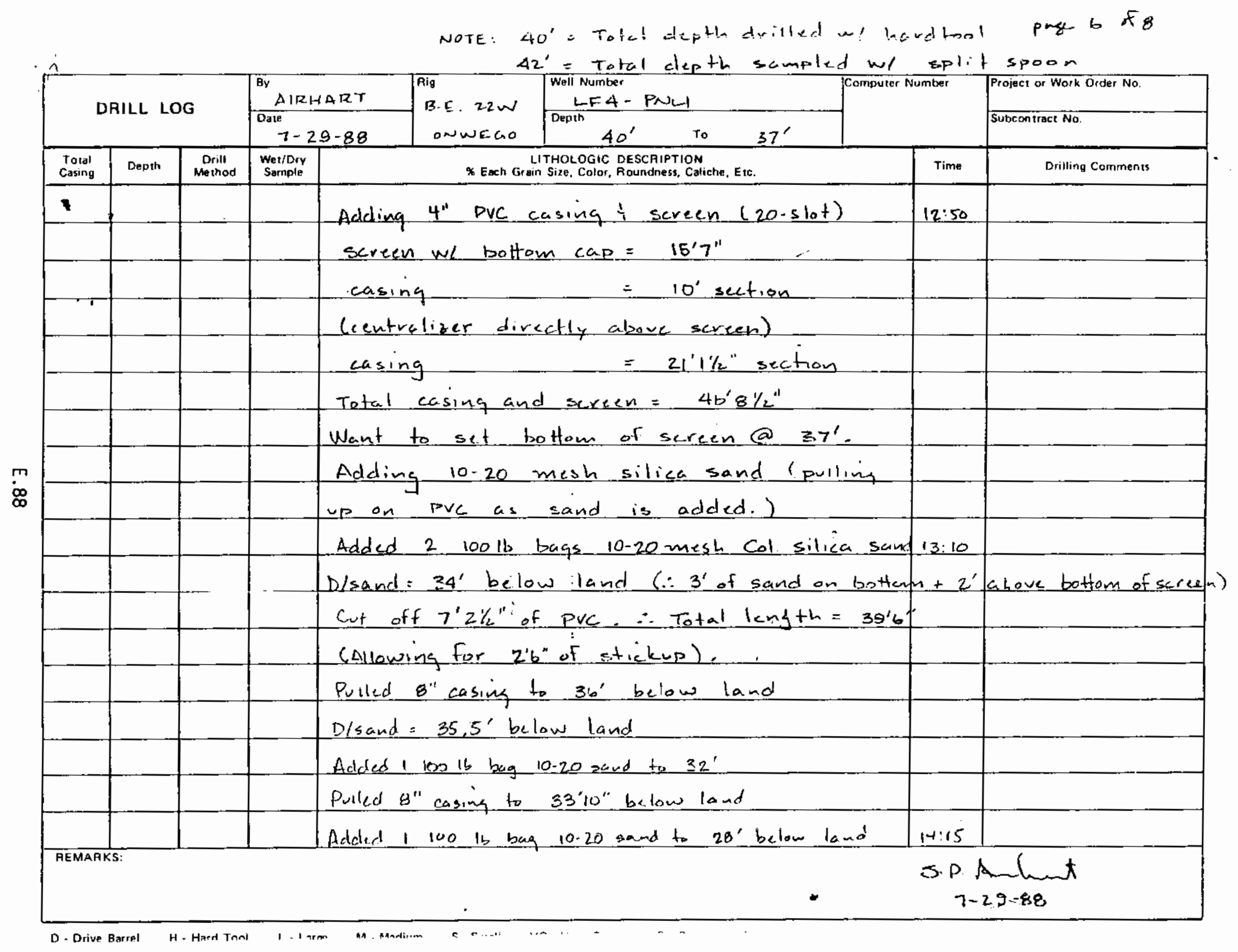


preg 7 at 8

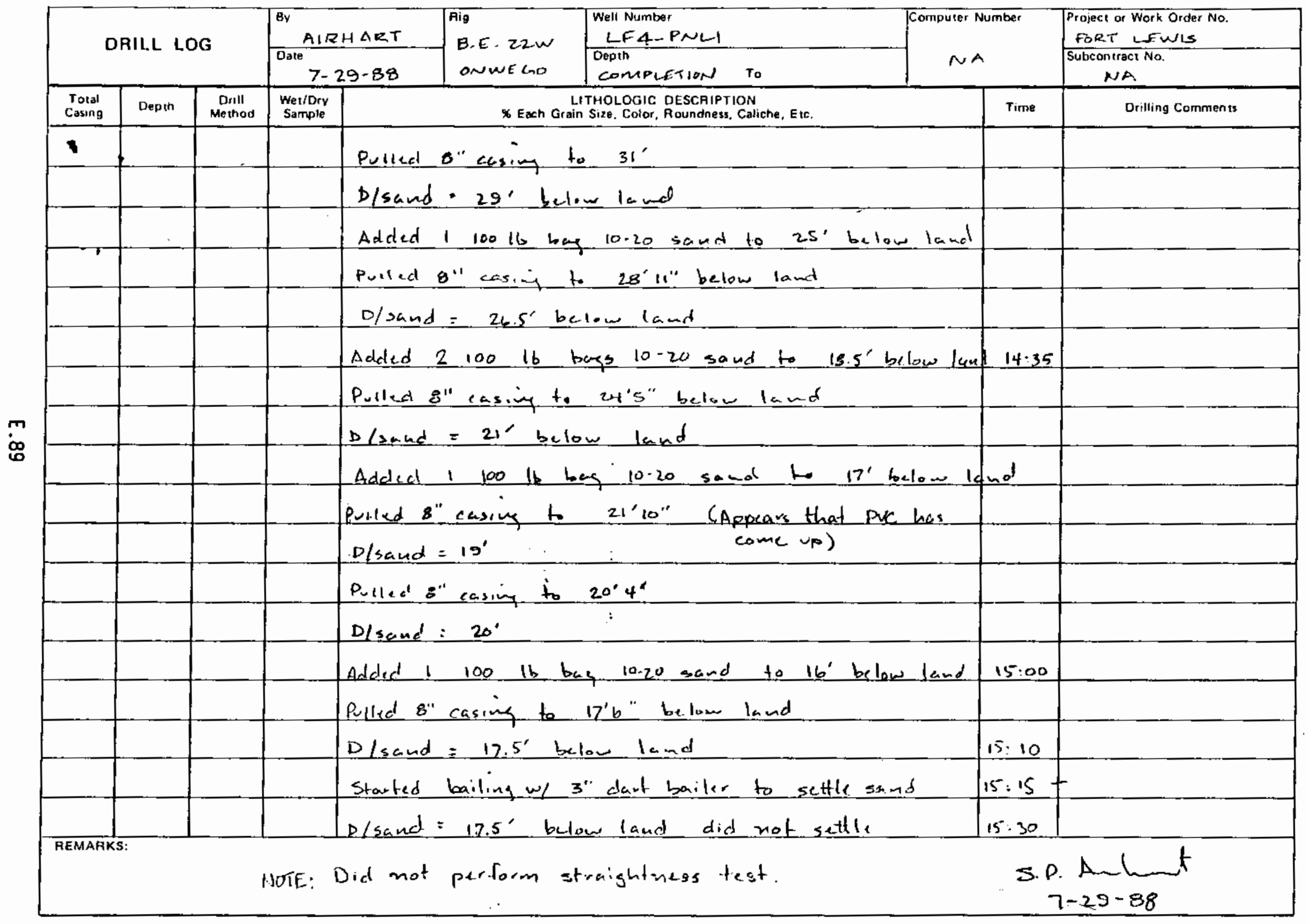

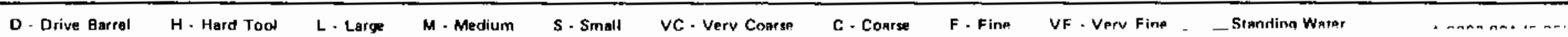


rex $B$ f 3

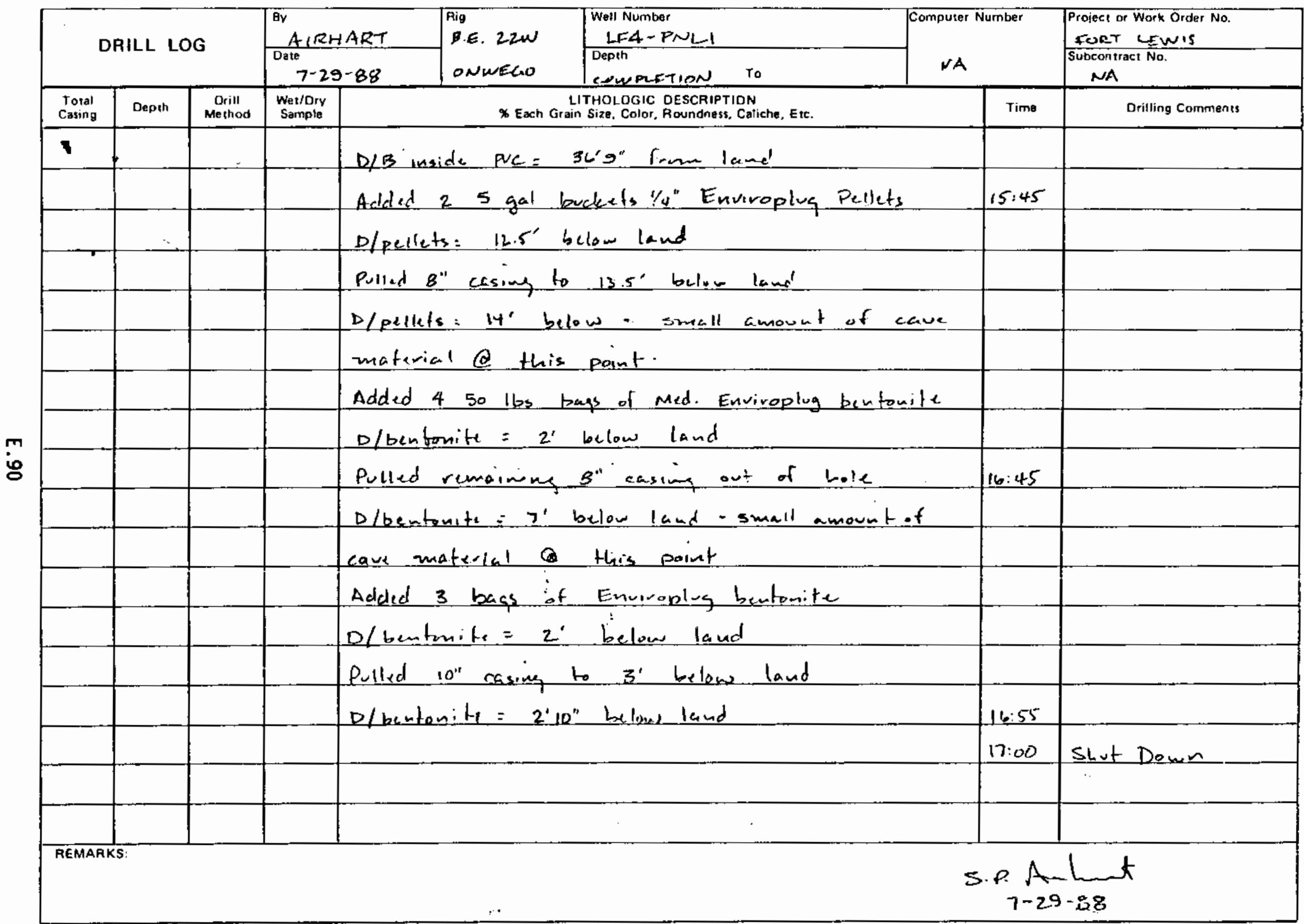

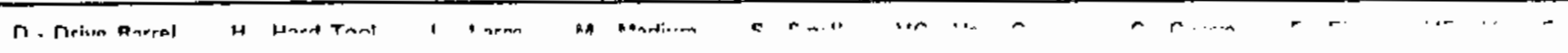


rougt 1 of 1

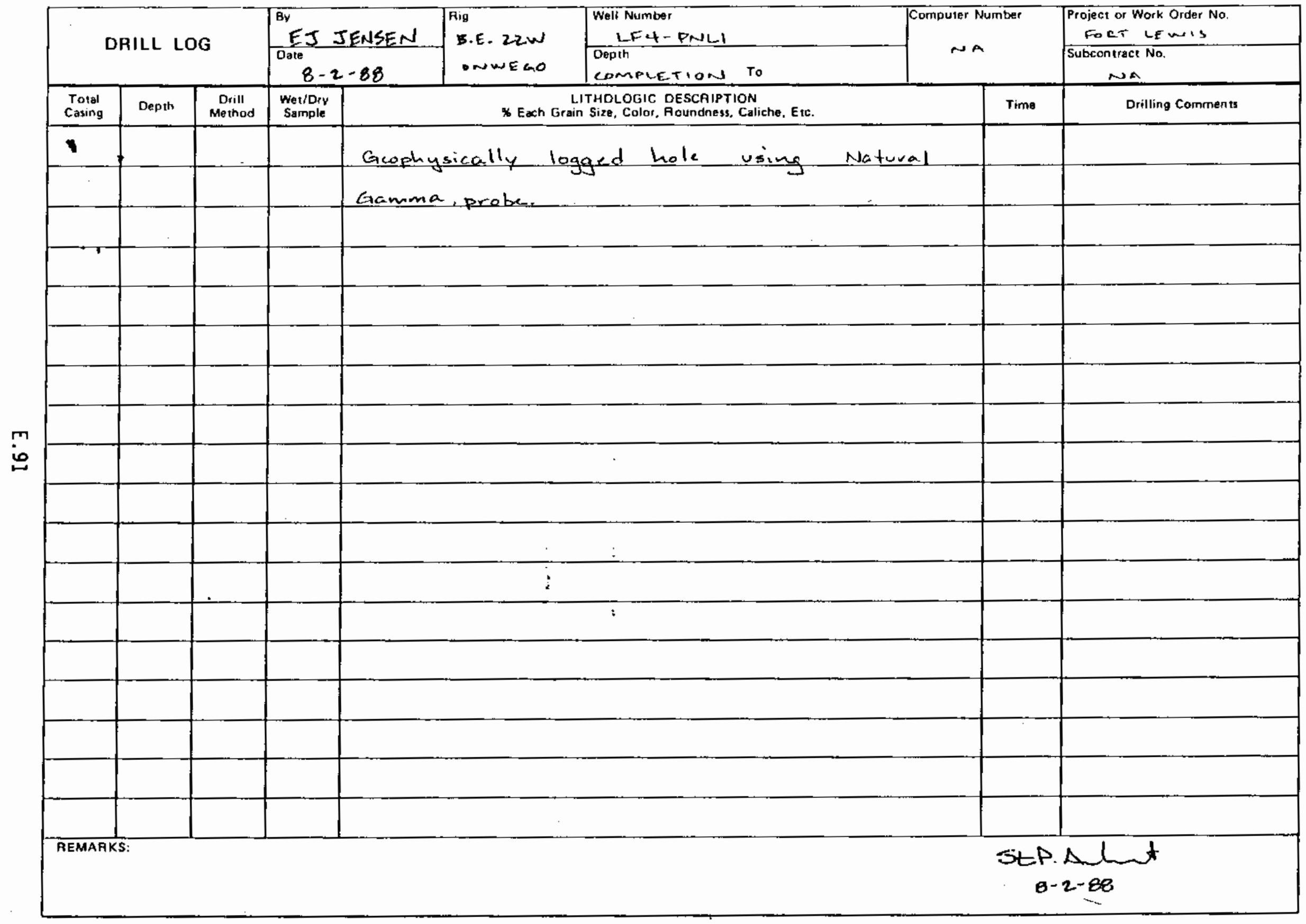

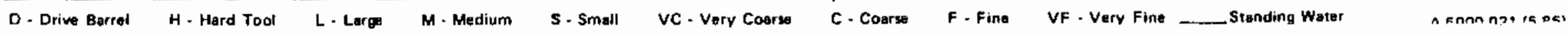


pare 1 of 1

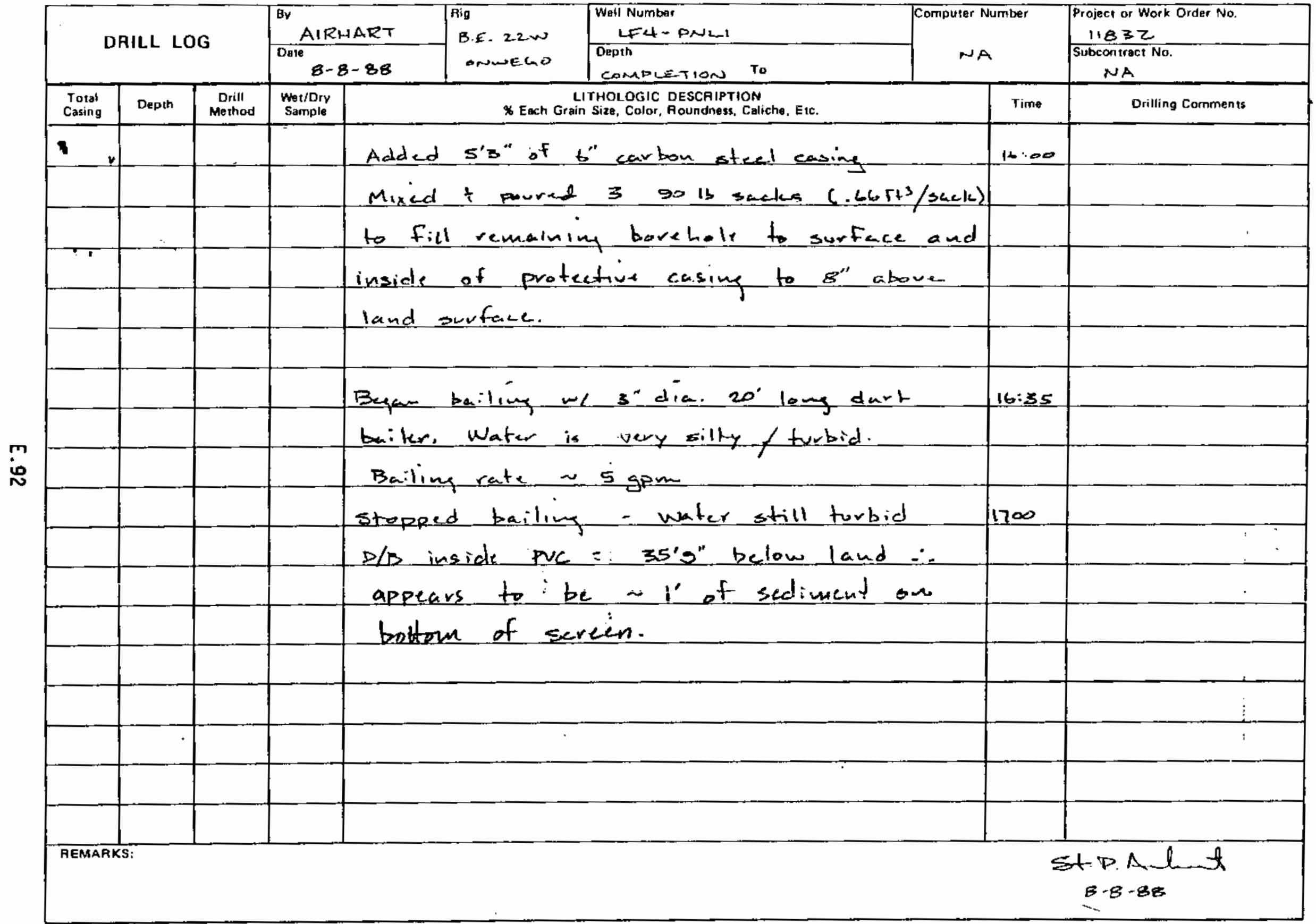

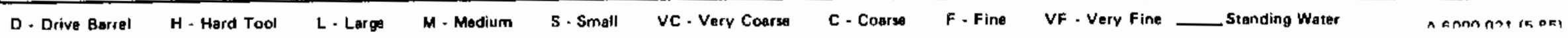


pose 1 .f 1

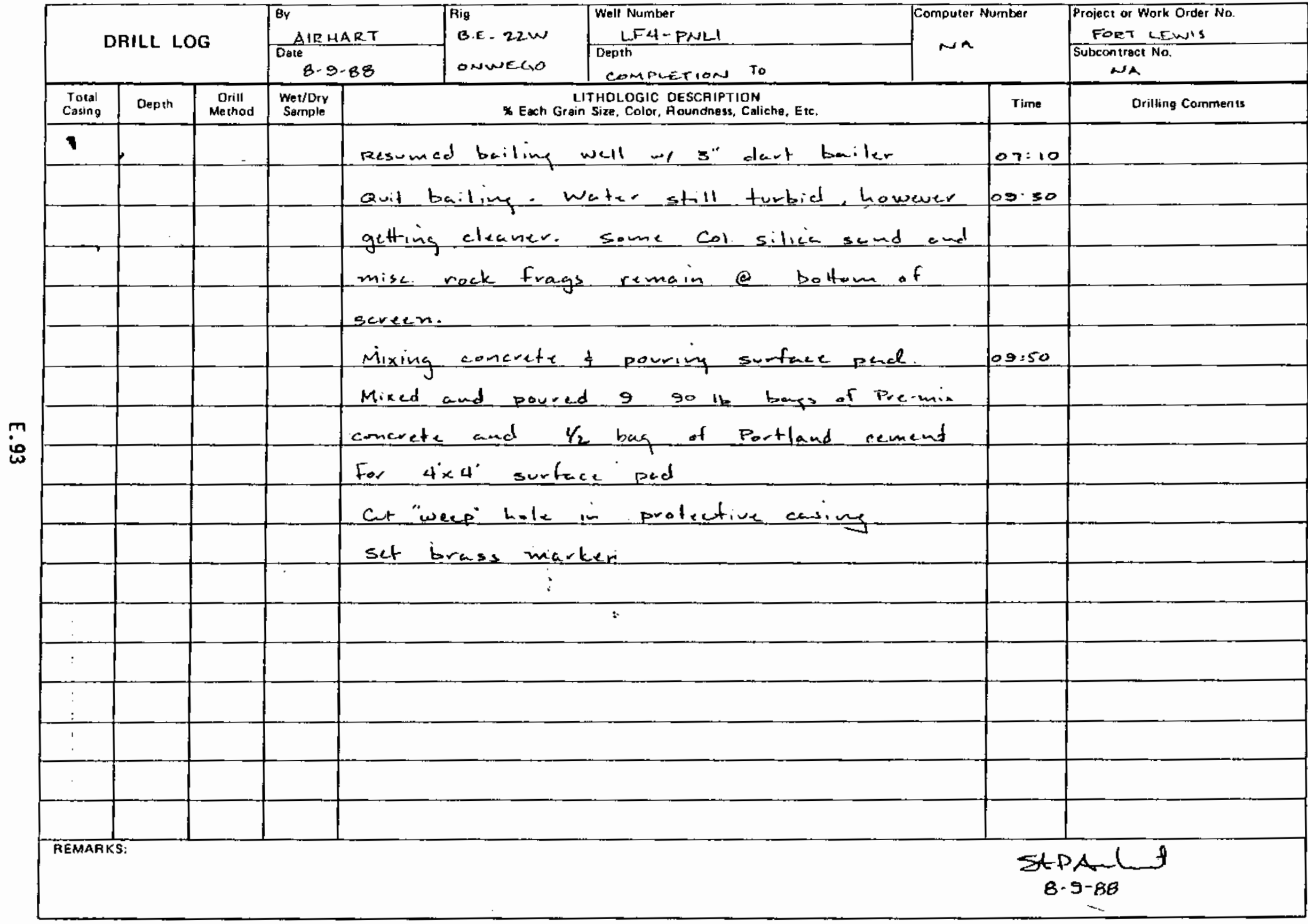

h. Hentand , tam

MA. Mndition

. $-n+\cdots$ 
pare $1 . f 1$

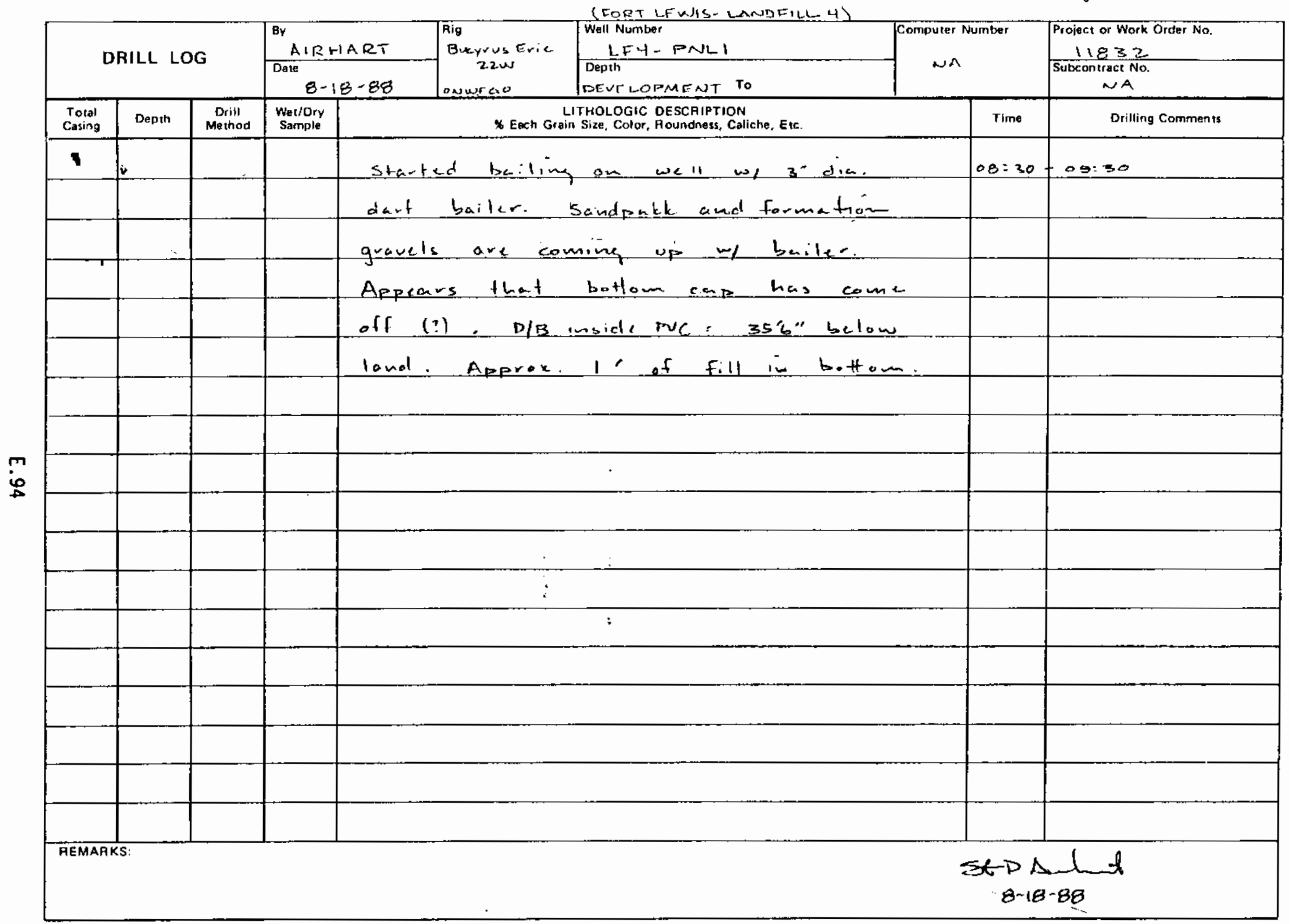
D - Drivo Barret
H. Hard Tool
L - Large
M. Medium
5. Small
vc - Verv coerse
c. Cosrse
F - Fine
VF - Verv Fine
- Sranctionn Watar 
page 1.t 1

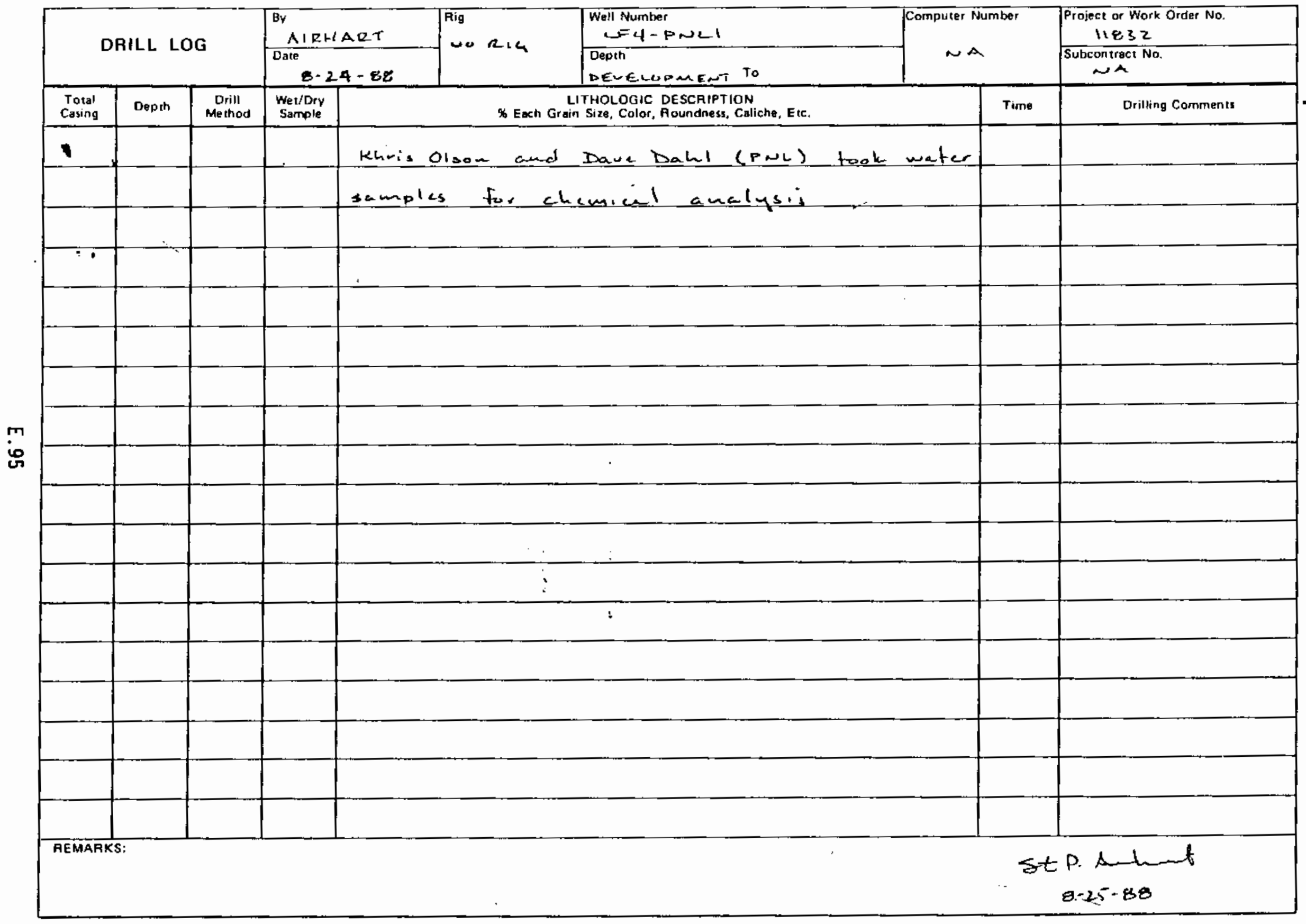

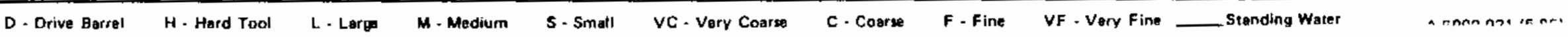




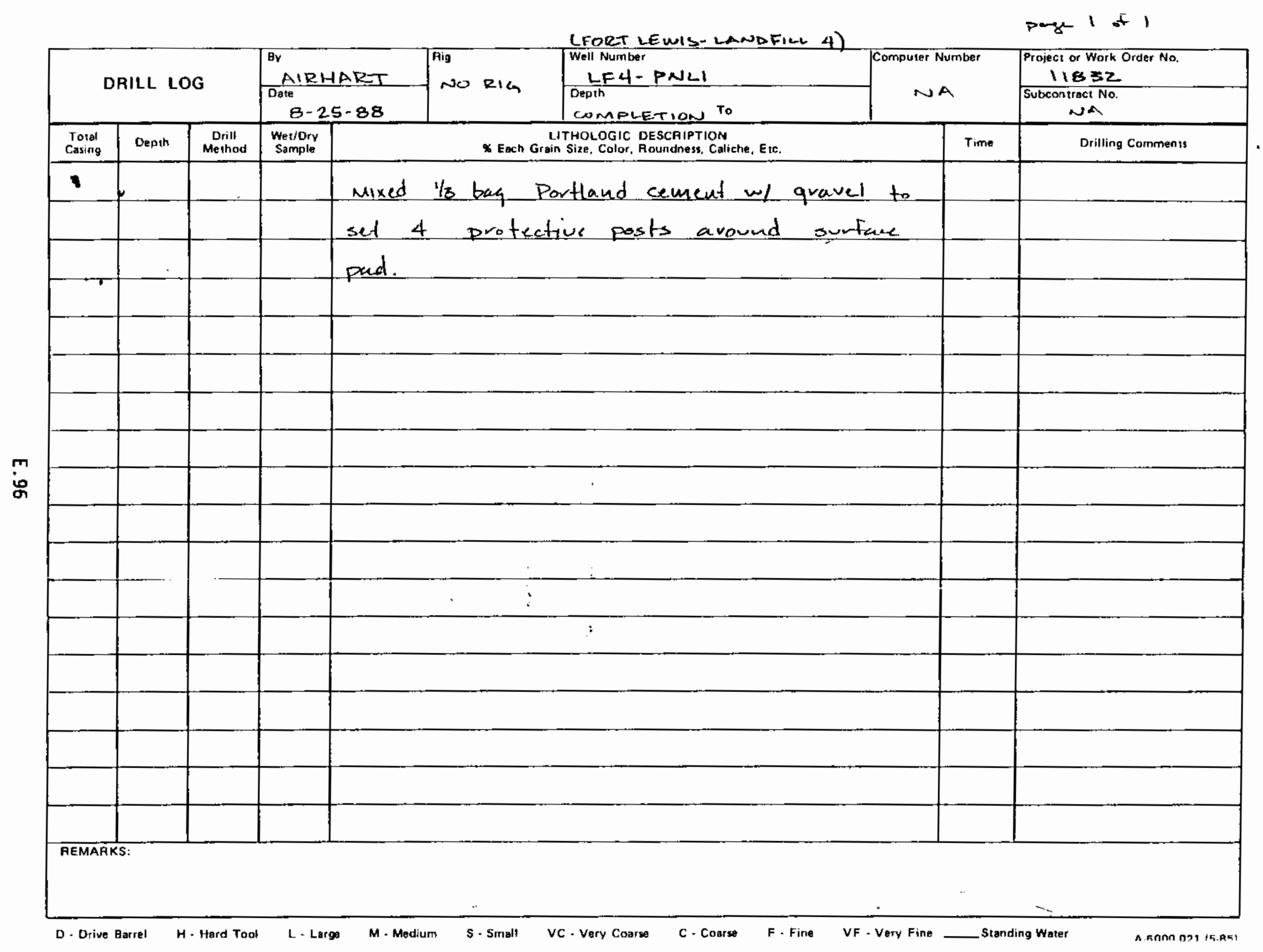


paye lof 1

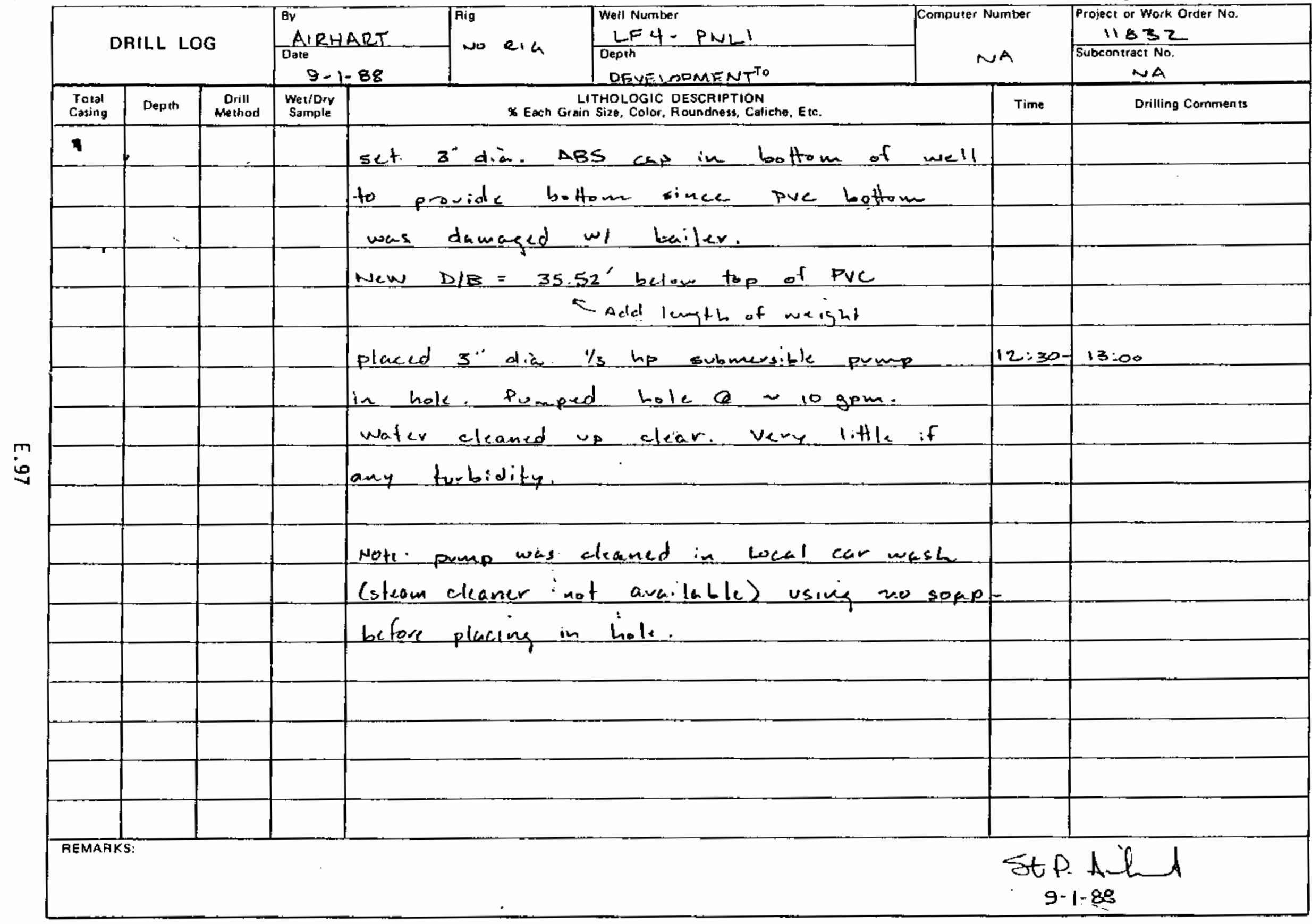

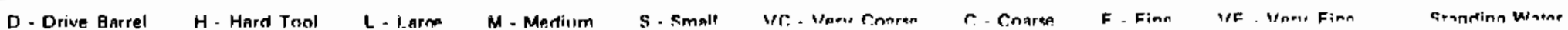


prong- : wet

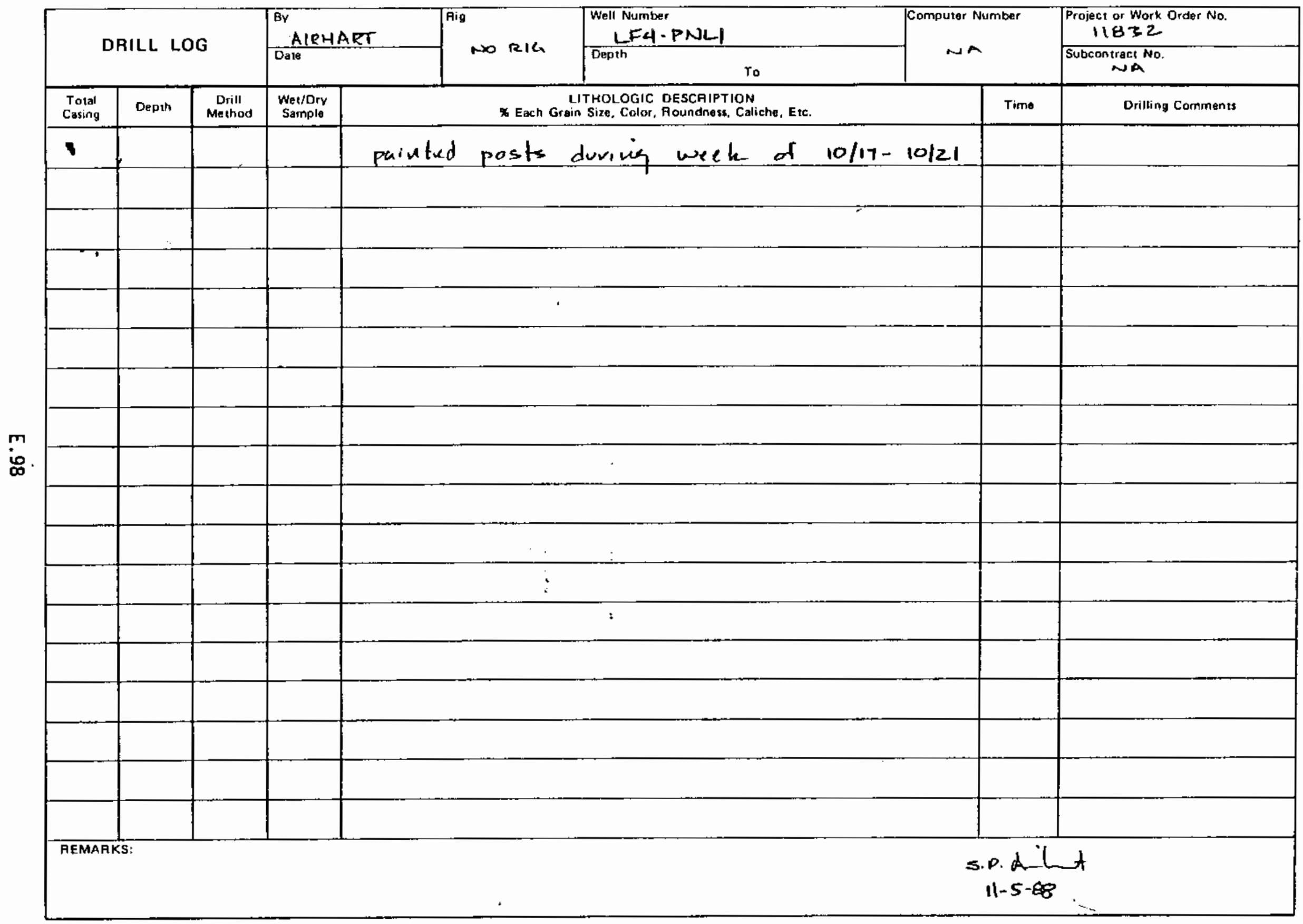

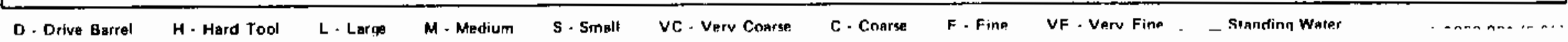


WELL LF4-PNL2 


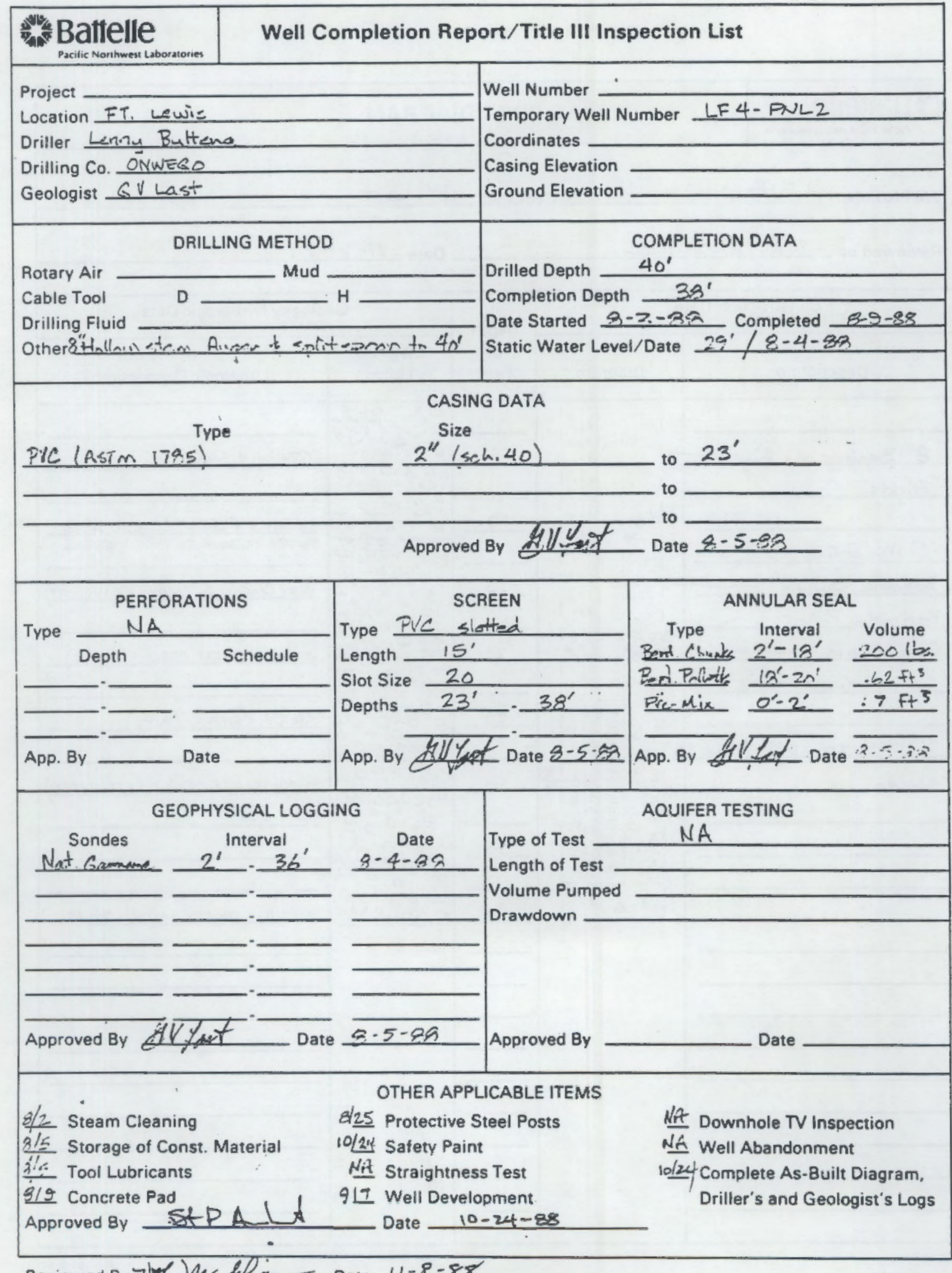

Reviewed By JWh. Wh c hlim Date 11-8-88 


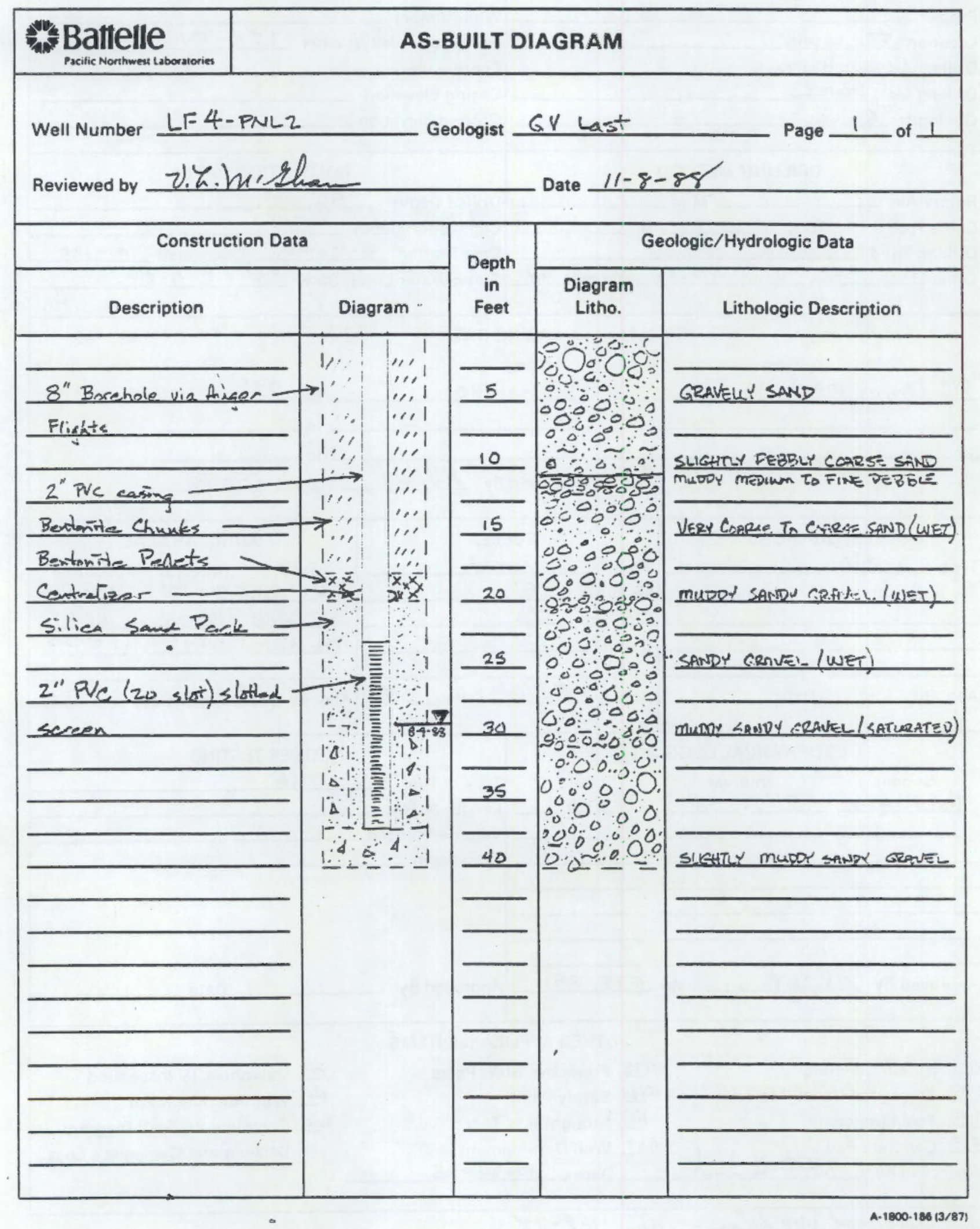




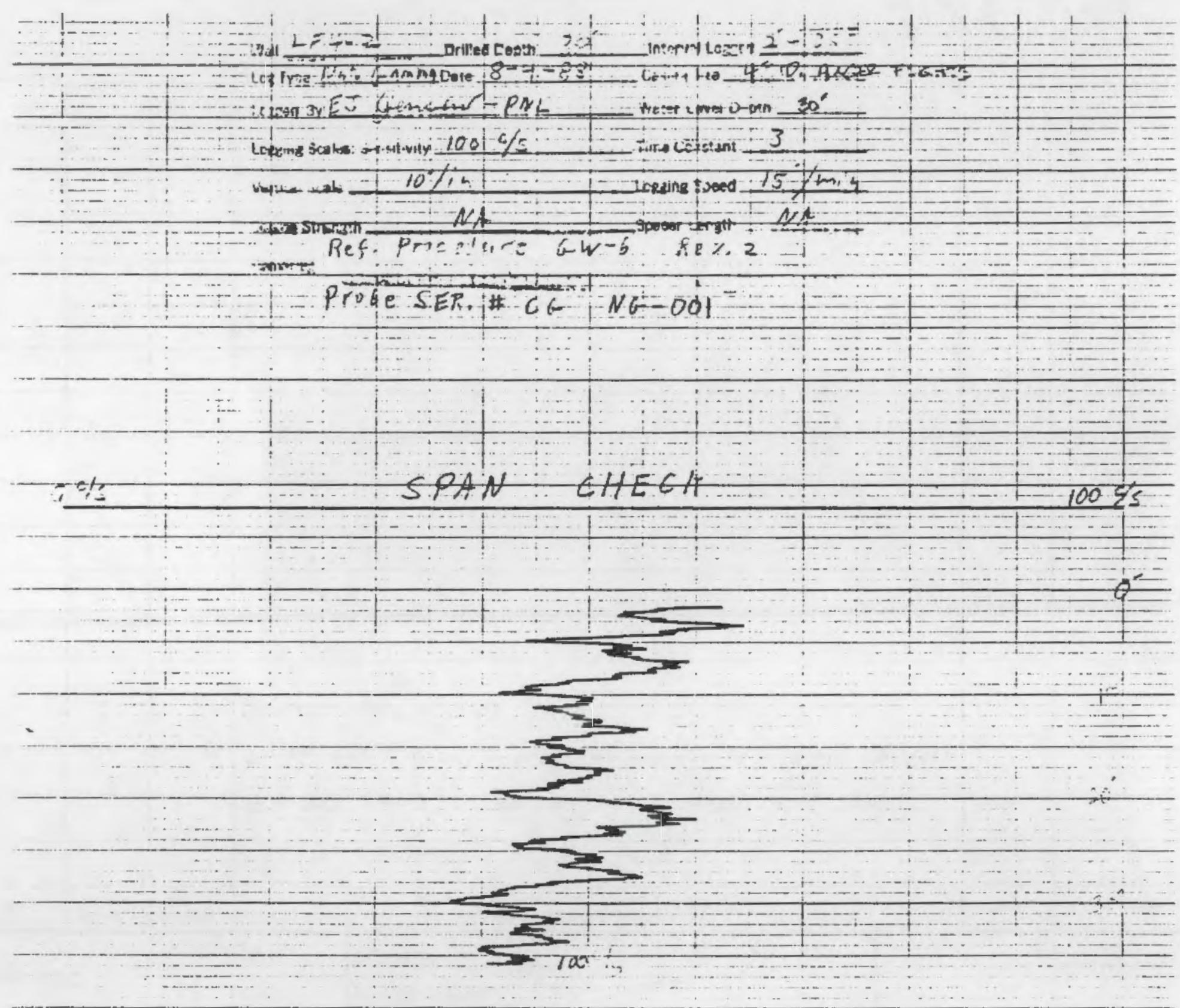


page i of

\begin{tabular}{|c|c|c|c|c|c|c|c|c|}
\hline \multirow{2}{*}{\multicolumn{3}{|c|}{ DRILL LOG }} & \multirow{2}{*}{\multicolumn{2}{|c|}{$\begin{array}{l}\text { Bv } F J \text { Jensen } \\
\text { Date } / 2 / 88 \\
8 / 2\end{array}$}} & \multirow{2}{*}{$\begin{array}{l}\text { Rig CHRAMm } \\
\text { S.CHR to Drill } \\
\text { Roto }\end{array}$} & \multirow{2}{*}{\multicolumn{2}{|c|}{$\begin{array}{l}\text { Well Number } \\
\begin{array}{llll}L F & 4-P N L 2 \\
\text { Depth } & 0^{\prime} \quad \text { To } & 5^{\prime}\end{array}\end{array}$}} & \multirow{3}{*}{\begin{tabular}{|c|} 
Project or Work Order No. \\
Subcontract No. \\
Drilling Comments \\
\end{tabular}} \\
\hline & & & & & & & & \\
\hline $\begin{array}{c}\text { Total } \\
\text { Casing }\end{array}$ & Depin & $\begin{array}{l}\text { Drill } \\
\text { Method } \\
\end{array}$ & 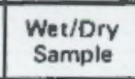 & & \multicolumn{2}{|c|}{ 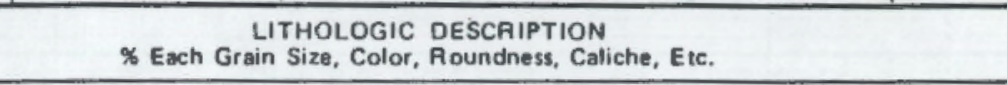 } & Time & \\
\hline \multirow[t]{5}{*}{ 、 } & & & & \multicolumn{3}{|c|}{ steam cleaned rotary rig. } & 0900 & \\
\hline & & & & \multicolumn{3}{|c|}{ 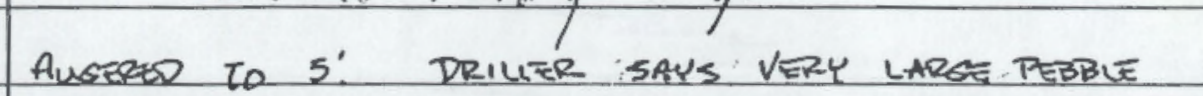 } & 1300 & \\
\hline & & & & \multicolumn{3}{|c|}{ 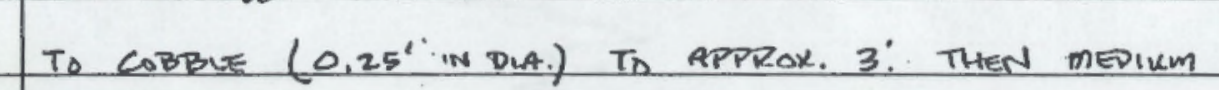 } & & \\
\hline & & & & & & \\
\hline & & & & \multicolumn{3}{|c|}{ PEBBLE $\left(0,1^{\prime}\right.$ in DiA.) $\tau_{0} 5^{\prime}$ : } & & \\
\hline & & & & & & & & \\
\hline & & & & & & & & \\
\hline & & & & & & & & \\
\hline & & & & & & 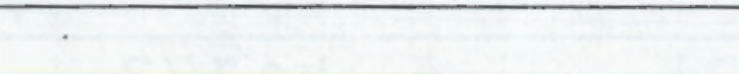 & & \\
\hline & & & & & & & & \\
\hline & & & & & & & & \\
\hline & & & & & is & & & \\
\hline & & & & & & $\because$ & & \\
\hline & & & & & & & & \\
\hline & & & & & & & & \\
\hline & & & & & & & & \\
\hline & & & & & & & & \\
\hline & & & & & & & & \\
\hline REMAR: & & & & & & S & $t$ & $13 / 38$ \\
\hline
\end{tabular}


fire 1 if 4

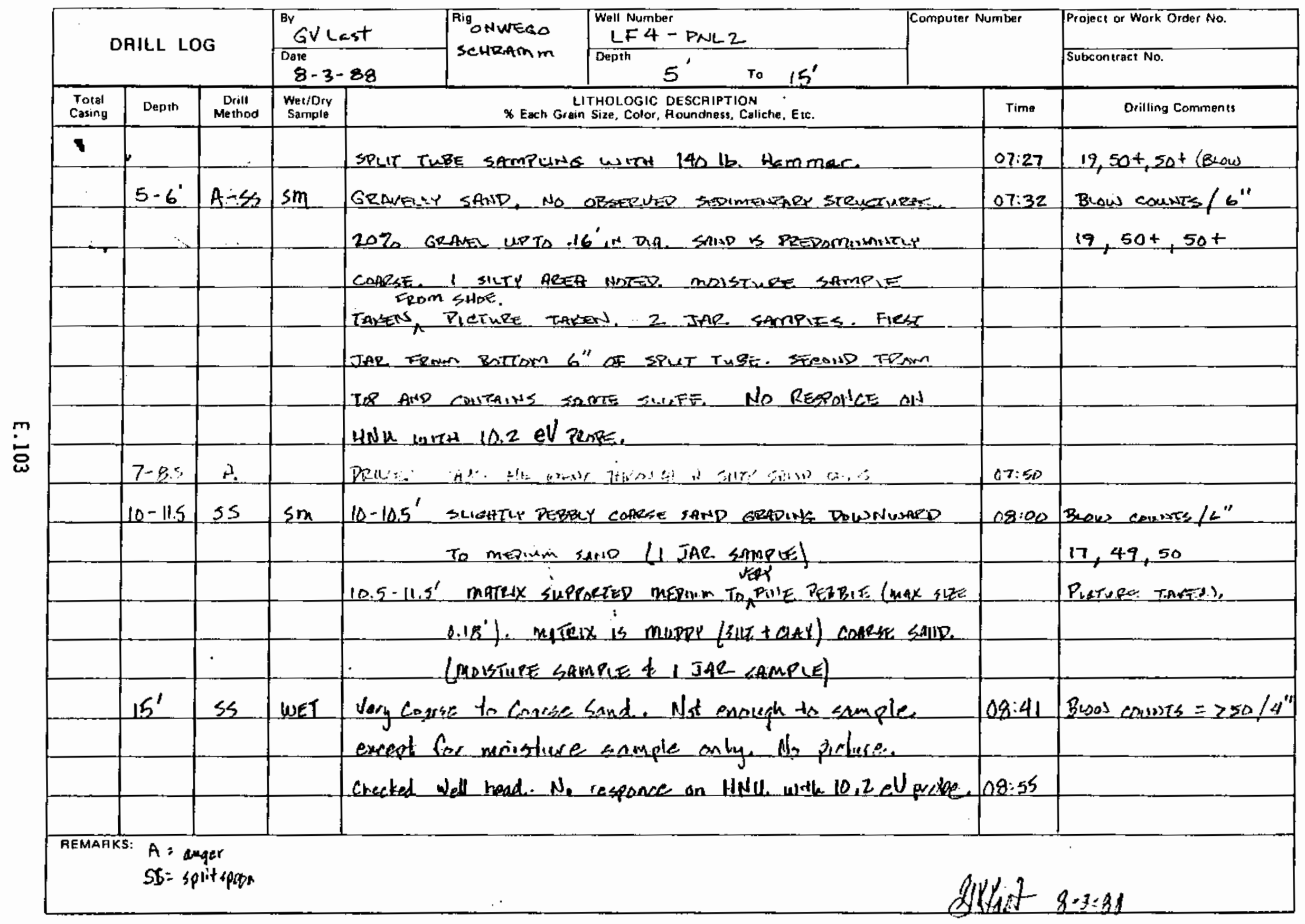

D. Drive Barrel H. Hard Tool L. Large M-Medium S-Small VC.Very Coarse C. Coarse F. Fine VF. Very Fine a 
rage 2.54

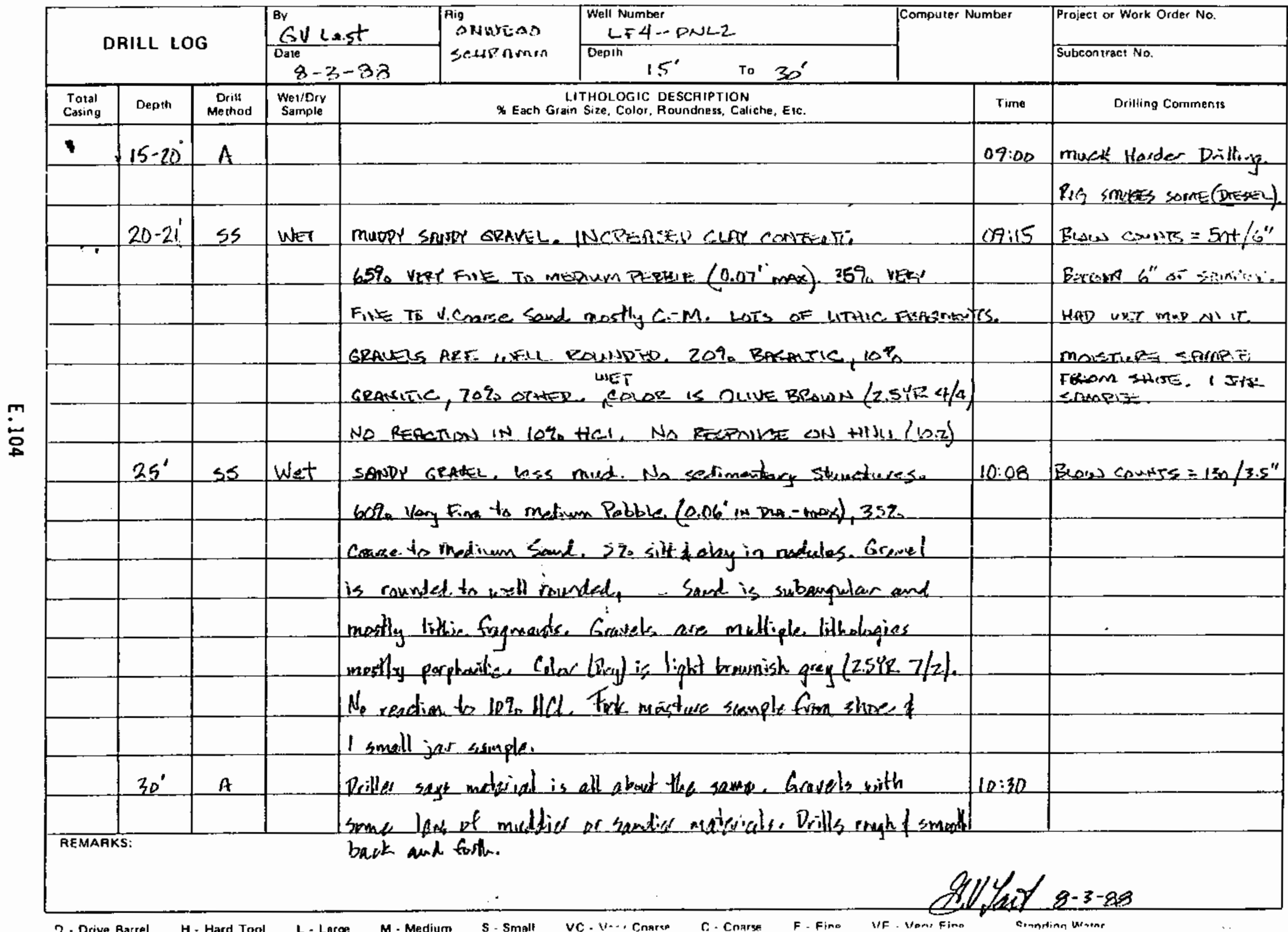


prye $=$ of 4

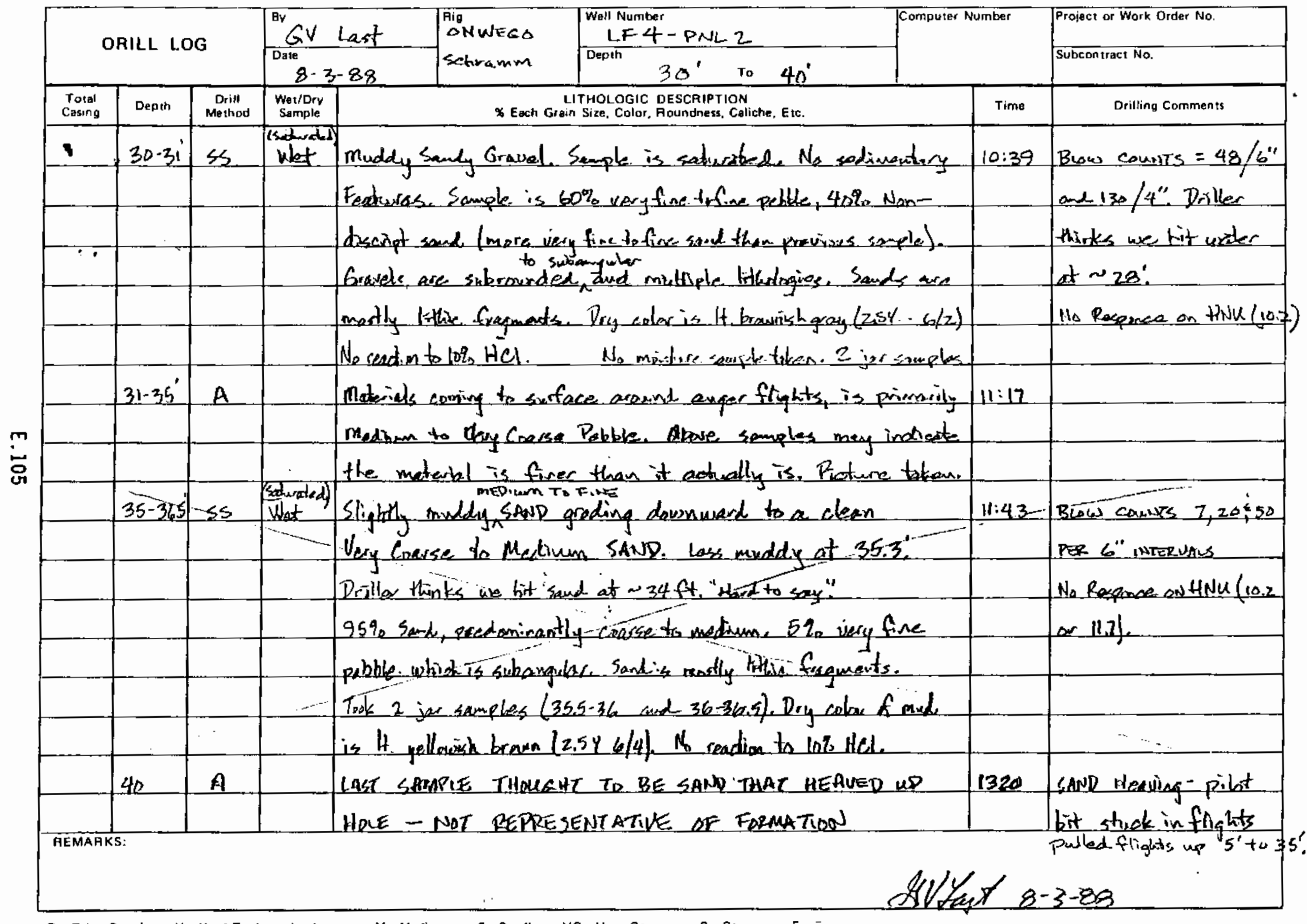

D. Drive Barrel H. Hard To

L. Lnrae M. Mertiltm 
prese 4 or 4

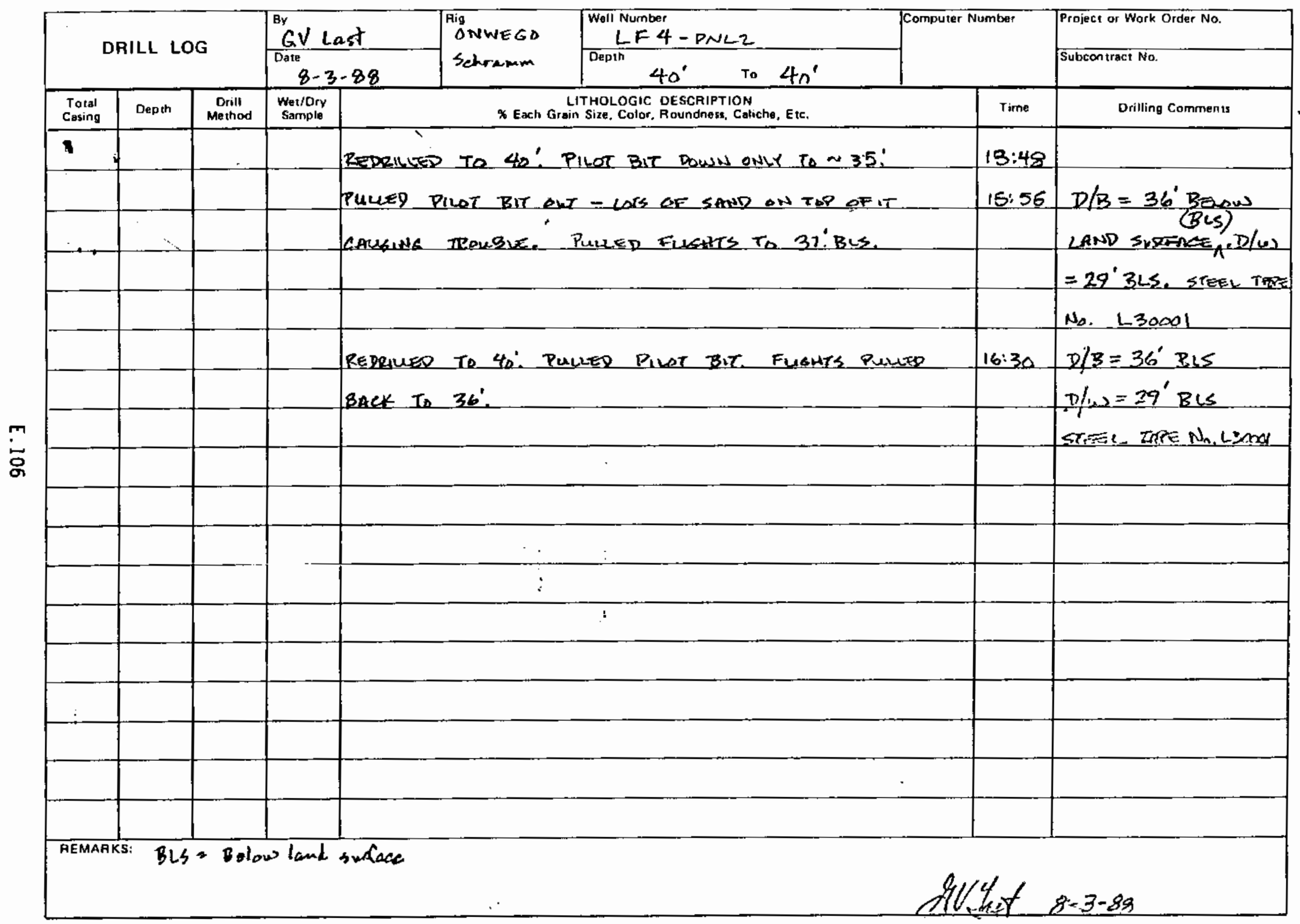
D. Drive Barrel H. Herd Tood
$s \cdot$ Small
vc - Very Cosrse
c - Corse
VF - Very Fine
Standing Woter

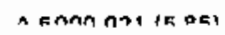




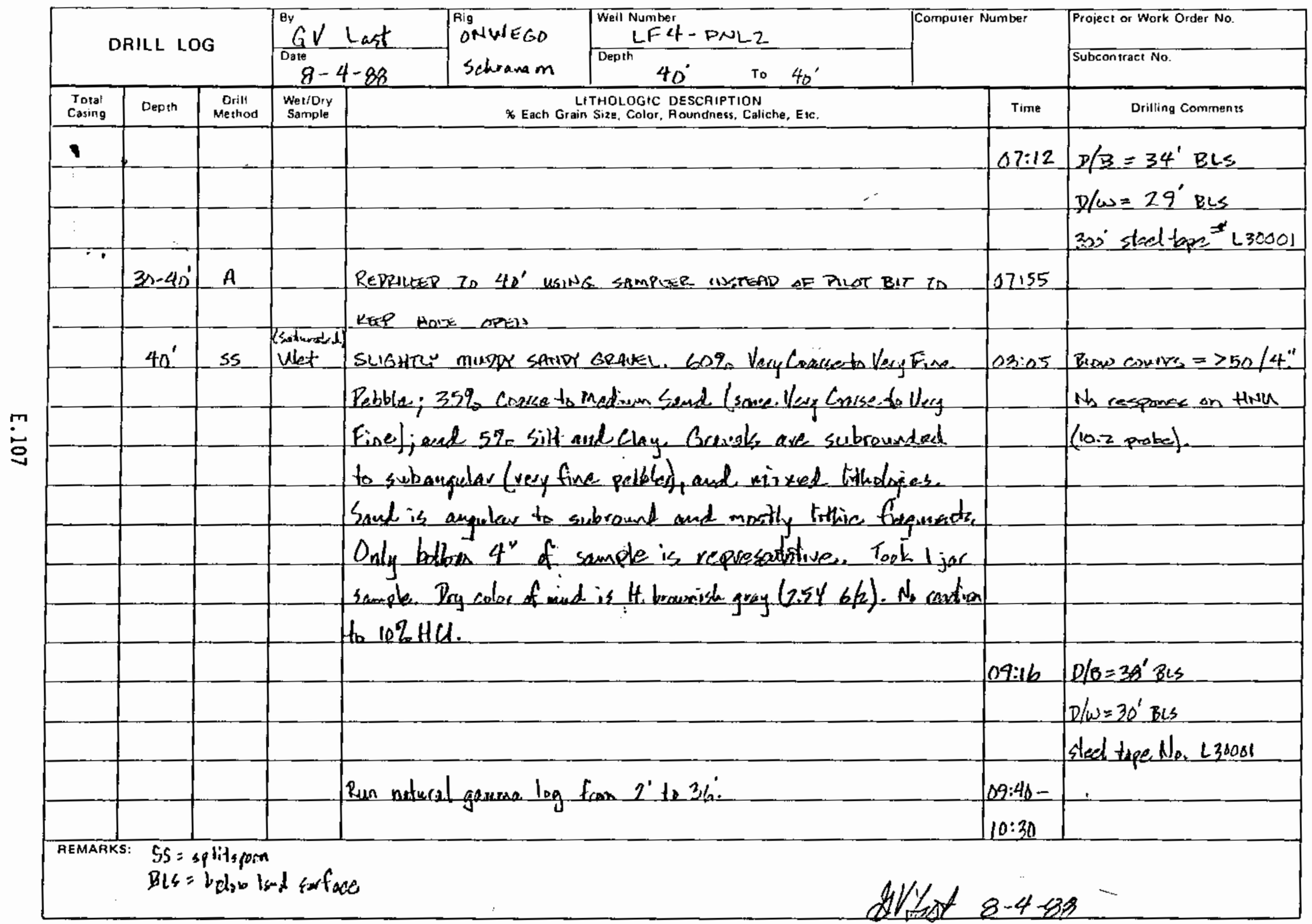

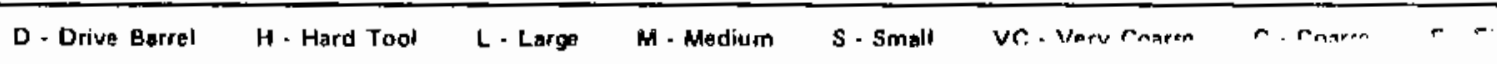




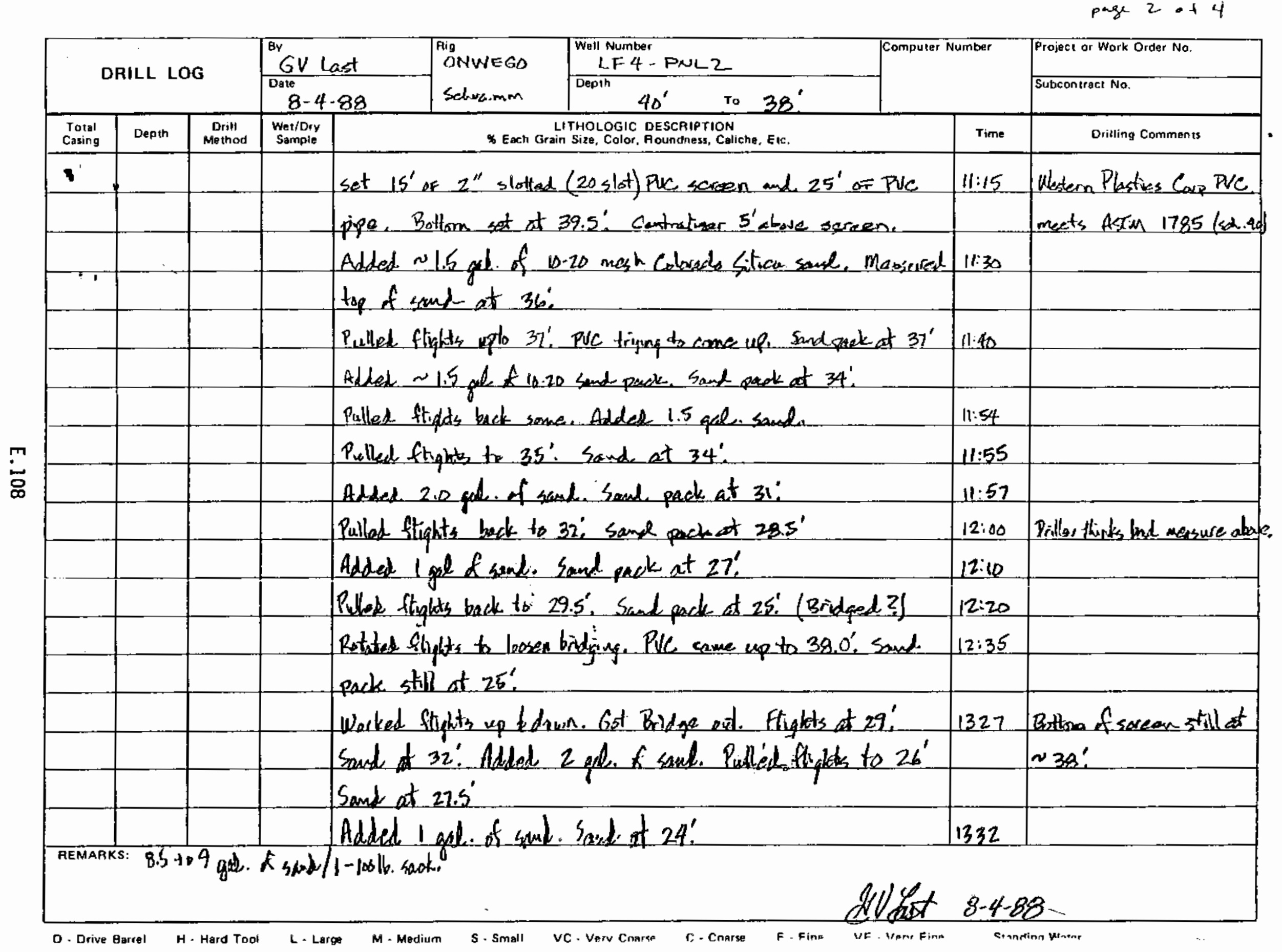




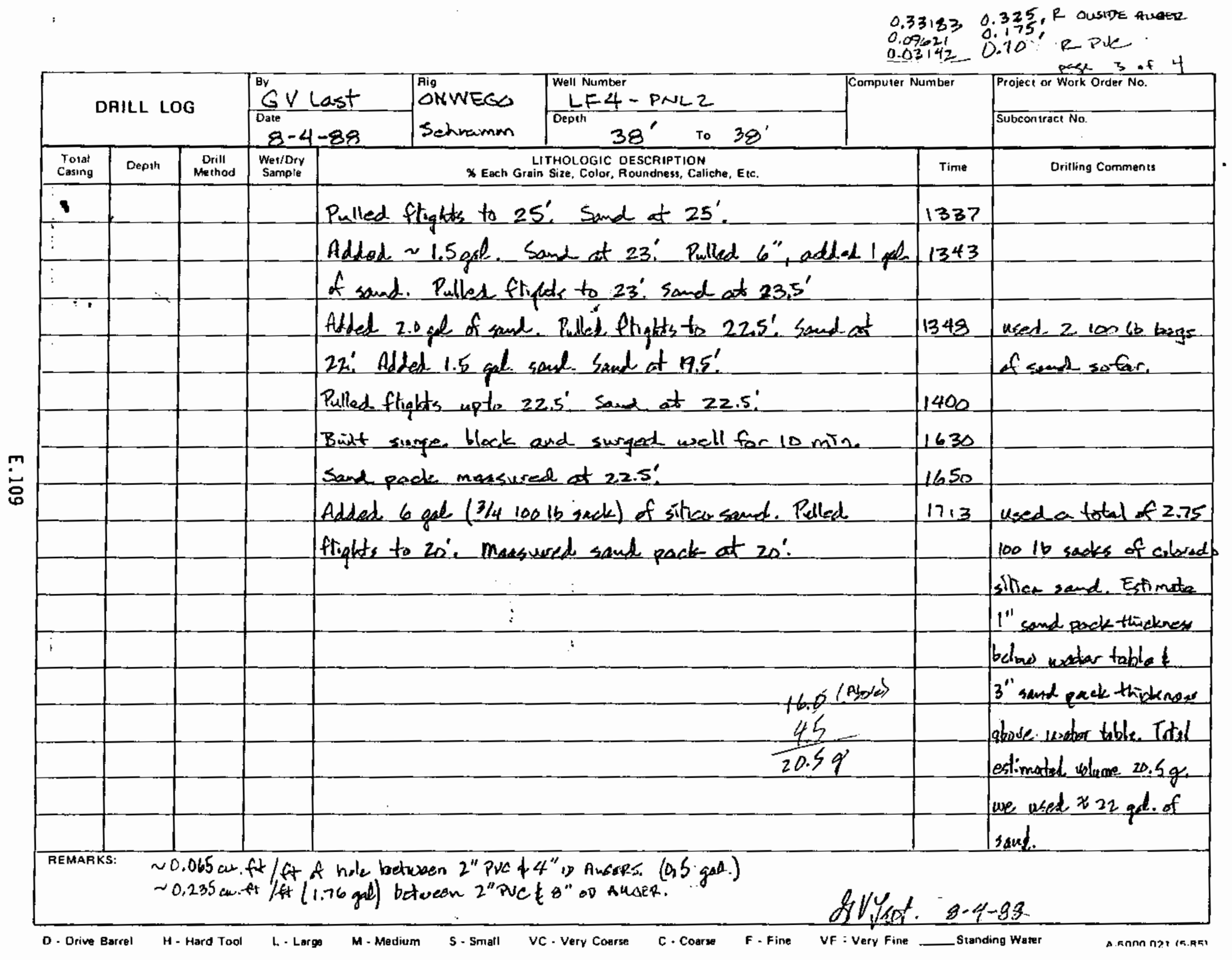


page 4 of 4

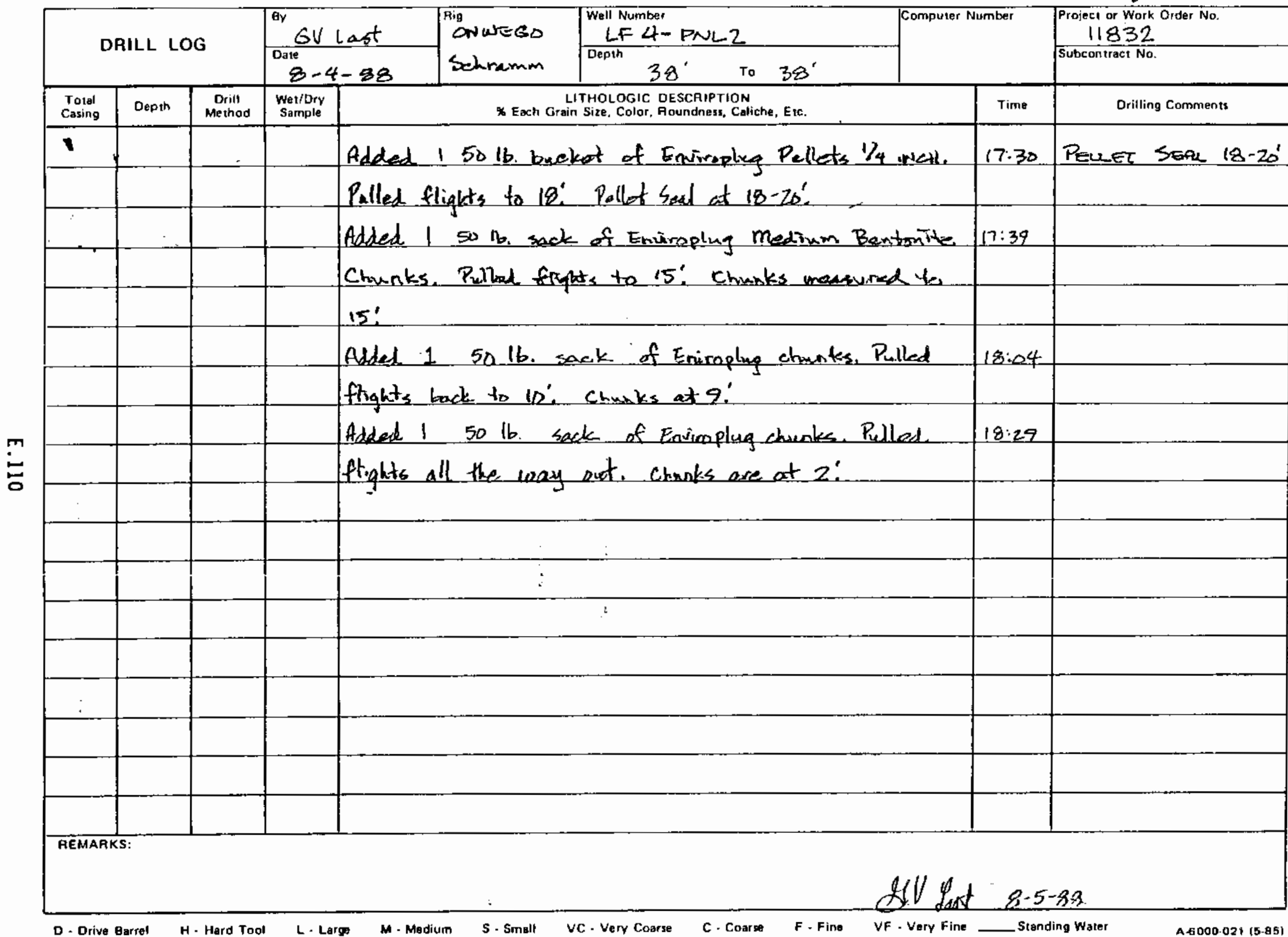


paye 1 of 1

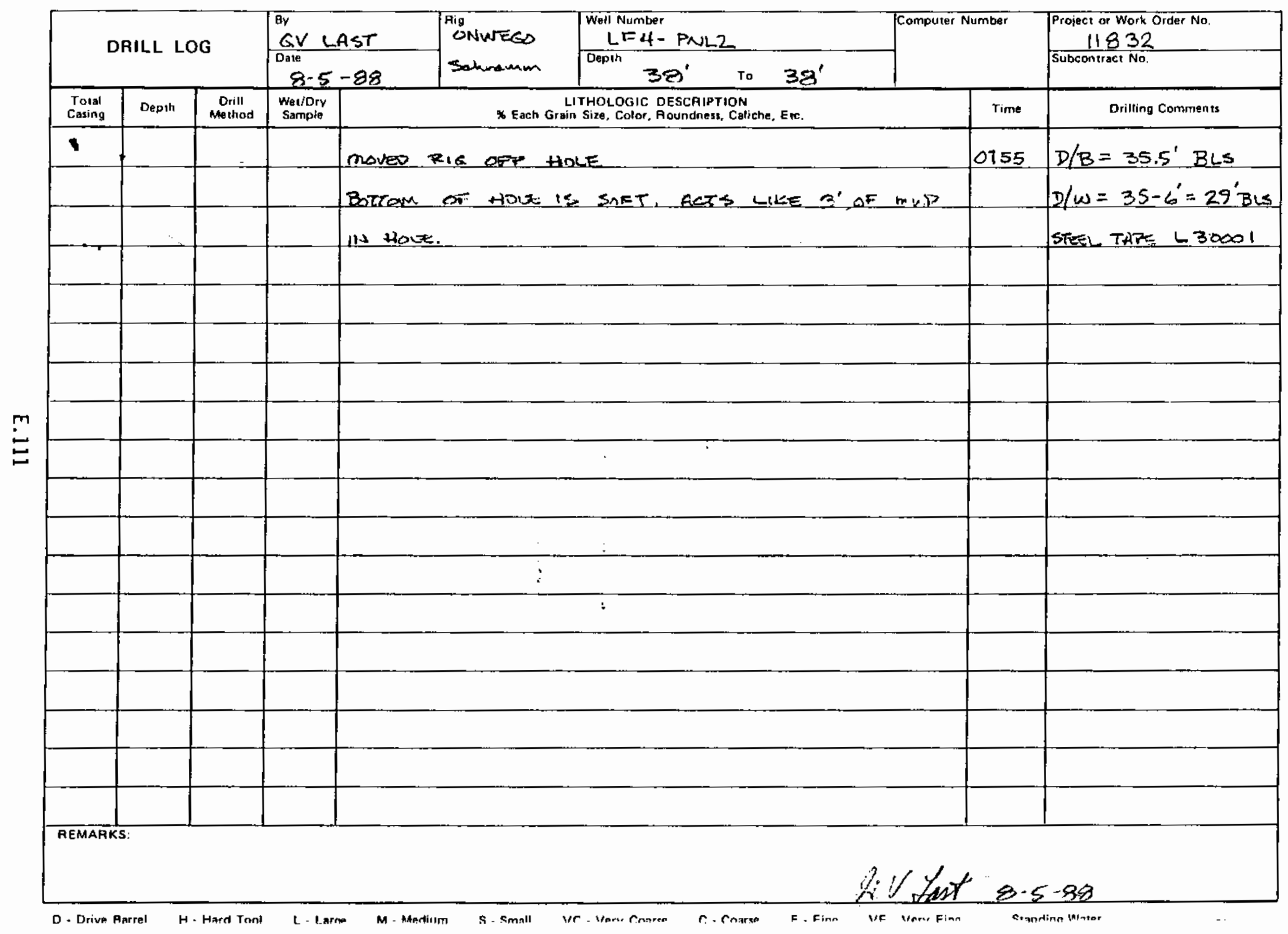




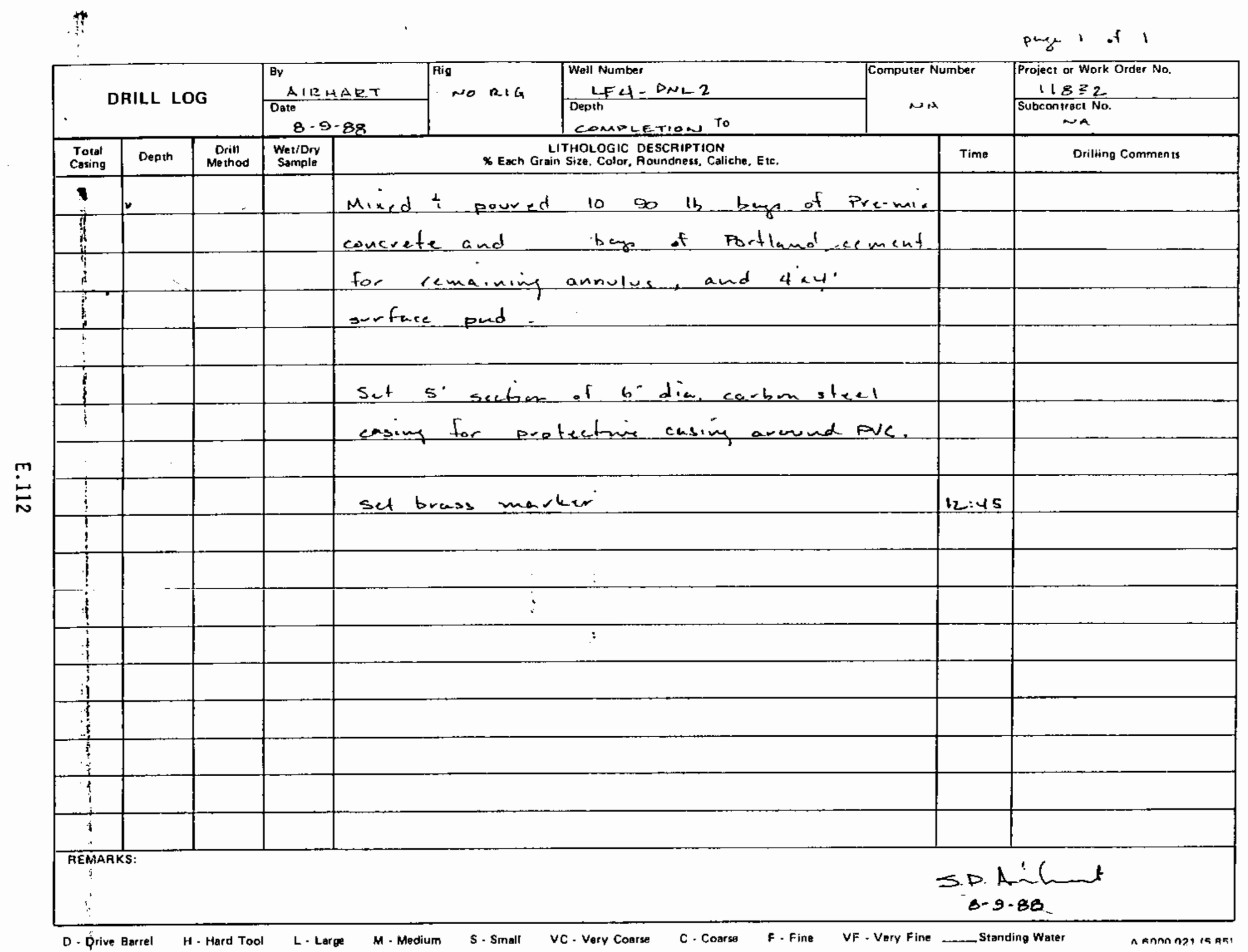


parg 1 of 1

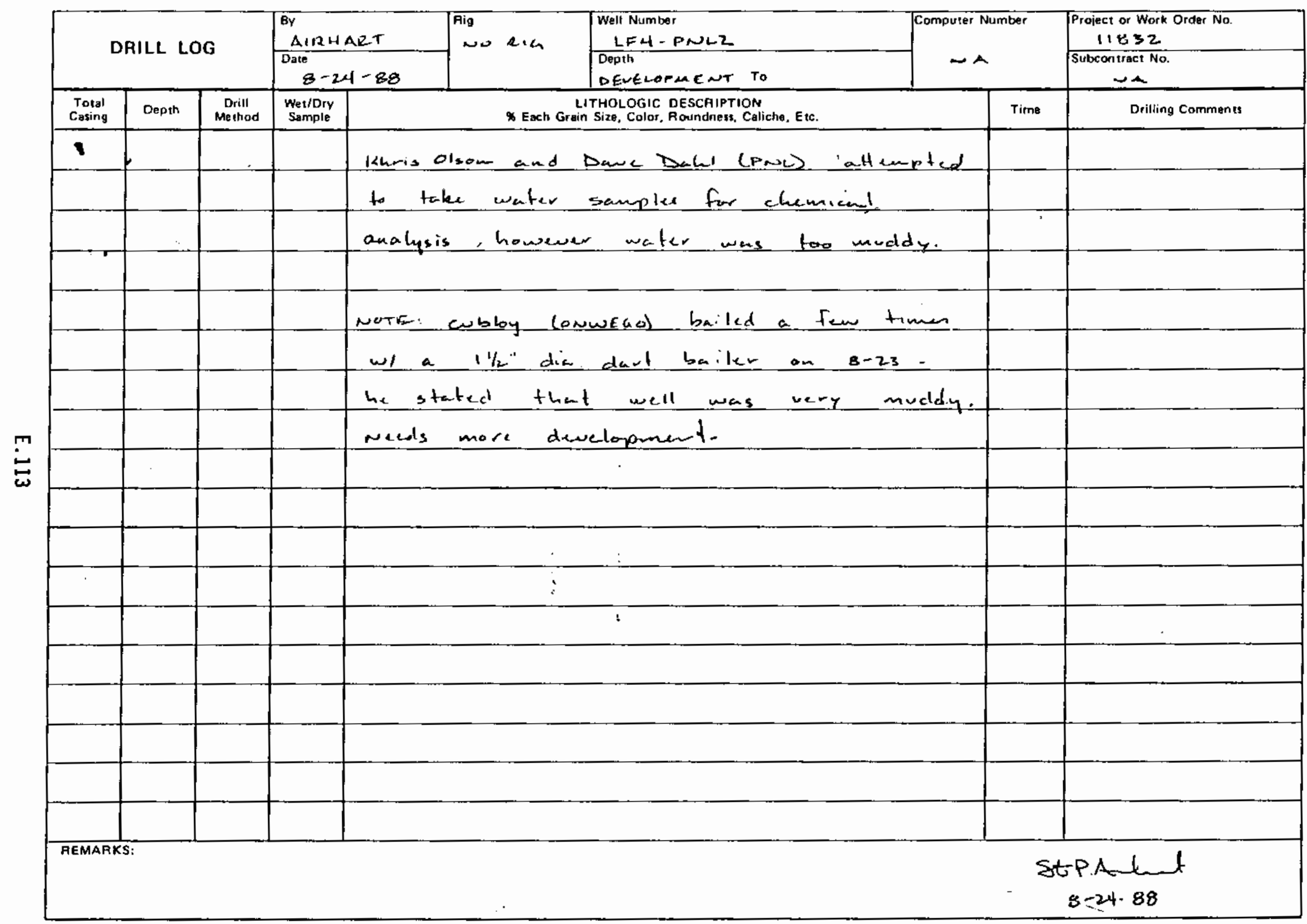

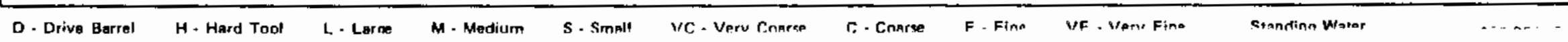


CFORT LEWIS - LANDFILLU)

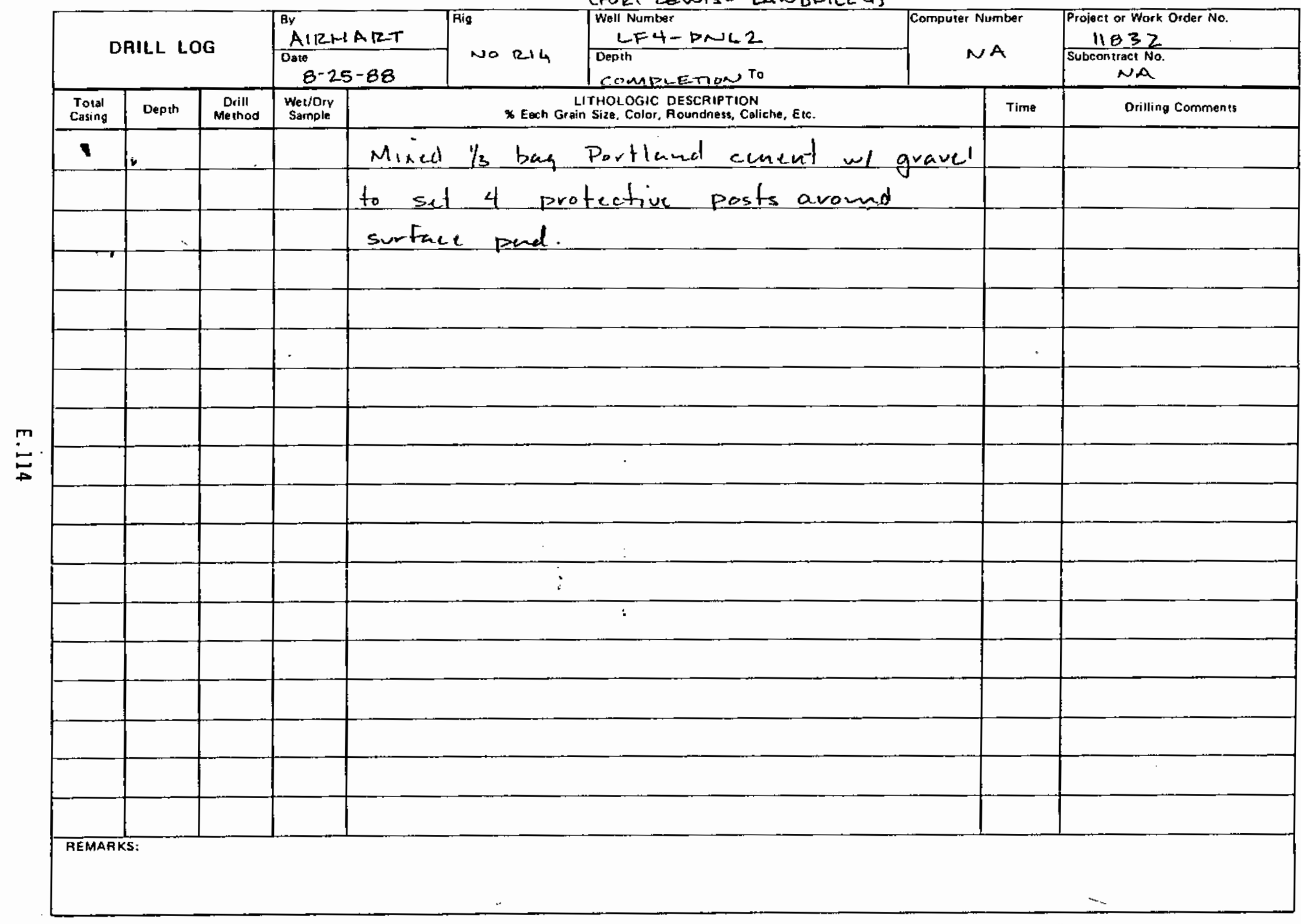

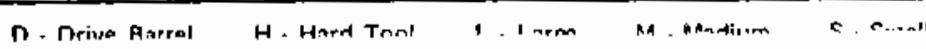




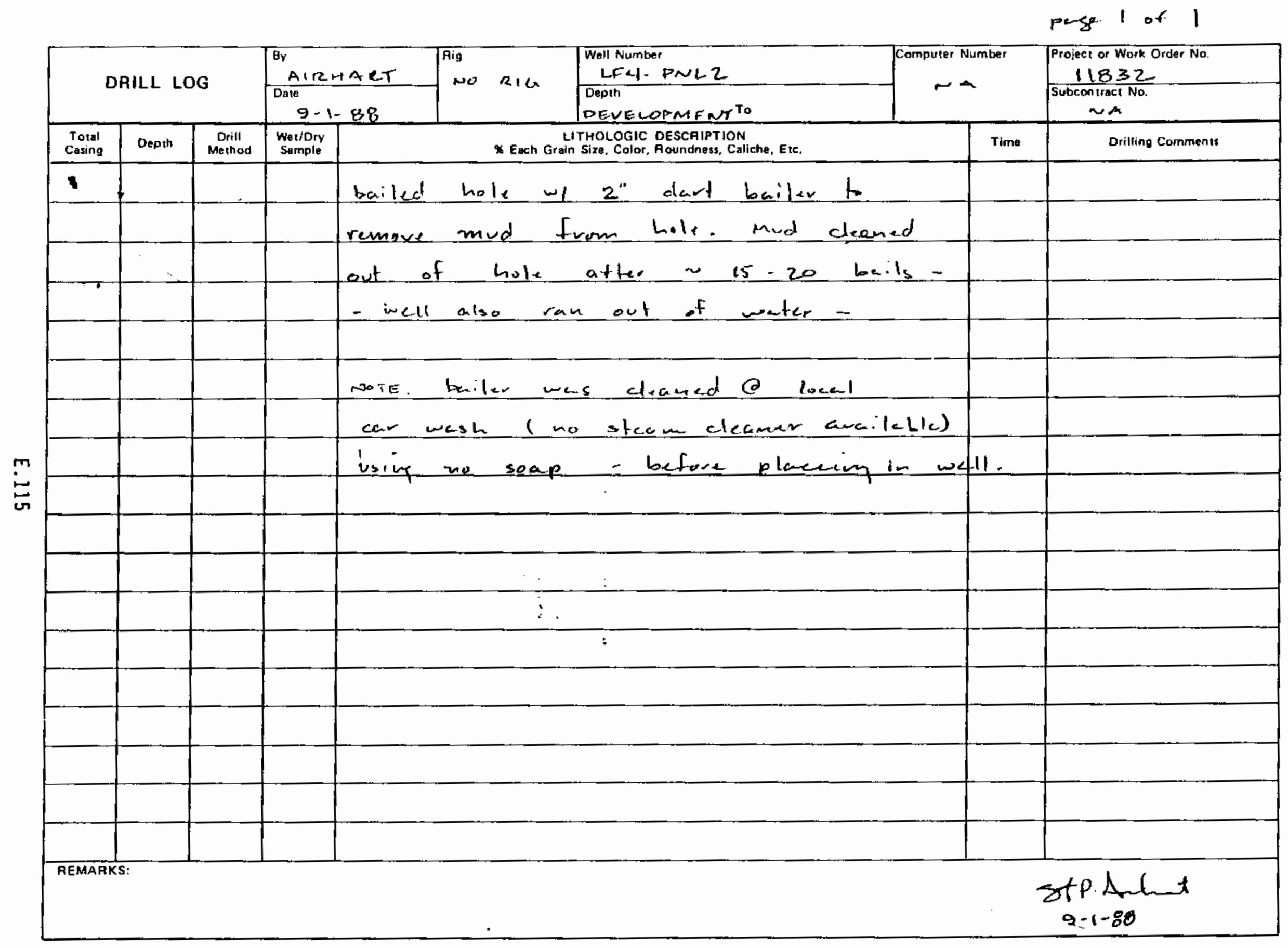




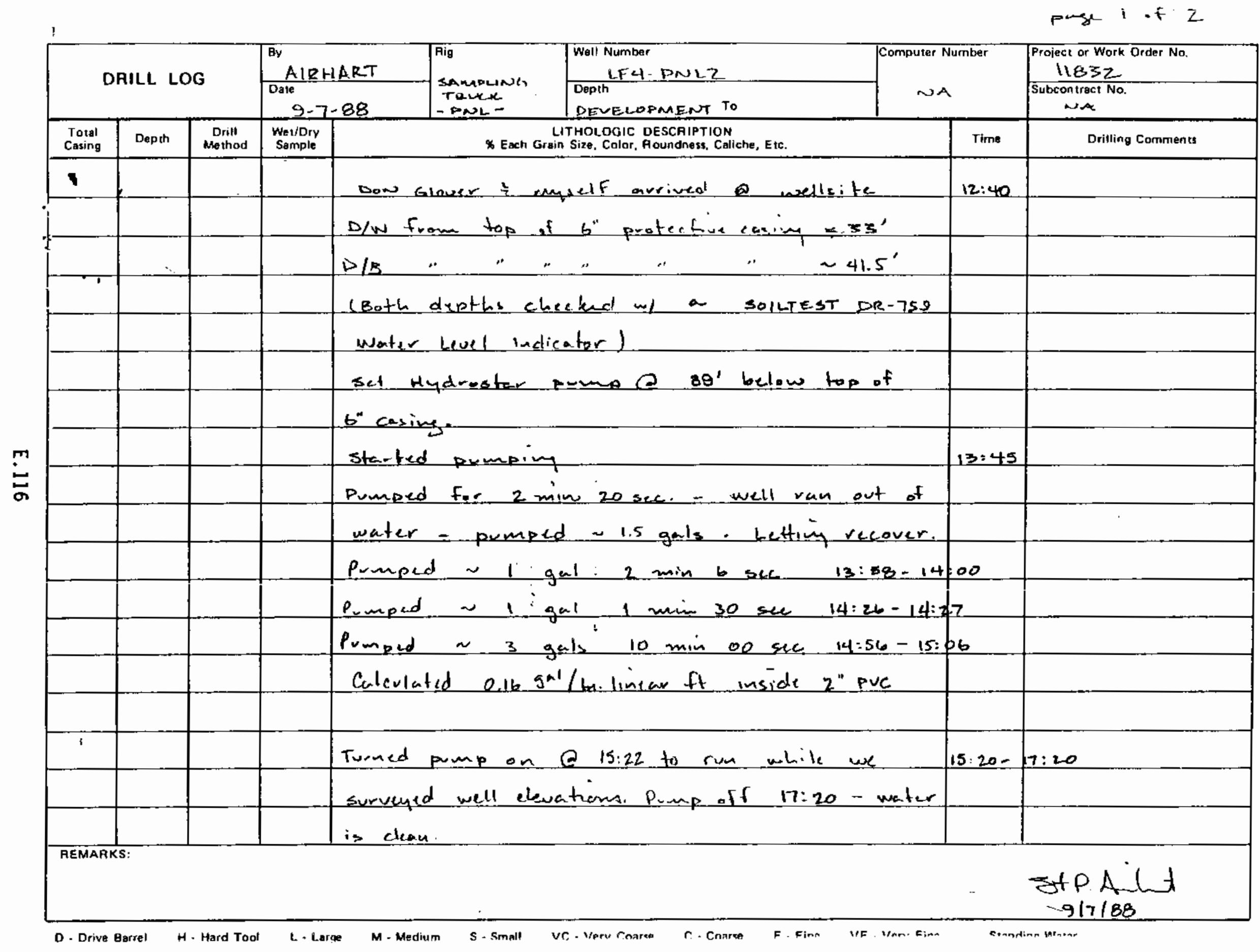


pang 2 . 2

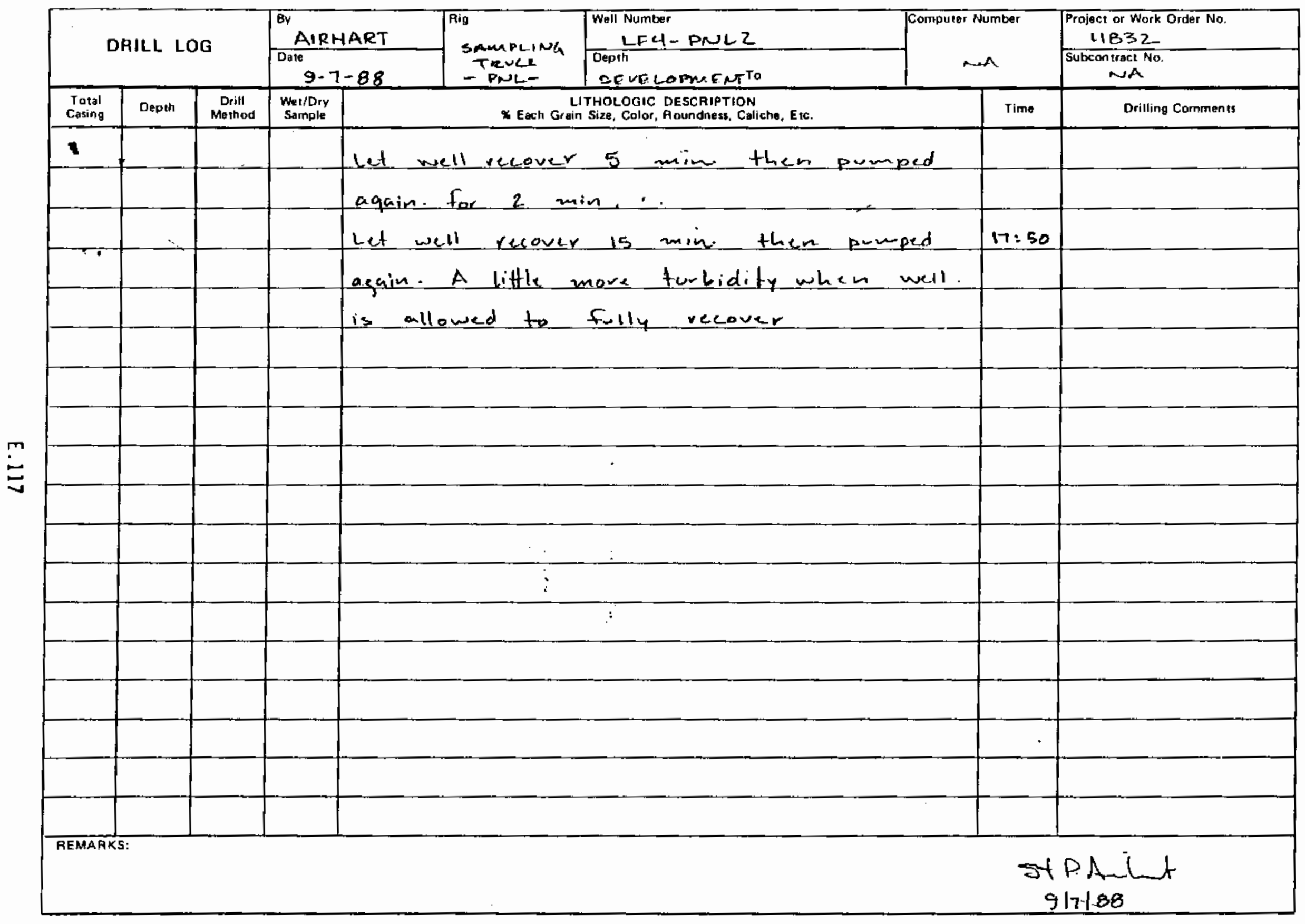

D. Drive Paret

RA. AAnction

S. Small

- Prores 
pare i wi

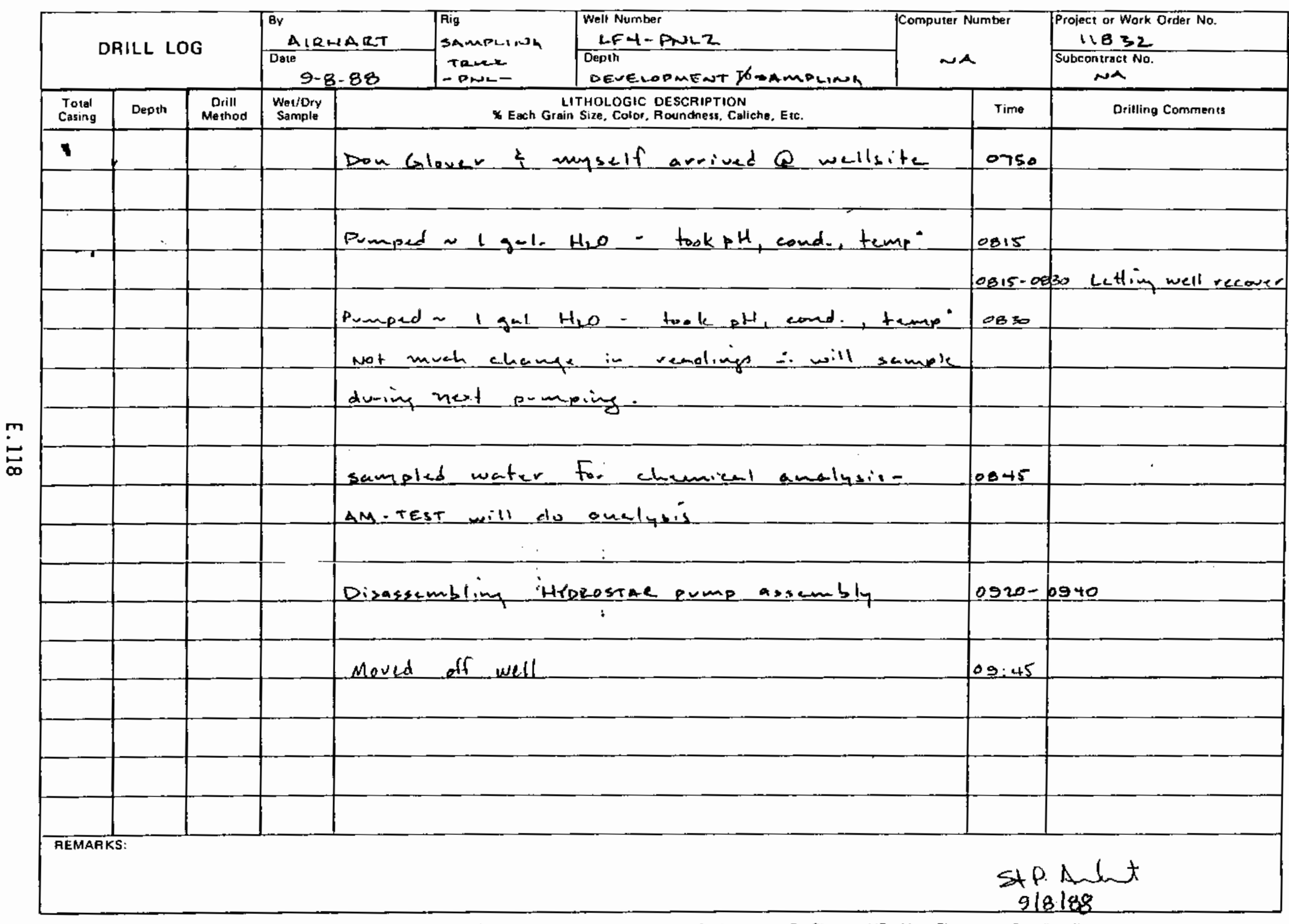

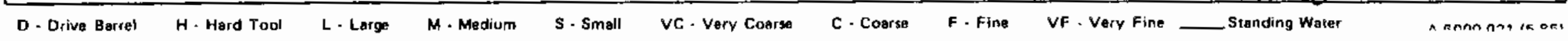


, $F$

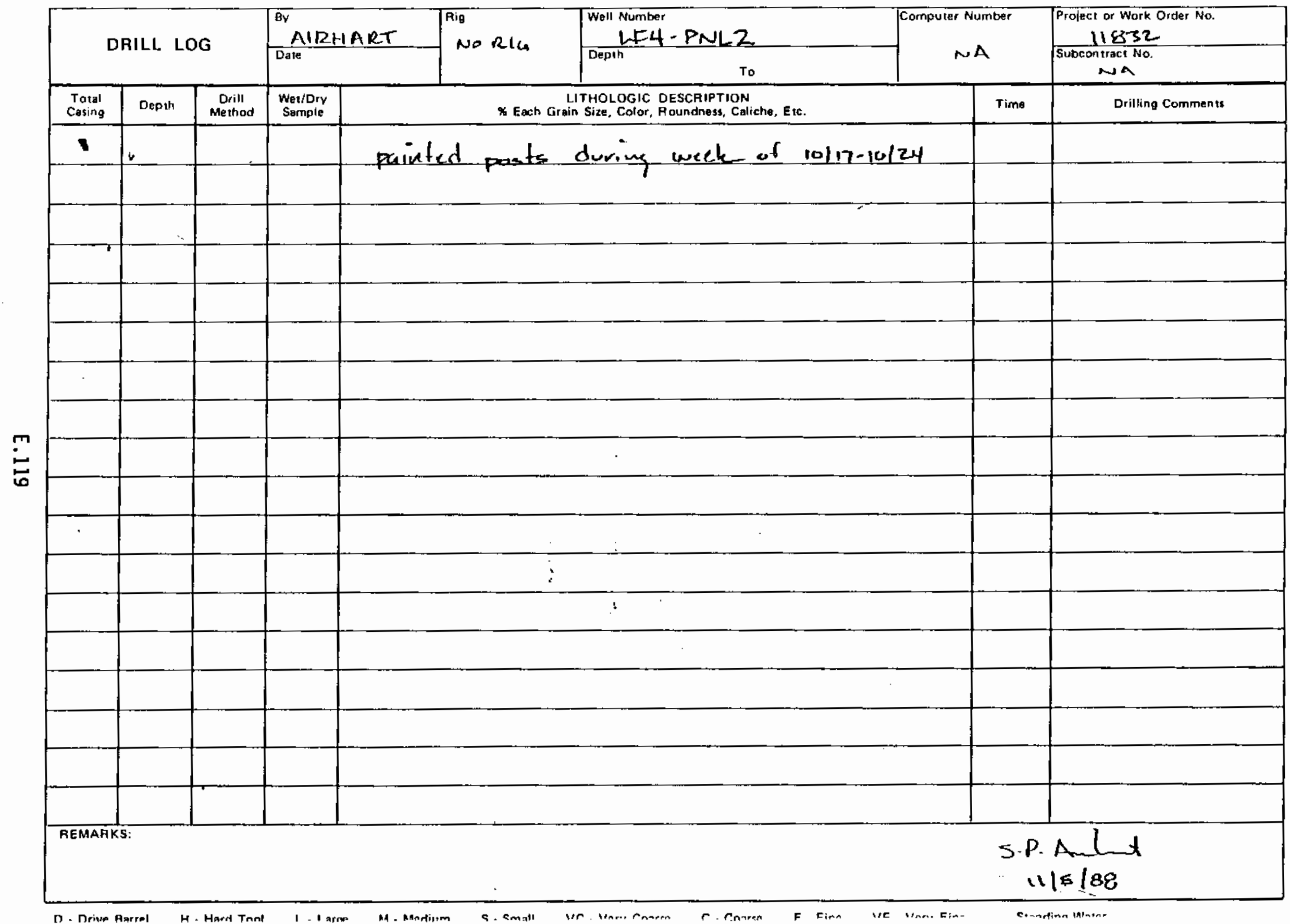



WELL LF4-PNL3 


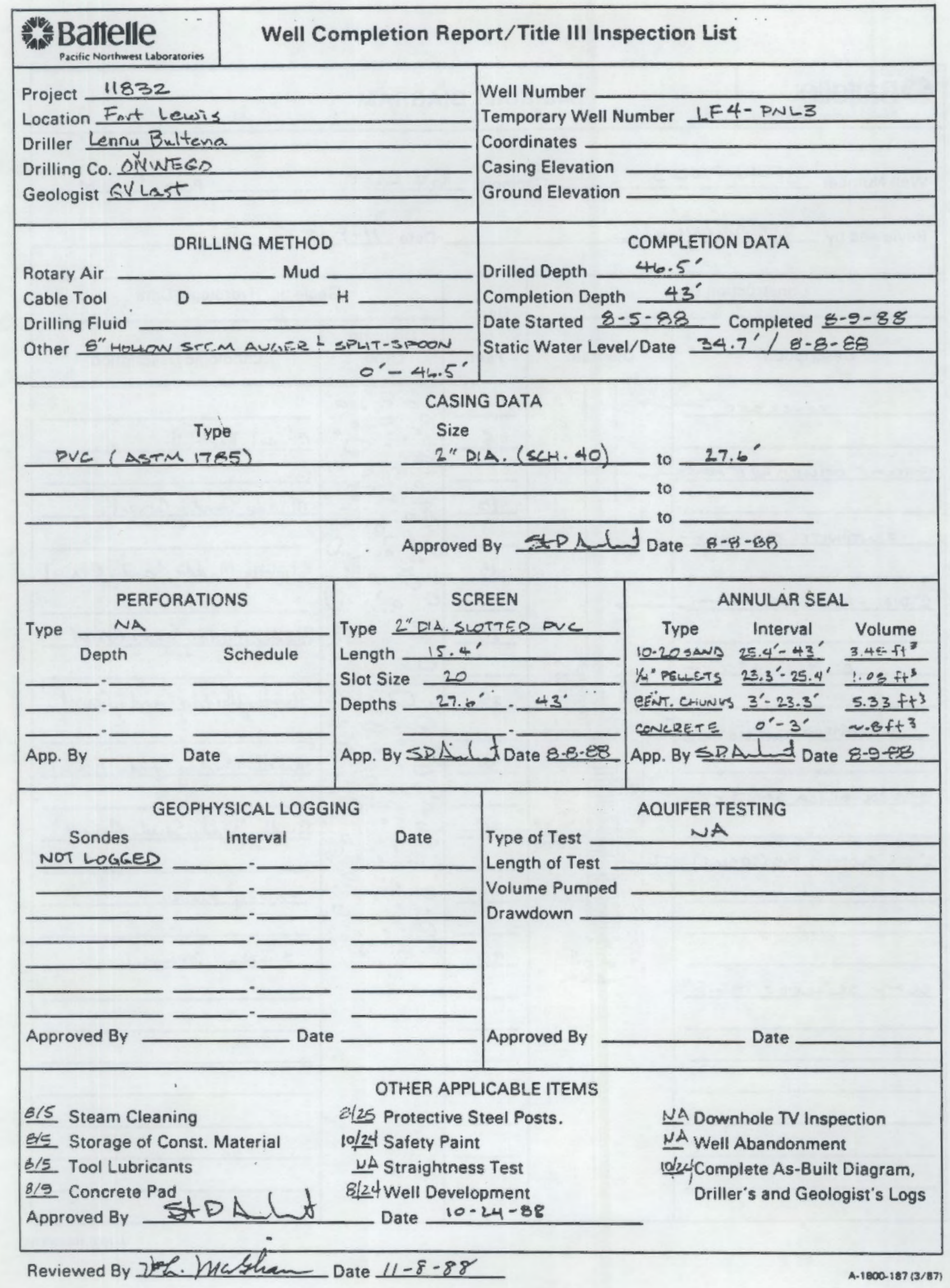




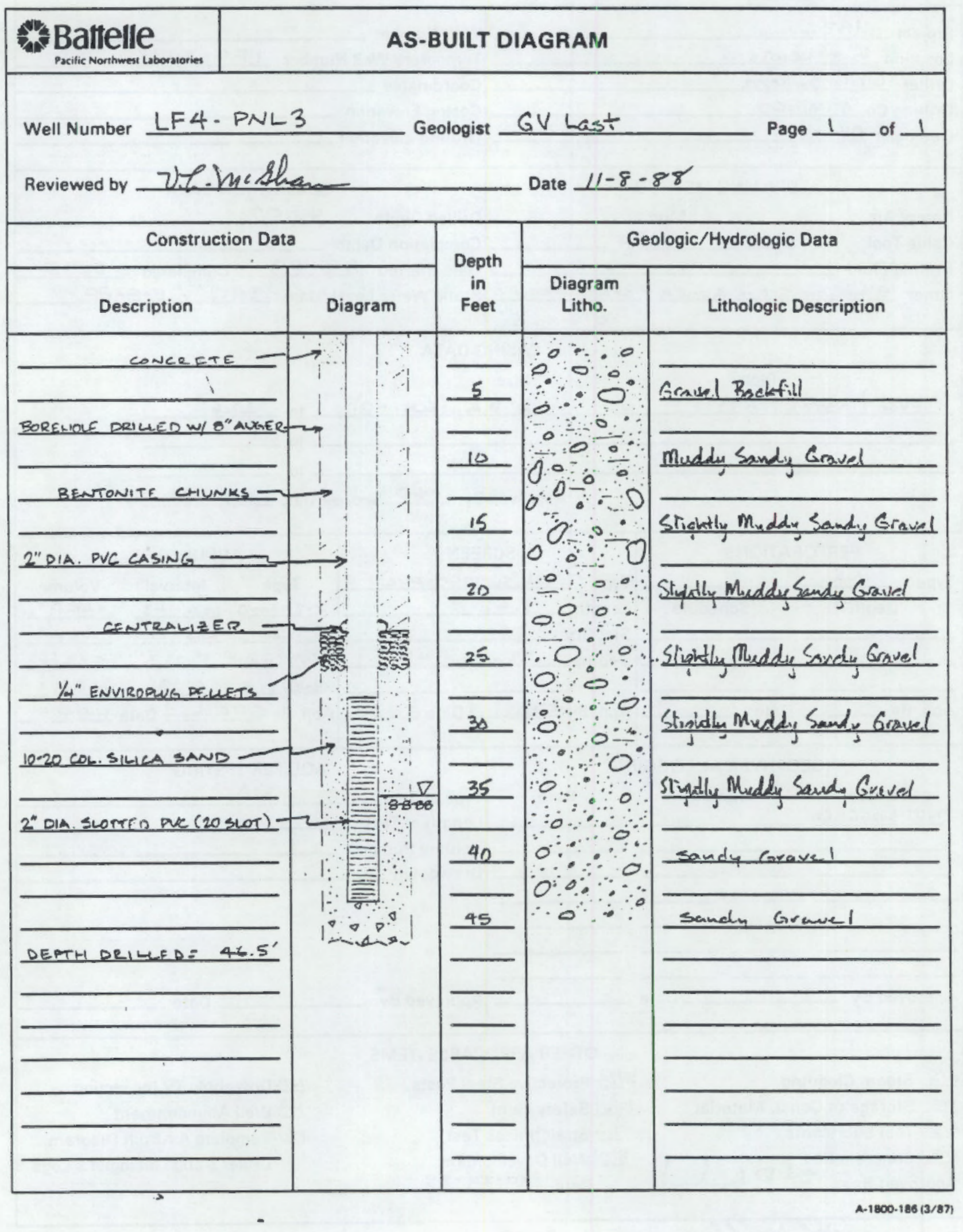


Dage 1 of 4

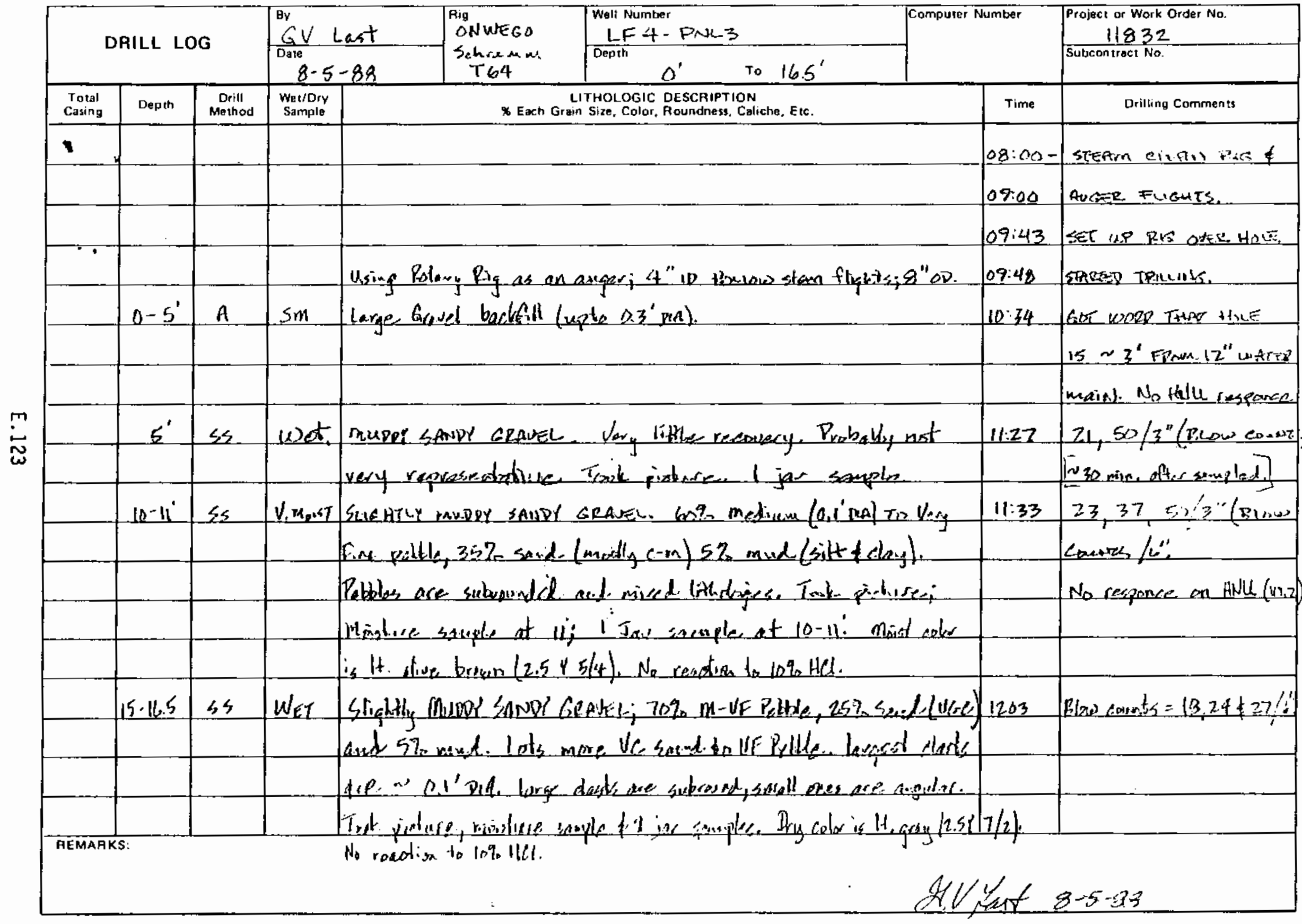


pange 7. of 4

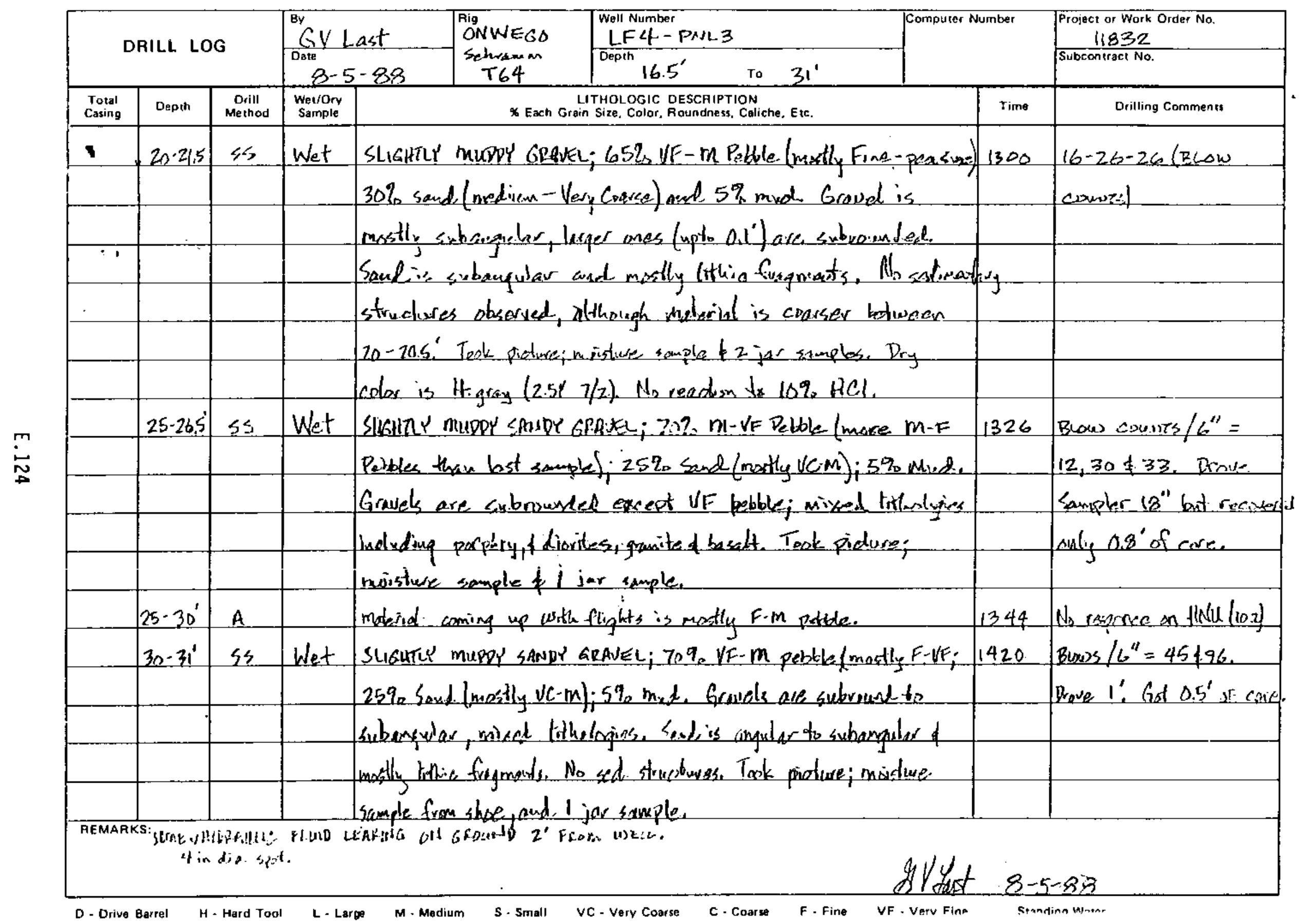


parg 3 if 4

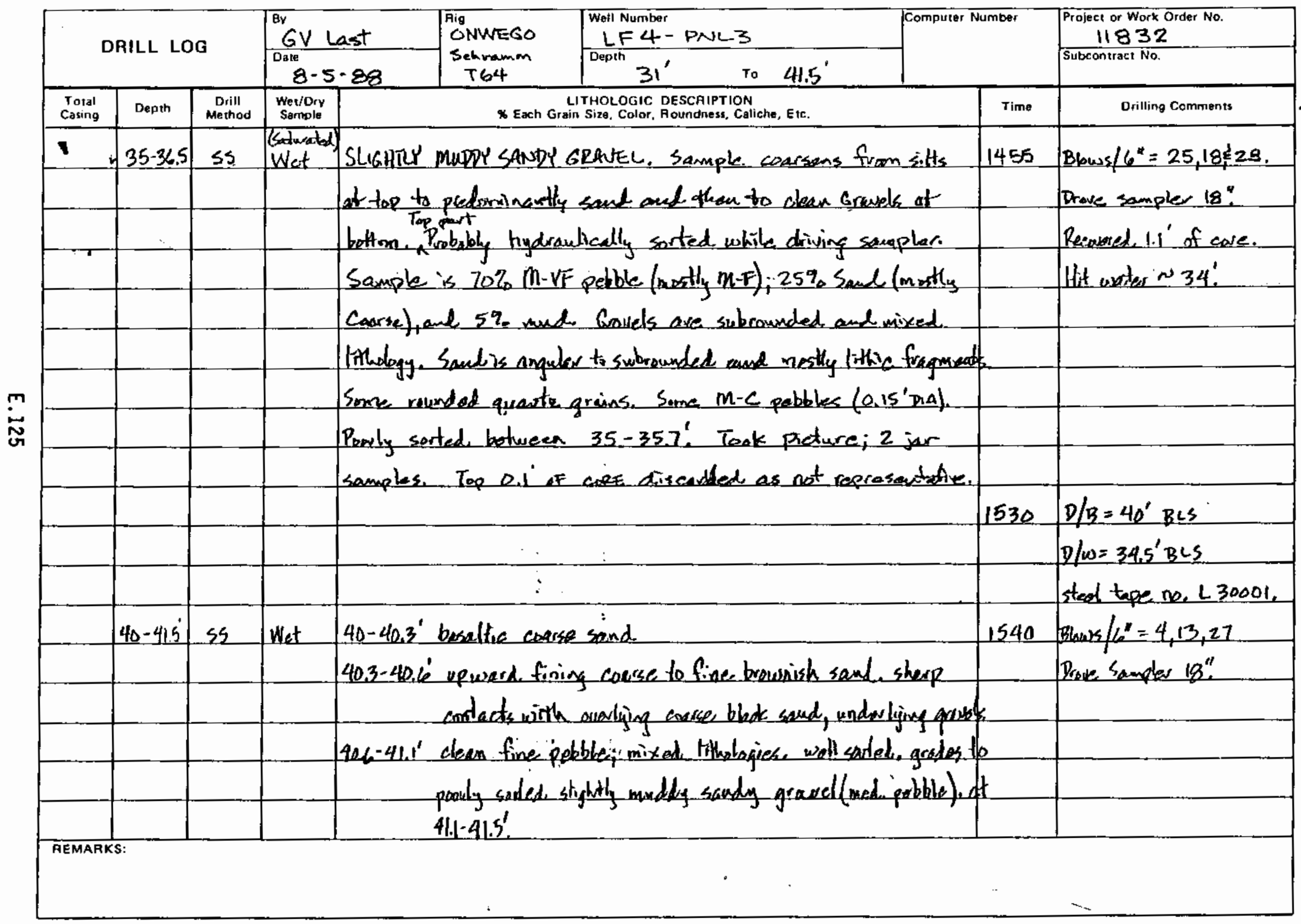


pinge 4 - 4

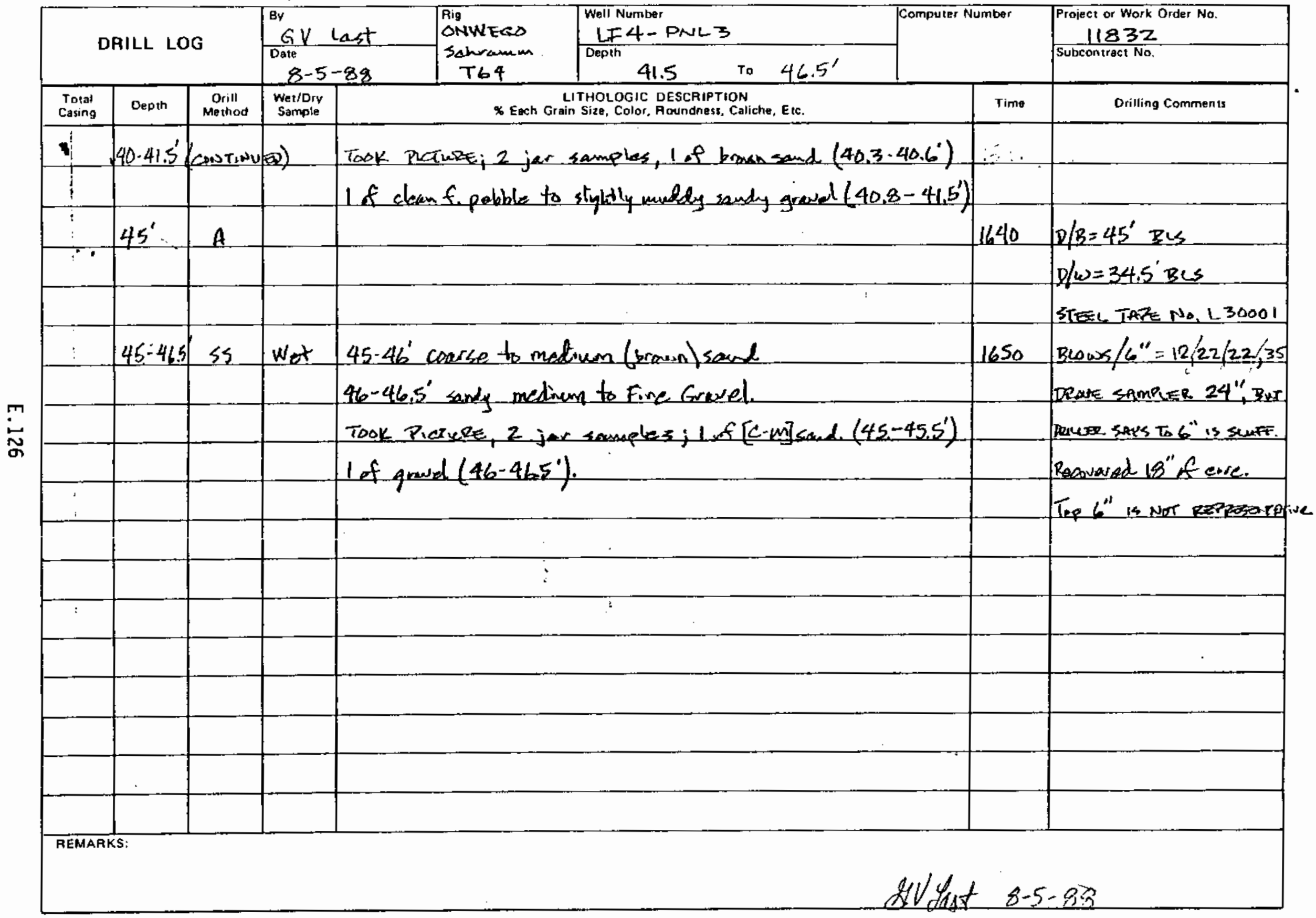


pare 1 of 3

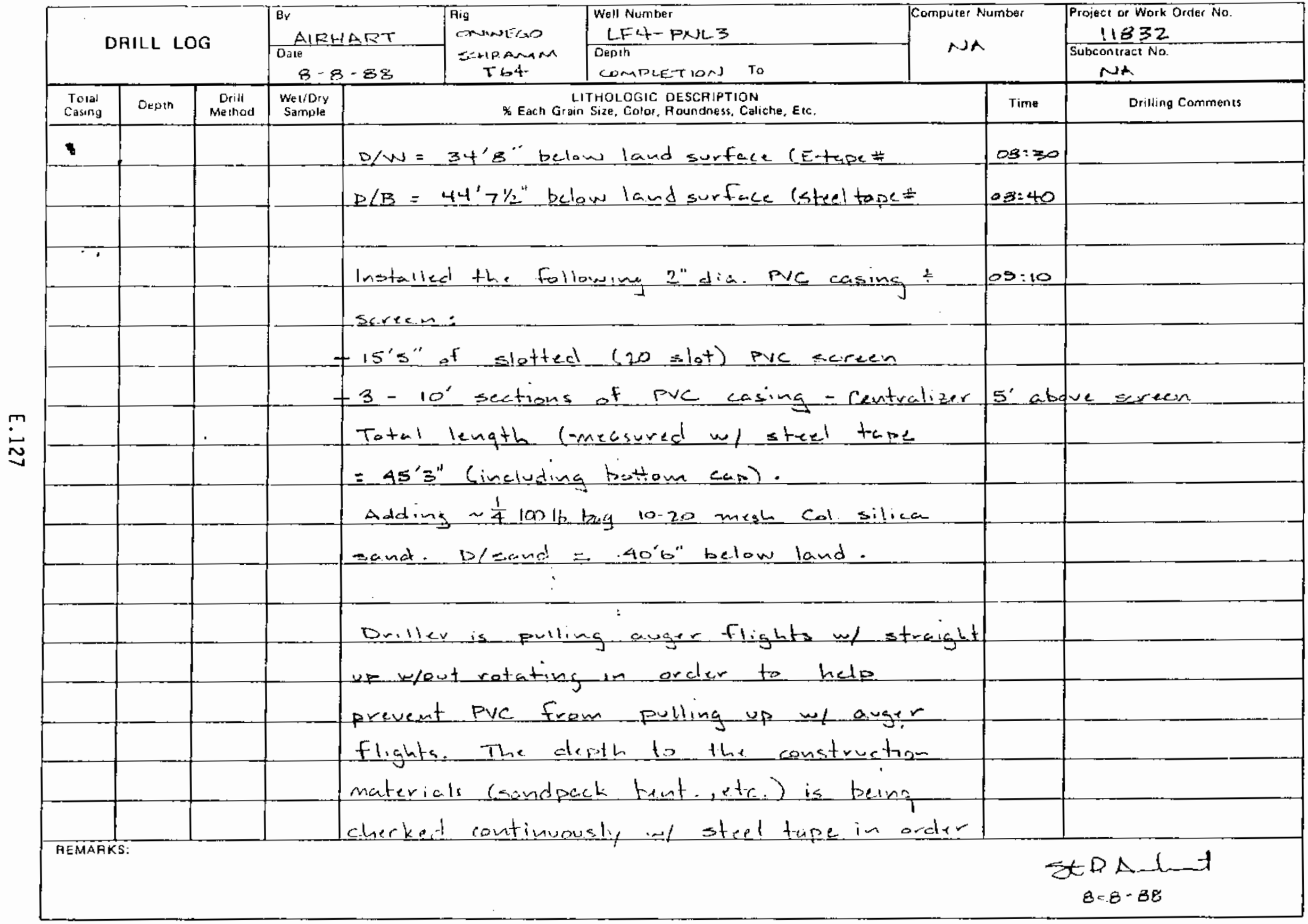

D-Drive Barrel H-Hard Tool 
puge $=$ \& 3

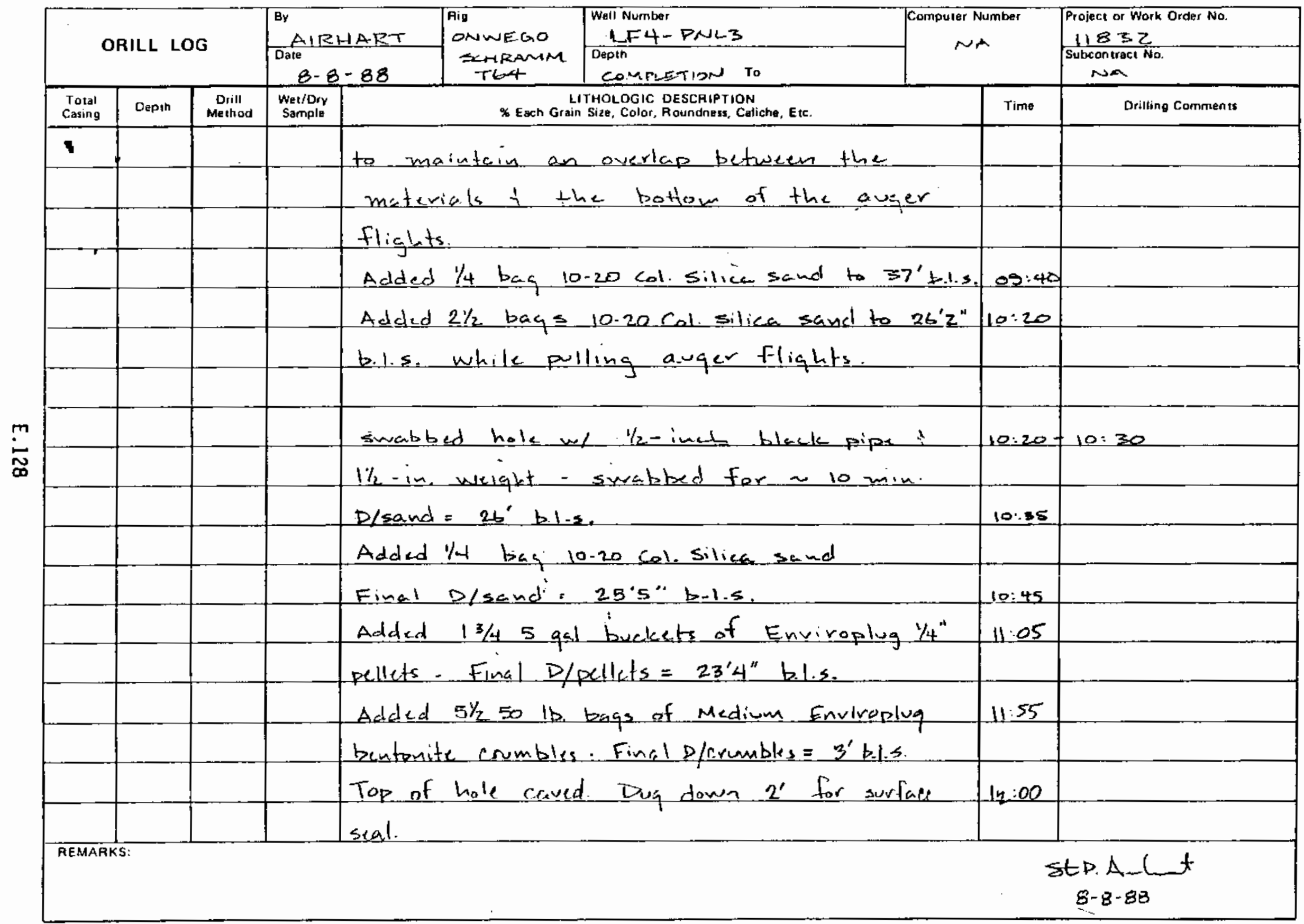

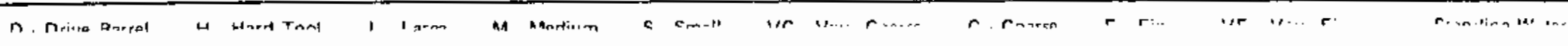




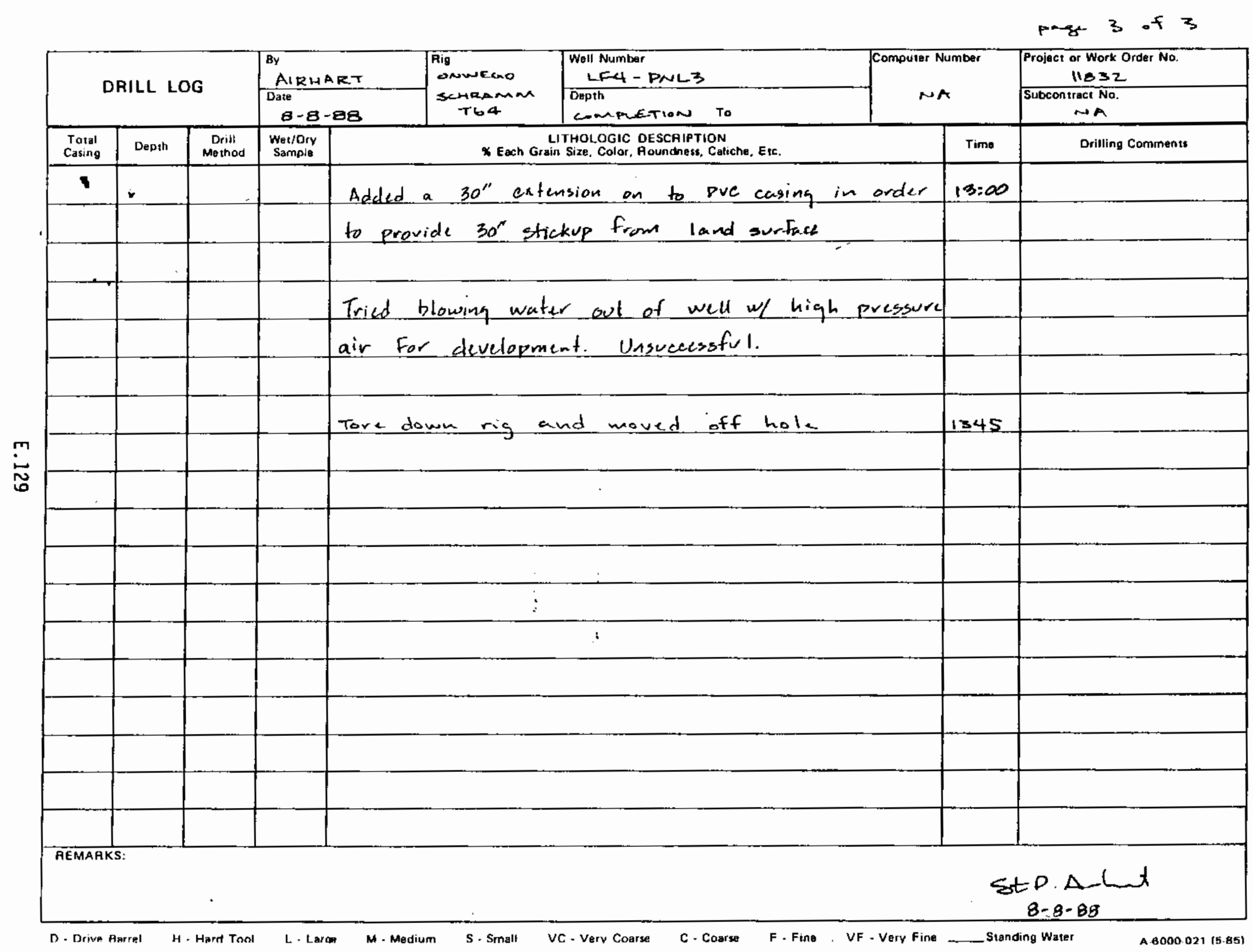




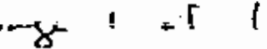

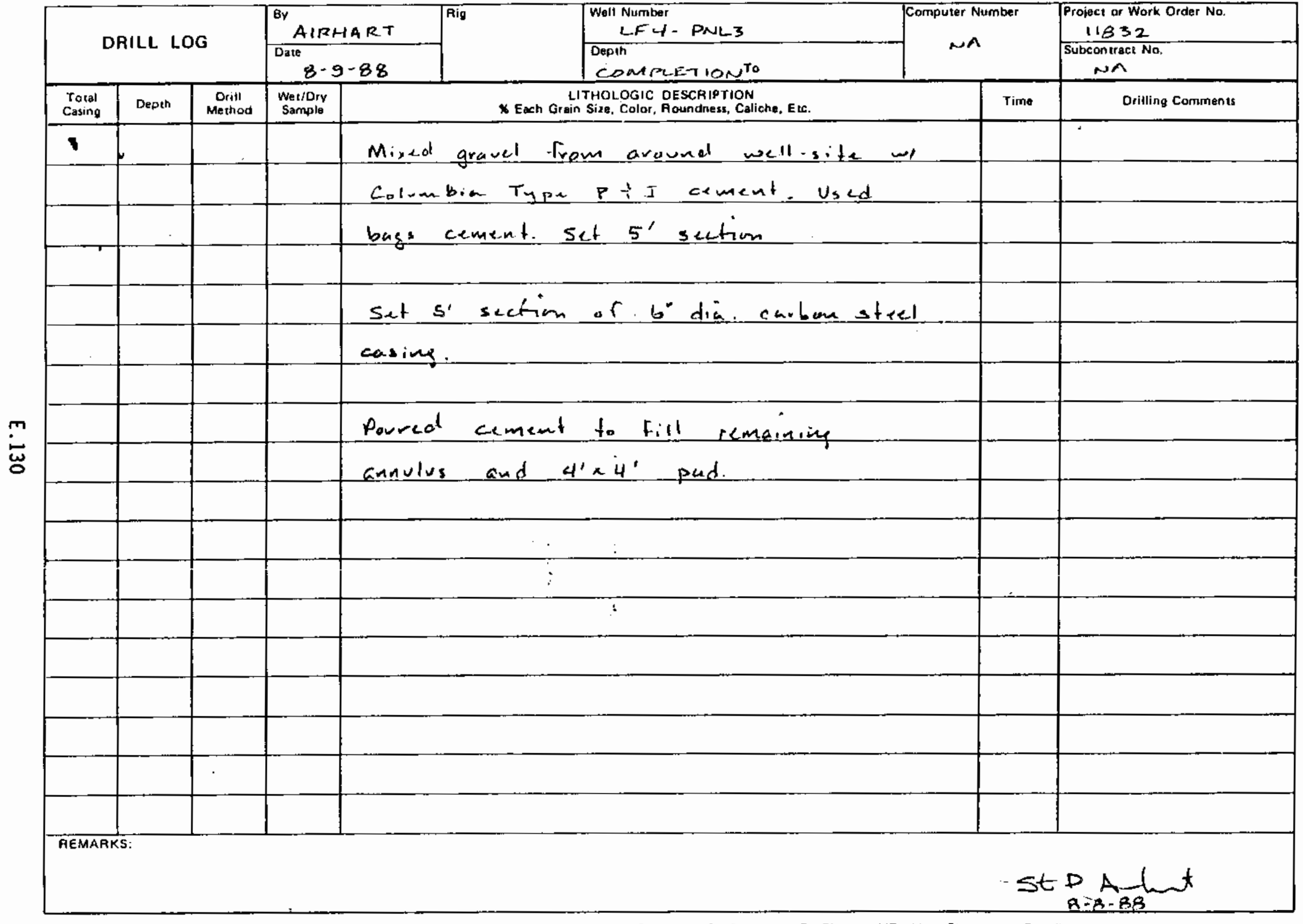
D. Drive Barrel
L. Large
M - Medium
S - Small
VC - Verv Coare
C. Coarse
F. Finn VF . Veru Fin
Standing wax 
pagt $1 \therefore 1$

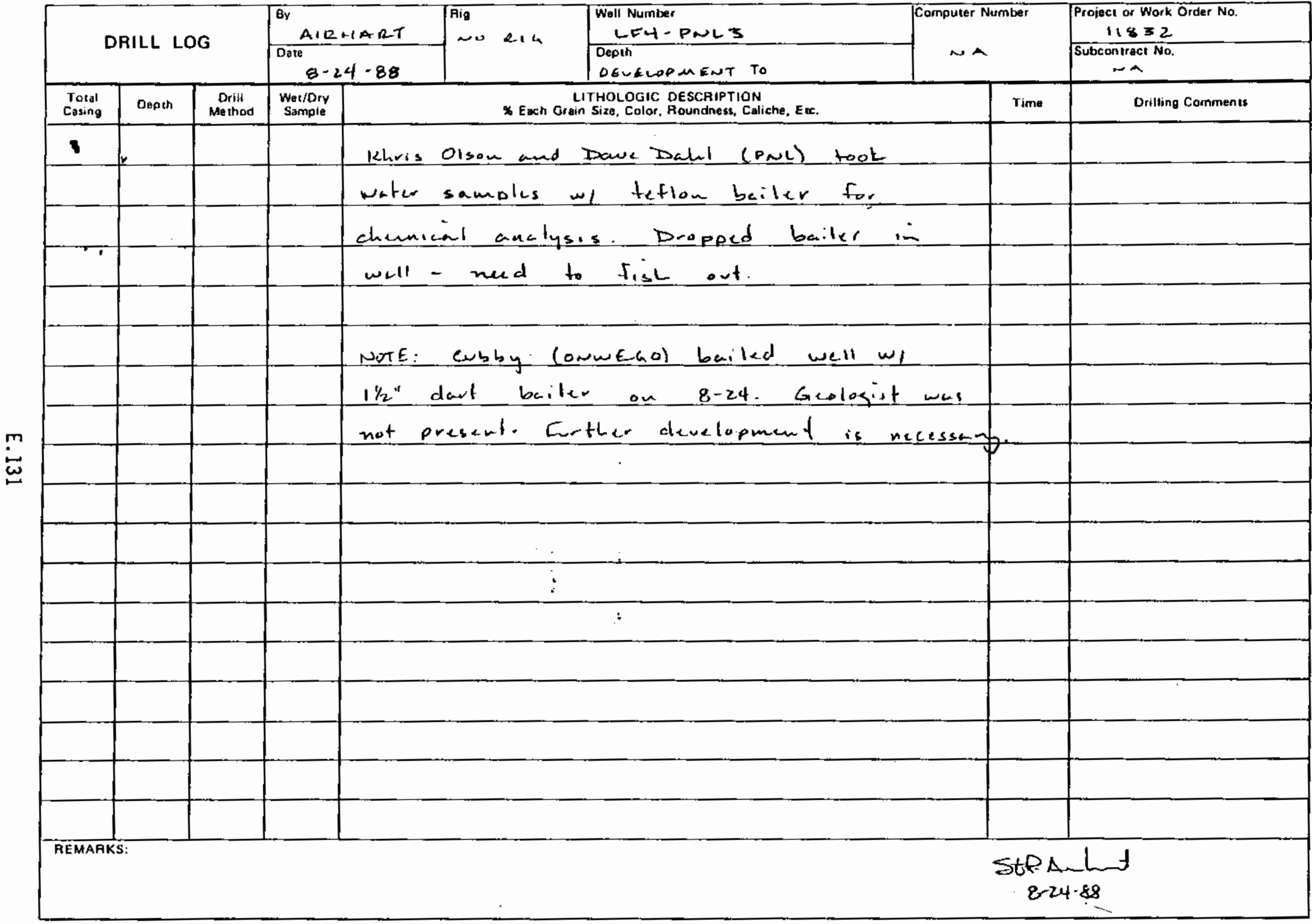

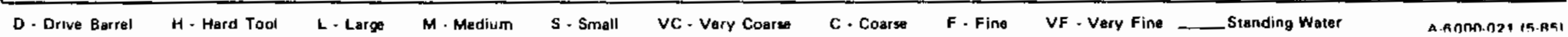




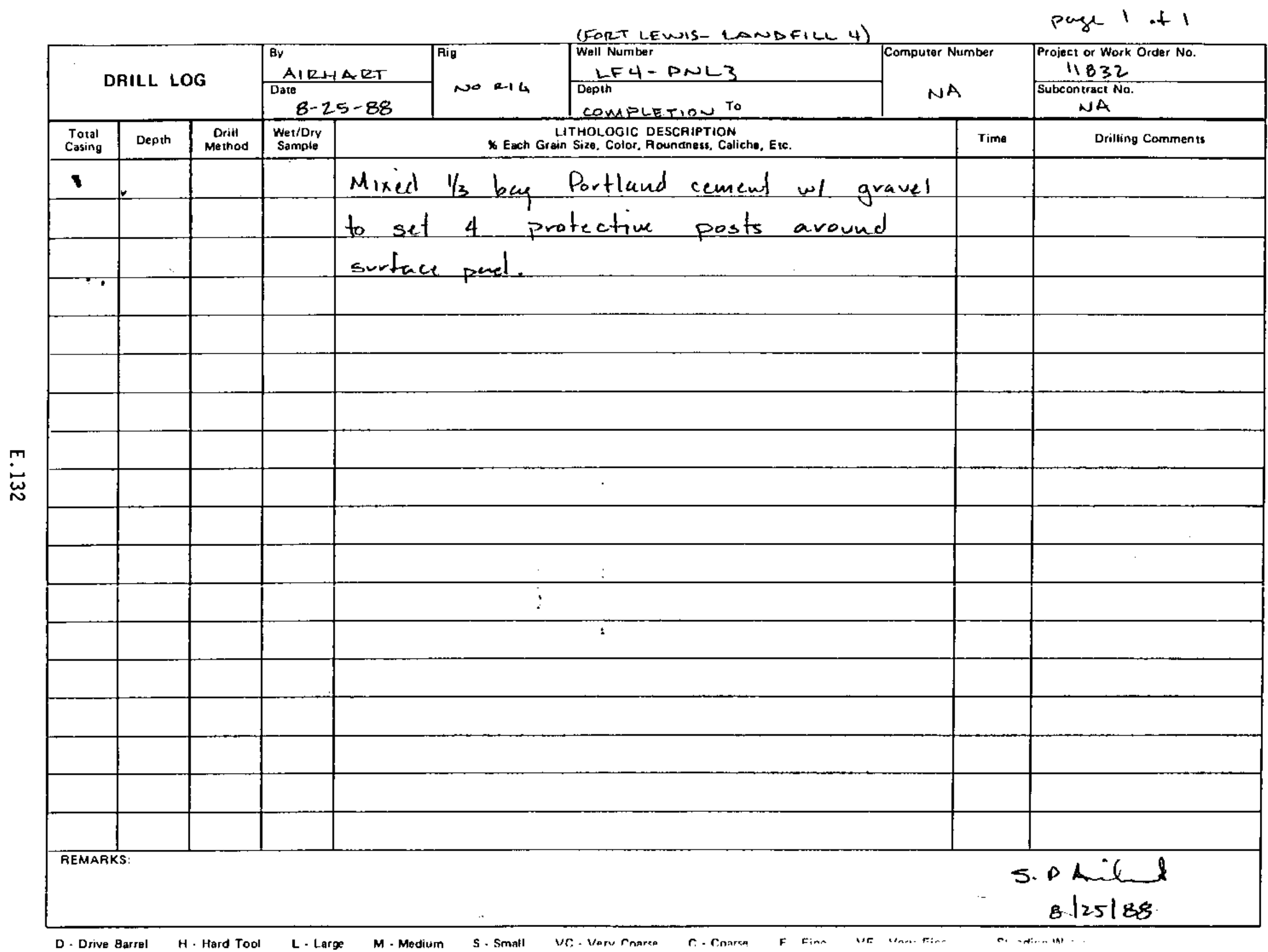


$p-\gamma|\cdot f|$

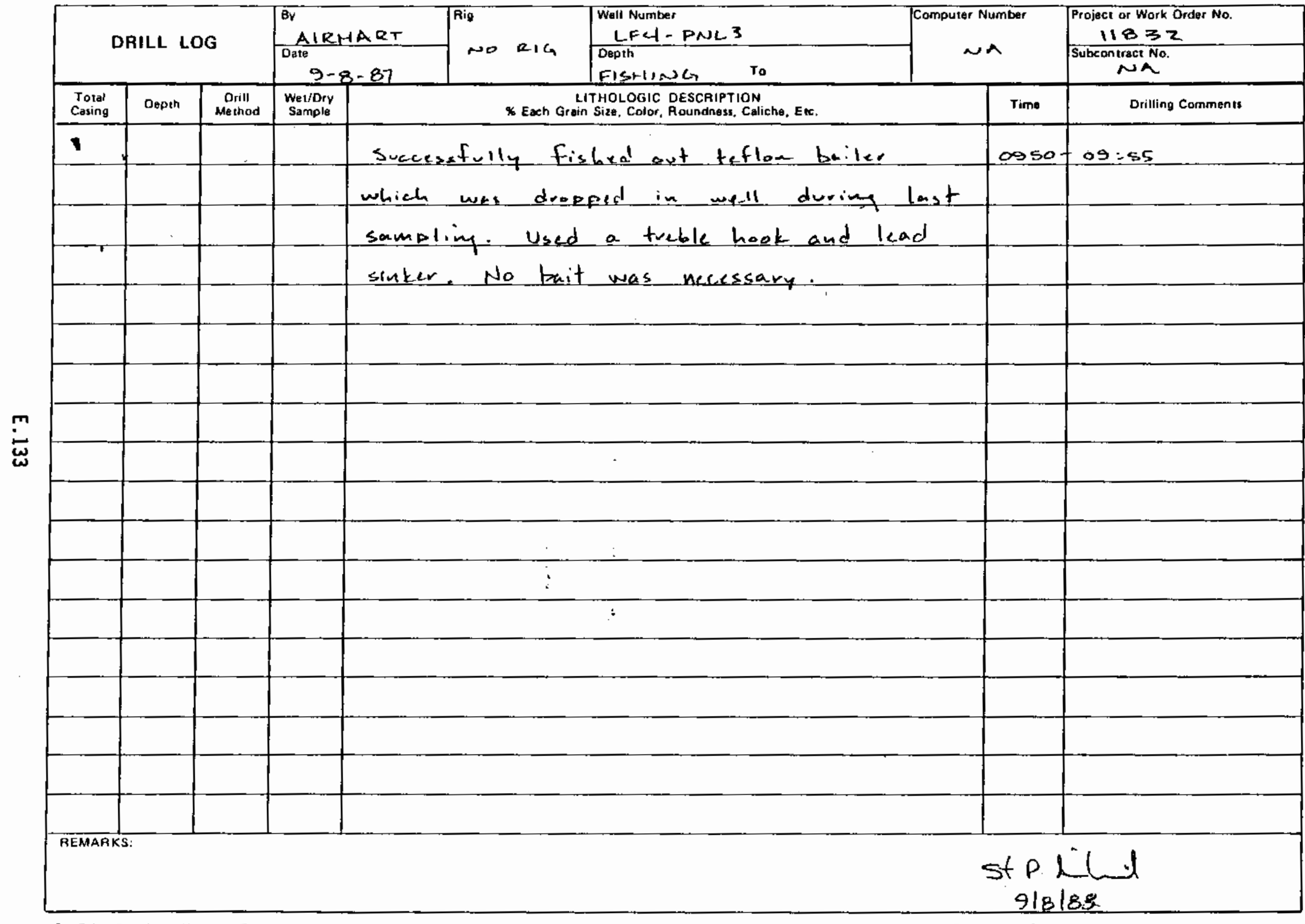
D. Orive Barrel
H. Hard Tool
L. Larne
M - Medium
S - Small
c. . Cnarse
F. Finn
VF - Varw Firia
standing Mtgtap 
1 of 1

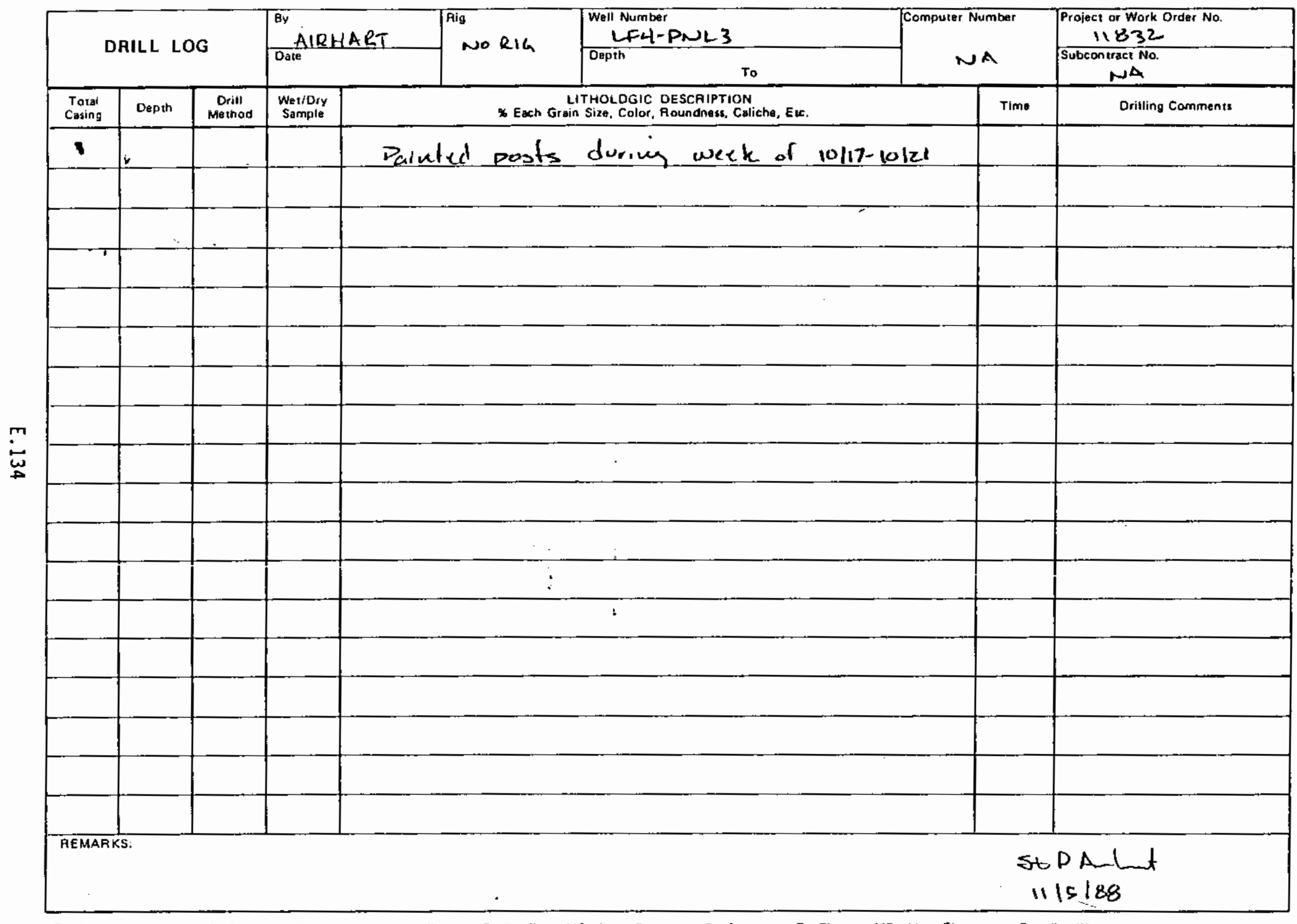

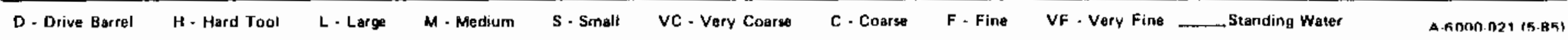


WELL LF4-PNL4 


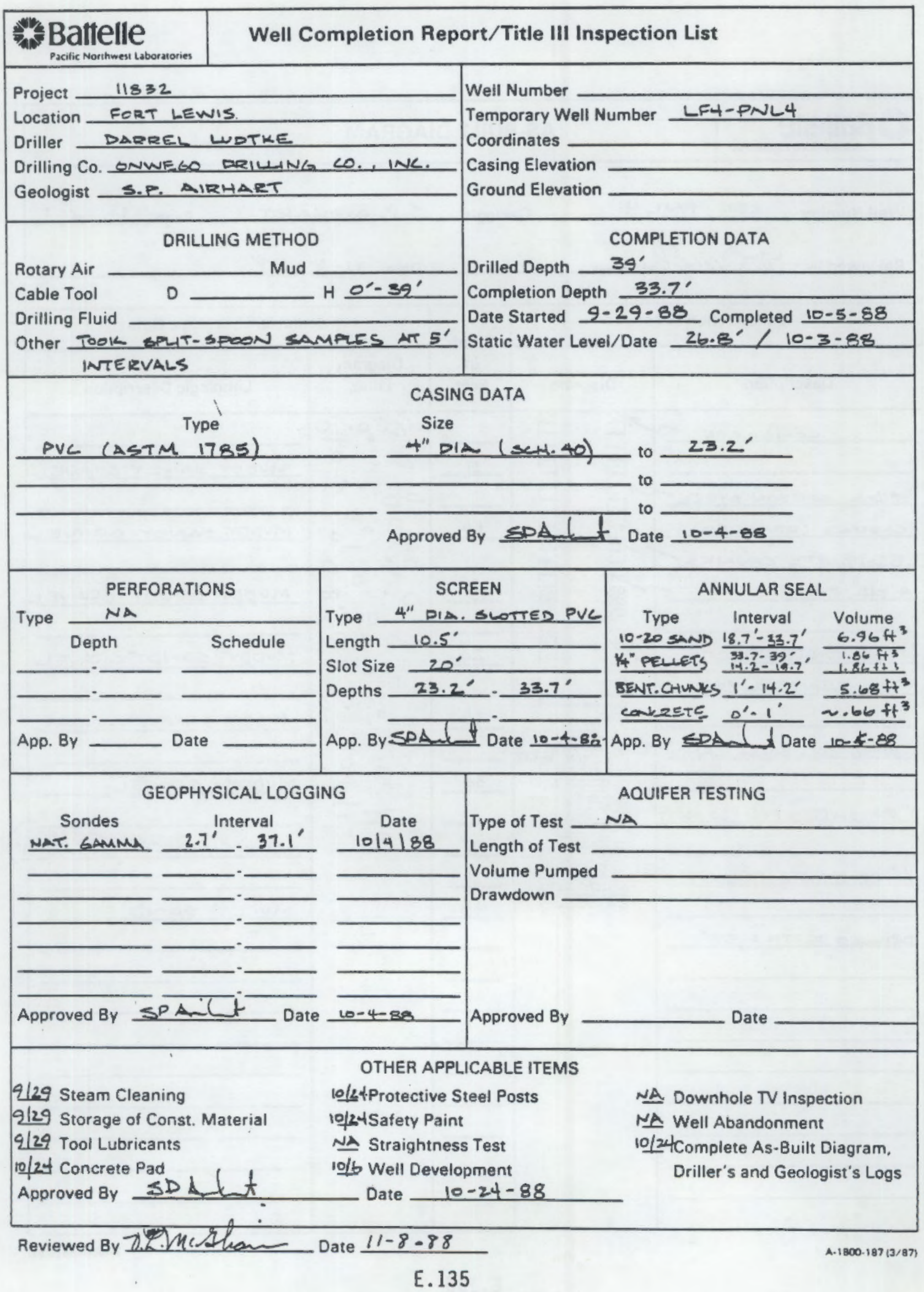




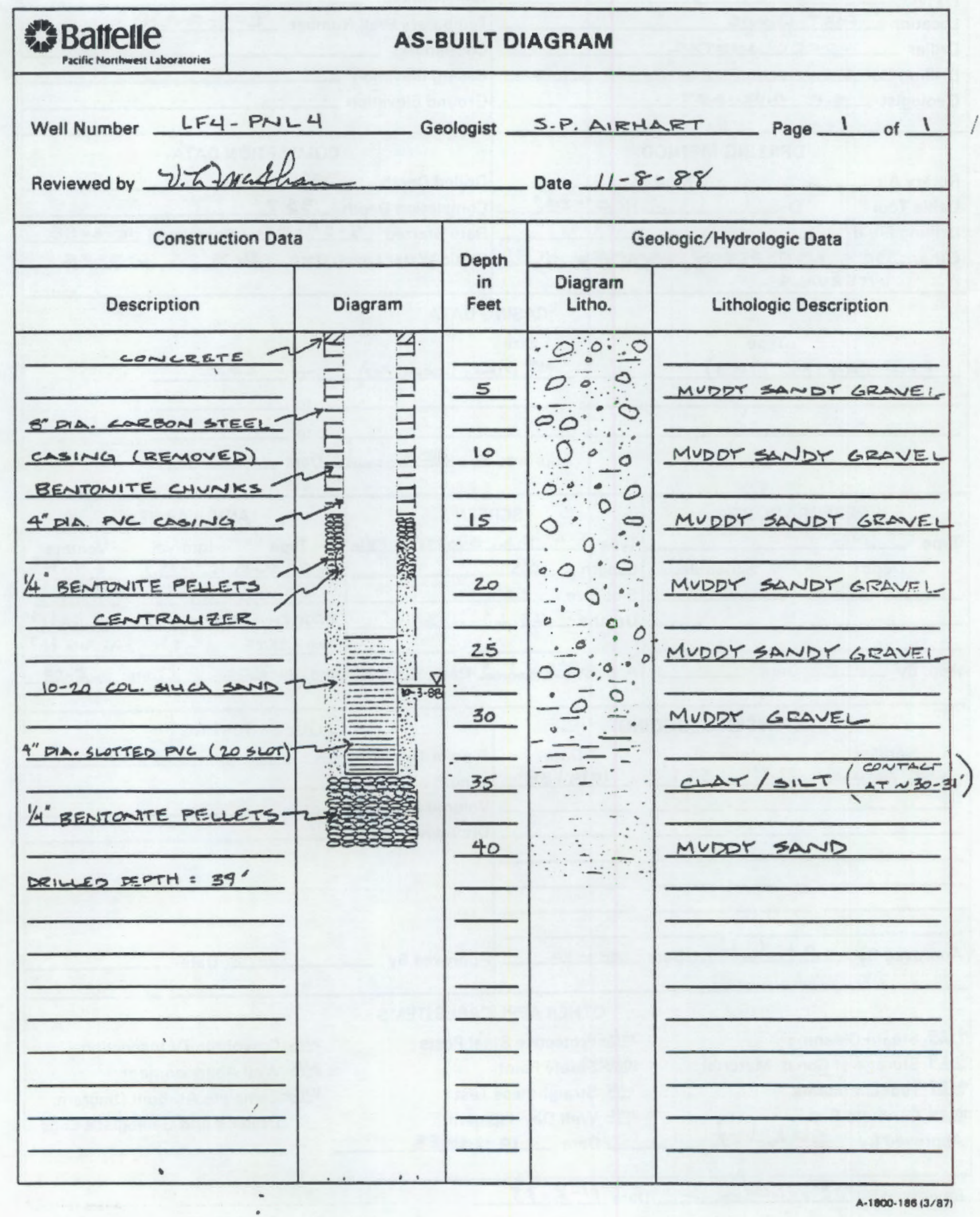

\section{E.136}




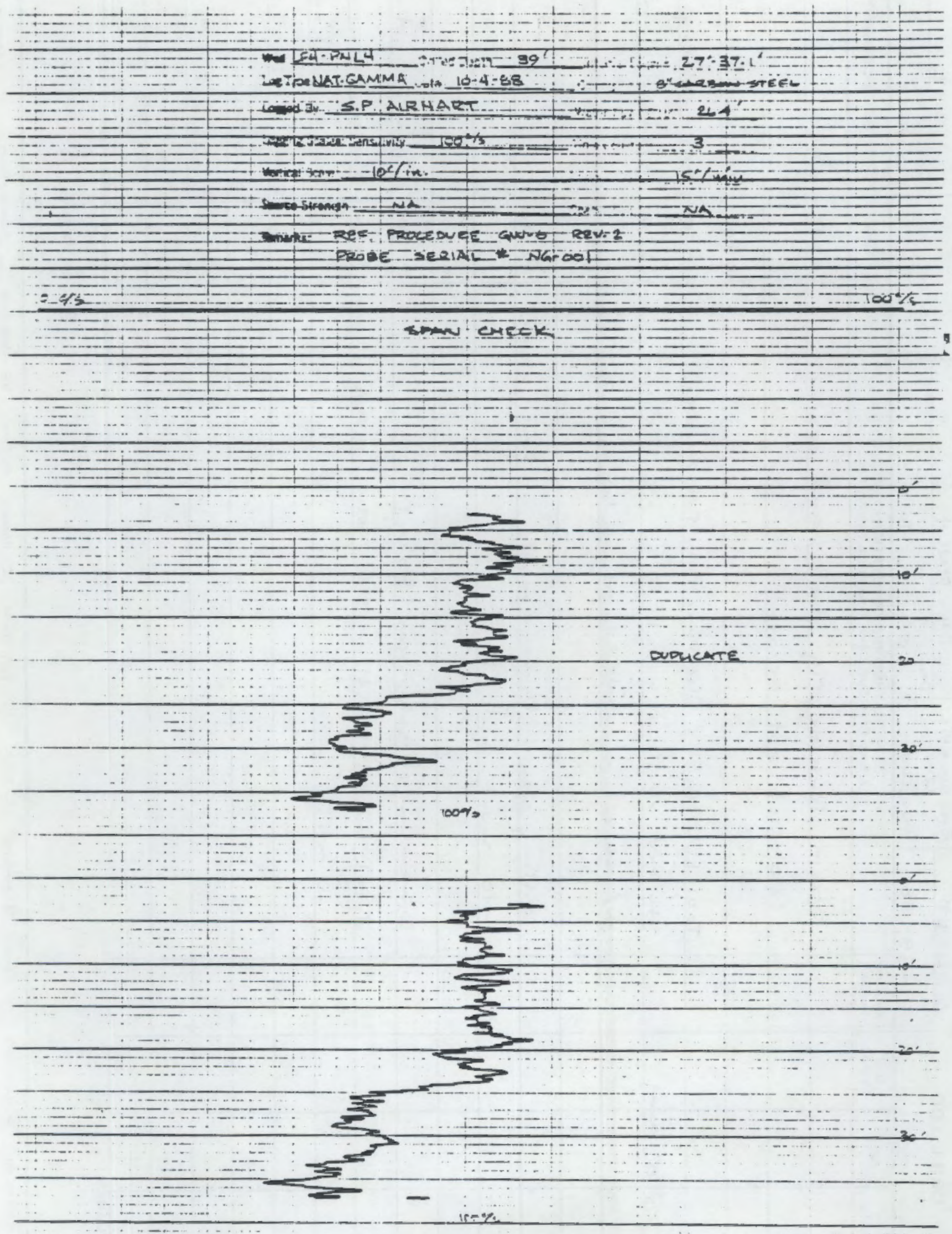




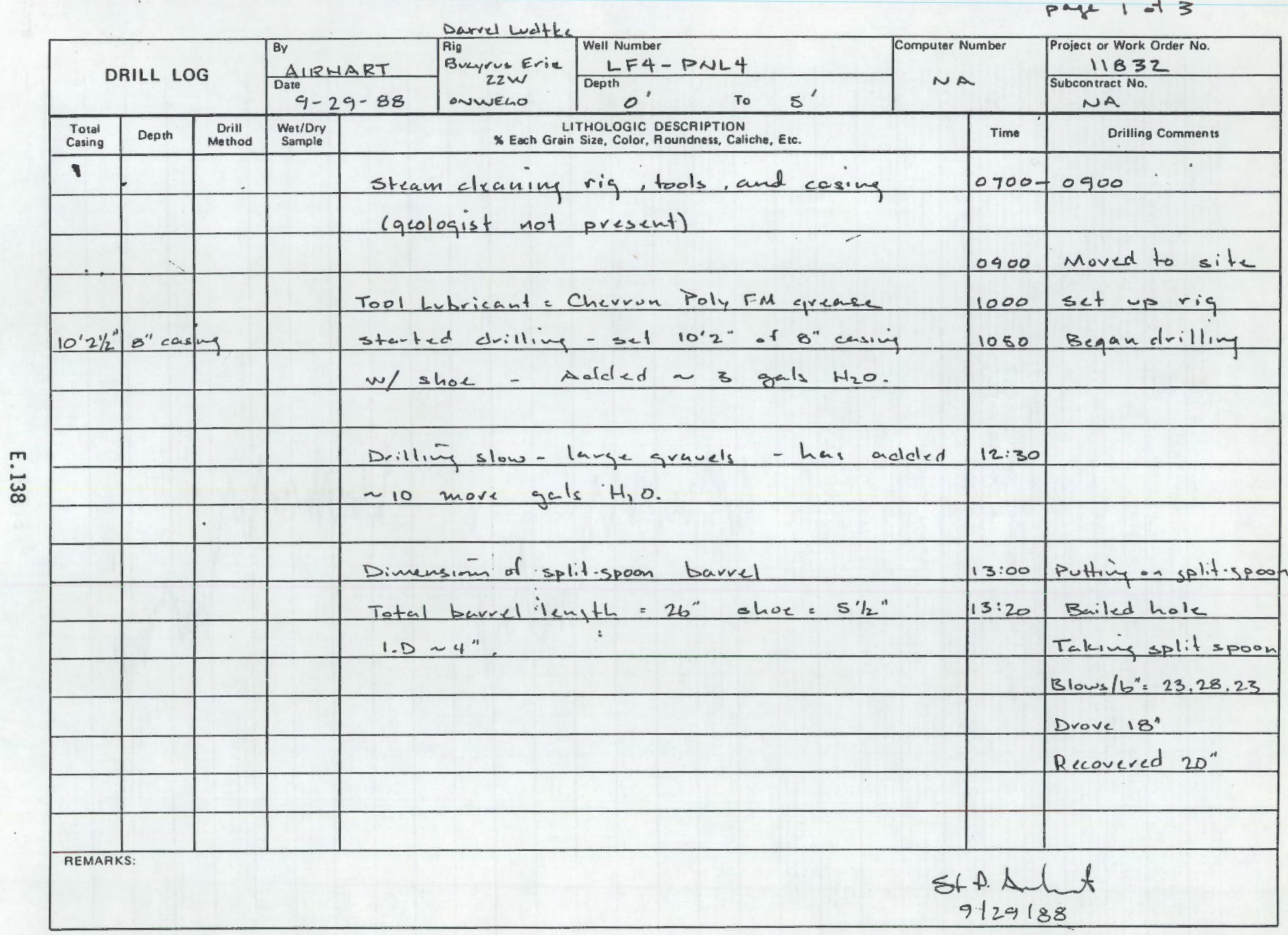
n. nniwn narmal
H. Hared Tnat
1 - I arne
M - Madium
S. Small
VC. Very Coarse
C. Coarse
F. Fine VF - Very Fine
Standing Water 
pure 2 of -

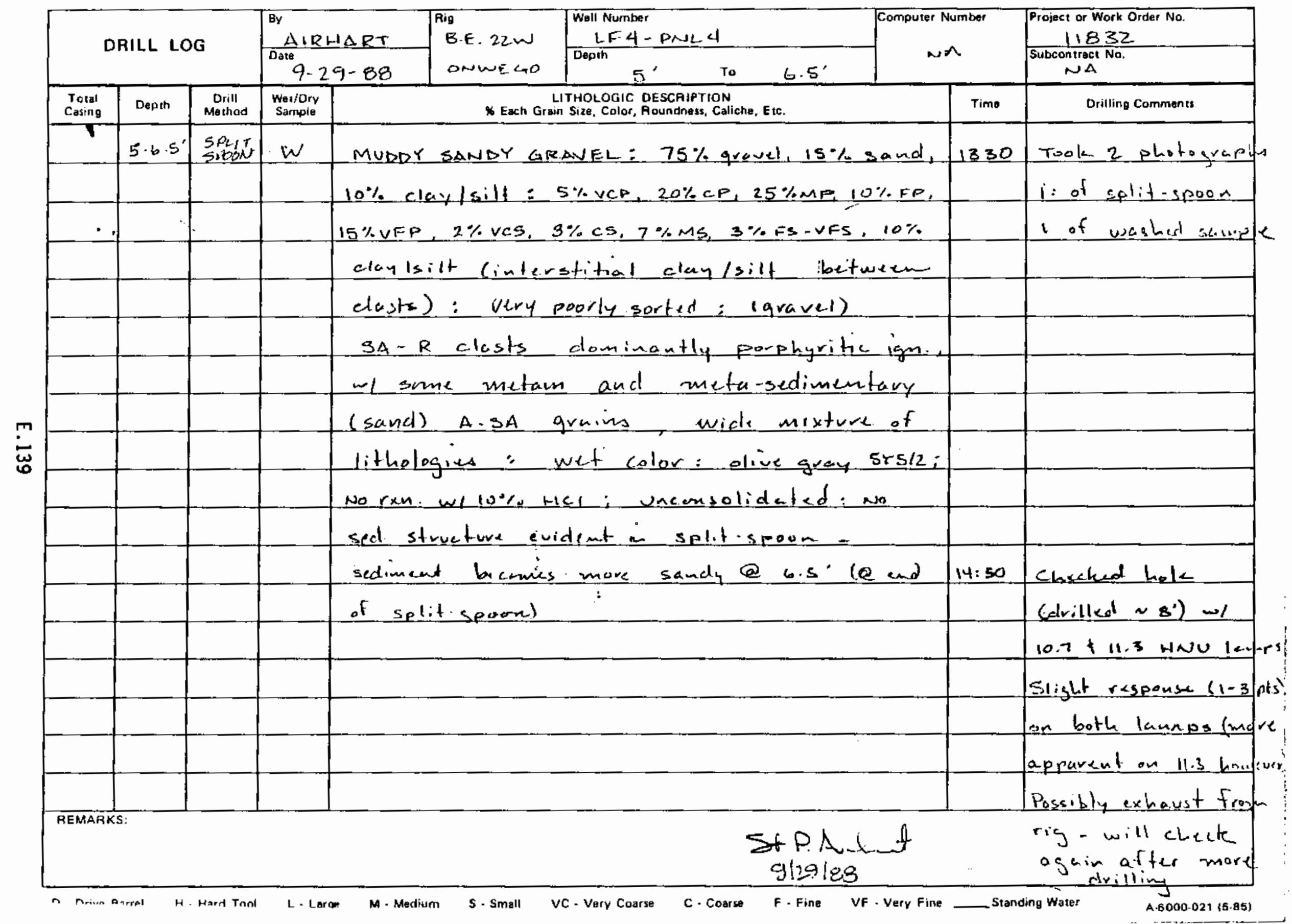


p-ge $\leq$ of 3

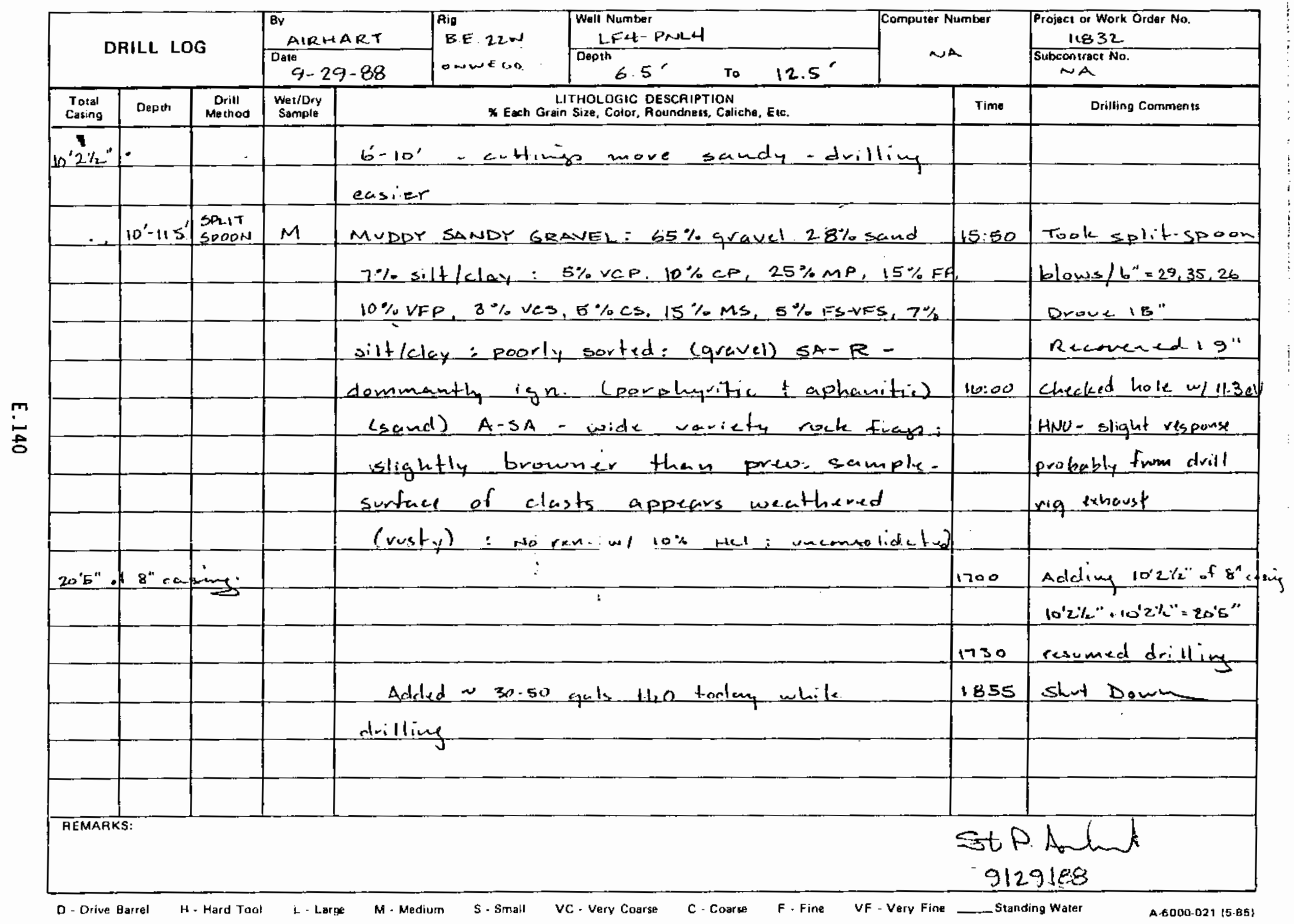


pang I n.

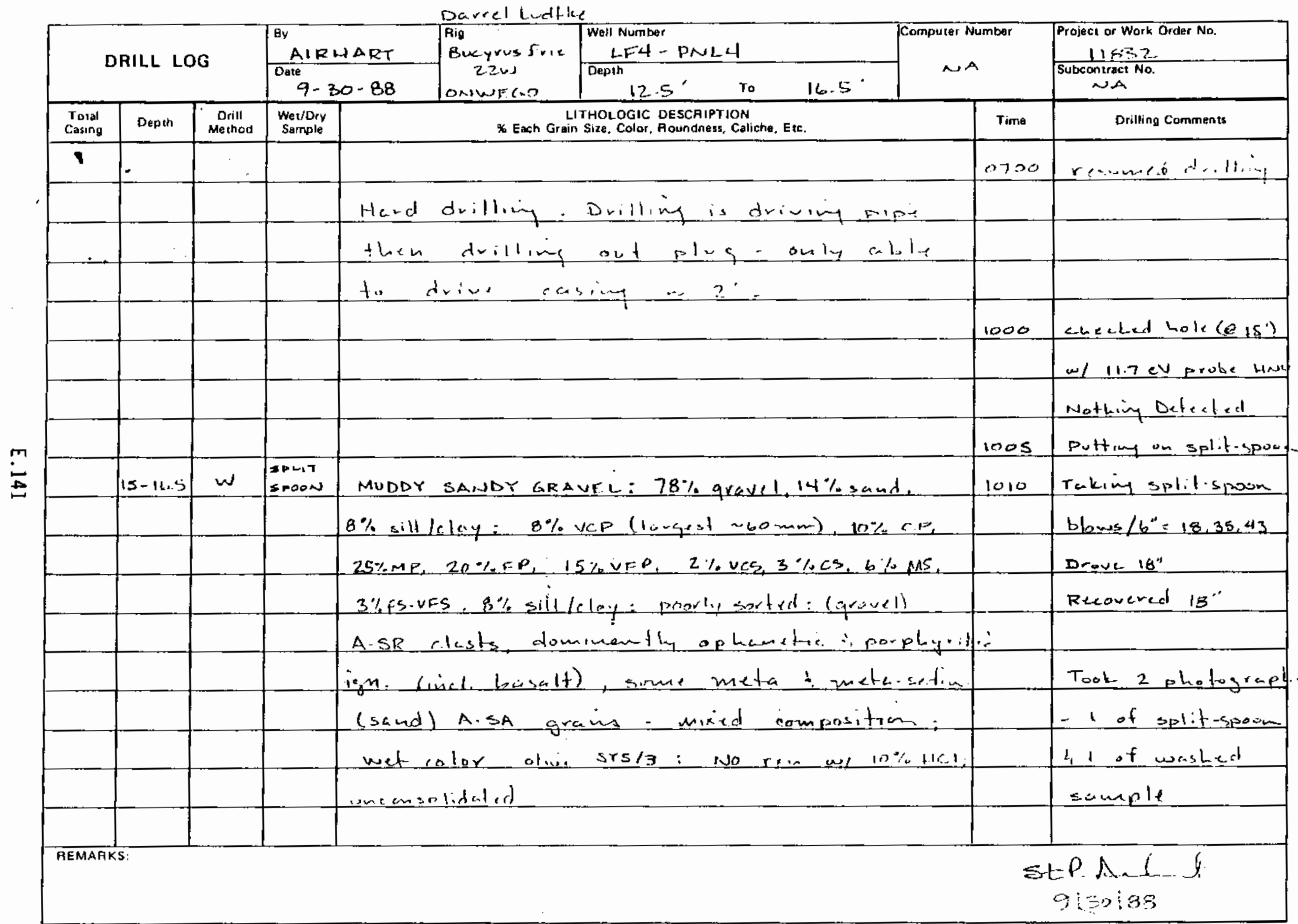


$F 2,7$.

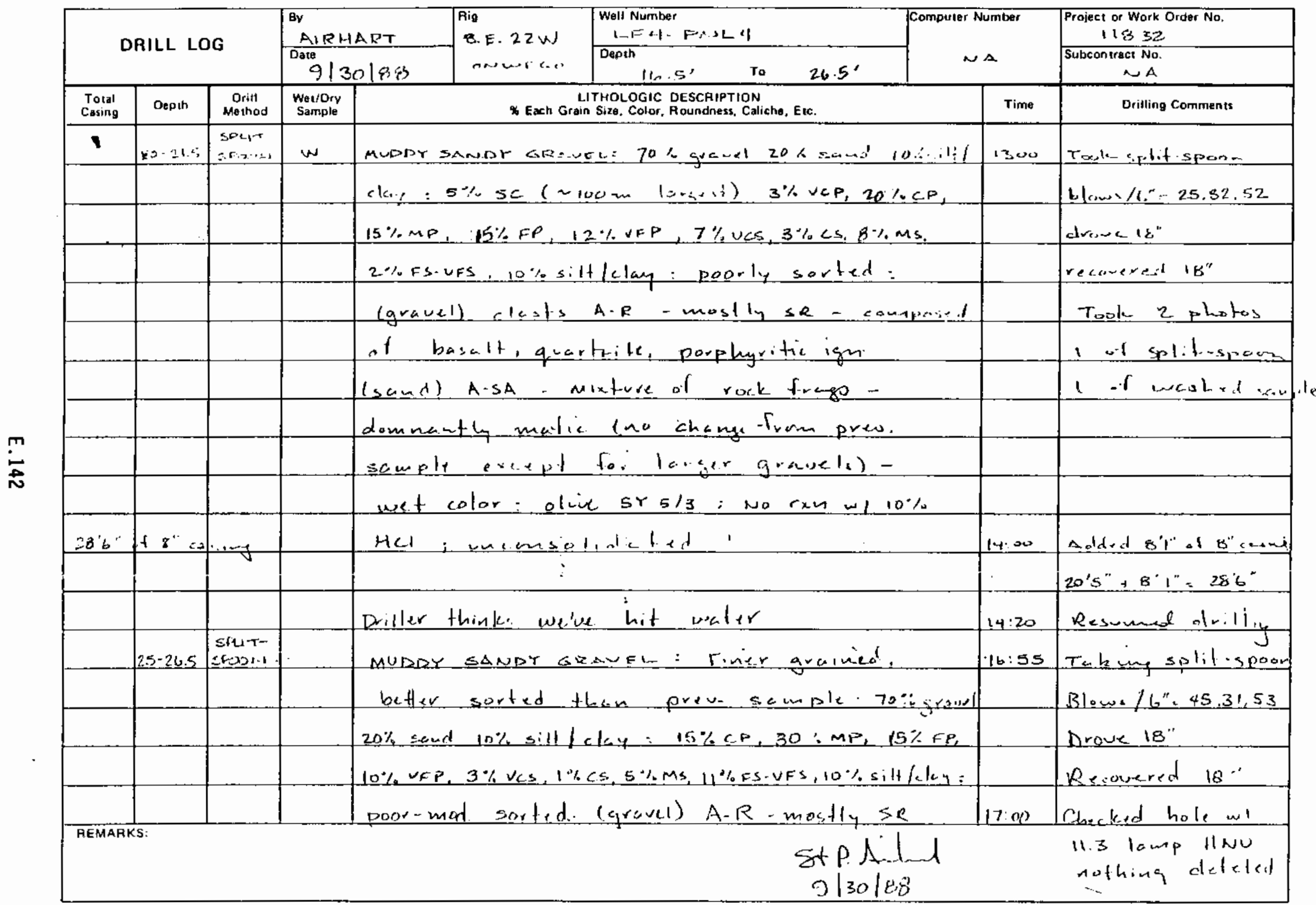

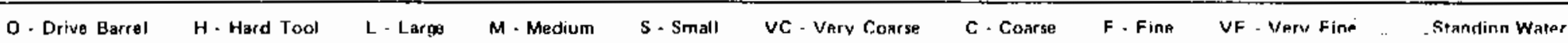


raye 3 of 5

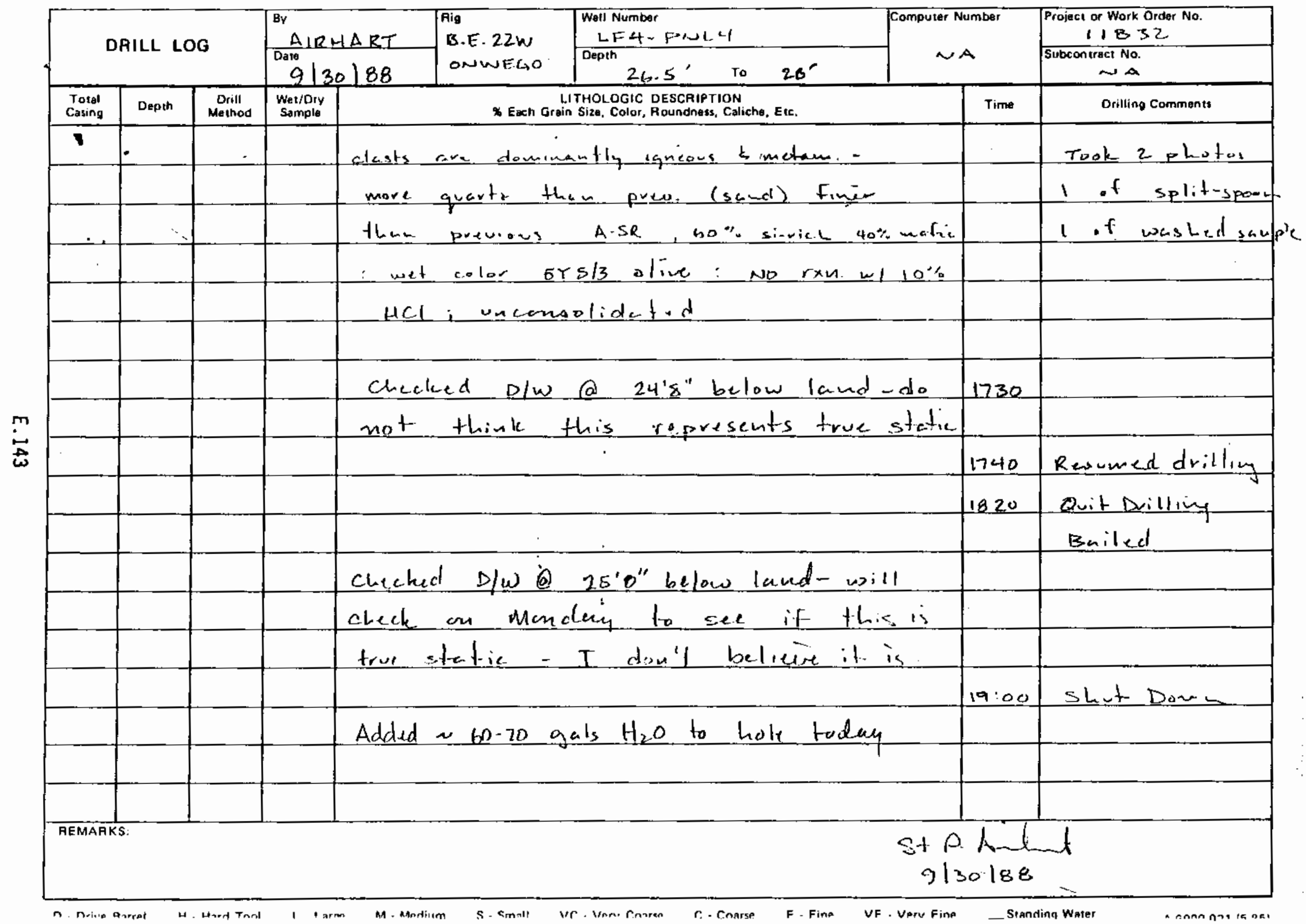




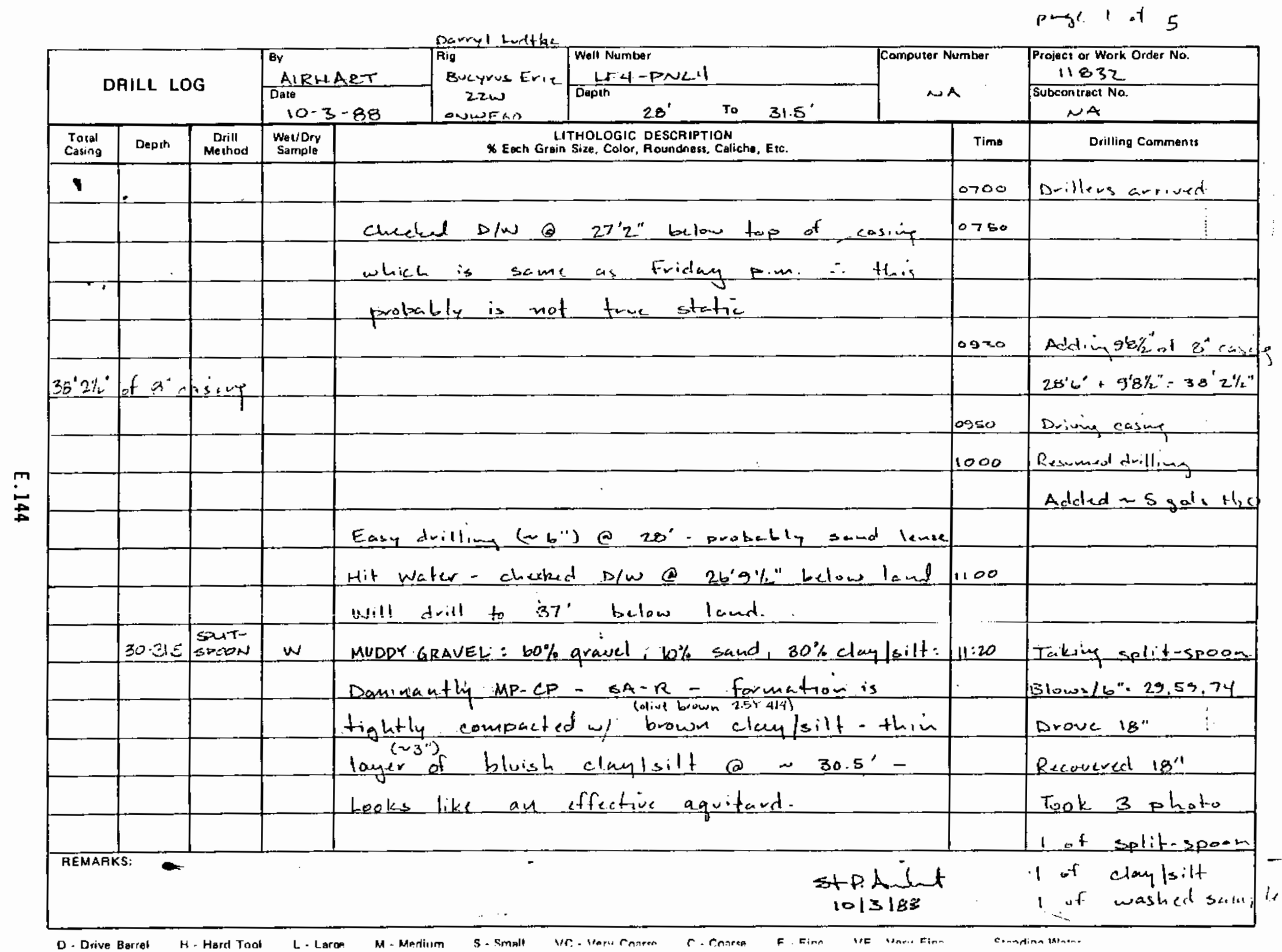


at: 2

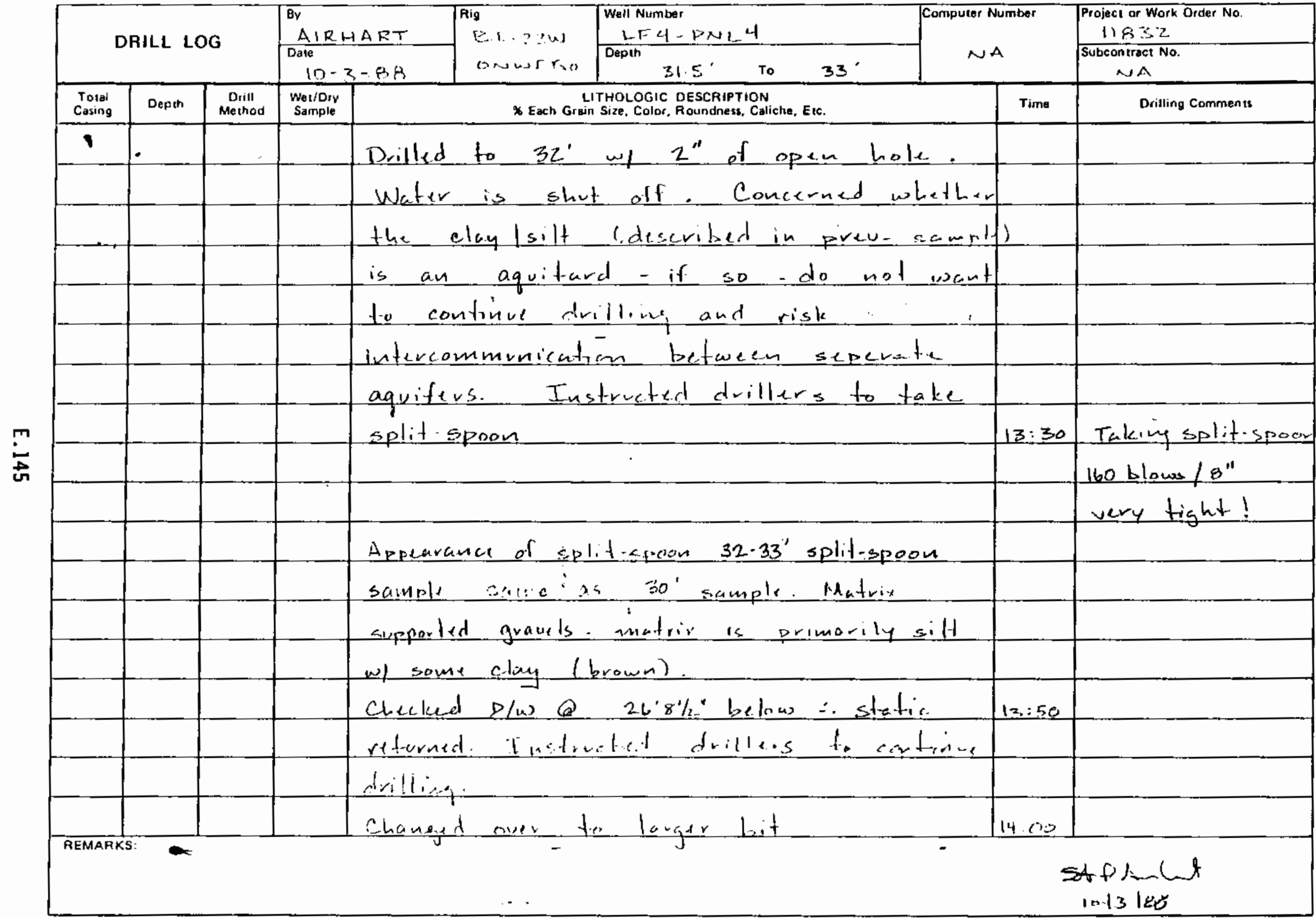

M. Medium

s - 5mall

VC - Very Coarse

C. Coarso

F - Fine

VF - Very Fine

Standing Water 
$1-4)+45$

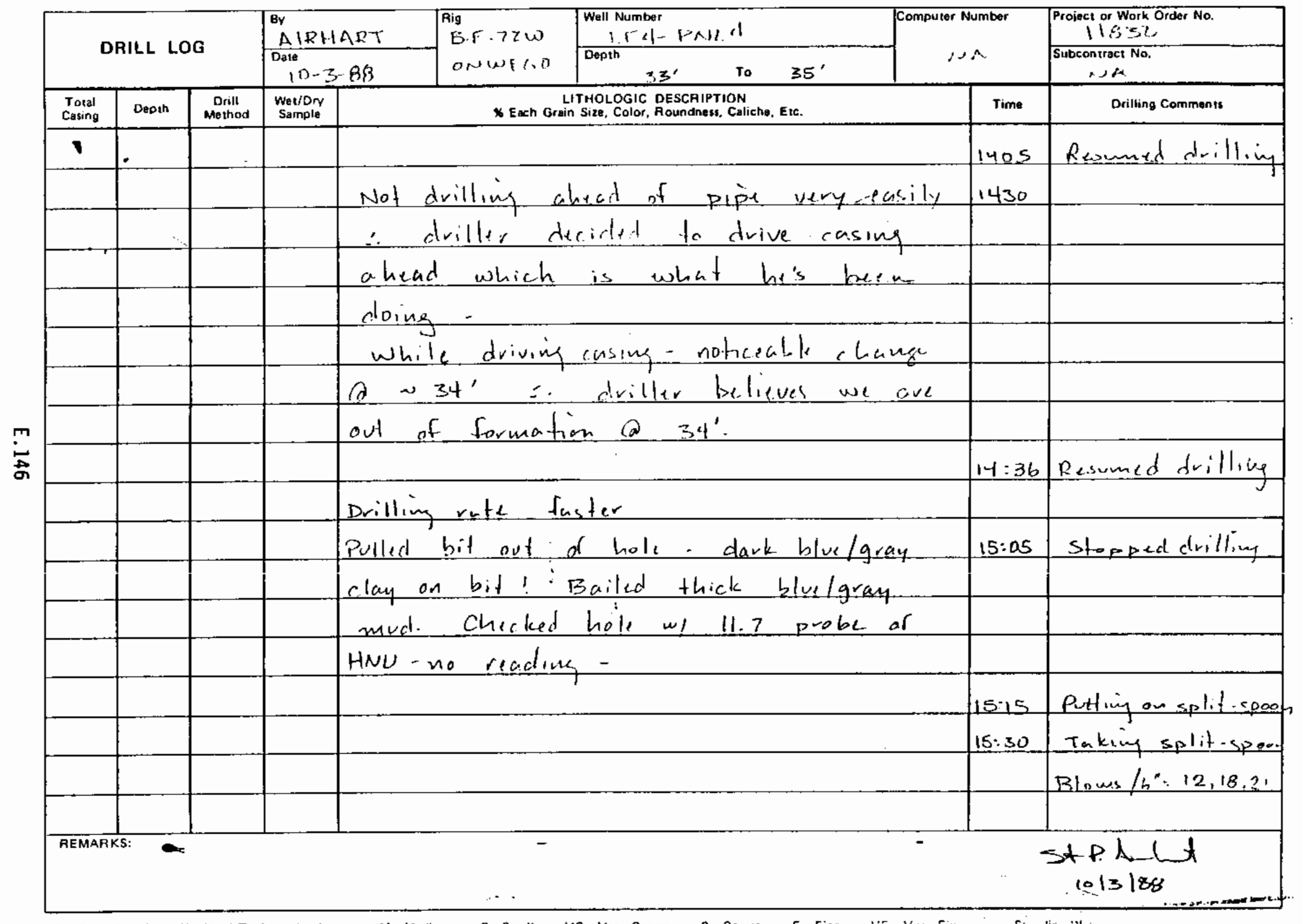




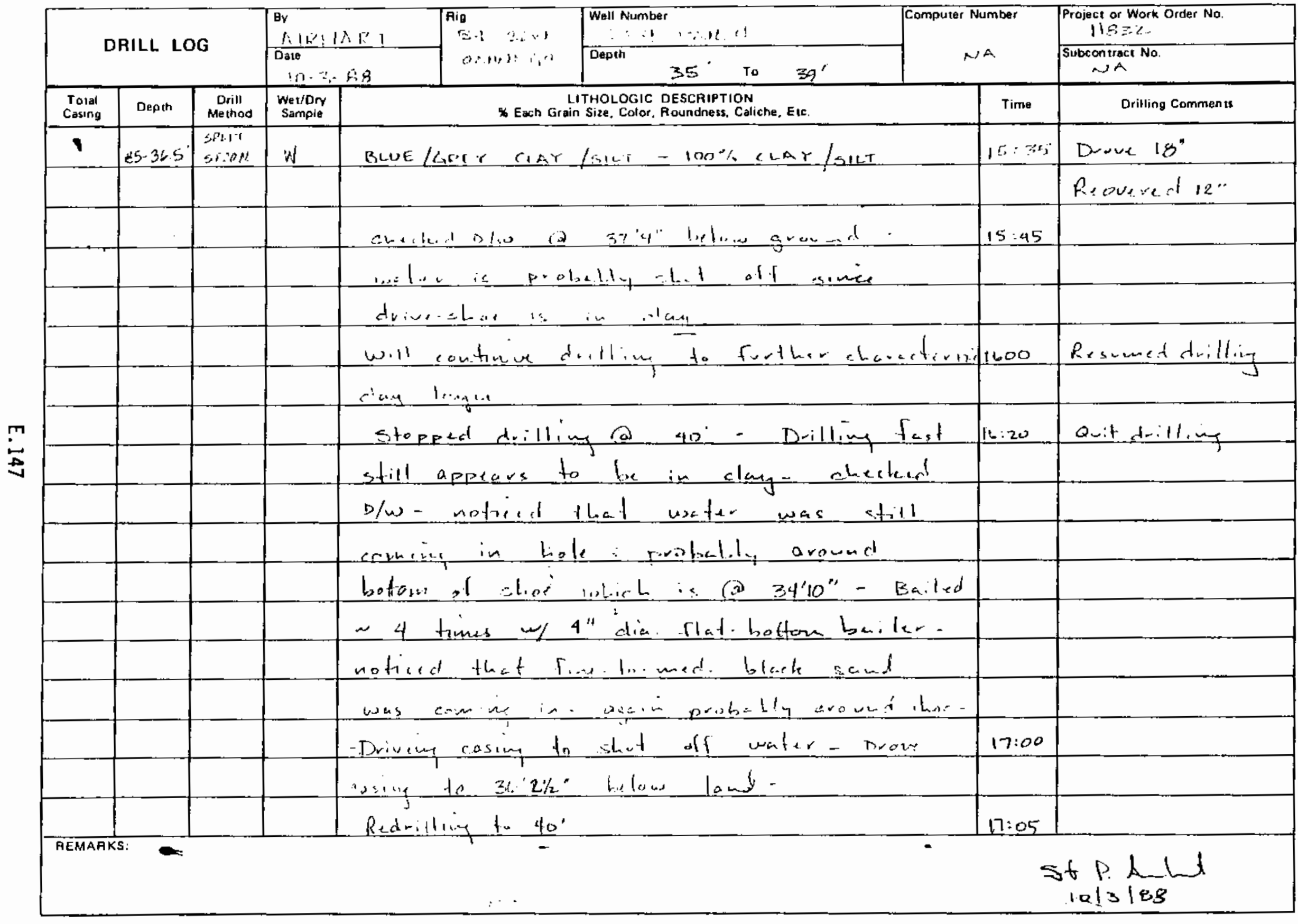


pose s ir 5

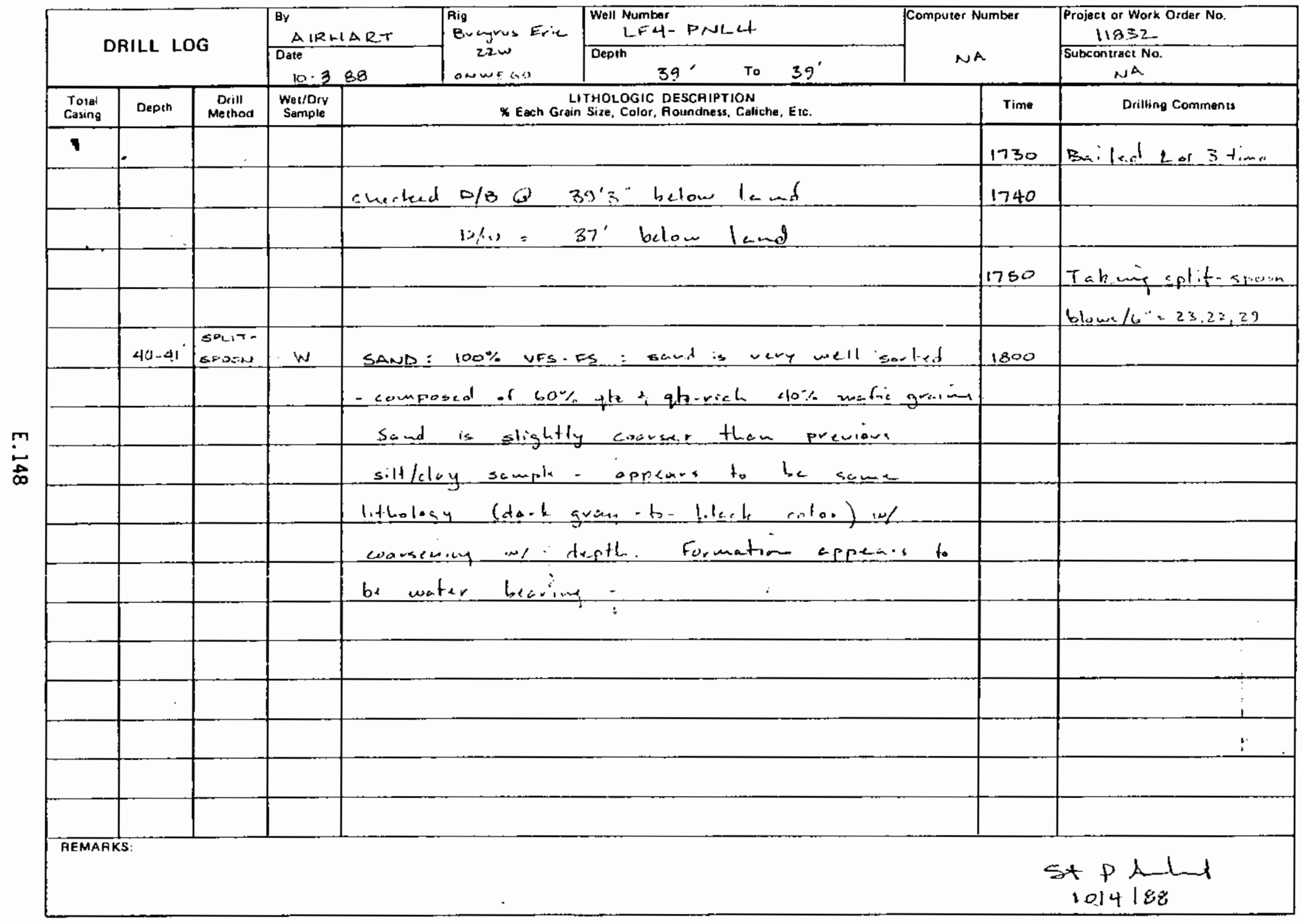

n. neim Daxpl 


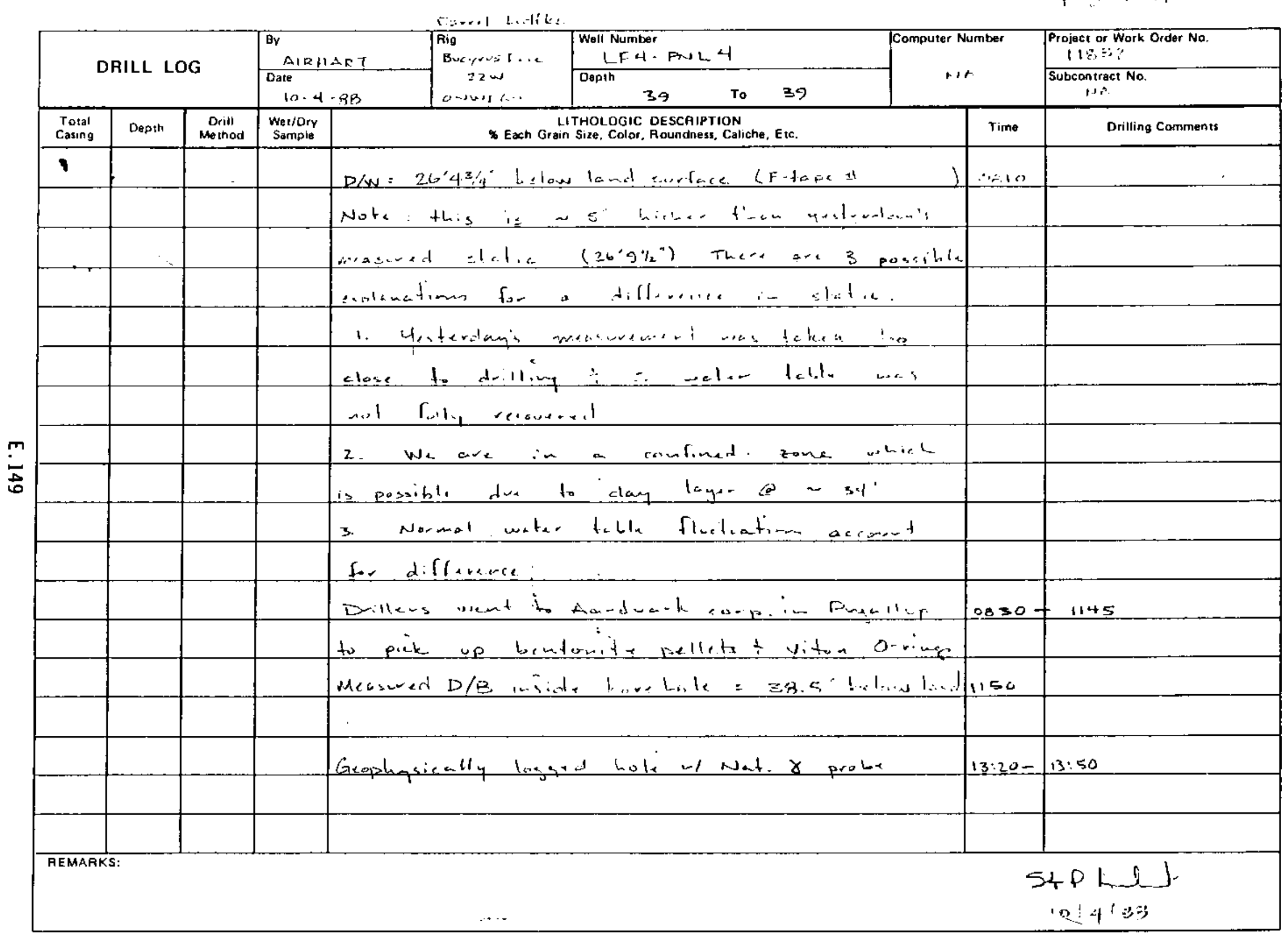


ras: $=.14$

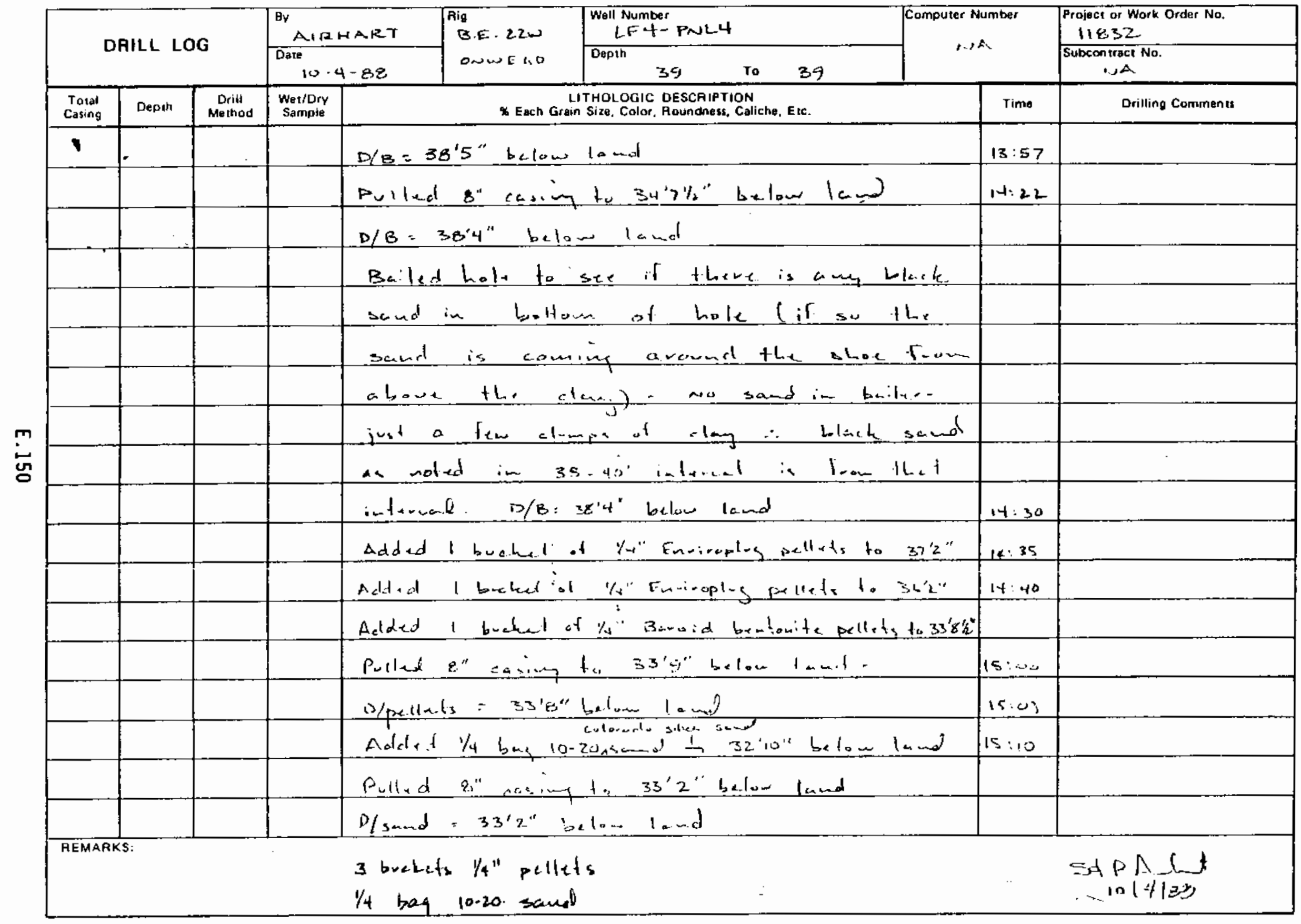




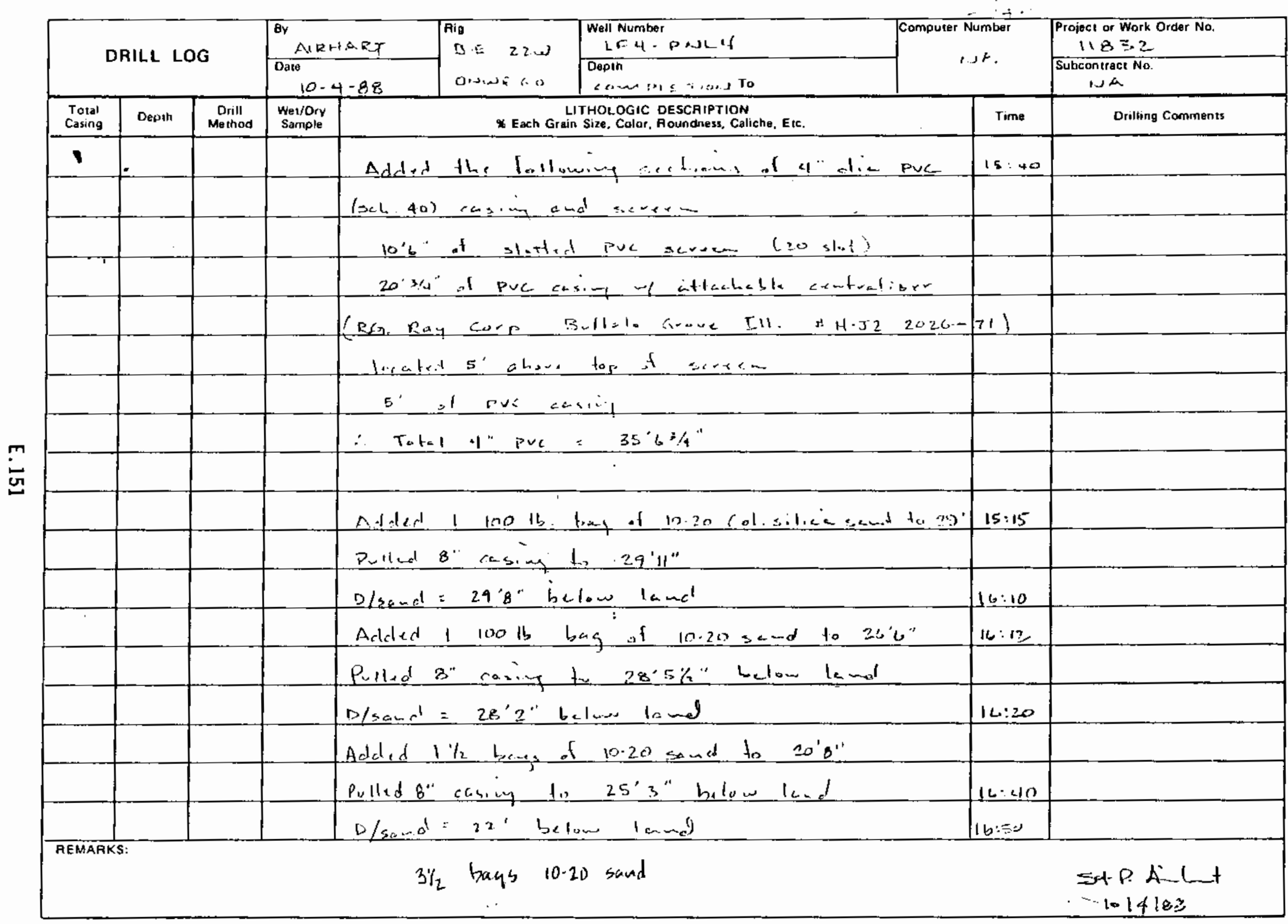

D-Drive Aarrel H. Harat Tn 


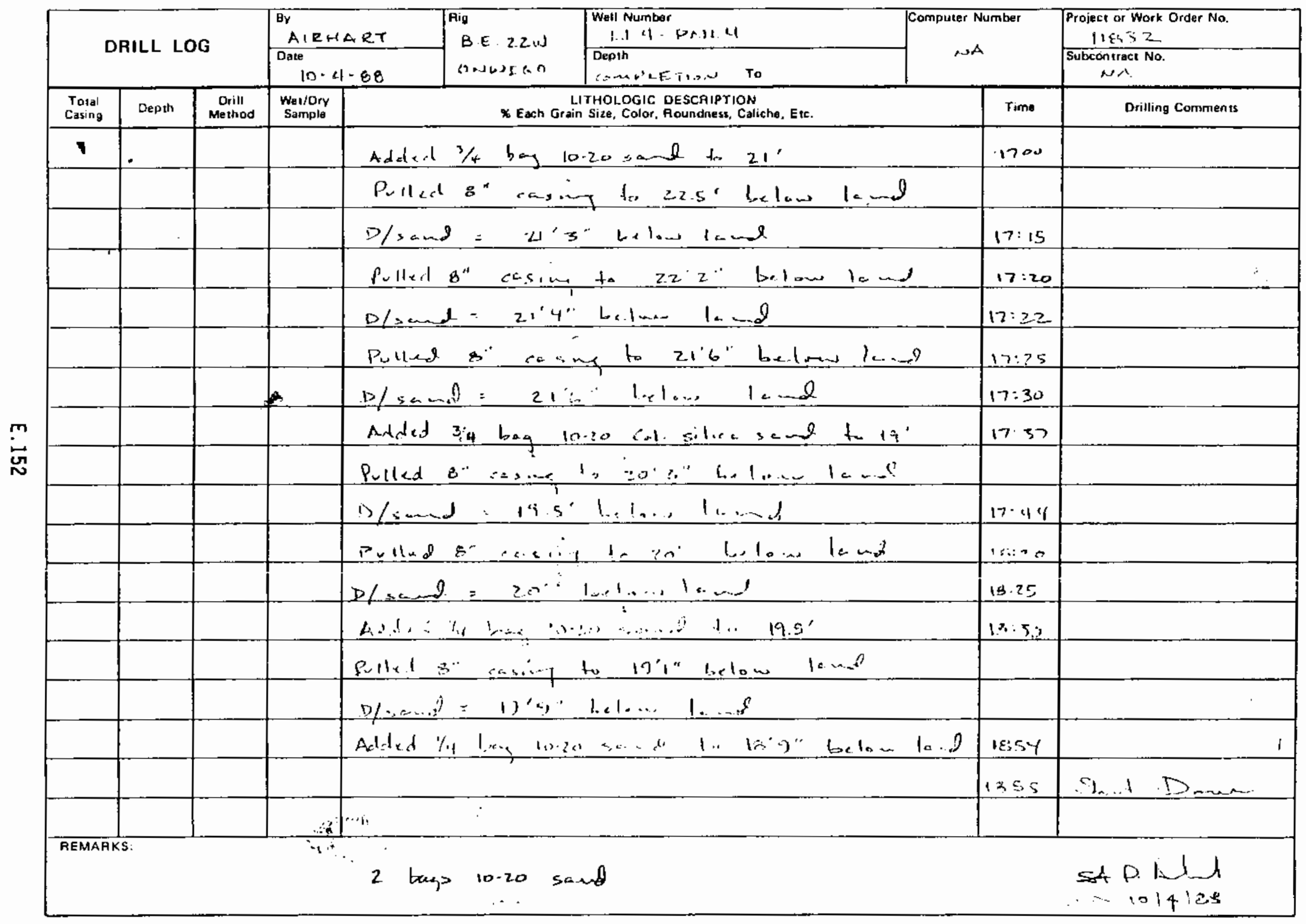


$T+x^{2} 1$ at

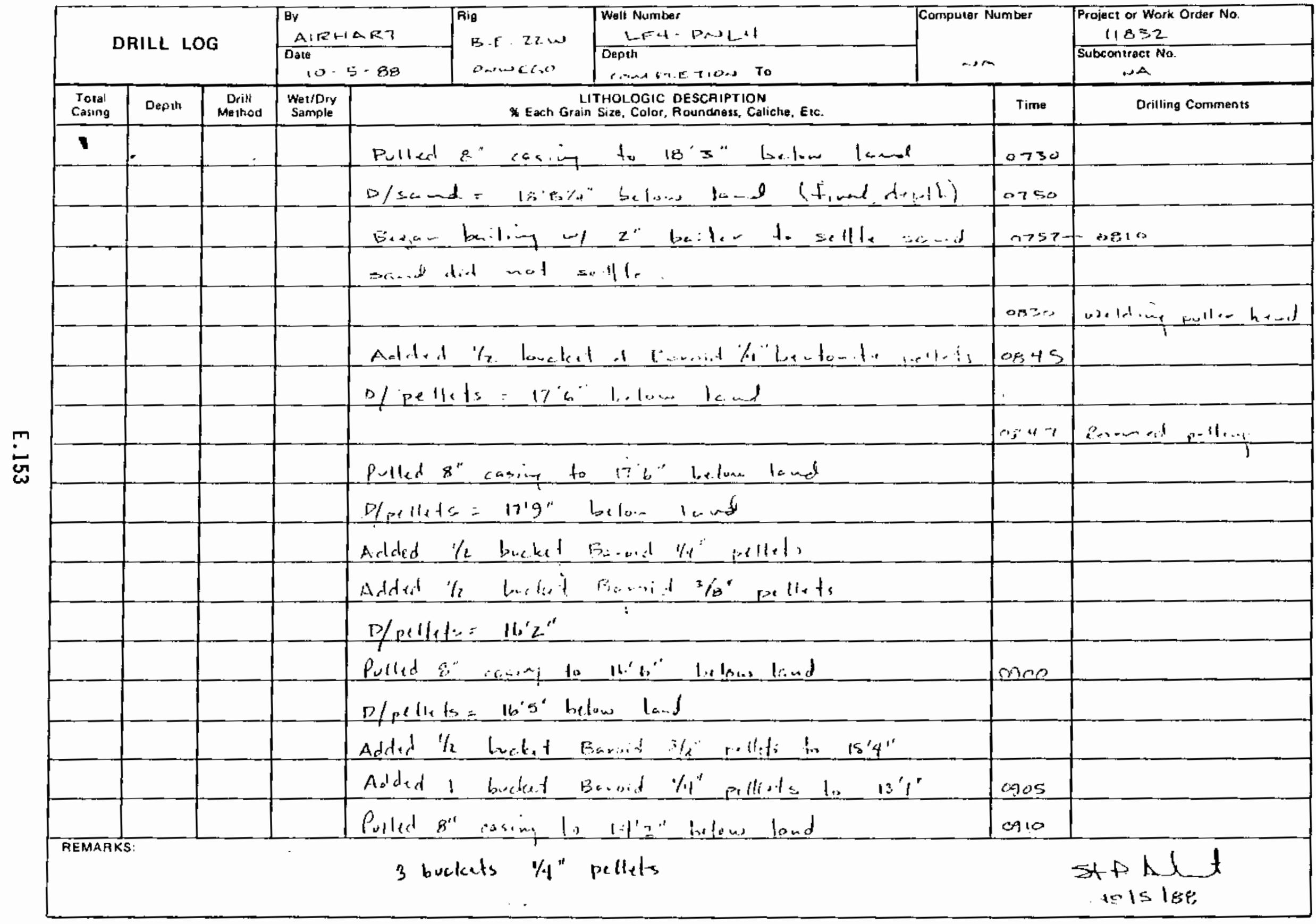


peoged $2 x$

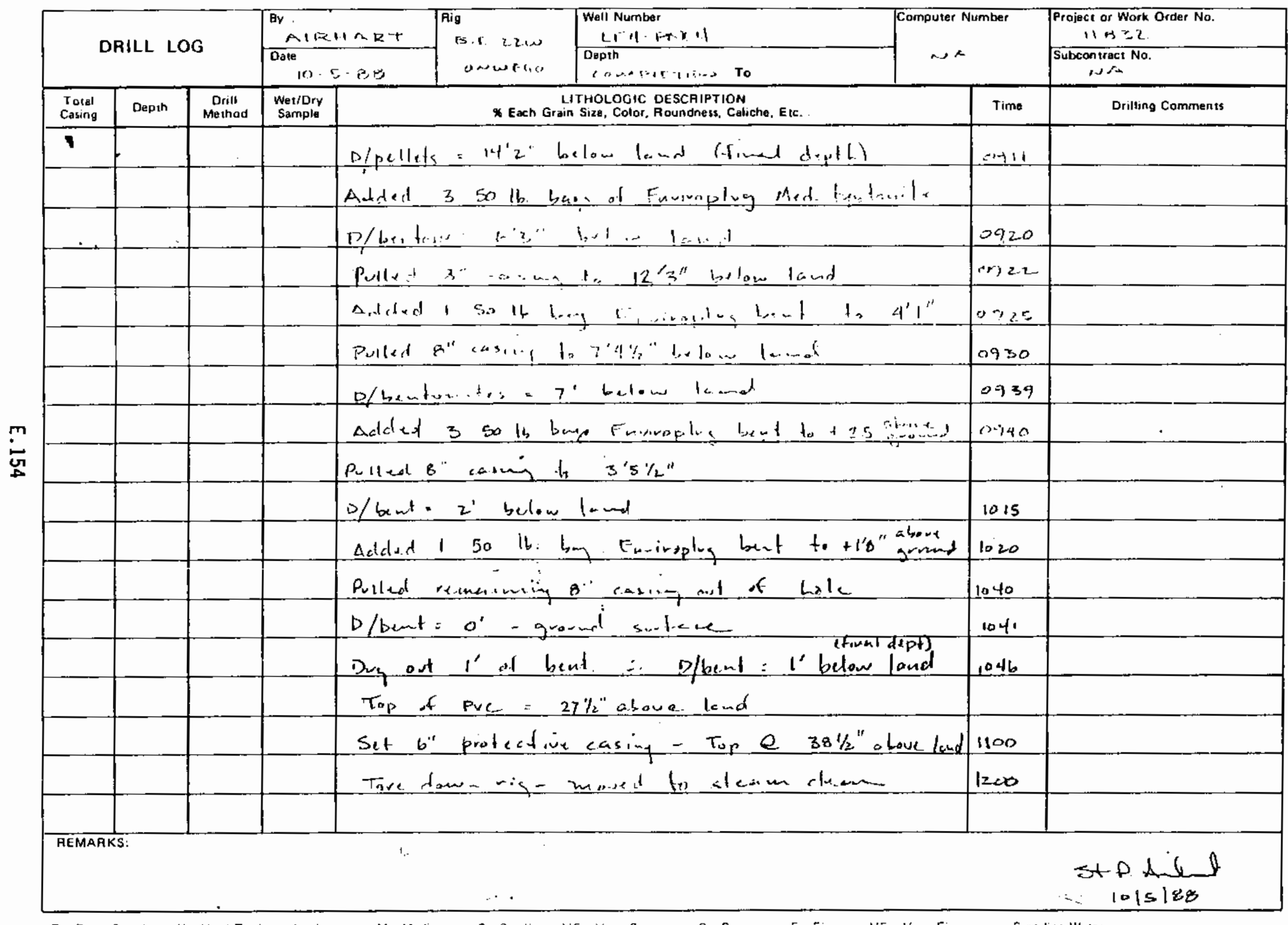




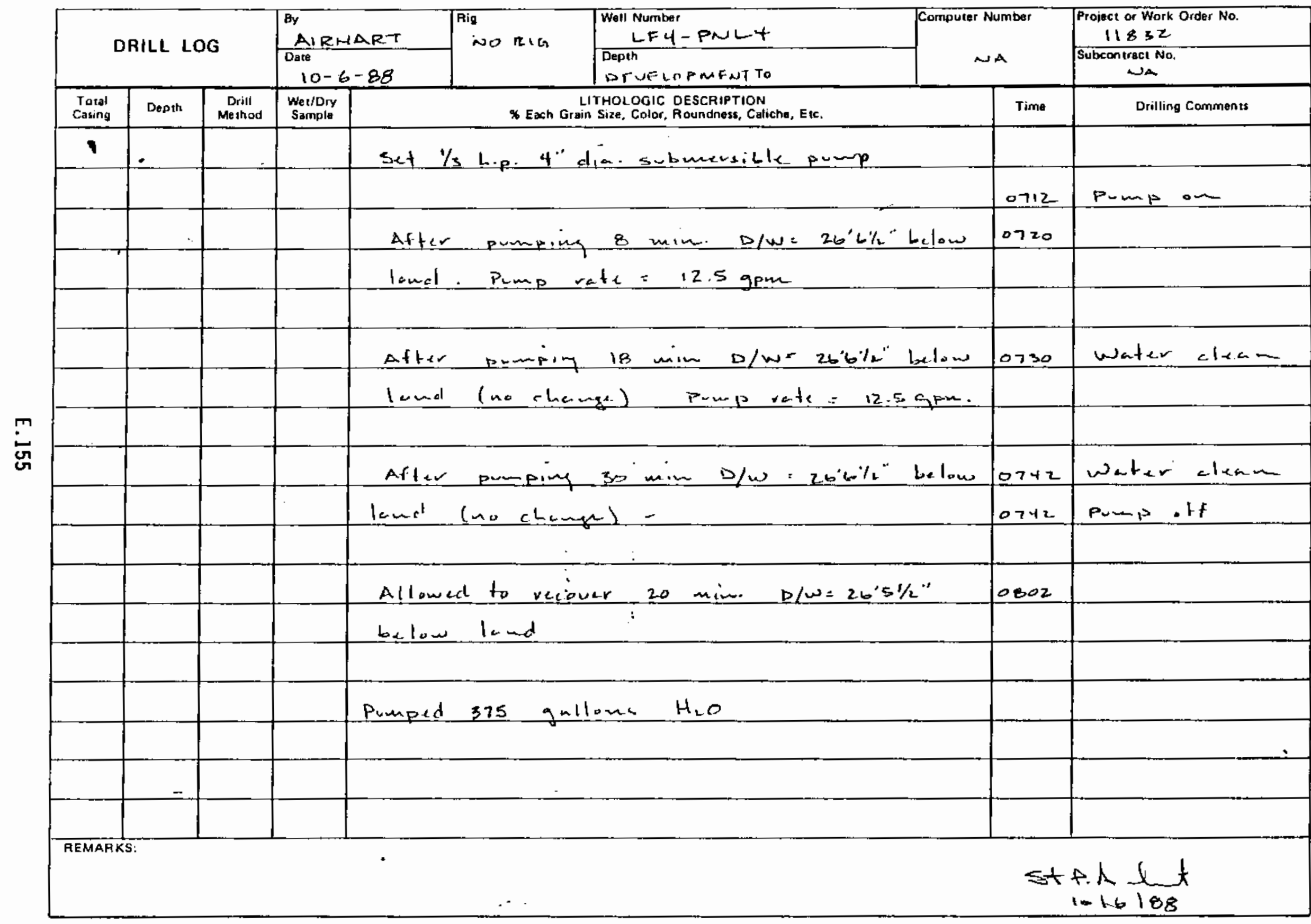

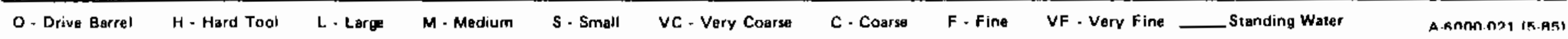


payg, of 1

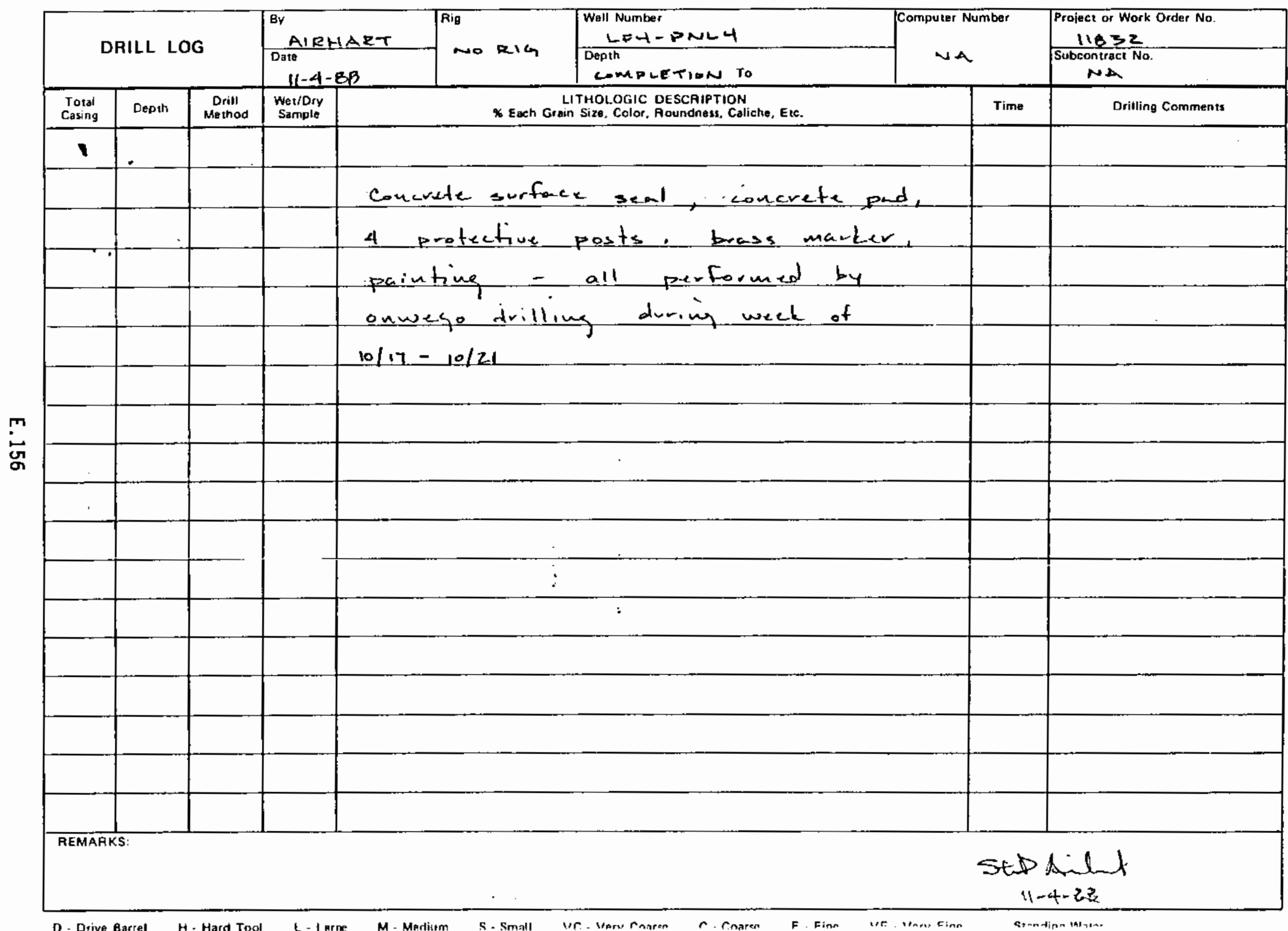


WELL LF4-PNL 5 


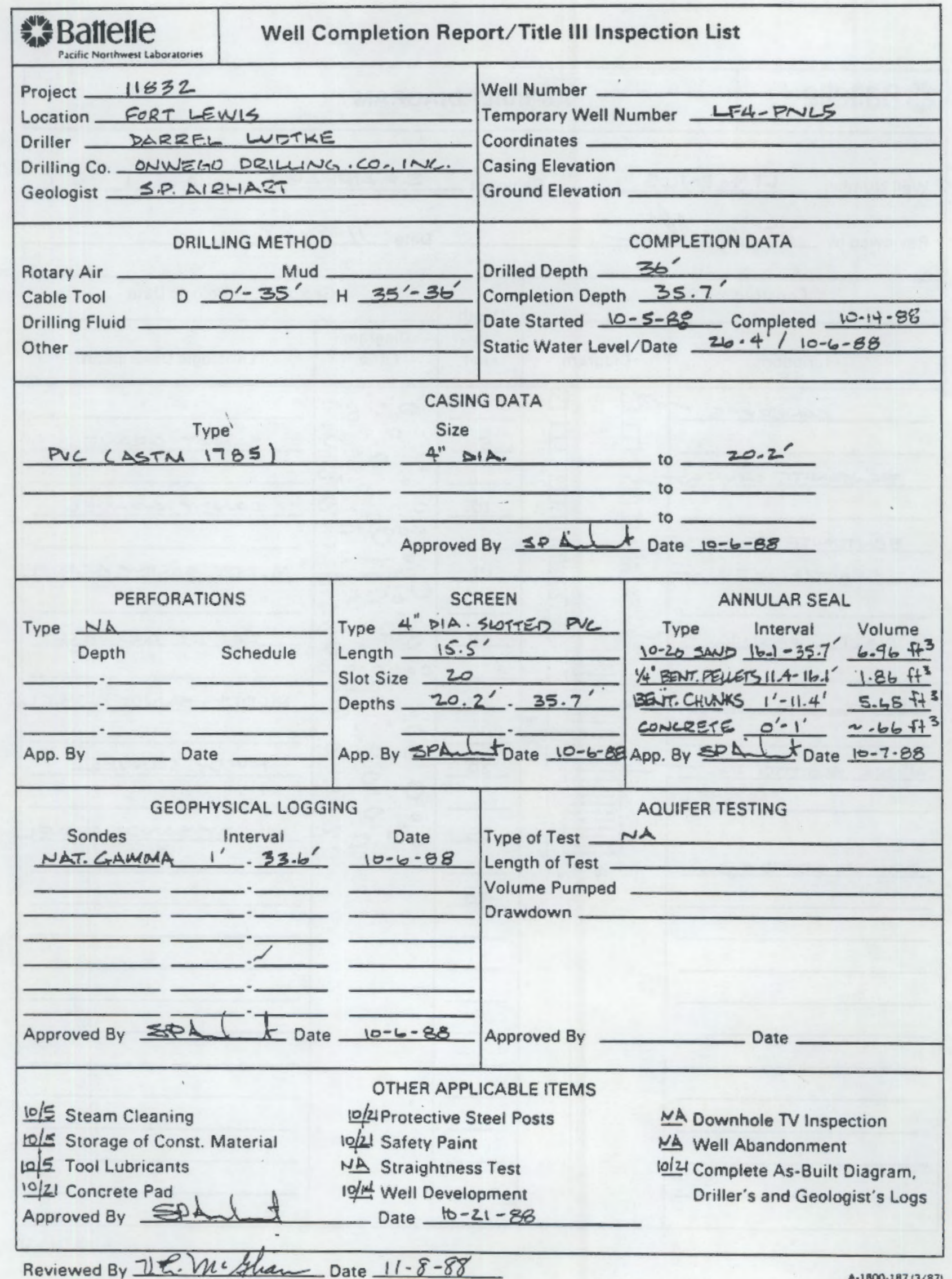




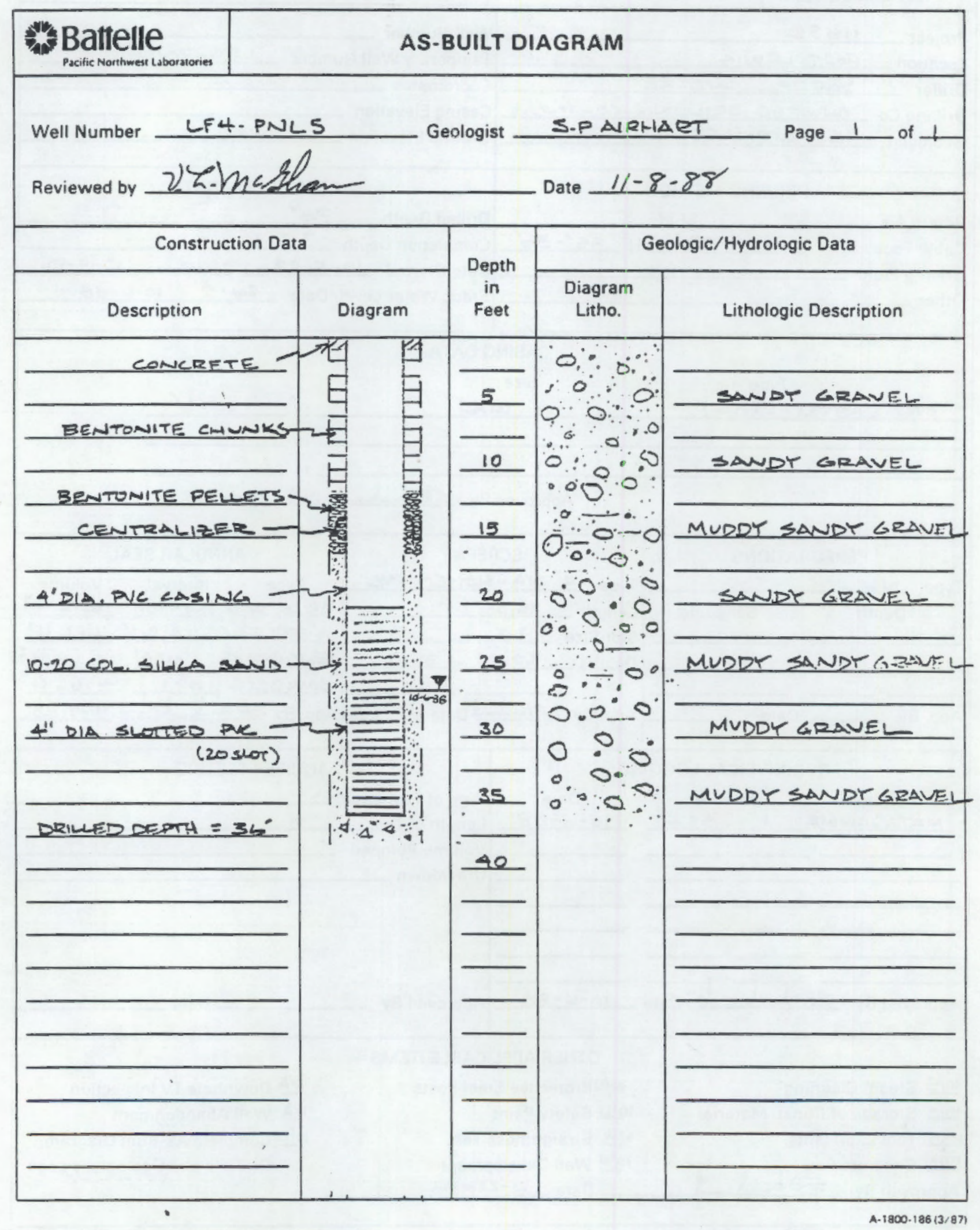

E. 158 


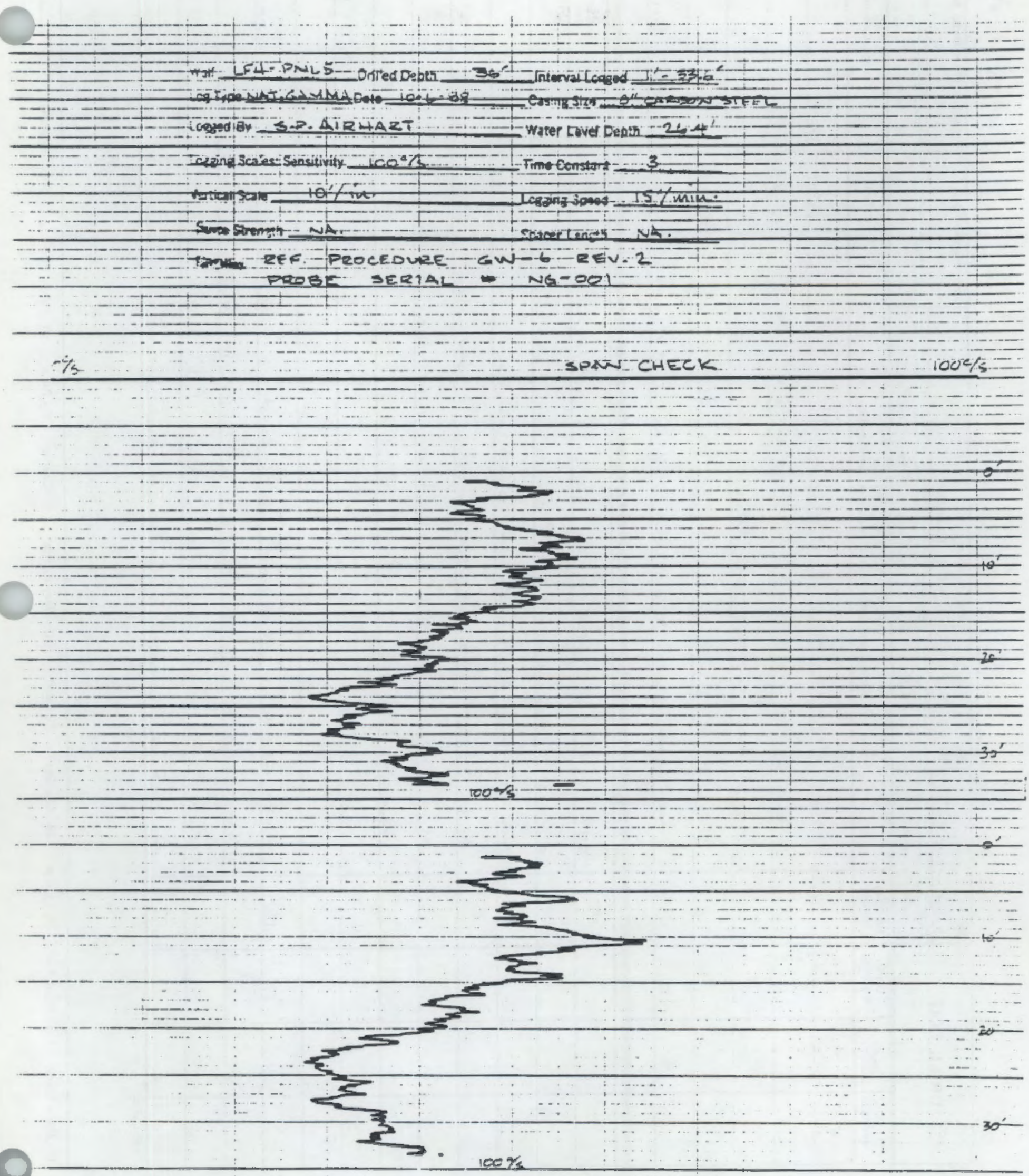


For $61+13$

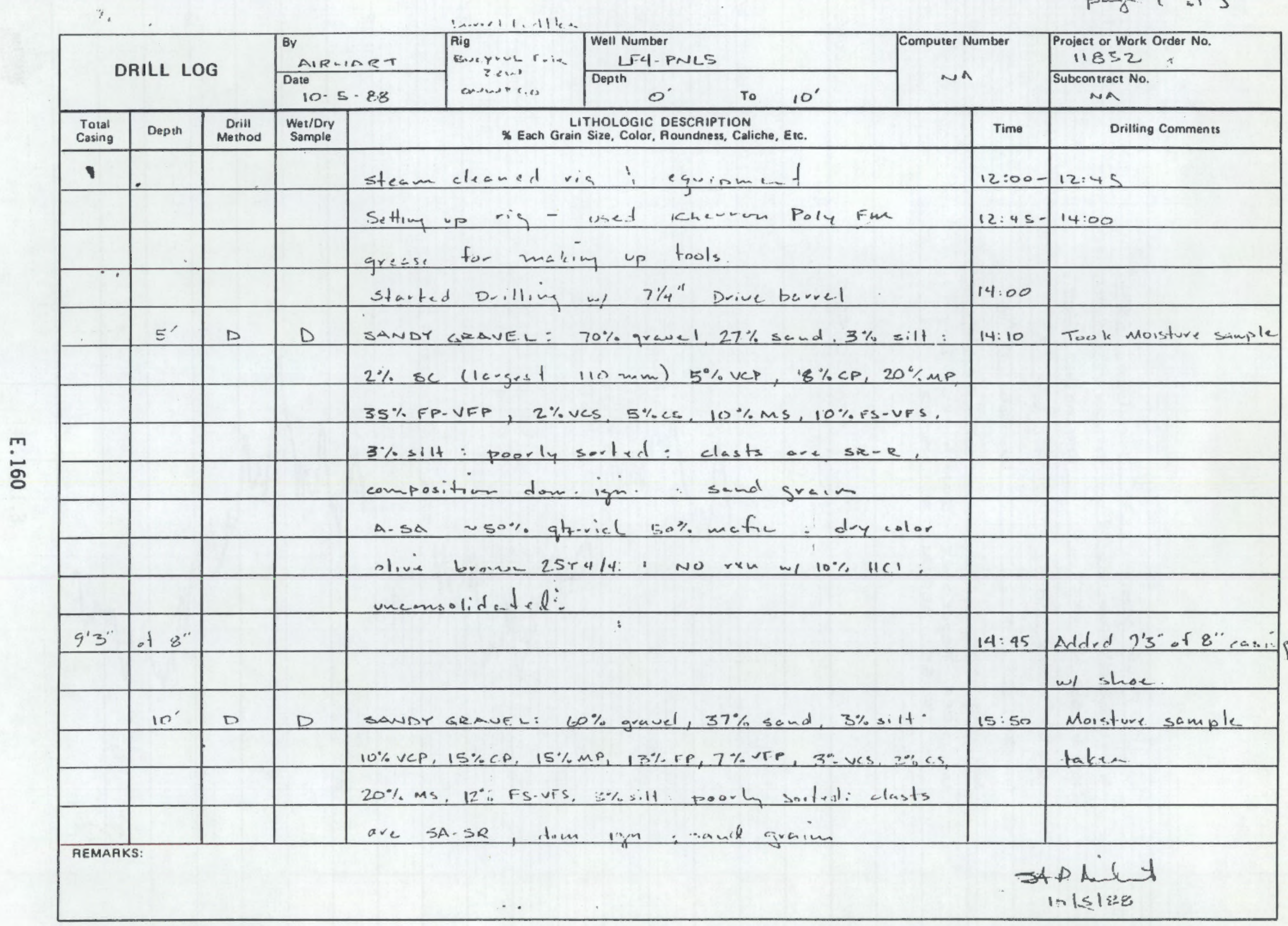

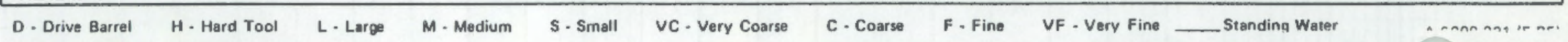




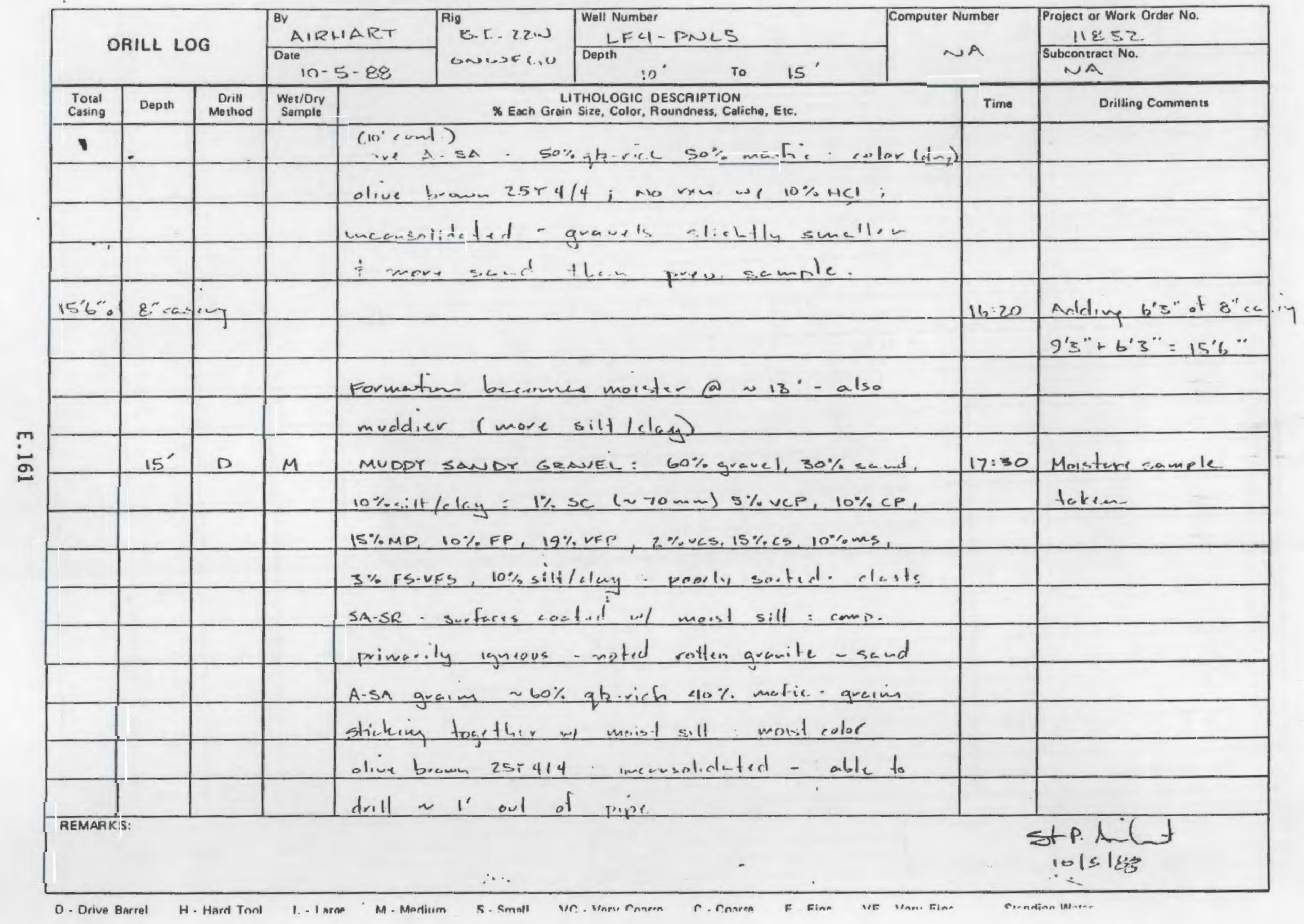


roy 3 of 3

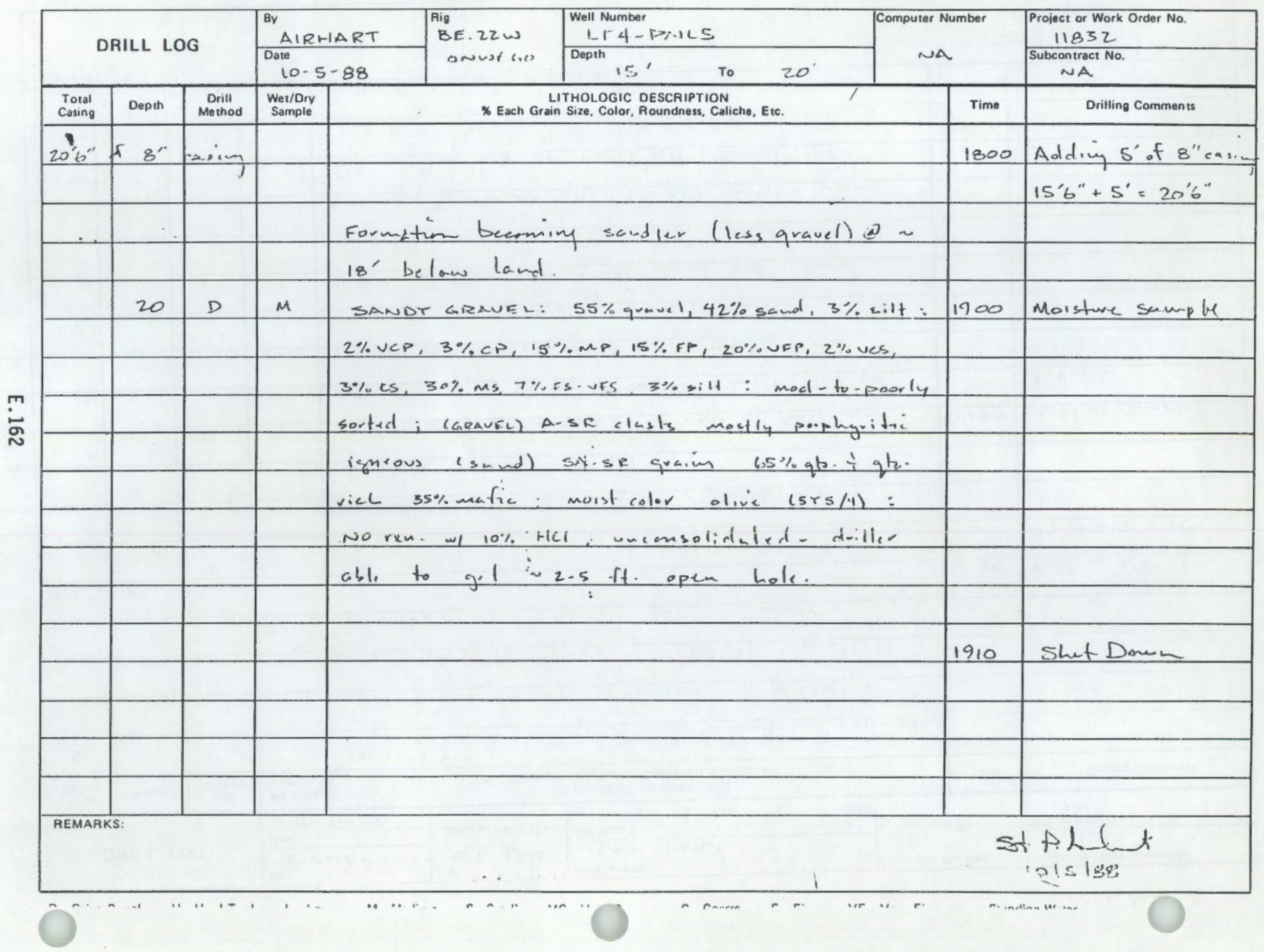




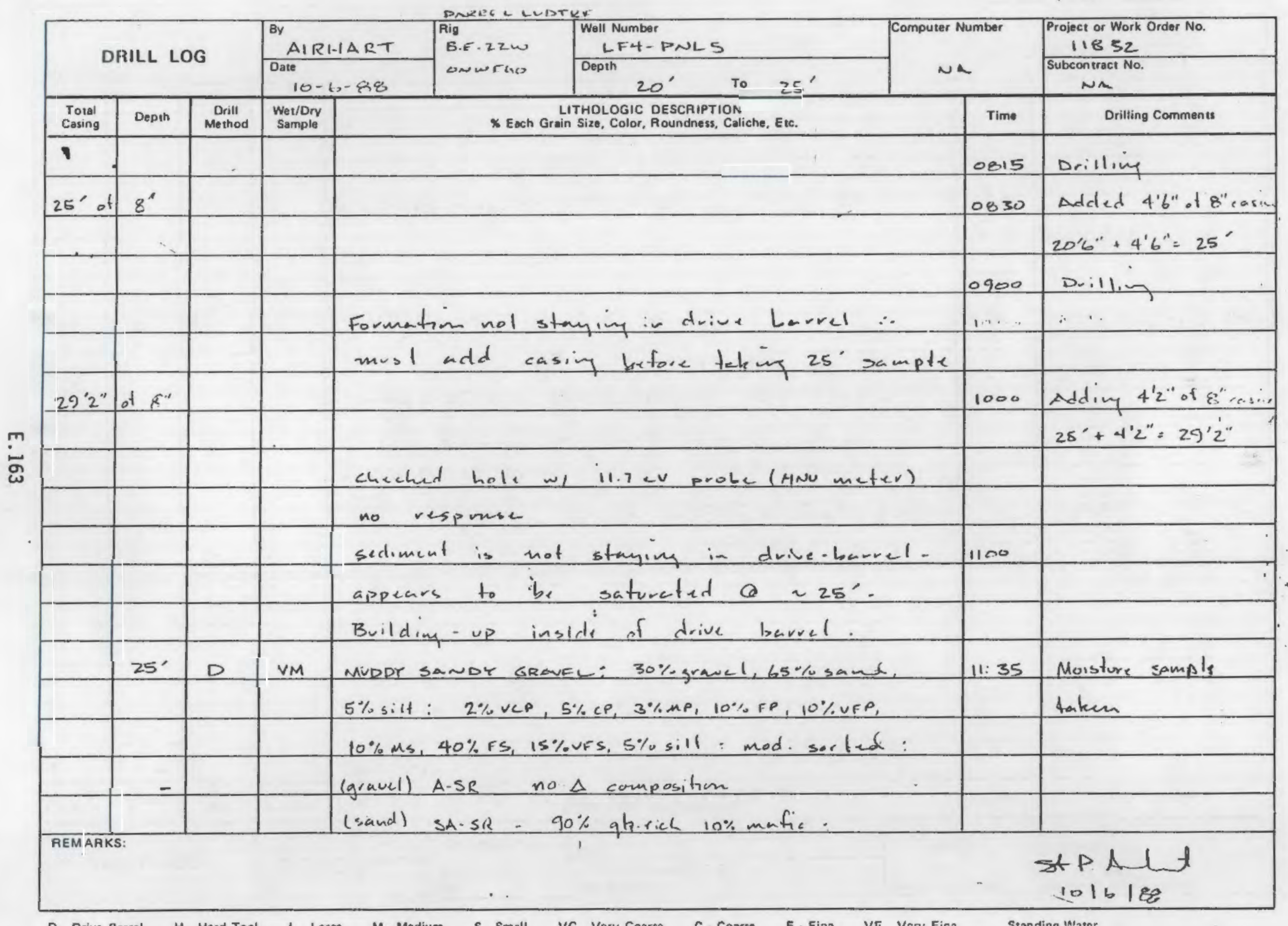

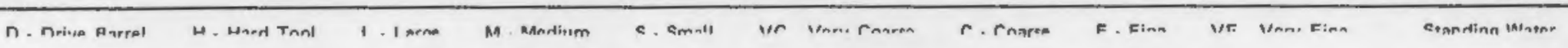




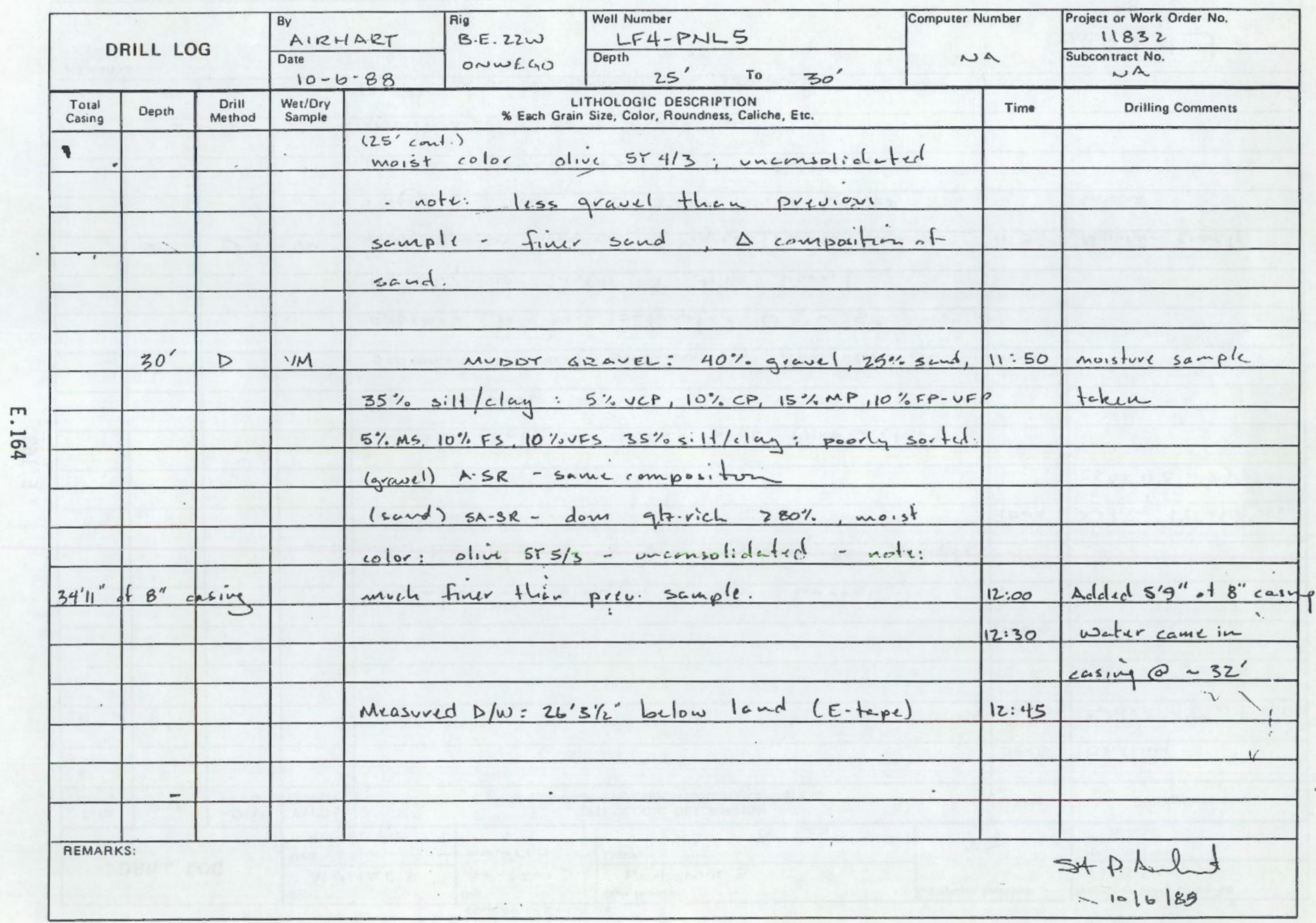




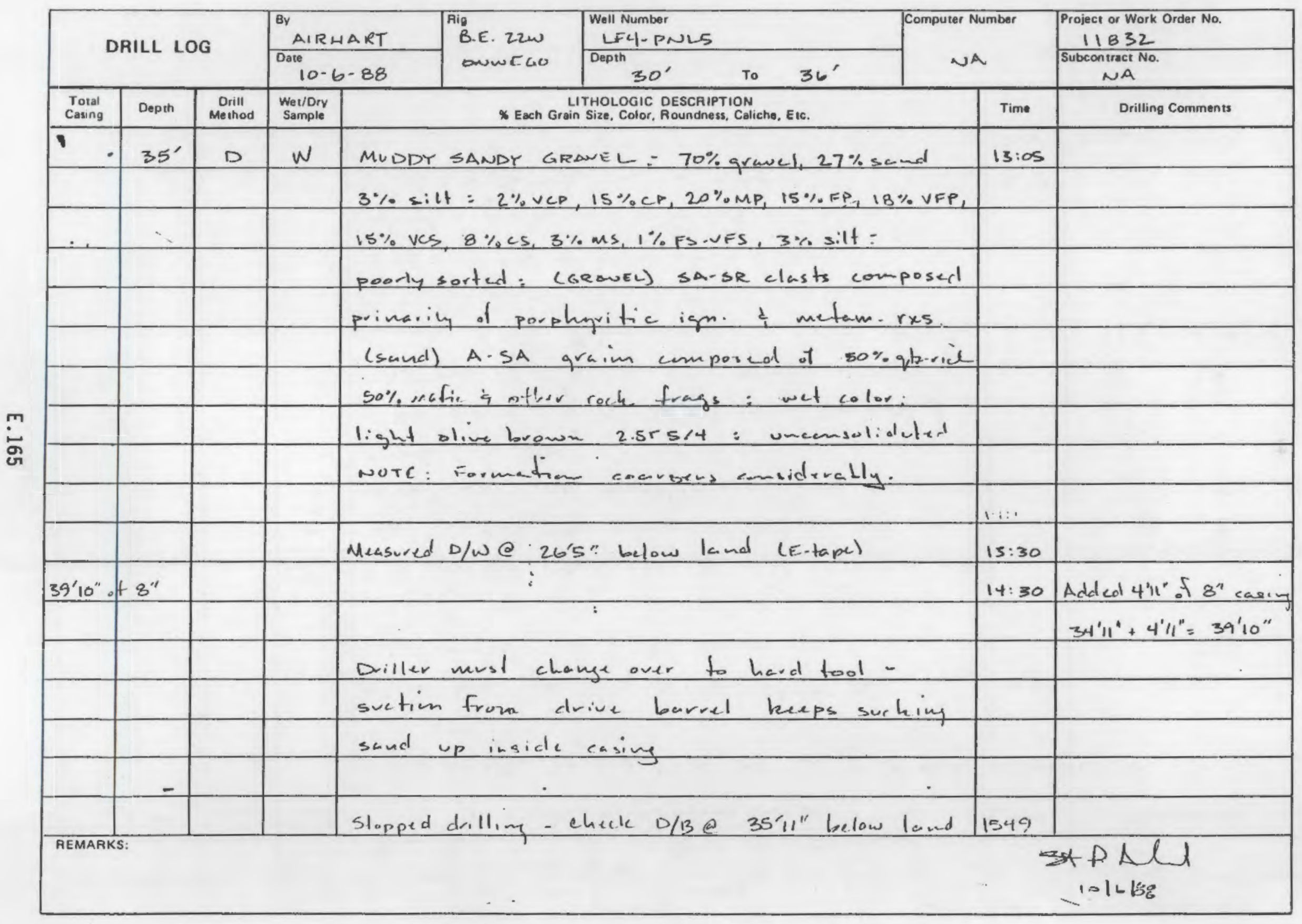




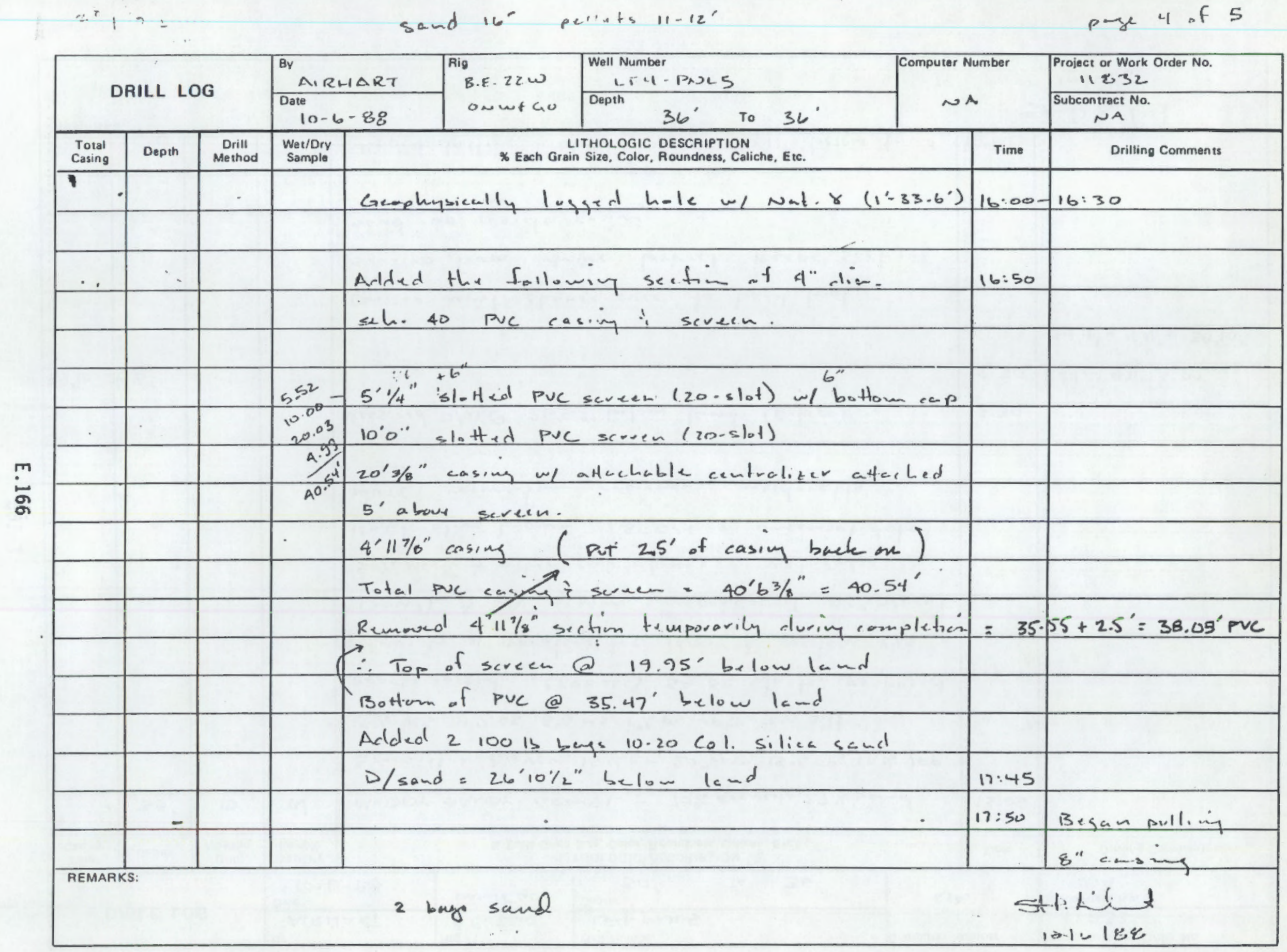




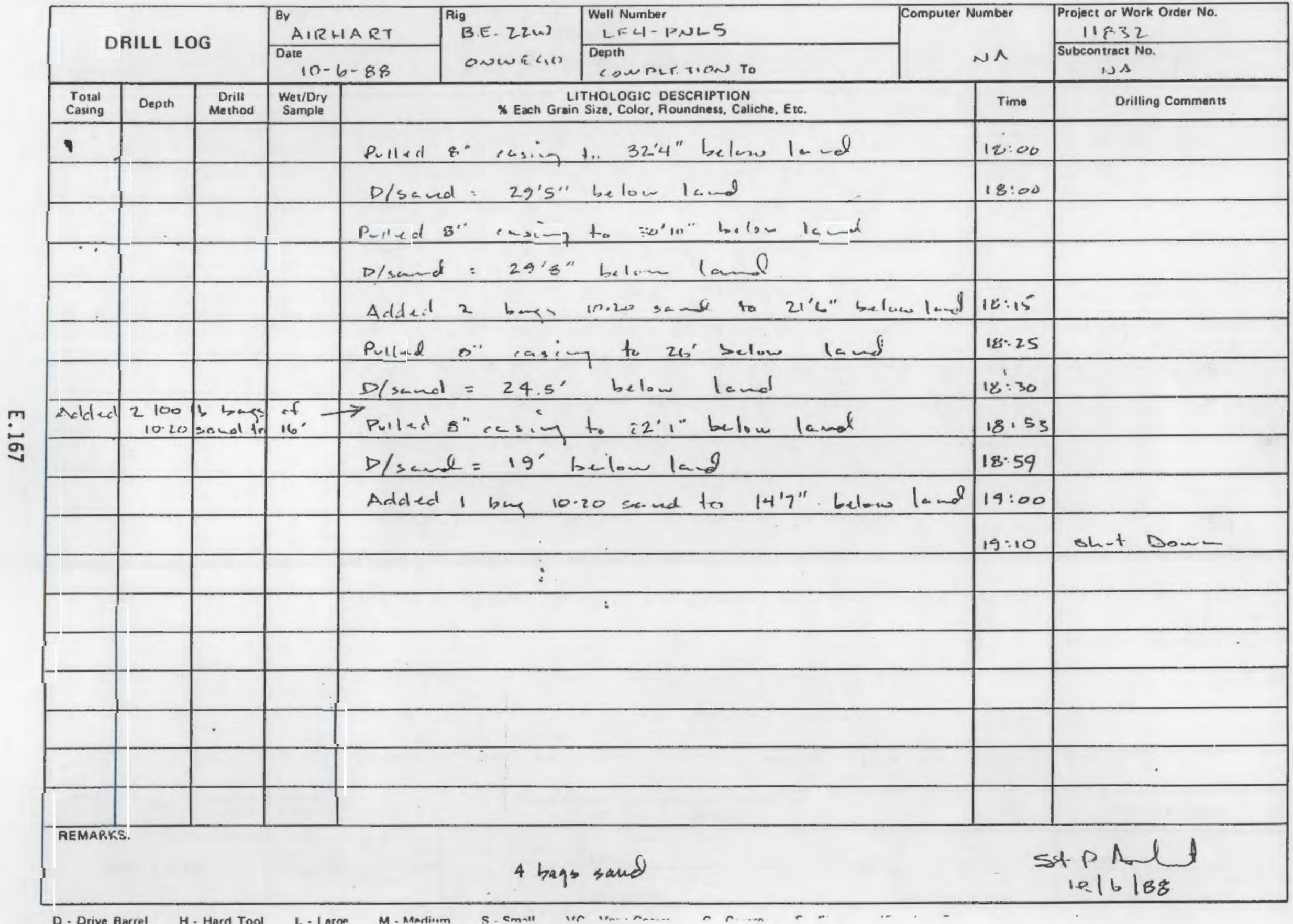


DALES L T.UDT:

pece 1 \& 2

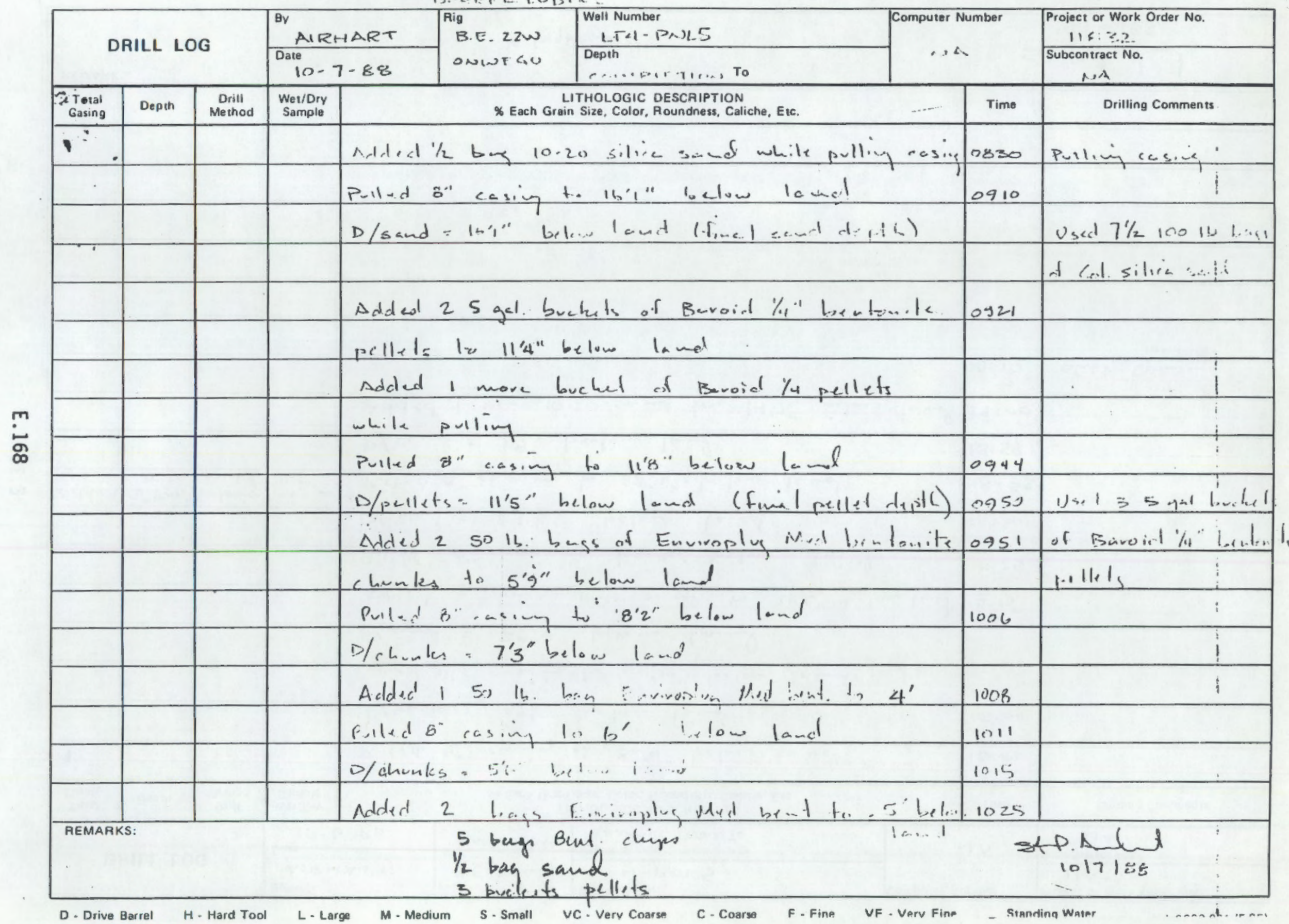




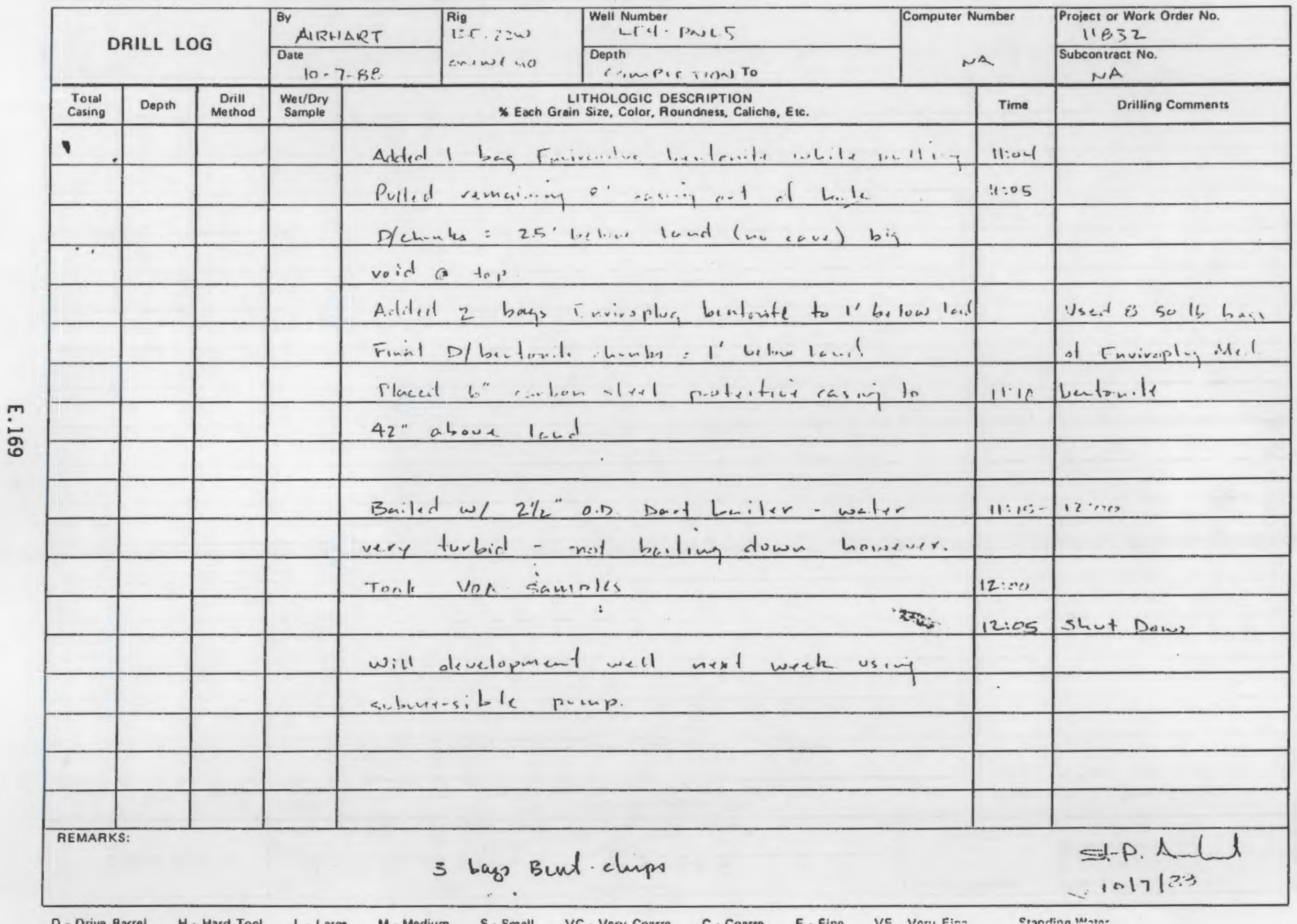




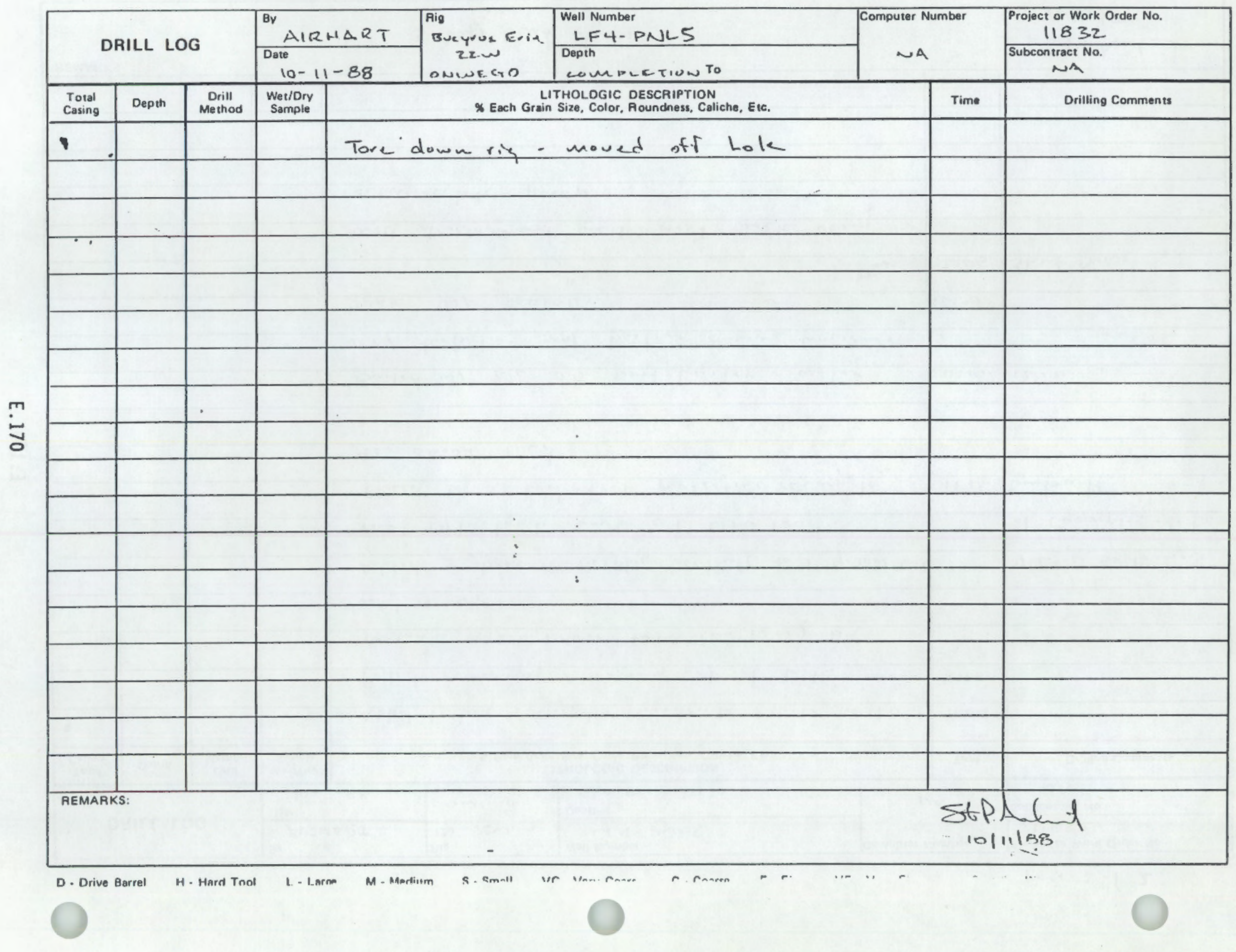


perge 1 of 2

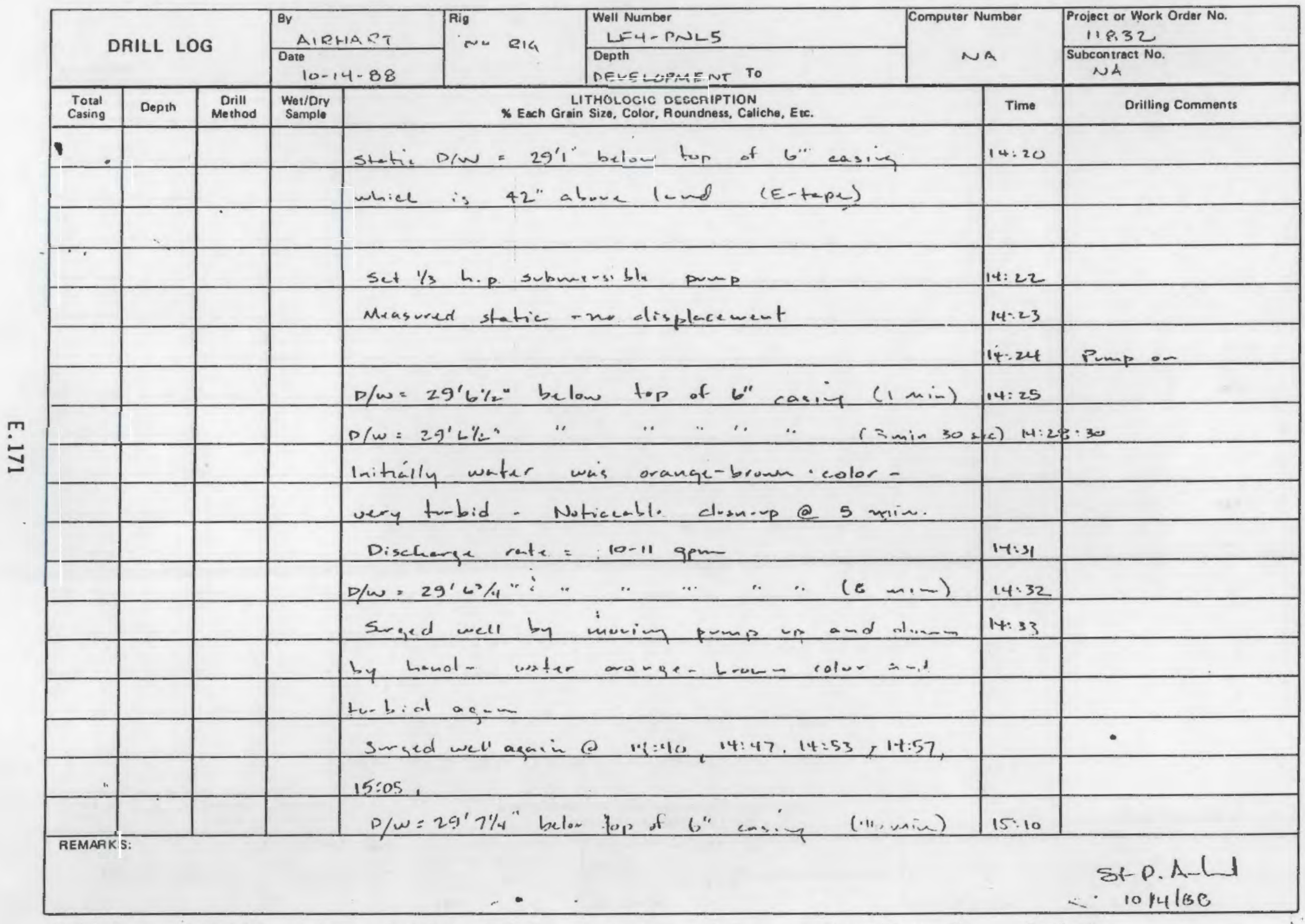

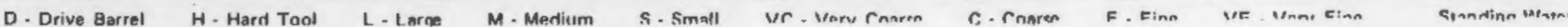




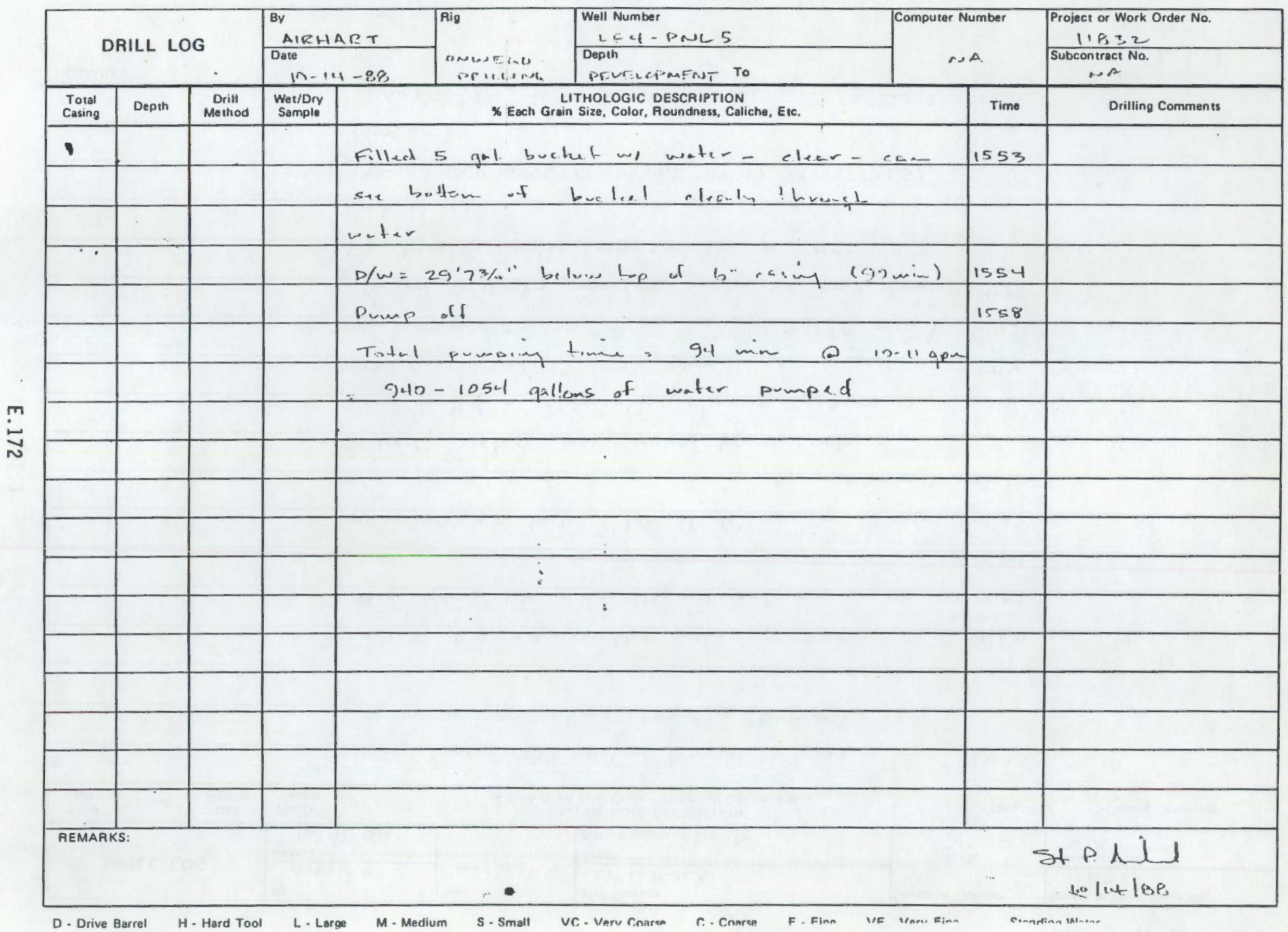
D. Drive Barre
- Hard Tool
Large
- Medium
Nentroare 


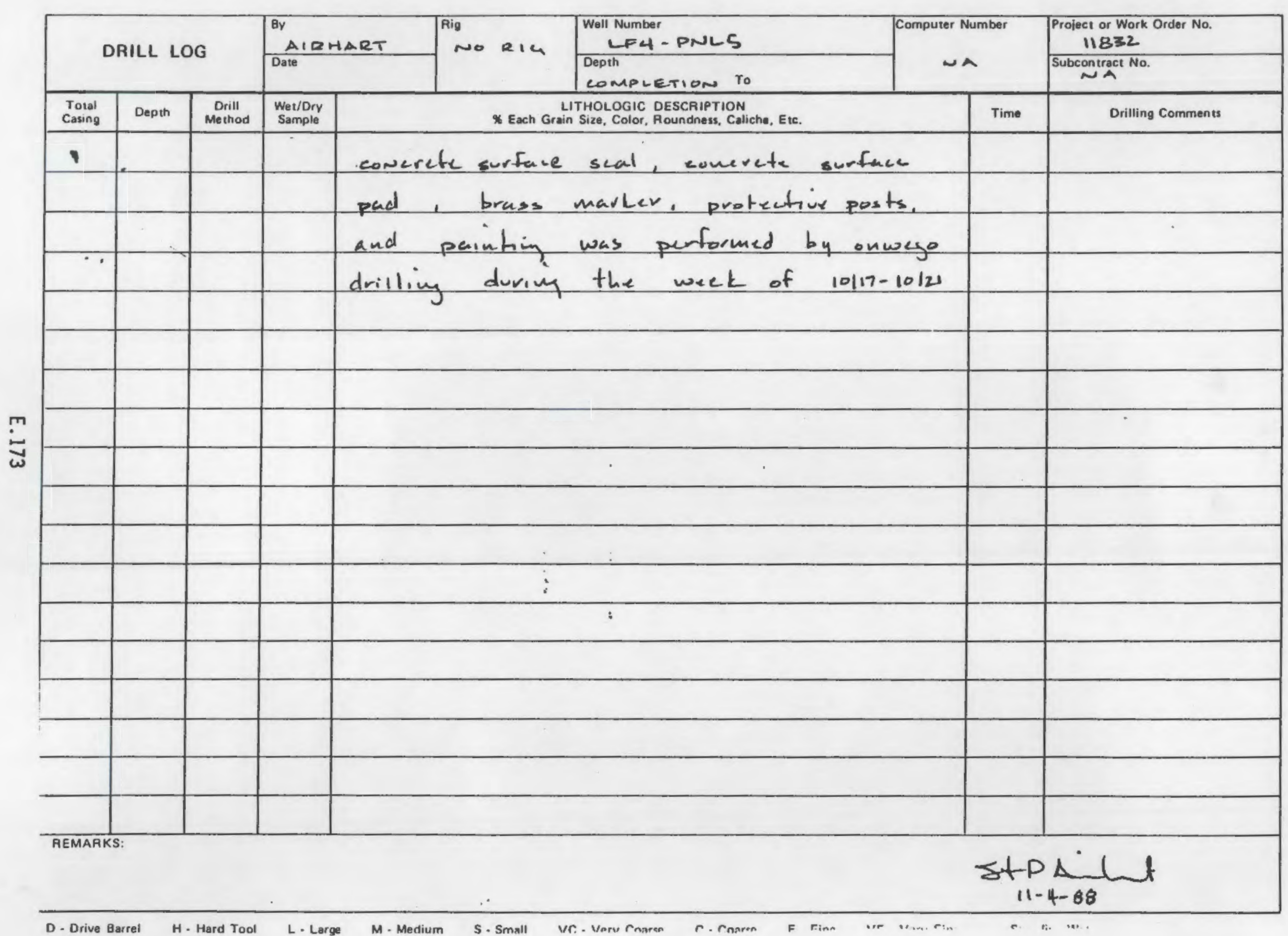



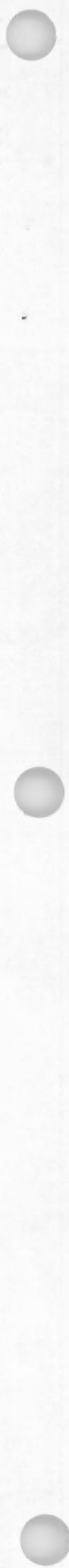
WELL LF4-PNL6 


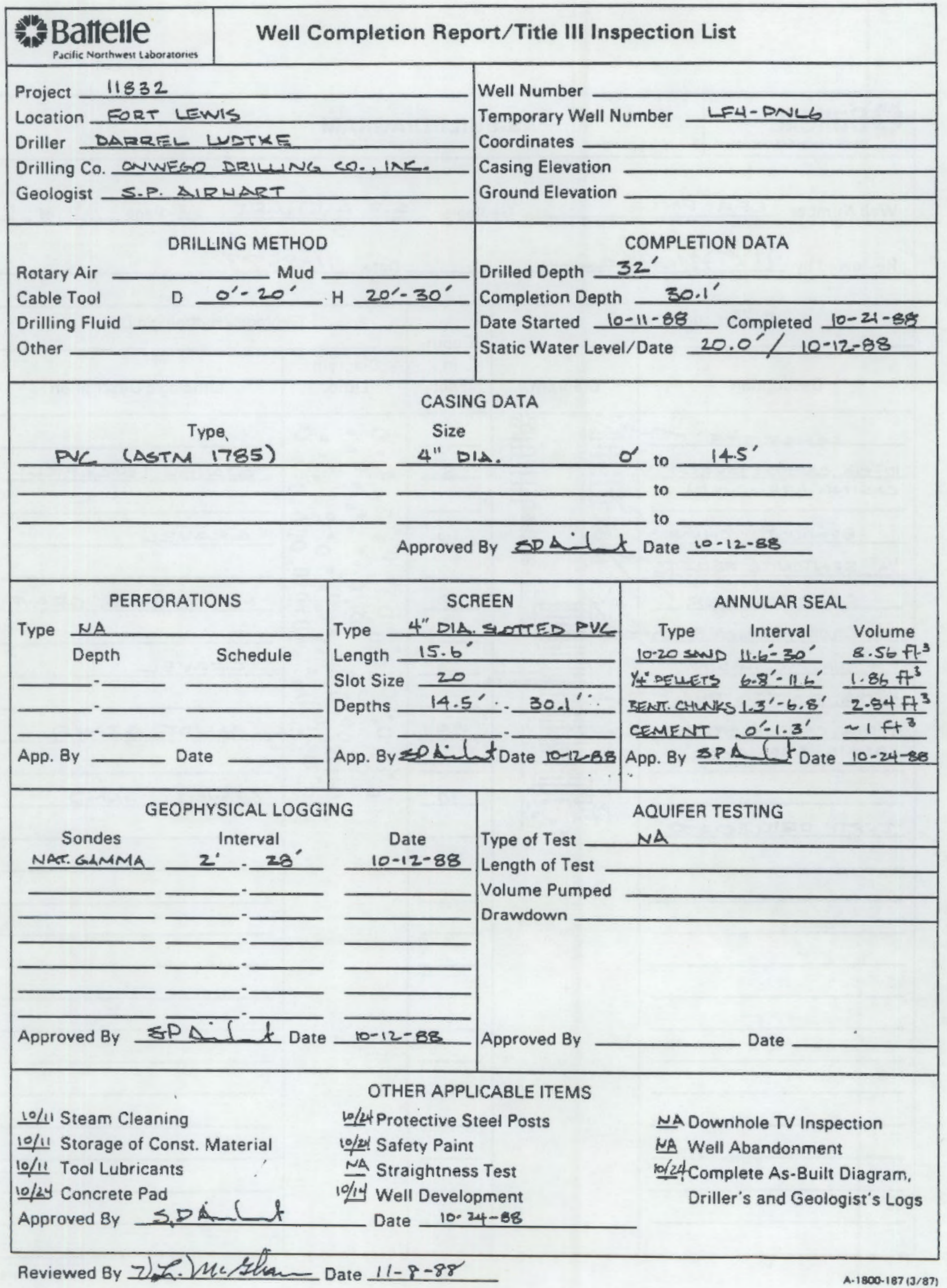




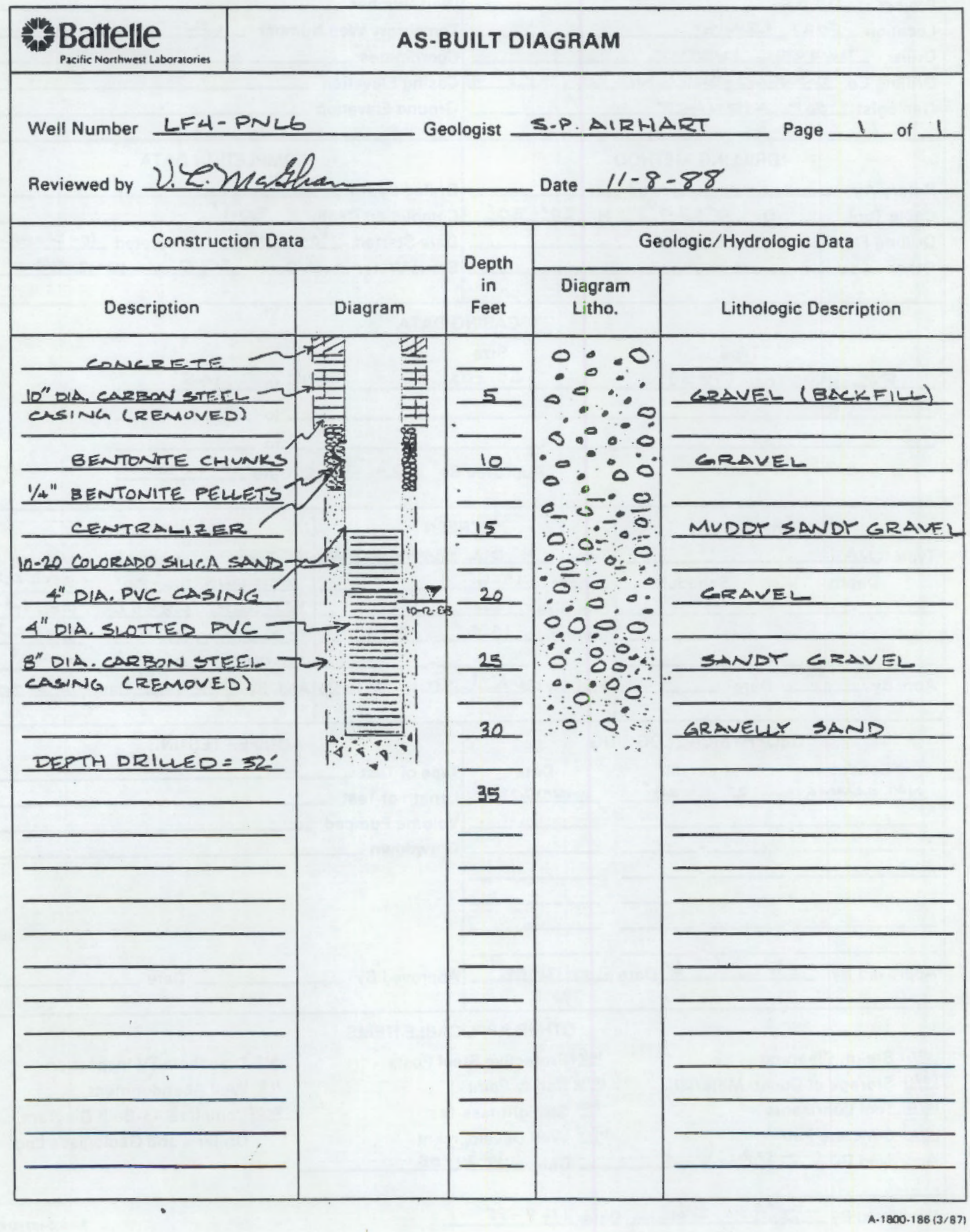

E.176 


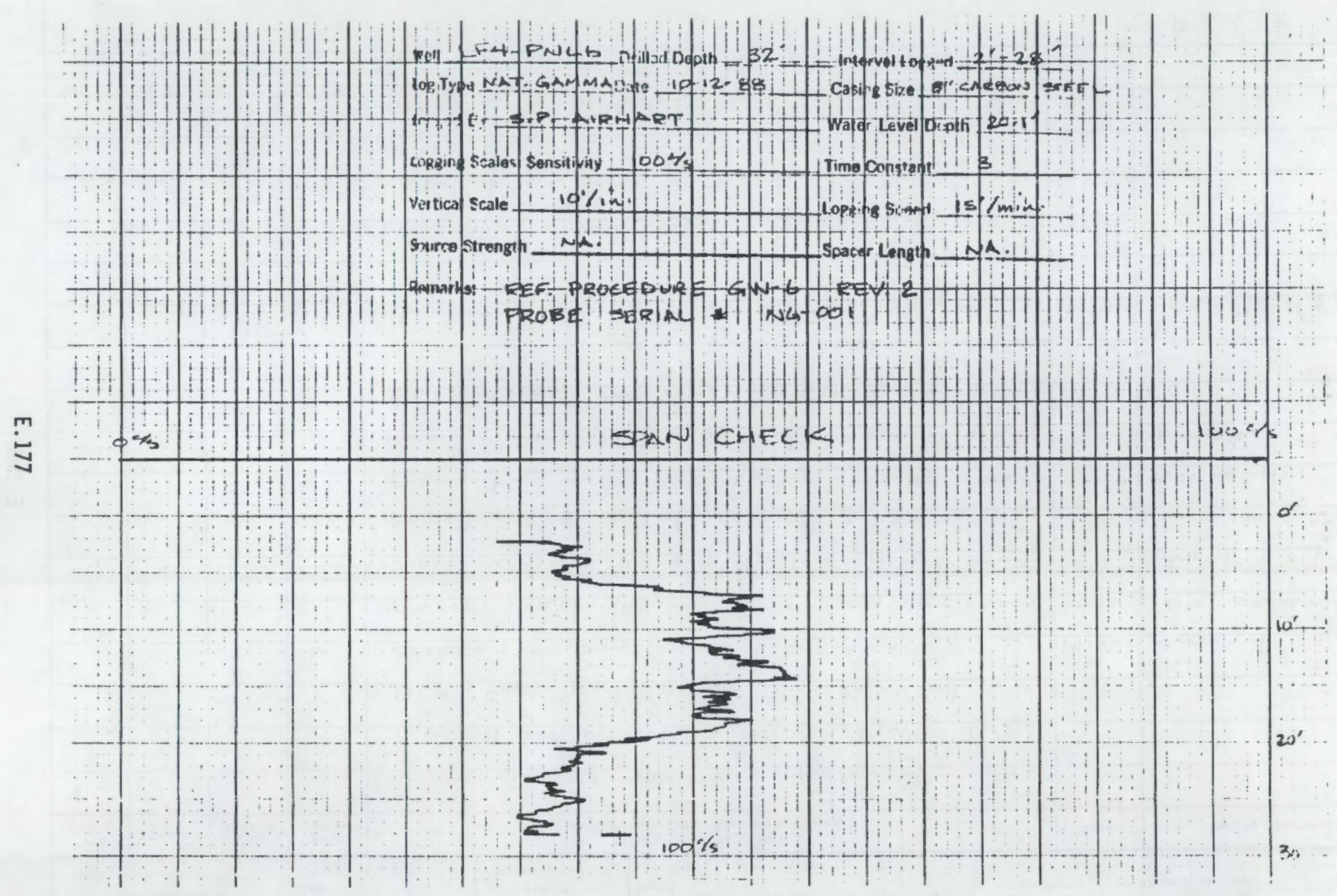


page $1 . t 3$

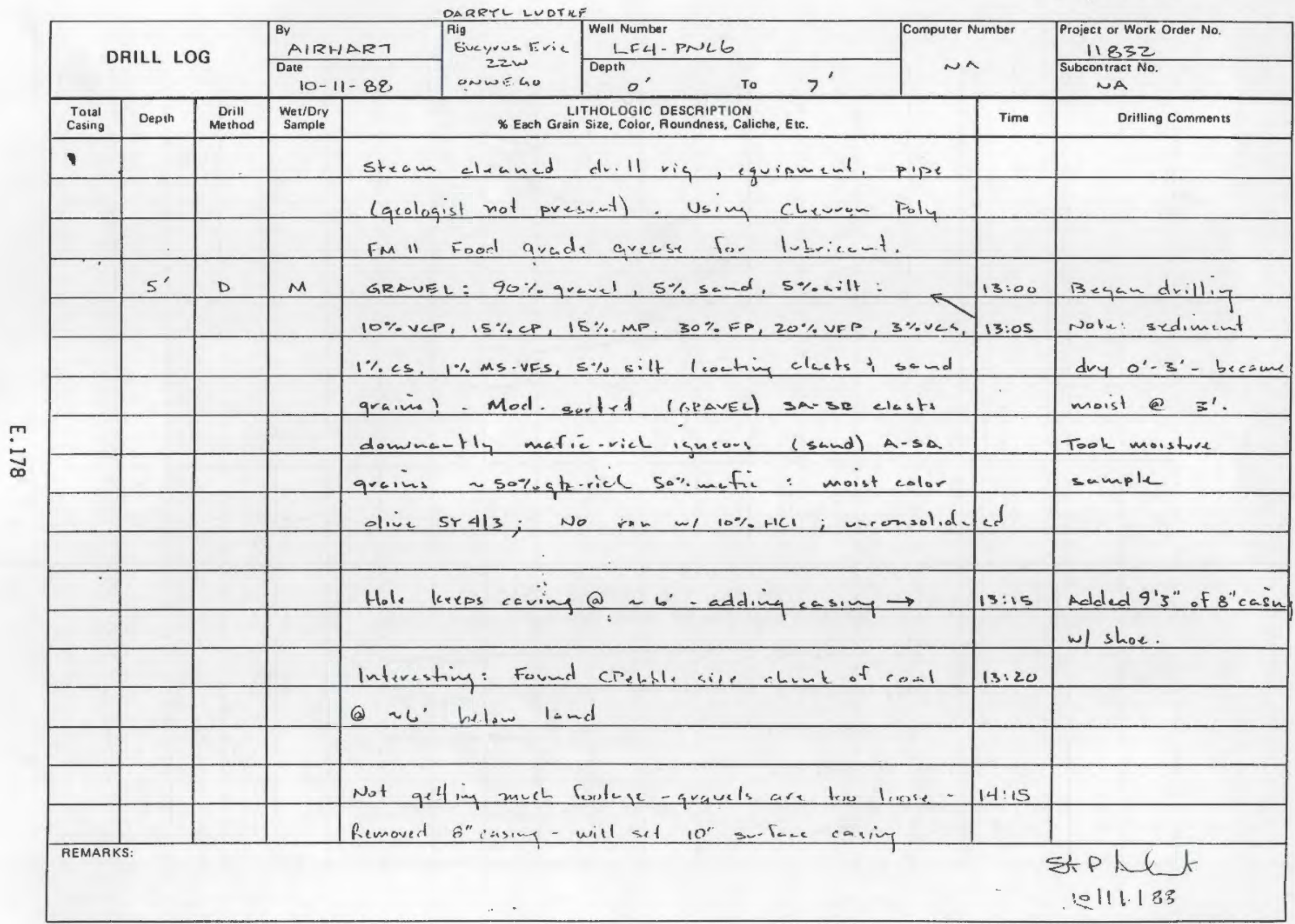


p-get $2 . t$

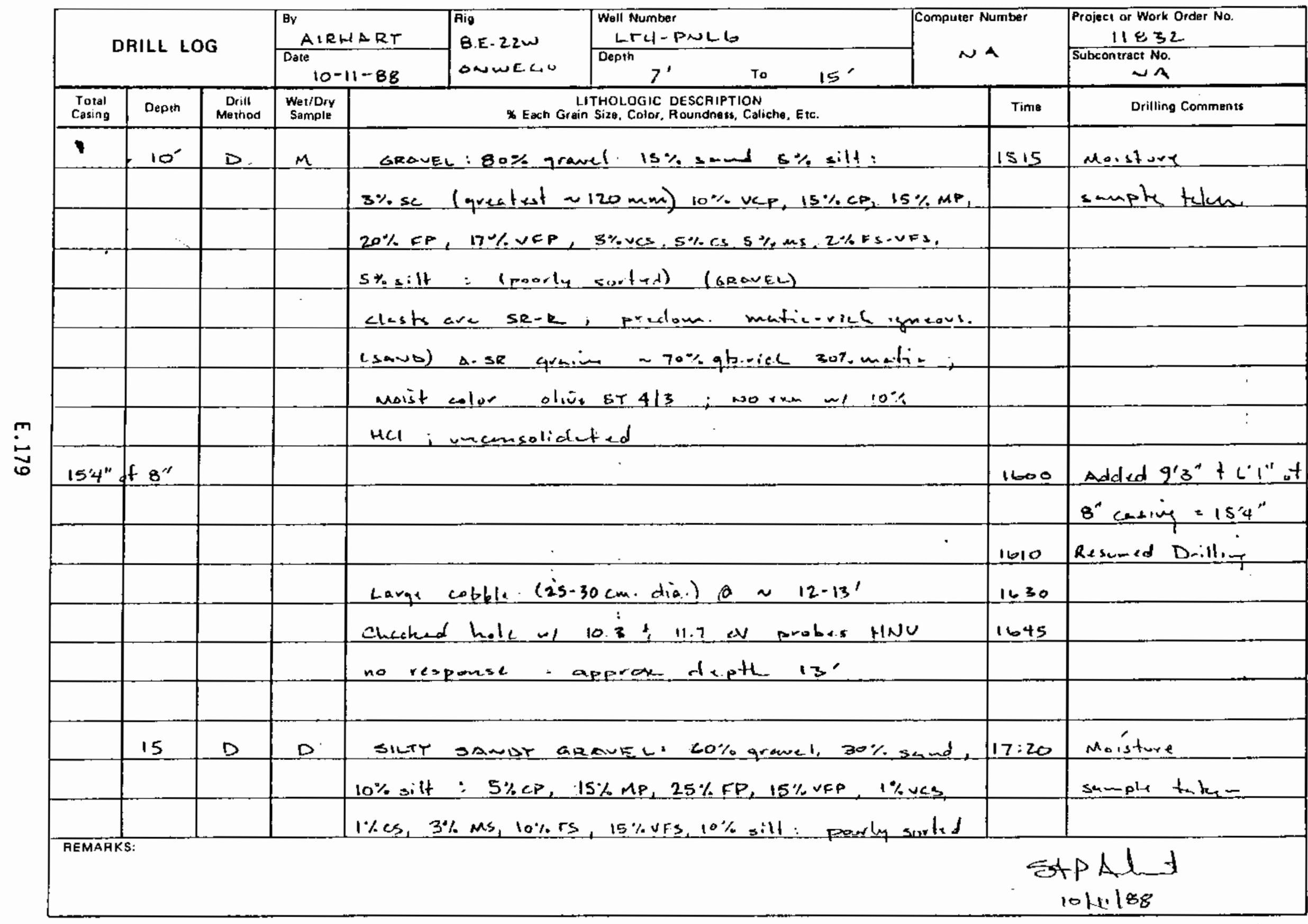

O. Drive Barret H. Hard Tool L-Large M-Medium S-Small VC-Very Coarse C-Coarse F. Fine VF-Verv Fine __ Standing Water 
page $=$ of 3

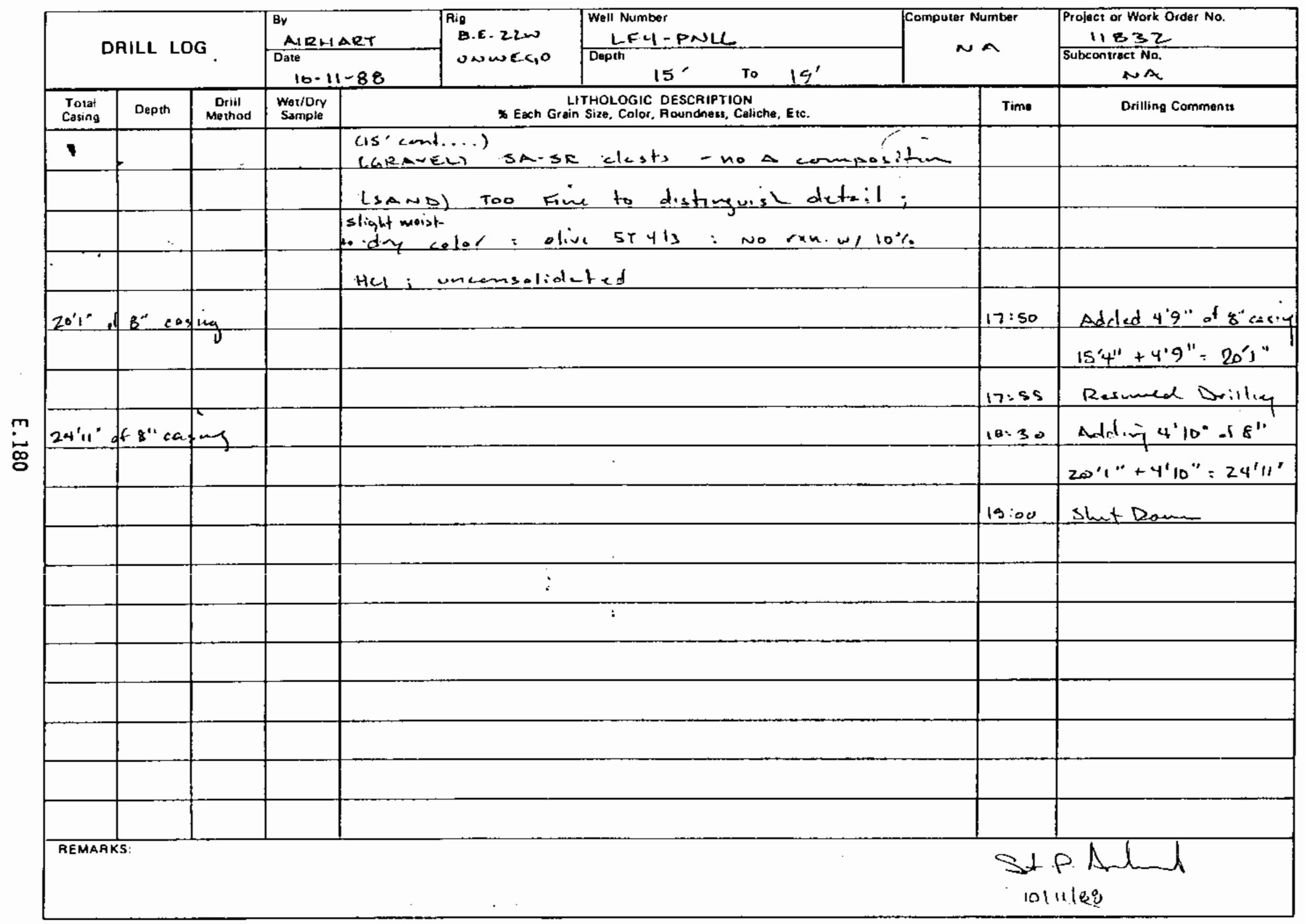

H. Hard Tanl

1. arme

M- Aandiium

ur wan n w w 
poge 1

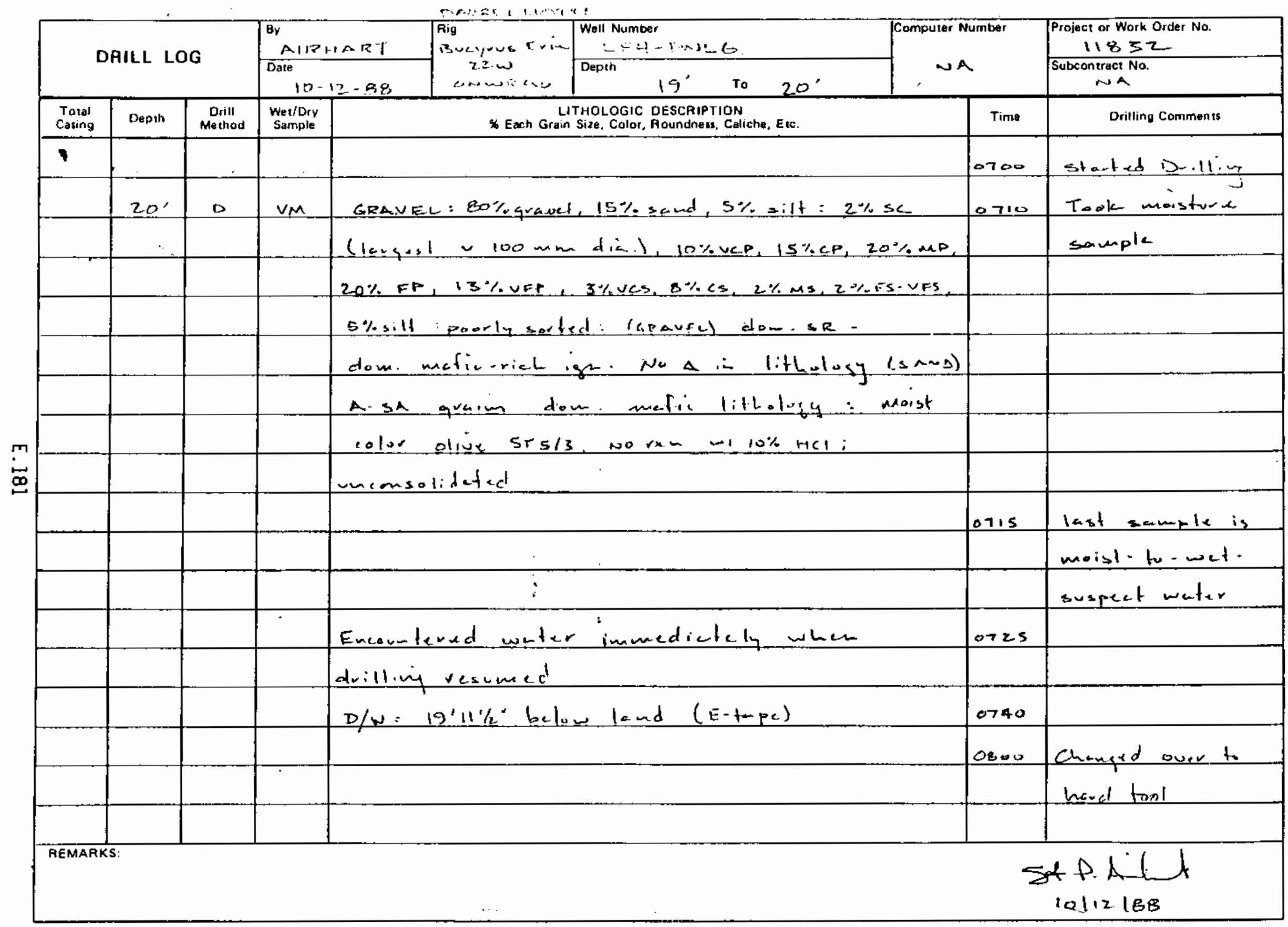

n. Drien Derrol is Mnedtand

th nantion e rate. 
pare 2 f $g$

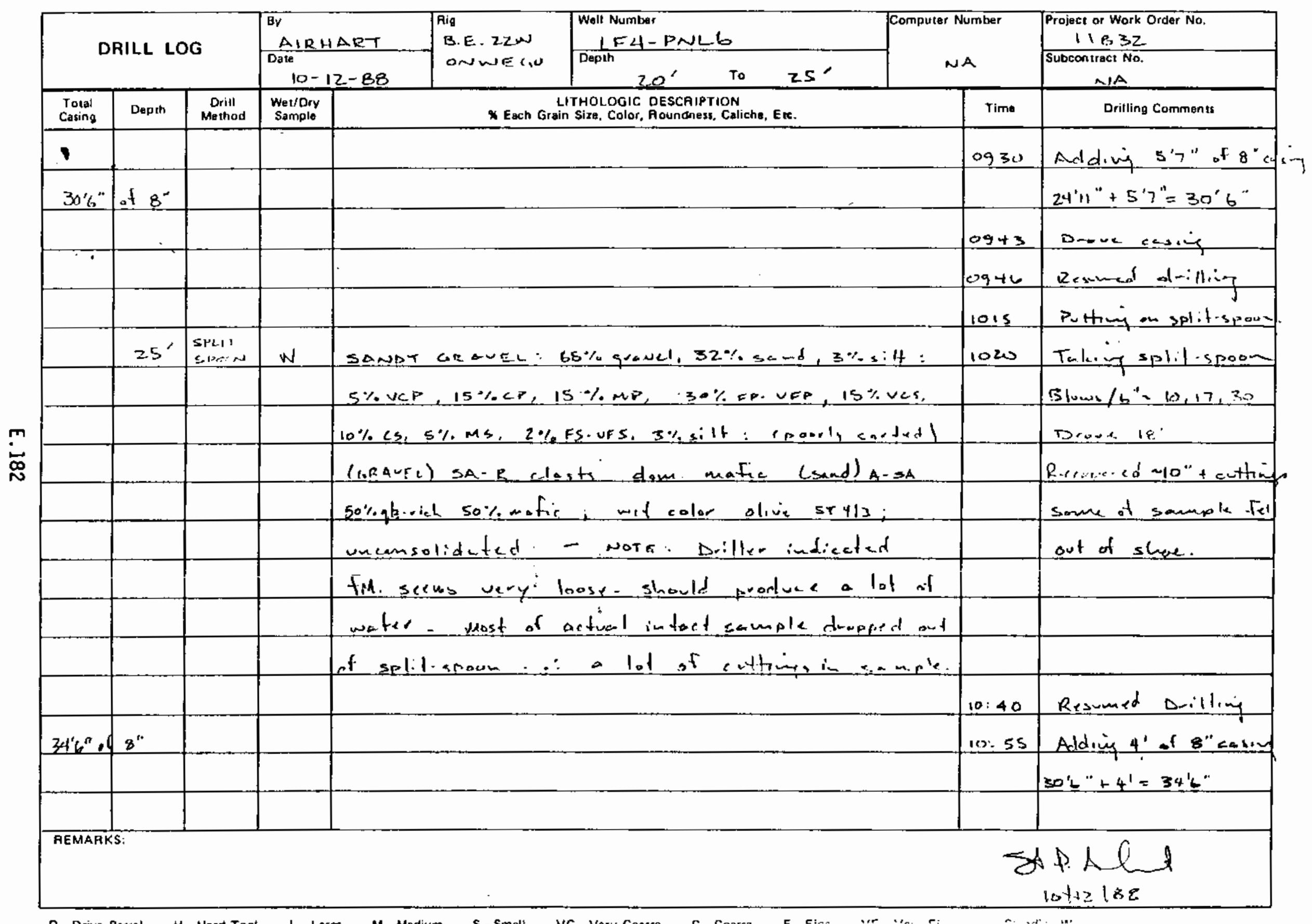

D. Drive Barrel H. Hard Toot

L. Larme M. Medisım 
pere 3.8

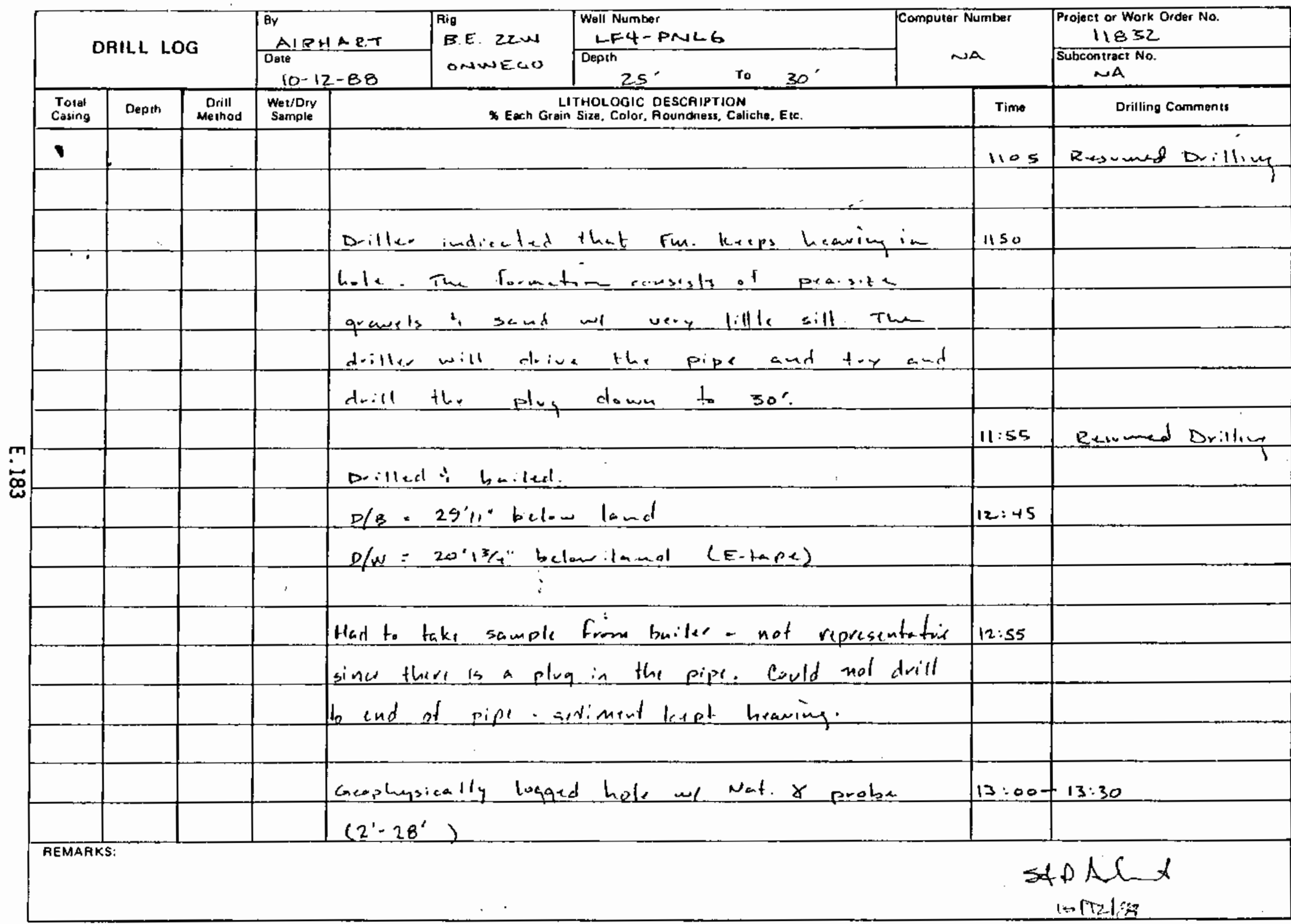

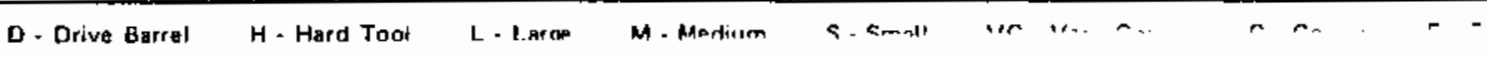


page $4 . f_{8}$

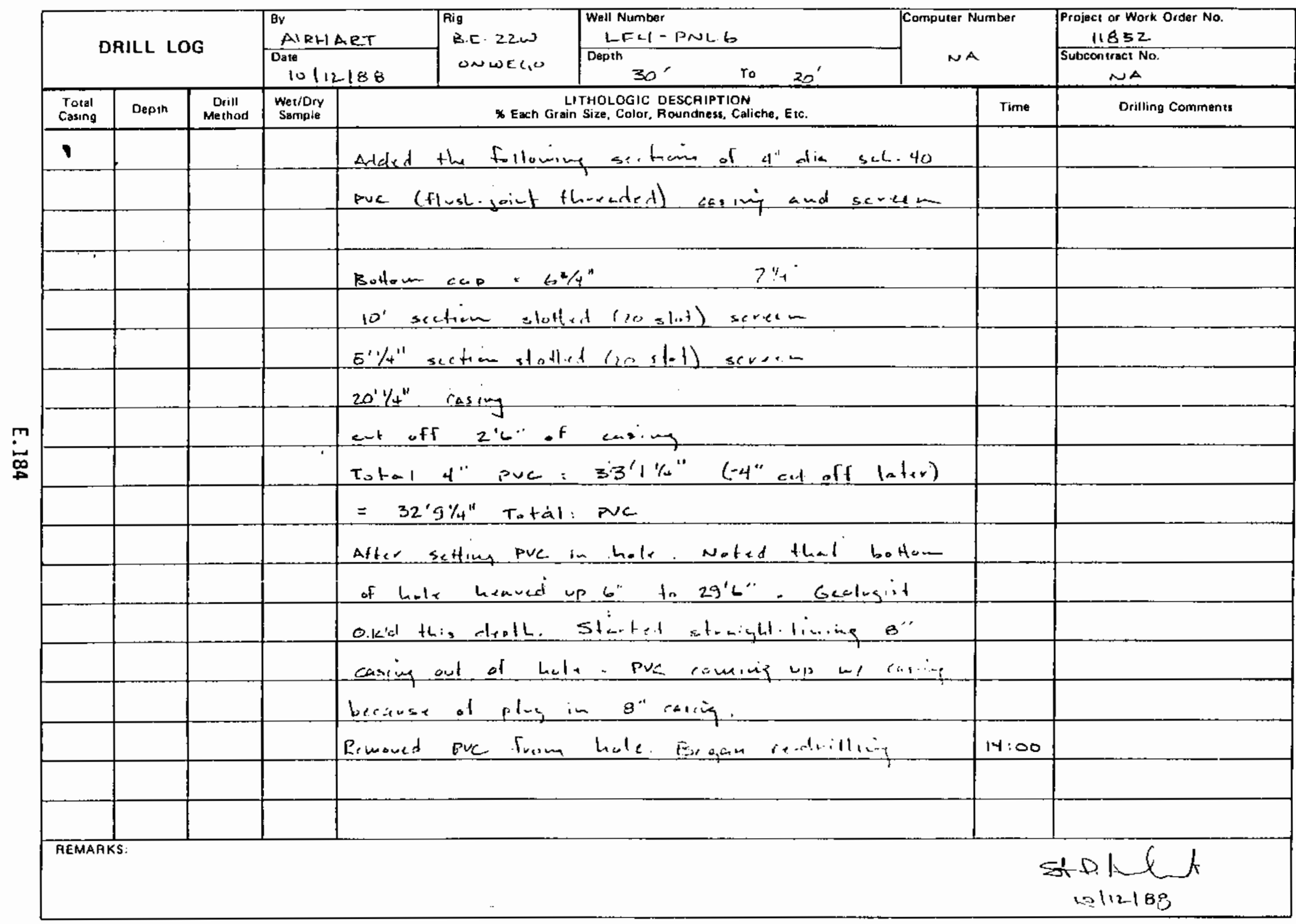

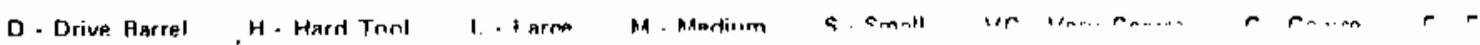




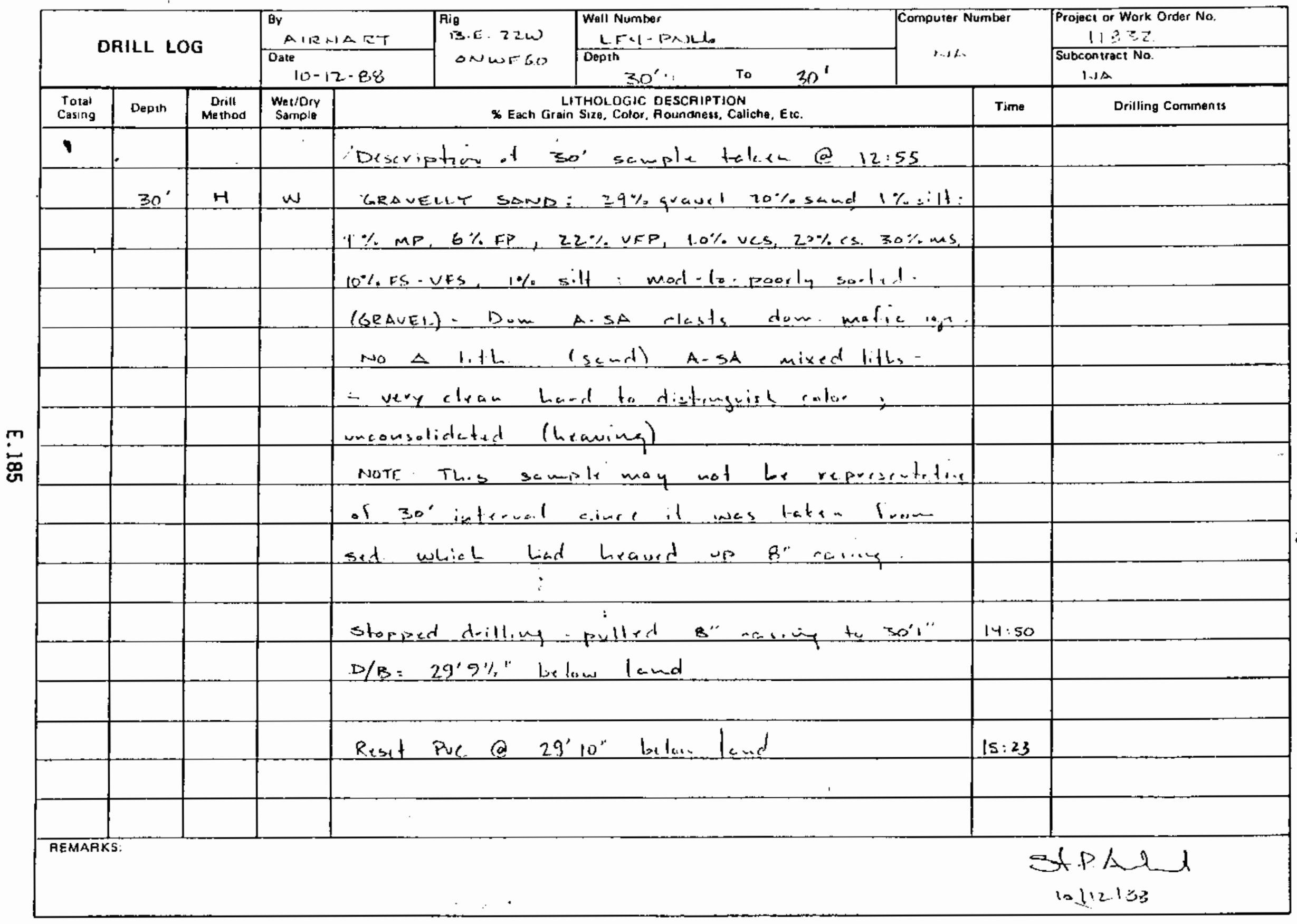

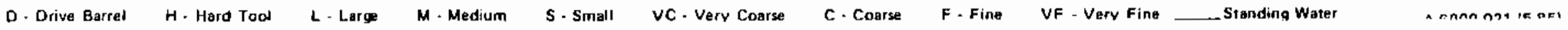


p=-y, of 8

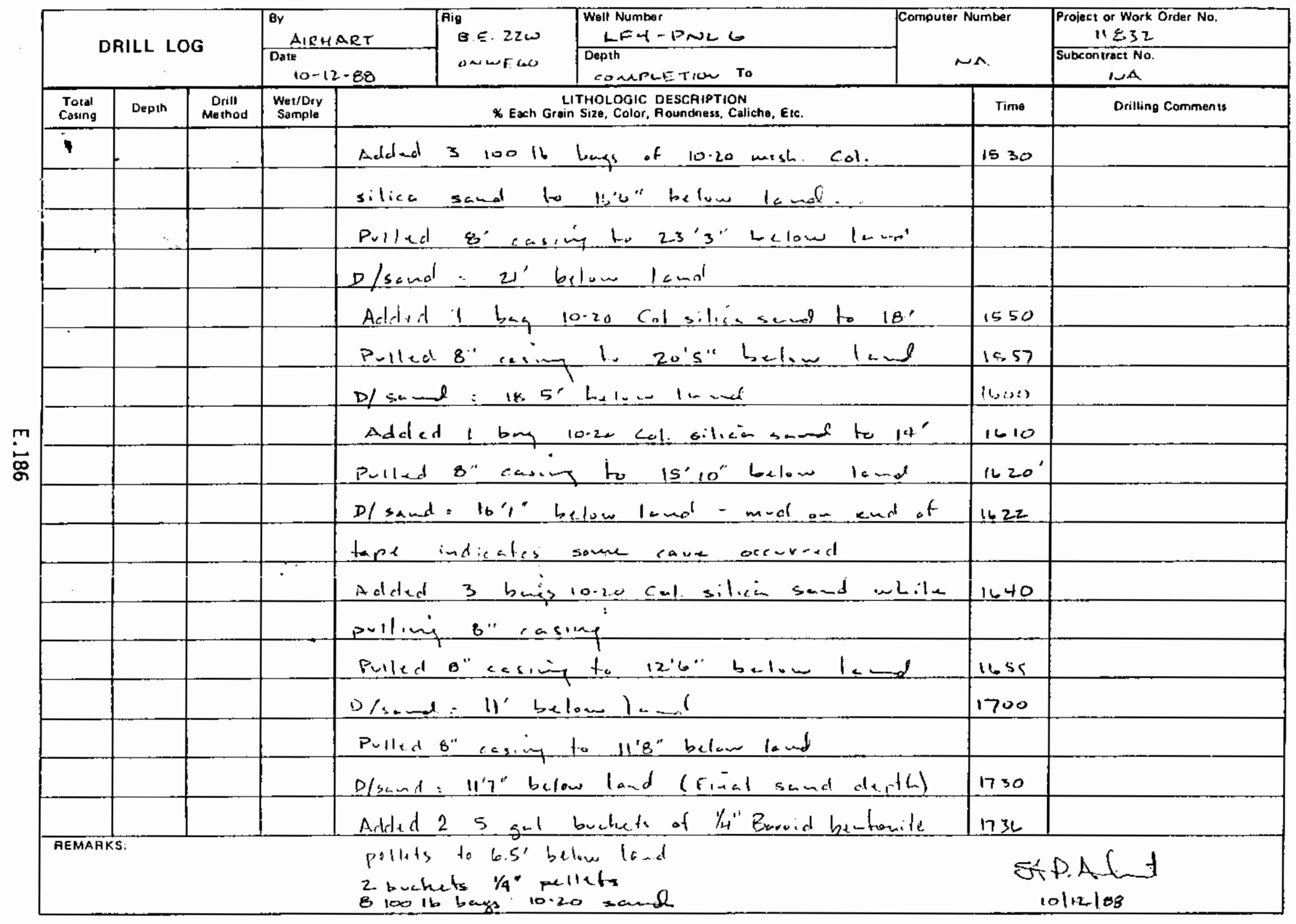

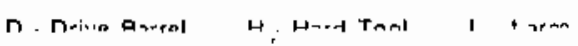




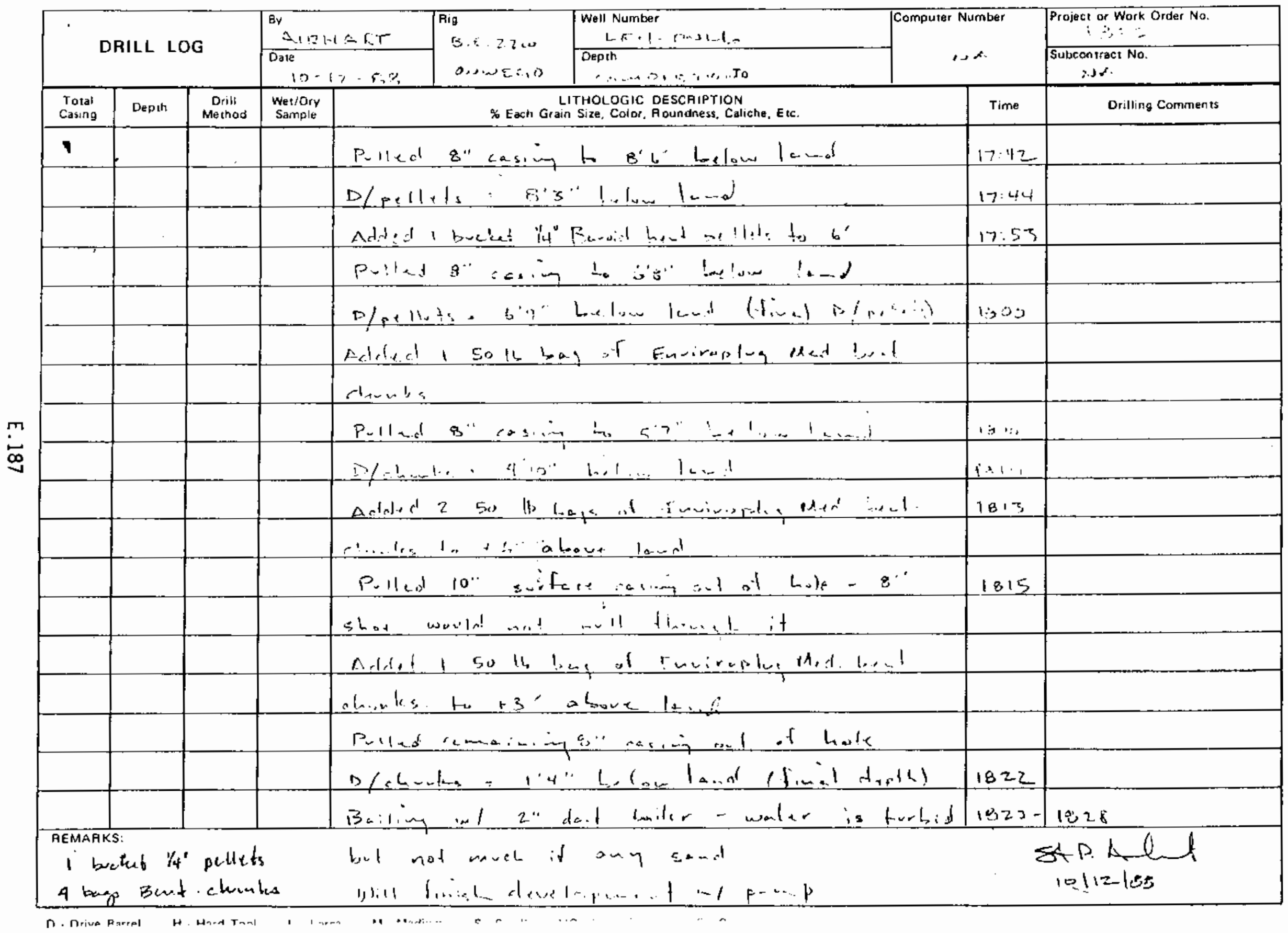


Hej $\&$ is

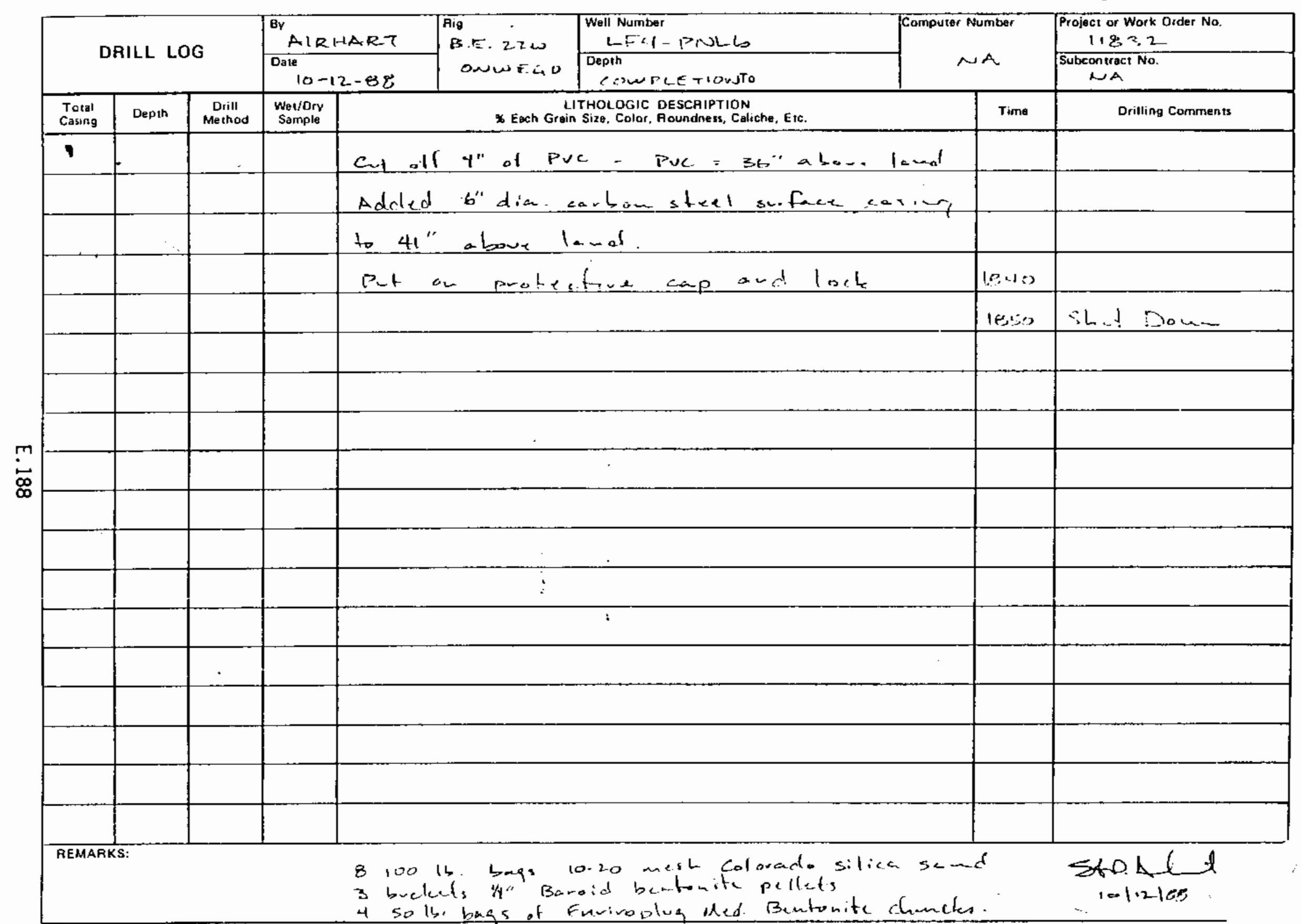

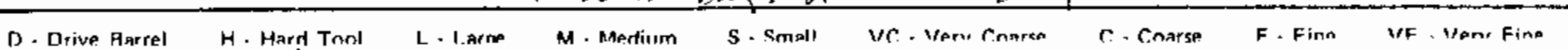

sandinn water 
pare -1 of 1

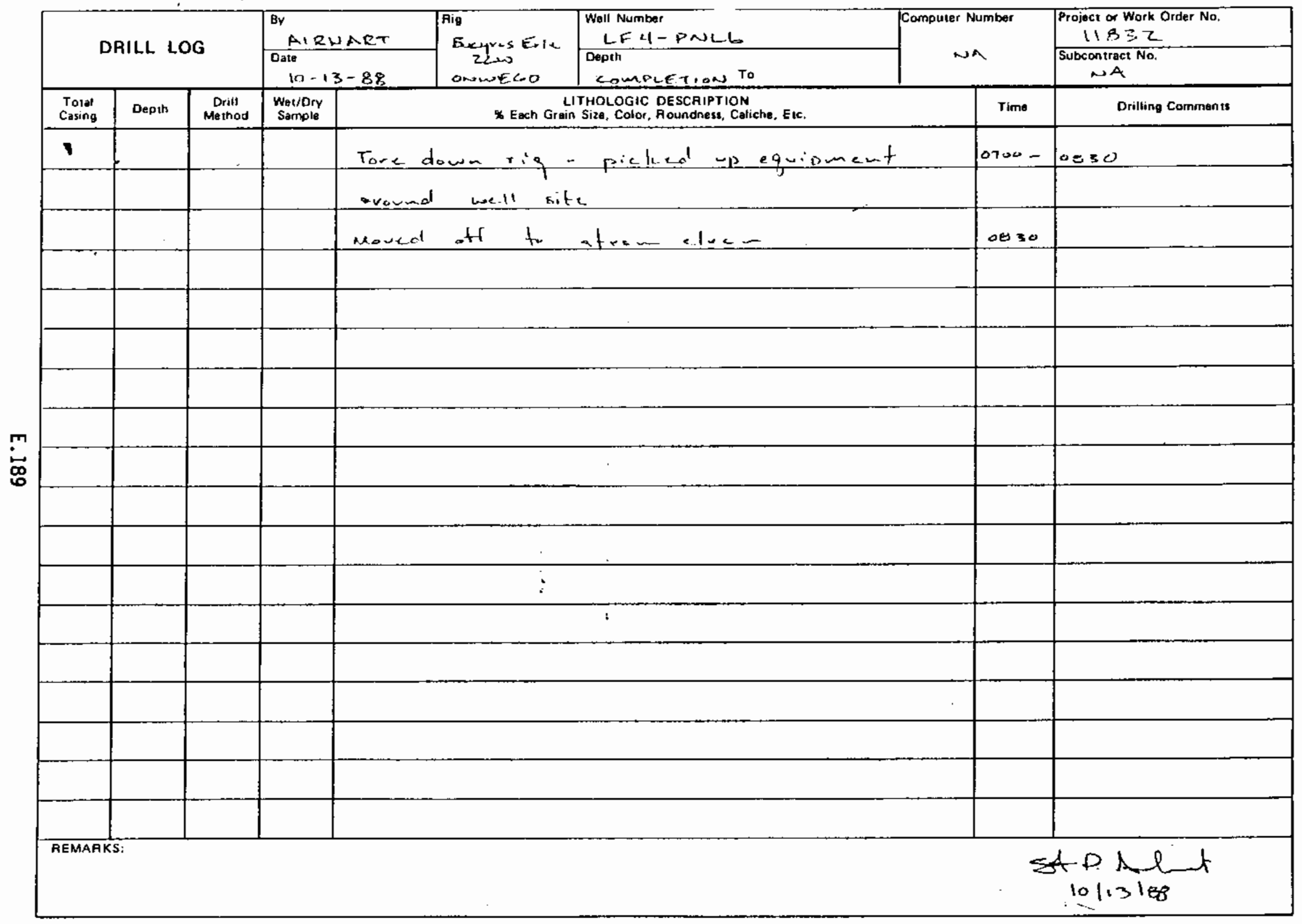
D. Drive Barrel
H - Hard Toot
L - Large
M. Medium
S - Small
VC. Very Coare
c. Coarse
F - Fine
VF - Very Fine
A.600ก.021 (6.85) 


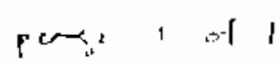

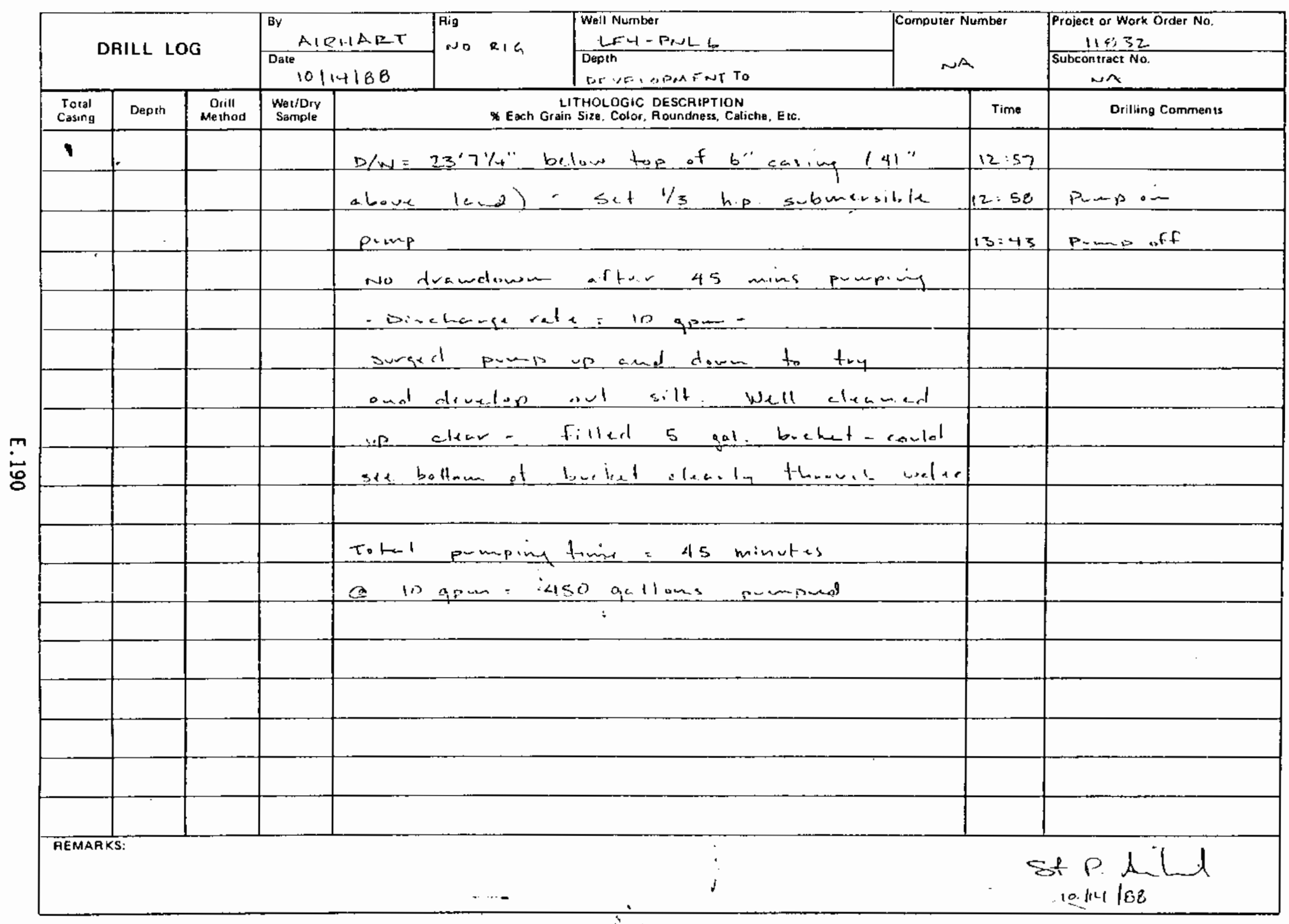

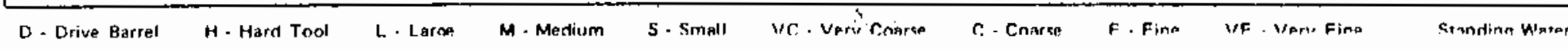


pag 1 of

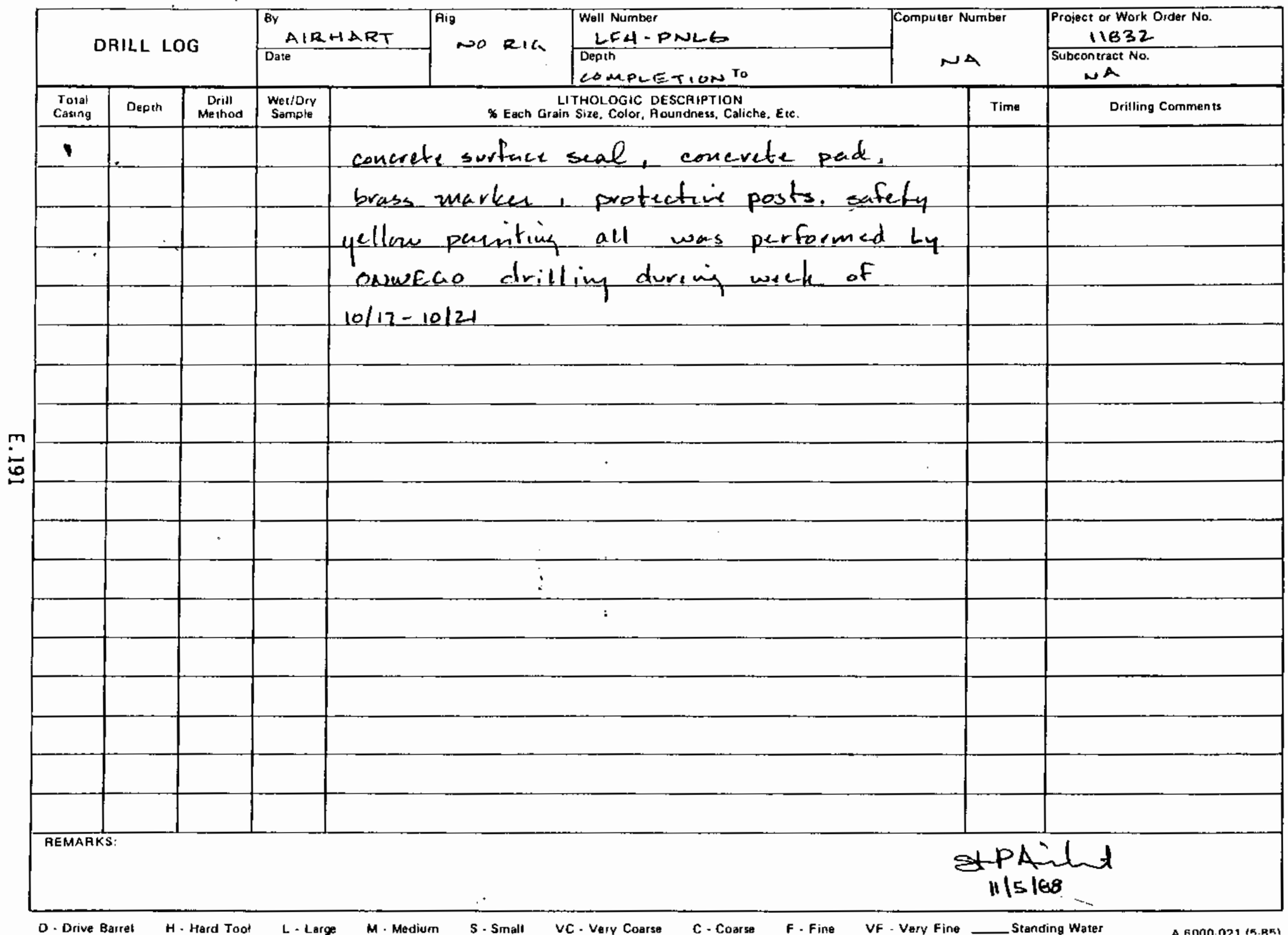


,

. 


\section{APPENDIX $F$}

MOISTURE CONTENT OF SEDIMENT SAMPLES 


\section{APPENDIX $F$}

\section{MOISTURE_CONTENT OF SEDIMENT SAMPLES}

The information below shows moisture content data for selected wells at Landfills 1 and 4 .

\begin{tabular}{|c|c|c|}
\hline Well Number & $\begin{array}{l}\text { Depth, feet Below } \\
\quad \text { Land Surface } \\
\end{array}$ & $\begin{array}{c}\text { Moisture Content, } \\
\text { wt.\% }\end{array}$ \\
\hline LFl-PNLI & $\begin{array}{r}6.0 \\
10.0 \\
15.0 \\
25.0 \\
30.0 \\
33.5\end{array}$ & $\begin{array}{l}2.42 \\
5.93 \\
4.63 \\
6.98 \\
5.69 \\
7.87\end{array}$ \\
\hline LFI-PNL2 & $\begin{array}{l}10.5 \\
15.0 \\
20.8\end{array}$ & $\begin{array}{l}4.58 \\
5.73 \\
8.75\end{array}$ \\
\hline LF1-PNL3 & $\begin{array}{r}5.0 \\
10.5\end{array}$ & $\begin{array}{l}8.24 \\
5.68\end{array}$ \\
\hline LF4-PNL2 & $\begin{array}{r}6.0 \\
11.5 \\
15.0 \\
21.0 \\
25.0\end{array}$ & $\begin{array}{l}2.22 \\
3.37 \\
6.44 \\
8.40 \\
6.88\end{array}$ \\
\hline LF4-PNL3 & $\begin{array}{l}11.0 \\
16.5 \\
21.5 \\
26.5 \\
31.0\end{array}$ & $\begin{array}{l}5.00 \\
5.57 \\
5.35 \\
5.43 \\
4.01\end{array}$ \\
\hline LF4-PNL5 & $\begin{array}{r}5.0 \\
10.0 \\
15.0 \\
20.0 \\
25.0 \\
30.0\end{array}$ & $\begin{array}{r}3.87 \\
3.25 \\
3.27 \\
4.79 \\
13.27 \\
12.75\end{array}$ \\
\hline L.F4-PNL6 & $\begin{array}{r}5.0 \\
10.0 \\
15.0 \\
20.0\end{array}$ & $\begin{array}{l}3.61 \\
2.58 \\
3.00 \\
4.77\end{array}$ \\
\hline
\end{tabular}




\section{.}


APPENDIX G

WATER-LEVEL MEASUREMENT PROCEDURE 


\section{APPENDIX G}

\section{WATER-LEVEL MEASUREMENT PROCEDURE}

This appendix contains the procedure used to measure water levels at the new wells near Landfills 1 and 4.

G.1 
Introduction

Equipment

Graduated Steel Tape Method
Water-ievel measurements are taken each time a well is sampled, before it is purged. These measurements are taken as depth-to-water from the top of the well casing. They must be subtracted from the surveyed elevation of the casing given in Hanford Wells to obtain the elevation of the water table. The water-table elevations obtained for all wells in the sampling network during a particular sampiing episode can be used to produce a contour map showing the ground-water surface at the time that the measurements were made. These contour maps can be used to help characterize the ground-water flow system and to ensure that the sampling network is adequate.

Graduated steel measuring tapes are more accurate than electrical tapes and so should be used for official measurements. However, an electrical tape can be used to determine the approximate depth to water.

The following equipment will be needed:

- steel measuring tape with attached weight

- blue carpenter's chalk

- a copy of Hanford Wells (PNL-5397)

- electrical tape

- engineer's measuring tape

- field record forms.

Chalk the l-ft section of steel, tape below the zero reading point.

Find the elevation of the measuring point and e estimated water level in Hanford Wells, or use an electris tape to find the approximate depth to water.

Lower the steel tape from the well's measuring point (marked with paint on the top of the casing) to the estimated water level. Note the amount of tape that is in the well by reading the tape at the measuring point. This value is referred to as the "hold point."

Remove the steel tape and check the wetted portion below the zero reading point.

NOTE: If the chalked portion is not wet, repeat the procedure, but allow mare of the tape to go down the. weli (i.e., use a greater hold point).

Add the unwetted length of the chalked portion of the tapa. to the hold point value to obtain the depth-to-water measurement.

\section{G.2}

SECTION 13.1

PAGE 1 of 2 
Electric Tape Method
If the chaiked portion is not wet, repeat until the water level is marked on the chalked portion of the tape.

Repeat the procedure until two steel tape measurements agree within $\pm 0.05 \mathrm{ft}$.

Record the depth-to-water measurements, time of measurements, measuring device, and the name of the person taking the measurements on the fieid record form.

Lower the electric tape from the measuring point into the borehole until the buzzer and the light indicate contact with the water.

Mark the electric tape at the measuring point and identify the nearest graduation on the electric tape.

If the water level is deeper than the nearest graduation marked on the tape, add the difference to the depth identified to obtain the true depth to water.

If the water jevel is shallower than the nearest graduation marked on the tape, subtract the difference from the depth identified to obtain the true depth to water.

Record the depth-to-water measurements, time of measurements, measuring device, and the name of the person taking the measurements on the fjeld record form.

NOTE: This measurement should be usad oniy as an approximate depth to water, because the electric tape is less reliable than the steel tape.
DATE ISSUED: $7-86$

SUPERSEDES

ISSUE DATED: NEW
PNL-MA- 580

SECTION

13.1

PAGE 2 of 2 

APPENDIX $\mathrm{H}$

WELL LOCATION AND ELEVATION SURVEY DATA 


\section{APPENDIX H}

\section{WELL LOCATION AND ELEVATION SURVEY DATA}

This appendix includes

- results of the 1988 and 1982 surveys of the existing (1981 and 1984) monitoring wells at Landfills I and 4

- results of the 1989 survey of both the new (1988) and the existing (1981 and 1984) monitoring wells at Landfills 1 and 4. 


\section{DEPARTMENT OF THE ARMY \\ SEATTLE DISTRICT, CORPS OF ENGINEERS \\ P.O. HOX C.3755 \\ SEATTLE, WASHINGTON $98124-2255$}

March 7, 1988

Geotechnical Branch

SUBJECT: Ft. Leuis, Washington Landfills 1 and 4, Horizontal and Vertical

Control for Existing Borings

Mr. Paul Eddy

Battelle Pacific Northwest Labs

Battelle Blvd.

Richland, Washington 99352

Dear Mr. Eddy:

Seattle District has completed the survey of existing ground water monitoring vells located at landfills 1 \& 4, Ft. Lewis, Washington. The survey

information is provided herein.

In reviewing existing information, our survey personnel disclosed a 1982 survey of the wells located at landfill 4. Information from the earlier survey is being provided here; the vells were not resurveyed. Landfill 1 required a new survey, that information is also attached. The vell casings associated with landfill 1 are in very poor condition, consequently all elevations are on $2 \times 2$ hubs placed approximately 2-feet from the casings. It was also discovered that wells $1 \& 2$ at landfill 4 were incorrectly identified (numbers reversed) on the map provided to you in our meeting of February 11.

If you any questions, or if we can be of further assistance, please contact Mr. Mike Bowlus/NPS-EG-H, telephone (206) 764-6744.

Enclosure

Copies furnished:

Randy Hanna/DEH AFZHEHQ

Fort Lewis, Washington
S.T. Biveng

E.T. Bailey

Chief, Hazardous Waste Mgrnt. Sect. 
LANDFILLS 1 \& 4

FT. LEWIS, WASHINGTON

WELL SURVEY

\begin{tabular}{|c|c|c|c|c|c|}
\hline WELL \# & ELEV. $1 /$ & LATITUDE & LONGITUDE & NORTHING & EASTING $2 /$ \\
\hline $\begin{array}{l}L F-1 \\
L F-2 \\
L F-3 \\
L F-4\end{array}$ & $\begin{array}{l}302.46 \\
302.14 \\
236.77 \\
311.46\end{array}$ & $\begin{array}{l}47.4 .11 .24535 \\
47.4 .02 .80571 \\
47.2 .52 .69644 \\
47.4 .01 .82937\end{array}$ & $\begin{array}{l}122.35 .18 .85152 \\
122.35 .18 .83797 \\
122.35 .16 .21592 \\
122.35 .11 .32028\end{array}$ & $\begin{array}{l}640125.080 \\
639270.330 \\
638241.710 \\
639157.680\end{array}$ & $\begin{array}{l}1479594.900 \\
1479573.200 \\
1479727.540 \\
1480090.810\end{array}$ \\
\hline \\
\hline $\begin{array}{l}\text { LANDFI } \\
\text { (1982 }\end{array}$ & 4 & ro & $n g, \cos$ & & ) \\
\hline WELL 非 & ELEV. & LATITUDE & LONGITUDE & NORTHING & EASTING \\
\hline $\begin{array}{r}1 \\
2 \\
3 \\
4 \\
11\end{array}$ & $\begin{array}{l}224.69 \\
219.19 \\
238.79 \\
235.93 \\
234.79\end{array}$ & $\begin{array}{l}47.6 .49 .98508 \\
47.6 .53 .18160 \\
47.7 .06 .21637 \\
47.7 .09 .37644 \\
47.7 .00 .66870\end{array}$ & $\begin{array}{l}122.36 .05 .55298 \\
122.36 .28 .23885 \\
122.36 .06 .17757 \\
122.35 .44 .09606 \\
122.36 .04 .44537\end{array}$ & $\begin{array}{l}656287.351 \\
656652.944 \\
657932.344 \\
658211.757 \\
657367.306\end{array}$ & $\begin{array}{l}1476791.728 \\
1475231.875 \\
1476792.355 \\
1478327.455 \\
1476897.139\end{array}$ \\
\hline
\end{tabular}

1/ NGVD 27

$\underline{2} /$ STATE gLANE COORDINATES ZONE 2 


\begin{tabular}{|c|c|c|c|c|}
\hline \multicolumn{2}{|c|}{ ROUTING AND TRANSMITTAL SLIP } & \multicolumn{3}{|c|}{ Date } \\
\hline \multicolumn{2}{|c|}{$\begin{array}{l}\text { To: (Name, office symbol, room number, } \\
\text { bulding, Agengylpost) }\end{array}$} & & Initials & Datt \\
\hline \multicolumn{3}{|l|}{2.} & & \\
\hline \multicolumn{3}{|l|}{3.} & & \\
\hline \multicolumn{3}{|l|}{4.} & & \\
\hline \multicolumn{3}{|l|}{5.} & & \\
\hline Action & File & \multicolumn{3}{|c|}{ Note and Retum } \\
\hline Approvat & For Clearance & \multicolumn{3}{|c|}{ Per Conversation } \\
\hline As Requested & For Correction & \multicolumn{3}{|c|}{ Prepare Reply } \\
\hline Circulate & For Your Information & \multicolumn{3}{|c|}{ Soe Mo } \\
\hline Comment & Investigato & \multicolumn{3}{|c|}{ Signature } \\
\hline Coordination & Justity & & & \\
\hline
\end{tabular}

\section{REMARKS}

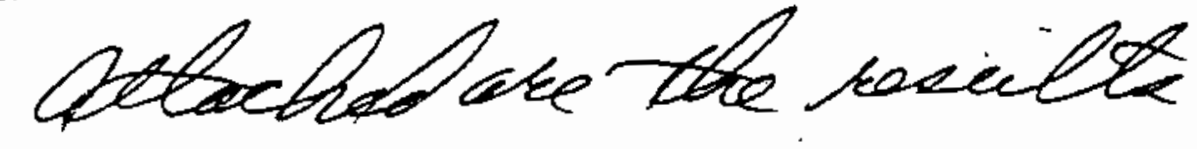
tete acescerfer<smiles>CCC12C=CC=C(CC1)C2(C)C</smiles>

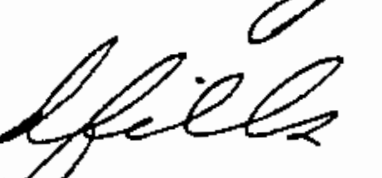

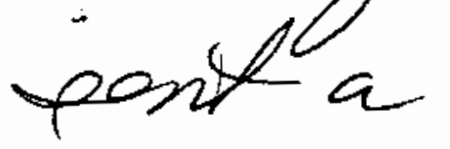

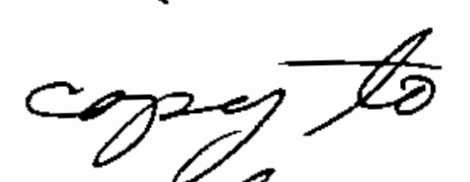

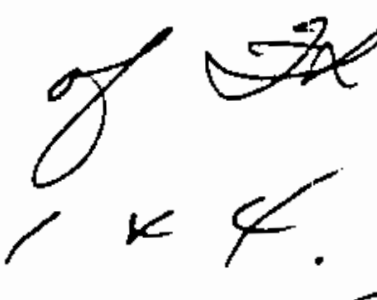

Peore<smiles>CC1CC1</smiles>

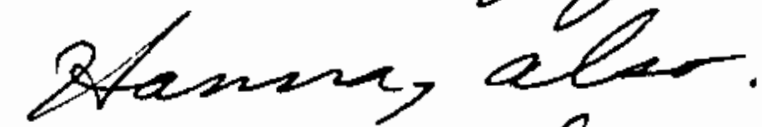<smiles>CC(C)(C)CCC1CCCC1C1CCCC1</smiles><smiles>CCCC1CCCCC1C</smiles>

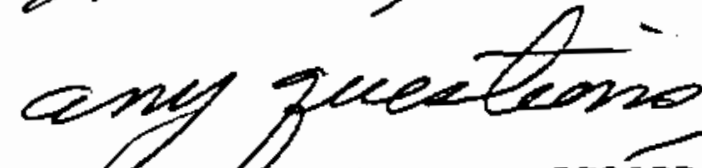

DO DOT US6 this form as a AECOAD of approvals, concurrences. disposals. clearances, and similar actions

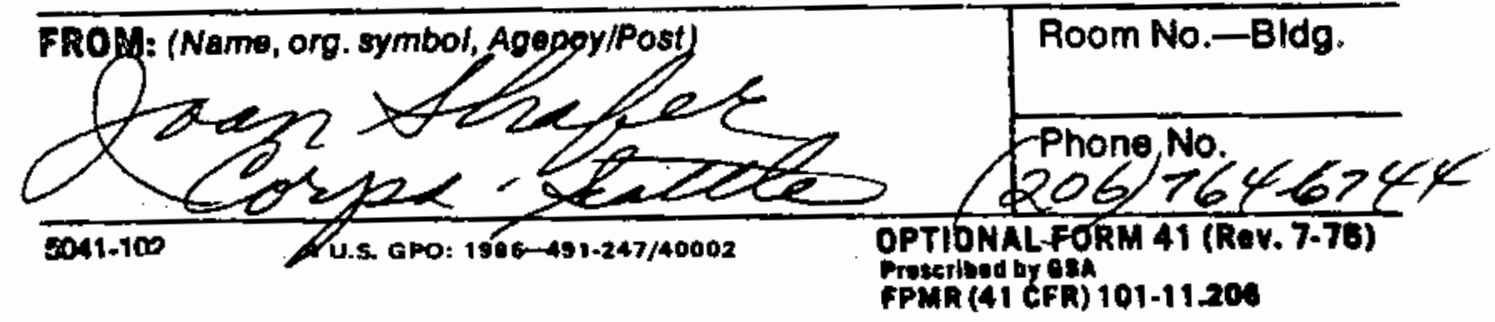

H. 4 
Port Lewis, WA

Well Location Survey

Landfills to. 1 s No. 4

Seattle District Corps of Engineers

\footnotetext{
For: The Department of Army

Seattle District Corps of Engineers

Contract DACA67-89-D-0003

Work Order No. 2

Horton Dennis and Associates, Inc.

May 22, 1989

HDA Job No. 8371.02
}

H. 5 
Horizontal Control: Washington coordinate system, south zone. Established from United States Army Corps of Engineers published control for 1987 aerial mapping project.

Vertical Control: National Geodetic Vertical Datum 1929 (NGVD 1929). Also established from USA COE 1987 published mapping control.

Explanation: Horizontal and vertical values were established for the center of existing brass caps at well locations. Vertical values were established for the North Rim of the existing PVC well pipe inside well casing. 
SEATTLE DISTRICT CORPS OF ENGINEERS

WORK ORDER NO. 2 COHTRACT DACA67-89-D-0003

HDA JOB NO. 8371.02

FORT LEWIS. WASHINGTON

WELL LOCATIONS LANDFILL $I \& 4$

\begin{tabular}{|c|c|c|c|c|c|}
\hline \multirow{2}{*}{ WELL NO } & \multicolumn{3}{|c|}{ LANDFILL NO. 1} & \multirow[b]{2}{*}{ EASTING } & \multirow[b]{2}{*}{ REMARK'S } \\
\hline & COMPUTER NO & ELEVATION & NORTHING & & \\
\hline \multirow[t]{2}{*}{ PNL- 1} & 136 & 306.47 & 640370.6733 & 1480.403 .3561 & BRASS CAP \\
\hline & & 308.66 & & & TCP PVC \\
\hline \multirow[t]{2}{*}{$L F \mid-4$} & 139 & 311.45 & 639158.6144 & 1480090.8904 & BRASS CAP \\
\hline & & 312.59 & & & TOP PVC \\
\hline \multirow[t]{2}{*}{ LFI-3 } & 142 & 296.90 & 638242.6336 & 1479727.4028 & BRASS CAP \\
\hline & & 297.69 & & & TOP PVC \\
\hline \multirow[t]{2}{*}{ PNL-2 } & 143 & 296.82 & 638284.9083 & 1479184.6580 & BRASS CAP \\
\hline & & 298.37 & & & TOP PVC \\
\hline \multirow[t]{2}{*}{ PNL-3 } & 144 & 306.30 & 638634.4298 & 1478887.6439 & BRASS CAP \\
\hline & & 307.74 & & & TOP PVC \\
\hline \multirow[t]{2}{*}{ LFI-2 } & 146 & 302.11 & 639271.3857 & 1479573.5543 & ERASS CAP \\
\hline & & 303.48 & & & TOP.PVC \\
\hline \multirow[t]{2}{*}[F]{-1} & 148 & 302.63 & 640126.0797 & 1479594.9728 & ERASS CAP \\
\hline & & 303.64 & & & TOP PVC \\
\hline \multirow[t]{2}{*}{ PNL-4 } & 150 & 303.19 & 640611.7514 & 1480157.4493 & BRASS CAP \\
\hline & & 305.00 & & & TOP PVC \\
\hline
\end{tabular}

NOTE: BRASS CAP IS A PRESET MONUMENT AT THE WELL SITE. ALL ELEVATIONS ON PVCS ARE TO THE MORTH RIM. 
PAGE 2 OF 2

\begin{tabular}{|c|c|c|c|c|c|}
\hline \multirow{3}{*}{$\begin{array}{l}\text { WELL HO. } \\
\text { LFA-PNL-4 }\end{array}$} & \multirow[t]{3}{*}{ CUMPUTER HO } & ELEVATION & \multirow{3}{*}{$\begin{array}{c}\text { NORTHING } \\
658162.3213\end{array}$} & \multirow{3}{*}{$\begin{array}{c}\text { EASTING } \\
1476174.4060\end{array}$} & \multirow{3}{*}{$\begin{array}{c}\text { REMARKS } \\
\text { BRASS CAP } \\
\text { TOP PVC }\end{array}$} \\
\hline & & 233.86 & & & \\
\hline & & 235.72 & & & \\
\hline \multirow{2}{*}{ LFA-PLN-1 } & 103 & 236.22 & 657439.0119 & 1476136.1599 & BRASS CAP \\
\hline & & 237.82 & & & TOP PVC \\
\hline \multirow{2}{*}{ LF4-II } & 105 & 232.06 & 657397.1543 & 1476813.5658 & BRASS CAP \\
\hline & & 234.05 & & & TOP PVC \\
\hline \multirow[t]{2}{*}{ LF 4-PNL-2 } & 107 & 238.22 & 658639.2226 & 1477139.2425 & BRASS CAP \\
\hline & & $240.4 \theta$ & & & TOP PVC \\
\hline \multirow[t]{2}{*}{ LF4-4 } & 110 & 233.96 & 658201.8046 & 1478294.0279 & BRASS CAP \\
\hline & & 235.41 & & & TOP PVC \\
\hline \multirow[t]{2}{*}{ LF 4-PNL-6 } & 114 & 229.55 & 656714.1107 & 1478619.1314 & BRASS CAP \\
\hline & & 232.19 & & & TOP PVC \\
\hline \multirow{2}{*}{ LF 4-PHL5 } & 120 & 235.12 & $\begin{array}{l}657661.29 \\
657574.677\end{array}$ & $\begin{array}{l}1475354.80 \\
+47568+3245\end{array}$ & BRASS CAP \\
\hline & & 237.46 & & & TOP PVC \\
\hline \multirow[t]{2}{*}{ LFA- } & 124 & 217.32 & 656696.7986 & 1475242.6186 & BRASS CAP \\
\hline & & 218.27 & & & TOP PVC \\
\hline \multirow{3}{*}{ LFA-ZZ } & & & & & \\
\hline & 129 & 223.31 & 656276.4394 & 1476795.1897 & BPASS CAP \\
\hline & & 225.37 & & & TOP PVC \\
\hline \multirow[t]{2}{*}{ LF 4-PNL-3 } & 132 & 244.37 & 656576.2839 & 1477954.7651 & BRA55 CAP \\
\hline & & 246.59 & & & TOP PVC \\
\hline
\end{tabular}

Note: CoRpections made pER VERBL communication with JoAN SAAFER, U.S. ARmi combs ON EKINERE, JUNE 13,1989. 


\section{APPENDIX I}

DRILL LOGS OF EXISTING WELLS AT LANDFILL SITES 1 AND 4 


\section{DRILL LOGS OF EXISTING WELLS AT LANDFILL SITES 1 AND 4}

This appendix contains drilling information for older wells at Landfills 1 and 4 , including

- for Landfill 1

- well 84-CD-LF 1

- well 84-CD-LF-2

- we]1 84-CD-LF-3

- well 84-CD-LF-4

- for Landfilir 4

- well Fort Lewis \#1

- well Fort Lewis \#2

- we11 Fort Lewis \#3

- well Fort Lewis \#4

- well Fort Lewis \#11. 


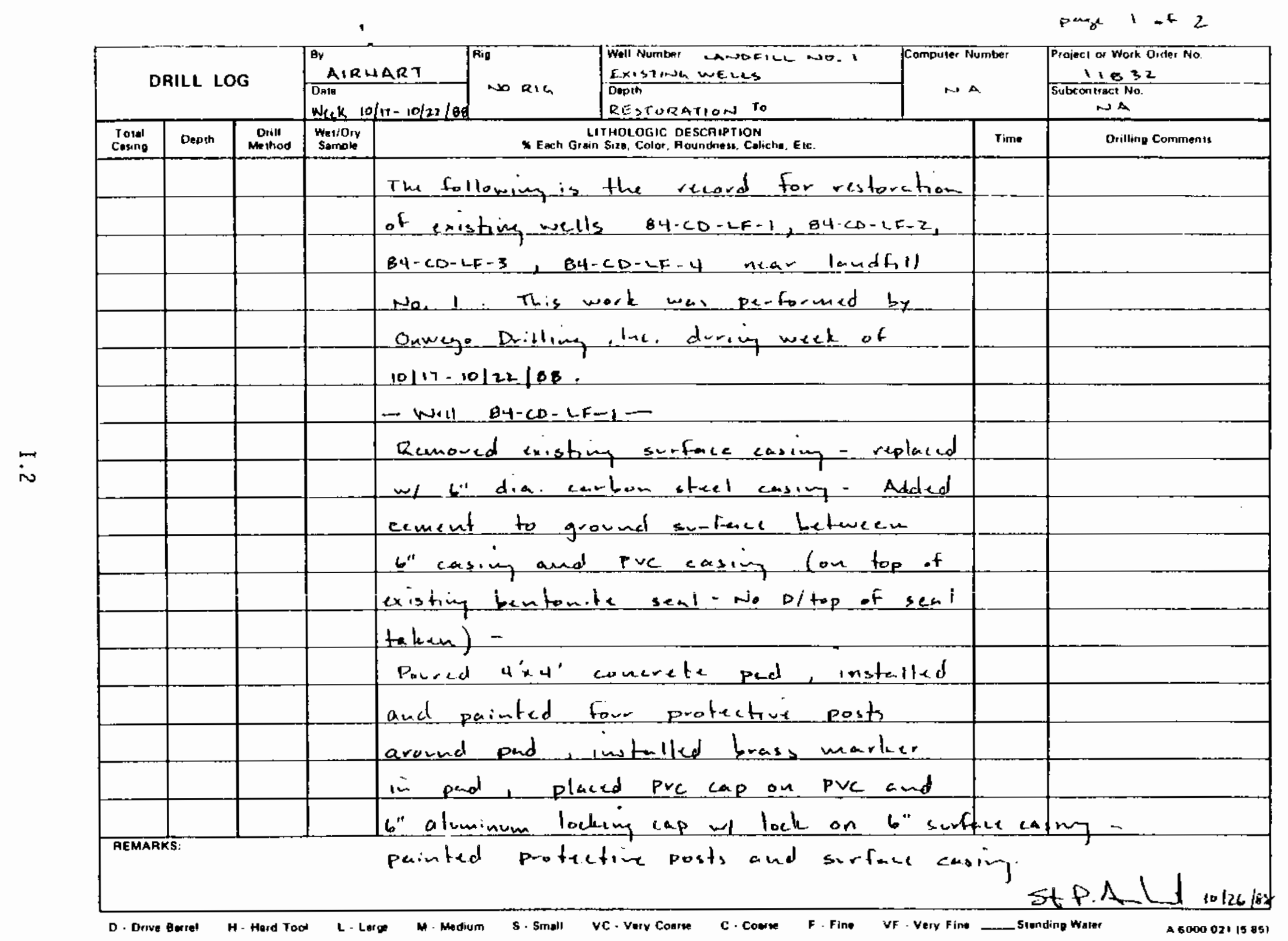




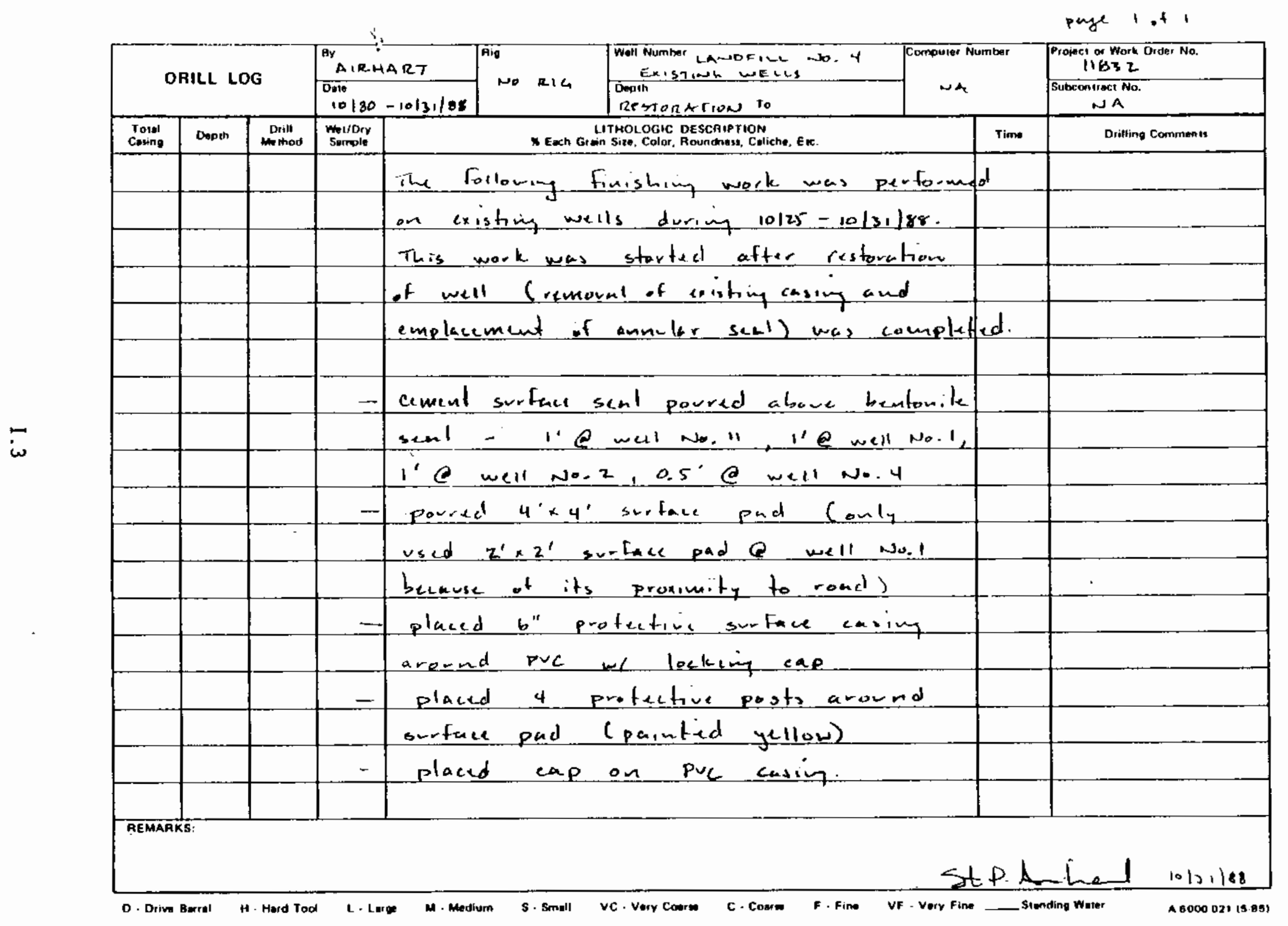


WELL 84-CD-LF 1 
liole lin.

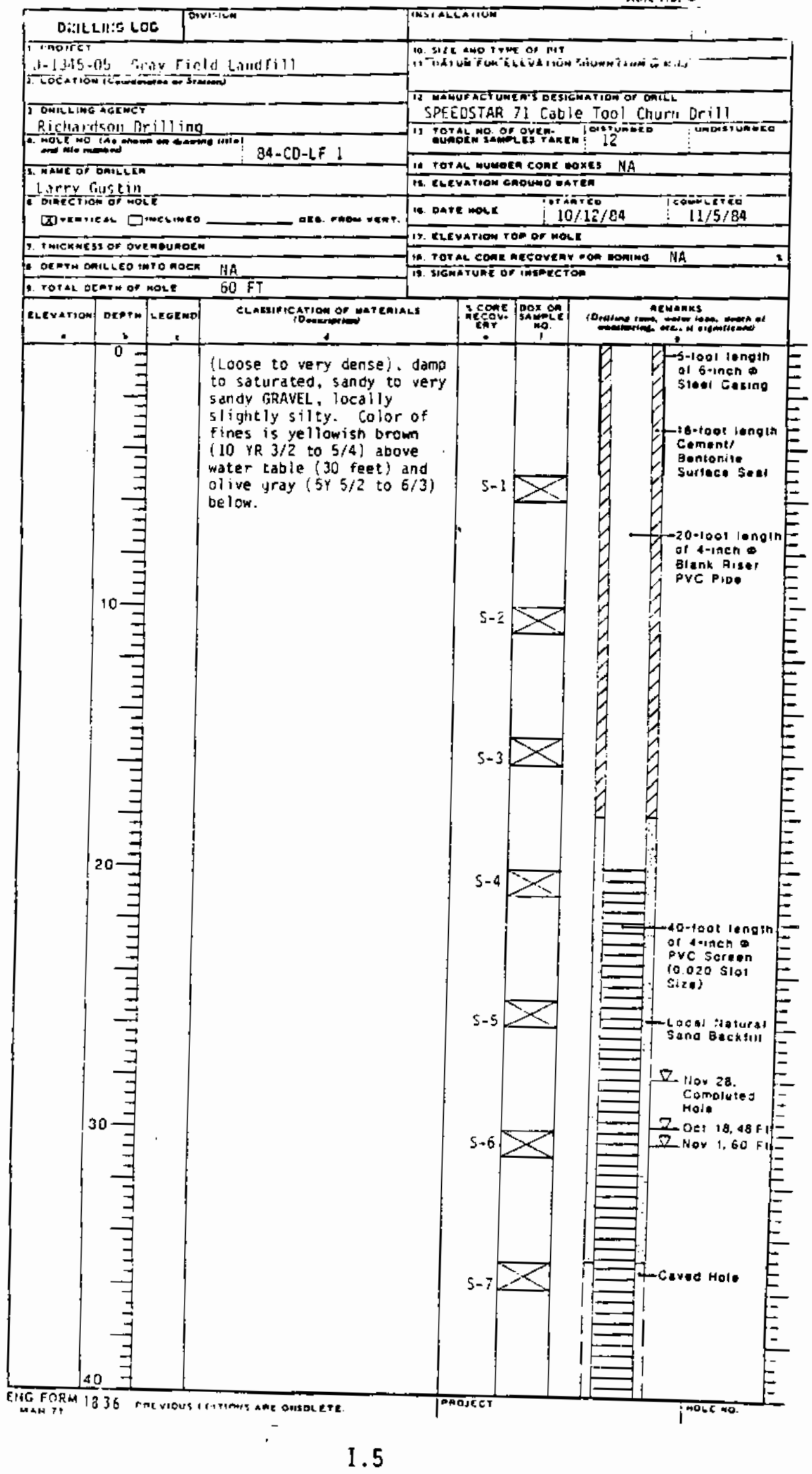




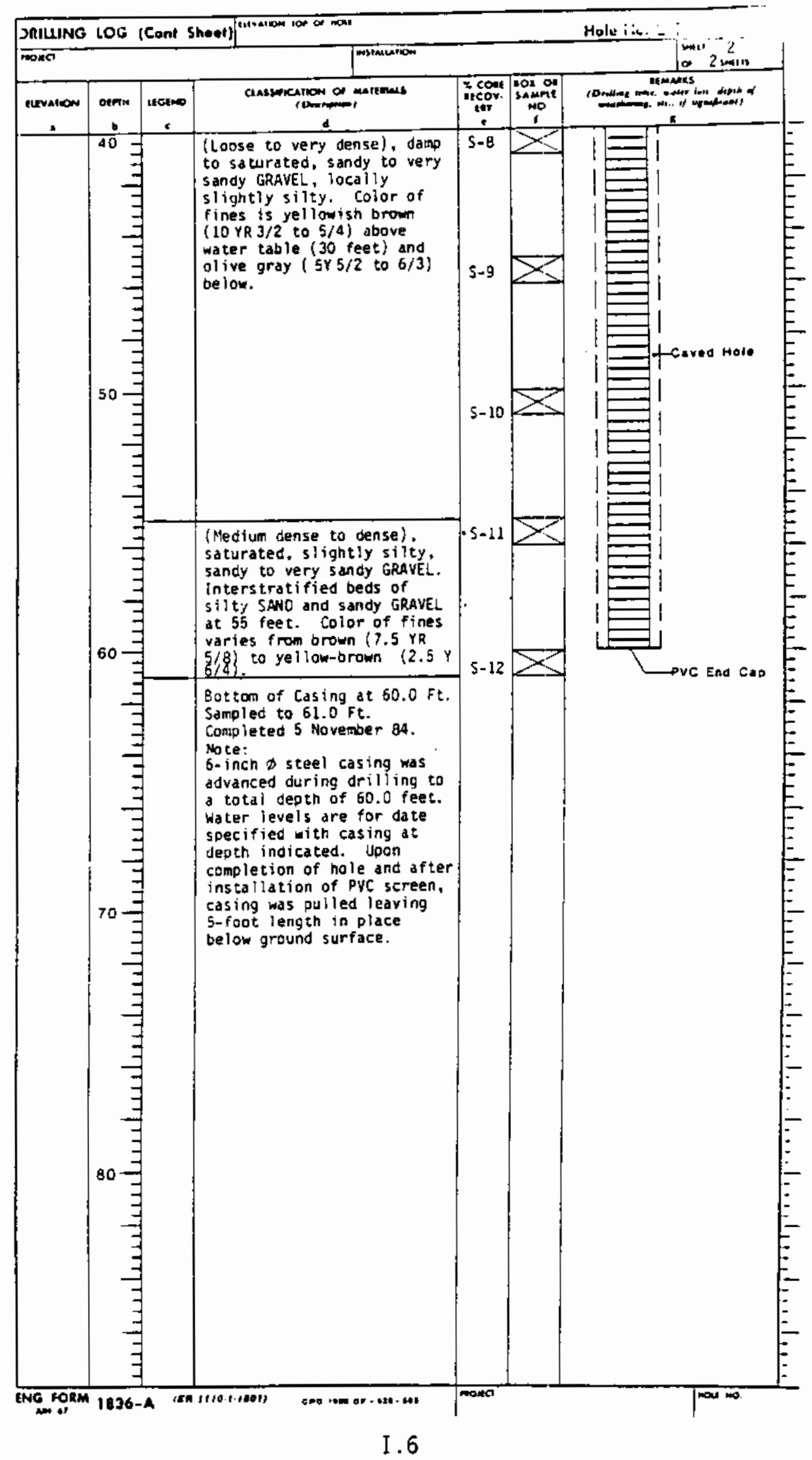


WELL 84-CD-LF-2 


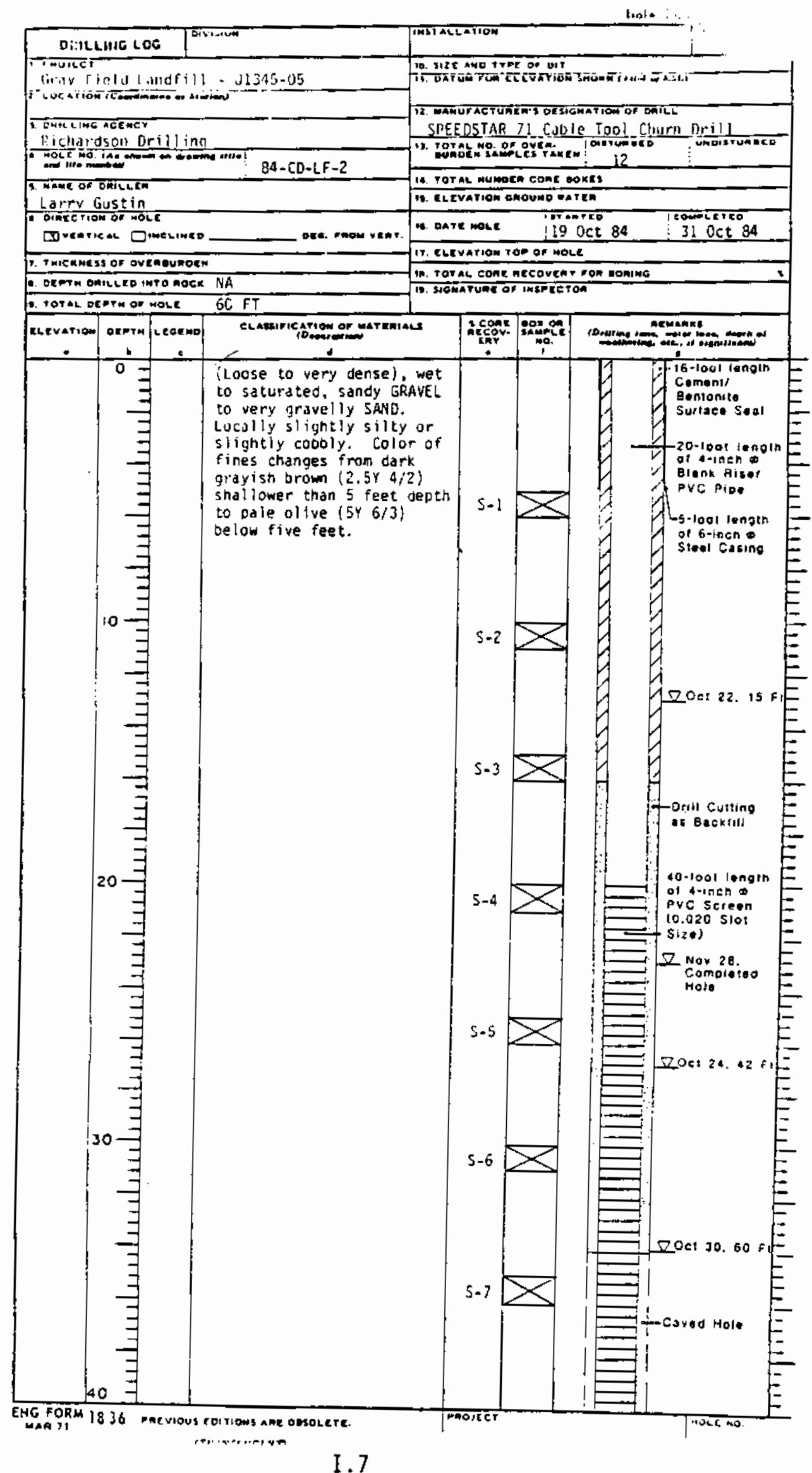




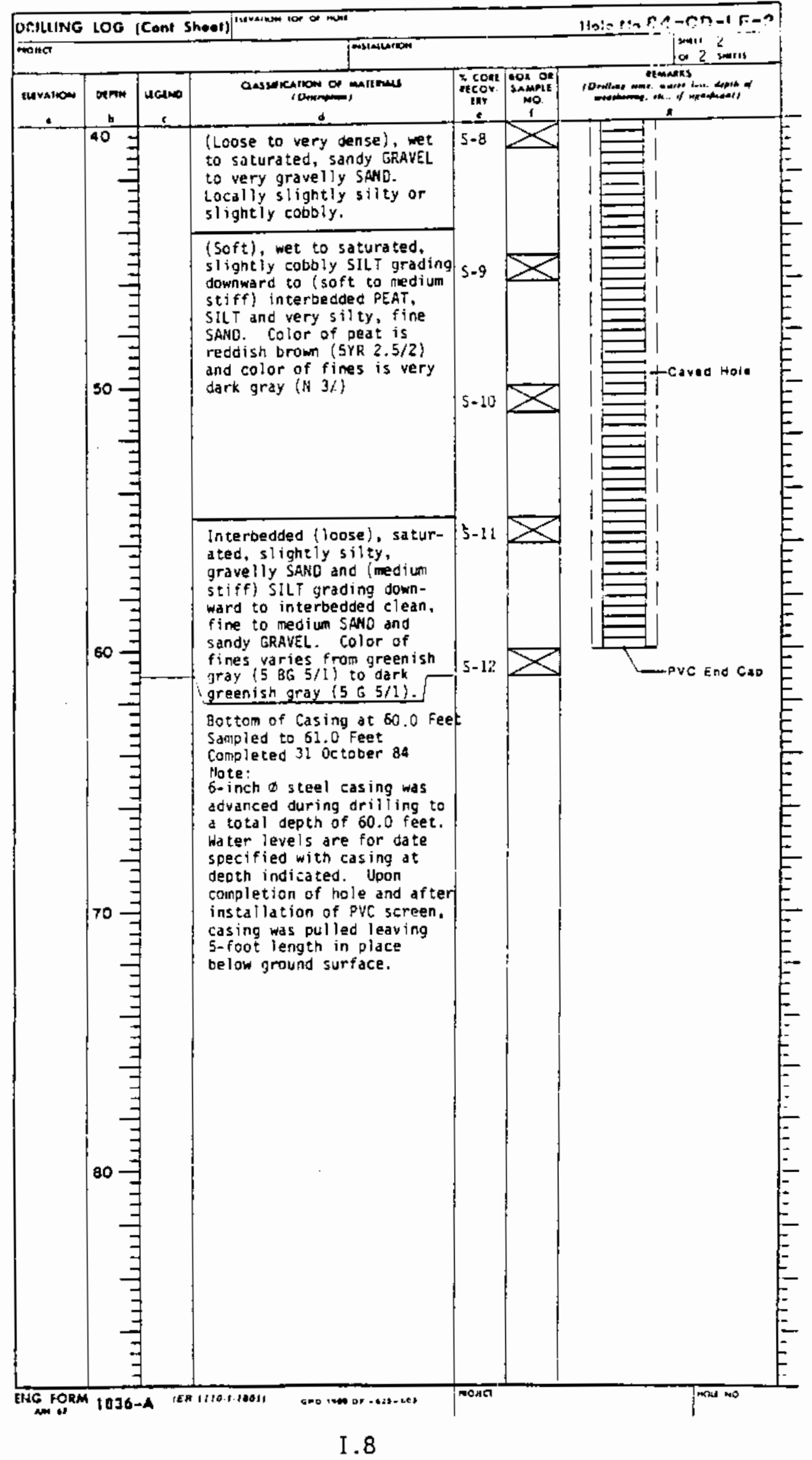




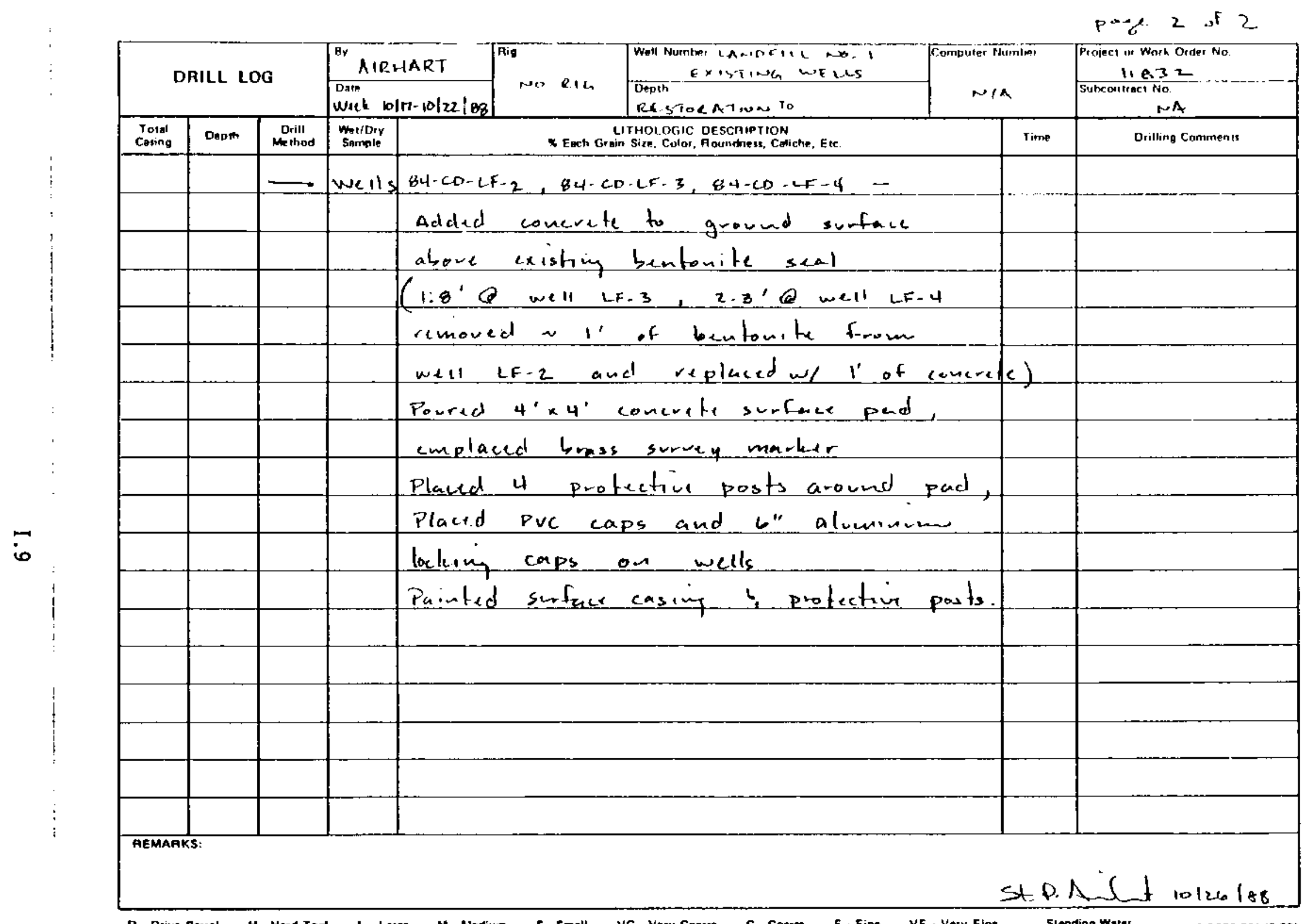




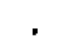


WELL $84-C D-L F-3$ 


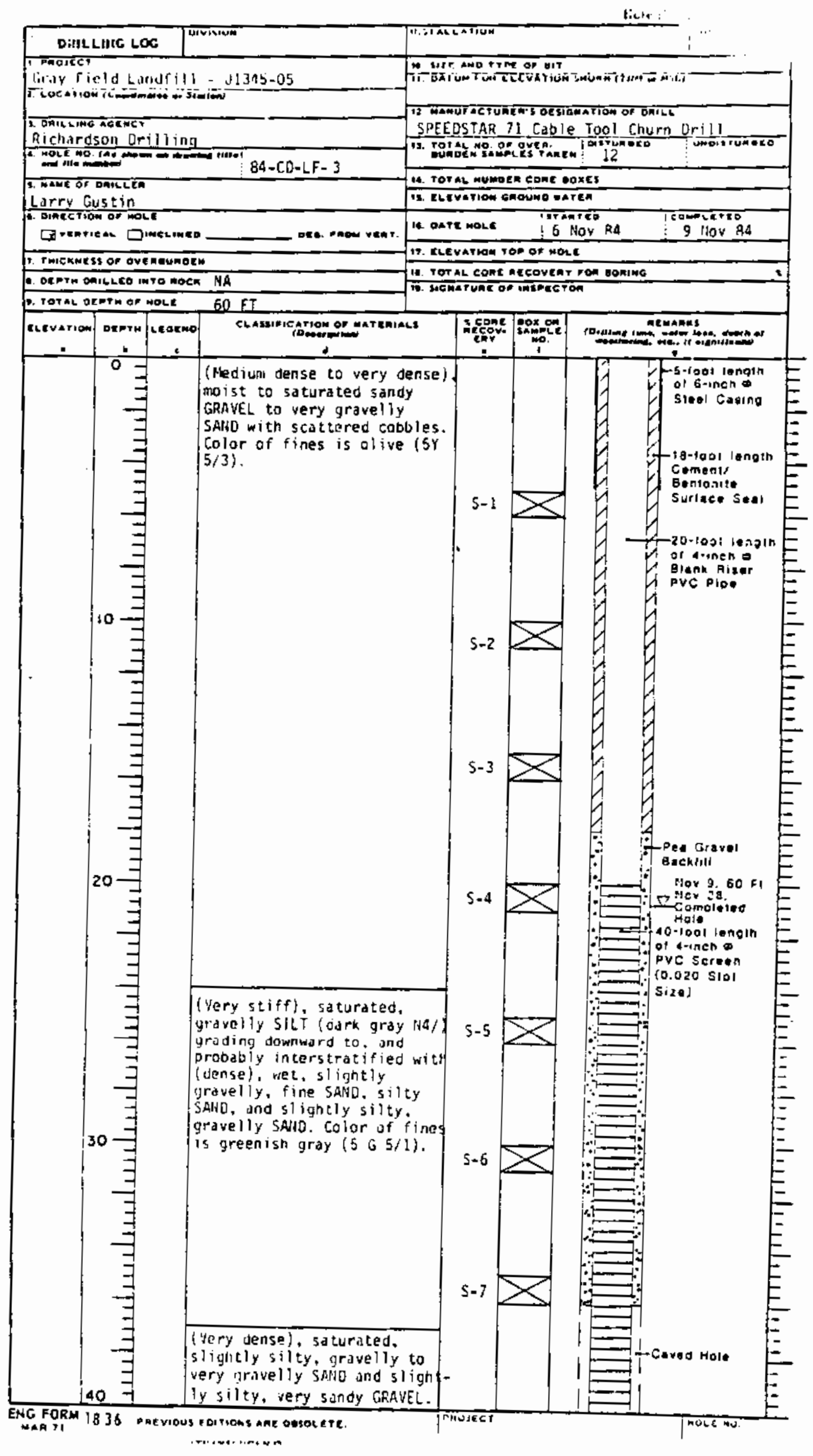




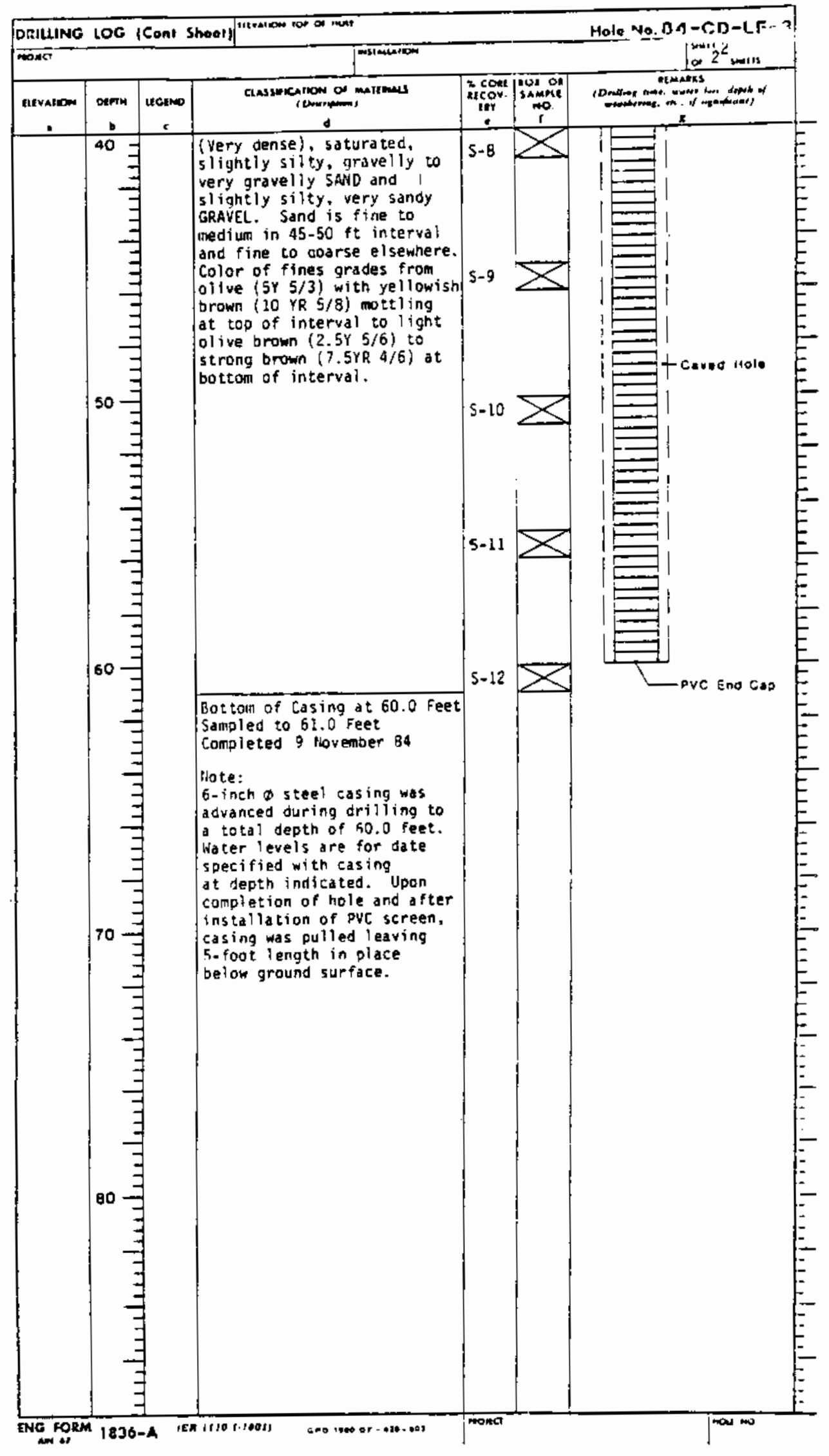

I. 12 
WELL 84-CD-LF-3 


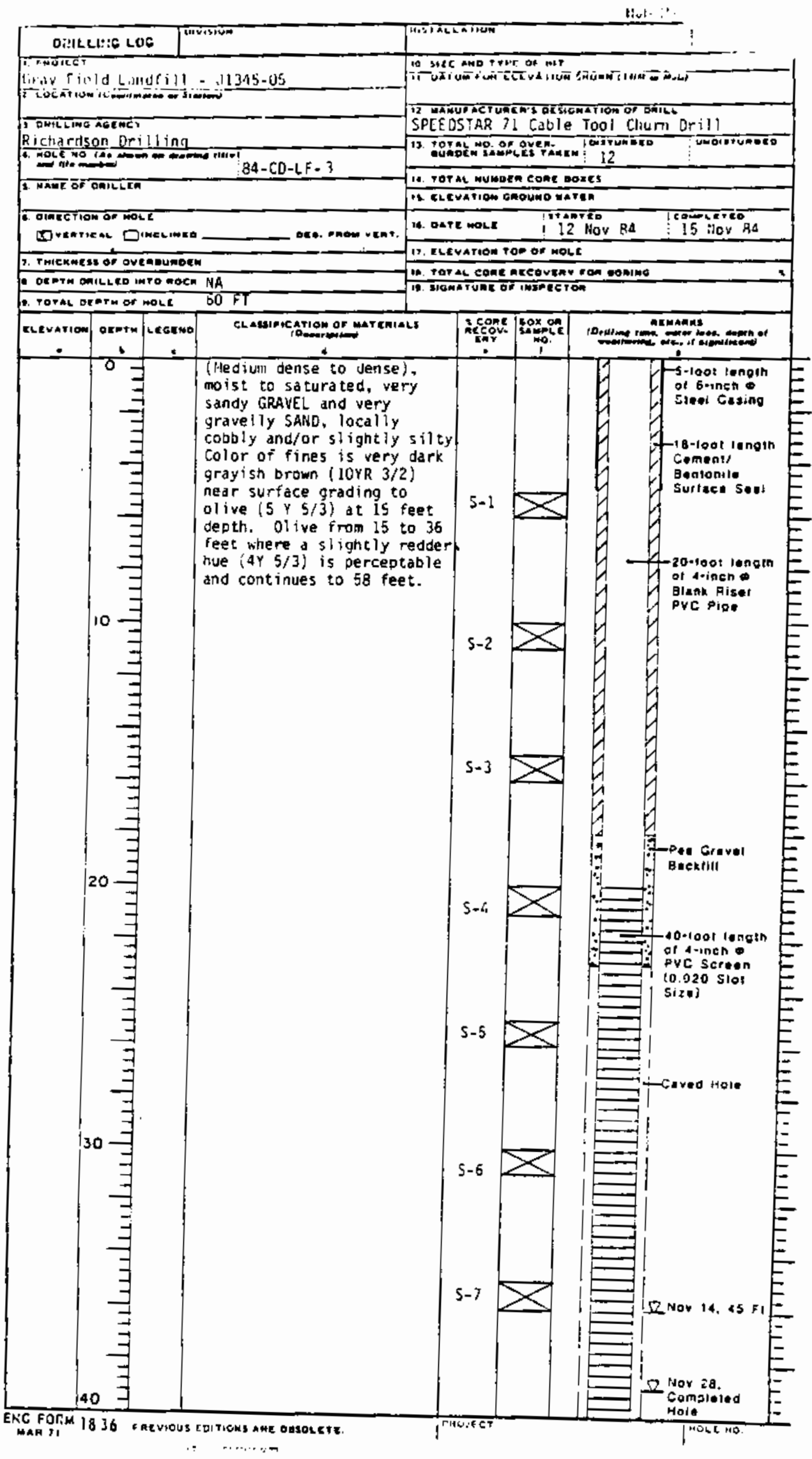

I. 13 


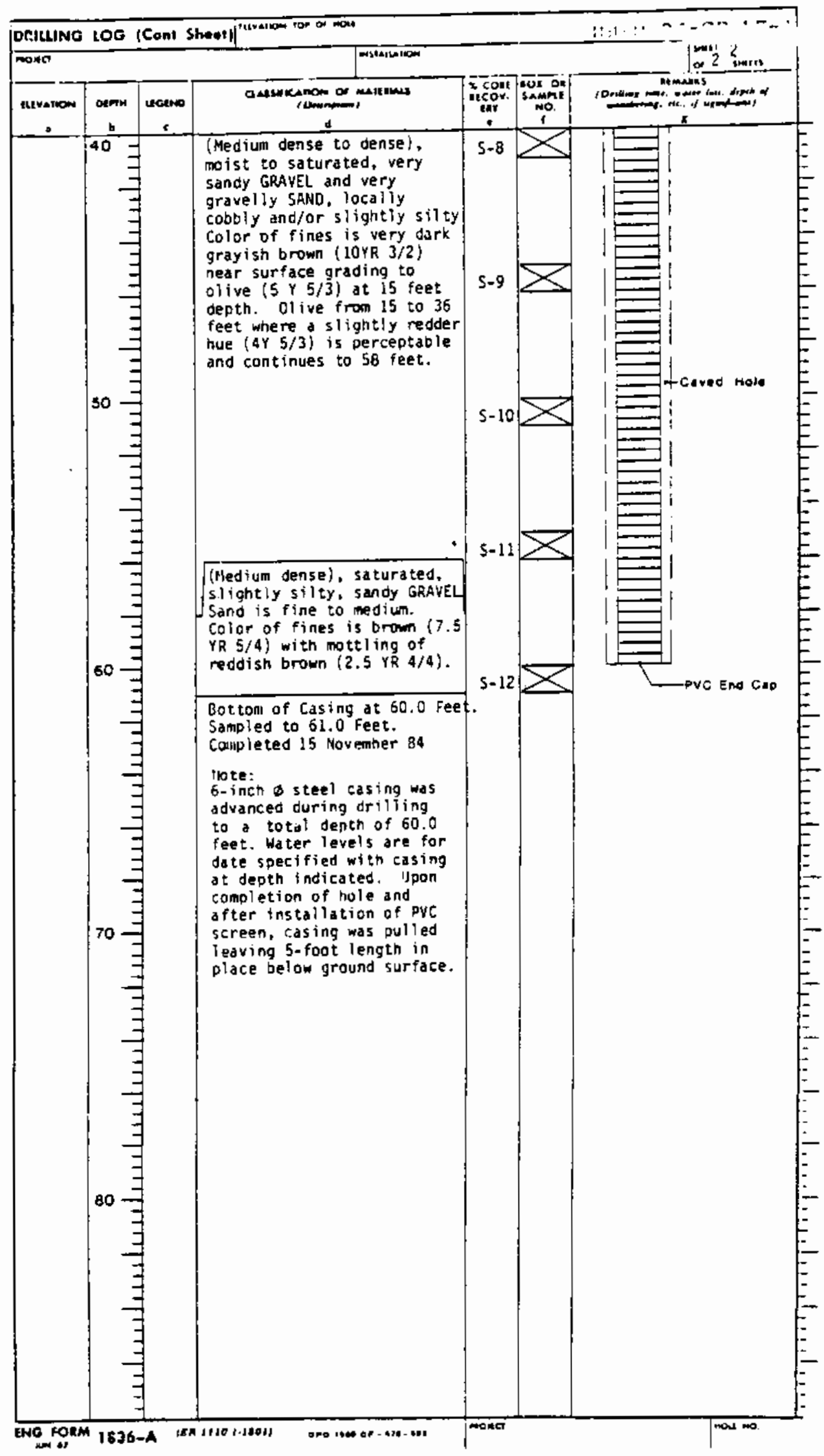

I. 14 
WELL FORT LEWIS \# 1 
WEIL LOG

\section{BY}

Tacoma Pump and Brilling Co., Inc.

30316 MOUNTAIN HIGHWAY

GRAHAM, WASHING TON 98338

PMONE 847 - 6087

Drillod for For

Addres SOUTH SIDE LANDILL//LAKE SEI/4, SW'/4, 19, T19N,RZE date stakted AFRIL 28,8 date completed APRIL Z8, 81 death $38^{\prime}$ around Level

stze of casing "I

RIG - cableg ROTARY

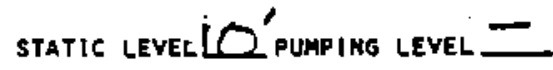
GPÄ 150 DRANDONA $\therefore$ screek 4 " DEC PEPE.

\section{PERFORATIONS}

\begin{tabular}{l|l}
\hline cA51 \\
\hline \\
\\
\\
\hline \\
\hline \\
\\
\hline
\end{tabular}

\section{USEDT TERTER}
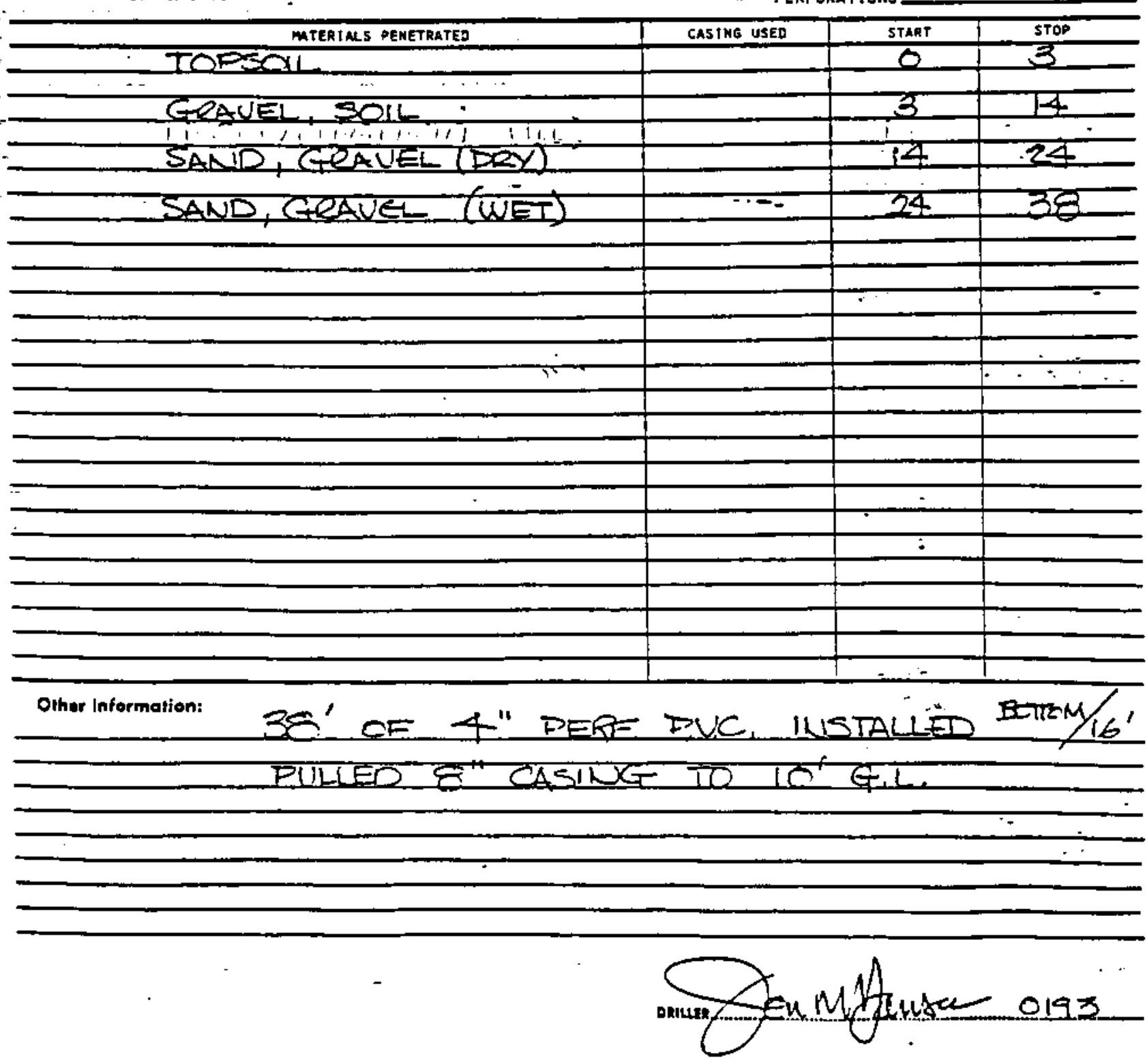


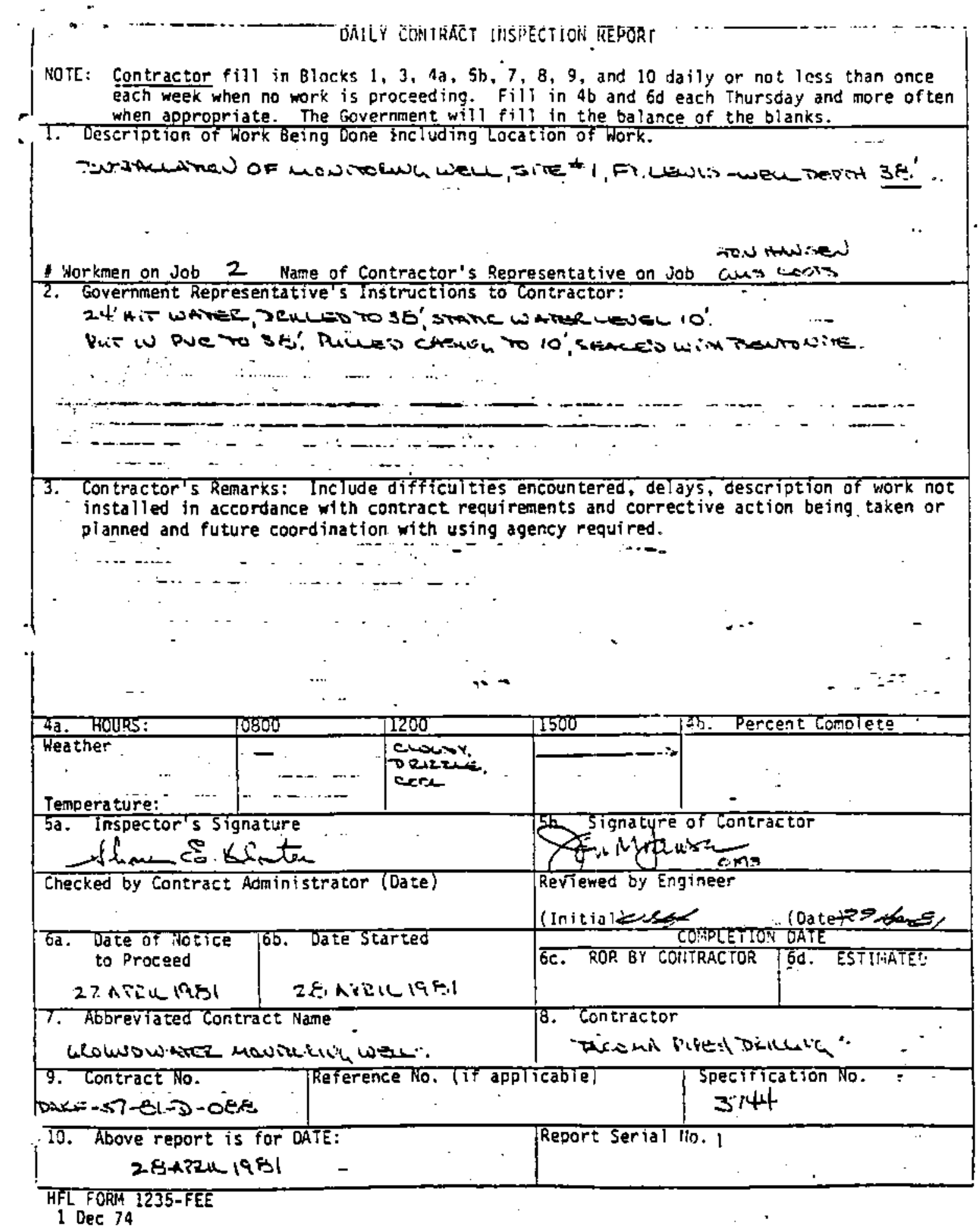




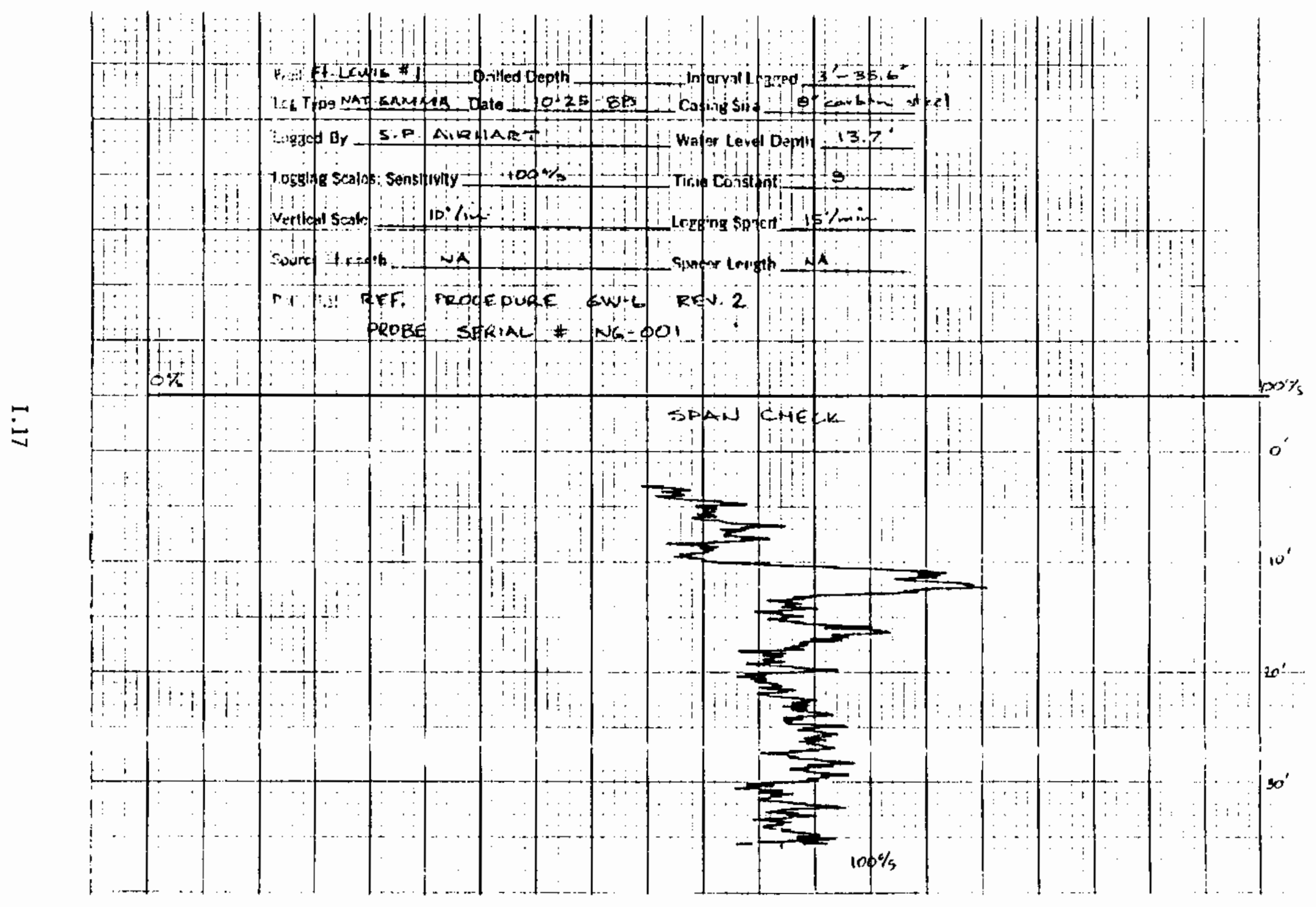


$p a r 1.62$

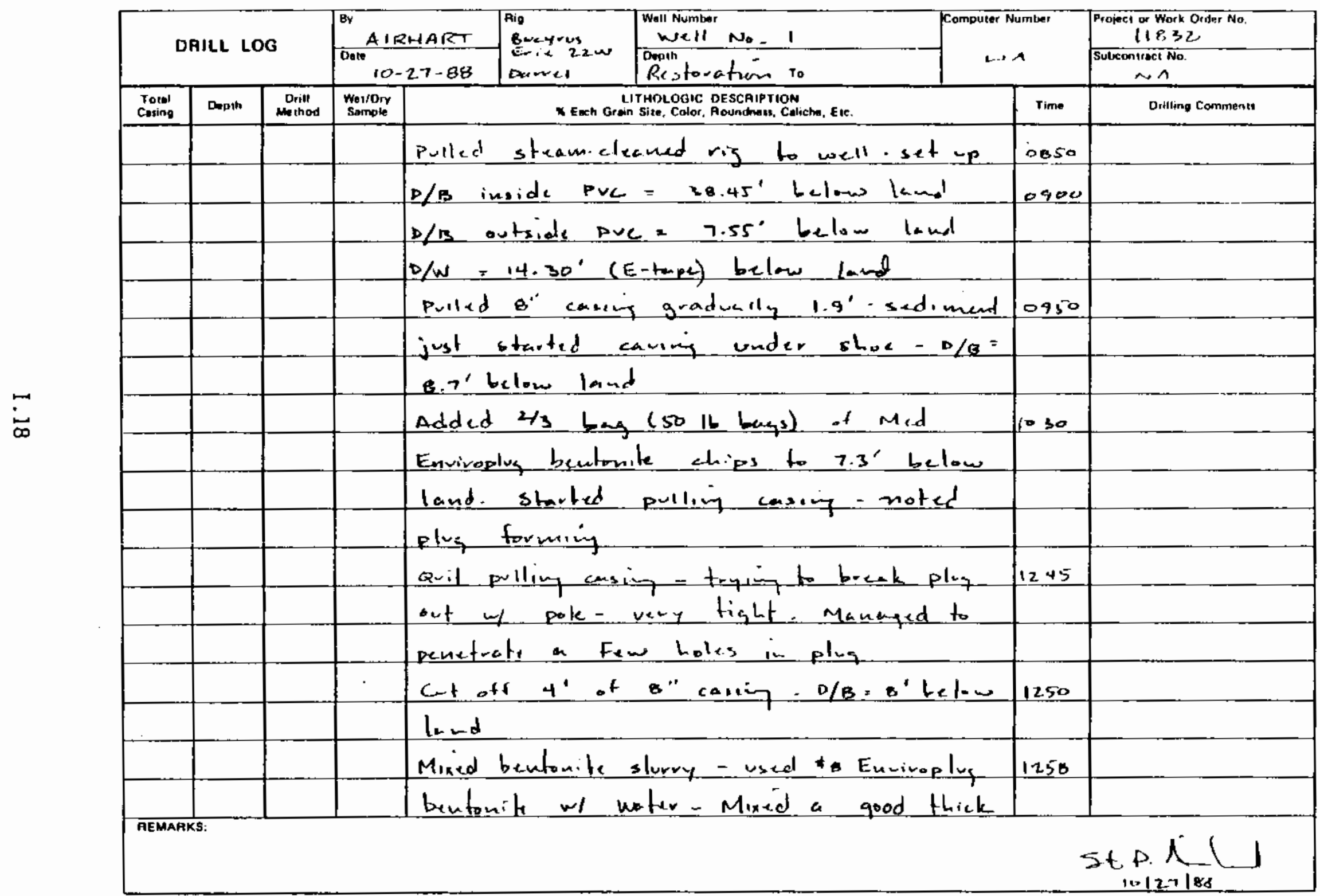

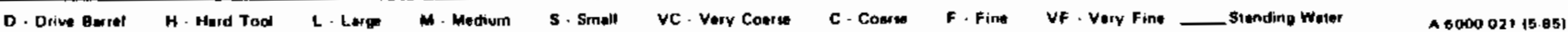


pang 2 of 2

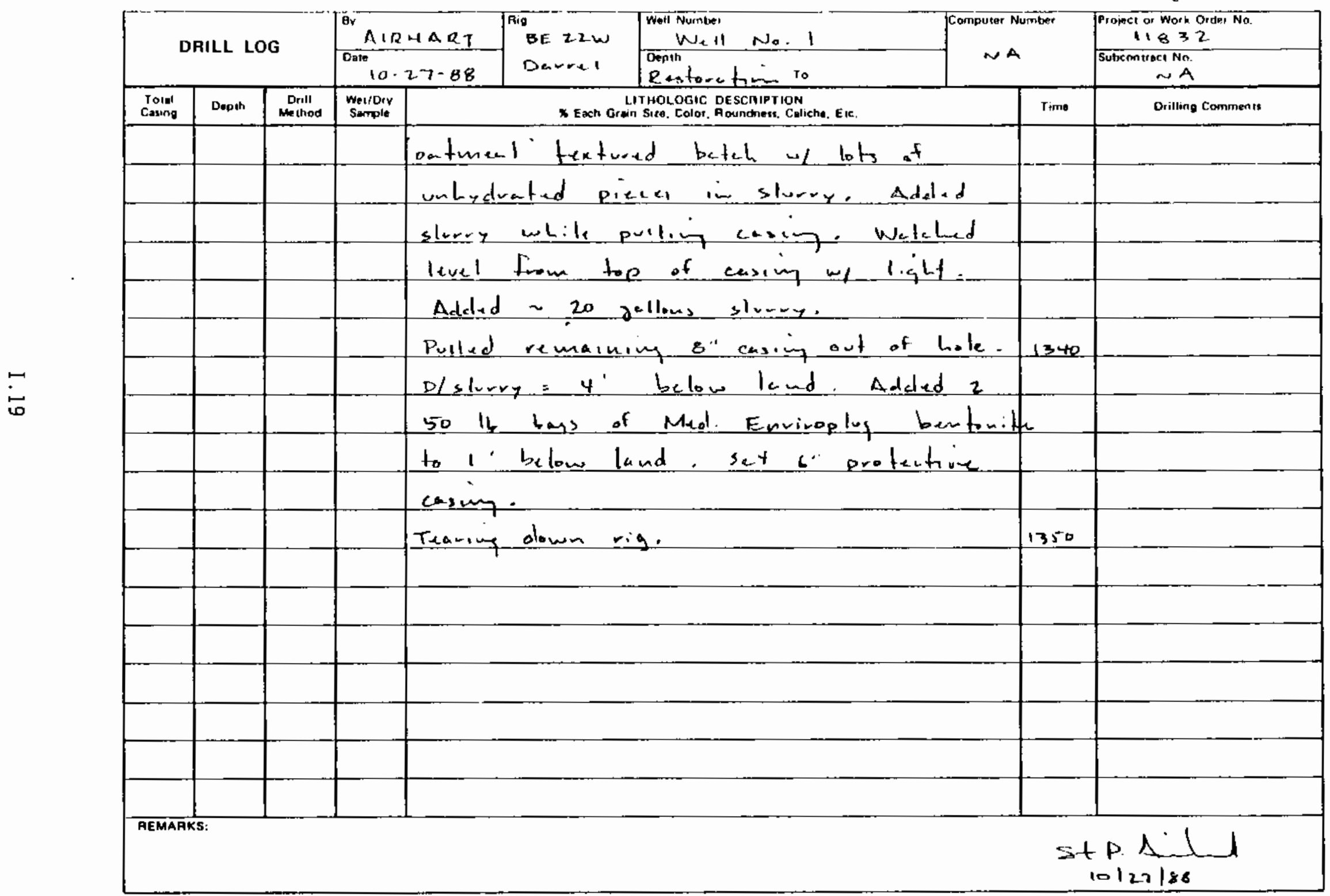

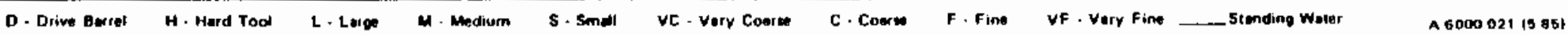




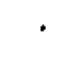

. 
WELL FORT LEWIS \#2 
WELI LOG

\section{BY}

Tacoma Pump and Drilling Co., Inc.

30316 MOUNTAIN HIGHWAY

GRAHAM, WASHINGTON 98330

PHONE 847 - 6087

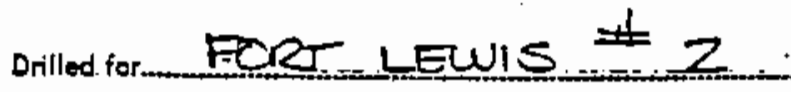

$\therefore$ Address date stakted ApRIL 29,81 DATE COMPLETED APRL Z9, 81 Depch 36 ' around bevel

size of casing

RIG - CABLED ROTARY STATIC LEVEL: 5 PUMPING LEVEL = GPM $10 \mathrm{O}+$ DRAWDONN screen A" PVC. FEDF. PERFORATIONS

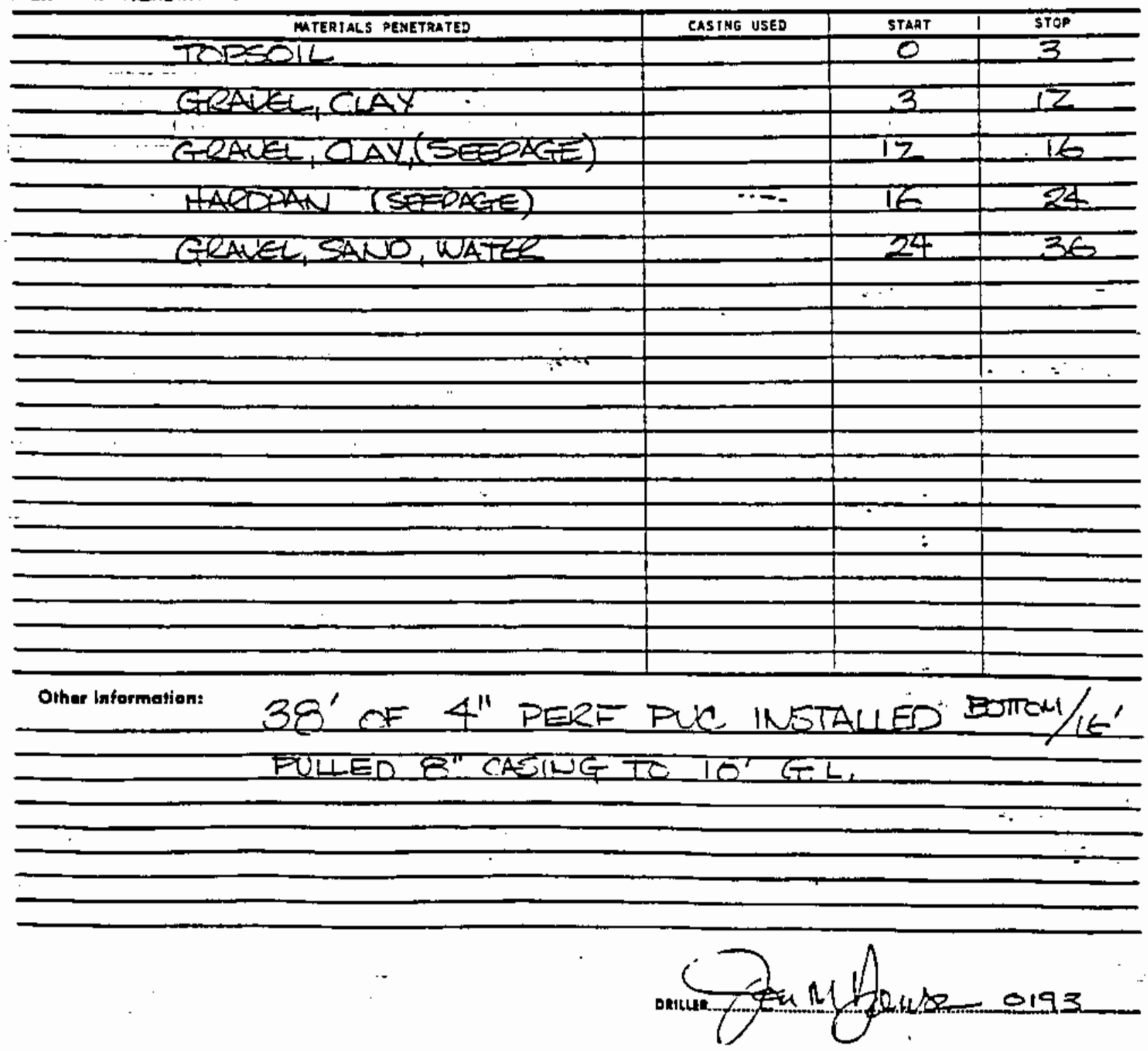


NOTE: Contractor fili in Blocks $1,3,4 a, 5 b, 7,3,9$, and 10 daily or not less than once each week when no work is proceeding. Fili in $4 b$ and $6 \mathrm{~d}$ each Thursday and more of ten when approoriate. The Government will fill in the balance of the blanks.

1. Description of Work being Done Including Location of Work.

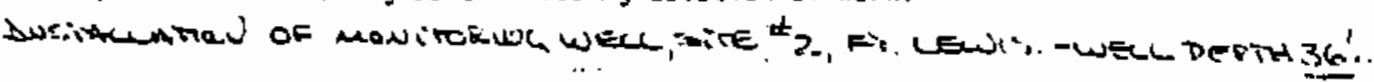

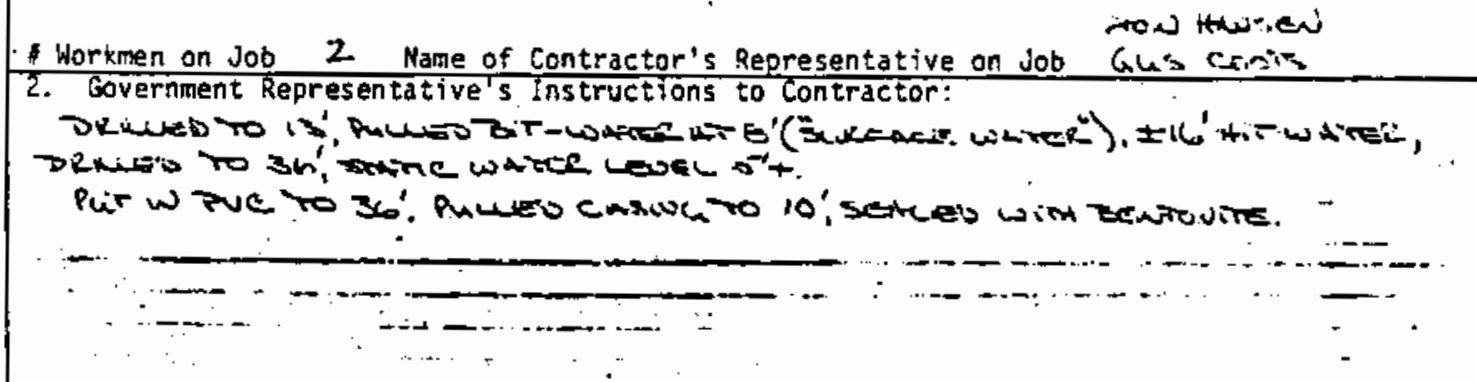

3. Contractor's Remariks: Include difficulties encountered, delays, description of work not instailed in accordance with contract requirements and corrective action being taken or planned and future coordination with using agency required.

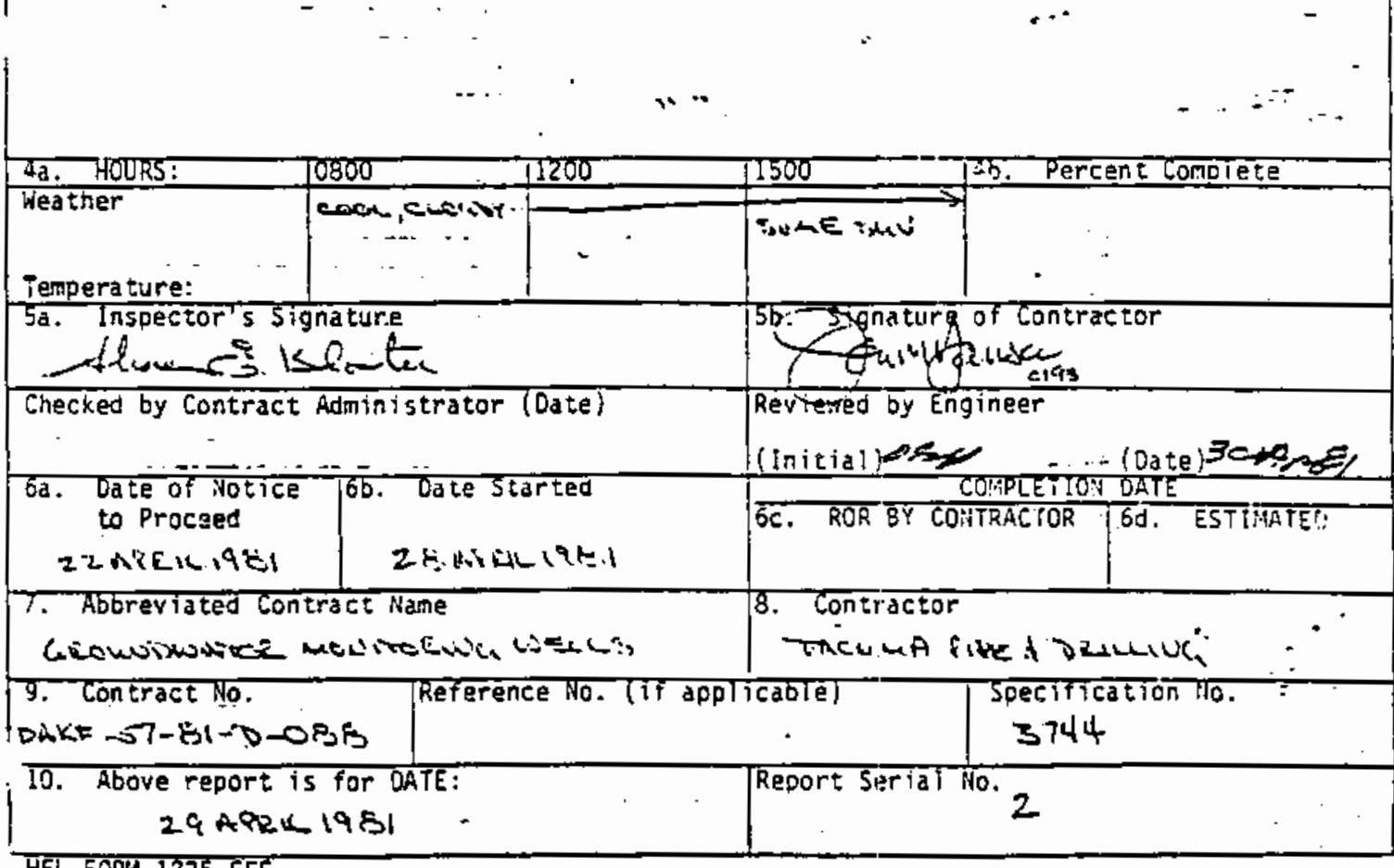

HFL FORP 1235+FEE

1 Dec 74 


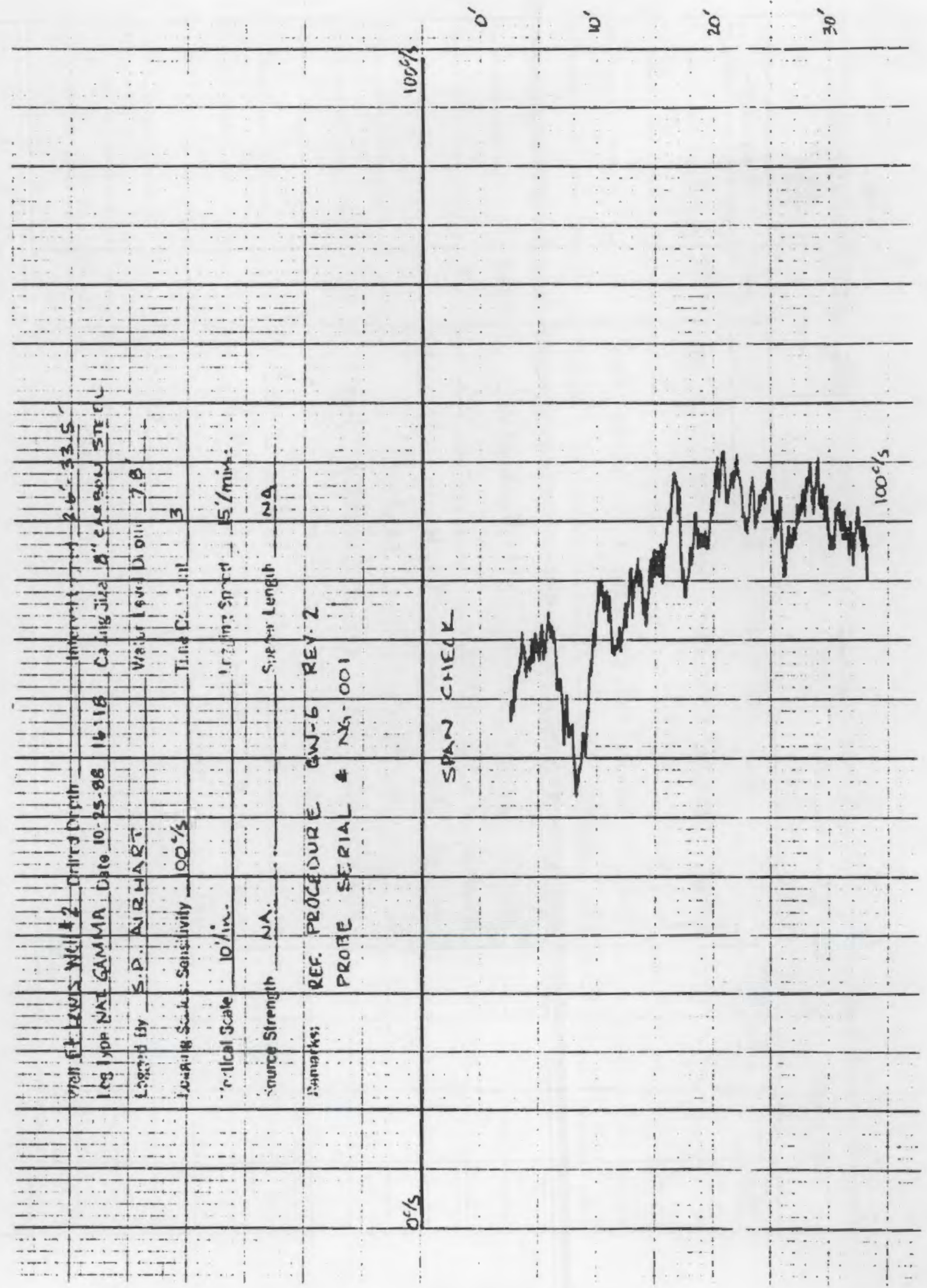




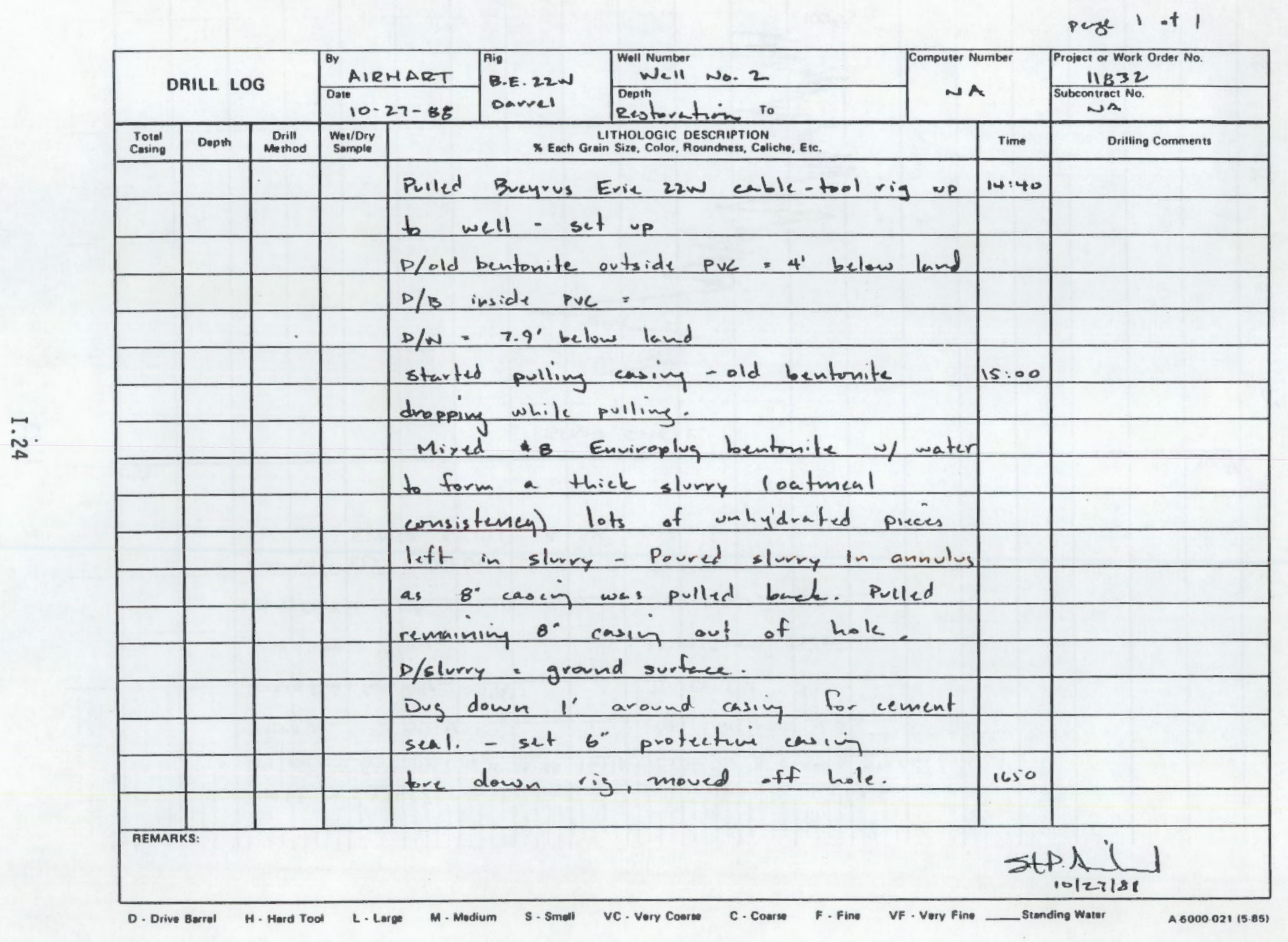


WELL FORT LEWIS \#3 
GELI LOG

BY

Tacoma Pump and Drilling Co., lac.

30316 MOUNTAIN HIGHWAY

GRAHAM, WASHINGTON 98338

PhoNe 047 - 6087

brilled for... FORT LEWIS 3

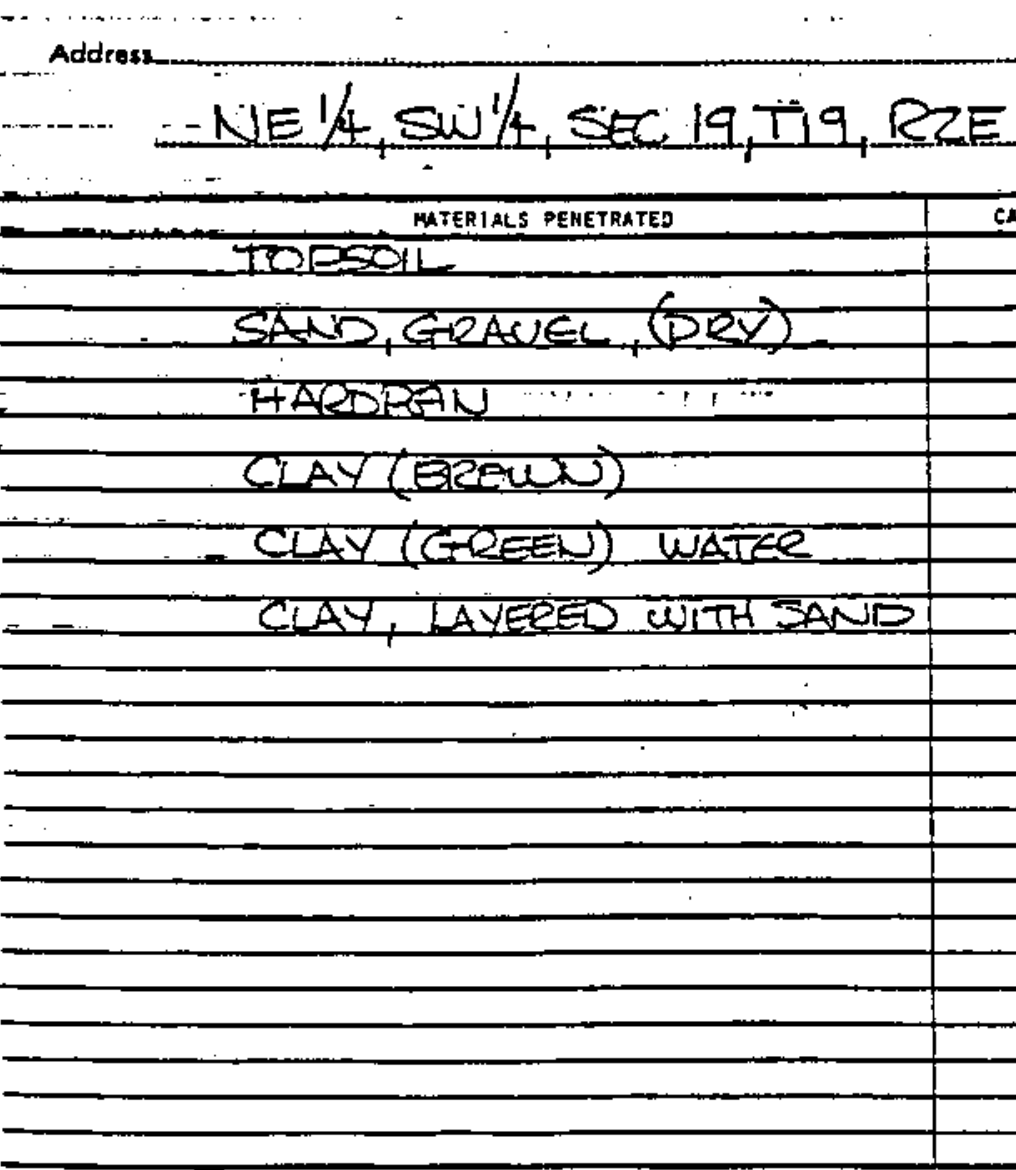

date started ARIL 30,8 date completed $A P Q L \quad 30,8$. Deoth $E Q^{\prime}$ Ground Level size of casing

RIG - CABLEL ROtary STATIC LEVELEL PUAP!HG LEVEL GPM $20+$ DRAMDOW SCREEM \&" PERF. PV

PERFORATIONS

Other Information:

S8' OF 4" PERF PUC IUSTALCED:BOTRM/16'

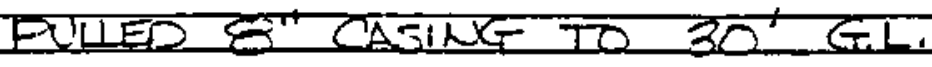

WATER GMIRG TPOOFH SAND LENSE ID

THE CLAY

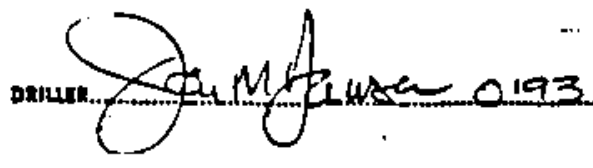


NOTE: Contractor fill in Blocks $1,3,40,56,7,8,9$, and 10 daily or not less than once edch week when no work is proceeding. Fili in $4 b$ and $6 d$ each Thurstay and more of ten when appropriate. The Government will fill in the balance of the blanks.

I. Description of Work Being Done Including Location of Work.

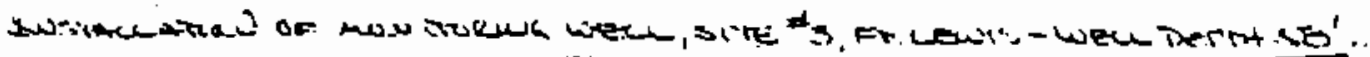

Workmen on Job $z$ Name of Contractor's Representative on Job row ithere.

2. Government Representative's Instructions to Contractor:

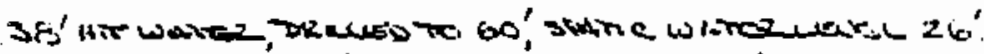

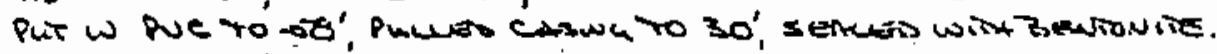

-

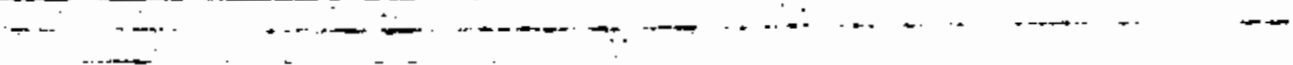

3. Contractor's Remarks: Include difficulties encountered, delays, description of work not installed in accordance with contract requirements and corrective action being taken or planned and future coordination with using agency required.

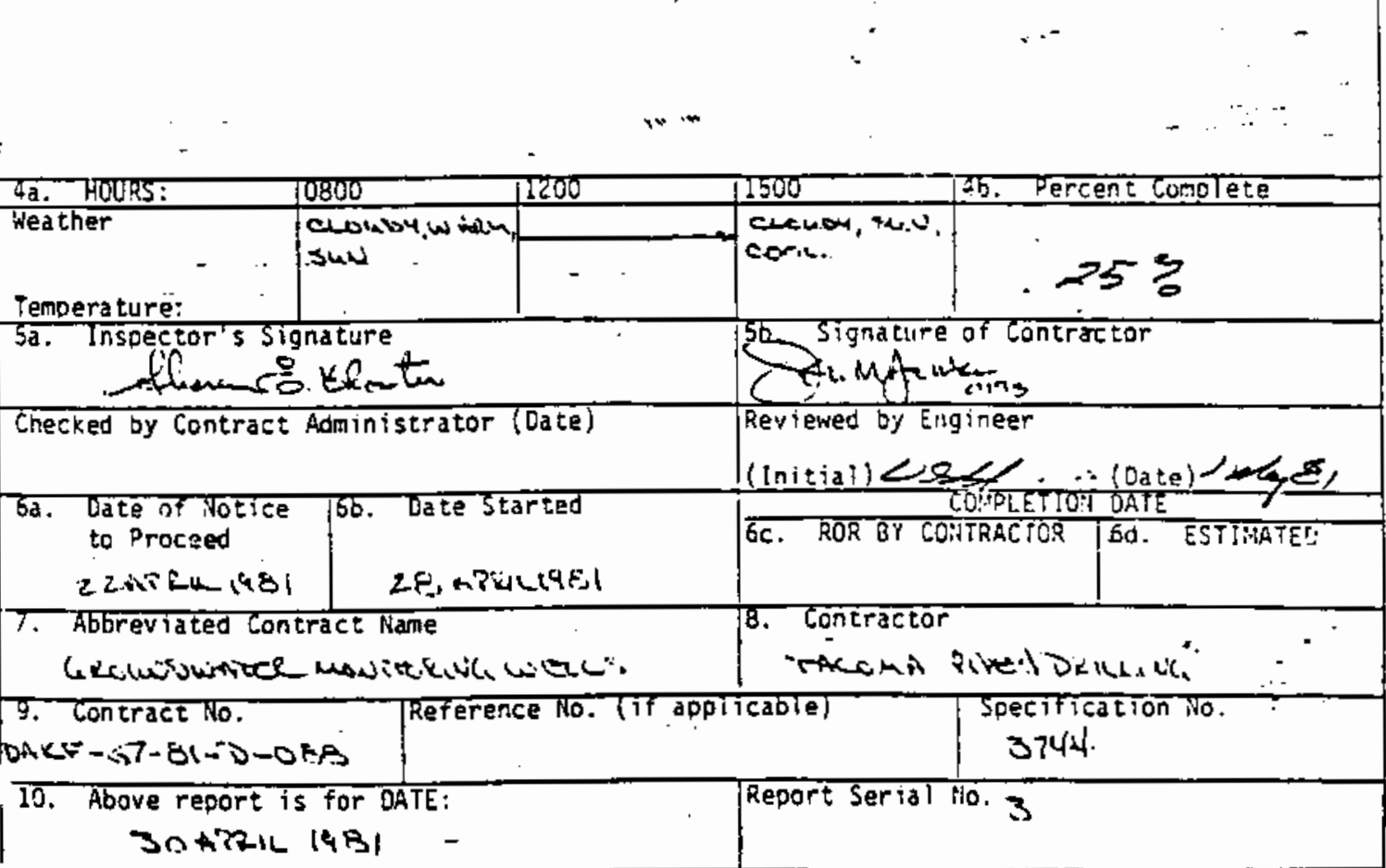

\footnotetext{
प्रFL FORM 1235-FEE
}

1 Dec 74 
rete 1 . t 3

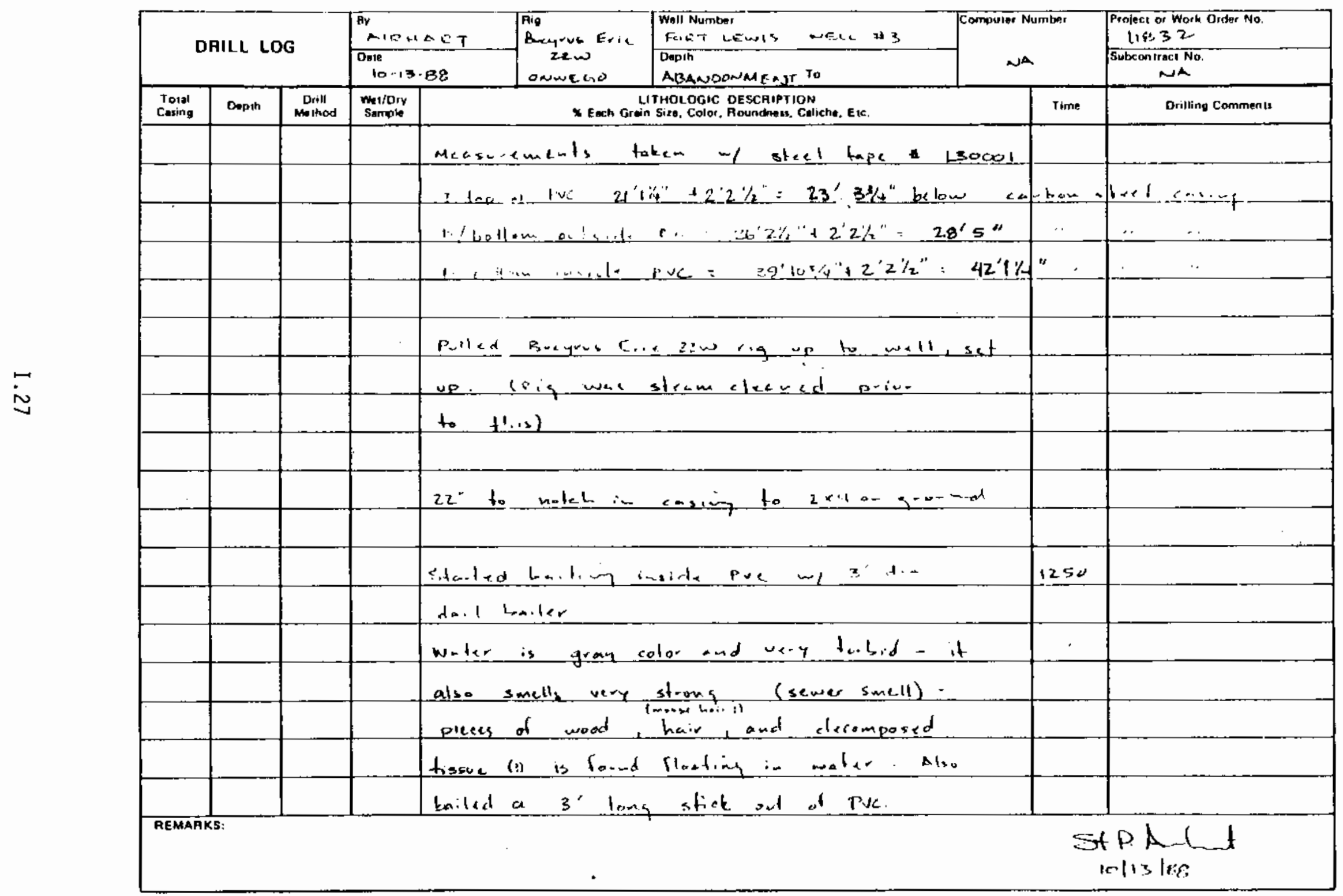

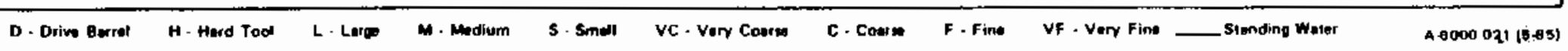




$$
r+13
$$

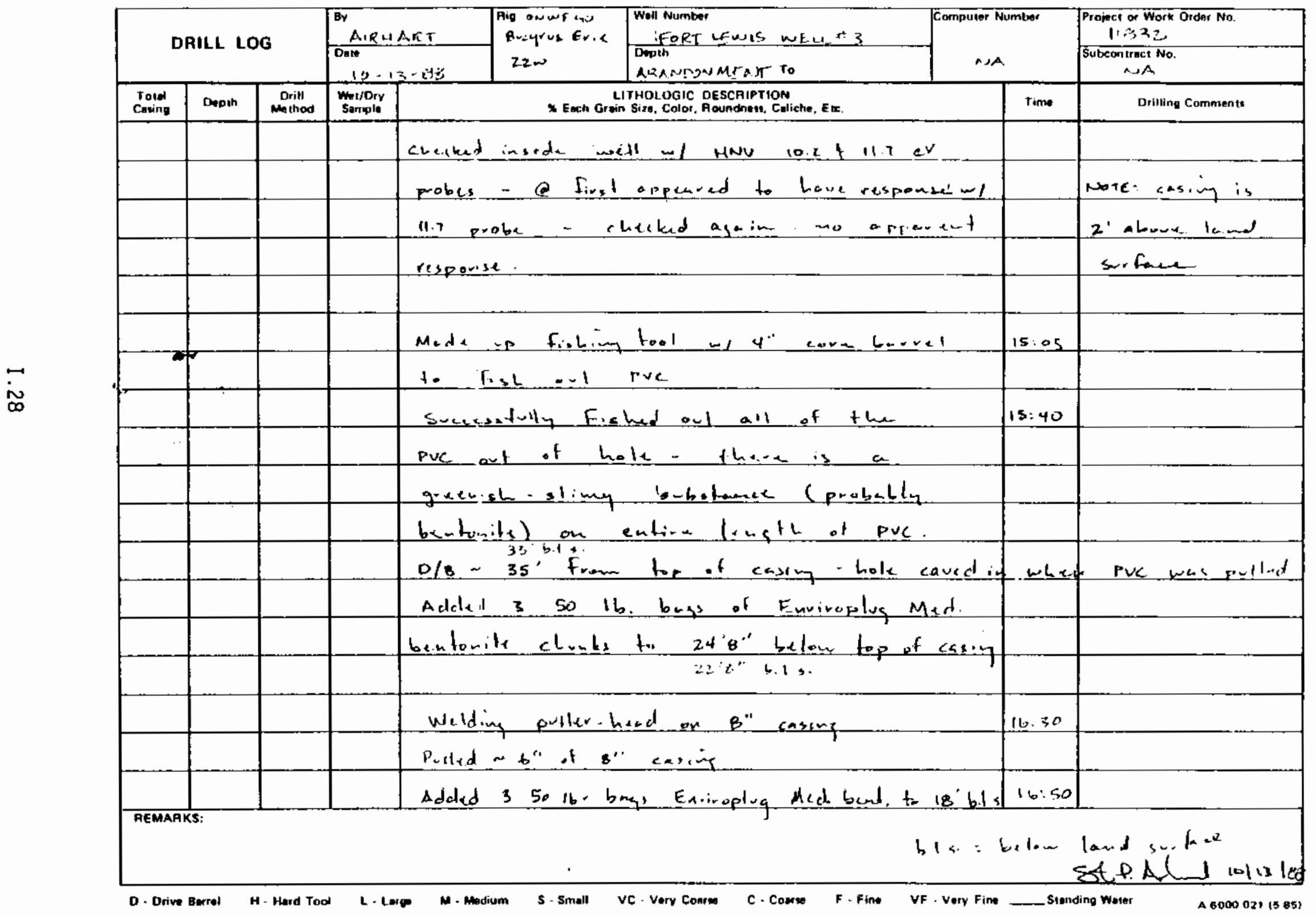




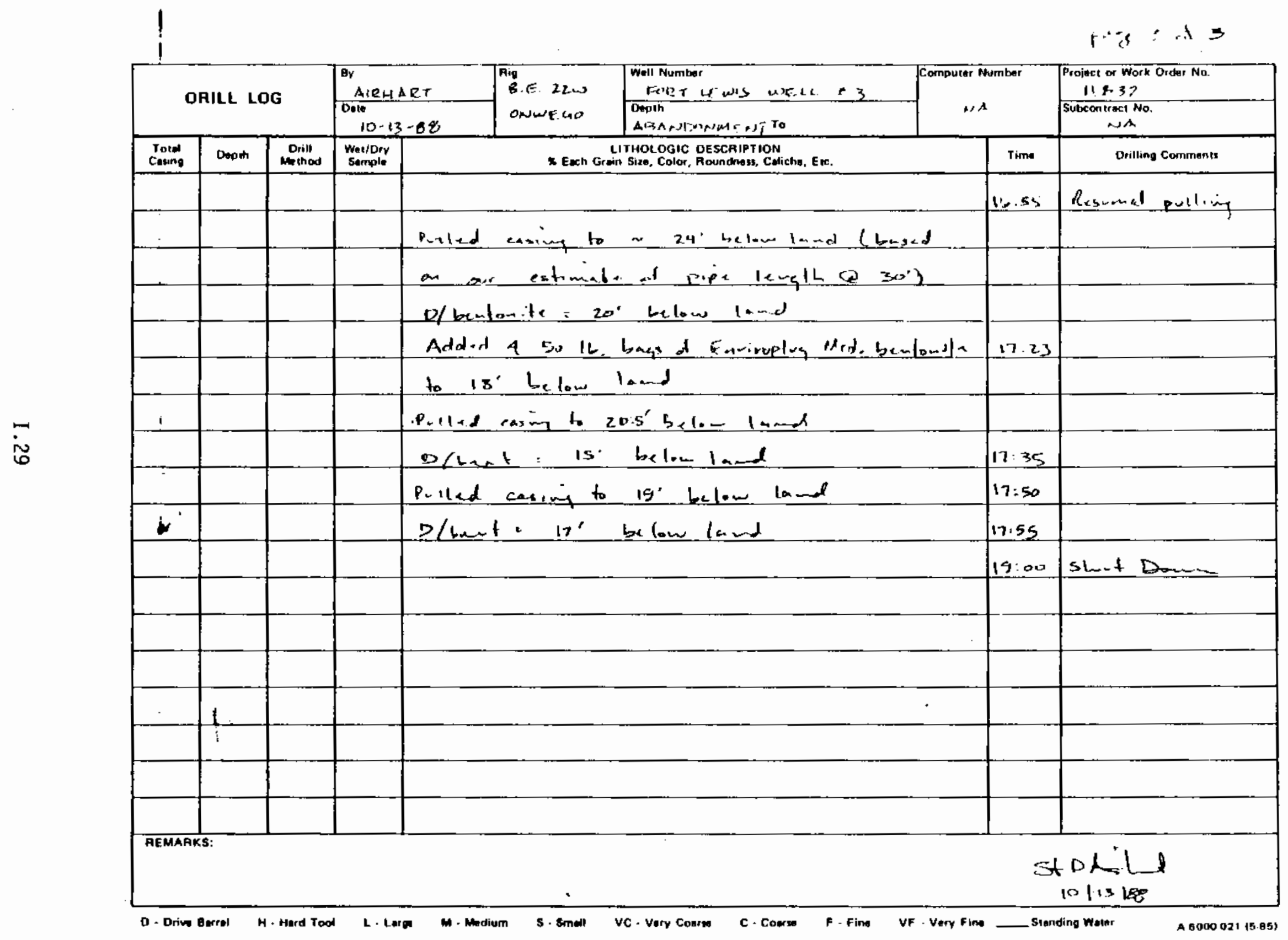




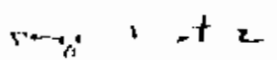

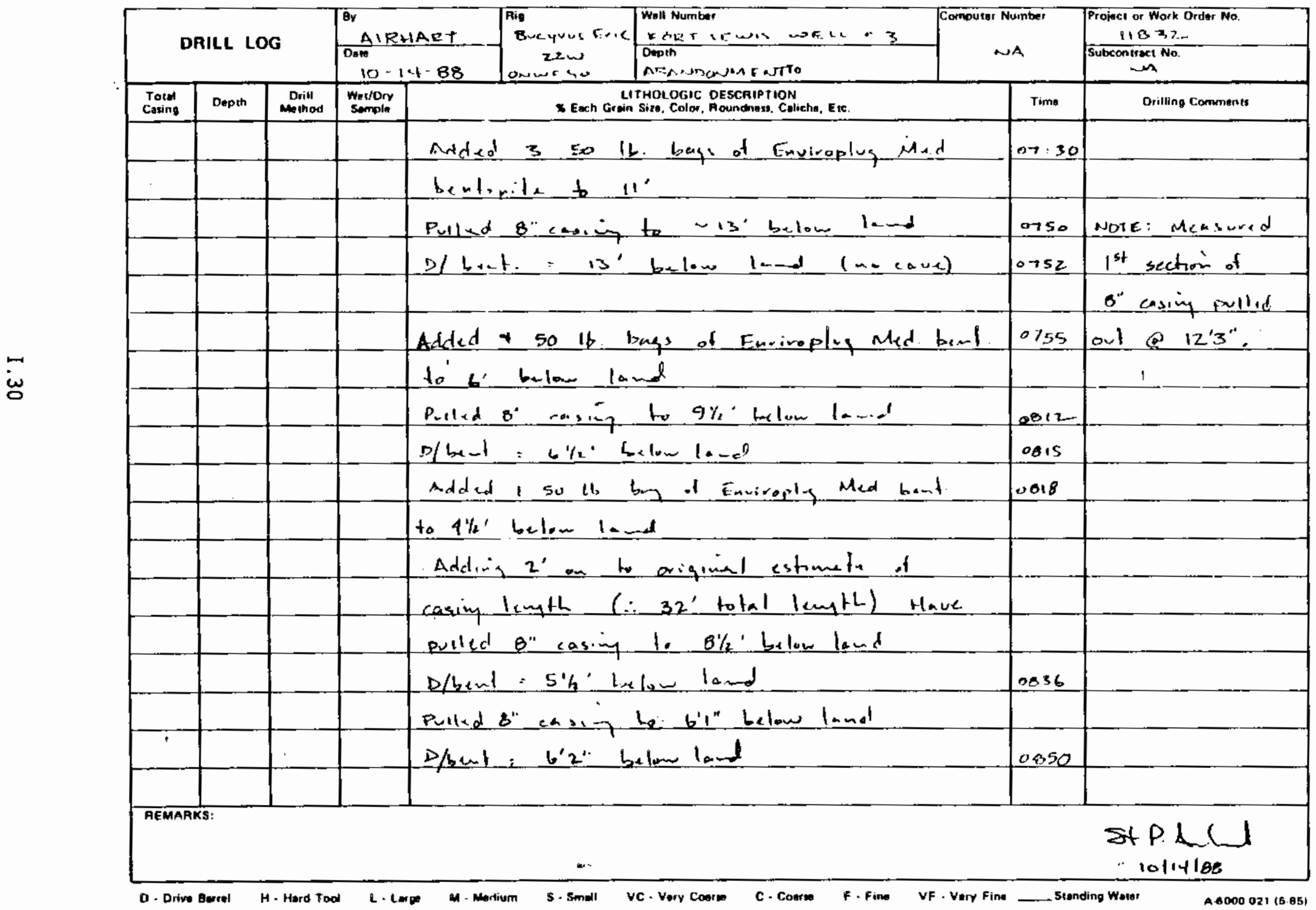


$\left.i^{\prime}\right)^{\prime} 12$

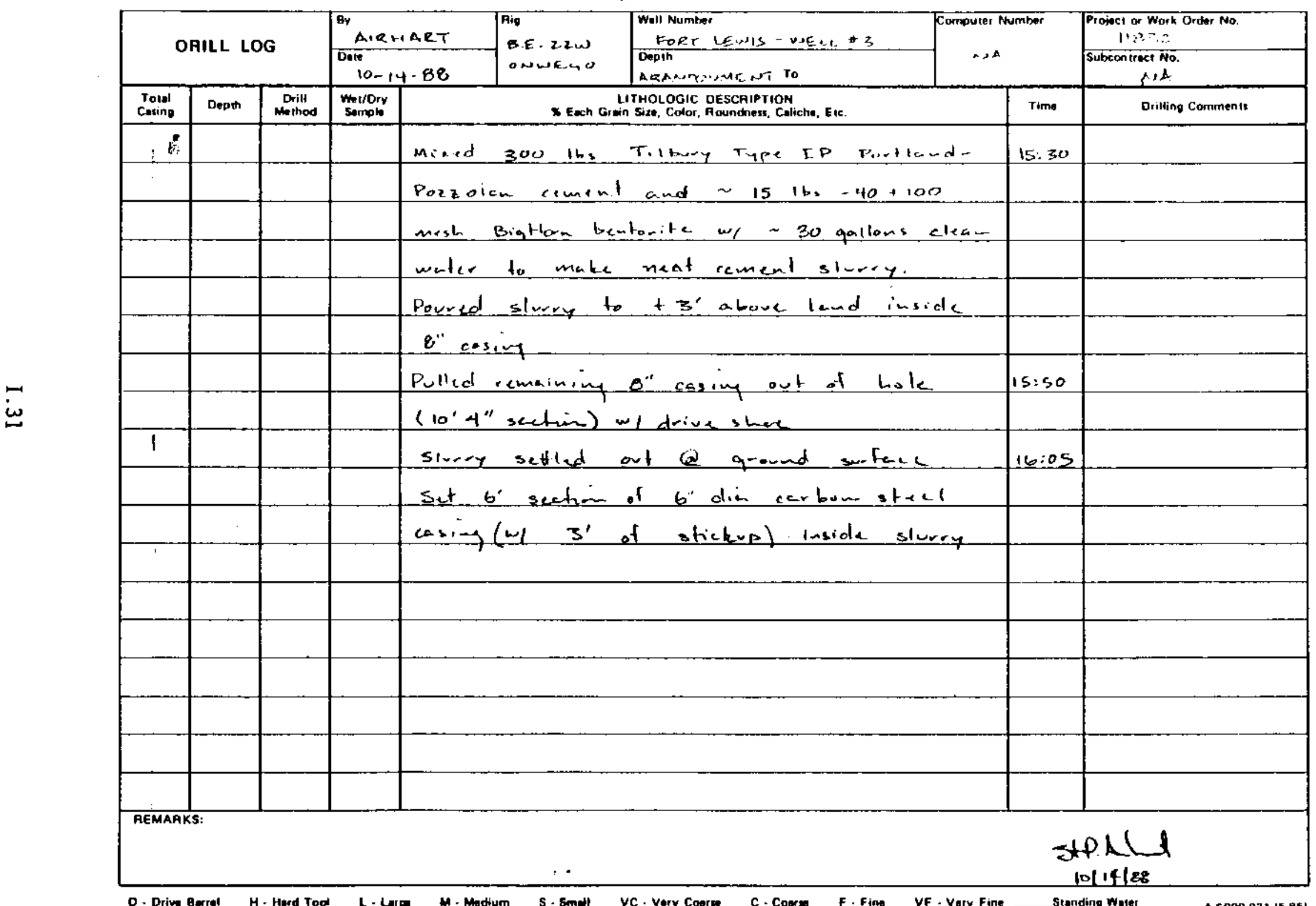


WELL FORT LEWIS \#4 
WEIL LOG

BY

Tacoma Pump and Drilling Co., Inc.

30316 MOUNTAIN HIGHWAY

GRAHAM, WASHINGTON 98338

Pmone 847-6087

Orillod for. FOOT LEUIS \#4

Address. date started AfRIL 30,81 date completed May I, Depth $98^{\prime}$ Ground Level

Size of cosing "

R1G - CABLED ROtary static level $2 Z_{\text {pumping level }}^{\prime}=$ GPM $\because \mathrm{O}+$ DRANDOHA SCREEN 4"! PERE FVC PERFORATIONS

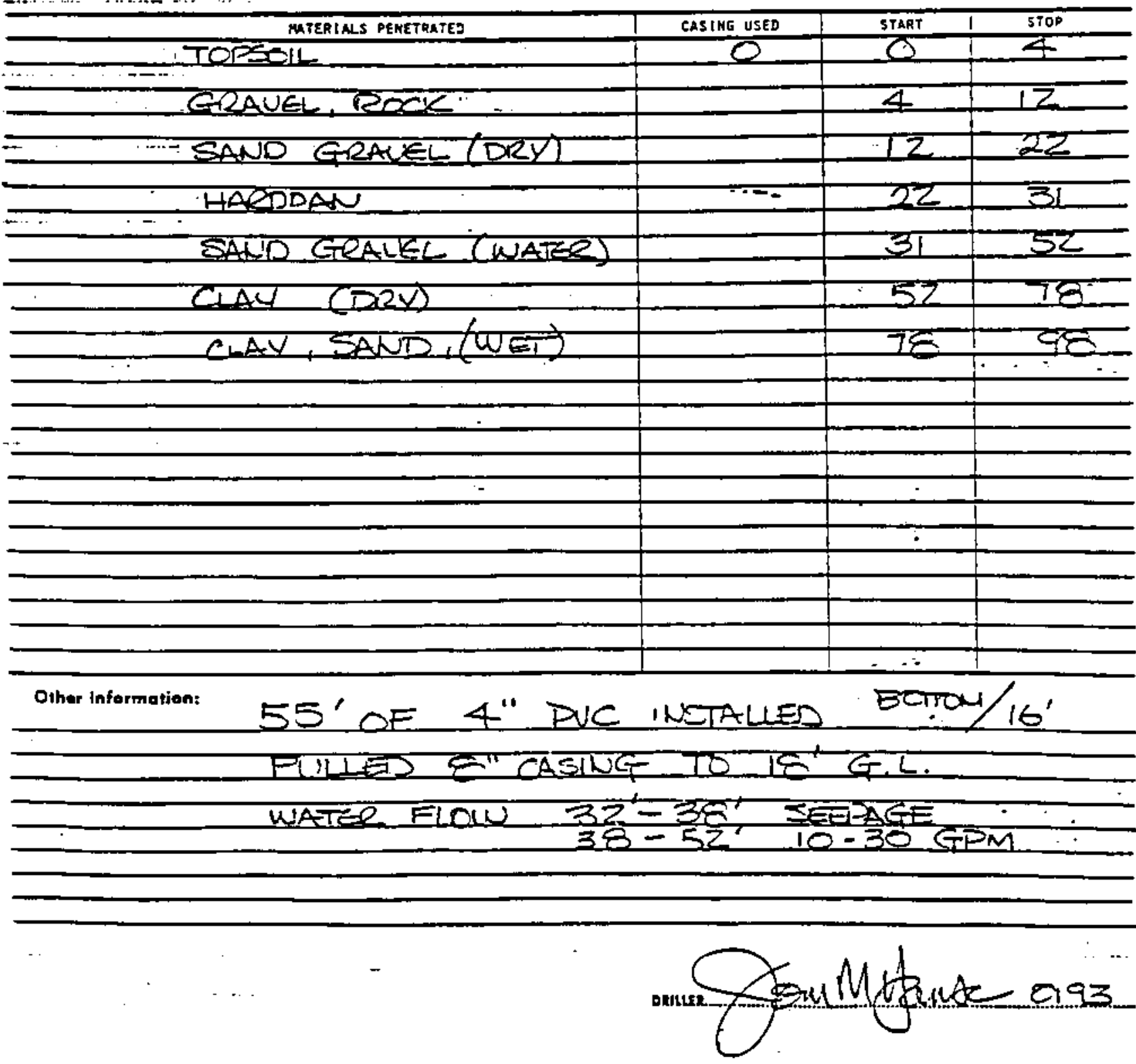


NOTE: Contractor fill in Blocks $1,3,4 \mathrm{~d}, 5 \mathrm{~b}, 7,3,9$, and 10 daily or not less than once each week when no work is proceeding. Fijl in $4 b$ and $5 d$ each Thursday and nore of ten when appropriate. The Government will fill in the balance of the blanks.

1. Description of Work Being Done Including Location of Work.

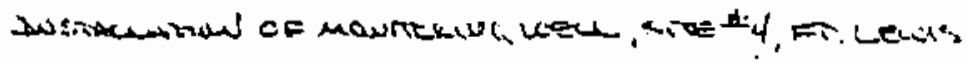

Workmen on Jab 2 . Name of Contractor's Representative on Job

TOW HWW:ES

2. Government Representative's Instructions to Contractor:

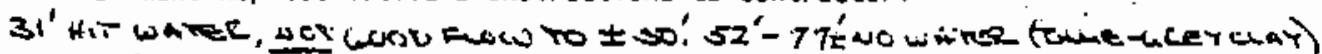

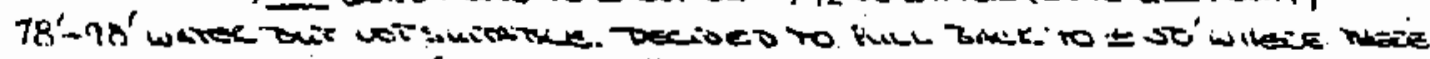

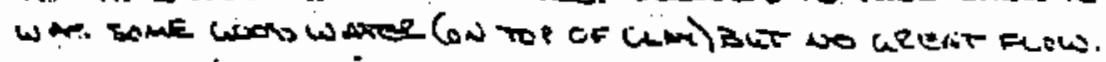

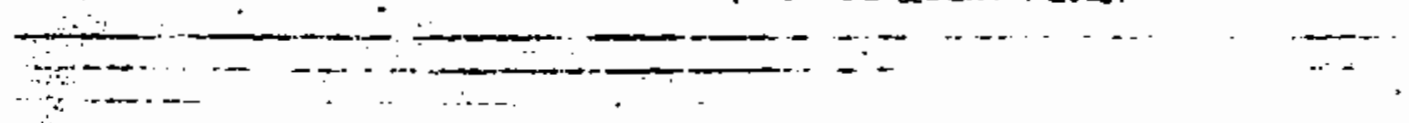

3. Contractor's Remarks: Inc?ude difficulties encountered, delays, description of work not installed in accordance with contract requirements and corrective action being. taken or planned and future coordination with using agency required.$$
\text { - - - - - }
$$

10800

Temperature: 1. .

5a. Inspector's Signature

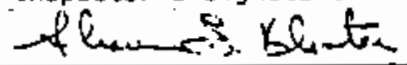

$-\quad 7$

DLiz

\begin{tabular}{l|l}
\hline Checked by Contract Administrator (Date) & Reritived by Engineer
\end{tabular}
ba. Uate of notice
to Proceed

6b. Oate Started

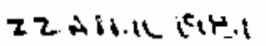

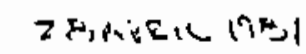

7. Abbreviated Contract Name

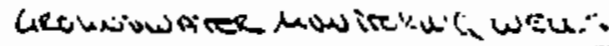

9. Contract ko. Reference No. (if applicable) (Initial) $<$ sese

57 signature of contractor

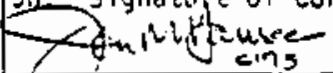

1 C. ROR BY COHTRAC SOR . $6 \mathrm{~d}$. (Date) $4 \pm 0 \times 5$

$D A K F-S T-E I-D-O E E$

10. Above report is for DATE:

8. Contractor iricomis rite A Delllute EST TPATE:

\section{ImaY 19\%?}

HFL FORM $1235-F E E$

$$
1 \text { Dec } 74
$$




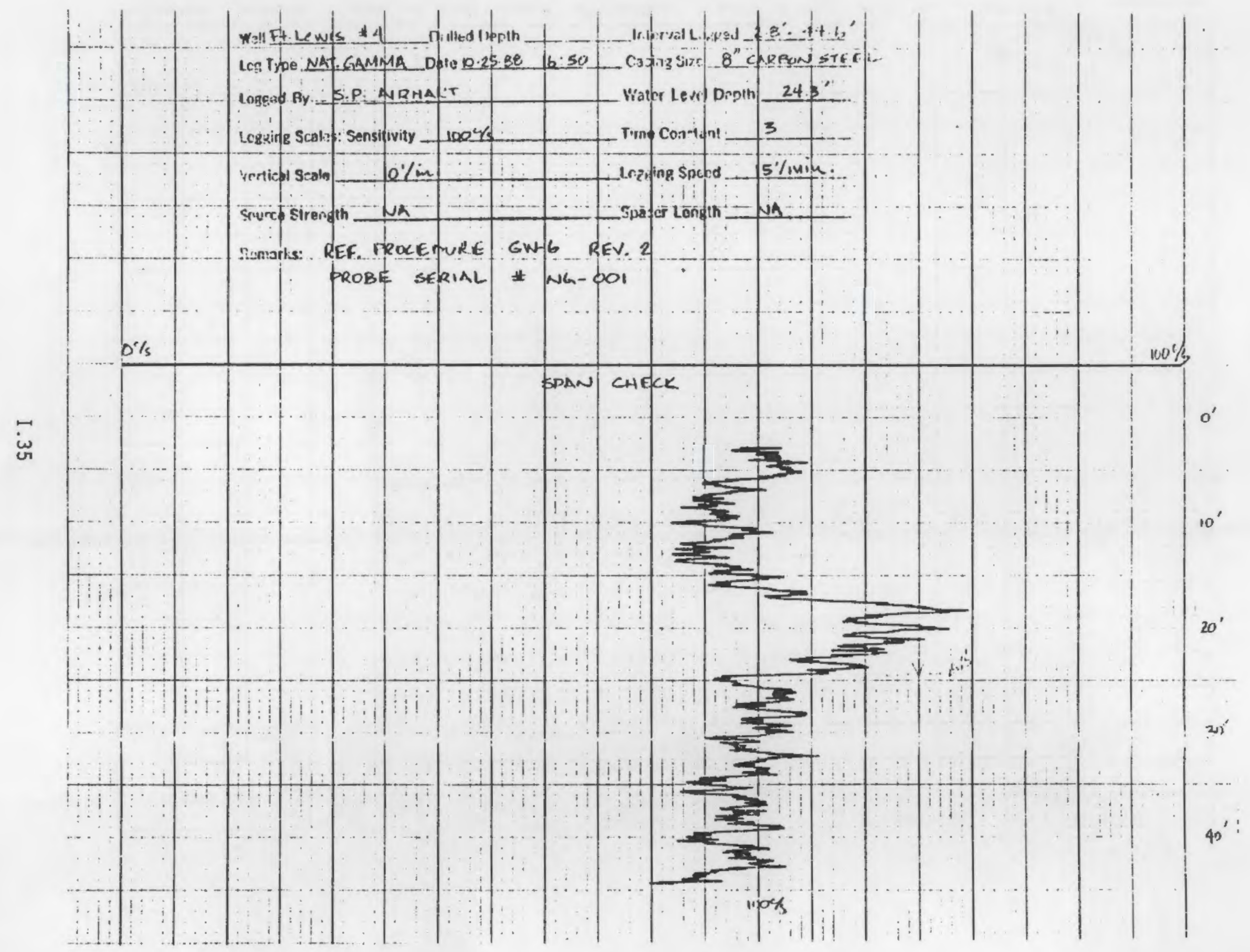


ringe 1 of 1

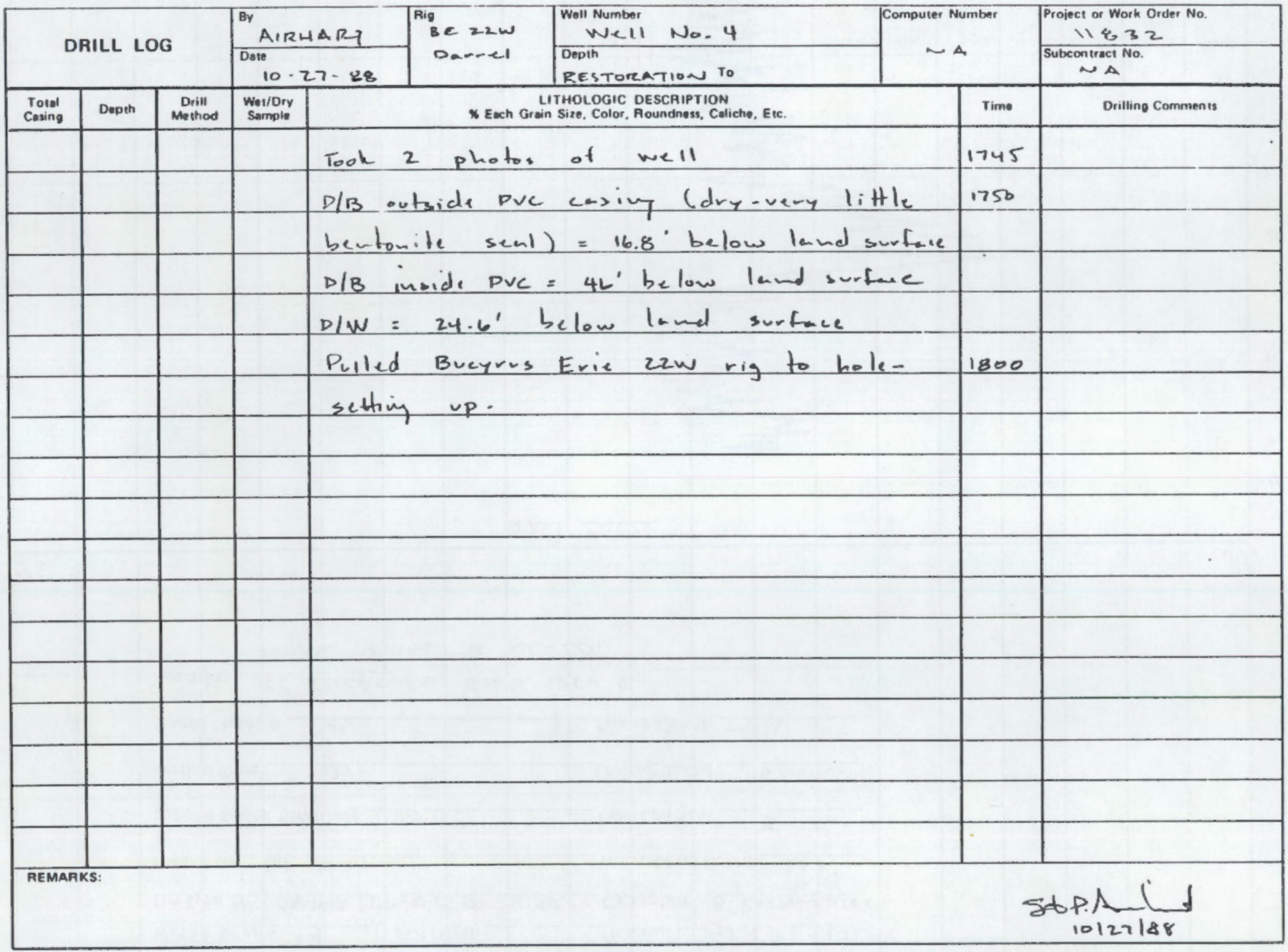

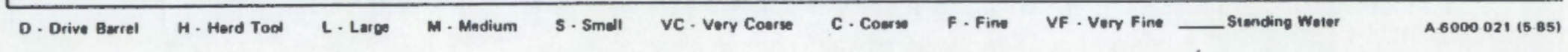


page 1 if z

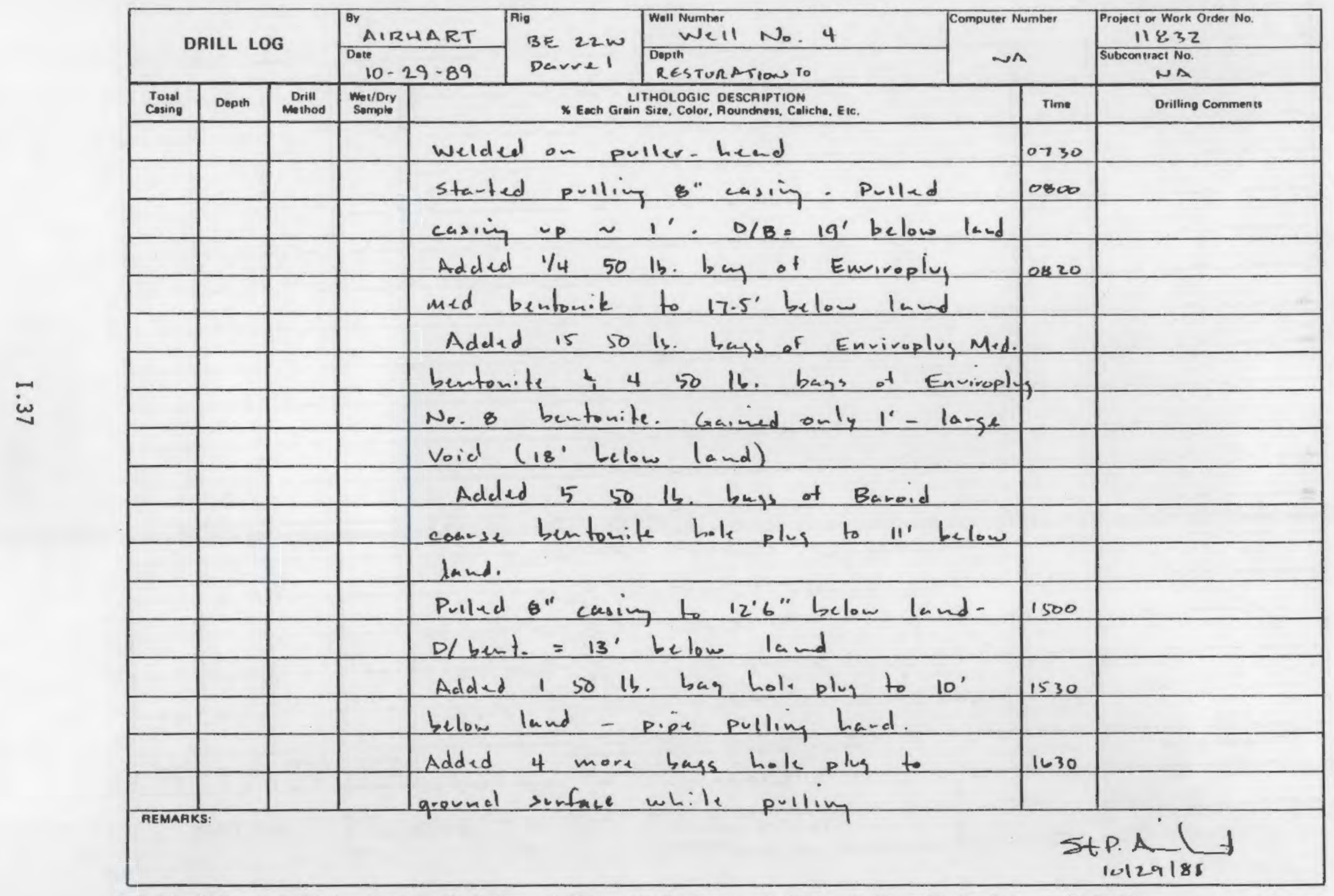

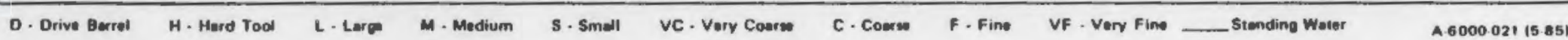


pange 2 \& 2

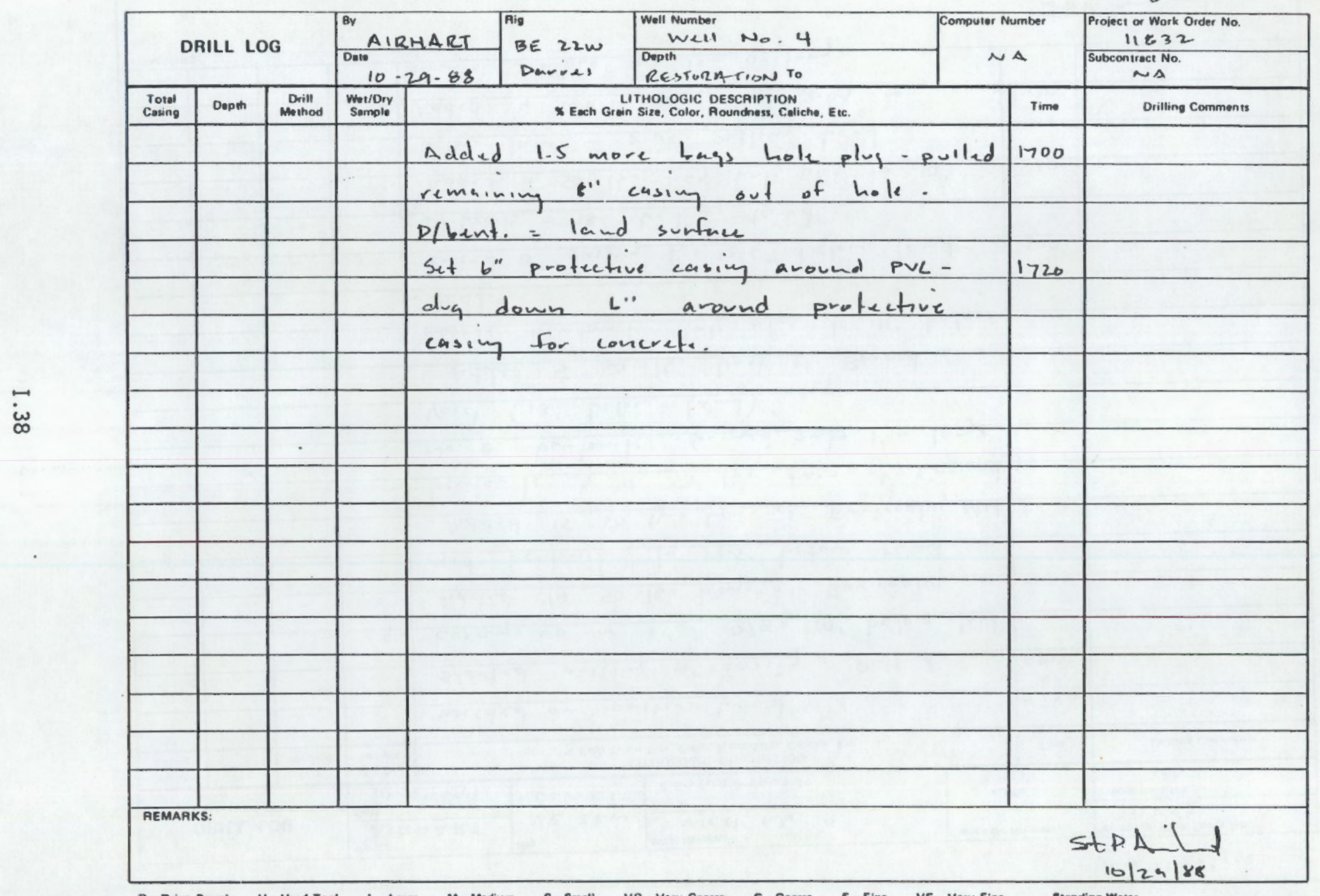

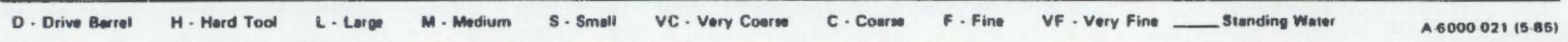


WELL FORT LEWIS \#11 


\section{WELL LOG \\ BY}

Tacoma Pump and Drilling Co., Inc.

30316 MOUNTAIN HIGHWAY

GRAHAM, WASHINGTON 98338

PhONE $847-6087$

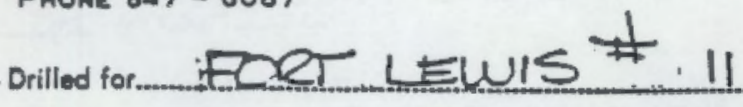

Address

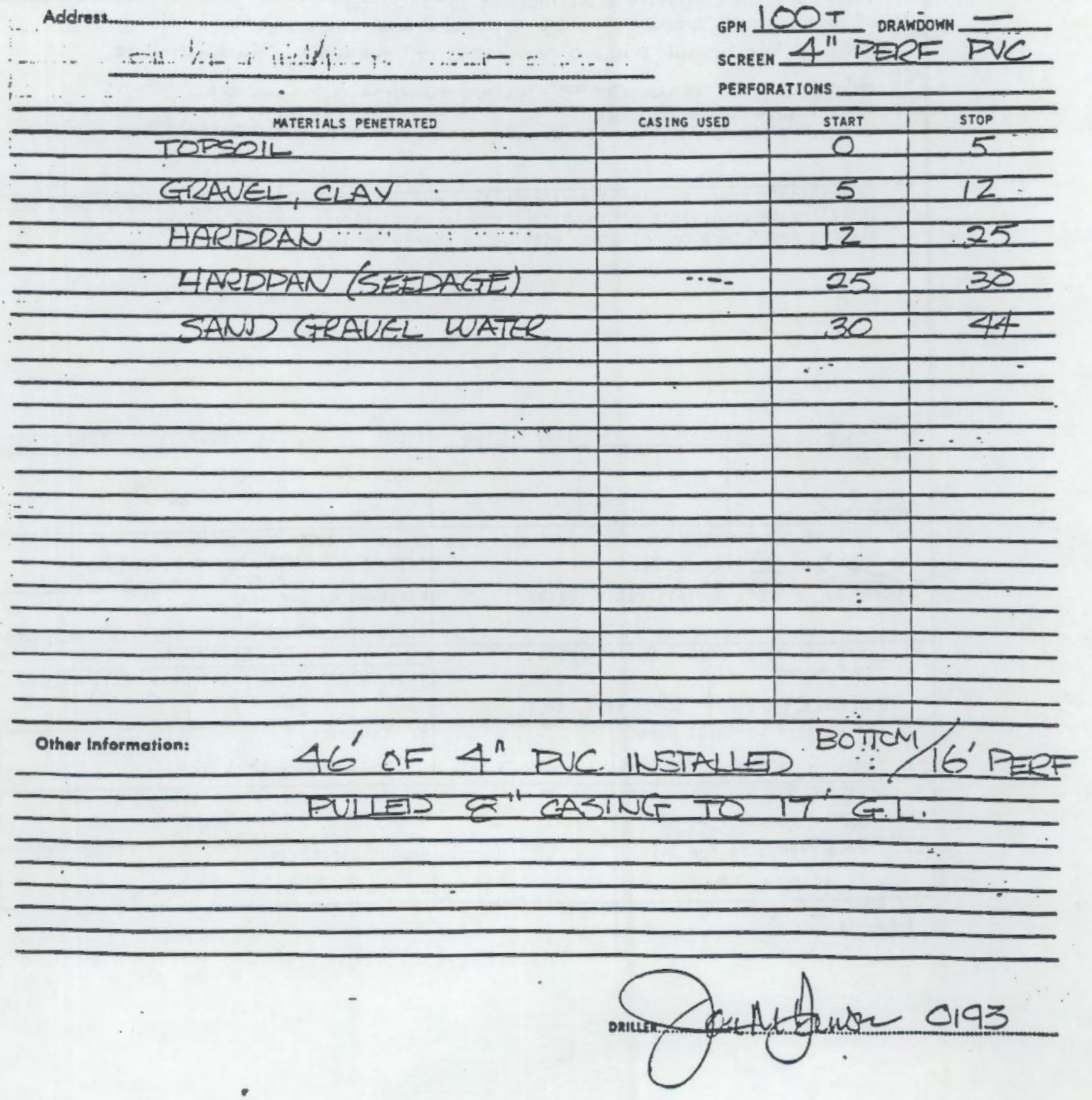

date started MAY 12,81 Date Conpleted MAY 12,81 Depth 14 Ground Level

size of casing

RIG - CABLED ROTARYY STATIC LEVEL 9 PUMPING LEVEL GPM $1 \mathrm{OOT}$ DRAMD̈OWH SCREEN 4 " PERE PVC PERFORATIOHS 
NOTE: Contractor fill in Blocks $1,3,4 \mathrm{~A}, 5 \mathrm{~h}, 7,8,9$, and 10 daily or not less than once each week when no work is proceeding. Fili in $4 b$ and $6 d$ each Thursday and more often when appropriate. The Government will fill in the balance of the blanks.

1. Description of Work Being Done Including Location of Work.

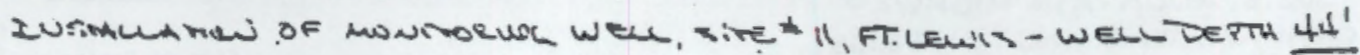

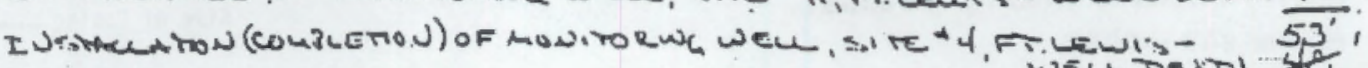

\#. Workmen on Job 2 Name of Contractor's Representative on Job Wustaris cas

2. Government Representative's Instructions to Contractor:

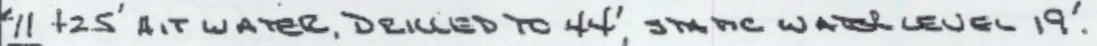

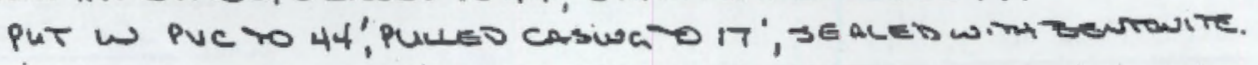

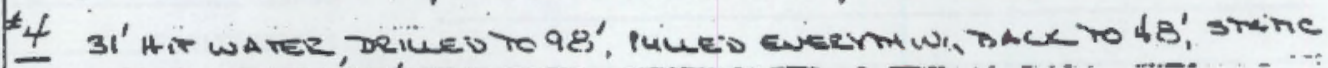

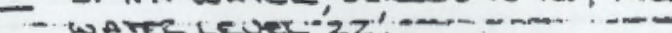

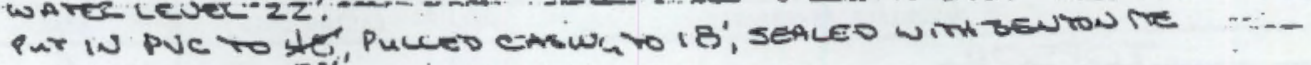

... PNC :

3. Contractor's Remarks: Include difficulties encountered, delays, description of work not installed in accordance with contract requirements and corrective action being taken or planned and future coordination with using agency required.

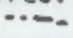

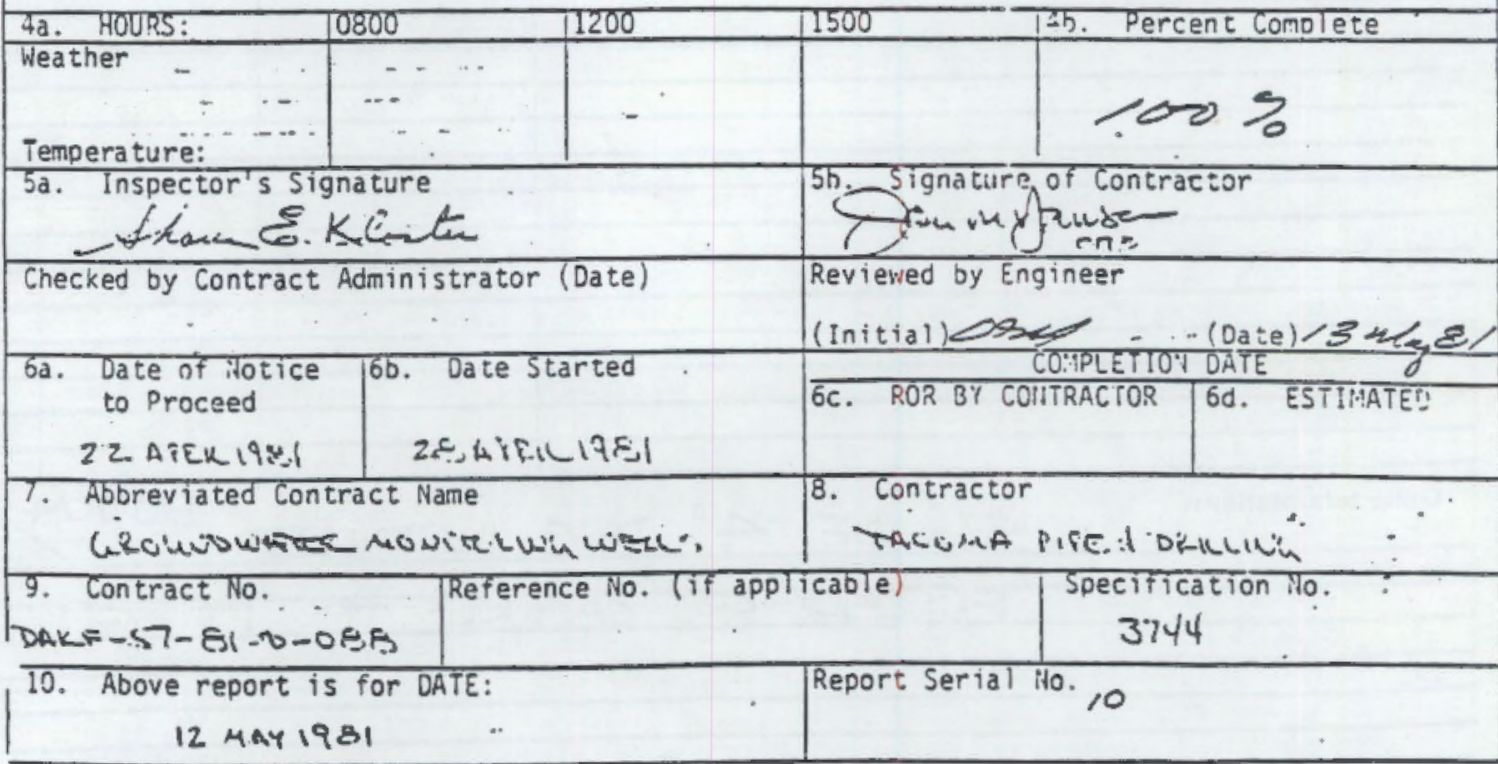

\section{HFL FORM 1235-FEE}

1 Dec 74 


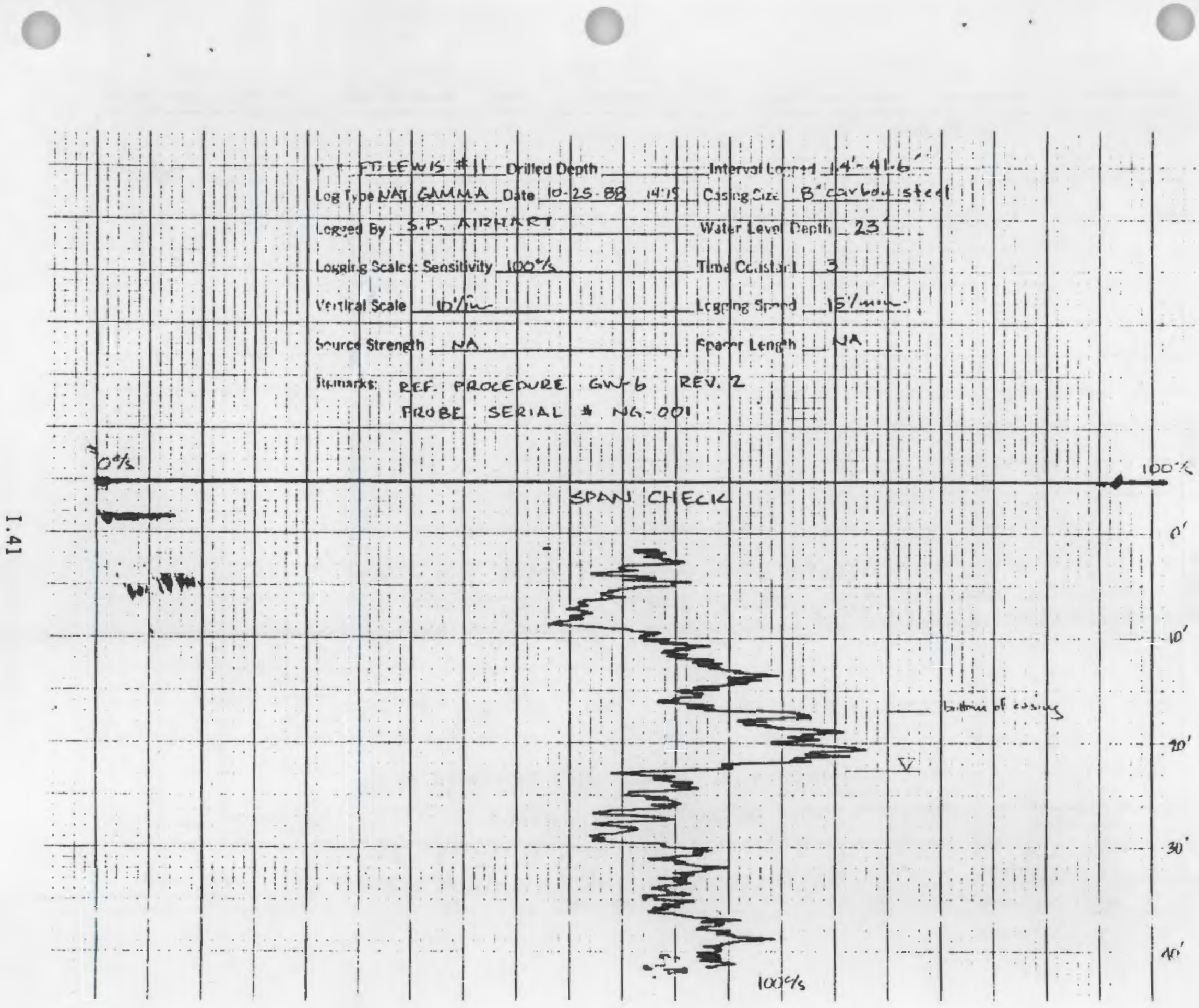




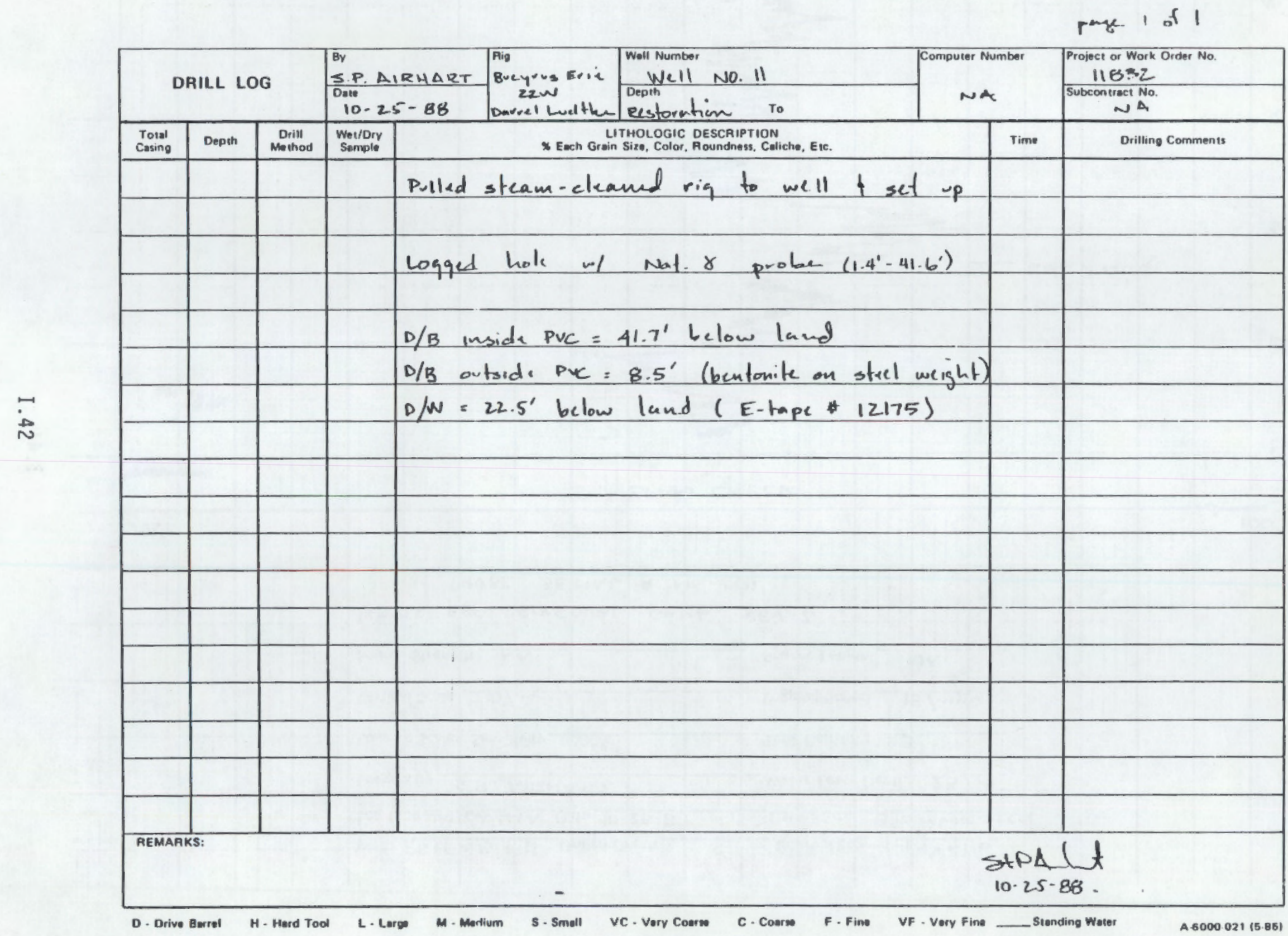

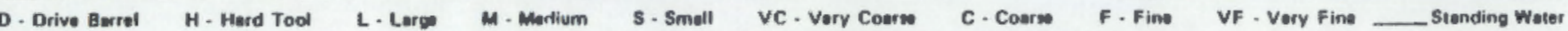

A.6000.021 (5.85) 
pog. 1 of 3

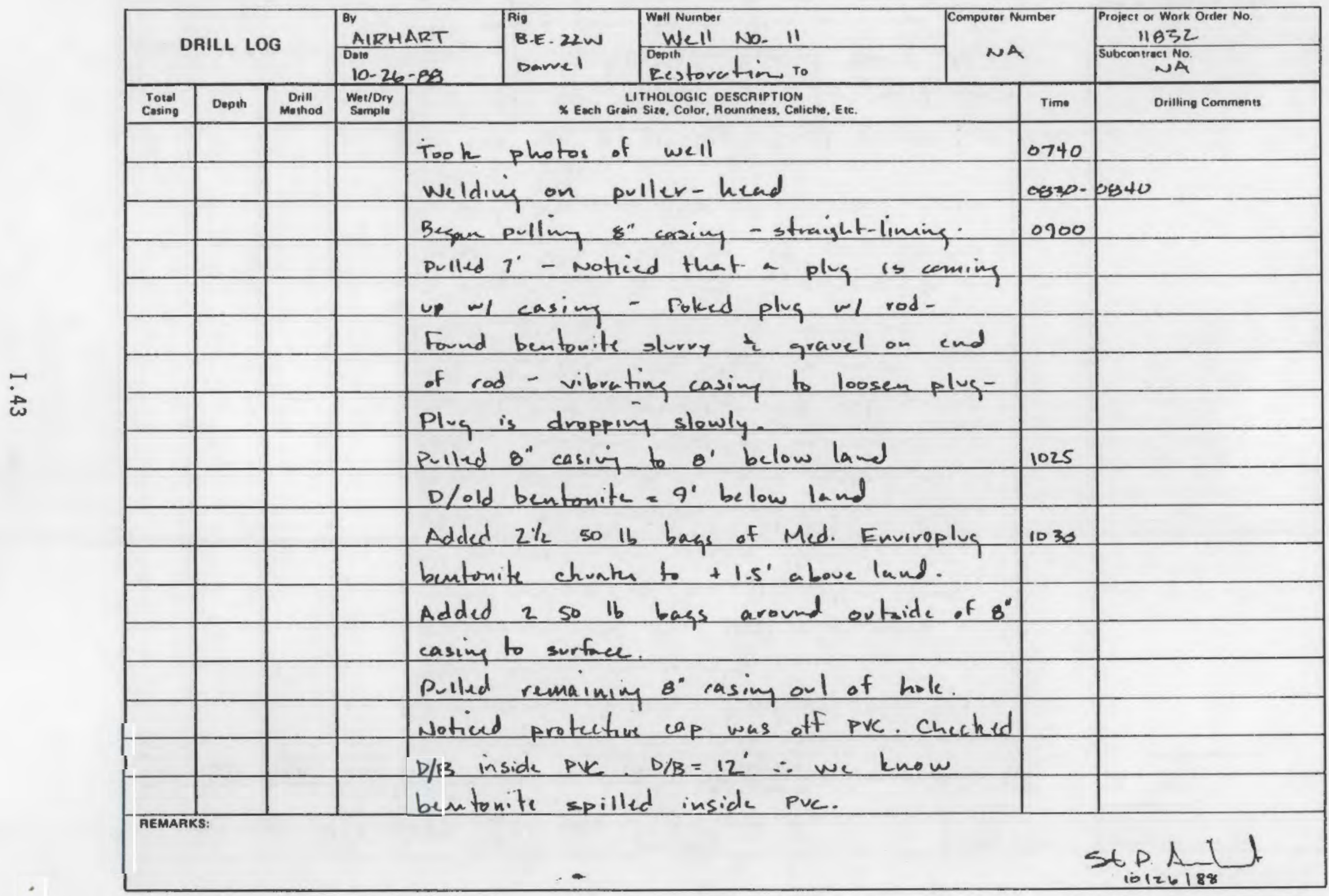

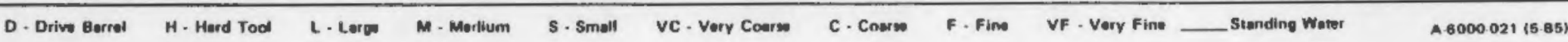


page 2 of 3

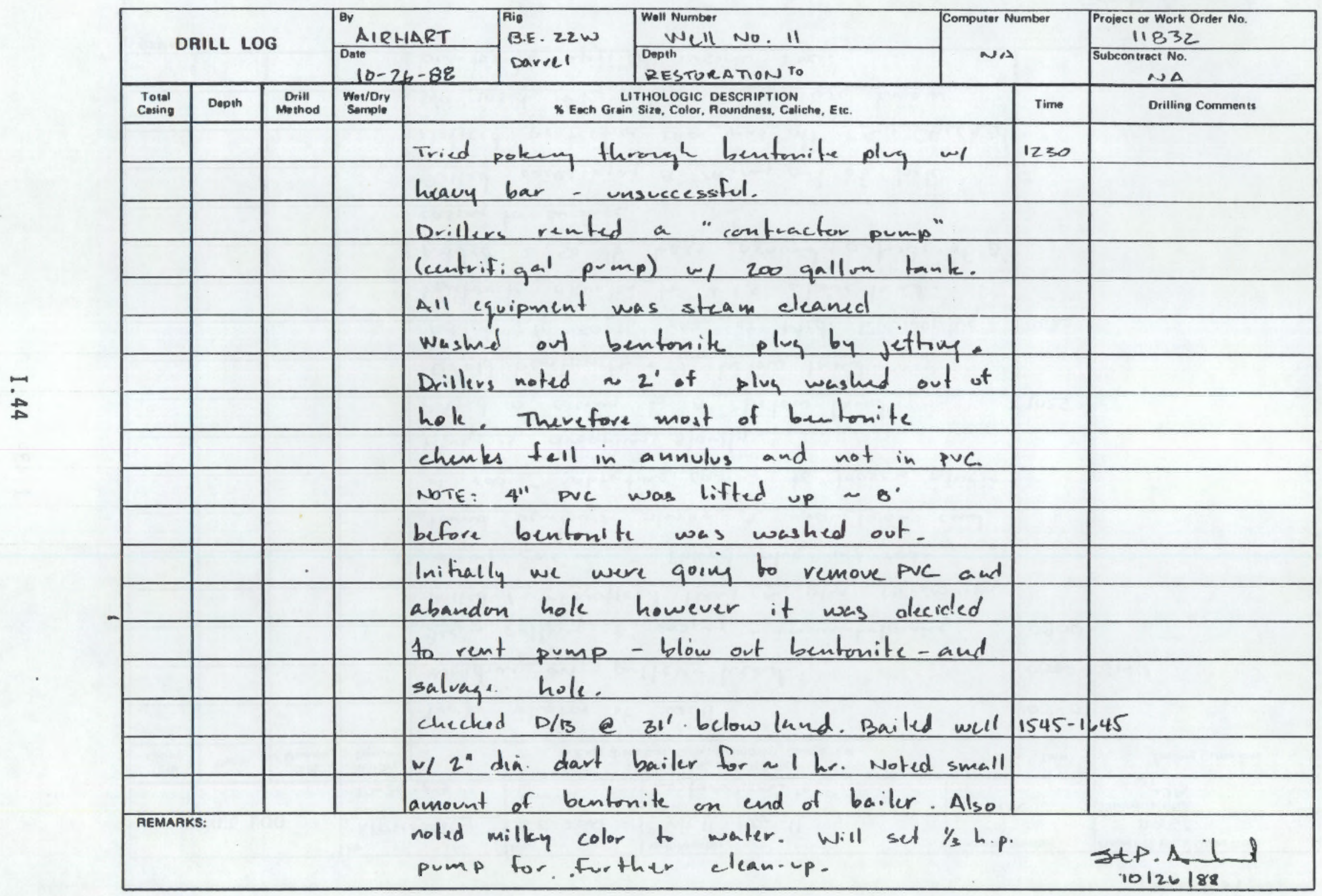

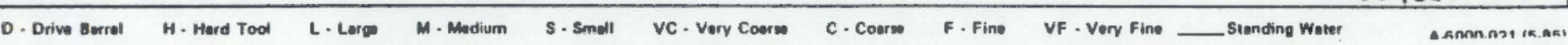



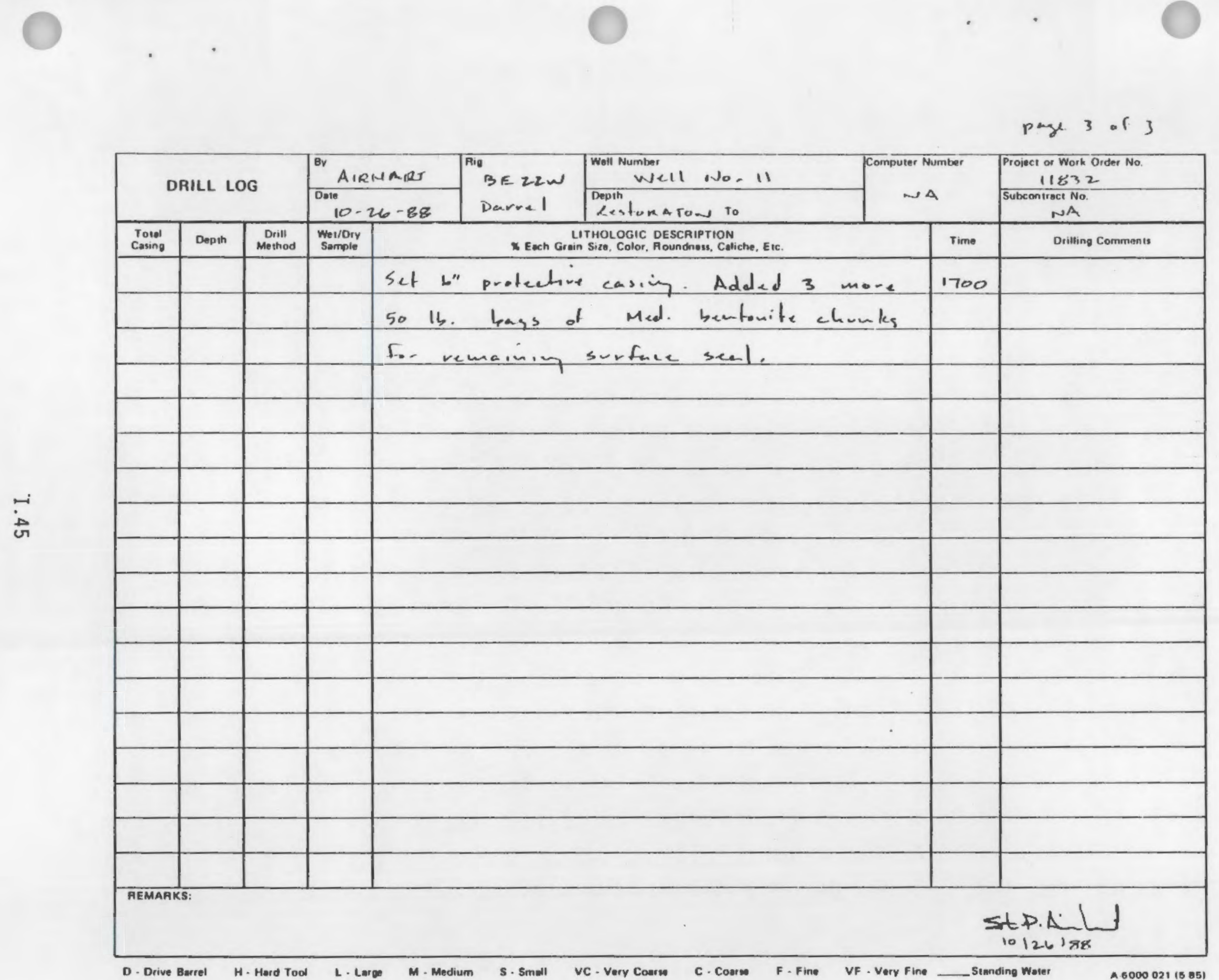

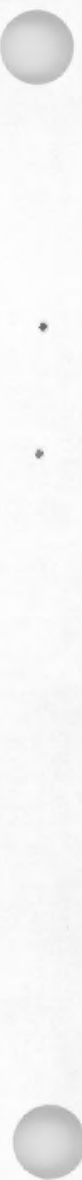

•

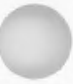


APPENDIX J

GROUND-WATER SAMPLE ANALYSES PROCEDURES 


\section{APPENDIX $J$}

\section{GROUND-WATER SAMPLE ANALYSES PROCEDURES}

This appendix lists procedures used to analyze ground-water samples from landfill wells for various constituents and the procedure used in obtaining ground-water samples from wells near Landfills 1 and 4 . The notation PNL-580 in the procedure refers to Environmental Monitoring Procedures (PNL 1990). 
CLIENT: Battelle

DATE REPORTED: $11 / 4 / 88$

REPORT TO: Paul Eddy

P.O. Box 999

Richland, WA 99352

PARAMETER

EPA/STANDARD METHODS

$\mathrm{pH}$

EPA 150.1

Pesticides + Herbicides \& PCB's

EPA 608

Conductivity

EPA 120.1

Coliform

Standard Method 909

Oil\& Grease

EPA 413.2

Total Organic Halides

EPA 450.1

Volatile organic Aromatics

EPA 601

Total Dissolved Solids

EPA 160.1

Total organic carbon

EPA 415.2

Metals

EPA 200.7

Cyanide

EPA 335.3

Chloride, Fluoride, Nitrate, sulfate

EPA 300.1

Phenol

EPA 420.1

JTD / pb

REPORTED

\section{BY}

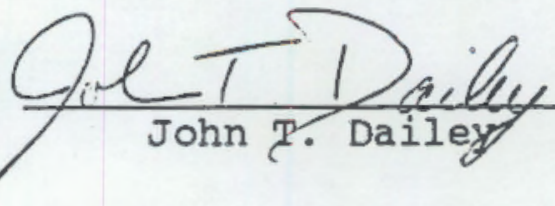

J. 2 
Sample Collection

Using the Hydrostar Pump
Upon arrival at the well head, immediately determine depth-to-water using the appropriate tapes, and record the determined values on the field record form.

- Wear gloves when taking sämples and when handling containers, especially those with added preservative.

Attaching the Pneumatic Cylinder Assembly

1. Insert the support for the pneumatic cylinder into the column support on the well head assembly* (Figure 1).

2. Pull the cylinder rod down until it is fully extended and has stopped.

3. Align the eyelet on the top portion of the turnbolt with the clevis pin hole on the lower portion of the cylinder rod.

4. Align the hole on the cylinder support with the column support on the well head so that the turnbolt eyelet and clevis pin hole on the cylinder rod are aligned when the piston is fully extended.

5. Insert the clevis pin through one of the intersecting pairs of holes on the column support and clip a hitch pin into the holes in the small end of the clevis pin.

6. Check the alignment on the turnbolt eyelet with the hole on the cylinder rod. The alignment must be nearly perfect, neither too high nor too low.

7. Adjust by rotating the turnbolt clockwise or counterclockwise.

* When inserting the cylinder support into the column support on the pump assembly, at least two holes on the cylinder support must overlap with two holes on the column support. If less than two holes overlap use the extension supplied with the Hydrostar pneumatic cylinder. Align the pumping system in the same manner as described above.

Operating the Pneumatic Cylinder

1. Attach either the purging hose (large diameter) or the teflon sampling hose to the outlet on the discharge tee of the sampling pump. 


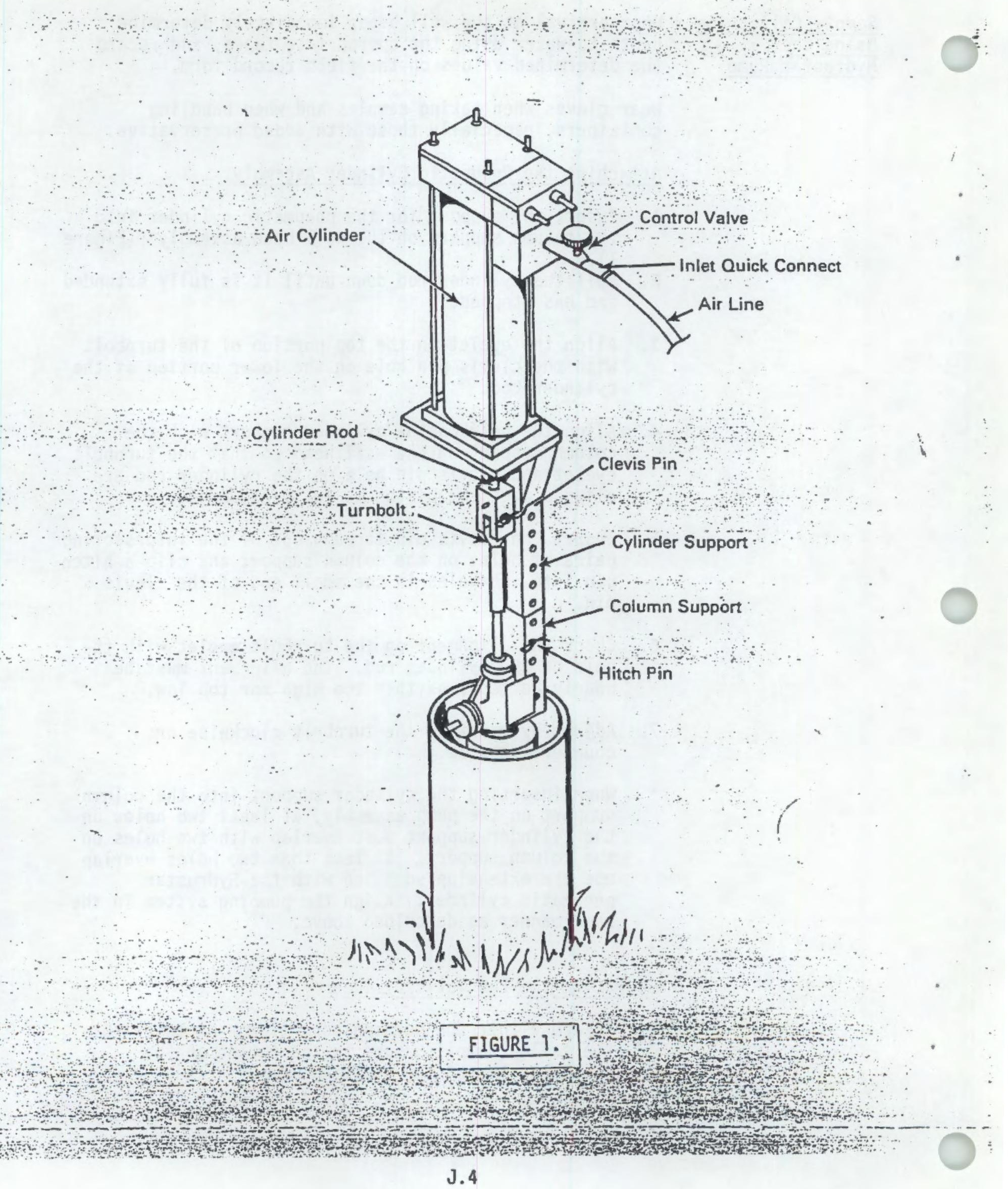


2. Attach the quick-connect on the supply-hose to the unattached end of the control valve on the pneumatic cylinder. The input air pressure should not exceed 120 psi.

3. Turn air supply on to the control valve.

4. Turn on the control valve on the pneumatic cylinder. The piston will begin to operate.

5. Adjust stroke rate to no more than 60 per minute*. The stroke speed of the pneumatic cylinder can be adjusted with the control valve located on the top of the pneumatic cylinder. A stroke is defined as one downward and one upward extension.

* If the pneumatic cylinder assembly is not operating correctly, and the problems are not due to the well or the pump in the well, the well may be hand pumped as described in "Manual Operation."

Sampling With Pneumatic Piston Assembly

1. Slow down the pumping rate until the piston operates smoothly. This rate will be less than 10 strokes a minute.

2. Attach the Teflon sampling hose and purge at this rate for a minimum of two-minutes.

3. Proceed with sampling all unfiltered samples according to PNL -580 .

4. Attach the filter assembly and purge the filter according to directions listed in PNL-580. If too much pressure is exerted across the filter the membrane will rupture, usually resulting in a popping noise. If this happens, replace the filter and restart the filtering procedure.

5. Dismantle the pneumatic pumping assembly as described below.

\section{Removing the Pneumatic Pumping Assembly}

1. Disconnect the air supply at the pneumatic cylinder.

2. Disassemble pneumatic cylinder in reverse order of steps 1 through 7 in the section "Attaching Pneumatic Cylinoier Assembly.". .

3. Replace well cap over top of well head. 


\section{Manual Operations}

1. Insert the handle support into the column support on the pump head assembly so that at least two holes on the handle support overlap with two holes on the column support (Figure 2).

2. Slide the clevis pin through one of the intersecting pairs of holes on the column support.

3. Clip the hitch pin into the hole in the small end of the clevis pin.

4. Remove the turnbolt on the top of the rod at the well head.

5. Attach the turnbolt on the end of the wire rope attached to the handle assembly onto the threaded rod at the top of the well head.

6. Lift the handle so that the flat edge of the cam nearest the shackle is approximately parallel with the ground.

7. Pull all the slack out of the wire rope.

8. Using either an adjustable or $9 / 16$ " open end wrench, tighten both nuts on the shackle until the sheath on the wire rope is compressed. Remembering to keep all the slack out of the wire rope.

Manual Well Sampling

1. Attach either the purging hose (large diameter) or the Teflon sampling hose (small diameter) to the outlet on the discharge tee of the sampling pump.

2. Begin pumping the operating handle with smooth, even strokes. For best performance, use 20 to 45 strokes per minute for purging the well. Use less than 10 strokes per minute during sampling... When the filter assembly is attached, special attention is required to prevent rapid build up of pressure across the filter. If too much pressure is exerted across the filter the membrane will rupture, usually resulting in a popping noise. If this happens, replace the filter and begin sampling for the filtered sample according to the written procedure.

3. When sampling is completed follow the direction below to disassemble the handle assembly from the well head.. 


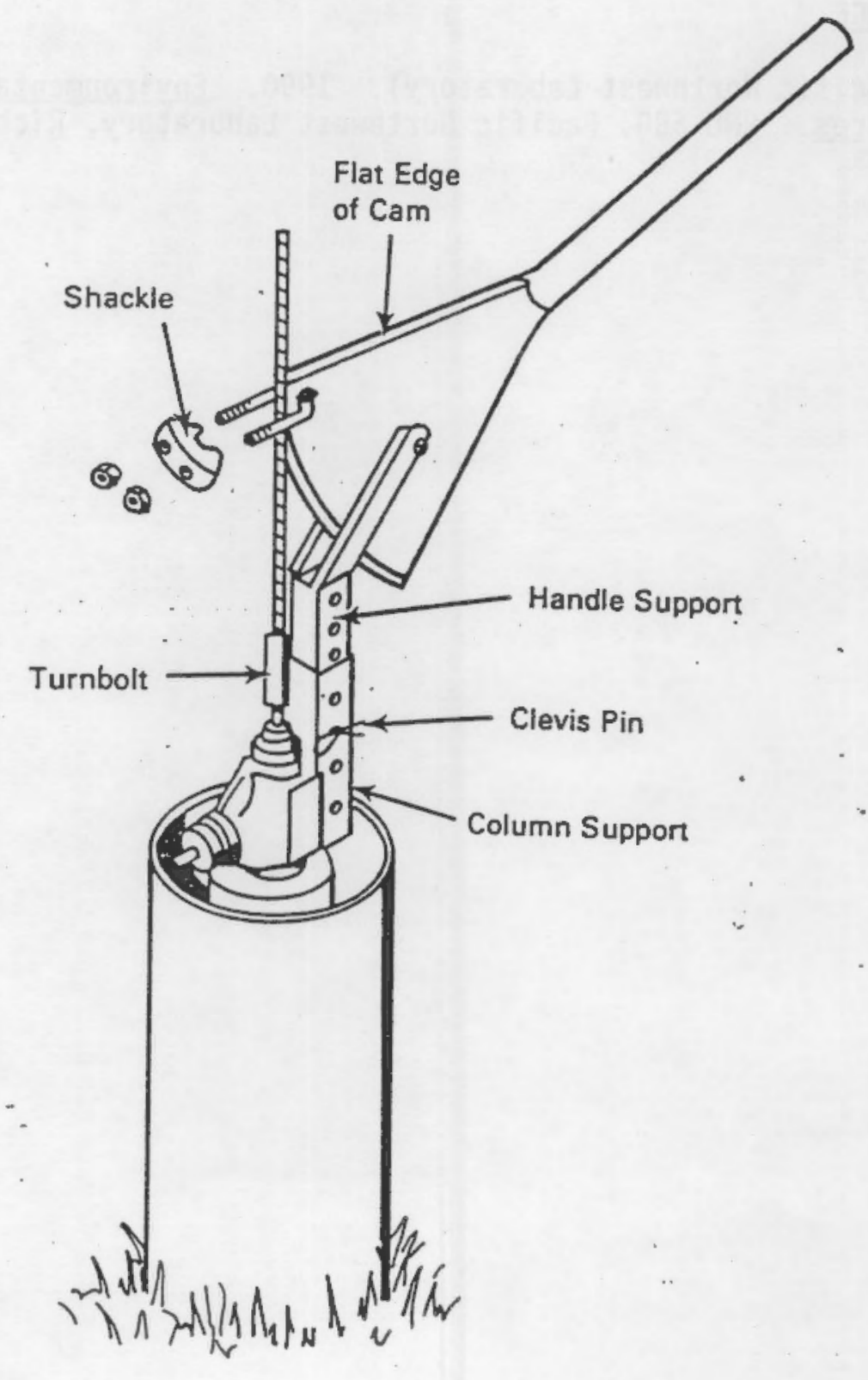

Figure 2

J.7 
Removing the Handle Assembly

Disassemble the handle assembly in reverse order of steps

1 through 8 in the section "Manual Operation."

\section{REFERENCE}

PNL (Pacific Northwest Laboratory). 1990. Environmental Monitoring

Procedures. PNL-580, Pacific Northwest Laboratory, Richland, Washington. 


\section{APPENDIX K}

GROUND-WATER SAMPLE ANALYSES RESULTS 


\section{APPENDIX K}

\section{GROUND-WATER SAMPLE ANALYSES RESULTS}

This appendix contains the results of ground-water sample analyses for wells at Landfills 1 and 4 .

K.1 
Fort Lewis Quality Control Samples Identifiers

Late August Sampling Event

LFA-PNLA-1-1-1

LFA-PNLB----

LFA-PNLC--

Early September Sampling Event

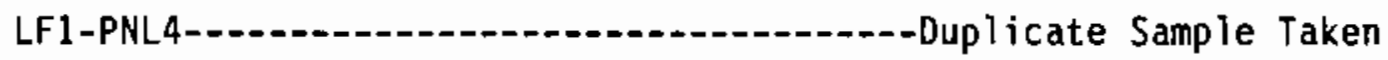

Early October Sampling Event

No QC Samples Taken

Late October Sampling Event

LF4-PNL4--1-1-1

Middle December Sampling

LF1-PNL5---1

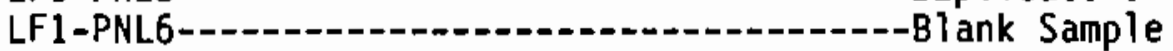

LF4-PNL7--_- USGS M1D0 Std 

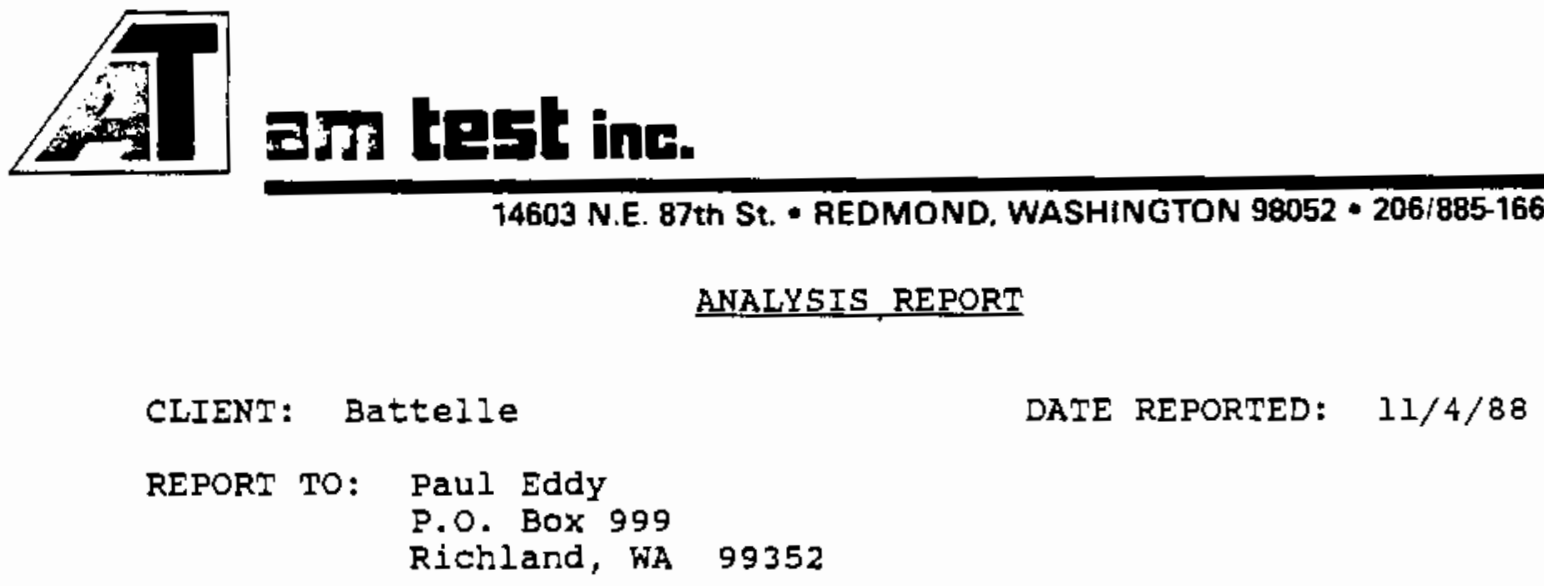

$\mathrm{JTD} / \mathrm{pb}$

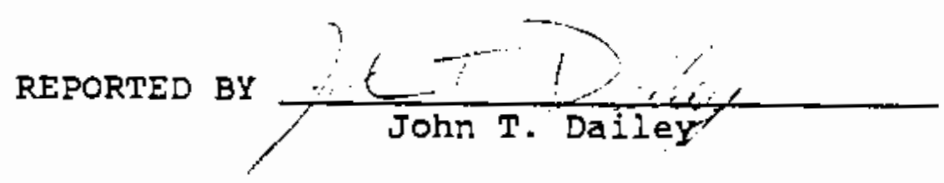


AM Test Analytical Results From Late August, 1988 Sampling Event 


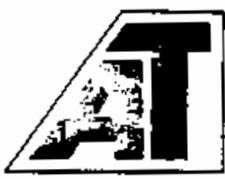

14603 N.E. 87th St. - REDMOND, WASHINGTON 98052 - 206/885-1664

\section{ANALYSIS REPORT}

CLIENT: Battelle - Pacific Northwest Labs

DATE REPORTED: $9 / 26 / 88$

REPORT TO: Paul Eddy

DATE REVISED: $10 / 27 / 88$

P.O. Box 999

Q.O. NO.: V5228 AD

Richland, WA 99352

Laboratory Sample Nos.

$816809 \quad 816810 \quad 816811$

client Identification

LFI-PNLI LF4-PNLI LF4-QNL3

$\mathrm{pH}$

6.35

6.25

6.20

Specific Conductance (umhos/cm a $25^{\circ} \mathrm{C}$ )

310.

390.

138 .

Total Coliform (MPN/100 mi)

220. 1,600 .

17.

0 il \& Grease $(\mathrm{mg} / 1)$

$<1.4$

$<1.3$

8.4

Total Organic Halides (ug/l as Cl-)

$<10$.

18.0

44.0

Gross Beta (pCI/L)

$<2$.

$<2$.

$<2$.

Radium ( $p C I / I)$

$<1$.

$<1$.

$<1$.

Gross Alpha (pCI/I)

$<1$.

$<1$.

$<1$.

Total Dissolved Solids (mg/l)

418.

295.

524 .

Total organic Carbon (mg/1)

3.33

3.27

$2.75\}$

1.14

chloride (mg/I)

4.89

15.6

4.20

Phenol (mg/1)

$<0.008<0.008<0.16$

Nitrate - Nitrogen (mg/1)

2.6

$<0.01$

0.771

Fluoride (mg/1)

$<0.1$

$<0.10$

$<0.10$ $<0.10$ ] 


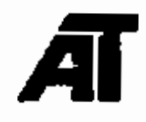

$-2-$

CLIENT: Battelle - Pacific Northwest Labs

DATE REPORTED: $9 / 26 / 88$

REPORT TO: Paul Eddy

DATE REVISED: $10 / 27 / 88$

P.O. NO.: V5228 AD

Laboratory Sample Nos.

816809

816810

816811

Client Identification

LFI-PNLI LF4-PNLI LF4-PNL3

Sulfate $(\mathrm{mg} / \mathrm{l})$

61.6

11.2

11.4

$10.4]$

Cyanide (mg/1)

$<0.006<0.006$

$<0.006$ 


$$
-3-
$$

CLIENT: Battelle - Pacific Northwest Labs

DATE REPORTED: $\quad 9 / 26 / 88$

DATE REVISED: $10 / 27 / 88$ REPORT TO: PaUl EddY

P.O. NO.: V5228 AD

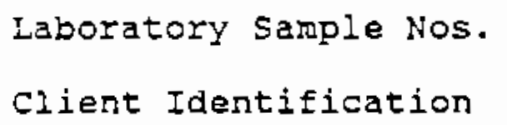

*Results to follow. 


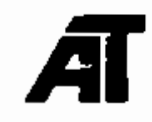

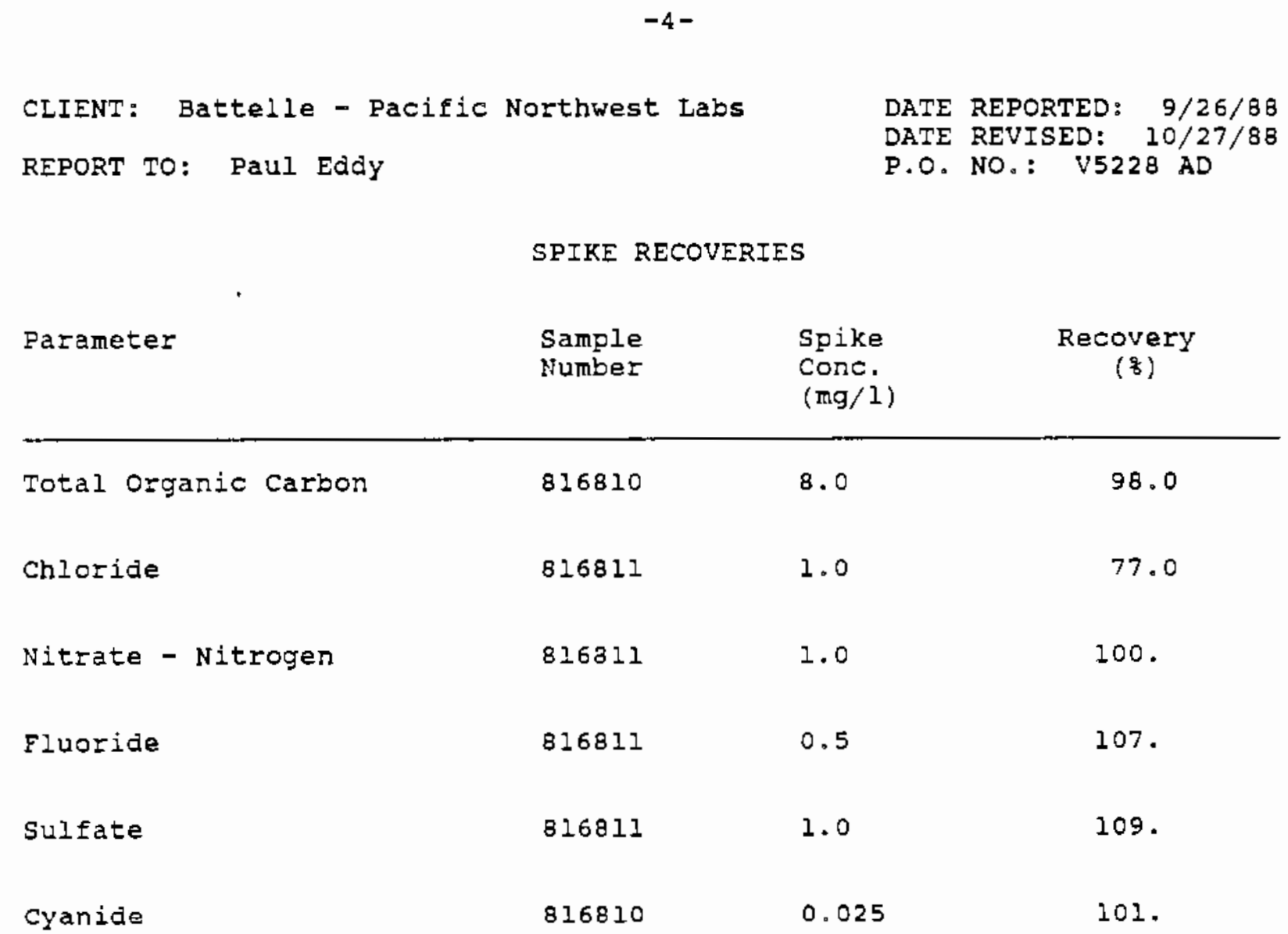




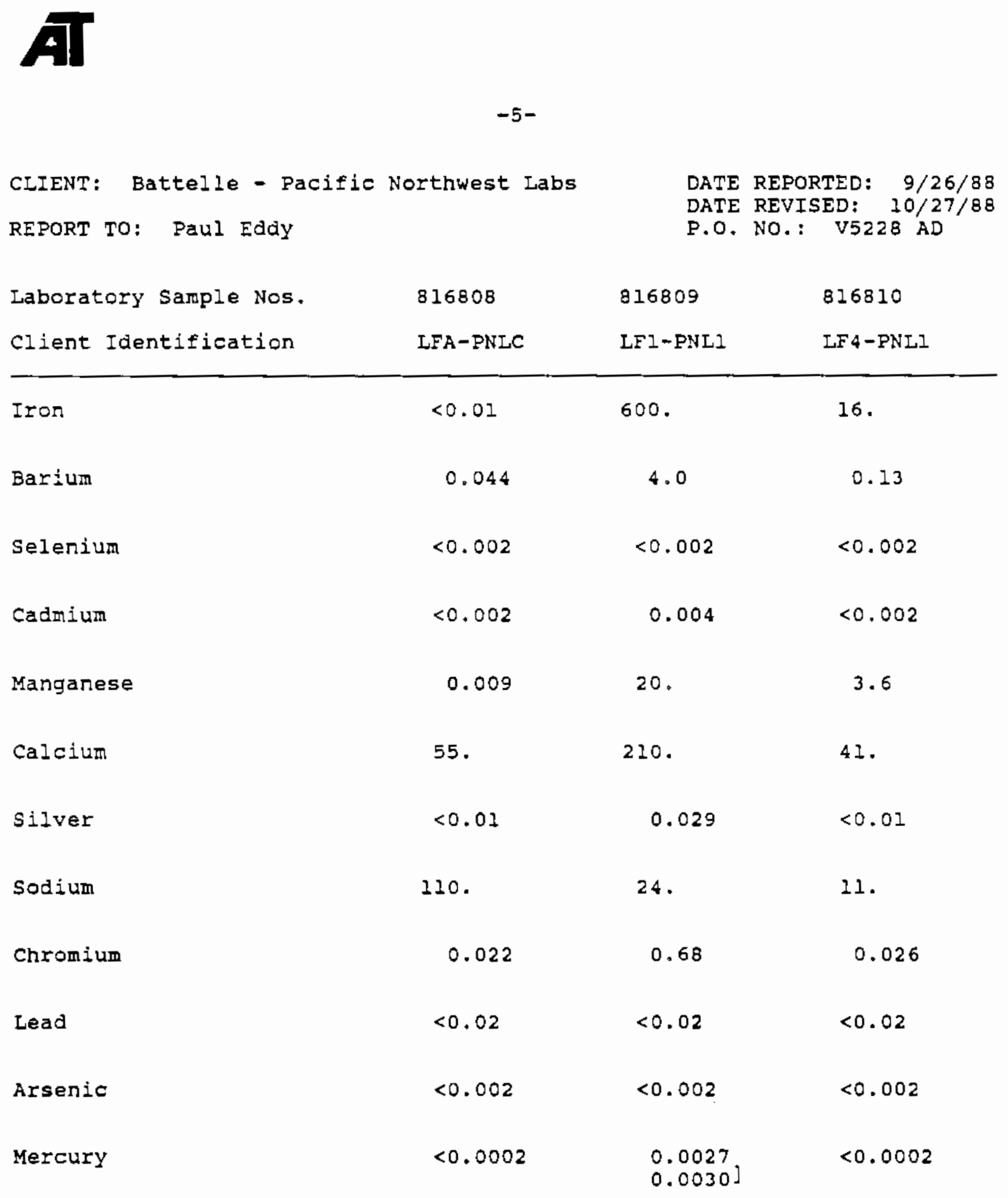

All values are reported in $\mathrm{mg} / \mathrm{l}$. 
CLIENT: Battelle - Pacific Northwest Labs

DATE REPORTED: $9 / 26 / 88$

DATE REVISED: $10 / 27 / 88$

REPORT TO: Paul Eddy

P.O. NO.: V5228 AD

\begin{tabular}{|c|c|c|c|}
\hline Laboratory Sample Nos. & 816811 & 816812 & 816813 \\
\hline client Identification & LF 4-PNL3 & LEA-PNLA & LFA-PNLB \\
\hline Iron & 29. & $\begin{array}{l}460 . \\
500.1\end{array}$ & 0.17 \\
\hline Barium & 0.36 & $\begin{array}{l}2.2 \\
2.32\end{array}$ & $<0.003$ \\
\hline Selenium & $<0.002$ & $\begin{array}{l}<0.002 \\
<0.002]\end{array}$ & $<0.002$ \\
\hline Cadmium & $<0.002$ & $\begin{array}{l}0.006 \\
0.004]\end{array}$ & $<0.002$ \\
\hline Manganese & 1.29 & $\begin{array}{l}12.6 \\
13.03\end{array}$ & 0.005 \\
\hline Calcium & 16. & $\begin{array}{l}118 . \\
110.1\end{array}$ & 0.23 \\
\hline silver & $<0.01$ & $\begin{array}{l}0.018 \\
0.030]\end{array}$ & $<0.01$ \\
\hline Sodium & 6.4 & $\begin{array}{l}17 . \\
16.3\end{array}$ & 2.7 \\
\hline Chromium & 0.037 & $\begin{array}{l}0.53 \\
0.49]\end{array}$ & $<0.006$ \\
\hline Lead & $<0.02$ & $\begin{array}{l}<0.02 \\
<0.02]\end{array}$ & $<0.02$ \\
\hline Arsenic & $<0.002$ & $\begin{array}{l}<0.002 \\
<0.002]\end{array}$ & $<0.002$ \\
\hline Mercury & $<0.0002$ & 0.0031 & $<0.0002$ \\
\hline
\end{tabular}

All values are reported in $\mathrm{mg} / \mathrm{l}$. 
$\boldsymbol{A T}$

$-7-$

CLIENT: Battelle - Pacific Northwest Labs

DATE REPORTED: $9 / 26 / 88$

REPORT TO: PaUl EddY

DATE REVISED: $10 / 27 / 88$

P.O. NO.: V5228 AD

SPIKE RECOVERIES

Element

Sample

Number

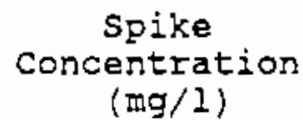

1.0

1.0

0.5

0.5

1.0

5.0

0.050

2.5

1.0

1.0

0.5
Recovery

(하)

Calcium

816813

916813

816813

816813

816813

Arsenic

97.0

97.0

66.0

94.0

95.5

93.4

88.0

112.

98.0

93.0

96.0 


\section{AT}

CLIENT: Battelle - Pacific Northwest Labs

DATE REPORTED: $9 / 26 / 88$

REPORT TO: PaUl Eddy

DATE REVISED: $10 / 27 / 88$

P.O. NO.: V5228 AD

GC ANALYSIS OF PURGEABLE HALOCARBONS BY EPA KETHOD 601

$\begin{array}{lllc}\text { Laboratory Sample Nos. } & 816809 & 816810 & \text { DETECTION } \\ \text { Client Identification } & \text { LF1-PNLI } & \text { LF4-PNLI } & \text { (ug/1) }\end{array}$

\begin{tabular}{|c|c|c|c|}
\hline Chloromethane & ND & ND & 2. \\
\hline Vinyl chloride & ND & ND & 2 . \\
\hline Bromomethane & ND & ND & 2. \\
\hline Chloroethane & ND & ND & 2. \\
\hline Dichlorodifluoromethane & ND & ND & 2. \\
\hline Trichlorofluoromethane & ND & ND & 2 。 \\
\hline 1,1 -Dichloroethylene & ND & ND & 2. \\
\hline Methylene Chloride & ND & ND & 10. \\
\hline Trans-1,2-Dichlorothylene & ND & 0.9 & 0.6 \\
\hline 1,1-Dichloroethane & ND & ND & 0.6 \\
\hline Chloroform & 1.4 & ND & 0.6 \\
\hline $1,1,1$-Trichloroethane & ND & ND & 0.6 \\
\hline Carbon Tetrachloride & ND & ND & 0.6 \\
\hline 1,2-Dichloroethane & ND & ND & 0.6 \\
\hline Trichloroethylene & ND & 13.9 & 0.6 \\
\hline 1,2-Dichloropropane & ND & ND & 0.6 \\
\hline Dichlorobromomethane & ND & ND & 0.6 \\
\hline Trans-1,3-Dichloropropene & ND & ND & 0.6 \\
\hline Cis-1,3-Dichloropropene & ND & ND & 0.6 \\
\hline $1,1,2$-Trichloroethane & ND & ND & 0.6 \\
\hline Tetrachloroethylene & ND & ND & 0.6 \\
\hline Dibromochloromethane & ND & ND & 0.6 \\
\hline Bromoform & ND & ND & 0.6 \\
\hline $1,1,2,2$-Tetrach 1 oroethane & ND & ND & 0.6 \\
\hline
\end{tabular}




\title{
AT
}$$
-9-
$$

CLIENT: Battelie - Pacific Northwest Labs

DATE REPORTED: $9 / 26 / 88$

DATE REVISED: $10 / 27 / 88$

REPORT TO: Paul Eddy

P.O. NO.: V5228 AD
\end{abstract}

GC ANALYSIS OF PURGEABLE HALOCARBONS BY EPA METHOD 601

$\begin{array}{llll}\text { Laboratory Sample Nos. } & 816811 & 816812 & \text { DETECTION } \\ \text { Client Identification } & \text { LF4-PNL3 } & \text { LFA-PNLA } & \text { (LIT/ }\end{array}$

Chloromethane

Vinyl Chloride

ND

Bromomethane

Chioroethane

Dichlorodifluoromethane

Trichlorofluoromethane

1,1-Dichloroethylene

Methylene Chloride

Trans-I, 2-Dichlorothylene

1,1-Dichloroethane

Chloroform

1,1,1-Trichloroethane

Carbon Tetrachloride

1,2-Dichloroethane

Trichloroethylene

1,2-Dichloropropane

Dichlorobromomethane

Trans-1, 3-Dichloropropene

Cis-1,3-Dichloropropene

1, 1,2-Trichloroethane

Tetrachloroethylene

Dibromochloromethane

ND

ND

ND

ND

ND

ND

ND

ND

ND

0.6

ND

ND

ND

13.

ND

ND

ND

ND

ND

ND

ND

Bromoform

1, 1,2,2-Tetrachloroethane

ND

ND

ND

ND

ND

ND

2.5

ND

ND

ND

ND

1. 4

ND

ND

ND

ND

ND

ND

ND

ND

ND

ND

ND

ND

ND

2.

2.

2 .

2.

2 .

2 .

2.

10.

0.6

0.6

0.6

0.6

0.6

0.6

0.6

0.6

0.6

0.6

0.6

0.6

0.6

0.6

0.6

0.6

ND $=$ Not Detected.

All results reported in ug/l. 


$$
-10-
$$

CLIENT: Battelle - Pacific Northwest Labs

DATE REPORTED: $9 / 26 / 88$

DATE REVISED: $10 / 27 / 88$

REPORT TO: Paul EddY

P.O. NO.: V5228 AD

GC ANALYSIS OF PURGEABLE HALOCARBONS BY EPA METHOD 601

$\begin{array}{llcc}\text { Laboratory Sample Nos. } & 816813 & 816813 & \text { DETECTION } \\ & & \text { DUPLICATE } & \text { LIMIT } \\ \text { Client Identification } & \text { LFA-PNLB } & \text { LEA-PNLB } & (\mathrm{ug} / \mathrm{I})\end{array}$

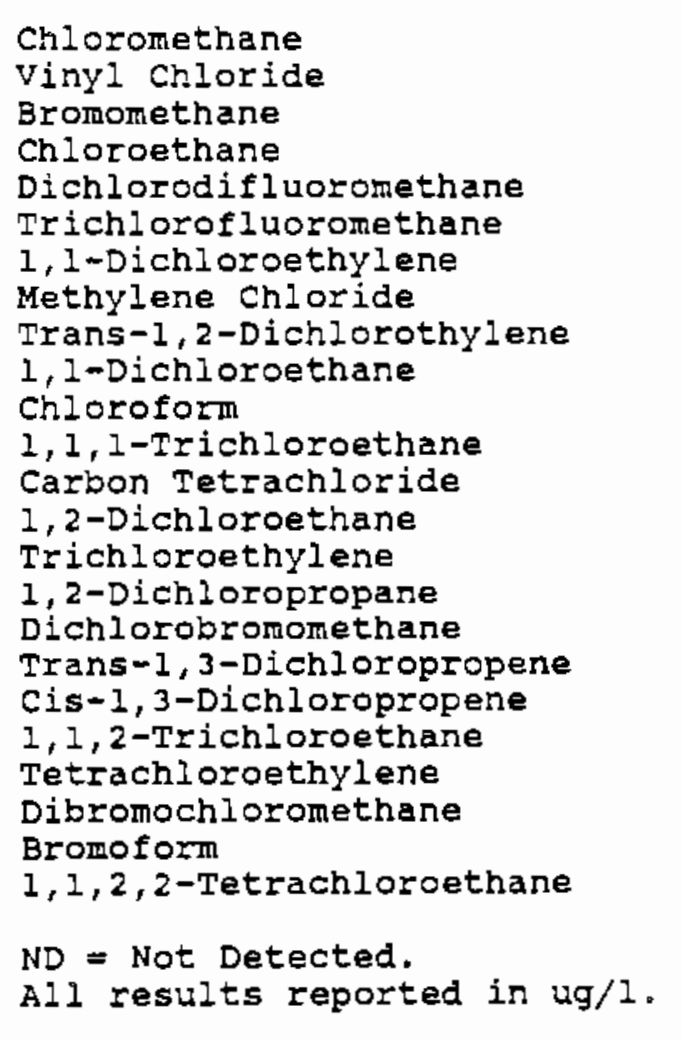

$\begin{array}{llc}\text { ND } & \text { ND } & 2 . \\ \text { ND } & \text { ND } & 2 . \\ \text { ND } & \text { ND } & 2 . \\ \text { ND } & \text { ND } & 2 . \\ \text { ND } & \text { ND } & 2 . \\ \text { ND } & \text { ND } & 2 . \\ \text { ND } & \text { ND } & 2 . \\ \text { ND } & \text { ND } & 10 . \\ \text { ND } & \text { ND } & 0.6 \\ \text { ND } & \text { ND } & 0.6 \\ \text { O.8 } & 0.9 & 0.6 \\ \text { ND } & \text { ND } & 0.6 \\ \text { ND } & \text { ND } & 0.6 \\ \text { ND } & \text { ND } & 0.6 \\ \text { ND } & \text { ND } & 0.6 \\ \text { ND } & \text { ND } & 0.6 \\ \text { ND } & \text { ND } & 0.6 \\ \text { ND } & \text { ND } & 0.6 \\ \text { ND } & \text { ND } & 0.6 \\ \text { ND } & \text { ND } & 0.6 \\ \text { ND } & \text { ND } & 0.6 \\ \text { ND } & \text { ND } & 0.6 \\ \text { ND } & \text { ND } & 0.6 \\ \text { ND } & \text { ND } & 0.6 \\ & & \end{array}$




\section{AT}

\section{$-11-$}

CLIENT: Battelle - Pacific Northwest Labs

DATE REPORTED: $9 / 26 / 88$

DATE REVISED: $10 / 27 / 88$

REPORT TO: PaUl EddY

P.O. NO.: V5228 AD

HERBICIDES IN WATER BY EPA METHOD 8150

\begin{tabular}{|c|c|c|c|c|}
\hline $\begin{array}{l}\text { Laboratory } \\
\text { Sample Nos. }\end{array}$ & $\begin{array}{l}\text { Client } \\
\text { Identification }\end{array}$ & $2,4-D$ & silvex & $2,4,5-\pi$ \\
\hline 816809 & $L F I-P N L I$ & ND & ND & ND \\
\hline 816810 & LF4-PNLI & ND & ND & ND \\
\hline 816811 & IF 4-PNL3 & ND & ND & ND \\
\hline 816812 & LFA-PNLA & ND & ND & ND \\
\hline 816813 & LFA-PNLB & ND & ND & ND \\
\hline DETECTION L & & 1.0 & 0.1 & 0.1 \\
\hline
\end{tabular}

K. 15 
$\boldsymbol{A T}$

$-12-$

CIIENT: Battelle - Pacific Northwest Labs

DATE REPORTED: $9 / 26 / 88$

REPORT TO: Paul Eddy

DATE REVISED: $10 / 27 / 88$

PCB'S \& PESTICIDES IN WAATER BY EPA METHOD 608

Laboratory Sample Nos.

$816809 \quad 816810$

Client Identification

LFI-PNLI

LF 4 -PNLI

DETECTION

LIMIT

$(u g / 1)$

\section{PESTICIDES}

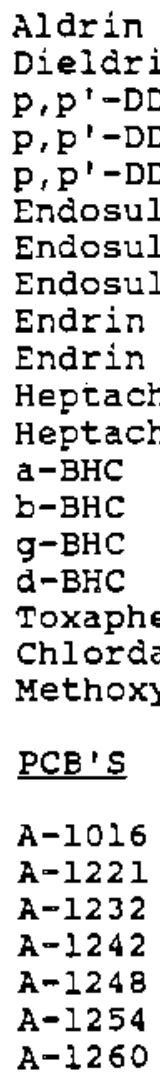

ND

ND

ND

ND

ND

ND

ND

ND

ND

ND

ND

ND

ND

ND

ND

ND

ND

ND

ND

ND

0.04

0.02

0.12

ND

ND

ND

0.04

0.11

0.14

0.04

ND

ND

ND

0.66

0.06

0.23

ND

ND

0.03

0.83

0.03

0.06

0.04

0.09

2.4

0.14

1.76

A- 1260

ND
ND
ND
ND
ND
ND

ND

ND

1.0

1.0

1.0

ND

1.0

ND

1.0

ND

1.0

1.0

ND $=$ Not Detected

All values are reported in ug/l. 
CLIENT: Battelle - Pacific Northwest Labs

DATE REPORTED: $9 / 26 / 88$

REPORT TO: Paul EddY

DATE REVISED: $10 / 27 / 88$

PCB'S PESTICIDES IN WAATER BY EPA METHOD 608

$\begin{array}{lllc}\text { Laboratory Sample Nos. } & 816811 & 816812 & \text { DETECTION } \\ \text { Client Identification } & \text { LF4-PNL3 } & \text { LFA-PNLA } & \text { (ug/1) }\end{array}$

PESTICIDES

Aldrin

Dieldrin

$\mathrm{P}, \mathrm{P}^{\prime}-\mathrm{DDT}$

$P, P^{\prime}-D D E$

$p, p^{\prime}-D D D$

Endosulfar I

Endosulfan II

Endosulfan Sulfate

LFA-PNLA

(ug/1)

Endrin

Endrin Aldehyde

Heptachlor

Heptachlor Epoxide

a-BHC

$\mathrm{b}-\mathrm{BHC}$

g-BHC

d-BHC

Toxaphene

Chlordane

Methoxychlor

PCB'S
ND

ND

ND

ND

ND

ND

ND

ND

ND

ND

ND

ND

ND

ND

ND

ND

ND

ND

ND

ND

ND

ND

ND

ND

ND

ND
ND

ND

ND

ND

ND

ND

ND

ND

ND

ND

ND

ND

ND

ND

ND

ND

ND

ND

ND
0.04

0.02

0.12

0.04

0.11

0.14

0.04

0.66

0.06

0.23

0.03

0.83

0.03

0.06

0.04

0.09

2.4

0.14

1.76

A- 1260

$\mathrm{ND}=$ Not Detected

All values are reported in $u g / l$.

ND

ND

ND

ND

ND

ND

ND
1.0

1.0

1.0

3.0

1.0

1.0

1.0 
$-14-$

CLIENT: Battelle - Pacific Northwest Labs

DATE REPORTED: $\quad 9 / 26 / 88$

DATE REVISED: $10 / 27 / 88$

REPORT TO: PaUl EddY

P.O. NO.: V5228 AD

PCB'S \& PESTICIDES IN WAATER BY EPA METHOD 608

Laboratory Sample Nos.

816813

DETECTION

client Identification

LFA-PNLB

LIMIT

(ug/l)

\section{PESTICIDES}

Aldrin

Dieldrin

$\mathrm{P}, \mathrm{P}^{i}-\mathrm{DDT}$

$p, p^{\prime}-D D E$

$p, p^{\prime}-D D D$

Endosulfan I

Endosulfan II

Endosulfan Sulfate

Endrin

Endrin Aldehyde

Heptachlor

Heptachlor Epoxide

a-BHC

b-BHC

g-BHC

d-BHC

Toxaphene

Chlordane

Methoxychlor

ND

ND

ND

ND

ND

ND

ND

ND

ND

ND

ND

ND

ND

ND

ND

ND

ND

ND

ND

$\underline{P C B}$ 'S
ND

ND

ND

ND

ND

ND

ND
0.04

0.02

0.12

0.04

0.11

0.14

0.04

0.66

0.06

0.23

0.03

0.83

0.03

0.06

0.04

0.09

2.4

0.14

1.76

ND I Not Detected

All values are reported in ug/l.

$\mathrm{JTD} / \mathrm{pb}$

REPORTED BY

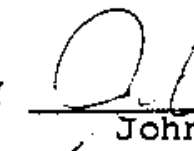

1.0

1.0

1.0

1.0

1.0

1.0

1.0 
Am Test Analytical Results From Early September, 1988 Sampling Event 


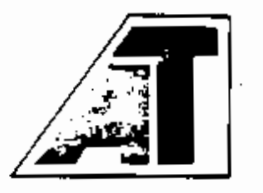

\section{am test inc.}

14603 N.E. 87th St. * REDMOND. WASHINGTON 98052 • 206/885-1664

\section{ANALYSIS REPORT}

CLIENT: Battelie - Pacific Northwest Labs

REPORT TO: PaUl Eddy

P.O. Box 999

Richland, WA 99352

Laboratory Sample Nos.

client Identification

$\mathrm{pH}$

Specific conductance (urhos/crin e $25^{\circ} \mathrm{C}$ )

Total Coliform (MPN/100 ml)

Oil Grease $(\mathrm{mg} / \mathrm{l})$

Total Organic Kalides (ug/l as $\mathrm{Cl}^{-}$)

Gross Beta (pCI/L)

Radium (PCI/L)

Gross Alpha (pCI/l)

Total Dissolved solids (mg/l)

Total organic Carbon ( $\mathrm{mg} / \mathrm{l}$ )

Chloride (mg/l)

Phenol (mg/l)

Nitrate - Nitrogen (mg/1)

Fluoride (mg/l)

sulfate (mg/l)

Cyanide (mg/1)
DATE REPORTED: $9 / 26 / 88$

DATE REVISED: $10 / 27 / 88$

P.O. NO.: V5228 AD

\begin{tabular}{ccc}
817883 & 817884 & 817885 \\
LF1-PNL2 & LF1-PNL3 & LF1-PNL4 \\
\hline 7.17 & 7.26 & $\begin{array}{l}6.39 \\
6.34\end{array}$
\end{tabular}

245.

120.

270 .

265$.

$<2.5$

20.

$<2.5$

$<1.2$

$<1.1$

$<1.2$

$<10$.

$<10$.

$<10$.

$<2$.

$<2$.

$<2$.

$<1$.

1.3

$<$ I.

$<1$.

$<1$.

$<1$.

151.

124.

177.

$\begin{array}{ccc}0.757 & \begin{array}{l}0.677 \\ 0.790]\end{array} & 2.12 \\ 2.74 & 4.60 & 3.65 \\ <0.008 & <0.008 & <0.008 \\ 0.23 & <0.01 & 1.83 \\ 0.72 & <0.01 & <0.01 \\ 3.51 & 12.5 & 23.6 \\ <0.006 & <0.006 & <0.006\end{array}$




\section{A}

CLIENT: Battelle - Pacific Northwest Labs

REPORT TO: Paul Eddy

DATE REPORTED: $9 / 26 / 88$

DATE REVISED: $10 / 27 / 88$

P.O. NO.: V5228 AD

Laboratory Sample Nos.

$817886 \quad 817887$

Client Identificaticn

DUP.

LF4-PNL2 LFI-PNL4

$\mathrm{pH}$

6.58

6.32

Specific conductance (umhos/cme $25^{\circ} \mathrm{C}$ )

440.

270 .

Total coliform (MPN/100 ml)

$<2.5$

$<2.5$

Cil \& Grease (mg/l)

$<1.2<1.2$

Total organic Halides (ug/l as $\mathrm{Cl}^{-}$)

$<10 .<10$.

Gross Beta (PCI/L)

$<1, \quad<1$.

Radium ( $\mathrm{PCI} / \mathrm{L})$

$<1$,

$<1$.

Gross Alpha (pCI/1)

$<1$

$<1$.

Total Dissolved Solids (mg/l)

293.

303$.

Total organic Carbon (mg/l)

2.28

Chloride (mg/l)

4.14

3.30

Phenol (mg/l)

$<0.008<0.008$

Nitrate - Nitrogen (mg/l)

$<0.01$

1.96

$1.92]$

Fluoride (mg/l)

$<0.01<0.01$

$<0.01]$

Sulfate (mg/l)

26.4

23.7

Cyanide (mg/l)

$<0.006$

$24.0]$

$<0.006$ 


$$
-3-
$$

CLIENT: Battelle - Pacific Northwest Labs

DATE REPORTED: $\quad 9 / 26 / 88$

DATE REVISED: $10 / 27 / 88$ REPORT TO: PAUL Eddy

P.O. NO.: V5228 AD

SPIKE RECOVERIES

\begin{tabular}{lccc} 
Parameter & $\begin{array}{c}\text { Sample } \\
\text { Number }\end{array}$ & $\begin{array}{c}\text { Spike } \\
\text { conc. } \\
(\mathrm{mg} / 1)\end{array}$ & $\begin{array}{c}\text { Recovery } \\
(8)\end{array}$ \\
\hline Total organic Halides & 817883 & 250. & 93.1 \\
Total organic Carbon & 817884 & 8.0 & 107.2 \\
Chloride & 817887 & 1.0 & 90.2 \\
Nitrate - Nitrogen & 817887 & 1.0 & 96.2 \\
Fluoride & 817887 & 0.5 & 86.4 \\
Sulfate & 817887 & 1.0 & 100. \\
Cyanide & 817884 & 0.025 & 95.0
\end{tabular}

$\star$ Reported in ug/l as C1-。 
AT

$-4-$

CLIENT: Battelle - Pacific Northwest Labs

DATE REPORTED: $\quad 9 / 26 / 88$

DATE REVISED: $20 / 27 / 88$

REPORT TO: PaUl Eddy

P.O. NO.: V5228 AD

\begin{tabular}{lccc} 
Laboratory Sample Nos. & 817883 & 817884 & 817885 \\
Client Identification & LFI-PNL2 & LFl-PNL3 & LFI-PNL4 \\
\hline Iron & 1.5 & 11. & 2.7 \\
Barium & 0.012 & 0.078 & 0.026 \\
Selenium & $<0.002$ & $<0.002$ & $<0.002$ \\
Cadmium & $<0.002$ & $<0.002$ & $<0.002$ \\
Manganese & 0.080 & 0.368 & 1.07 \\
Calcium & 23. & 13. & 30. \\
Silver & $<0.010$ & $<0.010$ & $<0.010$ \\
Sodium & 5.3 & 3.3 & 5.3 \\
Chromium & 0.073 & 0.10 & 0.11 \\
Lead & $<0.02$ & $<0.02$ & $<0.02$ \\
Arsenic & $<0.002$ & 0.004 & $<0.002$ \\
Mereury & $<0.0002$ & $<0.0002$ & $<0.0002$
\end{tabular}

All values are reported in $\mathrm{mg} / \mathrm{l}$. 
$-5-$

CLIENT: Battelle - Pacific Northwest Labs

DATE REPORTED: $9 / 26 / 88$

DATE REVISED: $20 / 27 / 88$

REPORT TO: PaUl Eddy

P.O. NO.: V5228 AD

\begin{tabular}{lcc} 
Laboratory Sample Nos. & $\begin{array}{l}817886 \\
\text { LF4-PNL2 }\end{array}$ & $\begin{array}{c}817887 \\
\text { DUP. } \\
\text { LFl-PNL4 }\end{array}$ \\
\hline Iron & 15. & 3.4 \\
Barium & 0.106 & 0.033 \\
Selenium & $<0.002$ & $<0.002$ \\
Cadmium & $<0.002$ & $<0.002$ \\
Manganese & 4.3 & 1.0 \\
Calcium & 54. & 29. \\
Silver & $<0.010$ & $<0.010$ \\
Sodium & 10.4 & 4.6 \\
Chromium & 0.20 & 0.10 \\
Lead & $<0.02$ & $<0.02$ \\
Arsenic & 0.003 & $<0.002$ \\
Mercury & $0.009]$ & $<0.0002$
\end{tabular}

All values are reported in $\mathrm{mg} / \mathrm{l}$. 
CLIENT: Battelle - Pacific Northwest Labs

DATE REPORTED: $9 / 26 / 88$

REPORT TO: Paul Eddy

DATE REVISED: $10 / 27 / 88$

P.O. NO.: V5228 AD

GC ANALYSIS OF PURGEABLE HALOCARBONS BY EPA METHOD 601

$\begin{array}{lllc}\text { Laboratory Sample Nos. } & 817883 & 817884 & \text { DETECTION } \\ \text { Client Identification } & \text { LFl-PNL2 } & \text { LFl-PNL3 } & \text { (ug/1) }\end{array}$

(ug/l)

Chloromethane

Vinyl Chloride

Bromomethane

Chloroethane

Dichlorodifluoromethane

Trichlorofluoromethane

1,1-Dichloroethylene

Methylene Chloride

Trans-1,2-Dichlorothylene

1,1-Dichloroethane

chloroform

1,1,1-Trichloroethane

Carbon Tetrachloride

1,2-Dichloroethane

TIlchloroethylene

1,2-Dichloropropane

Dichlorobromomethane

Trans-1,3-Dichloropropene

Cis-1,3-Dichloropropene

ND

ND

ND

ND

ND

ND

ND

ND

ND

ND

ND

ND

ND

ND

ND

ND

ND

ND

ND

1, 1, 2-Trichloroethane

Tetrachloroethylene

Dibromochloromethane

Bromoform

1,1,2,2-Tetrachloroethane

ND = Not Detected.

All results reported in ug/l.
ND

ND

ND

ND

ND

ND

ND

ND

ND

ND

ND

ND

ND

ND

ND

ND

ND

ND

ND

ND

ND

ND

ND

ND 3.
3.
3.
3.
3.
3.
3.
15.
2.
2.
2.
2.
2.
2.
2.
2.
2.
2.
2.
2.
2.
2.
2.
2. 


\section{AI}

CLIENT: Battelle - Pacific Northwest Labs

DATE REPORTED: $9 / 26 / 88$

DATE REVISED: $10 / 27 / 88$

REPORT TO: PAUI Eddy

P.O. NO.: V5228 AD

GC ANALYSIS OF PURGEABLE HALOCARBONS BY EPA METHOD 601

$\begin{array}{lllc}\text { Laboratory Sample Nos. } & 817885 & 817886 & \text { DETECTION } \\ \text { Client Identification } & \text { LFI-PNL4 } & \text { LF4-PNL2 } & \text { (ug/1) }\end{array}$

chloromethane

Vinyl Chloride

(ug/1)

Bromomethane

Chloroethane

Dichlorodifluoromethane

Trichlorofluoromethane

1,1-Dichloroethylene

Methylene Chloride

Trans-1,2-Dichlorothylene

1,1-Dichloroethane

chloroform

1,1,1-Trichloroethane

Carbon Tetrachloride

1,2-Dichloroethane

Trichloroethylene

1,2-Dichloropropane

Dichlorobromomethane

Trans-1,3-Dichloropropene

Cis-1,3-Dichloropropene

1, 1,2-Trichloroethane

Tetrachloroethylene

Dibromochloromethane

Bromoform

1, 1,2,2-Tetrachloroethane

ND

ND

ND

ND

ND

ND

ND

ND

ND

ND

ND

ND

ND

ND

ND

ND

ND

ND

ND

ND

ND

ND

ND

ND
ND

ND

ND

ND

ND

ND

ND

ND

ND

ND

ND

ND

ND

ND

5.7

ND

ND

ND

ND

ND

ND

ND

ND

ND
3.

3.

3.

3.

3.

3.

3.

25.

2.

2.

2 .

2 .

2.

2 .

2.

2 .

2.

2 .

2.

2.

2.

2.

2.

2 .

ND = Not Detected.

All results reported in ug/l. 
CLIENT: Battelle - Pacific Northwest Labs

DATE REPORTED: $9 / 26 / 88$

REPORT TO: Paul Eddy

DATE REVISED: $10 / 27 / 88$

P.O. NO.: V5228 AD

GC ANALYSIS OF PURGEABLE HALOCARBONS BY EPA METHOD 601

Laboratory Sample Nos.

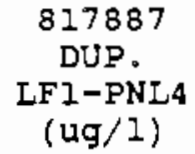

Chloromethane

vinyl Chloride

Bromomethane

Chloroethane

Dichlorodifluoromethane

Trichlorofluoromethane

1,1-Dichloroethylene

Methylene Chloride

Trans-1,2-Dichlorothylene

1,1-Dichloroethane

chloroform

1,1,1-Trichloroethane

Carbon Tetrachloride

1,2-Dichloroethane

Trichloroethylene

1,2-Dichloropropane

Dichlorobromomethane

Trans-1,3-Dichloropropene

Cis-1, 3-Dichloropropene

1, 1, 2-Trichloroethane

Tetrachloroethylene

Dibromochloromethane

Bromoform

$1,1,2,2$-Tetrachloroethane

ND = Not Detected.

$\begin{array}{cc}817887 & \text { DETECTION } \\ \text { SPIKE } & \text { LIMIT } \\ \text { RECOVERY } & \text { (ug/I) }\end{array}$

(z)

$\begin{array}{lrr}\text { ND } & - & 3 . \\ \text { ND } & - & 3 . \\ \text { ND } & - & 3 . \\ \text { ND } & - & 3 . \\ \text { ND } & - & 3 . \\ \text { ND } & - & 3 . \\ \text { ND } & - & 3 . \\ \text { ND } & - & 15 . \\ \text { ND } & 93 . & 2 . \\ \text { ND } & 82 . & 2 . \\ \text { ND } & 80 . & 2 . \\ \text { ND } & 105 . & 2 . \\ \text { ND } & 114 . & 2 . \\ \text { ND } & 75 . & 2 . \\ \text { ND } & 66 . & 2 . \\ \text { ND } & 76 . & 2 . \\ \text { ND } & 78 . & 2 . \\ \text { ND } & 115 . & 2 . \\ \text { ND } & 72 . & 2 . \\ \text { ND } & 63 . & 2 . \\ \text { ND } & 81 . & 2 . \\ \text { ND } & 71 . & 2 . \\ \text { ND } & - & \end{array}$




\section{AT}

$-9-$

CIIENT: Battelle - Pacific Northwest Labs

DATE REPORTED: $9 / 26 / 88$

DATE REVISED: $10 / 27 / 88$

REPORT TO: Paul Eddy

P.O. NO.: V5228 AD

PCB'S \& PESTICIDES IN WATER BY EPA METHOD 608

Laboratory Sample Nos.

817883

817884

client Identification

LF1-PNL2

LFI-PNL3

DETECTION

LIMIT

(ug/1)

\section{PESTICIDES}

Aldrin
Dieldrin
p'P'-DDT
p'p'-DDE
p'p'-DDD
Endosulfan I
Endosulfan II
Endosulfan Sulfate
Endrin
Endrin Aldehyde
Heptachlor
Heptachlor Epoxide
a-BHC
b-BHC
g-BHC
d-BHC
Toxaphene
Chlordane
Methoxychlor
PCB's

ND

ND

ND

ND

ND

ND

ND

ND

ND

ND

ND

ND

ND

ND

ND

ND

ND

ND

ND

A-1016

A-1221

A- 1232

$A-1242$

$A-1248$

$A-1254$

$A-1260$
ND

ND

ND

ND

ND

ND

ND

ND

ND

ND

ND

ND

ND

ND

ND

ND

ND

ND

ND

ND

ND

ND

ND

ND

ND

ND
0.04

0.02

0.12

0.04

0.11

0.14

0.04

0.66

0.06

0.23

0.03

0.83

0.03

0.06

0.04

0.09

2.4

0.14

1.76

ND = Not Detected

All values are reported in ug/l. 
CLIENT: Battelle - Pacific Northwest Labs

DATE REPORTED: $9 / 26 / 88$

REPORT TO: PaUl EddY

DATE REVISED: $10 / 27 / 88$

PCB'S \& PESTICIDES IN WATER BY EPA METHOD 608

Laboratory Sample Nos.

817885

817886

DETECTION

client Identification

LFI-PNL4

LF4-PNLI

LIMIT

(ug/l)

\section{PESTICIDES}

Aldrin

ND

Dieldrin

$p, p^{\prime}-D D T$

$p, p^{\prime}-D D E$

$p, p^{\prime}-D D D$

Endosulfan I

Endosulfan II

Endosulfan Sulfate

Endrin

Endrin Aldehyde

Heptachlor

Heptachlor Epoxide

a-BHC

$\mathrm{b}-\mathrm{BHC}$

g-BHC

d-BHC

Toxaphene

Chlordane

Methoxychlor

ND

ND

ND

ND

ND

ND

ND

ND

ND

ND

ND

ND

ND

ND

ND

ND

ND

ND

PCB'S

A-1016

A-1221

A- 1232

A- 1242

A- 1248

A- 1254

A-1260
ND

ND

ND

ND

ND

ND

ND
ND

ND

ND

ND

ND

ND

ND

ND

ND

ND

ND

ND

ND

ND

ND

ND

ND

ND

ND

ND

ND

ND

ND

ND

ND

ND
0.04

0.02

0.12

0.04

0.11

0.14

0.04

0.66

0.06

0.23

0.03

0.83

0.03

0.06

0.04

0.09

2.4

0.14

1.76

1.0

1.0

1.0

1.0

1.0

1.0

1.0

ND = Not Detected

All values are reported in $\mathrm{ug} / \mathrm{l}$. 
CLIENT: Battelle - Pacific Northwest Labs

DATE REPORTED: $9 / 26 / 88$

DATE REVISED: $10 / 27 / 88$

REPORT TO: PaUl Eddy

P.O. NO.: V5228 AD

PCB'S \& PESTICIDES IN WATER BY EPA METHOD 608

Laboratory Sample Nos.

817887

DUP.

DETECTION

LF $1-P N L 4$

LIMIT

Client Identification

$\mathrm{LF} 1-\mathrm{PNL} 4$

(ug/1)

\section{PESTICIDES}

$\begin{array}{lll}\text { Aldrin } & \text { ND } & 0.04 \\ \text { Dieldrin } & \text { ND } & 0.02 \\ \text { p,P'-DDT } & \text { ND } & 0.12 \\ \text { p, P'-DDE } & \text { ND } & 0.04 \\ \text { P,P'-DDD } & \text { ND } & 0.11 \\ \text { Endosulfan I } & \text { ND } & 0.14 \\ \text { Endosulfan II } & \text { ND } & 0.04 \\ \text { Endosulfan Sulfate } & \text { ND } & 0.66 \\ \text { Endrin } & \text { ND } & 0.06 \\ \text { Endrin Aldehyde } & \text { ND } & 0.23 \\ \text { Heptachlor } & \text { ND } & 0.03 \\ \text { Heptachlor Epoxide } & \text { ND } & 0.83 \\ \text { a-BHC } & \text { ND } & 0.03 \\ \text { b-BHC } & \text { ND } & 0.06 \\ \text { g-BHC } & \text { ND } & 0.04 \\ \text { d-BHC } & \text { ND } & 0.09 \\ \text { Toxaphene } & \text { ND } & 2.4 \\ \text { Ch10Idane } & \text { ND } & 0.14 \\ \text { Methoxychlor } & \text { ND } & 1.76 \\ \text { PCB'S } & & \\ \text { A-1016 } & & \\ \text { A-1221 } & & 1.0 \\ \text { A-I232 } & \text { ND } & 1.0 \\ \text { A-1242 } & \text { ND } & 1.0 \\ \text { A-1248 } & \text { ND } & 1.0 \\ \text { A-I254 } & \text { ND } & 1.0 \\ \text { A-1260 } & \text { ND } & 1.0 \\ \text { ND } & \text { ND } & 1.0\end{array}$

ND = Not Detected

All values are reported in ug/l. 


$$
-12-
$$

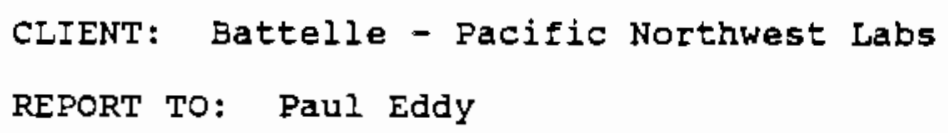

HERBICIDES IN WATER BY EPA METHOD 8150

\begin{tabular}{llccc}
$\begin{array}{l}\text { Laboratory } \\
\text { Sample Nos. }\end{array}$ & $\begin{array}{l}\text { Client } \\
\text { Identification }\end{array}$ & $2,4-D$ & Silvex & $2,4,5-T$ \\
\hline 817883 & LF1-PNL-2 & ND & ND & ND \\
817884 & LFI-PNL-3 & ND & ND & ND \\
817885 & LFI-PNL-4 & ND & ND & ND \\
817886 & LF4-PNL-2 & ND & ND & ND \\
817387 & LFI-PNL-4 & ND & ND & \\
& DUPIICate & & 0.1 & N.1
\end{tabular}

ND $=$ Not Detected

All values are reported in $\mathrm{ug} / \mathrm{l}$.

$\mathrm{JTD} / \mathrm{pb}$

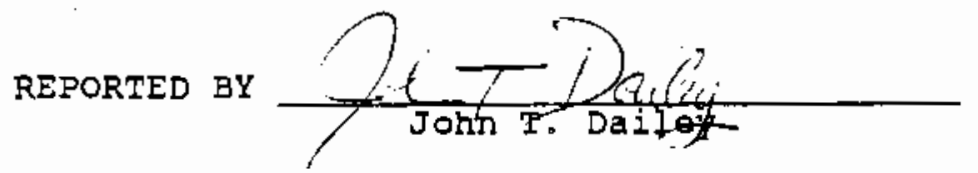


Am Test Analytical Results From Late October, 1988 Sampling Event 


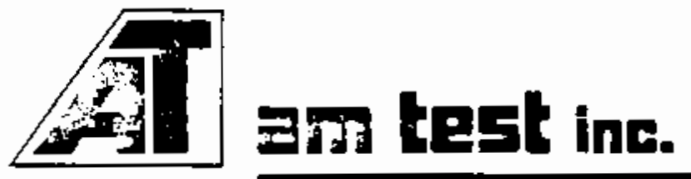

14603 N.E. 87th St. • REDMOND, WASHINGTON $98052 \cdot 206 / 885-1664$

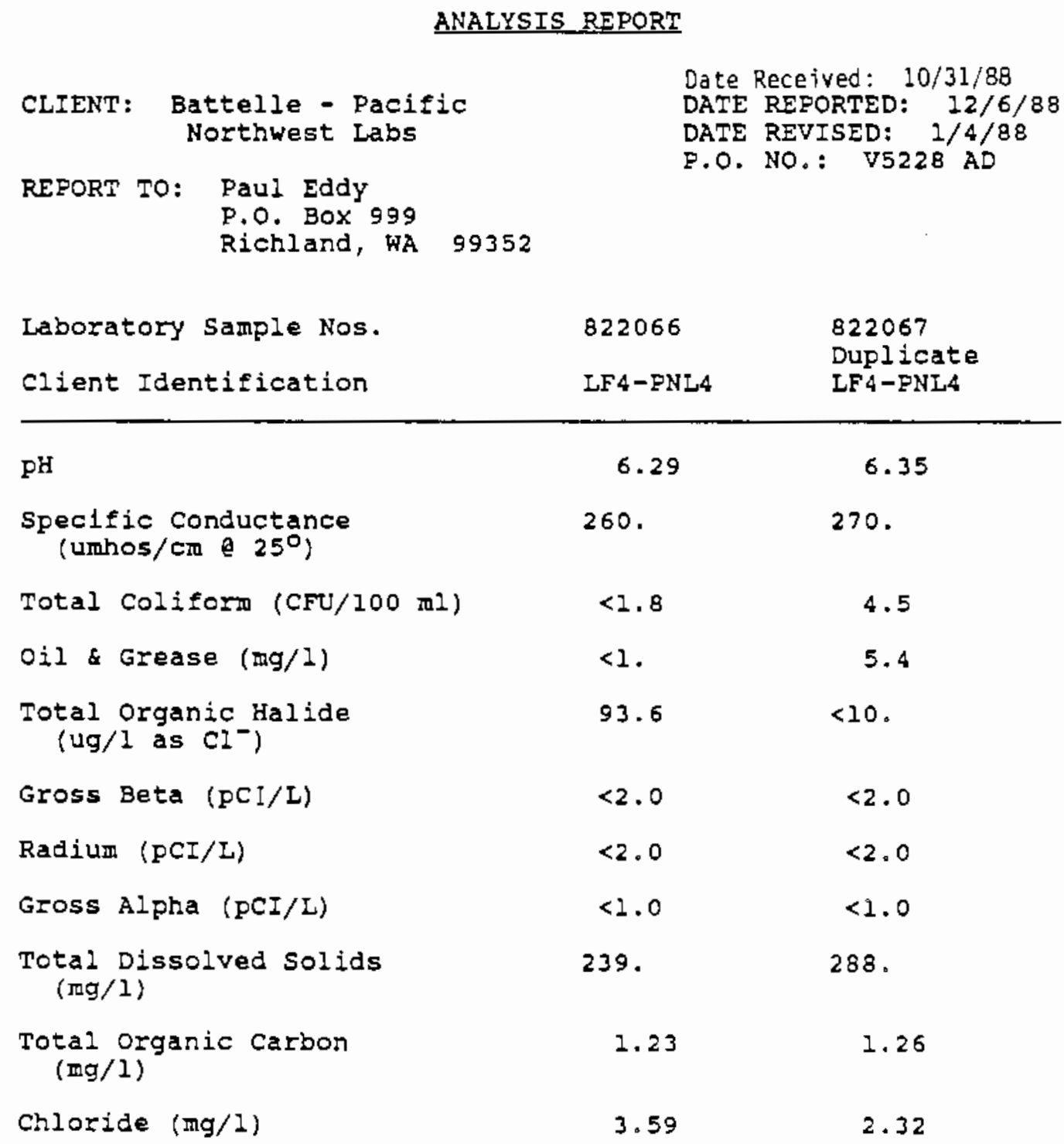




$$
-2-
$$

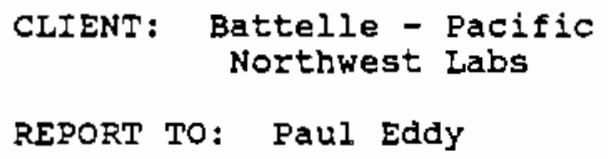

Laboratory Sample Nos.
Client Identification
Phenol (mg/l)
Nitrate - Nitrogen (mg/l)
Fluoride (mg/l)
Sulfate (mg/l)
Cyanide (mg/l)

822066

822067 Duplicate LF4-PNL4 LF4-PNL4

$\begin{array}{ll}<0.008 & <0.008 \\ 1.34 & 1.38 \\ <0.010 & <0.010 \\ 20.5 & 20.9 \\ <0.006 & <0.006\end{array}$




\begin{tabular}{|c|c|c|}
\hline $\begin{array}{c}\text { CLIENT: Battelle - Pacific } \\
\text { Northwest Labs }\end{array}$ & $\begin{array}{l}\text { DATE } \\
\text { DATE } \\
\text { P.O. }\end{array}$ & $\begin{array}{l}\text { REPORTED: } 12 / 6 / 88 \\
\text { REVISED: } 1 / 4 / 88 \\
\text { NO.: V5228 AD }\end{array}$ \\
\hline REPORT TO: Paul EddY & & \\
\hline Laboratory Sample Nos. & 822068 & 822069 \\
\hline client Identification & LF4-PNL5 & LF4-PNL6A \\
\hline $\mathrm{pH}$ & 6.67 & 6.88 \\
\hline $\begin{array}{c}\text { Specific Conductance } \\
\left.\text { (umbos/cm } 25^{\circ}\right)\end{array}$ & 92 . & 124. \\
\hline Total coliform (CFU/100 ml) & 23. & 49. \\
\hline Oiz \& Grease $(m g / 1)$ & 3.2 & 4.2 \\
\hline $\begin{array}{l}\text { Total organic Halide } \\
\text { (ug/l as Cl-) }\end{array}$ & 102. & 202. \\
\hline Gross Beta $(\mathrm{pCI} / \mathrm{L})$ & $<2.0$ & $<2.0$ \\
\hline Radium $(\rho C I / L)$ & $<2.0$ & $<2.0$ \\
\hline Gross Alpha (pCI/L) & $<1.0$ & $<1.0$ \\
\hline $\begin{array}{l}\text { Total Dissolved Solids } \\
(\mathrm{mg} / \mathrm{l})\end{array}$ & 150 . & 275. \\
\hline $\begin{array}{l}\text { Total organic Carbon } \\
(\mathrm{mg} / \mathrm{l})\end{array}$ & $\begin{array}{l}0.678 \\
0.752\end{array}$ & 0.693 \\
\hline Chloride (mg/l) & 1.41 & $\begin{array}{l}2.94 \\
2.90]\end{array}$ \\
\hline
\end{tabular}


AT

$-4-$

CIIENT: Battelle - Pacific

DATE REPORTED: $12 / 6 / 88$

Northwest Labs

REPORT TO: PaUl Eddy

DATE REVISED: $1 / 4 / 88$

P.O. NO.: V5228 AD

Laboratory Sample Nos.

822068

822069

client Identification

LF 4-PNL5

LF4-PNL6A

Pherol (mg/1)

$<0.008$

$<0.008$

Nitrate - Nitrogen (mg/l)

0.416

0.388

$0.366]$

Fluoride (mg/l)

$<0.010$

$<0.010$

sulfate (mg/1)

7.20

6.97

$6.72]$

Cyaride (mg/ 1 )

$<0.006$

$<0.006$

$<0.006$ ] 
CLIENT: Battelle - Pacific

Northuest Labs

REPORT TO: PaUl Eddy
DATE REPORTED: $12 / 6 / 88$

DATE REVISED: $1 / 4 / 88$

P.O. NO.: V5228 AD

SPIKE RECOVERIES

\begin{tabular}{lccr} 
Parameter & $\begin{array}{c}\text { Sample } \\
\text { Number }\end{array}$ & $\begin{array}{c}\text { Spike } \\
\text { Conc. } \\
(\text { ng/ })\end{array}$ & $\begin{array}{c}\text { Recovery } \\
(8)\end{array}$ \\
\hline Total organic Carbon & 822069 & 8.0 & 100.3 \\
Phenol & 822069 & $10 . \star$ & 98.2 \\
Nitrate - Nitrogen & 822069 & 0.25 & 97.7 \\
Fluoride & 822069 & 0.25 & 109.7 \\
Sulfate & 822069 & 0.5 & 107.6 \\
Cyanide & 822069 & 0.025 & 93.5
\end{tabular}

$\star$ Reported in ug. 
$-6-$

CLIENT: Battelle - Pacific Northwest Labs

REPORT TO: PaUl Eddy
DATE REPORTED: $12 / 6 / 86$

DATE REVISED: $1 / 4 / 88$

P.O. NO.: V5228 AD

Laboratory Sample Nos.

client Identification

822066

822067

Duplicate

LF 4-PNL4

LF 4-PNL4

Iron (mg/l)

0.22

0.073

Barium (mg/l)

0.011

0.010

Selenium (mg/1)

$<0.002$

$<0.002$

Cadmium (mg/1)

$<0.0005$

$<0.0005$

Manganese (mg/1)

0.027

0.020

Calciurn (mg/l)

32 .

32.

silver (ng/ $/ 1$ )

0.0008

0.0016

Sodium (mg/1)

B. 1

8.1

Chromium (mg/l)

0.013

0.012

Lead (mg/1)

$<0.001$

$<0.001$

Arsenic (mg/ $)$

$<0.002$

$<0.002$

Mercury (mg/1) 
CLIENT: Battelle - Pacific Northwest Labs

REPORT TO: PaUl EddY

Laboratory Sample Nos.

client Identification

Iron $(\pi g / 1)$

Barium (mg/I)

Selenium (mg/l)

Cadmium (mg/l)

Manganese (mg/l)

Calcium (mg/l)

Silver $(m g / 1)$

Sodium $(\mathrm{mg} / \mathrm{I})$

Chromiua (mg/1)

Lead (mg/1)

Arsenic (mg/l)

Mercury (mg/1)
DATE REPORTED: $12 / 6 / 88$

DATE REVISED: $1 / 4 / 88$

P.O. NO.: V5228 AD

LF 4-PNL5

LF4-PNL6A

0.27

6.6

$7.5]$

0.092

0.1043

0.006

$<0.002$

$<0.002$ ]

$<0.002$

$<0.0005$

0.0010 ]

$<0.0005$

0.333

0.007

10.

11.]

6.0

0.0018

0.0009 ]

0.0016

3.4

6.0

$4.4]$

$<0.006$

0.011 ]

0.004

$0.004]$

$<0.001$

0.005

$0.006]$

$<0.002$

$<0.0002$

$<0.0002$ 
$-8-$

$\begin{array}{ll}\text { CLIENT: Battelle - Pacific } & \text { DATE REPORTED: } 12 / 6 / 88 \\ \text { Northwest Labs } & \text { DATE REVISED: } 1 / 4 / 88 \\ \text { REPORT TO: Paul Eddy } & \text { P.O. NO.: V5228 AD }\end{array}$

SPIKE RECOVERIES

\begin{tabular}{lccc} 
Parameter & $\begin{array}{c}\text { Sample } \\
\text { Number }\end{array}$ & $\begin{array}{c}\text { Spike } \\
\text { Conc. } \\
(\text { mg/ })\end{array}$ & $\begin{array}{c}\text { Recovery } \\
(8)\end{array}$ \\
\hline Iron & 822069 & 1.0 & 85. \\
Bartum & 822069 & 1.0 & 104. \\
Selenium & 822069 & 0.25 & 106. \\
Cadmium & 822069 & 0.5 & 96. \\
Manganese & 822069 & 1.0 & 102. \\
Calcium & 822069 & 10. & 100. \\
Silver & 822069 & 0.040 & 93.2 \\
Sodium & 822069 & 5.0 & 92. \\
Chromium & 822069 & 1.0 & 200. \\
Lead & 822069 & 1.0 & 93. \\
Arsenic & 822069 & 0.5 & 96. \\
Mercury & 822067 & 0.0050 & 100.
\end{tabular}


CLIENT: Battelle - Pacific
Northwest Labs

REPORT TO: Paul Eddy
DATE REPORTED: $12 / 6 / 8 B$

DATE REVISED: $2 / 4 / 88$

P.O. NO.: V5228 AD

GC ANALYSIS OF PURGEABLE HALOCARBONS BY EPA METHOD 601

Laboratory Sample Nos.

BLANK

client Identification

DETECTION

IIMIT

(ug/1)

Chloromethane

vinyl Chloride

Bromomethane

chloroethane

Dichlorodifluoromethane

Trichlorofluoromethane

1,1-Dichloroethylene

Methylene Chloride

Trans-1, 2-Dichloroethylene

1, 1-Dichloroethane

Chloroforn

1,1,1-Trichloroethane

Carbon Tetrachloride

1,2-Dichloroethane

Trichloroethylene

1,2-Dichloropropane

Dichlorobromomethane

Trans-1,3-Dichloropropene

Cis-1,3-Dichloropropene

1, 1, 2-Irichloroethane

Tetrachloroethylene

Dibromochloromethane

Bromoform

$1,1,2,2$-Tetrachloroethane

ND
ND
ND
ND
ND
ND
8.0
ND
ND
1.0
ND
ND
ND
ND
ND
ND
ND
ND
ND
ND
ND
ND
ND

2.0

2.0

2.0

2.0

2.0

2.0

2. 0

1.5

0.7

0.7

0.7

0.7

0.7

0.7

0.7

0.7

0.7

0.7

0.7

0.7

0.7

0.7

0.7

0.7

All results are reported in ug/l. ND = Not Detected. 
CLIENT: Battelle - Pacific Northwest Labs

REPORT TO: Paul Eddy
DATE REPORTED: 12/6/88

DATE REVISED: $1 / 4 / 88$

P.O. NO.: V5228 AD

GC ANALYSIS OF PURGEABIE HALOCARBONS BY EPA METHOD 601

Laboratory Sample Nos.

822066

822067 Duplicate

DETECTION

Client Identification

LE4-PNL4

LF 4-PNL4

IIMIT

$(u g / 1)$

Chloromethane

Vinyl chloride

ND

Bromomethane

Chloroethane

ND

ND

ND

ND

ND

irichlorofluoromethane

1,1-Dichloroethylene

Methylene Chloride

ND

Trans-1,2-Dichloroethylene

1,1-Dichloroethane

Chloroform

1,1,1-Trichloroethane

carbon Tetrachloride

1,2-Dichloroethane

Trichloroethylene

26.

0.8

ND

ND

ND

ND

ND

22 .

1,2-Dichloropropane

Dichlorobromomethane

Trans-1, 3-Dichloropropene

ND

ND

ND

cis-1,3-Dichloropropene

ND

1, 1,2-Trichloroethare

Tetrachloroethylene

ND

ND

Dibromochloromethane

ND

Bromoform

$1,1,2,2$-Tetrachloroethane

ND

ND

ND

ND

ND

ND

ND

ND

ND

2.0

0.8

ND

ND

ND

ND

ND

19.

ND

ND

ND

ND

ND

ND

ND

ND

ND
2.0

2.0

2.0

2.0

2.0

2.0

2. 0

1. 5

0.7

0.7

0.7

0.7

0.7

0.7

0.7

0.7

0.7

0.7

0.7

0.7

0.7

0.7

0.7

0.7

All results are reported in ug/l. ND $=$ Not Detected. 


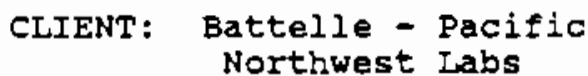

REPORT TO: PaUl EddY
DATE REPORTED: $12 / 6 / 88$

DATE REVISED: $1 / 4 / 88$

P.O. NO.: V5228 AD

GC ANALYSIS OF PURGEABLE HALOCARBONS BY EPA METHOD 601

Laboratory Sample Nos.

822068

822069

DETECTION

Client Identification

LF4 -PNL5

LF4-PNL6A

LIMIT

(ug/1)

Chloromethane

Vinyl Chloride

Bromomethane

Chloroethane

Dichlorodifluoromethane

Trichlorofluoromethane

1,1-Dichloroethylene

Methylene Chloride

Trans-1,2-Dichloroethylene

1,1-Dichloroethane

Chloroform

1,1,1-Trichloroethane

Carbon Tetrachloride

1,2-Dichloroethane

Trichloroethylene

1,2-Dichloropropane

Dichlorobromomethane

Trans-1,3-Dichloropropene

Cis-1,3-Dichloropropene

1,1,2-Irichloroethane

Tetrachloroethylene

Dibromochloromethane

Bromoform

$1,1,2,2$-Tetrachloroethane

$\begin{array}{lll}\text { ND } & \text { ND } & 2.0 \\ \text { ND } & \text { ND } & 2.0 \\ \text { ND } & \text { ND } & 2.0 \\ \text { ND } & \text { ND } & 2.0 \\ \text { ND } & \text { ND } & 2.0 \\ \text { ND } & \text { ND } & 2.0 \\ \text { ND } & \text { ND } & 2.0 \\ \text { 1.5 } & 2.5 & 1.5 \\ \text { ND } & \text { ND } & 0.7 \\ \text { ND } & \text { ND } & 0.7 \\ \text { ND } & 0.7 & 0.7 \\ \text { ND } & \text { ND } & 0.7 \\ \text { ND } & \text { ND } & 0.7 \\ \text { ND } & \text { ND } & 0.7 \\ \text { ND } & \text { ND } & 0.7 \\ \text { ND } & \text { ND } & 0.7 \\ \text { ND } & \text { ND } & 0.7 \\ \text { ND } & \text { ND } & 0.7 \\ \text { ND } & \text { ND } & 0.7 \\ \text { ND } & \text { ND } & 0.7 \\ \text { ND } & \text { ND } & 0.7 \\ \text { ND } & \text { ND } & 0.7 \\ \text { ND } & \text { ND } & 0.7 \\ \text { ND } & \text { ND } & 0.7\end{array}$

All results are reported in $u g / l$.

ND = Not Detected. 
CIIENT: Battelle - Pacific

Northwest Labs

REPORT TO: Paul Eddy
DATE REPORTED: $12 / 6 / 88$

DATE REVISED: $1 / 4 / 88$

P.O. NO.: V5228 AD

\begin{tabular}{lccc}
$\begin{array}{l}\text { Laboratory Sample Nos. } \\
\text { Client Identification }\end{array}$ & $\begin{array}{c}822066 \\
\text { LF4-PNL4 }\end{array}$ & $\begin{array}{c}822067 \\
\text { Duplicate } \\
\text { LF4 -PNL4 }\end{array}$ & $\begin{array}{c}\text { DETECTION } \\
\text { LIMIT } \\
\text { (Ug/1) }\end{array}$ \\
\hline PCB's* & ND & ND & 0.5 \\
Endrin & ND & ND & 0.06 \\
Lindane & ND & ND & 0.04 \\
Methoxychlor & ND & ND & 0.5 \\
Toxaphene & ND & ND & 5.0
\end{tabular}

«PCB's analyzed for Arochlor 1016, 1221, 1232, 1242, 1248, 1254, 1260 , 1262 .

ND $=$ Not Detected.

All values are reported in $u g / l$. 
CLIENT: $\begin{gathered}\text { Battelle - Pacific } \\ \text { Northwest Labs }\end{gathered}$
.

DATE REPORTED: $12 / 6 / 88$

DATE REVISED: $1 / 4 / 88$

REPORT TO: Paul EddY

P.O. NO.: V5228 AD

Laboratory Sample Nos.

822068

822069

DETECTION

client Identification

LF4-PNL5

IF4-DNI6A

LIMIT

PCB's*

ND

ND

0.5

Endrin

ND

ND

0.06

Lindane

ND

ND

0.04

Methoxychlor

ND

ND

0.5

Toxaphene

ND

ND

5.0

^PCB's analyzed for Arochlor 1016, 1221, 1232, 1242, 1248, 1254, 1260 , \& 1262 .

ND $=$ Not Detected.

All values are reported in $u g / l$. 
CLIENT: $\begin{gathered}\text { Battelle - Pacific } \\ \text { Northwest Labs }\end{gathered}$

REPORT TO: PaUl Eddy
DATE REPORTED: $12 / 6 / 88$

DATE REVISED: $1 / 4 / 88$

P.O. NO.: V5228 AD

HERBICIDES IN WATER BY EPA METHOD 8150

\begin{tabular}{llccc}
$\begin{array}{l}\text { Laboratory } \\
\text { Sample Nos. }\end{array}$ & $\begin{array}{l}\text { Client } \\
\text { Identification }\end{array}$ & $\begin{array}{c}2,4-D \\
(\mathrm{ug} / 1)\end{array}$ & $\begin{array}{c}\text { Silvex } \\
(\mathrm{ug} / \mathrm{l})\end{array}$ & $\begin{array}{r}2,4,5-\mathrm{T} \\
(\mathrm{ug} / 1)\end{array}$ \\
\hline 822066 & LF4-PNL4 & ND & ND & ND \\
822067 & $\begin{array}{l}\text { LF4-PNL4 } \\
\text { Duplicate }\end{array}$ & ND & ND & ND \\
822068 & LF4-PNL5 & ND & ND & ND \\
822069 & LF4-PNL6A & ND & ND & ND \\
DETECTION LIMIT & 1.0 & 0.1 & 0.1
\end{tabular}

ND = Not Detected.

$\mathrm{JTD} / \mathrm{pb}$

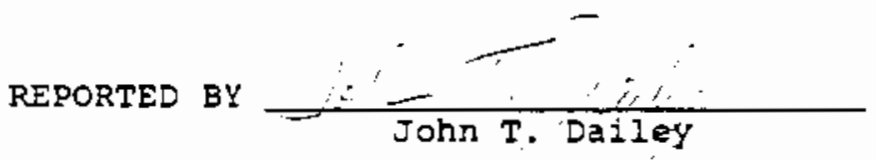


Am Test Analytical Results From Middle December, 1988 Sampling Event 


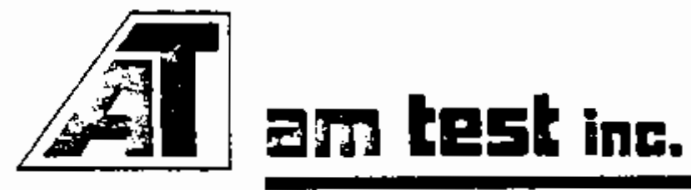

14603 N.E. 87th St. • REDMOND, WASHINGTON 98052 • 206/885-1664

\section{ANALYSIS REPORT}

CIIENT: Battelle Northwest Laboratories

DATE RECEIVED: $12 / 16 / 88$

REPORT TO: George Last

P.O. Box 999

Richland, WA 99352

DATE REPORTED: $12 / 31 / 88$

PROJECT: Fort Lewis

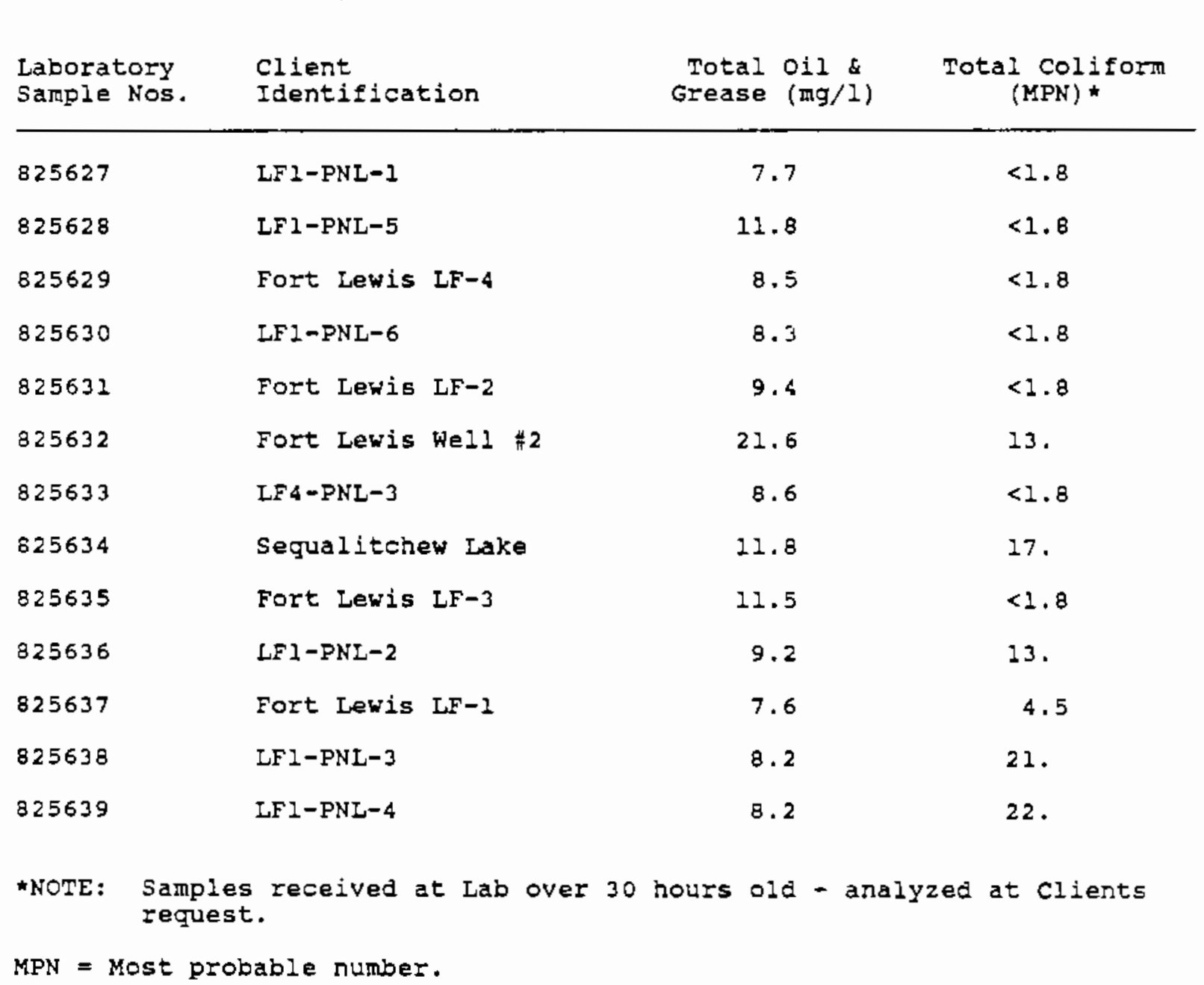

K. 45 


\section{AT}

CIIENT: Battelle Northwest Laboratories

REPORT TO: George Last

\author{
DATE RECEIVED: $12 / 16 / 88$ \\ DATE REPORTED: $12 / 31 / 88$ \\ PROJECT: Fort Lewis
}

GC/MS SEMIVOLATILES BY EPA METHOD 625

\author{
Laboratory Sample Nos. \\ client Identification
}

BNA

Method Method

Blank Blank

$12 / 19 / 88$
$12 / 21 / 88$
825627

LFI-PNL 1

DETECTION

LINIT
(Ug/I)
Ariline

2-Chlorophenol

Bis (2-Chloroethyl) Ether

Phenol

1,3-Dichlorobenzene

1,4-Dichlorobenzene

1,2-Dichlorobenzene

Benzyl Alcohol

Bis (2-Chloroisopropyl) Ether

2-Methylphenol

Hexachloroethane

$\mathrm{N}-\mathrm{Nitrosodipropylamine}$

Nitrobenzene

4 -Methyl phenol

Isopherone

2-Nitrophenol

2, 4-Dimethylphenol

Bis (2-chloroethoxy) Methane

2,4-Dichlorophenol

1,2,4-Trichlorobenzene

Naphthalene

Benzoic Acid

4-chloroaniline

Hexachlorobutadiene

2-Methylnaphthalene

4-Chloro-3-Methylphenol

Hexachlorocyclopentadiene

2, 4, 6-Trichlorophenol

$2,4,5-T r i c h l o r o p h e n o l$
ND

ND

ND

ND

ND

ND

ND

ND

ND

ND

ND

ND

ND

ND

ND

ND

ND

ND

ND

ND

ND

ND

ND

ND

ND

ND

ND

ND

ND
ND

ND

ND

ND

ND

ND

ND

ND

ND

ND

ND

ND

ND

ND

ND

ND

ND

ND

ND

ND

ND

ND

ND

ND

ND

ND

ND

ND

ND
ND

ND

ND

ND

ND

ND

ND

ND

ND

ND

ND

ND

ND

ND

ND

ND

ND

ND

ND

ND

ND

ND

ND

ND

ND

ND

ND

ND

ND
1.0

1.0

1.0

1.0

1.0

1.0

1. 0

1.0

1.0

1. 0

1.0

1.0

1.0

1.0

1.0

1. 0

1. 0

1. 0

1. 0

1.0

1.0

5.0

1.0

1. 0

1.0

1. 0

2.0

2.0

2.0

ND = Not Detected.

All values are reported in ug/l. 


\title{
AT
}

\begin{abstract}
$-3-$
CLIENT: Battelle Northwest Laboratories

DATE RECEIVED: $12 / 16 / 88$

REPORT TO: George Last

DATE REPORTED: $12 / 31 / 88$

PROJECT: Fort Lewis
\end{abstract}

GC/MS SEMIVOLATILES BY EPA METHOD 625

Laboratory Sample Nos.

BNA

Method Method

client Identification

Blank Blank

$12 / 19 / 88 \quad 12 / 21 / 88$
825627 DETECTION LIMIT
LFI-PNL-I (Ug/I)

\begin{tabular}{|c|c|c|c|c|}
\hline 2-chloronaphthalene & ND & ND & ND & 1.0 \\
\hline 2-Nitroaniline & ND & ND & ND & 2.0 \\
\hline Acenaphthylene & ND & ND & ND & 1.0 \\
\hline Dimethyl phthalate & ND & ND & ND & 1.0 \\
\hline 2,6-Dinitrotoluene & ND & ND & ND & 2.0 \\
\hline Aceraphthene & ND & ND & ND & 1.0 \\
\hline 3-Nitroaniline & ND & ND & ND & 2.0 \\
\hline 2,4-Dinitrophenol & ND & ND & ND & 5.0 \\
\hline Dibenzofuran & ND & ND & ND & 1.0 \\
\hline 2,4-Dinitrotoluene & ND & ND & ND & 2.0 \\
\hline 4-Nitrophenol & ND & ND & ND & 4.0 \\
\hline Fluorene & ND & ND & ND & 1.0 \\
\hline 4-Chlorophenyl Phenyl Ether & ND & ND & ND & 1.0 \\
\hline Diethyl Phthalate & ND & ND & ND & 1.0 \\
\hline 4-Nitroaniline & ND & ND & ND & 3.0 \\
\hline 2-Methyl-4,6-Dinitrophenol & ND & ND & ND & 5.0 \\
\hline N-Nitrosodiphenylanine & ND & ND & ND & 1.0 \\
\hline Azobenzene & ND & ND & ND & 1.0 \\
\hline 4-8romophenyl Phenyl Ether & ND & ND & ND & 2.0 \\
\hline Hexachlorobenzene & ND & ND & ND & 2.0 \\
\hline Pentachlorophenol & ND & ND & ND & 5.0 \\
\hline Phenanthrene & ND & ND & ND & 1.0 \\
\hline Anthracene & ND & ND & ND & 1.0 \\
\hline Di-N-Butyl Phthalate & ND & ND & ND & 1.0 \\
\hline Fluoranthene & ND & ND & ND & 1.0 \\
\hline Pyrene & ND & ND & ND & 1.0 \\
\hline Benzidine & ND & ND & ND & 3.0 \\
\hline Benzy Butyl phthalate & ND & ND & ND & 1.0 \\
\hline Benzo (a) Anthracene & ND & ND & ND & 1.0 \\
\hline
\end{tabular}

ND = Not Detected.

All values are reported in $\mathrm{ug} / \mathrm{l}$. 


\section{AT}

CLIENT: Battelle Northwest Laboratories

DATE RECEIVED:

$12 / 26 / 88$

DATE REPORTED: $12 / 31 / 88$

REPORT TO: George Last

PROJECT: Fort Lewis

GC/MS SEMIVOLATILES BY EPA METHOD 625

$\begin{array}{llll}\text { Laboratory Sample Nos. } & \text { BNA } & \text { BNA } & 825627 \\ \text { Client Identification } & \text { Method } & \text { Method } & \\ & \text { Blank } & \text { Blank } & \text { LFl-PNL·1 } \\ & 12 / 19 / 88 & 12 / 21 / 88 & \end{array}$

Compounds

Concentration (ug/1)

DETECTION

LIMIT

(ug/1)

\begin{tabular}{lllll}
\hline Chrysene & ND & ND & ND & 1.0 \\
$3,3-D i c h l o r o b e n z i d i n e$ & ND & ND & ND & 3.0 \\
Bis (2-Ethylhexy) Phthalate & ND & ND & ND & 1.0 \\
Di-N-Octyl Phthalate & ND & ND & ND & 1.0 \\
Benzo (b) Fluoranthene & ND & ND & ND & 1.0 \\
Benzo (k) Fluoranthene & ND & ND & ND & 1.0 \\
Benzo (a) Pyrene & ND & ND & ND & 1.0 \\
Indeno $(1,2,3-c d)$ Pyrene & ND & ND & ND & 1.0 \\
Dibenzo (a,h) Anthracene & ND & ND & ND & 1.0 \\
Benzo $(g, h, i)$ Perylene & ND & ND & ND & 1.0
\end{tabular}

Surrogate compounds

Recovery (f)

AMOUNT

SPIKED

(ug/1)

2-Fluorophenol

D6-Phenol

D5-Nitrobenzene

2-Fluorobiphenyl

2, 4, 6-Tribromophenol

D24-Terphenyl

37.

26.

48.

46 .

45.

91 .
46

32 .

73.

74 .

82.

200 .
42.

26.

72.

78 .

80.

95 .
50 .

50 .

25 .

25.

50 .

25 .

ND $=$ Not Detected. 


\section{Aा}

$-5-$

CLIENT: Battelle Northwest Laboratories

DATE RECEIVED: $12 / 16 / 88$

REPORT TO: George Last

DATE REPORTED: $12 / 31 / 88$

PROJECT: FOrt Lewis

GC/MS SEMIVOLATIIES BY EPA METHOD 625

Laboratory Sample Nos.

$825627 \quad 825628 \quad 825629$

Dup.

client Identification

LFI-PNL-1 LFI-PNL-5 Ft. Lewis (Ug/1)

$\mathrm{LF}-4$

Aniline

2-Chlorophenol

Bis (2-chloroethyl) Ether

Phenol

1,3-Dichlorobenzene

1,4-Dichlorobenzene

1,2-Dichlorobenzene

Benzyl Alcohol

Bis (2-Chloroisopropyl) Ether

2-Methylphenol

Hexachioroethane

N-Nitrosodipropylamine

Nitrobenzene

4-Methylphenol

Isopherone

2-Nitrophenol

2, 4-Dimethylphenol

Bis (2-chloroethoxy) Methane

2,4-Dichlorophenol

$1,2,4$-Trichlorobenzene

Naphthalene

Benzoic Acid

4-Chloroaniline

Hexachlorobutadiene

2-Methylnaphthalene

4-Chloro-3-Methylphenol

Hexachlorocyclopentadiene

$2,4,6$-Trichlorophenol

$2,4,5$-rrichlorophenol

ND $=$ Not Detected.

All values are reported in ug/l.
ND

ND

ND

ND

ND

ND

ND

ND

ND

ND

ND

ND

ND

ND

ND

ND

ND

ND

ND

ND

ND

ND

ND

ND

ND

ND

ND

ND

ND

\section{ND}

ND

ND

ND

ND

ND

ND

ND

ND

ND

ND

ND

ND

ND

ND

ND

ND

ND

ND

ND

ND

ND

ND

ND

ND

ND

ND

ND

ND
ND

ND

ND

ND

ND

ND

ND

ND

ND

ND

ND

ND

ND

ND

ND

ND

ND

ND

ND

ND

ND

ND

ND

ND

ND

ND

ND

ND

ND
1.0

1.0

1.0

1.0

1.0

1.0

1.0

1.0

1.0

1.0

1.0

1.0

1.0

1.0

1.0

1.0

3.0

1.0

1.0

1.0

1.0

5.0

1.0

1.0

1.0

1. 0

2. 0

2.0

2.0 


\title{
AI
}$$
-6-
$$

CLIENT: Battelle Northwest Laboratories

DATE RECEIVED: $12 / 16 / 88$

REPORT TO: George Last

DATE REPORTED: $12 / 31 / 88$

PROJECT: Fort Lewis
\end{abstract}

GC/MS SEMIVOLATILES BY EPA METHOD 625

$\begin{array}{lllll}\text { Laboratory Sample Nos. } & 825627 & 825628 & 825629 & \text { DETECTION } \\ \text { Client Identification } & \text { Dup. } & & & \text { LIMIT } \\ & \text { LFI-PNL-1 } & \text { LFl-PNL-5 } & \text { Ft. Lewis } & \text { (Ug/1) } \\ & & & \text { LF-4 } & \end{array}$

2-Chioronaphthalene

2-Nitroaniline

Acenaphthylene

Dimethyl Phthalate

2,6-Dinitrotoluene

Acenaphthene

3-Nitroaniline

2,4-Dinitrophenol

Dibenzofuran

2,4-Dinitrotoluene

4-Nitrophenol

Fluorene

4-Chlorophenyl Phenyl Ether

Diethyl Phthalate

4-Nitroaniline

2-Methyl-4,6-Dinitrophenol

$\mathrm{N}-\mathrm{Nitrosodiphenylamine}$

Azobenzene

4-Bromophenyl Phenyl Ether

Hexachlorobenzene

Pentachlorophenol

Phenanthrene

Anthracene

Di-N-Butyl Phthalate

Fluoranthene

Pyrene

Benzidine

Benzy Butyl Phthalate

Benzo (a) Anthracene

$\begin{array}{llll}\text { ND } & \text { ND } & \text { ND } & 1.0 \\ \text { ND } & \text { ND } & \text { ND } & 1.0 \\ \text { ND } & \text { ND } & \text { ND } & 1.0 \\ \text { ND } & \text { ND } & \text { ND } & 1.0 \\ \text { ND } & \text { ND } & \text { ND } & 2.0 \\ \text { ND } & \text { ND } & \text { ND } & 1.0 \\ \text { ND } & \text { ND } & \text { ND } & 2.0 \\ \text { ND } & \text { ND } & \text { ND } & 5.0 \\ \text { ND } & \text { ND } & \text { ND } & 1.0 \\ \text { ND } & \text { ND } & \text { ND } & 2.0 \\ \text { ND } & \text { ND } & \text { ND } & 4.0 \\ \text { ND } & \text { ND } & \text { ND } & 1.0 \\ \text { ND } & \text { ND } & \text { ND } & 1.0 \\ \text { ND } & \text { ND } & \text { ND } & 1.0 \\ \text { ND } & \text { ND } & \text { ND } & 3.0 \\ \text { ND } & \text { ND } & \text { ND } & 5.0 \\ \text { ND } & \text { ND } & \text { ND } & 1.0 \\ \text { ND } & \text { ND } & \text { ND } & 1.0 \\ \text { ND } & \text { ND } & \text { ND } & 2.0 \\ \text { ND } & \text { ND } & \text { ND } & 2.0 \\ \text { ND } & \text { ND } & \text { ND } & 5.0 \\ \text { ND } & \text { ND } & \text { ND } & 1.0 \\ \text { ND } & \text { ND } & \text { ND } & 1.0 \\ \text { ND } & \text { ND } & \text { ND } & 1.0 \\ \text { ND } & \text { ND } & \text { ND } & 1.0 \\ \text { ND } & \text { ND } & \text { ND } & 1.0 \\ \text { ND } & \text { ND } & \text { ND } & 3.0 \\ \text { ND } & \text { ND } & \text { ND } & 1.0 \\ \text { ND } & \text { ND } & \text { ND } & 1.0\end{array}$

ND $=$ Not Detected.

All values are reported in ug/l. 


\section{AT}

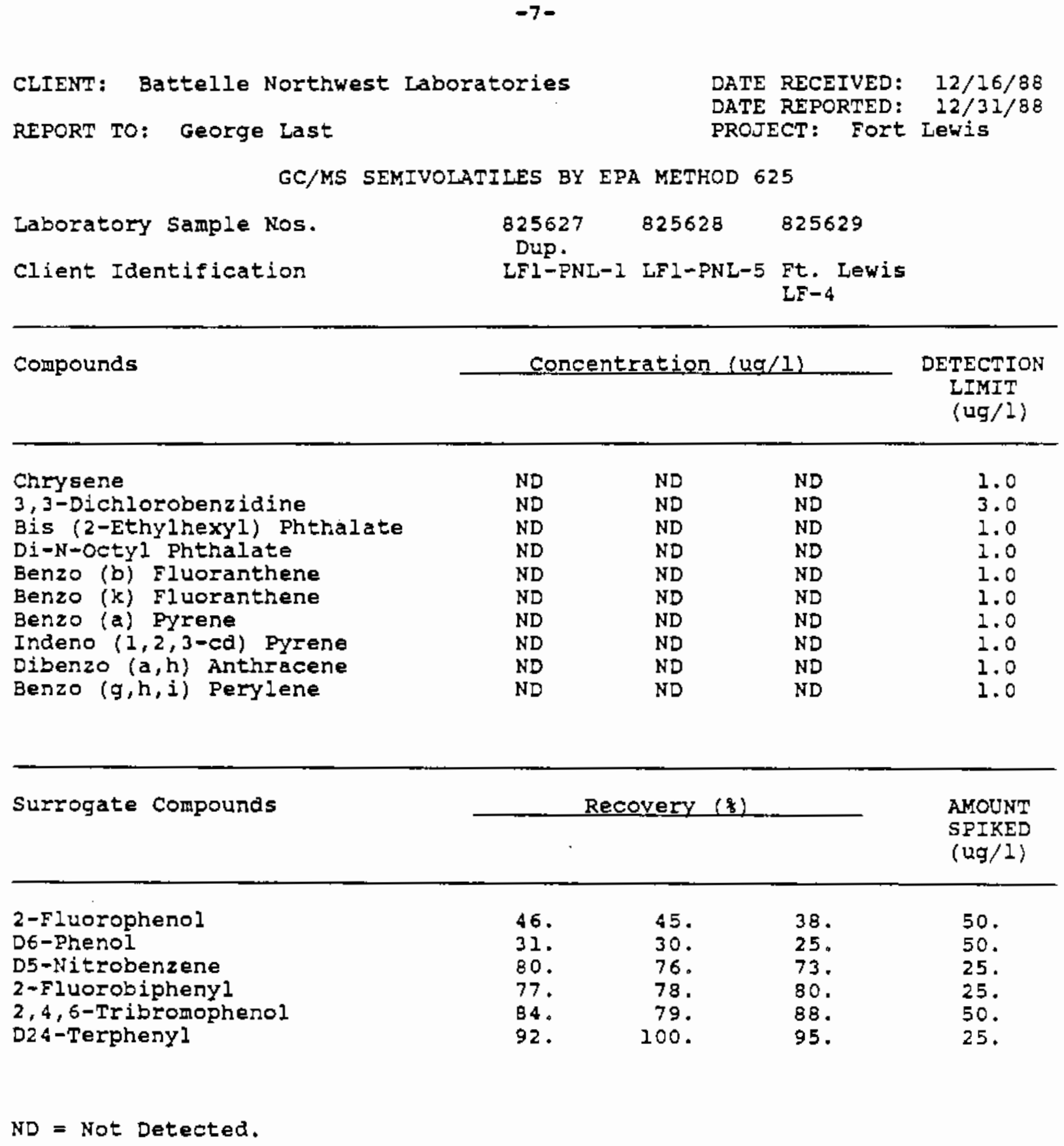


$-8-$

CLIENT: Battelle Northwest Laboratories

DATE RECEIVED: $12 / 16 / 88$

REPORT TO: George Last

DATE REPORTED: $12 / 31 / 88$

PROJECT: Fort Lewis

GC/MS SEMIVOLATILES BY EPA METHOD 625

$\begin{array}{lllll}\text { Laboratory Sample Nos. } & 825630 & 825631 & 825632 & \text { DETECTION } \\ \text { Client Identification } & \text { LF1-PNL-6 Ft. Lewis Ft. Lewis } & \text { LIMIT } \\ & & \text { LF-2 } & \text { Weil \#2 } & \text { (Ug) }\end{array}$

\begin{tabular}{|c|c|c|c|c|}
\hline Aniline & ND & ND & ND & 1.0 \\
\hline 2-Chlorophenol & ND & ND & ND & 1.0 \\
\hline Bis (2-chloroethyl) Ether & ND & ND & ND & 1.0 \\
\hline Phenol & ND & ND & ND & 1.0 \\
\hline 1,3-Dichlorobenzene & ND & ND & ND & 1.0 \\
\hline 1,4-Dichlorobenzene & ND & ND & ND & 1.0 \\
\hline 1,2-Dichlorobenzene & ND & ND & ND & 1.0 \\
\hline Benzyl Alcohol & ND & ND & ND & 1.0 \\
\hline Bis (2-Chloroisopropyl) Ether & ND & ND & ND & 1.0 \\
\hline 2-Methylphenol & ND & ND & ND & 1.0 \\
\hline Hexachloroethane & ND & ND & ND & 1.0 \\
\hline N-Nitrosodipropylamine & ND & ND & ND & 1.0 \\
\hline Nitrobenzene & ND & ND & ND & 1.0 \\
\hline 4-Methylphenol & ND & ND & ND & 1.0 \\
\hline Isopherone & ND & ND & ND & 1.0 \\
\hline 2-Nitrophenol & ND & ND & ND & 1.0 \\
\hline 2,4-Dimethylphenol & ND & ND & ND & 1.0 \\
\hline Bis (2-chloroethoxy) Methane & ND & ND & ND & 1.0 \\
\hline 2,4-Dichlorophenol & ND & ND & ND & 1.0 \\
\hline $1,2,4-T r$ ichlorobenzene & ND & ND & ND & 1,0 \\
\hline Naphthalene & ND & ND & ND & 1.0 \\
\hline Benzoic Acid & ND & ND & ND & 5.0 \\
\hline 4-Chloroaniline & ND & ND & ND & 1.0 \\
\hline Hexachlorobutadiene & ND & ND & ND & 1.0 \\
\hline 2-Methylnaphthalene & ND & ND & ND & 1.0 \\
\hline 4-Chloro-3-Methylphenol & ND & ND & ND & 1.0 \\
\hline Hexachlorocyclopentadiene & ND & ND & ND & 2.0 \\
\hline $2,4,6$-Trichlorophenol & ND & ND & ND & 2.0 \\
\hline $2,4,5$-Trichlorophenol & ND & ND & ND & 2.0 \\
\hline
\end{tabular}




\section{A}

-9-

CLIENT: Battelle Northwest Laboratories

REPORT TO: George Iast

DATE RECEIVED: $12 / 16 / 88$

DATE REPORTED: $12 / 31 / 88$

PROJECT: FOrt Lewis

GC/MS SEMIVOLATILES BY EPA METHOD 625

Laboratory Sample Nos.

825630

825631

825632

DETECTION

client Identification

LFI-PNL-6 Ft. Lewis

$\mathrm{LF}-2$

Ft. Lew

(ug/l)

WeIl 2

\begin{tabular}{|c|c|c|c|c|}
\hline 2-Chloronaphthalene & ND & ND & ND & 1.0 \\
\hline 2-Nitroaniline & ND & ND & ND & 1.0 \\
\hline Acenaphthylene & ND & ND & ND & 1.0 \\
\hline Dimethyl Phthalate & ND & ND & ND & 1.0 \\
\hline 2,6-Dinitrotoluene & ND & ND & ND & 2.0 \\
\hline Acenaphthene & ND & ND & ND & 1.0 \\
\hline 3-Nitroaniline & ND & ND & ND & 2.0 \\
\hline 2,4-Dinitrophenol & ND & ND & ND & 5.0 \\
\hline Dibenzoturan & ND & ND & ND & 1.0 \\
\hline 2, 4-Dinitrotoluene & ND & ND & ND & 2.0 \\
\hline 4-Nitrophenol & ND & ND & ND & 4.0 \\
\hline Fluorene & ND & ND & ND & 1.0 \\
\hline 4-Chlorophenyl Phenyl Ether & ND & ND & ND & 2.0 \\
\hline Diethyl Phthalate & ND & ND & ND & 1.0 \\
\hline 4-Nitroaniline & ND & ND & ND & 3.0 \\
\hline 2-Methyl-4,6-Dinitrophenol & ND & ND & ND & 5.0 \\
\hline N-Nitrosodiphenylamine & ND & ND & ND & 1.0 \\
\hline Azobenzene & ND & ND & ND & 1.0 \\
\hline 4-Bromophenyl Phenyl Ether & ND & ND & ND & 2.0 \\
\hline Hexachlorobenzene & ND & ND & ND & 2.0 \\
\hline Pentachlorophenol & ND & ND & ND & 5.0 \\
\hline Phenanthrene & ND & ND & ND & 1.0 \\
\hline Anthracene & ND & ND & ND & 1.0 \\
\hline Di-N-Butyl Phthalate & ND & ND & ND & 1.0 \\
\hline Fluoranthene & ND & $\mathrm{ND}$ & ND & 1.0 \\
\hline Pyrene & ND & ND & $\mathrm{ND}$ & 1.0 \\
\hline Benzidine & ND & ND & ND & 3.0 \\
\hline Benzy Butyl Phthalate & ND & ND & ND & 1.0 \\
\hline Benzo (a) Anthracene & ND & ND & ND & 1.0 \\
\hline
\end{tabular}

ND = Not Detected.

All values are reported in ug/l. 


\section{AI}

$-10-$

CLIENT: Battelle Northwest Laboratories

DATE RECEIVED:

$12 / 16 / 88$

DATE REPORTED: $12 / 31 / 88$

REPORT TO: George Last

PROJECT: Fort Lewis

GC/MS SEMIVOLATILES BY EPA METHOD 625

Laboratory Sample Nos.

825630

825631

825632

client Identification

LF1-PNL-6 Ft. Lewis Ft. Lewis

$L F-2$

Well \#2

\begin{tabular}{|c|c|c|}
\hline compounds & concentration $(u q / 2)$ & ETECTION \\
\hline
\end{tabular}

Chrysene

3,3-Dichlorobenzidine

Bis (2-Ethylhexyl) Phthalate

Di-N-OCtyl Phthalate

Benzo (b) Fluoranthene

Benzo (k) Fluoranthene

Benzo (a) Pyrene

Indeno $(1,2,3-c d)$ Pyrene

Dibenzo $(a, h)$ Anthracene

Benzo $(g, h, i)$ Perylene

$\begin{array}{llll}\text { ND } & \text { ND } & \text { ND } & 1.0 \\ \text { ND } & \text { ND } & \text { ND } & 3.0 \\ \text { ND } & \text { ND } & \text { ND } & 1.0 \\ \text { ND } & \text { ND } & \text { ND } & 1.0 \\ \text { ND } & \text { ND } & \text { ND } & 1.0 \\ \text { ND } & \text { ND } & \text { ND } & 1.0 \\ \text { ND } & \text { ND } & \text { ND } & 1.0 \\ \text { ND } & \text { ND } & \text { ND } & 1.0 \\ \text { ND } & \text { ND } & \text { ND } & 1.0 \\ \text { ND } & \text { ND } & \text { ND } & 1.0\end{array}$

Surrogate Compounds

Recovery (8)

AMOUNT

SPIKED

(ug/1)

2-Fluorophenol

D6-Phenol

D5-Nitrobenzene

2-Fluorobiphenyl

2, 4, 6-Tribromophenol

D24-Terphenyl

ND $=$ Not Detected.
45.

33.

80.

82.

70 .

96.

5.

29.

81.

83.

84.

100 .
50.

50.

25.

25.

50 .

25. 


\section{AT}

$-11-$

CIIENT: Battelle Northwest Laboratories

DATE RECEIVED: $\quad 12 / 16 / 88$

DATE REPORTED: $12 / 31 / 88$

REPORT TO: George Last

PROJECT: Fort Lewis

GC/MS SEMIVOLATILES BY EPA METHOD 625

$\begin{array}{lllll}\text { Laboratory Sample Nos. } & 825633 & 825634 & 825635 & \begin{array}{c}\text { DETECTION } \\ \text { LIMIT }\end{array} \\ \text { Client Identification } & \text { LF4-FNL-3 Seq. } & \text { Ft. Lewis } & \text { (ug/1) } \\ & & \text { LF-3 } & \text { LF-3 }\end{array}$

Aniline

2-Chlorophenol

Bis (2-Chloroethyl) Ether

Phenol

1,3-Dichlorobenzene

r.m.

1,4-Dichlorobenzene

1,2-Dichlorobenzene

Benzyl Alcohol

Bis (2-Chloroisopropyl) Ether

2-Methylpherol

Hexachloroethane

$\mathrm{N}$-Nitrosodipropylamine

ND

ND

ND

ND

ND

ND

ND

ND

ND

ND

ND

Nitrobenzene

ND

4-Methyl phenol

Isopherone

2-Nitrophenol

2,4-Dimethylphenol

Bis (2-chloroethoxy) Methane

ND

ND

ND

ND

ND

2,4-Dichlorophenol

2,2,4-Trichlorobenzene

ND

ND

Naphthalene

Benzoic Acid

4-Chloroaniline

Hexachlorobutadiene

2-Methylnaphthalene

4-Chloro-3-Methylphenol

Hexachlorocyclopentadiene

2, 4, 6-Trichlorophenol

2,4,5-Trichlorophenol

ND

ND

ND

ND

ND

ND

ND

ND

ND

ND

$\begin{array}{lll}\text { ND } & \text { ND } & 1.0 \\ \text { ND } & \text { ND } & 1.0 \\ \text { ND } & \text { ND } & 1.0 \\ \text { ND } & \text { ND } & 1.0 \\ \text { ND } & \text { ND } & 1.0 \\ \text { ND } & \text { ND } & 1.0 \\ \text { ND } & \text { ND } & 1.0 \\ \text { ND } & \text { ND } & 1.0 \\ \text { ND } & \text { ND } & 1.0 \\ \text { ND } & \text { ND } & 1.0 \\ \text { ND } & \text { ND } & 1.0 \\ \text { ND } & \text { ND } & 1.0 \\ \text { ND } & \text { ND } & 1.0 \\ \text { ND } & \text { ND } & 1.0 \\ \text { ND } & \text { ND } & 1.0 \\ \text { ND } & \text { ND } & 1.0 \\ \text { ND } & \text { ND } & 1.0 \\ \text { ND } & \text { ND } & 1.0 \\ \text { ND } & \text { ND } & 1.0 \\ \text { ND } & \text { ND } & 1.0 \\ \text { ND } & \text { ND } & 1.0 \\ \text { ND } & \text { ND } & 5.0 \\ \text { ND } & \text { ND } & 1.0 \\ \text { ND } & \text { ND } & 1.0 \\ \text { ND } & \text { ND } & 1.0 \\ \text { ND } & \text { ND } & 1.0 \\ \text { ND } & \text { ND } & 2.0 \\ \text { ND } & \text { ND } & 2.0 \\ \text { ND } & \text { ND } & 2.0\end{array}$

ND = Not Detected.

All values are reported in $u g / 1$.

*seq. = Sequalitchew 


\section{A}

CLIENT: Battelle Northwest Laboratories

DATE RECEIVED: $12 / 16 / 88$ DATE REPORTED: $12 / 31 / 88$ REPORT TO: George Last

PROJECT: Fort Lewis GC/MS SEMIVOLATILES BY EPA METHOD 625

Laboratory Sample Nos.

$\begin{array}{llll}825633 & 825634 & 825635 & \begin{array}{c}\text { DETECTION } \\ \text { LIMIT }\end{array} \\ \text { LF4-PNL-3 } & \begin{array}{l}\text { Seq. * } \\ \text { Lake }\end{array} & \text { Ft. Lewis } & \text { (ug/1) } \\ & \text { LF-3 } & \end{array}$

2-Chloronaphthalene

2-Nitroaniline

Acenaphthylene

Dimethyl Phthalate

2,6-Dinitrotoluene

Acenaphthene

3-Nitroaniline

2,4-Dinitrophenol

Dibenzofuran

2,4-Dinitrotoluene

4-Nitrophenol

Fluorene

4-Chlorophenyl Phenyl Ether

LF -3

Diethyl Phthalate

4-Nitroaniline

2-Methy1-4,6-Dinitrophenol

$N-N i t r o s o d$ iphenylamine

Azobenzene

4-Bromophenyl Phenyl Ether

Hexachlorobenzene

Pentachlorophenol

Phenanthrene

Anthracene

D1-N-Butyl Phthalate

Fluoranthene

Pyrene

Benzidine

Benzy Butyl Phthalate

Benzo (a) Arthracene

ND

ND

ND

ND

ND

ND

ND

ND

ND

ND

ND

ND

ND

ND

ND

ND

ND

ND

ND

ND

ND

ND

ND

ND

ND

ND

ND

ND

ND

$\begin{array}{lll}\text { ND } & \text { ND } & 1.0 \\ \text { ND } & \text { ND } & 1.0 \\ \text { ND } & \text { ND } & 1.0 \\ \text { ND } & \text { ND } & 1.0 \\ \text { ND } & \text { ND } & 2.0 \\ \text { ND } & \text { ND } & 1.0 \\ \text { ND } & \text { ND } & 2.0 \\ \text { ND } & \text { ND } & 5.0 \\ \text { ND } & \text { ND } & 1.0 \\ \text { ND } & \text { ND } & 2.0 \\ \text { ND } & \text { ND } & 4.0 \\ \text { ND } & \text { ND } & 1.0 \\ \text { ND } & \text { ND } & 1.0 \\ \text { ND } & \text { ND } & 1.0 \\ \text { ND } & \text { ND } & 3.0 \\ \text { ND } & \text { ND } & 5.0 \\ \text { ND } & \text { ND } & 1.0 \\ \text { ND } & \text { ND } & 1.0 \\ \text { ND } & \text { ND } & 2.0 \\ \text { ND } & \text { ND } & 2.0 \\ \text { ND } & \text { ND } & 5.0 \\ \text { ND } & \text { ND } & 1.0 \\ \text { ND } & \text { ND } & 1.0 \\ \text { ND } & \text { ND } & 1.0 \\ \text { ND } & \text { ND } & 1.0 \\ \text { ND } & \text { ND } & 1.0 \\ \text { ND } & \text { ND } & 3.0 \\ \text { ND } & \text { ND } & 1.0 \\ \text { ND } & \text { ND } & 1.0 \\ & & \end{array}$

ND $=$ Not Detected.

All values are reported in $u g / l$.

*Seq. I Sequalitchew 
CLIENT: Battelle Northwest Laboratories

REPORT TO: George Last
DATE RECEIVED: $12 / 16 / 88$

DATE REPORTED: $12 / 31 / 88$

PROJECT: Fort Lewis

GC/MS SEMIVOLATILES BY EPA METHOD 625

Laboratory Sample Nos.

client Identification
$825633 \quad 825634$

LF4-PNL-3 Seq. * Lake
825635

Ft. Lewis

LF -3

\section{Compounds}

Concentration $(u g / 1)$

ND

Chrysene

3,3-Dichlorobenzidine

Bis (2-Ethylhexyl) phthalate

Di-N-octyl Phthalate

Benzo (b) Fluoranthene

Benzo (k) Fluoranthene

Benzo (a) Pyrene

Indeno $(1,2,3-c d)$ Pyrene

Dibenzo $(a, h)$ Anthracene

Benzo $(g, h, i)$ Perylene
ND

ND

ND

ND

ND

ND

ND

ND

ND
DETECTION

LIMIT

(ug/1)
Surrogate compounds

\begin{tabular}{r} 
\\
\hline \\
51. \\
34 \\
78. \\
79. \\
90 \\
115.
\end{tabular}

Recovery ( 8$)$

ND

ND

ND

ND

ND

ND

ND

ND

ND

ND
1.0

3.0

2.0

1.0

1.0

1.0

1.0

1.0

1.0

1.0
2-Fluorophenol

D6-Phenol

D5-Nitrobenzene

2-Fluorobiphenyl

$2,4,6$-Tribromophenol

D2 4-Terpheny 1

*Seq. = Sequalitchew

ND $=$ Not Detected.
42.

30 .

79.

80.

90 .

98 .

15.
29.

19.

54.

59.

60 .

83.
50.

50 .

25.

25.

50 . 
CLIENT: Battelle Northwest Laboratories

DATE RECEIVED: $12 / 16 / 88$

DATE REPORTED: $12 / 31 / 88$

REPORT TO: George Last

PROJECT: FOrt Lewis

GC/MS SEMIVOLATILES BY EPA METHOD 625

$\begin{array}{llclc}\text { Laboratory Sample Nos. } & 825635 & 825636 & 825637 & \text { DETECTION } \\ \text { Client Identification } & \text { DFl-PIL-2 } & \text { LFl-PNL-2 } & \text { Ft. Lewis } & \text { LIMIT } \\ \text { (ug/1) }\end{array}$

\begin{tabular}{|c|c|c|c|c|}
\hline Aniline & ND & ND & ND & 1.0 \\
\hline 2-Chlorophenol & ND & ND & ND & 1.0 \\
\hline Bis (2-Chloroethyl) Ether & ND & ND & ND & 1.0 \\
\hline Phenol & ND & ND & ND & 1.0 \\
\hline 1,3-Dichlorobenzere & ND & ND & ND & 1.0 \\
\hline 1,4-Dichlorobenzene & ND & ND & ND & 1.0 \\
\hline 1,2-Dichlorobenzene & ND & ND & ND & 1.0 \\
\hline Benzyl Alcohol & ND & ND & ND & 1.0 \\
\hline Bis (2-Chloroisopropyl) Ether & ND & ND & ND & 1.0 \\
\hline 2-Methylpherol & ND & ND & ND & 1.0 \\
\hline Hexachloroethane & ND & ND & ND & 1.0 \\
\hline N-Nitrosodipropylamine & ND & ND & ND & 1.0 \\
\hline Nitrobenzene & ND & ND & ND & 1.0 \\
\hline 4-Methylphenol & ND & ND & ND & 1.0 \\
\hline Isopherone & ND & ND & ND & 1.0 \\
\hline 2-Nitrophenol & ND & ND & ND & 2.0 \\
\hline 2,4-Dimethylphenol & ND & ND & ND & 1.0 \\
\hline Bis (2-chloroethoxy) Methane & ND & ND & ND & 2.0 \\
\hline 2,4-Dichlorophenol & ND & ND & ND & 1.0 \\
\hline $1,2,4$-Trichlorobenzene & ND & ND & ND & 2.0 \\
\hline Naphthalene & ND & ND & ND & 1.0 \\
\hline Benzoic Acid & ND & ND & ND & 5.0 \\
\hline 4-Chloroaniline & ND & ND & ND & 1.0 \\
\hline Hexachlorobutadiene & ND & ND & ND & 1.0 \\
\hline 2-Methylnaphthalene & ND & ND & ND & 1.0 \\
\hline 4-Chloro-3-Methylphenol & ND & ND & ND & 1.0 \\
\hline Hexachlorocyclopertadiene & ND & ND & ND & 2.0 \\
\hline $2,4,6-\operatorname{Tr}$ ichlorophenol & ND & ND & ND & 2.0 \\
\hline 2,4,5-Trichlorophenol & ND & ND & ND & 2.0 \\
\hline
\end{tabular}

ND $=$ Not Detected.

All values are reported in $\mathrm{ug} / \mathrm{l}$. 


\section{AT}

$-15-$

CLIENT: Battelle Northwest Laboratories

DATE RECEIVED: $12 / 16 / 88$

REPORT TO: George Last

DATE REPORTED: $12 / 31 / 88$

FROJECT: Fort Lewis

GC/MS SEMIVOTATILES BY ERA METHOD 625

Laboratory Sample Nos.

Client Identification
$825636 \quad 825636$
Dup.
$(u g / 1)$
LF-I

$\begin{array}{lllll}\text { 2-Chioronaphthalene } & \text { ND } & \text { ND } & \text { ND } & 1.0 \\ \text { 2-Nitroaniline } & \text { ND } & \text { ND } & \text { ND } & 1.0 \\ \text { Acenaphthylene } & \text { ND } & \text { ND } & \text { ND } & 1.0 \\ \text { Dimethyl Phthalate } & \text { ND } & \text { ND } & \text { ND } & 1.0 \\ \text { 2,6-Dinitrotoluene } & \text { ND } & \text { ND } & \text { ND } & 2.0 \\ \text { Acenaphthene } & \text { ND } & \text { ND } & \text { ND } & 1.0 \\ \text { 3-Nitroaniline } & \text { ND } & \text { ND } & \text { ND } & 2.0 \\ \text { 2,4-Dinitrophenol } & \text { ND } & \text { ND } & \text { ND } & 5.0 \\ \text { Dibenzofuran } & \text { ND } & \text { ND } & \text { ND } & 1.0 \\ \text { 2,4-Dinitrotoluene } & \text { ND } & \text { ND } & \text { ND } & 2.0 \\ \text { 4-Nitrophenol } & \text { ND } & \text { ND } & \text { ND } & 4.0 \\ \text { Fluorene } & \text { ND } & \text { ND } & \text { ND } & 1.0 \\ \text { 4-Chlorophenyl Phenyl Ether } & \text { ND } & \text { ND } & \text { ND } & 1.0 \\ \text { Diethyl Fhthalate } & \text { ND } & \text { ND } & \text { ND } & 1.0 \\ \text { 4-Nitroaniline } & \text { ND } & \text { ND } & \text { ND } & 3.0 \\ \text { 2-Methyl-4,6-Dinitrophenol } & \text { ND } & \text { ND } & \text { ND } & 5.0 \\ \text { N-Nitrosodiphenylamine } & \text { ND } & \text { ND } & \text { ND } & 1.0 \\ \text { Azobenzene } & \text { ND } & \text { ND } & \text { ND } & 1.0 \\ \text { 4-Brorophenyl Fhenyl Ether } & \text { ND } & \text { ND } & \text { ND } & 2.0 \\ \text { Hexachlorobenzene } & \text { ND } & \text { ND } & \text { ND } & 2.0 \\ \text { Pentachlorophenol } & \text { ND } & \text { ND } & \text { ND } & 5.0 \\ \text { Phenanthrene } & \text { ND } & \text { ND } & \text { ND } & 1.0 \\ \text { Anthracene } & \text { ND } & \text { ND } & \text { ND } & 1.0 \\ \text { Di-N-Butyl Phthalate } & \text { ND } & \text { ND } & \text { ND } & 1.0 \\ \text { Fluoranthene } & \text { ND } & \text { ND } & \text { ND } & 1.0 \\ \text { Pyrene } & \text { ND } & \text { ND } & \text { ND } & 1.0 \\ \text { Benzidine } & \text { ND } & \text { ND } & \text { ND } & 3.0 \\ \text { Benzy Butyl Phthalate } & \text { ND } & \text { ND } & \text { ND } & 1.0 \\ \text { Benzo (a) Anthracene } & \text { ND } & \text { ND } & \text { ND } & 1.0 \\ & & & & \end{array}$

ND $=$ Not Detected.

All values are reported in ug/l. 
CLIENT: Battelle Northwest Laboratories

DATE RECEIVED:

DATE REPORTED:

PROJECT :

Fort Lewis

$16 / 88$

REPORT TO: George Last

$12 / 31 / 88$

GC/MS SEMIVOLATILES BY EPA METHOD 625

Laboratory Sample Nos.

825636

825636

825637

Client Identification

LFI-PNL-2 LFI-PNL-2 Ft. Lew is

LF -1

Compounds

Concentration (ug/1)

DETECTION

LIMIT

(ug/1)

Chrysene

3,3-Dichlorobenzidine

Bis (2-Ethylhexyl) Phthalate

Di-N-octyl Phthalate

Benzo (b) Fluoranthene

Benzo (k) Fluoranthene

Benzo (a) Pyrene

Indeno ( $1,2,3$-cd) Pyrene

Dibenzo (a,h) Anthracene

Berzo $(g, n, i)$ Perylene
ND

ND

ND

ND

ND

ND

ND

ND

ND

ND
ND

ND

ND

ND

ND

ND

ND

ND

ND

ND
1.0

3.0

1.0

1.0

1.0

1.0

1.0

1.0

1.0

1.0

Surrogate compounds

Recovery (8)

AMOUNT

SPIKED

(ug/l)

2-Fluorophenol

D6-Phenol

D5-Nitrobenzene

2-Fluorobiphenyl

2, 4, 6-Tribromophenol

D24-Terphenyl

ND $=$ Not Detected .
51.

35.

77 .

76.

95.

109.
47.

30.

76.

78 .

89.

102.
40.

27.

66.

68.

87.

99.
50.

50.

25.

25.

50.

25. 
CIIENT: Battelle Northwest Laboratories

DATE RECEIVED: $\quad 22 / 16 / 88$

DATE REPORTED: $12 / 31 / 88$

REPORT TO: George Last

PROJECT: FOrt Lewis

GC/MS SEMIVOLATILES BY EPA METHOD 625

$\begin{array}{lll}\text { Laboratory Sample Nos. } & 825638 & 825639 \\ \text { Client Identification } & \text { LFI-PNL-3 } & \text { LFi-PNL-4 }\end{array}$

DETECTION

LIMIT

$(u g / l)$

Aniline

2-Chlorophenol

Bis (2-chloroethyl) Ether

Phenol

1,3-Dichlorobenzene

1,4-Dichlorobenzene

1.0

1.0

1.0

1.0

1.0

1.0

1.0

Benzyl Alcohol

Bis (2-chloroisopropyl) Ether

2-Methylphenol

Hexachloroethane

N-Nitrosodipropy lamine

ND

ND

ND

ND

ND

ND

ND

ND ND

1.0

ND

ND

ND

ND

ND

ND

1.0

1.0

1.0

ND

ND

4-Methylphenol

Isopherone

2-Nitrophenol

2,4-Dimethylphenol

Bis (2-chloroethoxy) Methane

ND

ND

ND

ND

ND

1.0

1.0

ND

1.0

1.0

ND

1.0

ND

ND

1.0

ND

ND ND

1.0

2,4-Dichlorophenol

1,2,4-Trich lorobenzene

ND

ND

1.0

Naphthalene

Benzoic Acid

4-Chloroaniline

Hexachlorobutadiene

2-Methylnaphthalene

4-Chloro-3-Methylphenol

ND

ND

ND

ND

1.0

1.0

5.0

1.0

ND

1.0

ND

ND

1.0

ND

Hexachlorocyclopentadiene

ND

ND

ND

ND

1.0

$2,4,6-\mathrm{Tr}$ ich lorophenol

2,4,5-Trichlorophenol

ND

ND

2.0

2.0

2.0

ND = Not Detected.

All values are reported in ug/l. 


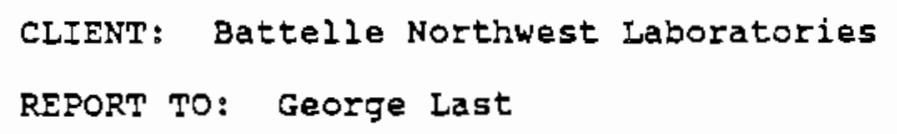

REPORT TO: George Last

$12 / 16 / 8$ 日

$12 / 31 / 88$

Lewis

GC/MS SEMIVOLATILES BY EPA METHOD 625

$\begin{array}{llc}\text { Laboratory Sample Nos. } & 825638 & 825639 \\ \text { Client Identification } & \text { LFI-PNL-3 } & \text { LFI-PNL-4 }\end{array}$

DETECTION

LIMIT

(ug/1)

2-Chloronaphthalene

2-Nitroaniline

Acenaphthylene

Dimethyl Phthalate

2,6-Dinitrotoluene

Acenaphthene

3-Nitroaniline

Lח

2,4-Dinitrophenol

Dibenzofuran

2,4-Dinitrotoluene

4-Nitrophenol

Fluorene

4-Chlorophenyl Phenyl Ether

Diethyl Phthalate

4-Nitroaniline

2-Methy1-4,6-Dinitrophenol

$\mathrm{N}-\mathrm{Nitrosodiphenylamine}$

Azobenzene

4-Bromophenyl Phenyl Ether

Hexachlorobenzene

Pentachlorophenol

Phenanthrene

Anthracene

Di-N-Butyl Phthalate

Fluoranthene

Pyrene

Benzidine

Benzy Butyl Phthalate

Benzo (a) Anthracene

$\begin{array}{lll}\text { ND } & \text { ND } & 1.0 \\ \text { ND } & \text { ND } & 1.0 \\ \text { ND } & \text { ND } & 1.0 \\ \text { ND } & \text { ND } & 1.0 \\ \text { ND } & \text { ND } & 2.0 \\ \text { ND } & \text { ND } & 1.0 \\ \text { ND } & \text { ND } & 2.0 \\ \text { ND } & \text { ND } & 5.0 \\ \text { ND } & \text { ND } & 1.0 \\ \text { ND } & \text { ND } & 2.0 \\ \text { ND } & \text { ND } & 4.0 \\ \text { ND } & \text { ND } & 1.0 \\ \text { ND } & \text { ND } & 1.0 \\ \text { ND } & \text { ND } & 1.0 \\ \text { ND } & \text { ND } & 3.0 \\ \text { ND } & \text { ND } & 5.0 \\ \text { ND } & \text { ND } & 1.0 \\ \text { ND } & \text { ND } & 1.0 \\ \text { ND } & \text { ND } & 2.0 \\ \text { ND } & \text { ND } & 2.0 \\ \text { ND } & \text { ND } & 5.0 \\ \text { ND } & \text { ND } & 1.0 \\ \text { ND } & \text { ND } & 1.0 \\ \text { ND } & \text { ND } & 1.0 \\ \text { ND } & \text { ND } & 1.0 \\ \text { ND } & \text { ND } & 1.0 \\ \text { ND } & \text { ND } & 3.0 \\ \text { ND } & \text { ND } & 1.0 \\ \text { ND } & \text { ND } & 1.0 \\ & & \end{array}$

ND $=$ Not Detected.

All values are reported in ug/l. 


\section{A}

$-19-$

CLIENT: Battelle Northwest Laboratories

DATE RECEIVED: $12 / 16 / 88$

DATE REPORTED: $12 / 31 / 88$

REPORT TO: George Last

PROJECT: FOIt Lewis

GC/MS SEMIVOLATILES BY EPA METHOD 625

Laboratory Sample Nos. $\quad 825638 \quad 825639$

Client Identification IFI-PNL-3 LFL-PNL-4

\begin{tabular}{|c|c|c|}
\hline compounds & Concentration (ug/l) & DETECTION \\
\hline
\end{tabular}

Chrysene

3,3-Dichlorobenzidine

Bis (2-Ethylhexyl) Phthalate

Di-N-octy I Phthalate

Benzo (b) Fluoranthene

Benzo (k) Fluoranthene

Benzo (a) Pyrene

Indeno $(1,2,3-c d)$ Pyrene

Dibenzo (a,h) Anthracene

Benzo $(g, h, i)$ Perylene

\section{ND}

ND

2.3

ND

ND

ND

ND

ND

ND

ND
ND

ND

ND

ND

ND

ND

ND

ND

ND

ND
1.0

3.0

1.0

1.0

1.0

1.0

1.0

1.0

1.0

1.0

\section{Surrogate compounds}

Recovery (8)

AMOUNT

SPIKED

(ug/1)

2-Eluorophenol

D6-Phenol

D5-Nitrobenzene

2-Fluorobiphenyl

2, 4,6-Tribromophenol

D24-Terpheny1

47.

33 .

70 .

73.

85.

96.
37 .

25.

60.

61.

75.

87.
50.

50 .

25.

25 .

50 .

25.

ND $=$ Not Detected. 
CLIENT: Battelle Northwest Laboratories REPORT TO: George Last

DATE RECEIVED: $12 / 16 / 88$ DATE REPORTED: $12 / 31 / 8 \mathrm{~B}$ SPIKE DATA

Laboratory Sample No.

825635 Matrix spike

Client Identification

Ft. Lewis LF-3

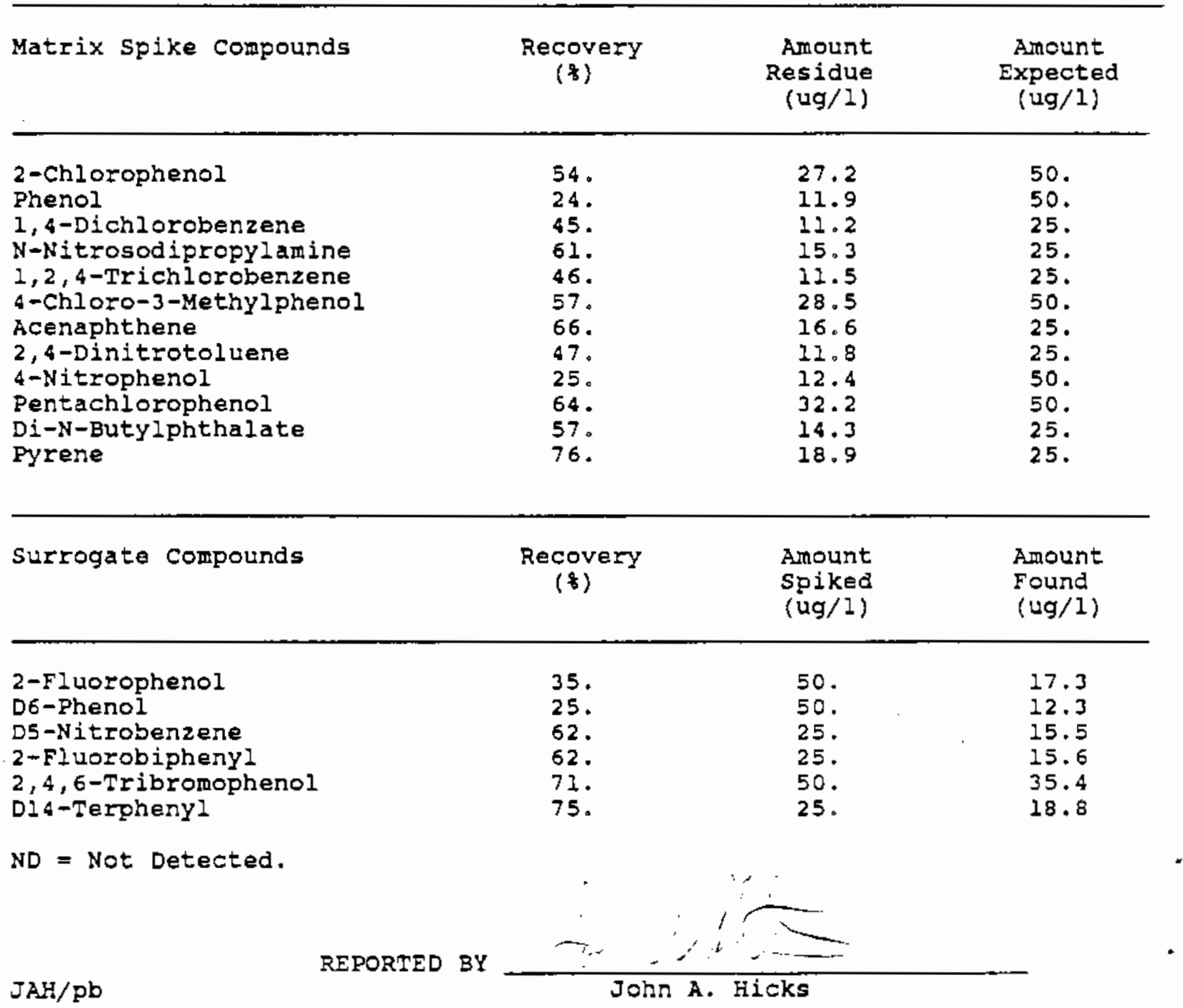


PNL -7613

UC -903

\section{DISTRIBUTIDN}

No. of

Copies

OFFSITE

12 DOE/Office of Scientific and Technical Information

13 K. Durbin $\mathrm{HQ}$, I Corps and Ft. Lewis

Attn: AFZH-DEQ

Fort Lewis, WA 98433-5000

ONSITE

DOE Richland Operations office

J. J. Sutey
No. of

Copies

18 Pacific Northwest Laboratory

S. P. Airhart

P. G. Doctor

J. T. Falco

J. M. Hales

P. C. Hays

G. V. Last (2)

K. B. Olsen

W. T. Penne\}1

J. R. Raymond

L. E. Rogers

R. L. Skaggs

Publishing Coordination

Technical Report Files (5) 
